Supporting Information

\title{
Enantioselective Dehydrative $\gamma$-Arylation of $\alpha$-Indolyl Propargylic Alcohols with Phenols: Access to Chiral Tetrasubstituted Allenes and Naphthopyrans
}

Wen-Run Zhu, Qiong Su, Hong-Juan Diao, Er-Xuan Wang, Feng Wu, Yun-Long Zhao, Jiang Weng, ${ }^{*}$ and Gui Lu*

Guangdong Provincial Key Laboratory of Chiral Molecule and Drug Discovery, School of Pharmaceutical Sciences, Sun Yat-sen University, Guangzhou 510006, P. R. China.

E-mail for J.W.: wengi2@mail.sysu.edu.cn. E-mail for G.L.: lugui@mail.sysu.edu.cn 


\section{Table of Contents}

1. General Information S3

2. Screening of Catalysts and Condition Optimization S3

3. Experimental Procedure and Characterization of substrates S5

a. General Procedure to Prepare Substrates 1a-1r, $8 \quad$ S5

b. Analytical Data for Substrates 1a-1r, $8 \quad$ S5

4. Experimental Procedure and Characterization of Products S12

a. General Procedure to Prepare Racemic Products 3a-3aa S12

b. General Procedure to Prepare Chiral Products 3a-3aa S12

c. Analytical Data for Products 3a-3aa $\quad \mathbf{S 1 2}$

d. General Procedure to Prepare Racemic Products 5a-5I S26

e. General Procedure to Prepare Chiral Products 5a-5I S26

f. Analytical Data for Products 5a-5I S26

5. The scale-up experiment $\quad$ S32

6. Experimental Procedure and Characterization Data of Compounds 11-17.

7. Proposed reaction pathways $\$$

8. Determination of the absolute configuration of 5d S37

$\begin{array}{lll}9 & \text { References } & \$ 37\end{array}$

10. Data for X-ray Crystal Structures of $3 z$ and $5 d \quad S 39$

11. Copies of NMR Spectra S43

$\begin{array}{lr}\text { 12. Copies of HPLC Spectra } & \mathbf{S 1 4 0}\end{array}$ 


\section{General Information}

All reactions were carried out in oven-dried reaction vessel unless otherwise noted and solvents were dried according to established procedures. Reactions were monitored by thin layer chromatography (TLC). Purification of reaction product was carried out by flash chromatography using Qing Dao Sea Chemical Reagent silica gel (200-300 mesh). ${ }^{1} \mathrm{H},{ }^{13} \mathrm{C}$ and ${ }^{19} \mathrm{~F}$ NMR spectra were recorded on Bruker $400 \mathrm{MHz}$ or 500 $\mathrm{MHz}$ spectrometer in $\mathrm{CDCl}_{3}$ unless otherwise noted. Chemical shifts in ${ }^{1} \mathrm{H}$ NMR spectra are reported in parts per million (ppm, $\delta$ ) downfield from the internal standard $\mathrm{Me}_{4} \mathrm{Si}(\mathrm{TMS}, \delta=0 \mathrm{ppm})$. Chemical shifts in ${ }^{13} \mathrm{C}$ NMR spectra are reported relative to the central line of the chloroform signal $(\delta=77.0 \mathrm{ppm})$. Data are presented as follows: chemical shift, integration, multiplicity ( $\mathrm{s}=$ singlet, $\mathrm{d}=$ doublet, $\mathrm{t}=$ triplet, $\mathrm{q}=$ quartet, $\mathrm{m}=$ multiplet) and coupling constant in Hertz $(\mathrm{Hz})$. HPLC analyses were conducted on an Agilent instrument using Daicel Chiralpak IA, IB, AD-H or Chiralcel OD-H columns. High resolution mass spectra were obtained with a Shimadzu LCMS-IT-TOF mass spectrometer. The single crystal X-ray diffraction studies were carried out on a Xcalibur Onyx Nova diffractometer equipped with CuK/a radiation.

Substrates $10^{1 \mathrm{a}}$ and $1 \mathrm{aa}^{1 \mathrm{~b}}$ were synthesized according to the literature method.

\section{Screening of Catalysts and Condition Optimization}

Table S1. Screening of catalysts and optimization of reaction conditions ${ }^{[a]}$<smiles>Oc1ccc2ccccc2c1</smiles>

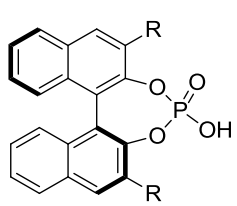

(S)-A1: $\mathrm{R}=2,4,6-{ }^{-} \mathrm{Pr}_{3} \mathrm{C}_{6} \mathrm{H}_{2}$ (S)-A2: $\mathrm{R}=3,5-\left(\mathrm{CF}_{3}\right)_{2} \mathrm{C}_{6} \mathrm{H}_{3}$

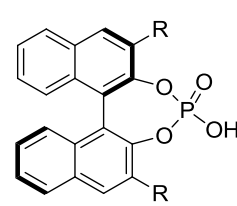

$(R)-\mathbf{A}$
(R)-A3: $\mathrm{R}=3,5-\mathrm{Ph}_{2} \mathrm{C}_{6} \mathrm{H}_{3}$ $(R)-\mathrm{A} 4: \mathrm{R}=9$-phenanthryl $(R)-\mathrm{A5}$ : $\mathrm{R}=9$-anthryl (R)-A6: $\mathrm{R}=1$-pyrenyl (R)-A7: $\mathrm{R}=1$-naphthyl (R)-A8: $\mathrm{R}=2$-naphthyl (R)-A9: $\mathrm{R}=4-\mathrm{NO}_{2} \mathrm{C}_{6} \mathrm{H}_{4}$ (R)-A10: $\mathrm{R}=4-{ }^{i} \mathrm{PrC}_{6} \mathrm{H}_{4}$ (R)-A11: $\mathrm{R}=4-\mathrm{ClC}_{6} \mathrm{H}_{4}$ (R)-A12: $\mathrm{R}=\mathrm{SiPh}_{3}$ (R)-A13: $\mathrm{R}=4-\mathrm{CF}_{3} \mathrm{C}_{6} \mathrm{H}_{4}$ (R)-A14: $\mathrm{R}=4-\mathrm{PhC}_{6} \mathrm{H}_{4}$

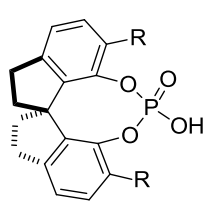

(R)-B1: $\mathrm{R}=3,5-\mathrm{Ph}_{2} \mathrm{C}_{6} \mathrm{H}_{3}$ (R)-B2: $\mathrm{R}=9$-phenanthryl (R)-B3: $\mathrm{R}=9$-anthryl $(R)-\mathrm{B} 3: \mathrm{R}=9$-anthryl
$(R)-\mathrm{B} 4: \mathrm{R}=1$-naphthyl

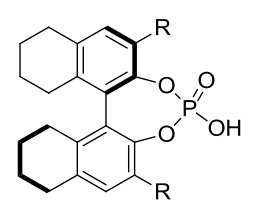

(R)-C1: R= 1-naphthyl

(R)-C2: $\mathrm{R}=2$-naphthyl

(R)-C3: R= 9-phenanthryl (R)-C4: $\mathrm{R}=4-\mathrm{PhC}_{6} \mathrm{H}_{4}$

\begin{tabular}{|c|c|c|c|c|c|c|c|c|}
\hline Entry & $\begin{array}{l}\text { Catalyst } \\
\text { (x mol\%) }\end{array}$ & $\begin{array}{c}\text { Solvent } \\
(\mathrm{x} \mathrm{mL})\end{array}$ & 1a: $4 a$ & $\begin{array}{c}\text { Temperature } \\
{\left[{ }^{\circ} \mathrm{C}\right]}\end{array}$ & $\begin{array}{l}\text { Additive } \\
(25 \mathrm{mg}) \\
\end{array}$ & Time & $\begin{array}{c}\text { Yield }^{[b]} \\
{[\%]}\end{array}$ & $e r^{[c]}$ \\
\hline 1 & $(R)-\mathrm{B} 1(10)$ & DCM (1) & $1: 1$ & RT & -- & $24 \mathrm{~h}$ & 99 & $37: 63$ \\
\hline 2 & $(R)-\mathbf{B} 2(10)$ & DCM (1) & $1: 1$ & RT & -- & $30 \mathrm{~h}$ & 92 & $36: 64$ \\
\hline 3 & $(R)$-B3 (10) & $\mathrm{DCM}(1)$ & $1: 1$ & RT & -- & $32 \mathrm{~h}$ & 86 & $39: 61$ \\
\hline 4 & $(R)-\mathrm{B} 4(10)$ & $\mathrm{DCM}(1)$ & $1: 1$ & RT & -- & $18 \mathrm{~h}$ & 96 & $45: 55$ \\
\hline 5 & (S)-A1 (10) & $\mathrm{DCM}(1)$ & $1: 1$ & RT & -- & $72 \mathrm{~h}$ & 90 & $40: 60$ \\
\hline 6 & $(S)-\mathbf{A} 2(10)$ & $\mathrm{DCM}(1)$ & $1: 1$ & $\mathrm{RT}$ & -- & $8 \mathrm{~h}$ & 98 & $45: 55$ \\
\hline 7 & $(R)-\mathbf{A} 3(10)$ & $\mathrm{DCM}(1)$ & $1: 1$ & RT & -- & $18 \mathrm{~h}$ & 93 & $65: 35$ \\
\hline 8 & $(R)-\mathbf{A 4}(10)$ & $\mathrm{DCM}(1)$ & $1: 1$ & $\mathrm{RT}$ & -- & $108 \mathrm{~h}$ & 92 & $55: 45$ \\
\hline 9 & $(R)-\mathbf{A} 5(10)$ & $\mathrm{DCM}(1)$ & $1: 1$ & RT & -- & $24 \mathrm{~h}$ & 96 & $70: 30$ \\
\hline 10 & $(R)-\mathbf{A 6}(10)$ & DCM (1) & $1: 1$ & $\mathrm{RT}$ & -- & $96 h$ & 93 & $61: 39$ \\
\hline 11 & $(R)-\mathbf{A} 7(10)$ & $\mathrm{DCM}(1)$ & $1: 1$ & RT & -- & $8 \mathrm{~h}$ & 95 & $80: 20$ \\
\hline 12 & $(R)-\mathbf{A 8}(10)$ & DCM (1) & $1: 1$ & RT & -- & $18 \mathrm{~h}$ & 99 & $74: 26$ \\
\hline
\end{tabular}




\begin{tabular}{|c|c|c|c|c|c|c|c|c|}
\hline 13 & $(R)-\mathbf{A 9}(10)$ & DCM (1) & $1: 1$ & RT & -- & $16 \mathrm{~h}$ & 98 & $65: 35$ \\
\hline 14 & $(R)-\mathbf{A} 10(10)$ & $\mathrm{DCM}(1)$ & $1: 1$ & RT & -- & $24 h$ & 95 & $60: 40$ \\
\hline 15 & $(R)-\mathbf{A} 11(10)$ & DCM (1) & $1: 1$ & RT & -- & $12 \mathrm{~h}$ & 98 & $71: 29$ \\
\hline 16 & $(R)-\mathbf{A 1 2}(10)$ & DCM (1) & $1: 1$ & RT & -- & $120 \mathrm{~h}$ & 82 & $51: 49$ \\
\hline 17 & $(R)-\mathbf{A} 13(10)$ & DCM (1) & $1: 1$ & RT & -- & $3 h$ & 95 & $75: 25$ \\
\hline 18 & $(R)-\mathbf{C} 1(10)$ & DCM (1) & $1: 1$ & $\mathrm{RT}$ & -- & $6 h$ & 93 & $80: 20$ \\
\hline 19 & $(R)-\mathbf{C 2}(10)$ & DCM (1) & $1: 1$ & RT & -- & $6 h$ & 93 & $79: 21$ \\
\hline 20 & $(R)-\mathbf{C} 3(10)$ & DCM (1) & $1: 1$ & RT & -- & $8 \mathrm{~h}$ & 95 & $71: 29$ \\
\hline 21 & $(R)-\mathbf{A} 7(10)$ & toluene (1) & $1: 1$ & RT & -- & $5 h$ & 95 & $75: 25$ \\
\hline 22 & $(R)-\mathbf{A} 7(10)$ & EtOAc (1) & $1: 1$ & RT & -- & $5 d$ & $\mathrm{NR}$ & -- \\
\hline 23 & $(R)-\mathbf{A} 7(10)$ & $\mathrm{CHCl}_{3}(1)$ & $1: 1$ & RT & -- & $12 \mathrm{~h}$ & 95 & $60: 40$ \\
\hline 24 & $(R)-\mathbf{A} 7(10)$ & DCE (1) & $1: 1$ & RT & -- & $8 \mathrm{~h}$ & 95 & $80: 20$ \\
\hline 25 & $(R)-\mathbf{A} 7(10)$ & $\operatorname{MeCN}(1)$ & $1: 1$ & RT & -- & $120 \mathrm{~h}$ & 46 & $53: 47$ \\
\hline 26 & $(R)-\mathbf{A} 7(10)$ & THF (1) & $1: 1$ & RT & -- & $5 d$ & $\mathrm{NR}$ & -- \\
\hline 27 & $(R)-\mathbf{A} 7(10)$ & DMF (1) & $1: 1$ & RT & -- & $5 d$ & $\mathrm{NR}$ & -- \\
\hline 28 & $(R)-\mathbf{A} 7(10)$ & DMSO (1) & $1: 1$ & RT & -- & $5 d$ & $\mathrm{NR}$ & -- \\
\hline 29 & $(R)-\mathbf{A} 7(10)$ & $\mathrm{CCl}_{4}(1)$ & $1: 1$ & RT & -- & $2 \mathrm{~h}$ & 92 & $75: 25$ \\
\hline 30 & $(R)-\mathbf{A} 14(10)$ & DCM (1) & $1: 1$ & RT & -- & $3 h$ & 95 & $83: 17$ \\
\hline 31 & $(R)-\mathbf{C 4}(10)$ & DCM (1) & $1: 1$ & RT & -- & $8 \mathrm{~h}$ & 95 & $83: 17$ \\
\hline 32 & $(R)-\mathbf{A} 14(5)$ & DCM (1) & $1: 1$ & RT & -- & $3 h$ & 95 & $82: 18$ \\
\hline 33 & $(R)-\mathbf{A} 14(20)$ & DCM (1) & $1: 1$ & RT & -- & $2.5 \mathrm{~h}$ & 90 & $86: 14$ \\
\hline 34 & $(R)-\mathbf{A} 14(20)$ & DCM $(0.5)$ & $1: 1$ & RT & -- & $2 \mathrm{~h}$ & 85 & $87: 13$ \\
\hline 35 & $(R)-\mathbf{A} 14(20)$ & $\mathrm{DCM}(2)$ & $1: 1$ & RT & -- & $3 h$ & 96 & $83: 17$ \\
\hline 36 & $(R)-\mathbf{A} 14(20)$ & DCM (4) & $1: 1$ & RT & -- & $12 \mathrm{~h}$ & 93 & $78: 22$ \\
\hline 37 & $(R)-\mathbf{A} 14(20)$ & $\mathrm{DCM}(0.5)$ & $1: 1.5$ & RT & -- & $3 h$ & 86 & $84: 16$ \\
\hline 38 & $(R)-\mathbf{A} 14(20)$ & $\mathrm{DCM}(0.5)$ & $1.5: 1$ & RT & -- & $3 h$ & 86 & $85: 15$ \\
\hline 39 & $(R)-\mathbf{A} 14(20)$ & $\mathrm{DCM}(0.5)$ & $1: 1$ & RT & $3 \AA ̊ M S$ & $5 d$ & $\mathrm{NR}$ & -- \\
\hline 40 & $(R)-\mathbf{A} 14(20)$ & $\mathrm{DCM}(0.5)$ & $1: 1$ & RT & $4 \AA ̊ M S$ & $3 h$ & 86 & $82: 18$ \\
\hline 41 & $(R)-\mathbf{A} 14(20)$ & DCM $(0.5)$ & $1: 1$ & $\mathrm{RT}$ & $5 \AA ̊ M S$ & $3 h$ & 86 & $84: 16$ \\
\hline 42 & $(R)-\mathbf{A} 14(20)$ & DCM $(0.5)$ & $1: 1$ & RT & $\mathrm{Na}_{2} \mathrm{SO}_{4}$ & $3 h$ & 90 & $85: 15$ \\
\hline 43 & $(R)-\mathbf{A} 14(20)$ & $\mathrm{DCM}(0.5)$ & $1: 1$ & RT & $\mathrm{MgSO}_{4}$ & $3 h$ & 90 & $85: 15$ \\
\hline 44 & $(R)-\mathbf{A} 14(20)$ & DCM $(0.5)$ & $1: 1$ & $10^{\circ} \mathrm{C}$ & -- & $18 \mathrm{~h}$ & 86 & $89: 11$ \\
\hline 45 & $(R)-\mathbf{A} 14(20)$ & DCM $(0.5)$ & $1: 1$ & $0^{\circ} \mathrm{C}$ & -- & $24 h$ & 86 & $90: 10$ \\
\hline 46 & $(R)-\mathbf{A 1 4}(20)$ & DCM $(0.5)$ & $1: 1$ & $-5^{\circ} \mathrm{C}$ & -- & $24 \mathrm{~h}$ & 86 & $90: 10$ \\
\hline 47 & $(R)-\mathbf{A} 14(20)$ & DCM $(0.5)$ & $1: 1$ & $-10^{\circ} \mathrm{C}$ & -- & $30 \mathrm{~h}$ & 90 & $89: 11$ \\
\hline 48 & $(R)-\mathbf{A} 14(20)$ & $\mathrm{DCM}(0.5)$ & $1: 1$ & $-20^{\circ} \mathrm{C}$ & -- & $4 d$ & 87 & $86: 14$ \\
\hline 49 & $(R)-\mathbf{A} 14(20)$ & $\operatorname{DCM}(0.3)$ & $1: 1$ & $0^{\circ} \mathrm{C}$ & -- & $24 \mathrm{~h}$ & 86 & $89: 11$ \\
\hline 50 & $(R)-\mathbf{A} 14(20)$ & DCM (1) & $1: 1$ & $0^{\circ} \mathrm{C}$ & -- & $24 \mathrm{~h}$ & 86 & $89: 11$ \\
\hline 51 & $(R)-\mathbf{A} 14(20)$ & DCM $(0.5)$ & $1: 1$ & $0^{\circ} \mathrm{C}$ & $5 \AA ̊$ M.S. & $3 h$ & 86 & $88: 12$ \\
\hline 52 & $(R)-\mathbf{A} 14(20)$ & DCM $(0.5)$ & $1: 1$ & $0^{\circ} \mathrm{C}$ & $\mathrm{Na}_{2} \mathrm{SO}_{4}$ & $3 h$ & 86 & $88: 12$ \\
\hline 53 & $(R)-\mathbf{A 1 4}(20)$ & DCM $(0.5)$ & $1: 1$ & $0^{\circ} \mathrm{C}$ & $\mathrm{H}_{2} \mathrm{O}(1 \mathrm{eq})$ & $30 \mathrm{~h}$ & 86 & $88: 12$ \\
\hline 54 & $(R)-\mathbf{A 1 4}(20)$ & DCM $(0.5)$ & $1: 1$ & $0^{\circ} \mathrm{C}$ & $\mathrm{H}_{2} \mathrm{O}(5 \mathrm{eq})$ & $30 \mathrm{~h}$ & 86 & $88: 12$ \\
\hline 55 & $(R)-\mathbf{A 1 4}(20)$ & DCM $(0.5)$ & $1: 1$ & $-20^{\circ} \mathrm{C}$ & $5 \AA ̊$ M.S. & $2 d$ & 82 & $80: 20$ \\
\hline 56 & $(R)-\mathbf{A} 7(20)$ & DCM (1) & $1: 1$ & $\mathrm{RT}$ & -- & $4 \mathrm{~h}$ & 98 & $84: 16$ \\
\hline 57 & $(R)-\mathbf{A} 7(20)$ & DCM (1) & $1: 1$ & $0^{\circ} \mathrm{C}$ & -- & $48 \mathrm{~h}$ & 90 & $89: 11$ \\
\hline 58 & $(R)-\mathbf{A} 7(20)$ & $\mathrm{DCM}(0.5)$ & $1: 1$ & $0^{\circ} \mathrm{C}$ & -- & $48 \mathrm{~h}$ & 95 & $93: 7$ \\
\hline 59 & $(R)-\mathrm{A} 7(20)$ & DCM (0.5) & $1: 1$ & $-10^{\circ} \mathrm{C}$ & -- & $4 d$ & 90 & $95: 5$ \\
\hline 60 & $(R)-\mathbf{A} 7(20)$ & DCM $(0.5)$ & $1: 1$ & $-20^{\circ} \mathrm{C}$ & -- & $8 d$ & 90 & $95: 5$ \\
\hline 61 & $(R)-\mathbf{C} 1(20)$ & DCM $(0.5)$ & $1: 1$ & $-10^{\circ} \mathrm{C}$ & -- & $6 d$ & 86 & $95: 5$ \\
\hline $62^{[\mathrm{d}]}$ & $(R)-\mathbf{A} 7(20)$ & $\operatorname{DCM}(0.5)$ & $1: 1$ & $-10^{\circ} \mathrm{C}$ & -- & $6 \mathrm{~d}$ & 95 & $94: 6$ \\
\hline
\end{tabular}

[a] Unless otherwise specified, all reactions were carried out with catalyst (x mol\%), 1a $(0.05 \mathrm{mmol})$ and $4 a$ $(0.05 \mathrm{mmol})$ in the indicated solvent $(\mathrm{x} \mathrm{mL})$ at room temperature. [b] Isolated yield of $5 \mathbf{a}$. [c] Determined by chiral-phase HPLC analysis. [d] $0.10 \mathrm{mmol} 1$ a was used. 


\section{Experimental Procedure and Characterization of Substrates \\ a. General Procedure to Prepare Substrates 1a-1r, 8}<smiles>[R]n1cc(C(=O)C(F)(F)F)c2cc[R1]cc21</smiles>

S1<smiles>[R]C#CC</smiles>

S2<smiles>[R]C#CC(O)(c1cn([R])c2c1[CH-][R1]C=C2)C(F)(F)F</smiles>

1 and 8

At $-78{ }^{\circ} \mathrm{C}$, under $\mathrm{N}_{2}$, to a flame-dried flask charged with a solution of the terminal alkyne S2 $(6 \mathrm{mmol}, 3$ equiv) in dry THF (15 mL) was added ${ }^{\mathrm{n}} \mathrm{BuLi}(6 \mathrm{mmol}, 2.5 \mathrm{~mL}, 2.4 \mathrm{M}$ in hexane) dropwise. The reaction was stirred for $0.5 \mathrm{~h}$ at the same temperature and then a solution of the corresponding indolyl-ketone $\mathbf{S} 1$ ( $2 \mathrm{mmol}$, 1.0 equiv) in THF (5 mL) was added via syringe. The reaction mixture was then slowly warmed up to room temperature and stirred for $32 \mathrm{~h}$. Upon completion, the reaction mixture was cooled to $0{ }^{\circ} \mathrm{C}$ and a saturated aqueous $\mathrm{NH}_{4} \mathrm{Cl}$ solution $(7 \mathrm{~mL}$ ) was added dropwise. The organic layer was separated. The aqueous layer was extracted with ethyl acetate $(3 \times 10 \mathrm{~mL})$. The combined organic layers were washed with brine $(30 \mathrm{~mL})$, dried over anhydrous $\mathrm{Na}_{2} \mathrm{SO}_{4}$, filtered, and concentrated. The crude product was purified directly by flash column chromatography on silica gel (petroleum ether/ ethyl acetate $=10: 1 \sim 5: 1$ ) or recrystallization to give the desired a-indolyl propargylic alcohols 1 and 8.

\section{b. Analytical Data for Substrates 1a-1r, 8}

1,1,1-trifluoro-2-(1 H-indol-3-yl)-4-phenylbut-3-yn-2-ol (1a):<smiles>OC(C#Cc1ccccc1)(c1c[nH]c2ccccc12)C(F)(F)F</smiles>

light brown solid, $554.9 \mathrm{mg}, 88 \%$ yield. Mp.131.7-132. $3^{\circ} \mathrm{C}$. purified by flash column chromatography on silica gel (petroleum ether/ ethyl acetate $=5: 1, \mathrm{Rf}=0.3$ ).

${ }^{1} \mathrm{H}$ NMR $\left(400 \mathrm{MHz}, \mathrm{CDCl}_{3}\right) \delta: 8.25(\mathrm{~s}, 1 \mathrm{H}), 8.03(\mathrm{~d}, \mathrm{~J}=7.9 \mathrm{~Hz}, 1 \mathrm{H}), 7.52(\mathrm{t}, \mathrm{J}=5.4 \mathrm{~Hz}, 3 \mathrm{H}), 7.43-7.32(\mathrm{~m}, 4 \mathrm{H})$, $7.26-7.16(\mathrm{~m}, 2 \mathrm{H}), 3.16(\mathrm{~s}, 1 \mathrm{H})$.

${ }^{13} \mathrm{C}$ NMR $\left(101 \mathrm{MHz}, \mathrm{CDCl}_{3}\right) \delta: 136.7,132.2,129.5,128.6,128.6,125.0,124.1$ (q, J = 285.4 Hz), 122.8, 121.3, $121.2,120.7,111.7,111.5,87.3,84.7,71.2$ (q, J = 34.3 Hz).

${ }^{19} \mathrm{~F}$ NMR $\left(376 \mathrm{MHz}, \mathrm{CDCl}_{3}\right) \delta:-79.92$.

HRMS (ESI): $m / z$ calcd. for $\mathrm{C}_{18} \mathrm{H}_{11} \mathrm{NOF}_{3}[\mathrm{M}-\mathrm{H}]=314.0798$; found: 314.0794 .

2-(4-chloro-1H-indol-3-yl)-1,1,1-trifluoro-4-phenylbut-3-yn-2-ol (1b):<smiles>CC(O)(c1c[nH]c2cccc(Cl)c12)C(F)(F)F</smiles>

light brown solid, $573.6 \mathrm{mg}, 82 \%$ yield. Mp.126.0-126.3 ${ }^{\circ} \mathrm{C}$. purified by flash column chromatography on silica gel (petroleum ether/ ethyl acetate $=5: 1, \mathrm{Rf}=0.4$ ).

${ }^{1} \mathbf{H}$ NMR $\left(400 \mathrm{MHz}, \mathrm{CDCl}_{3}\right) \delta: 8.52(\mathrm{~s}, 1 \mathrm{H}), 7.53-7.41(\mathrm{~m}, 3 \mathrm{H}), 7.37-7.24(\mathrm{~m}, 4 \mathrm{H}), 7.21(\mathrm{~d}, \mathrm{~J}=7.7 \mathrm{~Hz}, 1 \mathrm{H})$, $7.12(\mathrm{t}, \mathrm{J}=7.8 \mathrm{~Hz}, 1 \mathrm{H}), 4.42(\mathrm{~s}, 1 \mathrm{H})$.

${ }^{13} \mathrm{C}$ NMR $\left(101 \mathrm{MHz}, \mathrm{CDCl}_{3}\right) \delta: 138.5,132.0,129.3,128.4,125.9,124.3,124.0$ (q, J = 286.3 Hz), 123.6, 122.7, 
122.7, 121.6, 111.4, 110.9, 87.8, 85.3, 70.8 (q, J = 33.3 Hz).

${ }^{19} \mathrm{~F}$ NMR $\left(376 \mathrm{MHz}, \mathrm{CDCl}_{3}\right) \delta:-77.20$.

HRMS (ESI): $m / z$ calcd. for $\mathrm{C}_{18} \mathrm{H}_{10} \mathrm{NOF}_{3} \mathrm{Cl}[\mathrm{M}-\mathrm{H}]: 348.0409$; found: 348.0399 .

1,1,1-trifluoro-2-(5-fluoro-1 $H$-indol-3-yl)-4-phenylbut-3-yn-2-ol (1c):

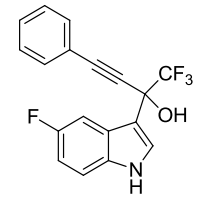

light brown solid, $619.9 \mathrm{mg}, 93 \%$ yield. Mp.132.2-133. ${ }^{\circ} \mathrm{C}$. purified by flash column chromatography on silica gel (petroleum ether/ ethyl acetate $=5: 1, \mathrm{Rf}=0.3$ ).

${ }^{1} \mathbf{H}$ NMR $\left(400 \mathrm{MHz}, \mathrm{CDCl}_{3}\right)$ ס: $8.32(\mathrm{~s}, 1 \mathrm{H}), 7.69(\mathrm{~d}, \mathrm{~J}=10.0 \mathrm{~Hz}, 1 \mathrm{H}), 7.59-7.48(\mathrm{~m}, 3 \mathrm{H}), 7.44-7.34(\mathrm{~m}, 3 \mathrm{H})$, 7.30 (dd, J = 8.9, $4.4 \mathrm{~Hz}, 1 \mathrm{H}), 7.00(\mathrm{td}, \mathrm{J}=9.0,2.4 \mathrm{~Hz}, 1 \mathrm{H}), 3.26(\mathrm{~s}, 1 \mathrm{H})$.

${ }^{13} \mathrm{C}$ NMR $\left(101 \mathrm{MHz}, \mathrm{CDCl}_{3}\right)$ ס: $158.3(\mathrm{~d}, \mathrm{~J}=235.1 \mathrm{~Hz}), 133.2,132.2,129.7,128.6,126.6,125.5$ (d, J = 10.7 Hz), 124.1 (q, J = 285.4 Hz), 121.1, 112.2 (d, J = 9.7 Hz), 111.9 (d, J = 4.5 Hz), 111.4 (d, J = 26.6 Hz), 106.4 (d, J = $25.0 \mathrm{~Hz}), 87.5,84.4,71.0(\mathrm{q}, \mathrm{J}=34.0 \mathrm{~Hz})$.

${ }^{19}$ F NMR $\left(376 \mathrm{MHz}, \mathrm{CDCl}_{3}\right) \delta:-80.13,-123.00$.

HRMS (ESI): $m / z$ calcd. for $\mathrm{C}_{18} \mathrm{H}_{10} \mathrm{NOF}_{4}[\mathrm{M}-\mathrm{H}]: 332.0704$; found: 332.0697.

2-(5-chloro-1H-indol-3-yl)-1,1,1-trifluoro-4-phenylbut-3-yn-2-ol (1d):<smiles>OC1(C(O)(C#Cc2ccccc2)C(F)(F)F)CNc2ccc(Cl)cc21</smiles>

light brown solid, $643.5 \mathrm{mg}, 92 \%$ yield. Mp. $113.5-114.8^{\circ} \mathrm{C}$. purified by flash column chromatography on silica gel (petroleum ether/ ethyl acetate $=5: 1, \mathrm{Rf}=0.3$ ).

${ }^{1} \mathrm{H}$ NMR $\left(400 \mathrm{MHz}, \mathrm{CDCl}_{3}\right)$ ס: $8.33(\mathrm{~s}, 1 \mathrm{H}), 8.00(\mathrm{~s}, 1 \mathrm{H}), 7.56-7.45(\mathrm{~m}, 3 \mathrm{H}), 7.42-7.31(\mathrm{~m}, 3 \mathrm{H}), 7.26(\mathrm{t}, \mathrm{J}=$ $8.5 \mathrm{~Hz}, 1 \mathrm{H}), 7.17(\mathrm{dd}, \mathrm{J}=8.7,1.9 \mathrm{~Hz}, 1 \mathrm{H}), 3.22(\mathrm{~s}, 1 \mathrm{H})$.

${ }^{13} \mathrm{C}$ NMR $\left(101 \mathrm{MHz}, \mathrm{CDCl}_{3}\right)$ ס: 135.1, 132.2, 129.7, 128.7, 126.5, 126.3, 126.1, 124.0 (q, J = 285.6 Hz), 123.3, $121.1,120.8,112.5,111.6,87.7,84.3,71.0$ (q, J = 34.4 Hz).

${ }^{19}$ F NMR $\left(376 \mathrm{MHz}, \mathrm{CDCl}_{3}\right) \delta:-80.10$.

HRMS (ESI): $m / z$ calcd. for $\mathrm{C}_{18} \mathrm{H}_{10} \mathrm{NOF}_{3} \mathrm{Cl}[\mathrm{M}-\mathrm{H}]: 348.0409$; found: 348.0399.

2-(5-bromo-1H-indol-3-yl)-1,1,1-trifluoro-4-phenylbut-3-yn-2-ol (1e):<smiles>OC1(C(O)(C#Cc2ccccc2)C(F)(F)F)CNc2ccc(Br)cc21</smiles>

light brown solid, $725.3 \mathrm{mg}, 92 \%$ yield. Mp.113.1-114. ${ }^{\circ} \mathrm{C}$. purified by flash column chromatography on silica gel (petroleum ether/ ethyl acetate $=5: 1, \mathrm{Rf}=0.3$ ).

${ }^{1} \mathrm{H}$ NMR $\left(400 \mathrm{MHz}, \mathrm{CDCl}_{3}\right)$ ס: $8.31(\mathrm{~s}, 1 \mathrm{H}), 8.19(\mathrm{~s}, 1 \mathrm{H}), 7.58-7.49(\mathrm{~m}, 3 \mathrm{H}), 7.43-7.31(\mathrm{~m}, 4 \mathrm{H}), 7.25(\mathrm{~d}, \mathrm{~J}=$ $8.5 \mathrm{~Hz}, 1 \mathrm{H}), 3.15(\mathrm{~s}, 1 \mathrm{H})$.

${ }^{13} \mathrm{C}$ NMR $\left(101 \mathrm{MHz}, \mathrm{CDCl}_{3}\right)$ ס: 135.3, 132.2, 129.7, 128.7, 126.7, 126.1, 125.9, 124.0 (q, J = 285.5 Hz), 123.9, $121.1,114.1,113.0,111.5,87.7,84.3,71.0(\mathrm{q}, \mathrm{J}=34.5 \mathrm{~Hz})$.

${ }^{19}$ F NMR $\left(376 \mathrm{MHz}, \mathrm{CDCl}_{3}\right) \delta:-80.11$.

HRMS (ESI): $m / z$ calcd. for $\mathrm{C}_{18} \mathrm{H}_{11} \mathrm{NOF}_{3} \mathrm{Br}$ [M-]: 392.9982 ; found: 393.0005 . 
1,1,1-trifluoro-2-(5-methyl-1H-indol-3-yl)-4-phenylbut-3-yn-2-ol (1f):

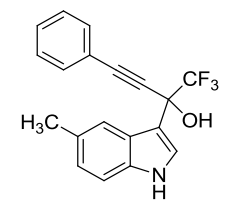

light brown solid, $632.3 \mathrm{mg}, 96 \%$ yield. Mp. $147.4-148.7^{\circ} \mathrm{C}$. purified by flash column chromatography on silica gel (petroleum ether/ ethyl acetate $=5: 1, \mathrm{Rf}=0.3$ ).

${ }^{1} \mathrm{H}$ NMR $\left(400 \mathrm{MHz}, \mathrm{CDCl}_{3}\right) \delta: 8.14(\mathrm{~s}, 1 \mathrm{H}), 7.80(\mathrm{~s}, 1 \mathrm{H}), 7.52(\mathrm{~d}, \mathrm{~J}=6.4 \mathrm{~Hz}, 2 \mathrm{H}), 7.45$ (d, J = 2.6 Hz, $\left.1 \mathrm{H}\right), 7.40-$ $7.31(\mathrm{~m}, 3 \mathrm{H}), 7.25(\mathrm{~d}, \mathrm{~J}=8.3 \mathrm{~Hz}, 1 \mathrm{H}), 7.06(\mathrm{~d}, \mathrm{~J}=8.2 \mathrm{~Hz}, 1 \mathrm{H}), 3.11(\mathrm{~s}, 1 \mathrm{H}), 2.45(\mathrm{~s}, 3 \mathrm{H})$.

${ }^{13} \mathrm{C}$ NMR $\left(101 \mathrm{MHz}, \mathrm{CDCl}_{3}\right) \delta: 135.1,132.2,130.0,129.5,128.6,125.2,125.1,124.5,124.2$ (q, J = 285.9 Hz), 121.4, 120.8, 111.2, 87.2, 84.9, 71.3 (q, J = 34.4 Hz), 21.8.

${ }^{19}$ F NMR $\left(376 \mathrm{MHz}, \mathrm{CDCl}_{3}\right) \delta:-79.81$.

HRMS (ESI): $\mathrm{m} / \mathrm{z}$ calcd. for $\mathrm{C}_{19} \mathrm{H}_{13} \mathrm{NOF}_{3}[\mathrm{M}-\mathrm{H}]: 328.0955$; found: 328.0941 .

1,1,1-trifluoro-2-(5-methoxy-1H-indol-3-yl)-4-phenylbut-3-yn-2-ol (1g):<smiles>COc1ccc2[nH]cc(C(O)(C#Cc3ccccc3)C(F)(F)F)c2c1</smiles>

light brown solid, $600.8 \mathrm{mg}, 87 \%$ yield. Mp.150.3-152.8 ${ }^{\circ} \mathrm{C}$. purified by flash column chromatography on silica gel (petroleum ether/ ethyl acetate $=5: 1, \mathrm{Rf}=0.4$ ).

${ }^{1}$ H NMR $(400 \mathrm{MHz}$, DMSO-d $)$ ס: $11.20(\mathrm{~s}, 1 \mathrm{H}), 7.62-7.49(\mathrm{~m}, 4 \mathrm{H}), 7.48-7.42(\mathrm{~m}, 3 \mathrm{H}), 7.33(\mathrm{~d}, \mathrm{~J}=8.6 \mathrm{~Hz}$, $2 \mathrm{H}), 6.80$ (dd, J = 8.9, $2.4 \mathrm{~Hz}, 1 \mathrm{H}), 3.71(\mathrm{~s}, 3 \mathrm{H})$.

${ }^{13} \mathrm{C}$ NMR (101 MHz, DMSO-d $\left.\mathrm{d}_{6}\right) \delta: 153.3,131.8,131.6,129.5,128.9,126.1,125.3,124.6$ (q, J = 286.0 Hz), $121.0,112.5,111.5,110.6,102.3,86.3,85.3,69.7$ (q, J = 32.8 Hz), 55.2.

${ }^{19} \mathrm{~F}$ NMR $\left(376 \mathrm{MHz}, \mathrm{DMSO}-\mathrm{d}_{6}\right) \delta:-78.90$.

HRMS (ESI): $m / z$ calcd. for $\mathrm{C}_{19} \mathrm{H}_{13} \mathrm{NO}_{2} \mathrm{~F}_{3}[\mathrm{M}-\mathrm{H}]: 344.0904$; found: 344.0892 .

2-(6-chloro-1H-indol-3-yl)-1,1,1-trifluoro-4-phenylbut-3-yn-2-ol (1h):<smiles>OC(C#Cc1ccccc1)(C1CNc2cc(Cl)ccc21)C(F)(F)F</smiles>

light brown solid, $629.5 \mathrm{mg}, 90 \%$ yield. Mp.114.2-115.9 ${ }^{\circ} \mathrm{C}$. purified by flash column chromatography on silica gel (petroleum ether/ ethyl acetate $=5: 1, \mathrm{Rf}=0.3$ ).

${ }^{1} \mathbf{H}$ NMR $\left(400 \mathrm{MHz}, \mathrm{CDCl}_{3}\right)$ ס: $8.30(\mathrm{~s}, 1 \mathrm{H}), 7.93(\mathrm{~d}, \mathrm{~J}=8.5 \mathrm{~Hz}, 1 \mathrm{H}), 7.59-7.47(\mathrm{~m}, 3 \mathrm{H}), 7.45-7.30(\mathrm{~m}, 4 \mathrm{H})$, $7.16(\mathrm{~d}, \mathrm{~J}=8.5 \mathrm{~Hz}, 1 \mathrm{H}), 3.27(\mathrm{~s}, 1 \mathrm{H})$.

${ }^{13} \mathrm{C} \mathrm{NMR}\left(101 \mathrm{MHz}, \mathrm{CDCl}_{3}\right) \delta: 137.0,132.2,129.7,128.8,128.7,125.6,124.0$ (q, J = 285.6 Hz), 123.6, 122.2, 121.5, 121.1, 112.0, 111.4, 87.6, 84.3, 71.0 (q, J = 34.7 Hz).

${ }^{19} \mathrm{~F}$ NMR $\left(376 \mathrm{MHz}, \mathrm{CDCl}_{3}\right) \delta:-80.07$.

HRMS (ESI): $m / z$ calcd. for $\mathrm{C}_{18} \mathrm{H}_{10} \mathrm{NOF}_{3} \mathrm{Cl}[\mathrm{M}-\mathrm{H}]: 348.0409$; found: 348.0400 .

2-(7-chloro-1 H-indol-3-yl)-1,1,1-trifluoro-4-phenylbut-3-yn-2-ol (1i): 


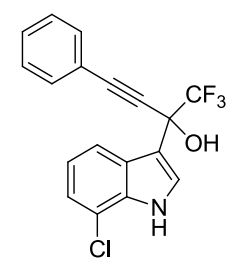

light brown solid, $657.5 \mathrm{mg}, 94 \%$ yield. Mp.83.3-84. $6^{\circ} \mathrm{C}$. purified by flash column chromatography on silica gel (petroleum ether/ ethyl acetate $=10: 1, \mathrm{Rf}=0.2$ ).

${ }^{1} \mathrm{H}$ NMR $\left(400 \mathrm{MHz}, \mathrm{CDCl}_{3}\right) \delta: 8.56(\mathrm{~s}, 1 \mathrm{H}), 7.92(\mathrm{~d}, \mathrm{~J}=8.0 \mathrm{~Hz}, 1 \mathrm{H}), 7.57(\mathrm{~d}, \mathrm{~J}=2.5 \mathrm{~Hz}, 1 \mathrm{H}), 7.51$ (d, J = 6.7 Hz, $2 \mathrm{H}), 7.41-7.30(\mathrm{~m}, 3 \mathrm{H}), 7.23(\mathrm{~d}, \mathrm{~J}=7.2 \mathrm{~Hz}, 1 \mathrm{H}), 7.11(\mathrm{t}, \mathrm{J}=7.9 \mathrm{~Hz}, 1 \mathrm{H}), 3.44(\mathrm{~s}, 1 \mathrm{H})$.

${ }^{13} \mathrm{C}$ NMR $\left(101 \mathrm{MHz}, \mathrm{CDCl}_{3}\right) \delta: 134.0,132.2,129.7,128.6,126.5,125.5,124.0$ (q, J = 285.7 Hz), 122.1, 121.5, $121.1,120.0,116.9,113.0,87.5,84.4,71.0$ (q, J = 34.2 Hz).

${ }^{19}$ F NMR $\left(376 \mathrm{MHz}, \mathrm{CDCl}_{3}\right) \delta:-80.09$.

HRMS (ESI): $m / z$ calcd. for $\mathrm{C}_{18} \mathrm{H}_{10} \mathrm{NOF}_{3} \mathrm{Cl}[\mathrm{M}-\mathrm{H}]: 348.0409$; found: 348.0401 .

1,1,1-trifluoro-4-(4-fluorophenyl)-2-(1H-indol-3-yl)but-3-yn-2-ol (1j):<smiles>OC(C#Cc1ccc(F)cc1)(C1CNc2ccccc21)C(F)(F)F</smiles>

light brown solid, $533.2 \mathrm{mg}, 80 \%$ yield. Mp.163.3-165.3 ${ }^{\circ} \mathrm{C}$. purified by flash column chromatography on silica gel (petroleum ether/ ethyl acetate $=5: 1, \mathrm{Rf}=0.3$ ).

${ }^{1}$ H NMR (400 MHz, DMSO-d $\left.\mathrm{d}_{6}\right) \delta: 11.36(\mathrm{~s}, 1 \mathrm{H}), 7.84(\mathrm{~d}, \mathrm{~J}=8.0 \mathrm{~Hz}, 1 \mathrm{H}), 7.67-7.55(\mathrm{~m}, 4 \mathrm{H}), 7.43(\mathrm{~d}, \mathrm{~J}=8.1 \mathrm{~Hz}$, $1 \mathrm{H}), 7.29(\mathrm{t}, \mathrm{J}=8.9 \mathrm{~Hz}, 2 \mathrm{H}), 7.13(\mathrm{t}, \mathrm{J}=7.1 \mathrm{~Hz}, 1 \mathrm{H}), 7.05(\mathrm{t}, \mathrm{J}=7.1 \mathrm{~Hz}, 1 \mathrm{H})$.

${ }^{13} \mathrm{C}$ NMR (101 MHz, DMSO-d 6 ) $\delta: 162.4(\mathrm{~d}, \mathrm{~J}=248.5 \mathrm{~Hz}), 136.7,134.1$ (d, J = 8.7 Hz), 125.7, 124.9, 124.5 (q, J $=286.4 \mathrm{~Hz}), 121.4,120.5,119.3,117.5(\mathrm{~d}, \mathrm{~J}=3.2 \mathrm{~Hz}), 116.2(\mathrm{~d}, \mathrm{~J}=22.3 \mathrm{~Hz}), 111.9,111.0,86.1,84.2,69.7$ (q, $\mathrm{J}=32.7 \mathrm{~Hz})$.

${ }^{19} \mathrm{~F}$ NMR $\left(376 \mathrm{MHz}, \mathrm{DMSO}-\mathrm{d}_{6}\right) \delta:-78.89,-109.46$.

HRMS (ESI): $m / z$ calcd. for $\mathrm{C}_{18} \mathrm{H}_{10} \mathrm{NOF}_{4}[\mathrm{M}-\mathrm{H}]: 332.0704$; found: 332.0701 .

4-(4-chlorophenyl)-1,1,1-trifluoro-2-(1H-indol-3-yl)but-3-yn-2-ol (1k):<smiles>OC(C#Cc1ccc(Cl)cc1)(C1CNc2ccccc21)C(F)(F)F</smiles>

light brown solid, $601.5 \mathrm{mg}, 86 \%$ yield. Mp.130.3-132. $2^{\circ} \mathrm{C}$. purified by flash column chromatography on silica gel (petroleum ether/ ethyl acetate $=5: 1, \mathrm{Rf}=0.3$ ).

${ }^{1} \mathrm{H}$ NMR $\left(400 \mathrm{MHz}, \mathrm{CDCl}_{3}\right) \delta: 8.25(\mathrm{~s}, 1 \mathrm{H}), 8.00(\mathrm{~d}, \mathrm{~J}=7.8 \mathrm{~Hz}, 1 \mathrm{H}), 7.74-7.14(\mathrm{~m}, 8 \mathrm{H}), 3.21(\mathrm{~s}, 1 \mathrm{H})$.

${ }^{13} \mathrm{C}$ NMR (101 MHz, $\mathrm{CDCl}_{3}$ ) $\delta: 136.7,135.7,133.7,133.4,129.0,127.4$ (q, J = 289.2 Hz), 124.9, 122.9, 121.1, 120.8, 119.7, 111.6, 111.5, 86.2, 85.6, 71.2 (q, J = 33.7 Hz).

${ }^{19} \mathrm{~F}$ NMR $\left(376 \mathrm{MHz}, \mathrm{CDCl}_{3}\right) \delta:-79.83$.

HRMS (ESI): $m / z$ calcd. for $\mathrm{C}_{18} \mathrm{H}_{10} \mathrm{NOF}_{3} \mathrm{Cl}[\mathrm{M}-\mathrm{H}]: 348.0409$; found: 348.0397.

1,1,1-trifluoro-2-(1H-indol-3-yl)-4-(p-tolyl)but-3-yn-2-ol (1I): 
<smiles></smiles>

light brown solid, $526.9 \mathrm{mg}, 80 \%$ yield. Mp.138.1-139.6 ${ }^{\circ} \mathrm{C}$. purified by flash column chromatography on silica gel (petroleum ether/ ethyl acetate $=5: 1, \mathrm{Rf}=0.3$ ).

${ }^{1} \mathrm{H}$ NMR $\left(400 \mathrm{MHz}, \mathrm{CDCl}_{3}\right) \delta: 8.21(\mathrm{~s}, 1 \mathrm{H}), 8.01(\mathrm{~d}, \mathrm{~J}=7.8 \mathrm{~Hz}, 1 \mathrm{H}), 7.47(\mathrm{~d}, \mathrm{~J}=2.2 \mathrm{~Hz}, 1 \mathrm{H}), 7.40(\mathrm{~d}, \mathrm{~J}=7.9 \mathrm{~Hz}$, 2H), $7.35(\mathrm{~d}, \mathrm{~J}=8.0 \mathrm{~Hz}, 1 \mathrm{H}), 7.26-7.11(\mathrm{~m}, 4 \mathrm{H}), 3.15(\mathrm{~s}, 1 \mathrm{H}), 2.35(\mathrm{~s}, 3 \mathrm{H})$.

${ }^{13} \mathrm{C}$ NMR $\left(101 \mathrm{MHz}, \mathrm{CDCl}_{3}\right) \delta: 139.9,136.7,132.1,129.3,125.1,125.0,124.1$ (q, J = 285.5 Hz), 122.8, 121.2, 120.7, 118.2, 111.8, 111.5, 87.5, 84.1, 71.2 (q, J = 34.4 Hz), 21.7.

${ }^{19}$ F NMR $\left(376 \mathrm{MHz}, \mathrm{CDCl}_{3}\right) \delta:-79.92$.

HRMS (ESI): $m / z$ calcd. for $\mathrm{C}_{19} \mathrm{H}_{13} \mathrm{NOF}_{3}[\mathrm{M}-\mathrm{H}]:$ : 328.0955; found: 328.0943 .

1,1,1-trifluoro-2-(1H-indol-3-yl)-4-(4-methoxyphenyl)but-3-yn-2-ol (1m):<smiles>COc1ccc(C#CC(O)(c2c[nH]c3ccccc23)C(F)(F)F)cc1</smiles>

light brown solid, $621.6 \mathrm{mg}, 90 \%$ yield. Mp.164.1-165.8 ${ }^{\circ} \mathrm{C}$. purified by flash column chromatography on silica gel (petroleum ether/ ethyl acetate $=5: 1, \mathrm{Rf}=0.4$ ).

${ }^{1} \mathrm{H}$ NMR $\left(400 \mathrm{MHz}, \mathrm{DMSO}_{-} \mathrm{d}_{6}\right) \delta: 11.33(\mathrm{~s}, 1 \mathrm{H}), 7.84(\mathrm{~d}, \mathrm{~J}=7.8 \mathrm{~Hz}, 1 \mathrm{H}), 7.66-7.32(\mathrm{~m}, 5 \mathrm{H}), 7.12(\mathrm{t}, \mathrm{J}=7.4 \mathrm{~Hz}$, $1 \mathrm{H}), 7.08-6.87(\mathrm{~m}, 3 \mathrm{H}), 3.79(\mathrm{~s}, 3 \mathrm{H})$.

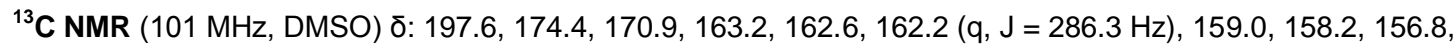
152.2, 150.5, 149.5, 149.0, 123.0, 122.6, 107.3 (q, J = 33.1 Hz), 93.0.

${ }^{19} \mathrm{~F}$ NMR (376 MHz, DMSO) $\delta:-78.93$.

HRMS (ESI): $m / z$ calcd. for $\mathrm{C}_{19} \mathrm{H}_{13} \mathrm{NO}_{2} \mathrm{~F}_{3}[\mathrm{M}-\mathrm{H}]: 344.0904$; found: 344.0901 .

4-(3-chlorophenyl)-1,1,1-trifluoro-2-(1H-indol-3-yl)but-3-yn-2-ol (1n):<smiles>OC(C#Cc1cccc(Cl)c1)(c1c[nH]c2ccccc12)C(F)(F)F</smiles>

light brown solid, $594.5 \mathrm{mg}, 85 \%$ yield. Mp. $114.1-115.4^{\circ} \mathrm{C}$. purified by flash column chromatography on silica gel (petroleum ether/ ethyl acetate $=5: 1, \mathrm{Rf}=0.3$ ).

${ }^{1} \mathrm{H}$ NMR $\left(400 \mathrm{MHz}, \mathrm{CDCl}_{3}\right)$ ס: $8.26(\mathrm{~s}, 1 \mathrm{H}), 7.99(\mathrm{~d}, \mathrm{~J}=7.8 \mathrm{~Hz}, 1 \mathrm{H}), 7.53-7.43(\mathrm{~m}, 2 \mathrm{H}), 7.41-7.31(\mathrm{~m}, 3 \mathrm{H})$, $7.29-7.14(\mathrm{~m}, 3 \mathrm{H}), 3.18(\mathrm{~s}, 1 \mathrm{H})$.

${ }^{13} \mathrm{C}$ NMR (101 MHz, $\left.\mathrm{CDCl}_{3}\right) \delta: 136.7,134.5,132.0,130.3,129.9,125.0,124.9,124.0$ (q, J = 285.4 Hz), 123.0, 122.9, 121.1, 120.8, 111.6, 111.4, 85.8, 85.7, 71.2 (q, J = 34.2 Hz).

${ }^{19}$ F NMR $\left(376 \mathrm{MHz}, \mathrm{CDCl}_{3}\right) \delta:-79.81$.

HRMS (ESI): $m / z$ calcd. for $\mathrm{C}_{18} \mathrm{H}_{10} \mathrm{NOF}_{3} \mathrm{Cl}[\mathrm{M}-\mathrm{H}]: 348.0409$; found: 348.0398 .

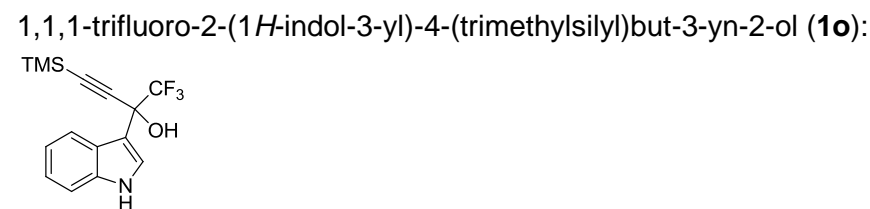

light brown solid, $417.2 \mathrm{mg}, 67 \%$ yield. Mp. $114.1-114.9^{\circ} \mathrm{C}$. purified by flash column chromatography on silica 
gel (petroleum ether/ ethyl acetate $=10: 1, \mathrm{Rf}=0.4$ ).

${ }^{1} \mathrm{H}$ NMR $\left(400 \mathrm{MHz}, \mathrm{CDCl}_{3}\right) \delta: 8.20(\mathrm{~s}, 1 \mathrm{H}), 7.95(\mathrm{~d}, \mathrm{~J}=6.9 \mathrm{~Hz}, 1 \mathrm{H}), 7.41(\mathrm{~s}, 1 \mathrm{H}), 7.36-7.31(\mathrm{~m}, 1 \mathrm{H}), 7.24-$ $7.13(\mathrm{~m}, 2 \mathrm{H}), 3.07(\mathrm{~s}, 1 \mathrm{H}), 0.24(\mathrm{~s}, 9 \mathrm{H})$.

${ }^{13} \mathrm{C}$ NMR $\left(101 \mathrm{MHz}, \mathrm{CDCl}_{3}\right) \delta: 137.3,125.7,125.5,124.5$ (q, $\left.J=285.7 \mathrm{~Hz}\right), 123.4,121.9,121.2,112.2,112.1$, 100.9, 93.9, 71.4 (q, $J=34.3 \mathrm{~Hz}), 0.3$.

${ }^{19} \mathrm{~F} \mathrm{NMR}\left(376 \mathrm{MHz}, \mathrm{CDCl}_{3}\right) \delta:-80.05$.

HRMS (ESI): $m / z$ calcd. for $\mathrm{C}_{15} \mathrm{H}_{15} \mathrm{NOF}_{3} \mathrm{Si}[\mathrm{M}-\mathrm{H}]: 310.0880$; found: 310.0871 .

4-cyclopropyl-1,1,1-trifluoro-2-(1H-indol-3-yl)but-3-yn-2-ol (1p):

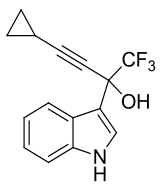

light brown solid, $491.5 \mathrm{mg}, 88 \%$ yield. Mp.97.4-99.2 ${ }^{\circ} \mathrm{C}$. purified by flash column chromatography on silica gel (petroleum ether/ ethyl acetate $=10: 1, \mathrm{Rf}=0.3$ ).

${ }^{1} \mathrm{H}$ NMR $\left(400 \mathrm{MHz}, \mathrm{CDCl}_{3}\right) \delta: 8.18(\mathrm{~s}, 1 \mathrm{H}), 7.93(\mathrm{~d}, \mathrm{~J}=7.8 \mathrm{~Hz}, 1 \mathrm{H}), 7.38(\mathrm{~d}, \mathrm{~J}=2.5 \mathrm{~Hz}, 1 \mathrm{H}), 7.33(\mathrm{~d}, \mathrm{~J}=8.0 \mathrm{~Hz}$, $1 \mathrm{H}), 7.26-7.12(\mathrm{~m}, 2 \mathrm{H}), 2.96(\mathrm{~s}, 1 \mathrm{H}), 1.41-1.29(\mathrm{~m}, 1 \mathrm{H}), 0.92-0.72(\mathrm{~m}, 4 \mathrm{H})$.

${ }^{13} \mathrm{C}$ NMR $\left(101 \mathrm{MHz}, \mathrm{CDCl}_{3}\right) \delta: 136.7,125.0,124.9,124.1$ (q, J = 287.5 Hz), 122.7, 121.2, 120.5, 112.2, 111.5, 91.8, 71.3, $70.7(\mathrm{q}, \mathrm{J}=34.1 \mathrm{~Hz}), 8.6,8.5,-0.5$.

${ }^{19}$ F NMR $\left(376 \mathrm{MHz}, \mathrm{CDCl}_{3}\right) \delta:-80.25$.

HRMS (ESI): $m / z$ calcd. for $\mathrm{C}_{15} \mathrm{H}_{11} \mathrm{NOF}_{3}[\mathrm{M}-\mathrm{H}]: 278.0798$; found: 278.0806 .

1,1,1-trifluoro-2-(1H-indol-3-yl)-5,5-dimethylhex-3-yn-2-ol (1q):<smiles>CC(C)(C)C#CC(O)(C1CNc2ccccc21)C(F)(F)F</smiles>

light brown solid, $484.3 \mathrm{mg}, 82 \%$ yield. Mp. $125.3-126.8^{\circ} \mathrm{C}$. purified by flash column chromatography on silica gel (petroleum ether/ ethyl acetate $=10: 1, \mathrm{Rf}=0.4$ ).

${ }^{1} \mathrm{H}$ NMR (400 MHz, DMSO-d $\left.\mathrm{d}_{6}\right)$ ס: $11.24(\mathrm{~s}, 1 \mathrm{H}), 7.80(\mathrm{~d}, \mathrm{~J}=7.9 \mathrm{~Hz}, 1 \mathrm{H}), 7.45-7.35(\mathrm{~m}, 2 \mathrm{H}), 7.20(\mathrm{~s}, 1 \mathrm{H}), 7.10$ $(\mathrm{t}, \mathrm{J}=7.5 \mathrm{~Hz}, 1 \mathrm{H}), 7.02(\mathrm{t}, \mathrm{J}=7.5 \mathrm{~Hz}, 1 \mathrm{H}), 1.26(\mathrm{~s}, 9 \mathrm{H})$.

${ }^{13}$ C NMR (126 MHz, DMSO-d $\left.\mathrm{d}_{6}\right) \delta: 137.1,125.8,125.4,125.0$ (q, J = 286.0 Hz), 121.7, 121.2, 119.4, 112.2, 112.1, 94.5, 76.5, 69.5 (q, J = 32.6 Hz), 30.8, 27.6.

${ }^{19}$ F NMR (376 MHz, DMSO-d 6 ) $\delta:-79.41$.

HRMS (ESI): $m / z$ calcd. for $\mathrm{C}_{16} \mathrm{H}_{15} \mathrm{NOF}_{3}[\mathrm{M}-\mathrm{H}]: 294.1111$; found: 294.1101 .

1,1,1-trifluoro-2-(1 H-indol-3-yl)oct-3-yn-2-ol (1r):<smiles>CCCCC#CC(O)(c1c[nH]c2ccccc12)C(F)(F)F</smiles>

light brown solid, $519.7 \mathrm{mg}$, $88 \%$ yield. Mp.68.8-70.1 ${ }^{\circ} \mathrm{C}$. purified by flash column chromatography on silica gel (petroleum ether/ ethyl acetate $=10: 1, \mathrm{Rf}=0.4$ ).

${ }^{1} \mathrm{H}$ NMR $\left(400 \mathrm{MHz}, \mathrm{CDCl}_{3}\right) \delta: 8.18(\mathrm{~s}, 1 \mathrm{H}), 7.95(\mathrm{~d}, \mathrm{~J}=7.8 \mathrm{~Hz}, 1 \mathrm{H}), 7.39(\mathrm{~d}, \mathrm{~J}=2.4 \mathrm{~Hz}, 1 \mathrm{H}), 7.32(\mathrm{~d}, \mathrm{~J}=8.1 \mathrm{~Hz}$, $1 \mathrm{H}), 7.25-7.11(\mathrm{~m}, 2 \mathrm{H}), 3.01(\mathrm{~s}, 1 \mathrm{H}), 2.32(\mathrm{t}, \mathrm{J}=7.0 \mathrm{~Hz}, 2 \mathrm{H}), 1.61-1.51(\mathrm{~m}, 2 \mathrm{H}), 1.49-1.37(\mathrm{~m}, 2 \mathrm{H}), 0.92(\mathrm{t}$, $\mathrm{J}=7.3 \mathrm{~Hz}, 3 \mathrm{H})$.

${ }^{13} \mathrm{C}$ NMR $\left(101 \mathrm{MHz}, \mathrm{CDCl}_{3}\right) \delta: 136.7,125.0,124.2(\mathrm{q}, \mathrm{J}=285.4 \mathrm{~Hz}), 122.7,121.3,120.5,112.1,111.5,88.8$, 
76.3, 70.7 (q, J = 34.1 Hz), 30.3, 22.1, 18.5, 13.7.

${ }^{19}$ F NMR $\left(376 \mathrm{MHz}, \mathrm{CDCl}_{3}\right) \delta:-80.27$.

HRMS (ESI): $m / z$ calcd. for $\mathrm{C}_{16} \mathrm{H}_{15} \mathrm{NOF}_{3}[\mathrm{M}-\mathrm{H}]: 294.1111$; found: 294.1098.

1,1,1-trifluoro-2-(1-methyl-1H-indol-3-yl)-4-phenylbut-3-yn-2-ol (8):

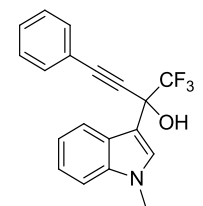

light brown solid, $573.0 \mathrm{mg}, 87 \%$ yield. Mp.156.3-158.6 ${ }^{\circ} \mathrm{C}$. purified by flash column chromatography on silica gel (petroleum ether/ ethyl acetate $=5: 1, \mathrm{Rf}=0.4$ ).

${ }^{1} \mathbf{H}$ NMR $\left(400 \mathrm{MHz}, \mathrm{CDCl}_{3}\right) \delta: 8.00(\mathrm{~d}, \mathrm{~J}=7.2 \mathrm{~Hz}, 1 \mathrm{H}), 7.52(\mathrm{~d}, \mathrm{~J}=6.1 \mathrm{~Hz}, 2 \mathrm{H}), 7.43-7.30(\mathrm{~m}, 5 \mathrm{H}), 7.29-7.14$ $(\mathrm{m}, 2 \mathrm{H}), 3.77(\mathrm{~s}, 3 \mathrm{H}), 3.13(\mathrm{~s}, 1 \mathrm{H})$.

${ }^{13} \mathrm{C}$ NMR $\left(101 \mathrm{MHz}, \mathrm{CDCl}_{3}\right)$ ס: 137.6, 132.2, 129.5, 129.4, 128.6, 125.5, 124.1 (q, J = 285.9 Hz), 122.4, 121.4, 121.3, 120.3, 109.9, 109.7, 87.1, 84.9, 71.1 (q, J = 34.1 Hz), 33.1.

${ }^{19}$ F NMR $\left(376 \mathrm{MHz}, \mathrm{CDCl}_{3}\right)$ ס: -79.96 .

HRMS (ESI): $\mathrm{m} / \mathrm{z}$ calcd. for $\mathrm{C}_{19} \mathrm{H}_{13} \mathrm{NOF}_{3}[\mathrm{M}-\mathrm{H}]$ ]: 328.0955; found: 328.0947. 


\section{Experimental Procedure and Characterization of Products}

\section{a. General Procedure to Prepare Racemic Products 3a-3aa}<smiles>[R]C#CC(O)(C#N)c1c[nH]c2cc[R1]cc12</smiles>

1<smiles>[H][R11]1c(O)c2c3c1C(CC2)C3</smiles>

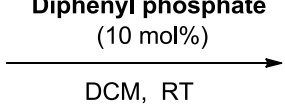

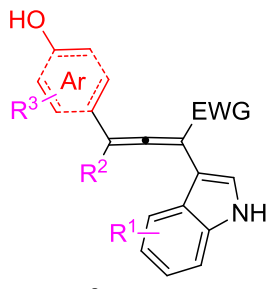

3

$$
\mathrm{Ph}-\mathrm{O}^{\mathrm{O}} \mathrm{P}-\mathrm{OH}-\mathrm{OH}
$$

Diphenyl phosphate

To a solution of $\alpha$-indolyl propargylic alcohols 1 ( $0.10 \mathrm{mmol}, 1.0$ equiv.), and catalyst diphenyl phosphate ( $0.01 \mathrm{mmol}, 10 \mathrm{~mol} \%)$ in DCM $(2 \mathrm{~mL})$ was added phenols $2(0.12 \mathrm{mmol}, 1.2$ equiv.). The mixture was stirred at room temperature until the reaction was completed (monitored by TLC analysis). The crude product was purified directly by flash column chromatography on silica gel (petroleum ether/ ethyl acetate $=5: 1 \sim 3: 1$ ) to give the desired racemic products $\mathbf{3} \mathbf{a}-\mathbf{3} \mathbf{a}$.

\section{b. General Procedure to Prepare Chiral Products 3a-3aa}<smiles>[R]C#CC(O)(C#N)c1c[nH]c2cc[R1]cc12</smiles>

1<smiles>[R17]c1ccc2c(O)c1CC2</smiles>

2

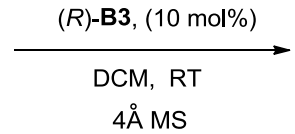

$4 \AA \mathrm{MS}$

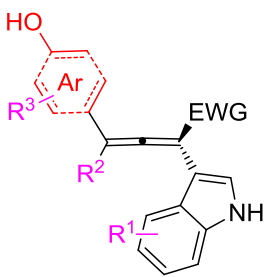

3

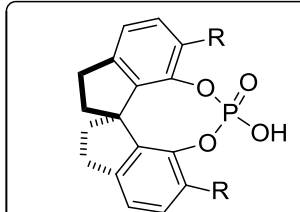

(R)-B3: R= 9-anthryl

To a solution of $\alpha$-indolyl propargylic alcohols 1 ( $0.10 \mathrm{mmol}, 1.0$ equiv.), $50 \mathrm{mg} 4 \AA$ molecular sieves, and catalyst $(\boldsymbol{R})$-B3 $(0.01 \mathrm{mmol}, 10 \mathrm{~mol} \%)$ in DCM $(2 \mathrm{~mL})$ was added phenols $2(0.12 \mathrm{mmol}, 1.2$ equiv.). The mixture was stirred at room temperature until the reaction was completed (monitored by TLC analysis). The crude product was purified directly by flash column chromatography on silica gel (petroleum ether/ ethyl acetate $=5: 1 \sim 3: 1)$ to give the desired chiral products 3a-3aa.

\section{c. Analytical Data for Products 3a-3aa}

(S)-4-(4,4,4-trifluoro-3-(1H-indol-3-yl)-1-phenylbuta-1,2-dien-1-yl)naphthalen-1-ol (3a):<smiles>Oc1ccc(C(=CC(c2ccccc2)C(F)(F)F)c2c[nH]c3ccccc23)c2ccccc12</smiles>

light brown oil, $42.4 \mathrm{mg}, 96 \%$ yield, $>99: 1 \mathrm{er},[a]_{D}^{20}=+31.62(\mathrm{c}=0.12, \mathrm{MeOH})$. purified by flash column chromatography on silica gel (petroleum ether/ ethyl acetate $=5: 1, \mathrm{Rf}=0.3$ ).

${ }^{1} \mathrm{H}$ NMR $\left(500 \mathrm{MHz}, \mathrm{CDCl}_{3}\right) \delta: 8.38-8.22(\mathrm{~m}, 2 \mathrm{H}), 7.93(\mathrm{~d}, \mathrm{~J}=8.2 \mathrm{~Hz}, 1 \mathrm{H}), 7.88(\mathrm{~d}, \mathrm{~J}=7.7 \mathrm{~Hz}, 1 \mathrm{H}), 7.55-7.23$ $(\mathrm{m}, 11 \mathrm{H}), 7.09(\mathrm{t}, \mathrm{J}=7.1 \mathrm{~Hz}, 1 \mathrm{H}), 6.85(\mathrm{~d}, \mathrm{~J}=7.0 \mathrm{~Hz}, 1 \mathrm{H}), 5.53(\mathrm{~s}, 1 \mathrm{H})$.

${ }^{13} \mathrm{C}$ NMR $\left(126 \mathrm{MHz}, \mathrm{CDCl}_{3}\right.$ ) $\delta: 205.4$ (q, J = $\left.2.9 \mathrm{~Hz}\right), 152.2,136.2,135.8,133.0,128.9,128.3,128.2,127.7$, 127.0, 126.4, 125.7, 125.5, 125.2, 124.9, 123.9 (q, J = 275.6 Hz), 123.4 (d, J = 2.3 Hz), 123.1, 122.2, 120.8, $120.1,115.6,111.5,108.5,104.8,98.3(q, J=35.4 \mathrm{~Hz})$. 
${ }^{19}$ F NMR (376 MHz, $\left.\mathrm{CDCl}_{3}\right) \delta:-61.33$.

HRMS (ESI): $m / z$ calcd. for $\mathrm{C}_{28} \mathrm{H}_{19} \mathrm{NOF}_{3}[\mathrm{M}+\mathrm{H}]^{+}:$: 442.1413; found: 442.1416 .

HPLC analysis: The enantiomeric excess was determined by HPLC with Chiralpak IA column (hexane/i-PrOH $=90 / 10$, flow rate $\left.1 \mathrm{~mL} \cdot \mathrm{min}^{-1}, \lambda=254 \mathrm{~nm}\right): t_{\text {major }}=15.6 \mathrm{~min}, \mathrm{t}_{\text {minor }}=19.1 \mathrm{~min}$.

(S)-4-(3-(4-chloro-1H-indol-3-yl)-4,4,4-trifluoro-1-phenylbuta-1,2-dien-1-yl)naphthalen-1-ol (3b):

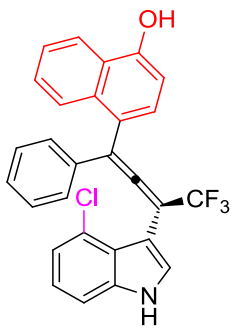

light brown oil, $29.5 \mathrm{mg}, 62 \%$ yield, $98: 2 \mathrm{er},[a]_{D}^{20}=-43.85(\mathrm{c}=0.10, \mathrm{MeOH})$. purified by flash column chromatography on silica gel (petroleum ether/ ethyl acetate $=5: 1, \mathrm{Rf}=0.3$ ).

${ }^{1} \mathrm{H}$ NMR $\left(400 \mathrm{MHz}, \mathrm{CDCl}_{3}\right) \delta: 8.30(\mathrm{~s}, 1 \mathrm{H}), 8.18(\mathrm{~d}, \mathrm{~J}=8.4 \mathrm{~Hz}, 1 \mathrm{H}), 7.65(\mathrm{~d}, \mathrm{~J}=8.5 \mathrm{~Hz}, 1 \mathrm{H}), 7.39(\mathrm{t}, \mathrm{J}=7.6 \mathrm{~Hz}$, $1 \mathrm{H}), 7.29-7.18(\mathrm{~m}, 8 \mathrm{H}), 7.16(\mathrm{~d}, \mathrm{~J}=2.6 \mathrm{~Hz}, 1 \mathrm{H}), 7.06-6.99(\mathrm{~m}, 2 \mathrm{H}), 6.78(\mathrm{~d}, \mathrm{~J}=7.7 \mathrm{~Hz}, 1 \mathrm{H}), 5.55(\mathrm{~s}, 1 \mathrm{H})$.

${ }^{13} \mathrm{C}$ NMR $\left(126 \mathrm{MHz}, \mathrm{CDCl}_{3}\right)$ ס: 207.2, 152.1, 137.0, 135.3, 133.0, 128.6, 128.3, 128.1, 127.8, 126.8, 126.4, 126.2, 126.1, 125.3, 124.7, 124.4, 124.2, 123.3 (q, J = 274.4 Hz), 123.2, 121.9, 121.6, 112.6, 110.0, 108.3, $105.1,95.6$ (q, $\mathrm{J}=36.5 \mathrm{~Hz})$.

${ }^{19} \mathrm{~F}$ NMR $\left(376 \mathrm{MHz}, \mathrm{CDCl}_{3}\right) \delta:-62.71$.

HRMS (ESI): $m / z$ calcd. for $\mathrm{C}_{28} \mathrm{H}_{18} \mathrm{NOF}_{3} \mathrm{Cl}[\mathrm{M}+\mathrm{H}]^{+}: 476.1024$; found: 476.1028 .

HPLC analysis: The enantiomeric excess was determined by HPLC with Chiralpak IA column (hexane/i-PrOH $=90 / 10$, flow rate $\left.1 \mathrm{~mL} \cdot \mathrm{min}^{-1}, \lambda=254 \mathrm{~nm}\right): t_{\text {major }}=14.2 \mathrm{~min}, \mathrm{t}_{\text {minor }}=11.6 \mathrm{~min}$.

(S)-4-(4,4,4-trifluoro-3-(5-fluoro-1 H-indol-3-yl)-1-phenylbuta-1,2-dien-1-yl)naphthalen-1-ol (3c):<smiles>Oc1ccc(C(=CC(=C(c2ccccc2)C(F)(F)F)c2c[nH]c3ccc(F)cc23)c2ccccc2)c2ccccc12</smiles>

light brown oil, $45.0 \mathrm{mg}, 98 \%$ yield, $99: 1 \mathrm{er},[a]_{D}^{20}=+25.00(\mathrm{c}=0.10, \mathrm{MeOH})$. purified by flash column chromatography on silica gel (petroleum ether/ ethyl acetate $=5: 1, \mathrm{Rf}=0.3$ ).

${ }^{1} \mathrm{H}$ NMR $\left(400 \mathrm{MHz}, \mathrm{CDCl}_{3}\right)$ ס: $8.34(\mathrm{~s}, 1 \mathrm{H}), 8.29(\mathrm{~d}, \mathrm{~J}=8.4 \mathrm{~Hz}, 1 \mathrm{H}), 7.93(\mathrm{~d}, \mathrm{~J}=8.3 \mathrm{~Hz}, 1 \mathrm{H}), 7.55-7.50(\mathrm{~m}, 2 \mathrm{H})$, 7.47 (dd, J = 8.1, $2.1 \mathrm{~Hz}, 3 \mathrm{H}), 7.40-7.29(\mathrm{~m}, 6 \mathrm{H}), 6.98(\mathrm{td}, \mathrm{J}=8.9,2.5 \mathrm{~Hz}, 1 \mathrm{H}), 6.90(\mathrm{~d}, \mathrm{~J}=7.7 \mathrm{~Hz}, 1 \mathrm{H}), 5.68$ $(\mathrm{s}, 1 \mathrm{H})$.

${ }^{13}$ C NMR (126 MHz, $\left.\mathrm{CDCl}_{3}\right)$ ס: 205.0, $158.3(\mathrm{~d}, \mathrm{~J}=236.1 \mathrm{~Hz}), 152.3,135.5,132.8,132.6,128.9,128.3,128.1$, 127.6, 127.1, 126.1, 126.0, 125.9, 125.5, 124.9, 124.8, 123.8 (q, J = 276.3 Hz), 122.1, 115.9, 112.0 (d, J = 9.7 $\mathrm{Hz}$ ), 111.4 (d, J = 26.4 Hz), 108.4, 105.2 (d, J = 25.0 Hz), 104.8 (d, J = 4.5 Hz), 97.9 (q, J = 35.9 Hz).

${ }^{19} \mathrm{~F}$ NMR $\left(471 \mathrm{MHz}, \mathrm{CDCl}_{3}\right) \delta:-61.35,-122.58$.

HRMS (ESI): $m / z$ calcd. for $\mathrm{C}_{28} \mathrm{H}_{18} \mathrm{NOF}_{4}[\mathrm{M}+\mathrm{H}]^{+}: 460.1319$; found: 460.1324 .

HPLC analysis: The enantiomeric excess was determined by HPLC with Chiralpak IC column (hexane/i-PrOH $=90 / 10$, flow rate $\left.1 \mathrm{~mL} \cdot \mathrm{min}^{-1}, \lambda=254 \mathrm{~nm}\right): t_{\text {major }}=13.0 \mathrm{~min}, \mathrm{t}_{\text {minor }}=16.0 \mathrm{~min}$. 
(S)-4-(3-(5-chloro-1 H-indol-3-yl)-4,4,4-trifluoro-1-phenylbuta-1,2-dien-1-yl)naphthalen-1-ol (3d):<smiles>Oc1ccc(C(=CC(=C(c2ccccc2)c2c[nH]c3ccc(Cl)cc23)C(F)(F)F)c2ccccc2)c2ccccc12</smiles>

light brown oil, $45.7 \mathrm{mg}, 96 \%$ yield, 99:1 er, $[a]_{D}^{20}=-28.00(\mathrm{c}=0.16, \mathrm{MeOH})$. purified by flash column chromatography on silica gel (petroleum ether/ ethyl acetate $=5: 1, \mathrm{Rf}=0.3$ ).

${ }^{1} \mathrm{H}$ NMR $\left(500 \mathrm{MHz}, \mathrm{CDCl}_{3}\right) \delta: 8.30(\mathrm{~s}, 1 \mathrm{H}), 8.25(\mathrm{~d}, \mathrm{~J}=8.1 \mathrm{~Hz}, 1 \mathrm{H}), 7.90(\mathrm{~d}, \mathrm{~J}=8.1 \mathrm{~Hz}, 1 \mathrm{H}), 7.80(\mathrm{~s}, 1 \mathrm{H}), 7.50-$ $7.44(\mathrm{~m}, 3 \mathrm{H}), 7.39-7.22(\mathrm{~m}, 7 \mathrm{H}), 7.12(\mathrm{~d}, \mathrm{~J}=8.5 \mathrm{~Hz}, 1 \mathrm{H}), 6.88(\mathrm{~d}, \mathrm{~J}=7.3 \mathrm{~Hz}, 1 \mathrm{H}), 5.71(\mathrm{~s}, 1 \mathrm{H})$.

${ }^{13} \mathrm{C}$ NMR $\left(126 \mathrm{MHz}, \mathrm{CDCl}_{3}\right)$ ס: 205.2, 152.3, 135.5, 134.4, 132.8, 128.8, 128.3, 128.1, 127.6, 127.3, 126.6, $126.5,126.1,125.5,124.9,124.8,124.4,123.6$ (q, $\mathrm{J}=275.3 \mathrm{~Hz}$ ), 123.3, 122.1, 119.6, 116.0, 112.3, 108.4, 104.5, 97.6 (q, J = 35.7 Hz).

${ }^{19}$ F NMR $\left(376 \mathrm{MHz}, \mathrm{CDCl}_{3}\right) \delta:-61.45$.

HRMS (ESI): $m / z$ calcd. for $\mathrm{C}_{28} \mathrm{H}_{18} \mathrm{NOF}_{3} \mathrm{Cl}[\mathrm{M}+\mathrm{H}]^{+}:$476.1024; found: 476.1022 .

HPLC analysis: The enantiomeric excess was determined by HPLC with Chiralpak IA column (hexane/i-PrOH $=90 / 10$, flow rate $\left.1 \mathrm{~mL} \cdot \mathrm{min}^{-1}, \lambda=254 \mathrm{~nm}\right): \mathrm{t}_{\text {major }}=12.5 \mathrm{~min}, \mathrm{t}_{\text {minor }}=16.5 \mathrm{~min}$.

(S)-4-(3-(5-bromo-1H-indol-3-yl)-4,4,4-trifluoro-1-phenylbuta-1,2-dien-1-yl)naphthalen-1-ol (3e):<smiles>Oc1ccc(C(=CC(=C(c2ccccc2)c2c[nH]c3ccc(Br)cc23)C(F)(F)F)C(F)(F)F)c2ccccc12</smiles>

light brown oil, $46.8 \mathrm{mg}, 90 \%$ yield, $98: 2 \mathrm{er},[a]_{D}^{20}=+15.55(\mathrm{c}=0.10, \mathrm{MeOH})$. purified by flash column chromatography on silica gel (petroleum ether/ ethyl acetate $=5: 1, \mathrm{Rf}=0.3$ ).

${ }^{1} \mathrm{H}$ NMR $\left(500 \mathrm{MHz}, \mathrm{CDCl}_{3}\right) \delta: 8.24(\mathrm{~d}, \mathrm{~J}=5.9 \mathrm{~Hz}, 2 \mathrm{H}), 7.96(\mathrm{~s}, 1 \mathrm{H}), 7.90(\mathrm{~d}, \mathrm{~J}=7.9 \mathrm{~Hz}, 1 \mathrm{H}), 7.50-7.44(\mathrm{~m}, 3 \mathrm{H})$, $7.38-7.23(\mathrm{~m}, 7 \mathrm{H}), 7.17(\mathrm{~d}, \mathrm{~J}=8.4 \mathrm{~Hz}, 1 \mathrm{H}), 6.88(\mathrm{~d}, \mathrm{~J}=7.5 \mathrm{~Hz}, 1 \mathrm{H}), 5.49(\mathrm{~s}, 1 \mathrm{H})$.

${ }^{13} \mathrm{C}$ NMR $\left(126 \mathrm{MHz}, \mathrm{CDCl}_{3}\right) \delta:$ 205.2, 152.2, 135.5, 134.7, 132.9, 128.9, 128.4, 128.1, 127.6, 127.4, 127.2, 126.1, 125.9, 125.5, 124.9, 124.8, 124.2 (d, J = 2.2 Hz), 123.6 (q, J = 275.2 Hz), 122.7, 122.1, 116.0, 114.1, $112.7,108.4,104.5,97.6$ (q, J = 35.5 Hz).

${ }^{19}$ F NMR $\left(471 \mathrm{MHz}, \mathrm{CDCl}_{3}\right) \delta:-61.45$.

HRMS (ESI): $\mathrm{m} / z$ calcd. for $\mathrm{C}_{28} \mathrm{H}_{18} \mathrm{NOF}_{3} \mathrm{Br}[\mathrm{M}+\mathrm{H}]^{+}: 520.0518$; found: 520.0524 .

HPLC analysis: The enantiomeric excess was determined by HPLC with Chiralpak IA column (hexane/i-PrOH $=90 / 10$, flow rate $\left.1 \mathrm{~mL} \cdot \mathrm{min}^{-1}, \lambda=254 \mathrm{~nm}\right): t_{\text {major }}=12.3 \mathrm{~min}, \mathrm{t}_{\text {minor }}=16.8 \mathrm{~min}$.

(S)-4-(4,4,4-trifluoro-3-(5-methyl-1 $H$-indol-3-yl)-1-phenylbuta-1,2-dien-1-yl)naphthalen-1-ol (3f): 
<smiles>Cc1ccc2[nH]cc(C(=C=C(c3ccccc3)c3ccc(O)c4ccccc34)C(F)(F)F)c2c1</smiles>

light brown oil, $44.6 \mathrm{mg}, 98 \%$ yield, $98: 2 \mathrm{er},[a]_{D}^{20}=-31.25(\mathrm{c}=0.14, \mathrm{MeOH})$. purified by flash column chromatography on silica gel (petroleum ether/ ethyl acetate $=5: 1, \mathrm{Rf}=0.3$ ).

${ }^{1} \mathrm{H}$ NMR $\left(400 \mathrm{MHz}, \mathrm{CDCl}_{3}\right) \delta: 8.29(\mathrm{~d}, \mathrm{~J}=8.1 \mathrm{~Hz}, 1 \mathrm{H}), 8.22(\mathrm{~s}, 1 \mathrm{H}), 7.94(\mathrm{~d}, \mathrm{~J}=8.4 \mathrm{~Hz}, 1 \mathrm{H}), 7.59(\mathrm{~s}, 1 \mathrm{H}), 7.54-$ $7.49(\mathrm{~m}, 2 \mathrm{H}), 7.46-7.42(\mathrm{~m}, 1 \mathrm{H}), 7.40-7.26(\mathrm{~m}, 7 \mathrm{H}), 7.05(\mathrm{~d}, \mathrm{~J}=8.3 \mathrm{~Hz}, 1 \mathrm{H}), 6.88(\mathrm{~d}, \mathrm{~J}=7.7 \mathrm{~Hz}, 1 \mathrm{H}), 5.57(\mathrm{~s}$, $1 \mathrm{H}), 2.23(\mathrm{~s}, 3 \mathrm{H})$.

${ }^{13} \mathrm{C}$ NMR $\left(126 \mathrm{MHz}, \mathrm{CDCl}_{3}\right)$ ठ: 205.7, 152.2, 136.0, 134.5, 133.0, 130.1, 128.9, 128.3, 128.2, 127.7, 127.1, 126.4, 126.0, 125.5, 125.3, 125.0, 124.6, 123.9 (q, J = 270.6 Hz), 123.4 (d, J = 2.1 Hz), 122.2, 119.8, 115.6, 111.0, 108.5, 104.2, 98.2 (q, J = 35.2 Hz), 21.5.

${ }^{19}$ F NMR (376 MHz, $\left.\mathrm{CDCl}_{3}\right) \delta:-61.43$.

HRMS (ESI): $m / z$ calcd. for $\mathrm{C}_{29} \mathrm{H}_{21} \mathrm{NOF}_{3}[\mathrm{M}+\mathrm{H}]^{+}:$: 456.1570; found: 456.1564 .

HPLC analysis: The enantiomeric excess was determined by HPLC with Chiralpak IA column (hexane/i-PrOH $=90 / 10$, flow rate $\left.1 \mathrm{~mL} \cdot \mathrm{min}^{-1}, \lambda=285 \mathrm{~nm}\right): \mathrm{t}_{\text {major }}=12.3 \mathrm{~min}, \mathrm{t}_{\text {minor }}=16.5 \mathrm{~min}$.

(S)-4-(4,4,4-trifluoro-3-(5-methoxy-1H-indol-3-yl)-1-phenylbuta-1,2-dien-1-yl)naphthalen-1-ol (3g):<smiles>COc1ccc2[nH]cc(C(=CC(=C(c3ccccc3)c3ccc(O)c4ccccc34)C(F)(F)F)C(F)(F)F)c2c1</smiles>

light brown oil, $45.3 \mathrm{mg}, 96 \%$ yield, $98: 2 \mathrm{er},[a]_{D}^{20}=+101.54(\mathrm{c}=0.13, \mathrm{MeOH})$. purified by flash column chromatography on silica gel (petroleum ether/ ethyl acetate $=5: 1, \mathrm{Rf}=0.3$ ).

${ }^{1} \mathrm{H}$ NMR $\left(400 \mathrm{MHz}, \mathrm{CDCl}_{3}\right) \delta: 8.23(\mathrm{~d}, \mathrm{~J}=8.4 \mathrm{~Hz}, 2 \mathrm{H}), 7.85(\mathrm{~d}, \mathrm{~J}=8.4 \mathrm{~Hz}, 1 \mathrm{H}), 7.50-7.44(\mathrm{~m}, 1 \mathrm{H}), 7.42-7.19$ (m, 9H), $7.14(\mathrm{~s}, 1 \mathrm{H}), 6.91-6.75(\mathrm{~m}, 2 \mathrm{H}), 5.53(\mathrm{~s}, 1 \mathrm{H}), 3.29(\mathrm{~s}, 3 \mathrm{H})$.

${ }^{13} \mathrm{C}$ NMR $\left(126 \mathrm{MHz}, \mathrm{CDCl}_{3}\right) \delta: 205.3,154.9,152.3,136.1,133.0,131.1,128.9,128.4,128.3,127.8,127.1$, 127.0, 126.3, 125.5, 125.0, 124.9, 123.9 (q, J = 275.7 Hz), 123.7 (d, J = 2.3 Hz), 122.2, 115.5, 113.9, 112.3, 108.4, 104.6, 101.0, 98.3 (q, J = 35.5 Hz), 55.2.

${ }^{19}$ F NMR $\left(376 \mathrm{MHz}, \mathrm{CDCl}_{3}\right) \delta:-61.59$.

HRMS (ESI): $\mathrm{m} / \mathrm{z}$ calcd. for $\mathrm{C}_{29} \mathrm{H}_{21} \mathrm{NO}_{2} \mathrm{~F}_{3}[\mathrm{M}+\mathrm{H}]^{+}:$472.1519; found: 472.1515 .

HPLC analysis: The enantiomeric excess was determined by HPLC with Chiralpak IA column (hexane/i-PrOH $=90 / 10$, flow rate $\left.1 \mathrm{~mL} \cdot \mathrm{min}^{-1}, \lambda=254 \mathrm{~nm}\right): \mathrm{t}_{\text {major }}=15.9 \mathrm{~min}, \mathrm{t}_{\text {minor }}=21.8 \mathrm{~min}$.

(S)-4-(3-(6-chloro-1H-indol-3-yl)-4,4,4-trifluoro-1-phenylbuta-1,2-dien-1-yl)naphthalen-1-ol (3h): 
<smiles>Oc1ccc(C(=C(c2ccccc2)C(F)(F)F)c2c[nH]c3cc(Cl)ccc23)c2ccccc12</smiles>

light brown oil, $45.7 \mathrm{mg}, 96 \%$ yield, $98: 2 \mathrm{er},[a]_{D}^{20}=+36.68(\mathrm{c}=0.10, \mathrm{MeOH})$. purified by flash column chromatography on silica gel (petroleum ether/ ethyl acetate $=5: 1, \mathrm{Rf}=0.3$ ).

${ }^{1} \mathrm{H} \mathrm{NMR}\left(400 \mathrm{MHz}, \mathrm{CDCl}_{3}\right)$ ס: $8.26(\mathrm{~d}, \mathrm{~J}=7.8 \mathrm{~Hz}, 2 \mathrm{H}), 7.86(\mathrm{~d}, \mathrm{~J}=8.4 \mathrm{~Hz}, 1 \mathrm{H}), 7.71(\mathrm{~d}, \mathrm{~J}=8.6 \mathrm{~Hz}, 1 \mathrm{H}), 7.51(\mathrm{t}$, $J=7.6 \mathrm{~Hz}, 1 \mathrm{H}), 7.46-7.40(\mathrm{~m}, 2 \mathrm{H}), 7.39-7.28(\mathrm{~m}, 7 \mathrm{H}), 7.01(\mathrm{dd}, \mathrm{J}=8.6,1.8 \mathrm{~Hz}, 1 \mathrm{H}), 6.85(\mathrm{~d}, \mathrm{~J}=7.7 \mathrm{~Hz}, 1 \mathrm{H})$, $5.50(\mathrm{~s}, 1 \mathrm{H})$.

${ }^{13} \mathrm{C}$ NMR (126 MHz, $\mathrm{CDCl}_{3}$ ) $\delta: 205.2,152.2,136.5,135.4,132.8,129.0,128.9,128.4,128.1,127.6,127.0$, 126.1, 125.5, 124.8, 124.2, $123.8(\mathrm{~d}, \mathrm{~J}=2.3 \mathrm{~Hz}), 123.7,123.6(\mathrm{q}, \mathrm{J}=275.4 \mathrm{~Hz}), 122.1,121.4,120.8,115.7$, 111.3, 108.4, 105.0, $97.8(\mathrm{q}, \mathrm{J}=35.4 \mathrm{~Hz})$.

${ }^{19}$ F NMR $\left(376 \mathrm{MHz}, \mathrm{CDCl}_{3}\right) \delta:-61.44$.

HRMS (ESI): $\mathrm{m} / z$ calcd. for $\mathrm{C}_{28} \mathrm{H}_{18} \mathrm{NOF}_{3} \mathrm{Cl}[\mathrm{M}+\mathrm{H}]^{+}: 476.1024$; found: 476.1022 .

HPLC analysis: The enantiomeric excess was determined by HPLC with Chiralpak IA column (hexane/i-PrOH $=90 / 10$, flow rate $1 \mathrm{~mL} \cdot \mathrm{min}^{-1}, \lambda=254 \mathrm{~nm}$ ): $\mathrm{t}_{\text {major }}=13.4 \mathrm{~min}, \mathrm{t}_{\text {minor }}=16.8 \mathrm{~min}$.

(S)-4-(3-(7-chloro-1 H-indol-3-yl)-4,4,4-trifluoro-1-phenylbuta-1,2-dien-1-yl)naphthalen-1-ol (3i):<smiles>Oc1ccc(C(=C(c2ccccc2)c2ccccc2Cl)c2c[nH]c3c(Cl)cccc23)c2ccccc12</smiles>

light brown oil, $32.4 \mathrm{mg}, 68 \%$ yield, $97: 3 \mathrm{er},[a]_{D}^{20}=+20.56(\mathrm{c}=0.10, \mathrm{MeOH})$. purified by flash column chromatography on silica gel (petroleum ether/ ethyl acetate $=10: 1, \mathrm{Rf}=0.2$ ).

${ }^{1} \mathrm{H}$ NMR $\left(500 \mathrm{MHz}, \mathrm{CDCl}_{3}\right)$ ס: $8.55(\mathrm{~s}, 1 \mathrm{H}), 8.28(\mathrm{~d}, \mathrm{~J}=7.3 \mathrm{~Hz}, 1 \mathrm{H}), 7.87(\mathrm{~d}, \mathrm{~J}=7.7 \mathrm{~Hz}, 1 \mathrm{H}), 7.72(\mathrm{~d}, \mathrm{~J}=5.8 \mathrm{~Hz}$, $1 \mathrm{H}), 7.56-7.21(\mathrm{~m}, 10 \mathrm{H}), 6.99(\mathrm{t}, \mathrm{J}=7.7 \mathrm{~Hz}, 1 \mathrm{H}), 6.88(\mathrm{~d}, \mathrm{~J}=7.4 \mathrm{~Hz}, 1 \mathrm{H}), 5.46(\mathrm{~s}, 1 \mathrm{H})$.

${ }^{13} \mathrm{C}$ NMR $\left(126 \mathrm{MHz}, \mathrm{CDCl}_{3}\right)$ ס: 205.3 (q, J = $\left.3.6 \mathrm{~Hz}\right), 152.2,135.4,133.5,132.8,128.8,128.3,128.1,127.6$, 127.0, 126.9, 126.1, 125.5, 124.8, 123.7, 123.7 (q, J = 290.5 Hz), 123.7, 122.4, 122.1, 121.5, 118.6, 116.9, 115.7, 108.4, 106.0, 97.8 (q, J = 36.6 Hz).

${ }^{19} \mathrm{~F} \mathrm{NMR}\left(471 \mathrm{MHz}, \mathrm{CDCl}_{3}\right) \delta:-61.47$.

HRMS (ESI): $\mathrm{m} / z$ calcd. for $\mathrm{C}_{28} \mathrm{H}_{18} \mathrm{NOF}_{3} \mathrm{Cl}[\mathrm{M}+\mathrm{H}]^{+}: 476.1024$; found: 476.1024 .

HPLC analysis: The enantiomeric excess was determined by HPLC with Chiralpak IA column (hexane/i-PrOH $=90 / 10$, flow rate $1 \mathrm{~mL} \cdot \mathrm{min}^{-1}, \lambda=285 \mathrm{~nm}$ ): $\mathrm{t}_{\text {major }}=9.4 \mathrm{~min}, \mathrm{t}_{\text {minor }}=10.9 \mathrm{~min}$.

(S)-4-(4,4,4-trifluoro-1-(4-fluorophenyl)-3-(1 H-indol-3-yl)buta-1,2-dien-1-yl)naphthalen-1-ol (3j): 
<smiles>Oc1ccc(C(=C(c2c[nH]c3ccccc23)C(F)(F)F)c2ccc(F)cc2)c2ccccc12</smiles>

light brown oil, $41.8 \mathrm{mg}, 91 \%$ yield, $99: 1 \mathrm{er},[a]_{D}^{20}=+54.00(\mathrm{c}=0.10, \mathrm{MeOH})$. purified by flash column chromatography on silica gel (petroleum ether/ ethyl acetate $=5: 1, \mathrm{Rf}=0.3$ ).

${ }^{1} \mathrm{H}$ NMR $\left(400 \mathrm{MHz}, \mathrm{CDCl}_{3}\right) \delta: 8.27(\mathrm{~s}, 1 \mathrm{H}), 8.23(\mathrm{~d}, \mathrm{~J}=7.9 \mathrm{~Hz}, 1 \mathrm{H}), 7.84(\mathrm{~d}, \mathrm{~J}=8.3 \mathrm{~Hz}, 1 \mathrm{H}), 7.79(\mathrm{~d}, \mathrm{~J}=8.0 \mathrm{~Hz}$, $1 \mathrm{H}), 7.48(\mathrm{t}, \mathrm{J}=7.6 \mathrm{~Hz}, 1 \mathrm{H}), 7.45-7.34(\mathrm{~m}, 4 \mathrm{H}), 7.33-7.28(\mathrm{~m}, 2 \mathrm{H}), 7.25-7.19(\mathrm{~m}, 1 \mathrm{H}), 7.05(\mathrm{t}, \mathrm{J}=7.6 \mathrm{~Hz}$, $1 \mathrm{H}), 6.97(\mathrm{t}, \mathrm{J}=8.7 \mathrm{~Hz}, 2 \mathrm{H}), 6.81(\mathrm{~d}, \mathrm{~J}=7.7 \mathrm{~Hz}, 1 \mathrm{H}), 5.44(\mathrm{~s}, 1 \mathrm{H})$.

${ }^{13} \mathrm{C}$ NMR $\left(126 \mathrm{MHz}, \mathrm{CDCl}_{3}\right) \delta: 205.1,162.8(\mathrm{~d}, \mathrm{~J}=248.3 \mathrm{~Hz}), 152.3,136.2,132.8,131.9(\mathrm{~d}, \mathrm{~J}=3.2 \mathrm{~Hz}), 129.4$ $(\mathrm{d}, \mathrm{J}=8.2 \mathrm{~Hz}$ ), 128.2, 127.1, 126.2, 125.7, 125.6, 125.0, 124.9, 123.8 (q, J = 276.6 Hz), 123.4 (d, J = 2.4 Hz), 123.1, 122.2, 120.9, 120.0, $115.9(\mathrm{~d}, \mathrm{~J}=21.8 \mathrm{~Hz}), 114.7,111.6,108.5,104.7,98.4$ (q, J = 35.4 Hz).

${ }^{19}$ F NMR $\left(376 \mathrm{MHz}, \mathrm{CDCl}_{3}\right) \mathrm{\delta}:-61.37(\mathrm{~d}, \mathrm{~J}=9.5 \mathrm{~Hz}),-113.53$.

HRMS (ESI): $m / z$ calcd. for $\mathrm{C}_{28} \mathrm{H}_{18} \mathrm{NOF}_{4}[\mathrm{M}+\mathrm{H}]^{+}: 460.1319$; found: 460.1318 .

HPLC analysis: The enantiomeric excess was determined by HPLC with Chiralpak IA column (hexane/i-PrOH $=90 / 10$, flow rate $\left.1 \mathrm{~mL} \cdot \mathrm{min}^{-1}, \lambda=285 \mathrm{~nm}\right): t_{\text {major }}=15.8 \mathrm{~min}, t_{\text {minor }}=17.7 \mathrm{~min}$.

(S)-4-(1-(4-chlorophenyl)-4,4,4-trifluoro-3-(1H-indol-3-yl)buta-1,2-dien-1-yl)naphthalen-1-ol (3k):<smiles>Oc1ccc(C(=CC(=C2C=CCC2)c2ccc(Cl)cc2)C(F)(F)F)c2ccccc12</smiles>

light brown oil, $39.0 \mathrm{mg}, 82 \%$ yield, $>99: 1 \mathrm{er},[a]_{D}^{20}=-19.76(\mathrm{c}=0.10, \mathrm{MeOH})$. purified by flash column chromatography on silica gel (petroleum ether/ ethyl acetate $=5: 1, \mathrm{Rf}=0.3$ ).

${ }^{1} \mathrm{H}$ NMR $\left(400 \mathrm{MHz}, \mathrm{CDCl}_{3}\right)$ ס: $8.34(\mathrm{~s}, 1 \mathrm{H}), 8.25(\mathrm{~d}, \mathrm{~J}=8.2 \mathrm{~Hz}, 1 \mathrm{H}), 7.81(\mathrm{~d}, \mathrm{~J}=15.5 \mathrm{~Hz}, 1 \mathrm{H}), 7.79(\mathrm{~d}, \mathrm{~J}=15.2$ $\mathrm{Hz}, 1 \mathrm{H}), 7.51-7.37(\mathrm{~m}, 5 \mathrm{H}), 7.33-7.18(\mathrm{~m}, 5 \mathrm{H}), 7.05(\mathrm{t}, \mathrm{J}=7.6 \mathrm{~Hz}, 1 \mathrm{H}), 6.82(\mathrm{~d}, \mathrm{~J}=7.7 \mathrm{~Hz}, 1 \mathrm{H}), 5.81(\mathrm{~s}, 1 \mathrm{H})$. ${ }^{13} \mathrm{C}$ NMR $\left(101 \mathrm{MHz}, \mathrm{CDCl}_{3}\right) \delta: 205.4(\mathrm{q}, \mathrm{J}=3.7 \mathrm{~Hz}), 152.5,136.2,134.4,134.2,132.8,129.1,129.0,128.3$, 127.1, 126.1, 125.6, 125.0, 124.6, 123.8 (q, J = 271.8 Hz), 123.6, 123.5 (d, J = 2.5 Hz), 123.1, 122.3, 120.9, 119.9, 114.8, 111.6, 108.5, 104.5, 98.6 (q, J = 35.3 Hz).

${ }^{19}$ F NMR $\left(376 \mathrm{MHz}, \mathrm{CDCl}_{3}\right) \delta:-61.31$.

HRMS (ESI): $\mathrm{m} / z$ calcd. for $\mathrm{C}_{28} \mathrm{H}_{18} \mathrm{NOF}_{3} \mathrm{Cl}[\mathrm{M}+\mathrm{H}]^{+}: 476.1024$; found: 476.1026 .

HPLC analysis: The enantiomeric excess was determined by HPLC with Chiralpak IA column (hexane/ $i$-PrOH $=90 / 10$, flow rate $\left.1 \mathrm{~mL} \cdot \mathrm{min}^{-1}, \lambda=254 \mathrm{~nm}\right): t_{\text {major }}=12.9 \mathrm{~min}, t_{\text {minor }}=14.8 \mathrm{~min}$.

(S)-4-(4,4,4-trifluoro-3-(1 H-indol-3-yl)-1-(p-tolyl)buta-1,2-dien-1-yl)naphthalen-1-ol (3I): 
<smiles>Cc1ccc(C(=CC(F)(C(F)(F)F)C(F)(F)F)c2c[nH]c3ccccc23)cc1</smiles>

light brown oil, $44.6 \mathrm{mg}, 98 \%$ yield, 99:1 er, $[a]_{D}^{20}=+15.69(\mathrm{c}=0.10, \mathrm{MeOH})$. purified by flash column chromatography on silica gel (petroleum ether/ ethyl acetate $=5: 1, \mathrm{Rf}=0.3$ ).

${ }^{1} \mathrm{H}$ NMR $\left(400 \mathrm{MHz}, \mathrm{CDCl}_{3}\right)$ ס: $8.29-8.18(\mathrm{~m}, 2 \mathrm{H}), 7.87$ (dd, J = 18.7, 8.1 Hz, 2H), $7.47(\mathrm{t}, \mathrm{J}=7.6 \mathrm{~Hz}, 1 \mathrm{H}), 7.42$ - $7.32(\mathrm{~m}, 4 \mathrm{H}), 7.25-7.17(\mathrm{~m}, 3 \mathrm{H}), 7.10-7.00(\mathrm{~m}, 3 \mathrm{H}), 6.80(\mathrm{~d}, \mathrm{~J}=7.7 \mathrm{~Hz}, 1 \mathrm{H}), 5.39(\mathrm{~s}, 1 \mathrm{H}), 2.32(\mathrm{~s}, 3 \mathrm{H})$.

${ }^{13} \mathrm{C} \mathrm{NMR}\left(126 \mathrm{MHz}, \mathrm{CDCl}_{3}\right)$ ס: 205.1 (q, J = $\left.3.3 \mathrm{~Hz}\right), 152.1,138.3,136.2,133.0,132.8,129.7,128.1,127.7$, 127.0, 126.4, 125.7, 125.5, 125.4, 124.9, 123.9 (q, J = 263.9 Hz), 123.3 (d, J = 2.4 Hz), 123.0, 122.1, 120.8, 120.2, 115.4, 111. 5, 108.5, 105.0, $98.1(\mathrm{q}, \mathrm{J}=35.1 \mathrm{~Hz}), 21.4$.

${ }^{19} \mathrm{~F}$ NMR $\left(376 \mathrm{MHz}, \mathrm{CDCl}_{3}\right) \delta:-61.33$.

HRMS (ESI): $\mathrm{m} / z$ calcd. for $\mathrm{C}_{29} \mathrm{H}_{21} \mathrm{NOF}_{3}[\mathrm{M}+\mathrm{H}]^{+}: 456.1570$; found: 456.1564 .

HPLC analysis: The enantiomeric excess was determined by HPLC with Chiralpak IA column (hexane/ $i-\mathrm{PrOH}$ $=90 / 10$, flow rate $\left.1 \mathrm{~mL} \cdot \mathrm{min}^{-1}, \lambda=285 \mathrm{~nm}\right): t_{\text {major }}=14.2 \mathrm{~min}, t_{\operatorname{minor}}=17.0 \mathrm{~min}$.

(S)-4-(4,4,4-trifluoro-3-(1 H-indol-3-yl)-1-(4-methoxyphenyl)buta-1,2-dien-1-yl)naphthalen-1-ol (3m):<smiles>COc1ccc(C(=CC(F)(C(F)(F)F)C(F)(F)F)c2c[nH]c3ccccc23)cc1</smiles>

light brown oil, $40.5 \mathrm{mg}, 86 \%$ yield, 99:1 er, $[a]_{D}^{20}=+12.32(\mathrm{c}=0.12, \mathrm{MeOH})$. purified by flash column chromatography on silica gel (petroleum ether/ ethyl acetate $=10: 1, \mathrm{Rf}=0.4$ ).

${ }^{1} \mathrm{H} \mathrm{NMR}\left(400 \mathrm{MHz}, \mathrm{CDCl}_{3}\right)$ ס: $8.31(\mathrm{~s}, 1 \mathrm{H}), 8.24(\mathrm{~d}, \mathrm{~J}=8.4 \mathrm{~Hz}, 1 \mathrm{H}), 7.91(\mathrm{~d}, \mathrm{~J}=8.3 \mathrm{~Hz}, 1 \mathrm{H}), 7.85(\mathrm{~d}, \mathrm{~J}=8.1 \mathrm{~Hz}$, $1 \mathrm{H}), 7.48(\mathrm{t}, \mathrm{J}=7.5 \mathrm{~Hz}, 1 \mathrm{H}), 7.45-7.34(\mathrm{~m}, 4 \mathrm{H}), 7.27(\mathrm{~d}, \mathrm{~J}=9.4 \mathrm{~Hz}, 2 \mathrm{H}), 7.22(\mathrm{t}, \mathrm{J}=7.6 \mathrm{~Hz}, 1 \mathrm{H}), 7.06(\mathrm{t}, \mathrm{J}=$ $7.6 \mathrm{~Hz}, 1 \mathrm{H}), 6.83(\mathrm{~d}, \mathrm{~J}=8.9 \mathrm{~Hz}, 3 \mathrm{H}), 5.67(\mathrm{~s}, 1 \mathrm{H}), 3.78(\mathrm{~s}, 3 \mathrm{H})$.

${ }^{13} \mathrm{C}$ NMR (126 MHz, $\left.\mathrm{CDCl}_{3}\right)$ ס: 204.9, 159.8, 152.2, 136.2, 133.0, 129.0, 128.1, 128.0, 126.9, 126.4, 125.7, 125.5, 125.4, 124.9, 123.9 (q, J = 275.0 Hz), 123.2 (d, J = $2.5 \mathrm{~Hz}), 123.0,122.2,120.8,120.2,115.1,114.4$, 111.5, 108.5, 105.2, $98.0(q, J=35.1 \mathrm{~Hz}), 55.4$.

${ }^{19} \mathrm{~F} \mathrm{NMR}\left(376 \mathrm{MHz}, \mathrm{CDCl}_{3}\right)$ ס: -61.42 .

HRMS (ESI): $\mathrm{m} / \mathrm{z}$ calcd. for $\mathrm{C}_{29} \mathrm{H}_{21} \mathrm{NO}_{2} \mathrm{~F}_{3}[\mathrm{M}+\mathrm{H}]^{+}: 472.1519$; found: 472.1514 .

HPLC analysis: The enantiomeric excess was determined by HPLC with Chiralpak IA column (hexane/ $/ \mathrm{-PrOH}$ $=90 / 10$, flow rate $\left.1 \mathrm{~mL} \cdot \mathrm{min}^{-1}, \lambda=285 \mathrm{~nm}\right): t_{\text {major }}=21.1 \mathrm{~min}, t_{\text {minor }}=30.6 \mathrm{~min}$.

(S)-4-(1-(3-chlorophenyl)-4,4,4-trifluoro-3-(1 H-indol-3-yl)buta-1,2-dien-1-yl)naphthalen-1-ol (3n): 
<smiles>Oc1ccc(C(=Cc2c[nH]c3ccccc23)C(F)(F)F)c2ccccc12</smiles>

light brown oil, $39.5 \mathrm{mg}, 83 \%$ yield, $91: 9 \mathrm{er},[a]_{D}^{20}=-13.62(\mathrm{c}=0.10, \mathrm{MeOH})$. purified by flash column chromatography on silica gel (petroleum ether/ ethyl acetate $=5: 1, \mathrm{Rf}=0.3$ ).

${ }^{1} \mathrm{H}$ NMR $\left(400 \mathrm{MHz}, \mathrm{CDCl}_{3}\right)$ ס: $8.33(\mathrm{~s}, 1 \mathrm{H}), 8.25(\mathrm{~d}, \mathrm{~J}=8.3 \mathrm{~Hz}, 1 \mathrm{H}), 7.80$ (dd, J = 23.3, 8.2 Hz, 2H), $7.55-7.46$ $(\mathrm{m}, 1 \mathrm{H}), 7.46-7.32(\mathrm{~m}, 4 \mathrm{H}), 7.31-7.18(\mathrm{~m}, 5 \mathrm{H}), 7.06(\mathrm{t}, \mathrm{J}=7.6 \mathrm{~Hz}, 1 \mathrm{H}), 6.83(\mathrm{~d}, \mathrm{~J}=7.7 \mathrm{~Hz}, 1 \mathrm{H}), 5.55(\mathrm{~s}, 1 \mathrm{H})$. ${ }^{13} \mathrm{C}$ NMR $\left(126 \mathrm{MHz}, \mathrm{CDCl}_{3}\right) \delta: 205.4,152.3,137.8,136.1,134.9,132.7,130.0,128.3,128.2,127.4,127.1$, 126.0, 125.8, 125.5, 125.4, 124.8, 124.4, 123.6 (q, J = 275.6 Hz), 123.4 (d, J = 2.3 Hz), 123.0, 122.2, 120.8, $119.8,114.5,111.4,108.4,104.3,98.6$ (q, J = 35.4 Hz).

${ }^{19} \mathrm{~F}$ NMR $\left(376 \mathrm{MHz}, \mathrm{CDCl}_{3}\right) \delta:-61.26$.

HRMS (ESI): $m / z$ calcd. for $\mathrm{C}_{28} \mathrm{H}_{18} \mathrm{NOF}_{3} \mathrm{Cl}[\mathrm{M}+\mathrm{H}]^{+}:$476.1024; found: 476.1028 .

HPLC analysis: The enantiomeric excess was determined by HPLC with Chiralpak IA column (hexane/i-PrOH $=90 / 10$, flow rate $\left.1 \mathrm{~mL} \cdot \mathrm{min}^{-1}, \lambda=254 \mathrm{~nm}\right): t_{\text {major }}=13.9 \mathrm{~min}, t_{\text {minor }}=15.6 \mathrm{~min}$.

(R)-4-(4,4,4-trifluoro-3-(1H-indol-3-yl)-1-(trimethylsilyl)buta-1,2-dien-1-yl)naphthalen-1-ol (3o):<smiles>CS(=O)(=O)C(=C(c1c[nH]c2ccccc12)C(F)(F)F)C(C(F)(F)F)C(F)(F)F</smiles>

light brown oil, $37.6 \mathrm{mg}, 86 \%$ yield, $98: 2 \mathrm{er},[a]_{D}^{20}=+118.64(\mathrm{c}=0.13, \mathrm{MeOH})$. purified by flash column chromatography on silica gel (petroleum ether/ ethyl acetate $=5: 1, \mathrm{Rf}=0.4$ ).

${ }^{1} \mathrm{H}$ NMR $\left(400 \mathrm{MHz}, \mathrm{CDCl}_{3}\right) \delta: 8.35-8.16(\mathrm{~m}, 2 \mathrm{H}), 8.17-8.12(\mathrm{~m}, 1 \mathrm{H}), 7.92(\mathrm{~d}, \mathrm{~J}=8.0 \mathrm{~Hz}, 1 \mathrm{H}), 7.58-7.47(\mathrm{~m}$, $2 \mathrm{H}), 7.39(\mathrm{~d}, \mathrm{~J}=8.1 \mathrm{~Hz}, 1 \mathrm{H}), 7.31-7.21(\mathrm{~m}, 3 \mathrm{H}), 7.15(\mathrm{t}, \mathrm{J}=8.0 \mathrm{~Hz}, 1 \mathrm{H}), 6.85(\mathrm{~d}, \mathrm{~J}=7.7 \mathrm{~Hz}, 1 \mathrm{H}), 5.32(\mathrm{~s}, 1 \mathrm{H})$, $0.25(\mathrm{~s}, 9 \mathrm{H})$.

${ }^{13} \mathrm{C}$ NMR $\left(126 \mathrm{MHz}, \mathrm{CDCl}_{3}\right) \delta: 205.6,152.1,137.6,133.8,128.0,127.7,127.6,127.1,126.9,126.7$ (q, J = $258.8 \mathrm{~Hz}$ ), 126.3, 126.2, 124.1, 123.8, 123.3, 121.6, 121.5, 112.8, 109.8, 108.9, 106.1, 92.1 (q, J = 35.5 Hz), 0.2 .

${ }^{19}$ F NMR $\left(376 \mathrm{MHz}, \mathrm{CDCl}_{3}\right) \delta:-60.60$.

HRMS (ESI): $m / z$ calcd. for $\mathrm{C}_{25} \mathrm{H}_{23} \mathrm{NOF}_{3} \mathrm{Si}[\mathrm{M}+\mathrm{H}]^{+}:$438.1496; found: 438.1493 .

HPLC analysis: The enantiomeric excess was determined by HPLC with Chiralpak IA column (hexane/ $i$-PrOH $=90 / 10$, flow rate $\left.1 \mathrm{~mL} \cdot \mathrm{min}^{-1}, \lambda=285 \mathrm{~nm}\right): t_{\text {major }}=9.3 \mathrm{~min}, t_{\text {minor }}=8.7 \mathrm{~min}$.

(S)-4-(1-cyclopropyl-4,4,4-trifluoro-3-(1H-indol-3-yl)buta-1,2-dien-1-yl)naphthalen-1-ol (3p): 


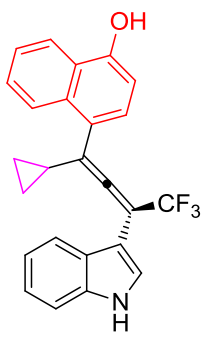

light brown oil, $33.2 \mathrm{mg}, 82 \%$ yield, $>99: 1 \mathrm{er},[a]_{D}^{20}=+66.15(\mathrm{c}=0.13, \mathrm{MeOH})$. purified by flash column chromatography on silica gel (petroleum ether/ ethyl acetate $=5: 1, \mathrm{Rf}=0.3$ ).

${ }^{1}$ H NMR $\left(400 \mathrm{MHz}, \mathrm{CDCl}_{3}\right) \delta: 8.42-8.07(\mathrm{~m}, 3 \mathrm{H}), 7.92(\mathrm{~d}, \mathrm{~J}=8.0 \mathrm{~Hz}, 1 \mathrm{H}), 7.62-7.45(\mathrm{~m}, 2 \mathrm{H}), 7.45-7.35(\mathrm{~m}$, $2 \mathrm{H}), 7.33(\mathrm{~s}, 1 \mathrm{H}), 7.27-7.22(\mathrm{~m}, 1 \mathrm{H}), 7.14(\mathrm{t}, \mathrm{J}=7.5 \mathrm{~Hz}, 1 \mathrm{H}), 6.80(\mathrm{~d}, \mathrm{~J}=7.7 \mathrm{~Hz}, 1 \mathrm{H}), 5.50(\mathrm{~s}, 1 \mathrm{H}), 1.89-1.71$ $(\mathrm{m}, 1 \mathrm{H}), 0.99-0.93(\mathrm{~m}, 1 \mathrm{H}), 0.91-0.84(\mathrm{~m}, 1 \mathrm{H}), 0.83-0.76(\mathrm{~m}, 1 \mathrm{H}), 0.76-0.68(\mathrm{~m}, 1 \mathrm{H})$.

${ }^{13} \mathrm{C}$ NMR $\left(126 \mathrm{MHz}, \mathrm{CDCl}_{3}\right)$ ס: 151.8, 136.2, 132.5 (d, J = 4.1 Hz), 126.9, 126.7, 126.5, 125.9, 125.8, 125.5, 124.8, 123.9 (q, J = 274.4 Hz), 123.0 (d, J = 2.5 Hz), 122.9, 122.1, 120.6, 120.5, 120.2, 116.6, 111.4, 108.3, 105.4, 97.5 (q, J = 34.5 Hz), 14.9, 9.0, 7.6.

${ }^{19}$ F NMR $\left(376 \mathrm{MHz}, \mathrm{CDCl}_{3}\right) \delta:-61.73$.

HRMS (ESI): $m / z$ calcd. for $\mathrm{C}_{25} \mathrm{H}_{19} \mathrm{NOF}_{3}[\mathrm{M}+\mathrm{H}]^{+}:$: 406.1413; found: 406.1405 .

HPLC analysis: The enantiomeric excess was determined by HPLC with Chiralpak IA column (hexane/ $i-\mathrm{PrOH}$ $=90 / 10$, flow rate $\left.1 \mathrm{~mL} \cdot \mathrm{min}^{-1}, \lambda=254 \mathrm{~nm}\right): t_{\text {major }}=13.5 \mathrm{~min}, t_{\text {minor }}=11.2 \mathrm{~min}$.

(S)-4-(6,6,6-trifluoro-5-(1 H-indol-3-yl)-2,2-dimethylhexa-3,4-dien-3-yl)naphthalen-1-ol (3q):<smiles></smiles>

light brown oil, $40.0 \mathrm{mg}, 95 \%$ yield, $>99: 1 \mathrm{er},[a]_{D}^{20}=+336.00(\mathrm{c}=0.15, \mathrm{MeOH})$. purified by flash column chromatography on silica gel (petroleum ether/ ethyl acetate $=5: 1, \mathrm{Rf}=0.4$ ).

${ }^{1}$ H NMR $\left(400 \mathrm{MHz}, \mathrm{CDCl}_{3}\right) \delta: 8.35-8.14(\mathrm{~m}, 3 \mathrm{H}), 8.00(\mathrm{~d}, \mathrm{~J}=8.0 \mathrm{~Hz}, 1 \mathrm{H}), 7.59-7.49(\mathrm{~m}, 2 \mathrm{H}), 7.39(\mathrm{~d}, \mathrm{~J}=8.0$ $\mathrm{Hz}, 1 \mathrm{H}), 7.36(\mathrm{~d}, \mathrm{~J}=7.7 \mathrm{~Hz}, 1 \mathrm{H}), 7.32-7.26(\mathrm{~m}, 2 \mathrm{H}), 7.21(\mathrm{t}, \mathrm{J}=7.5 \mathrm{~Hz}, 1 \mathrm{H}), 6.86(\mathrm{~d}, \mathrm{~J}=7.7 \mathrm{~Hz}, 1 \mathrm{H}), 5.39(\mathrm{~s}$, $1 \mathrm{H}), 1.30(\mathrm{~s}, 9 \mathrm{H})$.

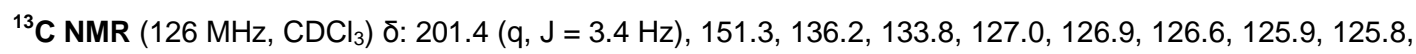
125.3, 124.7, 124.5 (q, J = 274.8 Hz), 123.1 (d, J = 2.0 Hz), 122.8, 121.8, 120.4, 120.3, 120.2, 111.4, 107.9, 105.6, 95.7 (q, J = 34.9 Hz), 37.2, 29.6.

${ }^{19}$ F NMR $\left(376 \mathrm{MHz}, \mathrm{CDCl}_{3}\right) \delta:-61.09$.

HRMS (ESI): $m / z$ calcd. for $\mathrm{C}_{26} \mathrm{H}_{23} \mathrm{NOF}_{3}[\mathrm{M}+\mathrm{H}]^{+}: 422.1726$; found: 422.1728.

HPLC analysis: The enantiomeric excess was determined by HPLC with Chiralpak IA column (hexane/ $i-\mathrm{PrOH}$ $=90 / 10$, flow rate $\left.1 \mathrm{~mL} \cdot \mathrm{min}^{-1}, \lambda=254 \mathrm{~nm}\right): t_{\text {major }}=11.1 \mathrm{~min}, t_{\text {minor }}=12.2 \mathrm{~min}$

(S)-4-(1,1,1-trifluoro-2-(1H-indol-3-yl)octa-2,3-dien-4-yl)naphthalen-1-ol (3r): 


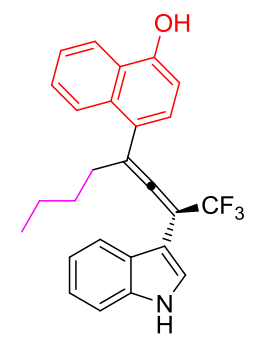

light brown oil, $38.8 \mathrm{mg}, 92 \%$ yield, $96: 4 \mathrm{er},[a]_{D}^{20}=-86.00(\mathrm{c}=0.11, \mathrm{MeOH})$. purified by flash column chromatography on silica gel (petroleum ether/ ethyl acetate $=10: 1, \mathrm{Rf}=0.3$ ).

${ }^{1}$ H NMR $\left(400 \mathrm{MHz}, \mathrm{CDCl}_{3}\right) \delta: 8.35-8.19(\mathrm{~m}, 2 \mathrm{H}), 8.18-8.12(\mathrm{~m}, 1 \mathrm{H}), 7.83(\mathrm{~d}, \mathrm{~J}=8.0 \mathrm{~Hz}, 1 \mathrm{H}), 7.56-7.47(\mathrm{~m}$, $2 \mathrm{H}), 7.41-7.28(\mathrm{~m}, 3 \mathrm{H}), 7.26-7.19(\mathrm{~m}, 1 \mathrm{H}), 7.11(\mathrm{t}, \mathrm{J}=7.6 \mathrm{~Hz}, 1 \mathrm{H}), 6.79(\mathrm{~d}, \mathrm{~J}=7.7 \mathrm{~Hz}, 1 \mathrm{H}), 5.37(\mathrm{~s}, 1 \mathrm{H})$, $2.80-2.56(\mathrm{~m}, 2 \mathrm{H}), 1.66-1.57(\mathrm{~m}, 2 \mathrm{H}), 1.42(\mathrm{dq}, \mathrm{J}=14.5,7.3 \mathrm{~Hz}, 2 \mathrm{H}), 0.89(\mathrm{t}, \mathrm{J}=7.3 \mathrm{~Hz}, 3 \mathrm{H})$.

${ }^{13} \mathrm{C}$ NMR $\left(126 \mathrm{MHz}, \mathrm{CDCl}_{3}\right)$ ס: 202.8 (q, J = $\left.3.5 \mathrm{~Hz}\right), 151.5,136.2,132.3,127.6,126.9,125.9,125.8,125.6$, 125.5, 124.9, 124.2 (q, J = 274.8 Hz), 123.0 (d, J = 2.4 Hz), 122.9, 122.2, 120.5, 120.1, 113.4, 111.4, 108.3, 105.5, 95.8 (q, J = 35.2 Hz), 35.4, 30.0, 22.5, 14.0.

${ }^{19}$ F NMR $\left(376 \mathrm{MHz}, \mathrm{CDCl}_{3}\right) \delta:-61.55$.

HRMS (ESI): $m / z$ calcd. for $\mathrm{C}_{26} \mathrm{H}_{23} \mathrm{NOF}_{3}[\mathrm{M}+\mathrm{H}]^{+}:$: 422.1726; found: 422.1721 .

HPLC analysis: The enantiomeric excess was determined by HPLC with Chiralcel OD column (hexane/ $i-\mathrm{PrOH}$ $=95 / 5$, flow rate $\left.1 \mathrm{~mL} \cdot \mathrm{min}^{-1}, \lambda=254 \mathrm{~nm}\right): t_{\text {major }}=28.3 \mathrm{~min}, t_{\text {minor }}=30.7 \mathrm{~min}$.

(S)-5-(benzyloxy)-4-(4,4,4-trifluoro-3-(1H-indol-3-yl)-1-phenylbuta-1,2-dien-1-yl)naphthalen-1-ol (3s):<smiles>Oc1ccc(C(=C=C(c2c[nH]c3ccccc23)C(F)(F)F)c2ccccc2)c2c(O)cccc12</smiles>

light brown oil, $49.3 \mathrm{mg}, 90 \%$ yield, $97: 3 \mathrm{er},[a]_{D}^{20}=+48.71(\mathrm{c}=0.19, \mathrm{MeOH})$. purified by flash column chromatography on silica gel (petroleum ether/ ethyl acetate $=3: 1, \mathrm{Rf}=0.4$ ).

${ }^{1} \mathrm{H}$ NMR $\left(400 \mathrm{MHz}, \mathrm{CDCl}_{3}\right) \delta: 8.24(\mathrm{~s}, 1 \mathrm{H}), 7.83(\mathrm{~d}, \mathrm{~J}=8.4 \mathrm{~Hz}, 1 \mathrm{H}), 7.77(\mathrm{~d}, \mathrm{~J}=8.0 \mathrm{~Hz}, 1 \mathrm{H}), 7.55-6.99(\mathrm{~m}$, $13 \mathrm{H}), 6.93(\mathrm{~s}, 1 \mathrm{H}), 6.81(\mathrm{~d}, \mathrm{~J}=6.1 \mathrm{~Hz}, 1 \mathrm{H}), 6.73(\mathrm{~d}, \mathrm{~J}=7.7 \mathrm{~Hz}, 1 \mathrm{H}), 6.61(\mathrm{~s}, 2 \mathrm{H}), 5.36(\mathrm{~s}, 1 \mathrm{H}), 4.79(\mathrm{~s}, 1 \mathrm{H})$, $4.76(\mathrm{~s}, 1 \mathrm{H})$.

${ }^{13} \mathrm{C}$ NMR $\left(126 \mathrm{MHz}, \mathrm{CDCl}_{3}\right) \delta: 204.2,155.1,152.0,138.0,136.8,136.2,129.9,128.5,128.2,127.3,127.2$, 127.0, 126.8, 126.7, 126.1, 125.9, 125.2, 124.1 (q, J = 275.3 Hz), 123.1 (d, J = 1.5 Hz), 123.0, 122.9, 120.6, 120.5, 120.0, 114.4, 111.3, 109.1, 108.2, 105.9, 97.3 (q, J = 38.0 Hz), 70.4.

${ }^{19}$ F NMR (376 MHz, $\left.\mathrm{CDCl}_{3}\right)$ ס: -61.33.

HRMS (ESI): $m / z$ calcd. for $\mathrm{C}_{35} \mathrm{H}_{24} \mathrm{NO}_{2} \mathrm{~F}_{3} \mathrm{Na}[\mathrm{M}+\mathrm{Na}]^{+}: 570.1651$; found: 570.1657 .

HPLC analysis: The enantiomeric excess was determined by HPLC with Chiralpak IA column (hexane/ $i-\mathrm{PrOH}$ $=90 / 10$, flow rate $\left.1 \mathrm{~mL} \cdot \mathrm{min}^{-1}, \lambda=254 \mathrm{~nm}\right): t_{\text {major }}=28.0 \mathrm{~min}, t_{\text {minor }}=18.5 \mathrm{~min}$.

(S)-tert-butyl(5-hydroxy-8-(4,4,4-trifluoro-3-(1H-indol-3-yl)-1-phenylbuta-1,2-dien-1-yl)naphthalen-1-yl)carbama te $(3 t)$ : 
<smiles>O=C(Nc1cccc2c(O)ccc(C(=C=C(c3c[nH]c4ccccc34)C(F)(F)F)c3c[nH]c4ccccc34)c12)c1ccccc1</smiles>

light brown oil, $52.3 \mathrm{mg}, 94 \%$ yield, $97: 3 \mathrm{er},[a]_{D}^{20}=+71.52(\mathrm{c}=0.17, \mathrm{MeOH})$. purified by flash column chromatography on silica gel (petroleum ether/ ethyl acetate $=5: 1, \mathrm{Rf}=0.2$ ).

${ }^{1} \mathrm{H}$ NMR $\left(400 \mathrm{MHz}, \mathrm{CDCl}_{3}\right) \delta: 8.45(\mathrm{~s}, 1 \mathrm{H}), 8.04(\mathrm{~d}, \mathrm{~J}=8.3 \mathrm{~Hz}, 1 \mathrm{H}), 7.99-7.77(\mathrm{~m}, 2 \mathrm{H}), 7.69(\mathrm{~d}, \mathrm{~J}=7.9 \mathrm{~Hz}, 1 \mathrm{H})$, $7.51-7.38(\mathrm{~m}, 3 \mathrm{H}), 7.29-7.22(\mathrm{~m}, 5 \mathrm{H}), 7.18-6.99(\mathrm{~m}, 2 \mathrm{H}), 6.63(\mathrm{~d}, \mathrm{~J}=7.6 \mathrm{~Hz}, 1 \mathrm{H}), 5.79(\mathrm{~s}, 1 \mathrm{H}), 1.29(\mathrm{~s}$, $9 \mathrm{H})$.

${ }^{13} \mathrm{C}$ NMR $\left(126 \mathrm{MHz}, \mathrm{CDCl}_{3}\right) \delta: 203.1,153.4,152.9,136.3,135.2,133.6,130.6,129.0,128.7,127.3,126.5$, 125.7, 125.6, 125.1 (q, J = 293.9 Hz), 123.6, 123.1, 123.0, 122.2, 121.1, 120.7, 120.0, 119.0, 118.7, 111.7, 108.3, 104.8, 99.0 (q, J = 33.9 Hz), 80.0, 28.1.

${ }^{19}$ F NMR $\left(376 \mathrm{MHz}, \mathrm{CDCl}_{3}\right) \delta:-60.74$.

HRMS (ESI): $m / z$ calcd. for $\mathrm{C}_{33} \mathrm{H}_{28} \mathrm{~N}_{2} \mathrm{O}_{3} \mathrm{~F}_{3}[\mathrm{M}+\mathrm{H}]^{+}:$557.2047; found: 557.2050.

HPLC analysis: The enantiomeric excess was determined by HPLC with Chiralcel OD column (hexane/i-PrOH $=90 / 10$, flow rate $\left.1 \mathrm{~mL} \cdot \mathrm{min}^{-1}, \lambda=254 \mathrm{~nm}\right): t_{\text {major }}=11.3 \mathrm{~min}, t_{\text {minor }}=9.9 \mathrm{~min}$.

(S)-2-methyl-4-(4,4,4-trifluoro-3-(1H-indol-3-yl)-1-phenylbuta-1,2-dien-1-yl)naphthalen-1-ol (3u):<smiles>Cc1cc(C(=CC(=C2C=Nc3ccccc32)C(F)(F)F)c2ccccc2)c2ccccc2c1O</smiles>

light brown oil, $41.0 \mathrm{mg}, 90 \%$ yield, $97: 3 \mathrm{er},[a]_{D}^{20}=+23.10(\mathrm{c}=0.15, \mathrm{MeOH})$. purified by flash column chromatography on silica gel (petroleum ether/ ethyl acetate $=5: 1, \mathrm{Rf}=0.4$ ).

${ }^{1} \mathrm{H}$ NMR $\left(400 \mathrm{MHz}, \mathrm{CDCl}_{3}\right) \delta: 8.30(\mathrm{~s}, 1 \mathrm{H}), 8.18(\mathrm{~d}, J=8.4 \mathrm{~Hz}, 1 \mathrm{H}), 7.84(\mathrm{dd}, J=7.9,5.8 \mathrm{~Hz}, 2 \mathrm{H}), 7.46(\mathrm{t}, J=$ $7.6 \mathrm{~Hz}, 1 \mathrm{H}), 7.41-7.33(\mathrm{~m}, 5 \mathrm{H}), 7.30-7.18(\mathrm{~m}, 5 \mathrm{H}), 7.04(\mathrm{t}, J=7.6 \mathrm{~Hz}, 1 \mathrm{H}), 5.22(\mathrm{~s}, 1 \mathrm{H}), 2.40(\mathrm{~s}, 3 \mathrm{H})$.

${ }^{13} \mathrm{C}$ NMR $\left(101 \mathrm{MHz}, \mathrm{CDCl}_{3}\right) \delta: 205.3,149.4,136.2,135.8,131.6,131.1,128.9,128.3,127.7,126.3,126.0$, 125.7, 125.6, 124.8, 124.7, 123.9 (q, $J=275.2 \mathrm{~Hz}), 123.3$ (d, $J=1.6 \mathrm{~Hz}), 123.1,121.5,120.8,120.2,116.1$, 115.4, 111.4, 104.9, 98.2 (q, $J=35.4 \mathrm{~Hz}), 15.7$.

${ }^{19}$ F NMR $\left(376 \mathrm{MHz}, \mathrm{CDCl}_{3}\right) \delta:-61.34$.

HRMS (ESI): $m / z$ calcd. for $\mathrm{C}_{29} \mathrm{H}_{21} \mathrm{NOF}_{3}[\mathrm{M}+\mathrm{H}]^{+}:$456.1570; found: 456.1567 .

HPLC analysis: The enantiomeric excess was determined by HPLC with Chiralpak IA column (hexane/i-PrOH $=90 / 10$, flow rate $\left.1 \mathrm{~mL} \cdot \mathrm{min}^{-1}, \lambda=254 \mathrm{~nm}\right): t_{\text {major }}=16.3 \mathrm{~min}, t_{\text {minor }}=19.1 \mathrm{~min}$.

(S)-4-(4,4,4-trifluoro-3-(1 H-indol-3-yl)-1-phenylbuta-1,2-dien-1-yl)-5,6,7,8-tetrahydronaphthalen-1-ol (3v): 
<smiles>Oc1ccc(C(=C(c2ccccc2)C(F)(F)F)c2c[nH]c3ccccc23)c2c1CCCC2</smiles>

light brown oil, $41.0 \mathrm{mg}, 90 \%$ yield, $99: 1 \mathrm{er},[a]_{D}^{20}=+38.00(\mathrm{c}=0.10, \mathrm{MeOH})$. purified by flash column chromatography on silica gel (petroleum ether/ ethyl acetate $=5: 1, \mathrm{Rf}=0.4$ ).

${ }^{1} \mathrm{H}$ NMR $\left(400 \mathrm{MHz}, \mathrm{CDCl}_{3}\right) \delta: 8.30(\mathrm{~s}, 1 \mathrm{H}), 7.77(\mathrm{~d}, \mathrm{~J}=8.0 \mathrm{~Hz}, 1 \mathrm{H}), 7.42-7.28(\mathrm{~m}, 7 \mathrm{H}), 7.23(\mathrm{t}, \mathrm{J}=7.6 \mathrm{~Hz}, 1 \mathrm{H})$, $7.14-7.04(\mathrm{~m}, 2 \mathrm{H}), 6.67(\mathrm{~d}, \mathrm{~J}=8.1 \mathrm{~Hz}, 1 \mathrm{H}), 4.82(\mathrm{~s}, 1 \mathrm{H}), 2.69(\mathrm{t}, \mathrm{J}=6.4 \mathrm{~Hz}, 2 \mathrm{H}), 2.66-2.48(\mathrm{~m}, 2 \mathrm{H}), 1.86-$ $1.75(\mathrm{~m}, 2 \mathrm{H}), 1.72-1.64(\mathrm{~m}, 2 \mathrm{H})$.

${ }^{13} \mathrm{C}$ NMR $\left(126 \mathrm{MHz}, \mathrm{CDCl}_{3}\right) \mathrm{\delta}: 204.4,153.7,137.9,136.2,135.5,128.9,128.3,128.2,127.4,126.8,125.8$, 124.2, 123.9 (q, J = 274.6 Hz), 123.3 (d, J = 1.7 Hz), 123.0, 120.7, 120.1, 116.0, 112.2, 111.5, 105.1, 98.0 (q, J $=32.9 \mathrm{~Hz}), 27.9,23.3,22.7,22.4$.

${ }^{19}$ F NMR (376 MHz, $\left.\mathrm{CDCl}_{3}\right)$ ס: -61.29 .

HRMS (ESI): $m / z$ calcd. for $\mathrm{C}_{28} \mathrm{H}_{23} \mathrm{NOF}_{3}[\mathrm{M}+\mathrm{H}]^{+}:$: 446.1726; found: 446.1727 .

HPLC analysis: The enantiomeric excess was determined by HPLC with Chiralpak IA column (hexane/i-PrOH $=90 / 10$, flow rate $\left.1 \mathrm{~mL} \cdot \mathrm{min}^{-1}, \lambda=254 \mathrm{~nm}\right): t_{\text {major }}=11.8 \mathrm{~min}, t_{\text {minor }}=17.0 \mathrm{~min}$.

(S)-7-(4,4,4-trifluoro-3-(1 H-indol-3-yl)-1-phenylbuta-1,2-dien-1-yl)-2,3-dihydro-1H-inden-4-ol (3w):<smiles>Oc1ccc(C(=CC(c2ccccc2)C(F)(F)F)c2c[nH]c3ccccc23)c2c1CCC2</smiles>

light brown oil, $35.4 \mathrm{mg}, 82 \%$ yield, $95: 5 \mathrm{er},[a]_{D}^{20}=+18.25(\mathrm{c}=0.16, \mathrm{MeOH})$. purified by flash column chromatography on silica gel (petroleum ether/ ethyl acetate $=5: 1, \mathrm{Rf}=0.4$ ).

${ }^{1} \mathrm{H}$ NMR $\left(400 \mathrm{MHz}, \mathrm{CDCl}_{3}\right) \delta: 8.34(\mathrm{~s}, 1 \mathrm{H}), 7.73(\mathrm{~d}, \mathrm{~J}=8.1 \mathrm{~Hz}, 1 \mathrm{H}), 7.42-7.31(\mathrm{~m}, 7 \mathrm{H}), 7.21(\mathrm{t}, \mathrm{J}=7.6 \mathrm{~Hz}, 1 \mathrm{H})$, 7.06 (dd, J = 14.2, 7.3 Hz, 2H), $6.67(\mathrm{~d}, \mathrm{~J}=8.2 \mathrm{~Hz}, 1 \mathrm{H}$ ), $4.86(\mathrm{~s}, 1 \mathrm{H}), 2.86(\mathrm{t}, \mathrm{J}=7.4 \mathrm{~Hz}, 2 \mathrm{H}), 2.74(\mathrm{t}, \mathrm{J}=7.5 \mathrm{~Hz}$, $2 \mathrm{H}), 2.07-1.98(\mathrm{~m}, 2 \mathrm{H})$.

${ }^{13} \mathrm{C}$ NMR (126 MHz, $\mathrm{CDCl}_{3}$ ) $\delta: 205.1$ (q, J = $\left.3.3 \mathrm{~Hz}\right), 152.3,146.1,136.3,135.8,130.3,129.3,128.8,128.3$, 128.3, 128.2, 125.8, 123.9, 123.8 (q, J = 275.1 Hz), 123.2 (d, J = 2.5 Hz), 123.0, 120.8, 120.2, 113.4, 111.4, 105.0, 98.3 (q, J = 35.3 Hz), 33.1, 29.0, 25.1.

${ }^{19}$ F NMR $\left(376 \mathrm{MHz}, \mathrm{CDCl}_{3}\right) \delta:-61.51$.

HRMS (ESI): $m / z$ calcd. for $\mathrm{C}_{27} \mathrm{H}_{21} \mathrm{NOF}_{3}[\mathrm{M}+\mathrm{H}]^{+}:$432.1570; found: 432.1573.

HPLC analysis: The enantiomeric excess was determined by HPLC with Chiralpak AD-H column (hexane $/ \mathrm{i}-\mathrm{PrOH}=90 / 10$, flow rate $1 \mathrm{~mL} \cdot \mathrm{min}^{-1}, \lambda=254 \mathrm{~nm}$ ): $t_{\text {major }}=17.8 \mathrm{~min}, t_{\text {minor }}=21.7 \mathrm{~min}$.

(S)-2,3-dimethyl-4-(4,4,4-trifluoro-3-(1 H-indol-3-yl)-1-phenylbuta-1,2-dien-1-yl)phenol (3x): 
<smiles>Cc1c(O)ccc(C(=C(c2ccccc2)C(F)(F)F)c2c[nH]c3ccccc23)c1C</smiles>

light brown oil, $35.2 \mathrm{mg}, 84 \%$ yield, $95: 5 \mathrm{er},[a]_{D}^{20}=-95.34(\mathrm{c}=0.17, \mathrm{MeOH})$. purified by flash column chromatography on silica gel (petroleum ether/ ethyl acetate $=5: 1, \mathrm{Rf}=0.3$ ).

${ }^{1} \mathrm{H} \mathrm{NMR}\left(400 \mathrm{MHz}, \mathrm{CDCl}_{3}\right) \delta: 8.30(\mathrm{~s}, 1 \mathrm{H}), 7.79(\mathrm{~d}, \mathrm{~J}=8.0 \mathrm{~Hz}, 1 \mathrm{H}), 7.44-7.38(\mathrm{~m}, 2 \mathrm{H}), 7.38-7.32(\mathrm{~m}, 4 \mathrm{H})$, $7.32-7.28(\mathrm{~m}, 1 \mathrm{H}), 7.23(\mathrm{t}, \mathrm{J}=8.1 \mathrm{~Hz}, 1 \mathrm{H}), 7.13-7.04(\mathrm{~m}, 2 \mathrm{H}), 6.67(\mathrm{~d}, \mathrm{~J}=8.2 \mathrm{~Hz}, 1 \mathrm{H}), 4.78(\mathrm{~s}, 1 \mathrm{H}), 2.21(\mathrm{~s}$, $3 \mathrm{H}), 2.17(\mathrm{~s}, 3 \mathrm{H})$.

${ }^{13} \mathrm{C}$ NMR $\left(126 \mathrm{MHz}, \mathrm{CDCl}_{3}\right)$ ס: 204.5 (q, J = 3.6 Hz), 153.7, 137.3, 136.2, 135.7, 128.9, 128.4, 128.2, 127.5, 127.3, 125.8, 124.0 (q, J = 274.8 Hz), 123.6, 123.2 (d, J = 2.5 Hz), 123.0, 120.8, 120.1, 116.6, 112.9, 111.5, 105.1, $98.1(\mathrm{q}, \mathrm{J}=35.2 \mathrm{~Hz}), 17.6,12.1$.

${ }^{19} \mathrm{~F} \mathrm{NMR}\left(376 \mathrm{MHz}, \mathrm{CDCl}_{3}\right) \delta:-61.29$.

HRMS (ESI): $\mathrm{m} / z$ calcd. for $\mathrm{C}_{26} \mathrm{H}_{21} \mathrm{NOF}_{3}[\mathrm{M}+\mathrm{H}]^{+}: 420.1570$; found: 420.1572 .

HPLC analysis: The enantiomeric excess was determined by HPLC with Chiralcel OD-H column (hexane $/ \mathrm{i}-\mathrm{PrOH}=95 / 5$, flow rate $1 \mathrm{~mL} \cdot \mathrm{min}^{-1}, \lambda=254 \mathrm{~nm}$ ): $t_{\text {major }}=31.8 \mathrm{~min}, t_{\text {minor }}=36.6 \mathrm{~min}$.

(S)-3,5-dimethyl-4-(4,4,4-trifluoro-3-(1H-indol-3-yl)-1-phenylbuta-1,2-dien-1-yl)phenol (3y):<smiles>CC=C(C=C(c1c(C)cc(O)cc1C)c1c[nH]c2ccccc12)C(F)(F)F</smiles>

light brown oil, $30.2 \mathrm{mg}, 72 \%$ yield, 92:8 er, $[a]_{D}^{20}=-107.56(\mathrm{c}=0.16, \mathrm{MeOH})$. purified by flash column chromatography on silica gel (petroleum ether/ ethyl acetate $=5: 1, \mathrm{Rf}=0.3$ ).

${ }^{1} \mathrm{H} \mathrm{NMR}\left(400 \mathrm{MHz}, \mathrm{CDCl}_{3}\right)$ ס: $8.32(\mathrm{~s}, 1 \mathrm{H}), 7.65(\mathrm{~d}, \mathrm{~J}=8.1 \mathrm{~Hz}, 1 \mathrm{H}), 7.41-7.29(\mathrm{~m}, 7 \mathrm{H}), 7.21(\mathrm{t}, \mathrm{J}=7.6 \mathrm{~Hz}, 1 \mathrm{H})$, $7.03(\mathrm{t}, \mathrm{J}=7.6 \mathrm{~Hz}, 1 \mathrm{H}), 6.58(\mathrm{~s}, 2 \mathrm{H}), 4.79(\mathrm{~s}, 1 \mathrm{H}), 2.12(\mathrm{~s}, 6 \mathrm{H})$.

${ }^{13} \mathrm{C}$ NMR (126 MHz, $\left.\mathrm{CDCl}_{3}\right)$ ס: 204.1 (q, J = 3.2 Hz), 155.3, 139.2, 136.1, 134.5, 129.1, 128.4, 127.0, 126.0, $125.9,124.1$ (q, J = 274.0 Hz), 123.4 (d, J = 2.2 Hz), 123.0, 120.6, 120.2, 114.7, 113.4, 111.4, 105.2, 97.8 (q, J $=35.6 \mathrm{~Hz}), 20.6$.

${ }^{19} \mathrm{~F} \mathrm{NMR}\left(376 \mathrm{MHz}, \mathrm{CDCl}_{3}\right) \delta:-60.88$.

HRMS (ESI): $\mathrm{m} / z$ calcd. for $\mathrm{C}_{26} \mathrm{H}_{21} \mathrm{NOF}_{3}[\mathrm{M}+\mathrm{H}]^{+}: 420.1570$; found: 420.1576 .

HPLC analysis: The enantiomeric excess was determined by HPLC with Chiralpak IA column (hexane/i-PrOH $=90 / 10$, flow rate $\left.1 \mathrm{~mL} \cdot \mathrm{min}^{-1}, \lambda=254 \mathrm{~nm}\right): t_{\text {major }}=22.9 \mathrm{~min}, t_{\operatorname{minor}}=16.0 \mathrm{~min}$.

(S)-3,5-dimethoxy-4-(4,4,4-trifluoro-3-(1 H-indol-3-yl)-1-phenylbuta-1,2-dien-1-yl)phenol (3z): 
<smiles></smiles>

light brown oil, $27.1 \mathrm{mg}, 60 \%$ yield, $85: 15 \mathrm{er},[a]_{D}^{20}=-34.55(\mathrm{c}=0.11, \mathrm{MeOH})$. purified by flash column chromatography on silica gel (petroleum ether/ ethyl acetate $=3: 1, \mathrm{Rf}=0.3$ ).

${ }^{1} \mathrm{H} \mathrm{NMR}\left(400 \mathrm{MHz}, \mathrm{CDCl}_{3}\right)$ ס: $8.26(\mathrm{~s}, 1 \mathrm{H}), 8.16(\mathrm{~d}, \mathrm{~J}=8.1 \mathrm{~Hz}, 1 \mathrm{H}), 7.45-7.32(\mathrm{~m}, 4 \mathrm{H}), 7.29(\mathrm{t}, \mathrm{J}=7.4 \mathrm{~Hz}, 2 \mathrm{H})$, $7.22(\mathrm{dd}, \mathrm{J}=16.4,7.4 \mathrm{~Hz}, 2 \mathrm{H}), 7.06(\mathrm{t}, \mathrm{J}=7.6 \mathrm{~Hz}, 1 \mathrm{H}), 6.12(\mathrm{~s}, 2 \mathrm{H}), 4.96(\mathrm{~s}, 1 \mathrm{H}), 3.68(\mathrm{~s}, 6 \mathrm{H})$.

${ }^{13} \mathrm{C} \mathrm{NMR}\left(126 \mathrm{MHz}, \mathrm{CDCl}_{3}\right)$ ס: 205.6 (q, J = 3.2 Hz), 159.3, 157.7, 136.2, 135.6, 128.6, 127.6, 127.0, 125.7, $124.0(q, J=276.1 \mathrm{~Hz}), 123.1(\mathrm{~d}, \mathrm{~J}=2.8 \mathrm{~Hz}), 122.9,121.3,120.5,111.0,109.5,105.1,104.7,98.0$ (q, J = 34.9 $\mathrm{Hz}), 92.3,55.8$.

${ }^{19} \mathrm{~F} \mathrm{NMR}\left(376 \mathrm{MHz}, \mathrm{CDCl}_{3}\right) \delta:-60.96$.

HRMS (ESI): $\mathrm{m} / z$ calcd. for $\mathrm{C}_{26} \mathrm{H}_{21} \mathrm{NO}_{3} \mathrm{~F}_{3}[\mathrm{M}+\mathrm{H}]^{+}: 452.1468$ found: 452.1469 .

HPLC analysis: The enantiomeric excess was determined by HPLC with Chiralcel OD-H column (hexane $/ \mathrm{i}-\mathrm{PrOH}=95 / 5$, flow rate $1 \mathrm{~mL} \cdot \mathrm{min}^{-1}, \lambda=254 \mathrm{~nm}$ ): $t_{\text {major }}=77.0 \mathrm{~min}, t_{\text {minor }}=70.3 \mathrm{~min}$.

(S)-ethyl 4-(4-hydroxynaphthalen-1-yl)-2-(1H-indol-3-yl)-4-phenylbuta-2,3-dienoate. (3aa):

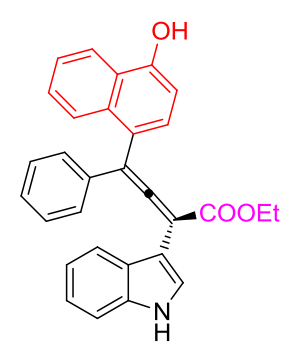

White foam, $38.3 \mathrm{mg}, 86 \%$ yield, $90: 10 \mathrm{er},[a]_{D}^{20}=+49.56(\mathrm{c}=0.11$, MeOH). purified by flash column chromatography on silica gel (petroleum ether/ ethyl acetate $=3: 1, \mathrm{Rf}=0.3$ ).

${ }^{1} \mathrm{H} \mathrm{NMR}\left(400 \mathrm{MHz}, \mathrm{CDCl}_{3}\right)$ ס: $8.28(\mathrm{~s}, 1 \mathrm{H}), 8.23(\mathrm{~d}, J=8.3 \mathrm{~Hz}, 1 \mathrm{H}), 8.03(\mathrm{~d}, J=8.3 \mathrm{~Hz}, 1 \mathrm{H}), 7.92(\mathrm{~d}, J=2.6 \mathrm{~Hz}$, $1 \mathrm{H}$ ), $7.88(\mathrm{~d}, J=8.0 \mathrm{~Hz}, 1 \mathrm{H}), 7.46(\mathrm{dd}, J=13.7,7.8 \mathrm{~Hz}, 2 \mathrm{H}), 7.41-7.33(\mathrm{~m}, 4 \mathrm{H}), 7.30-7.23(\mathrm{~m}, 3 \mathrm{H}), 7.17(\mathrm{t}, J$ $=7.3 \mathrm{~Hz}, 1 \mathrm{H}), 7.03(\mathrm{t}, J=7.6 \mathrm{~Hz}, 1 \mathrm{H}), 6.80(\mathrm{~d}, J=7.7 \mathrm{~Hz}, 1 \mathrm{H}), 5.63(\mathrm{~s}, 1 \mathrm{H}), 4.33(\mathrm{qd}, J=7.1,1.9 \mathrm{~Hz}, 2 \mathrm{H}), 1.37$ (t, $J=7.1 \mathrm{~Hz}, 3 \mathrm{H}$ );

${ }^{13} \mathrm{C} \mathrm{NMR}\left(126 \mathrm{MHz}, \mathrm{CDCl}_{3}\right)$ ס: 166.9, 152.1, 136.3, 136.1, 133.3, 128.8, 128.3, 127.8, 127.7, 126.8, 126.7, 126.0, 125.5, 125.4, 125.3, 125.0, 122.5, 122.1, 120.4, 120.3, 112.2, 111.3, 108.5, 106.6, 99.0, 61.5, 14.5 .

HRMS (ESI): $\mathrm{m} / z$ calcd. for $\mathrm{C}_{30} \mathrm{H}_{24} \mathrm{NO}_{3}[\mathrm{M}+\mathrm{H}]^{+}: 446.1751$; found: 446.1744 .

HPLC analysis: The enantiomeric excess was determined by HPLC with Chiralpak IB column (hexane/i-PrOH $=80 / 20$, flow rate $\left.1 \mathrm{~mL} \cdot \mathrm{min}^{-1}, \lambda=285 \mathrm{~nm}\right): t_{\text {major }}=14.5 \mathrm{~min}, t_{\text {minor }}=8.2 \mathrm{~min}$. 


\section{d. General Procedure to Prepare Racemic Products 5a-5I}<smiles>[R]C#CC(O)(c1c[nH]c2cc[R1]cc12)C(F)(F)F</smiles><smiles>Oc1ccc2ccccc2c1</smiles>

4a
Diphenyl phosphate (10 mol\%)

DCM, RT

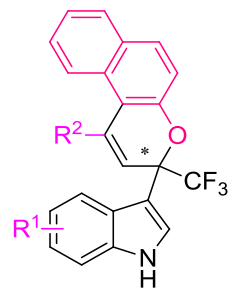

$\mathrm{Ph}-\mathrm{O}_{\mathrm{O}}^{\mathrm{O}} \mathrm{P}-\mathrm{OH}-\mathrm{Ph}$

Diphenyl phosphate

To a solution of $\alpha$-indolyl propargylic alcohols 1 ( $0.05 \mathrm{mmol}, 1.0$ equiv.), and catalyst diphenyl phosphate ( $0.005 \mathrm{mmol}, 10 \mathrm{~mol} \%)$ in DCM (1 mL) was added 2-naphthols 4 a $(0.05 \mathrm{mmol}, 1.0$ equiv.). The mixture was stirred at room temperature until the reaction was completed (monitored by TLC analysis). The crude product was purified directly by flash column chromatography on silica gel (petroleum ether/ ethyl acetate = 10:1 5:1) to give the desired racemic products $5 \mathbf{a}-5 \mathbf{l}$.

\section{e. General Procedure to Prepare Chiral Products 5a-5I}<smiles>[R]C#CC(O)(c1c[nH]c2cc[R1]cc12)C(F)(F)F</smiles>

1<smiles>Oc1ccc2ccccc2c1</smiles>

$4 a$

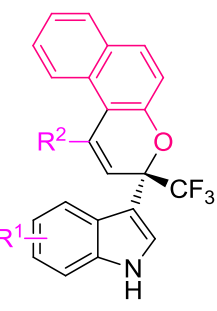

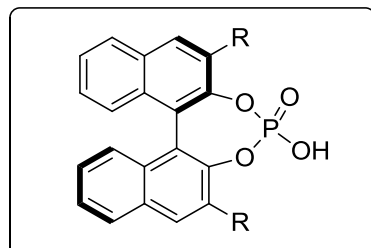

(R)-A7: R= 1-naphthyl

To a solution of $\alpha$-indolyl propargylic alcohols 1 ( $0.05 \mathrm{mmol}, 1.0$ equiv.) and catalyst $(\boldsymbol{R})$-A7 (0.01 mmol, 20 mol\%) in DCM $(0.5 \mathrm{~mL})$ was added 2-naphthols $4 a\left(0.05 \mathrm{mmol}, 1.0\right.$ equiv.). The mixture was stirred at $-10{ }^{\circ} \mathrm{C}$ until the reaction was completed (monitored by TLC analysis). The crude product was purified directly by flash column chromatography on silica gel (petroleum ether/ ethyl acetate $=10: 1 \sim 5: 1$ ) to give the desired chiral products 5a-5l.

\section{f. Analytical Data for Products 5a-5I}

(S)-3-(1-phenyl-3-(trifluoromethyl)-3H-benzo[f]chromen-3-yl)-1H-indole (5a):<smiles>FC(F)(F)[C@]1(c2c[nH]c3ccccc23)C=C(c2ccccc2)c2c(ccc3ccccc23)O1</smiles>

White foam, $19.9 \mathrm{mg}, 90 \%$ yield, $95: 5 \mathrm{er},[a]_{D}^{20}=+498.26(\mathrm{c}=0.13, \mathrm{MeOH})$. purified by flash column chromatography on silica gel (petroleum ether/ ethyl acetate $=10: 1, \mathrm{Rf}=0.6$ ).

${ }^{1} \mathrm{H}$ NMR $\left(400 \mathrm{MHz}, \mathrm{CDCl}_{3}\right) \delta: 8.09(\mathrm{~d}, J=7.9 \mathrm{~Hz}, 1 \mathrm{H}), 8.06(\mathrm{~s}, 1 \mathrm{H}), 7.69(\mathrm{~d}, J=8.8 \mathrm{~Hz}, 1 \mathrm{H}), 7.64(\mathrm{~d}, J=8.1 \mathrm{~Hz}$, $1 \mathrm{H}), 7.48-7.26(\mathrm{~m}, 7 \mathrm{H}), 7.25-7.14(\mathrm{~m}, 4 \mathrm{H}), 7.04-6.94(\mathrm{~m}, 2 \mathrm{H}), 6.35(\mathrm{~s}, 1 \mathrm{H})$.

${ }^{13} \mathrm{C}$ NMR $\left(101 \mathrm{MHz}, \mathrm{CDCl}_{3}\right) \delta: 151.5,140.8,140.0,136.4,131.5,130.7,129.8,128.8,128.6,128.2,128.1$, 128.0, 126.4, 126.2, 126.1, 125.4, 124.9 (q, J = 284.2 Hz), 123.7, 122.6, 121.6, 121.5, 120.6, 119.6, 118.2, $116.0,111.4,111.0,79.1(q, J=31.4 \mathrm{~Hz})$ 
${ }^{19} \mathrm{~F}$ NMR $\left(376 \mathrm{MHz}, \mathrm{CDCl}_{3}\right) \delta:-77.75$

HRMS (ESI): $m / z$ calcd. for $\mathrm{C}_{28} \mathrm{H}_{19} \mathrm{NOF}_{3}[\mathrm{M}+\mathrm{H}]^{+}:$:442.1413; found: 442.1410 .

HPLC analysis: The enantiomeric excess was determined by HPLC with Chiralpak IB column (hexane/ $i-\mathrm{PrOH}$ $=90 / 10$, flow rate $\left.1 \mathrm{~mL} \cdot \mathrm{min}^{-1}, \lambda=285 \mathrm{~nm}\right): t_{\text {major }}=8.5 \mathrm{~min}, t_{\text {minor }}=7.2 \mathrm{~min}$.

(S)-5-fluoro-3-(1-phenyl-3-(trifluoromethyl)-3H-benzo[f]chromen-3-yl)-1H-indole (5b):

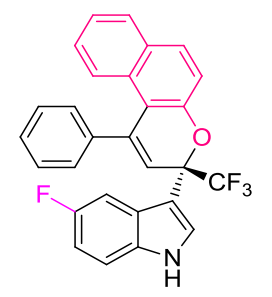

White foam, $20.7 \mathrm{mg}, 90 \%$ yield, $96: 4 \mathrm{er},[a]_{D}^{20}=+554.55$ (c $\left.=0.11, \mathrm{MeOH}\right)$. purified by flash column chromatography on silica gel (petroleum ether/ ethyl acetate $=10: 1, \mathrm{Rf}=0.6$ ).

${ }^{1} \mathrm{H}$ NMR $\left(400 \mathrm{MHz}, \mathrm{CDCl}_{3}\right) \delta: 8.06(\mathrm{~s}, 1 \mathrm{H}), 7.74(\mathrm{~d}, \mathrm{~J}=10.1 \mathrm{~Hz}, 1 \mathrm{H}), 7.70(\mathrm{~d}, \mathrm{~J}=8.9 \mathrm{~Hz}, 1 \mathrm{H}), 7.65(\mathrm{~d}, \mathrm{~J}=8.1$ $\mathrm{Hz}, 1 \mathrm{H}), 7.52-7.23(\mathrm{~m}, 7 \mathrm{H}), 7.18(\mathrm{ddd}, \mathrm{J}=8.0,6.3,1.7 \mathrm{~Hz}, 1 \mathrm{H}), 7.13(\mathrm{dd}, \mathrm{J}=8.9,4.4 \mathrm{~Hz}, 1 \mathrm{H}), 7.06-6.95(\mathrm{~m}$, $2 \mathrm{H}), 6.90$ (td, $\mathrm{J}=9.0,2.5 \mathrm{~Hz}, 1 \mathrm{H}), 6.30(\mathrm{~s}, 1 \mathrm{H})$.

${ }^{13} \mathrm{C}$ NMR $\left(101 \mathrm{MHz}, \mathrm{CDCl}_{3}\right) \delta: 158.2(\mathrm{~d}, \mathrm{~J}=235.0 \mathrm{~Hz}), 151.4,140.7,140.3,132.9,131.6,130.7,129.8,128.8$, 128.6, 128.3, 128.0, 127.8, 126.4, 125.5, 124.8 (q, J = 281.5 Hz), 123.8, 119.2, 118.2, 116.1, 112.1, 112.0, 111.4, 111.3 (d, J = 4.7 Hz), 111.1, 106.7 (d, J = 2.0 Hz), 106.5 (d, J = 1.3 Hz), 78.8 (q, J = 31.3 Hz).

${ }^{19}$ F NMR (376 MHz, $\left.\mathrm{CDCl}_{3}\right)$ $\delta:-77.72,-123.10$.

HRMS (ESI): $m / z$ calcd. for $\mathrm{C}_{28} \mathrm{H}_{18} \mathrm{NOF}_{4}[\mathrm{M}+\mathrm{H}]^{+}: 460.1319$; found: 460.1311 .

HPLC analysis: The enantiomeric excess was determined by HPLC with Chiralpak IB column (hexane/i-PrOH $=90 / 10$, flow rate $\left.1 \mathrm{~mL} \cdot \mathrm{min}^{-1}, \lambda=285 \mathrm{~nm}\right): t_{\text {major }}=9.0 \mathrm{~min}, t_{\text {minor }}=8.0 \mathrm{~min}$.

(S)-5-chloro-3-(1-phenyl-3-(trifluoromethyl)-3H-benzo[f]chromen-3-yl)-1H-indole (5c):

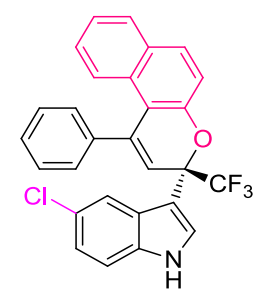

White foam, $21.9 \mathrm{mg}, 92 \%$ yield, $96: 4 \mathrm{er},[a]_{D}^{20}=+514.29(\mathrm{c}=0.14, \mathrm{MeOH})$. purified by flash column chromatography on silica gel (petroleum ether/ ethyl acetate $=10: 1, \mathrm{Rf}=0.6$ ).

${ }^{1} \mathrm{H}$ NMR $\left(400 \mathrm{MHz}, \mathrm{CDCl}_{3}\right)$ ס: $8.06(\mathrm{~s}, 2 \mathrm{H}), 7.70(\mathrm{~d}, \mathrm{~J}=8.8 \mathrm{~Hz}, 1 \mathrm{H}), 7.64(\mathrm{~d}, \mathrm{~J}=7.1 \mathrm{~Hz}, 1 \mathrm{H}), 7.44-7.35(\mathrm{~m}, 5 \mathrm{H})$, $7.33-7.21(\mathrm{~m}, 2 \mathrm{H}), 7.20-7.15(\mathrm{~m}, 1 \mathrm{H}), 7.09(\mathrm{~s}, 2 \mathrm{H}), 7.05-6.94(\mathrm{~m}, 2 \mathrm{H}), 6.30(\mathrm{~s}, 1 \mathrm{H})$.

${ }^{13} \mathrm{C}$ NMR $\left(101 \mathrm{MHz}, \mathrm{CDCl}_{3}\right) \delta: 151.3,140.6,140.3,134.7,131.7,130.7,129.8,129.0,128.8,128.6,128.3$, 128.1, 128.0, 127.9, 127.5, 127.1, 126.4, 125.5, 124.8 (q, J = 297.7 Hz), 123.8, 123.1, 121.0, 119.1, 118.1, 116.0, 112.4, 111.0, 78.8 (q, $\mathrm{J}=31.7 \mathrm{~Hz})$.

${ }^{19}$ F NMR $\left(376 \mathrm{MHz}, \mathrm{CDCl}_{3}\right) \delta:-77.70$.

HRMS (ESI): $m / z$ calcd. for $\mathrm{C}_{28} \mathrm{H}_{18} \mathrm{NOF}_{3} \mathrm{Cl}[\mathrm{M}+\mathrm{H}]^{+}: 476.1024$; found: 476.1014 .

HPLC analysis: The enantiomeric excess was determined by HPLC with Chiralpak IB column (hexane/ $i-\mathrm{PrOH}$ $=90 / 10$, flow rate $\left.1 \mathrm{~mL} \cdot \mathrm{min}^{-1}, \lambda=285 \mathrm{~nm}\right): t_{\text {major }}=8.7 \mathrm{~min}, t_{\text {minor }}=8.0 \mathrm{~min}$.

(S)-5-bromo-3-(1-phenyl-3-(trifluoromethyl)-3H-benzo[f]chromen-3-yl)-1 $H$-indole (5d): 
<smiles>FC(F)(F)c1c[nH]c2ccc(Br)cc12</smiles>

White foam, $23.4 \mathrm{mg}, 90 \%$ yield, $95: 5 \mathrm{er},[a]_{D}^{20}=+502.11$ (c $\left.=0.19, \mathrm{MeOH}\right)$. purified by flash column chromatography on silica gel (petroleum ether/ ethyl acetate $=10: 1, \mathrm{Rf}=0.6$ ).

${ }^{1} \mathrm{H}$ NMR $\left(400 \mathrm{MHz}, \mathrm{CDCl}_{3}\right) \delta: 8.23(\mathrm{~s}, 1 \mathrm{H}), 8.03(\mathrm{~s}, 1 \mathrm{H}), 7.71(\mathrm{~d}, \mathrm{~J}=8.9 \mathrm{~Hz}, 1 \mathrm{H}), 7.64(\mathrm{~d}, \mathrm{~J}=8.1 \mathrm{~Hz}, 1 \mathrm{H}), 7.50-$ $7.25(\mathrm{~m}, 7 \mathrm{H}), 7.24-7.15(\mathrm{~m}, 2 \mathrm{H}), 7.07-6.95(\mathrm{~m}, 3 \mathrm{H}), 6.30(\mathrm{~s}, 1 \mathrm{H})$.

${ }^{13} \mathrm{C}$ NMR $\left(101 \mathrm{MHz}, \mathrm{CDCl}_{3}\right) \delta: 151.3,140.6,140.3,135.0,131.7,130.7,129.8,128.8,128.6,128.4,128.1$, 128.0, 127.7, 127.3, 126.4, 125.6, 125.5, 124.7 (q, J = 287.0 Hz), 124.2, 124.1, 123.8, 119.1, 118.1, 116.0, 114.0, 112.8, 110.9, 78.7 (q, J = 31.6 Hz).

${ }^{19}$ F NMR $\left(376 \mathrm{MHz}, \mathrm{CDCl}_{3}\right) \delta:-77.69$.

HRMS (ESI): $m / z$ calcd. for $\mathrm{C}_{28} \mathrm{H}_{18} \mathrm{NOF}_{3} \mathrm{Br}[\mathrm{M}+\mathrm{H}]^{+}: 520.0518$; found: 520.0525 .

HPLC analysis: The enantiomeric excess was determined by HPLC with Chiralpak IB column (hexane/ $i-\mathrm{PrOH}$ $=90 / 10$, flow rate $\left.1 \mathrm{~mL} \cdot \mathrm{min}^{-1}, \lambda=285 \mathrm{~nm}\right): t_{\text {major }}=8.8 \mathrm{~min}, t_{\text {minor }}=8.1 \mathrm{~min}$.

(S)-5-methyl-3-(1-phenyl-3-(trifluoromethyl)-3H-benzo[f]chromen-3-yl)-1H-indole (5e):

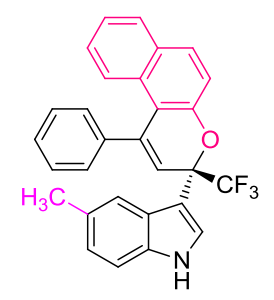

White foam, $19.6 \mathrm{mg}, 86 \%$ yield, $92: 8 \mathrm{er},[a]_{D}^{20}=+462.83(\mathrm{c}=0.12, \mathrm{MeOH})$. purified by flash column chromatography on silica gel (petroleum ether/ ethyl acetate $=10: 1, \mathrm{Rf}=0.6$ ).

${ }^{1} \mathrm{H}$ NMR $\left(400 \mathrm{MHz}, \mathrm{CDCl}_{3}\right) \delta: 7.95(\mathrm{~s}, 1 \mathrm{H}), 7.87(\mathrm{~s}, 1 \mathrm{H}), 7.70(\mathrm{~d}, \mathrm{~J}=8.8 \mathrm{~Hz}, 1 \mathrm{H}), 7.65(\mathrm{~d}, \mathrm{~J}=8.1 \mathrm{~Hz}, 1 \mathrm{H}), 7.46-$ $7.24(\mathrm{~m}, 7 \mathrm{H}), 7.17$ (ddd, J = 8.0, 6.4, $1.5 \mathrm{~Hz}, 1 \mathrm{H}), 7.11$ (d, J = 8.3 Hz, 1H), $7.05-6.95(\mathrm{~m}, 3 \mathrm{H}), 6.34(\mathrm{~s}, 1 \mathrm{H})$, $2.53(\mathrm{~s}, 3 \mathrm{H})$.

${ }^{13} \mathrm{C}$ NMR $\left(101 \mathrm{MHz}, \mathrm{CDCl}_{3}\right) \delta: 151.5,140.9,139.9,134.7,131.5,130.7,129.9,129.8,128.8,128.7,128.6$, 128.2, 128.1, 128.0, 126.5, 126.3, 125.4, 124.9 (q, J = 280.5 Hz), 124.2, 123.6, 121.2, 121.1, 119.7, 118.2, 116.0, 111.0, 110.5, 79.1 (q, J = 33.9 Hz), 21.9.

${ }^{19}$ F NMR $\left(376 \mathrm{MHz}, \mathrm{CDCl}_{3}\right) \delta:-77.66$.

HRMS (ESI): $m / z$ calcd. for $\mathrm{C}_{29} \mathrm{H}_{21} \mathrm{NOF}_{3}[\mathrm{M}+\mathrm{H}]^{+}:$456.1570; found: 456.1564 .

HPLC analysis: The enantiomeric excess was determined by HPLC with Chiralpak IB column (hexane/ $i$-PrOH $=90 / 10$, flow rate $\left.1 \mathrm{~mL} \cdot \mathrm{min}^{-1}, \lambda=285 \mathrm{~nm}\right): t_{\text {major }}=7.3 \mathrm{~min}, t_{\text {minor }}=6.7 \mathrm{~min}$.

(S)-5-methoxy-3-(1-phenyl-3-(trifluoromethyl)-3H-benzo[f]chromen-3-yl)-1H-indole (5f):<smiles>COc1ccc2[nH]cc([C@]3(C(F)(F)F)C=C(c4ccccc4)c4c(ccc5ccccc45)O3)c2c1</smiles> 
White foam, $19.3 \mathrm{mg}, 82 \%$ yield, $93: 7 \mathrm{er},[a]_{D}^{20}=+465.00$ (c $\left.=0.10, \mathrm{MeOH}\right)$. purified by flash column chromatography on silica gel (petroleum ether/ ethyl acetate $=10: 1, \mathrm{Rf}=0.6$ ).

${ }^{1} \mathrm{H}$ NMR $\left(400 \mathrm{MHz}, \mathrm{CDCl}_{3}\right) \delta: 8.00(\mathrm{~s}, 1 \mathrm{H}), 7.67(\mathrm{~d}, \mathrm{~J}=15.4 \mathrm{~Hz}, 1 \mathrm{H}), 7.65(\mathrm{~d}, \mathrm{~J}=14.7 \mathrm{~Hz}, 1 \mathrm{H}), 7.53(\mathrm{~s}, 1 \mathrm{H})$, $7.42-7.33(\mathrm{~m}, 5 \mathrm{H}), 7.32-7.22(\mathrm{~m}, 2 \mathrm{H}), 7.19-7.14(\mathrm{~m}, 1 \mathrm{H}), 7.12(\mathrm{dd}, \mathrm{J}=8.8,1.8 \mathrm{~Hz}, 1 \mathrm{H}), 6.99(\mathrm{q}, \mathrm{J}=8.6 \mathrm{~Hz}$, $2 \mathrm{H}), 6.81(\mathrm{~d}, \mathrm{~J}=8.8 \mathrm{~Hz}, 1 \mathrm{H}), 6.30(\mathrm{~s}, 1 \mathrm{H}), 3.93(\mathrm{~s}, 3 \mathrm{H})$.

${ }^{13} \mathrm{C}$ NMR $\left(101 \mathrm{MHz}, \mathrm{CDCl}_{3}\right)$ ס: 154.4, 151.3, 140.7, 139.9, 131.4, 131.3, 130.6, 129.7, 128.6, 128.5, 128.4,

128.1, 127.9, 126.7, 126.5, 126.3, 125.3, 124.8 (q, J = 285.0 Hz), 123.5, 119.5, 118.0, 116.0, 112.8, 111.9,

110.6, 103.3, 103.3, 78.9 (q, J = 26.1 Hz), 56.0.

${ }^{19}$ F NMR $\left(376 \mathrm{MHz}, \mathrm{CDCl}_{3}\right) \delta:-77.57$.

HRMS (ESI): $m / z$ calcd. for $\mathrm{C}_{29} \mathrm{H}_{21} \mathrm{NO}_{2} \mathrm{~F}_{3}[\mathrm{M}+\mathrm{H}]^{+}:$472.1519; found: 472.1511 .

HPLC analysis: The enantiomeric excess was determined by HPLC with Chiralpak IB column (hexane/ $i$-PrOH $=90 / 10$, flow rate $\left.1 \mathrm{~mL} \cdot \mathrm{min}^{-1}, \lambda=285 \mathrm{~nm}\right): t_{\text {major }}=9.1 \mathrm{~min}, t_{\text {minor }}=8.5 \mathrm{~min}$.

(S)-6-chloro-3-(1-phenyl-3-(trifluoromethyl)-3H-benzo[f]chromen-3-yl)-1H-indole (5g):

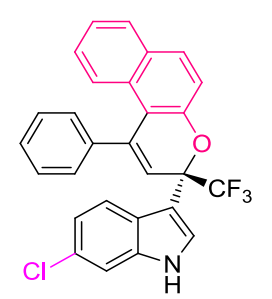

White foam, $21.4 \mathrm{mg}, 90 \%$ yield, $96: 4 \mathrm{er},[a]_{D}^{20}=+332.78$ (c $\left.=0.18, \mathrm{MeOH}\right)$. purified by flash column chromatography on silica gel (petroleum ether/ ethyl acetate $=10: 1, \mathrm{Rf}=0.6$ ).

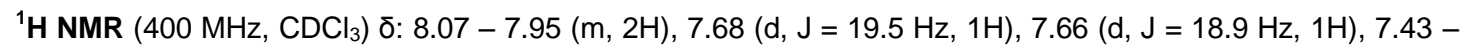
$7.24(\mathrm{~m}, 7 \mathrm{H}), 7.22-7.13(\mathrm{~m}, 3 \mathrm{H}), 7.08-6.94(\mathrm{~m}, 2 \mathrm{H}), 6.31(\mathrm{~s}, 1 \mathrm{H})$.

${ }^{13} \mathrm{C}$ NMR $\left(101 \mathrm{MHz}, \mathrm{CDCl}_{3}\right) \delta: 151.3,140.6,140.3,136.8,131.7,130.7,129.8,128.8,128.6,128.6,128.3$, 128.2, 127.9, 126.9, 126.4, 125.5, 124.7 (q, J = 298.0 Hz), 124.7, 123.8, 122.5, 122.5, 121.5, 119.2, 118.1, $116.1,111.4,111.3,78.8(\mathrm{q}, \mathrm{J}=31.6 \mathrm{~Hz})$.

${ }^{19} \mathrm{~F}$ NMR $\left(376 \mathrm{MHz}, \mathrm{CDCl}_{3}\right) \delta:-77.74$.

HRMS (ESI): $m / z$ calcd. for $\mathrm{C}_{28} \mathrm{H}_{18} \mathrm{NOF}_{3} \mathrm{Cl}[\mathrm{M}+\mathrm{H}]^{+}: 476.1024$; found: 476.1033 .

HPLC analysis: The enantiomeric excess was determined by HPLC with Chiralpak IB column (hexane/i-PrOH $=90 / 10$, flow rate $\left.1 \mathrm{~mL} \cdot \mathrm{min}^{-1}, \lambda=245 \mathrm{~nm}\right): t_{\text {major }}=7.6 \mathrm{~min}, t_{\text {minor }}=6.7 \mathrm{~min}$.

(S)-3-(1-(4-fluorophenyl)-3-(trifluoromethyl)-3H-benzo[f]chromen-3-yl)-1H-indole (5h):

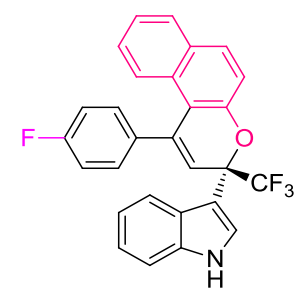

White foam, $20.0 \mathrm{mg}, 87 \%$ yield, $92: 8 \mathrm{er},[a]_{D}^{20}=+452.00$ (c $\left.=0.10, \mathrm{MeOH}\right)$. purified by flash column chromatography on silica gel (petroleum ether/ ethyl acetate $=10: 1, \mathrm{Rf}=0.5$ ).

${ }^{1} \mathrm{H}$ NMR $\left(400 \mathrm{MHz}, \mathrm{CDCl}_{3}\right) \delta: 8.08(\mathrm{~s}, 1 \mathrm{H}), 8.05(\mathrm{~d}, \mathrm{~J}=7.8 \mathrm{~Hz}, 1 \mathrm{H}), 7.68(\mathrm{~d}, \mathrm{~J}=8.9 \mathrm{~Hz}, 1 \mathrm{H}), 7.63(\mathrm{~d}, \mathrm{~J}=8.1 \mathrm{~Hz}$, $1 \mathrm{H}), 7.36(\mathrm{~d}, \mathrm{~J}=8.8 \mathrm{~Hz}, 1 \mathrm{H}), 7.33(\mathrm{~d}, \mathrm{~J}=2.7 \mathrm{~Hz}, 1 \mathrm{H}), 7.23(\mathrm{~d}, \mathrm{~J}=9.9 \mathrm{~Hz}, 3 \mathrm{H}), 7.20-7.12(\mathrm{~m}, 3 \mathrm{H}), 7.06(\mathrm{t}, \mathrm{J}=$ $8.2 \mathrm{~Hz}, 2 \mathrm{H}), 7.02-6.94(\mathrm{~m}, 2 \mathrm{H}), 6.29(\mathrm{~s}, 1 \mathrm{H})$. 
${ }^{13} \mathrm{C}$ NMR (101 MHz, $\left.\mathrm{CDCl}_{3}\right) \delta: 162.8(\mathrm{~d}, \mathrm{~J}=247.5 \mathrm{~Hz}), 151.6,139.0,136.7$ (d, J = 3.4 Hz), 136.4, 131.7, 130.7, 129.7, 129.7, 128.7, 126.3, 126.1, 126.0, 125.5, 124.8 (q, J = 283.6 Hz), 123.7, 122.6, 121.5, 121.5, 120.6, $119.5,118.2,115.8,115.8,115.7,111.5,110.9,79.1$ (q, J = 31.8 Hz).

${ }^{19} \mathrm{~F}$ NMR $\left(376 \mathrm{MHz}, \mathrm{CDCl}_{3}\right) \delta:-77.77,-113.68$.

HRMS (ESI): $m / z$ calcd. for $\mathrm{C}_{28} \mathrm{H}_{18} \mathrm{NOF}_{4}[\mathrm{M}+\mathrm{H}]^{+}: 460.1319$; found: 460.1310 .

HPLC analysis: The enantiomeric excess was determined by HPLC with Chiralpak IB column (hexane/i-PrOH $=90 / 10$, flow rate $\left.1 \mathrm{~mL} \cdot \mathrm{min}^{-1}, \lambda=254 \mathrm{~nm}\right): t_{\text {major }}=9.0 \mathrm{~min}, t_{\text {minor }}=7.4 \mathrm{~min}$.

(S)-3-(1-(4-chlorophenyl)-3-(trifluoromethyl)-3H-benzo[f]chromen-3-yl)-1H-indole (5i):

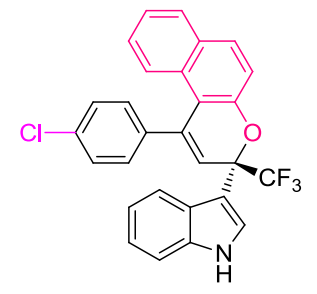

White foam, $20.2 \mathrm{mg}, 85 \%$ yield, $93: 7 \mathrm{er},[a]_{D}^{20}=+463.00(\mathrm{c}=0.10, \mathrm{MeOH})$. purified by flash column chromatography on silica gel (petroleum ether/ ethyl acetate $=10: 1, \mathrm{Rf}=0.6$ ).

${ }^{1}$ H NMR $\left(400 \mathrm{MHz}, \mathrm{CDCl}_{3}\right) \delta: 8.13-8.02(\mathrm{~m}, 2 \mathrm{H}), 7.70(\mathrm{~d}, \mathrm{~J}=8.9 \mathrm{~Hz}, 1 \mathrm{H}), 7.65(\mathrm{~d}, \mathrm{~J}=8.1 \mathrm{~Hz}, 1 \mathrm{H}), 7.40-7.33$ (m, 4H), $7.27-7.14(\mathrm{~m}, 6 \mathrm{H}), 7.07-6.97(\mathrm{~m}, 2 \mathrm{H}), 6.33(\mathrm{~s}, 1 \mathrm{H})$.

${ }^{13} \mathrm{C}$ NMR $\left(101 \mathrm{MHz}, \mathrm{CDCl}_{3}\right)$ $\delta: 139.2,139.0,136.4,134.2,131.8,130.7,130.2,129.6,129.4,129.3,129.0$, 128.8, 128.7, 126.3, 126.1, 126.0, 125.6, 124.8 (q, J = 283.9 Hz), 123.8, 122.7, 121.5, 120.7, 119.9, 118.2, 115.6, 111.5, 110.8, 79.1 (q, J = 31.6 Hz).

${ }^{19}$ F NMR $\left(376 \mathrm{MHz}, \mathrm{CDCl}_{3}\right) \delta:-77.73$.

HRMS (ESI): $\mathrm{m} / z$ calcd. for $\mathrm{C}_{28} \mathrm{H}_{18} \mathrm{NOF}_{3} \mathrm{Cl}[\mathrm{M}+\mathrm{H}]^{+}:$476.1024; found: 476.1025 .

HPLC analysis: The enantiomeric excess was determined by HPLC with Chiralpak IB column (hexane/ $i-\mathrm{PrOH}$ $=90 / 10$, flow rate $\left.1 \mathrm{~mL} \cdot \mathrm{min}^{-1}, \lambda=254 \mathrm{~nm}\right): t_{\text {major }}=8.5 \mathrm{~min}, t_{\text {minor }}=7.2 \mathrm{~min}$.

(S)-3-(1-(p-tolyl)-3-(trifluoromethyl)-3H-benzo[f]chromen-3-yl)-1H-indole (5j):

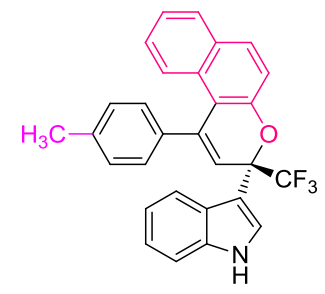

White foam, $21.2 \mathrm{mg}, 93 \%$ yield, $95: 5 \mathrm{er},[a]_{D}^{20}=+451.67(\mathrm{c}=0.12, \mathrm{MeOH})$. purified by flash column chromatography on silica gel (petroleum ether/ ethyl acetate $=10: 1, \mathrm{Rf}=0.7$ ).

${ }^{1} \mathrm{H}$ NMR $\left(400 \mathrm{MHz}, \mathrm{CDCl}_{3}\right) \delta: 8.09(\mathrm{~d}, \mathrm{~J}=7.8 \mathrm{~Hz}, 1 \mathrm{H}), 8.03(\mathrm{~s}, 1 \mathrm{H}), 7.68(\mathrm{~d}, \mathrm{~J}=8.8 \mathrm{~Hz}, 1 \mathrm{H}), 7.64(\mathrm{~d}, \mathrm{~J}=8.0 \mathrm{~Hz}$, $1 \mathrm{H}), 7.42-7.34(\mathrm{~m}, 2 \mathrm{H}), 7.32-7.11(\mathrm{~m}, 8 \mathrm{H}), 7.08(\mathrm{~d}, \mathrm{~J}=8.4 \mathrm{~Hz}, 1 \mathrm{H}), 7.03-6.97(\mathrm{~m}, 1 \mathrm{H}), 6.31(\mathrm{~s}, 1 \mathrm{H}), 2.43(\mathrm{~s}$ $3 \mathrm{H})$.

${ }^{13} \mathrm{C}$ NMR $\left(101 \mathrm{MHz}, \mathrm{CDCl}_{3}\right) \delta: 151.5,139.8,138.1,137.9,136.4,131.4,130.7,129.9,129.4,128.5,127.9$, 126.5, 126.3, 126.1, 125.3, 124.9 (q, J = 283.8 Hz), 123.6, 122.6, 121.6, 120.6, 119.2, 118.2, 116.1, 111.4, 111.2, 79.0 (q, J = 31.5 Hz), 21.5 .

${ }^{19}$ F NMR $\left(376 \mathrm{MHz}, \mathrm{CDCl}_{3}\right) \delta:-77.74$.

HRMS (ESI): $m / z$ calcd. for $\mathrm{C}_{29} \mathrm{H}_{21} \mathrm{NOF}_{3}[\mathrm{M}+\mathrm{H}]^{+}:$456.1570; found: 456.1564 . 
HPLC analysis: The enantiomeric excess was determined by HPLC with Chiralpak IB column (hexane/i-PrOH $=90 / 10$, flow rate $\left.1 \mathrm{~mL} \cdot \mathrm{min}^{-1}, \lambda=280 \mathrm{~nm}\right): t_{\text {major }}=8.4 \mathrm{~min}, t_{\text {minor }}=7.0 \mathrm{~min}$.

(S)-3-(1-(4-methoxyphenyl)-3-(trifluoromethyl)-3H-benzo[f]chromen-3-yl)-1H-indole (5k):

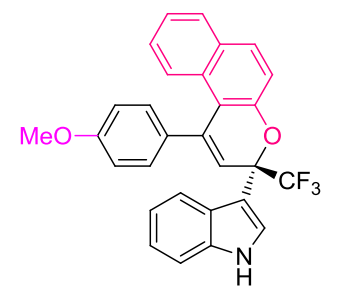

White foam, $22.6 \mathrm{mg}, 96 \%$ yield, $95: 5 \mathrm{er},[a]_{D}^{20}=+513.37$ (c $\left.=0.14, \mathrm{MeOH}\right)$. purified by flash column chromatography on silica gel (petroleum ether/ ethyl acetate $=10: 1, \mathrm{Rf}=0.7$ ).

${ }^{1} \mathrm{H}$ NMR $\left(400 \mathrm{MHz}, \mathrm{CDCl}_{3}\right) \delta: 8.12-8.01(\mathrm{~m}, 2 \mathrm{H}), 7.66(\mathrm{~d}, \mathrm{~J}=8.8 \mathrm{~Hz}, 1 \mathrm{H}), 7.62(\mathrm{~d}, \mathrm{~J}=8.1 \mathrm{~Hz}, 1 \mathrm{H}), 7.35(\mathrm{~d}, \mathrm{~J}=$ $8.8 \mathrm{~Hz}, 2 \mathrm{H}), 7.27-7.11(\mathrm{~m}, 6 \mathrm{H}), 7.07(\mathrm{~d}, \mathrm{~J}=8.7 \mathrm{~Hz}, 1 \mathrm{H}), 7.02-6.97(\mathrm{~m}, 1 \mathrm{H}), 6.90(\mathrm{~d}, \mathrm{~J}=8.1 \mathrm{~Hz}, 2 \mathrm{H}), 6.27(\mathrm{~s}$, $1 \mathrm{H}), 3.85(\mathrm{~s}, 3 \mathrm{H})$.

${ }^{13} \mathrm{C}$ NMR (101 MHz, CDCl $)$ ): 159.7, 151.5, 139.4, 136.4, 133.2, 131.4, 130.7, 129.9, 129.2, 128.5, 126.5, 126.3, 126.1, 125.4, 124.9 (d, J = 284.2 Hz), 123.6, 122.6, 121.6, 120.6, 118.7, 118.2, 116.2, 114.1, 111.4, $111.2,79.0$ (q, J = 31.7 Hz), 55.5 .

${ }^{19} \mathrm{~F}$ NMR $\left(376 \mathrm{MHz}, \mathrm{CDCl}_{3}\right) \delta:-77.77$.

HRMS (ESI): $m / z$ calcd. for $\mathrm{C}_{29} \mathrm{H}_{21} \mathrm{NO}_{2} \mathrm{~F}_{3}[\mathrm{M}+\mathrm{H}]^{+}:$472.1519; found: 472.1513.

HPLC analysis: The enantiomeric excess was determined by HPLC with Chiralpak IB column (hexane/ $i-\mathrm{PrOH}$ $=90 / 10$, flow rate $\left.1 \mathrm{~mL} \cdot \mathrm{min}^{-1}, \lambda=280 \mathrm{~nm}\right): t_{\text {major }}=10.5 \mathrm{~min}, t_{\text {minor }}=8.8 \mathrm{~min}$.

(S)-3-(1-cyclopropyl-3-(trifluoromethyl)-3H-benzo[f]chromen-3-yl)-1 $H$-indole (5I):<smiles>FC(F)(F)[C@H]1C=C(c2ccccc2)c2c(ccc3ccccc23)O1</smiles>

White foam, $19.5 \mathrm{mg}, 96 \%$ yield, $90: 10 \mathrm{er},[a]_{D}^{20}=+415.38$ ( $\mathrm{c}=0.13$, MeOH). purified by flash column chromatography on silica gel (petroleum ether/ ethyl acetate $=10: 1, \mathrm{Rf}=0.7$ ).

${ }^{1} \mathrm{H}$ NMR $\left(400 \mathrm{MHz}, \mathrm{CDCl}_{3}\right) \delta: 8.66(\mathrm{~d}, \mathrm{~J}=8.7 \mathrm{~Hz}, 1 \mathrm{H}), 7.94(\mathrm{~d}, \mathrm{~J}=8.3 \mathrm{~Hz}, 2 \mathrm{H}), 7.65(\mathrm{~d}, \mathrm{~J}=8.1 \mathrm{~Hz}, 1 \mathrm{H}), 7.60$ (d, $\mathrm{J}=8.8 \mathrm{~Hz}, 1 \mathrm{H}), 7.36(\mathrm{t}, \mathrm{J}=7.7 \mathrm{~Hz}, 1 \mathrm{H}), 7.29-7.26(\mathrm{~m}, 1 \mathrm{H}), 7.25-7.21(\mathrm{~m}, 1 \mathrm{H}), 7.21-7.15(\mathrm{~m}, 1 \mathrm{H}), 7.16-$ $6.97(\mathrm{~m}, 3 \mathrm{H}), 5.98(\mathrm{~s}, 1 \mathrm{H}), 2.14-1.97(\mathrm{~m}, 1 \mathrm{H}), 1.27-1.20(\mathrm{~m}, 1 \mathrm{H}), 1.06-0.93(\mathrm{~m}, 2 \mathrm{H}), 0.88-0.82(\mathrm{~m}, 1 \mathrm{H})$. ${ }^{13} \mathrm{C}$ NMR $\left(101 \mathrm{MHz}, \mathrm{CDCl}_{3}\right) \delta: 150.5,140.6,136.3,131.1,130.7,130.6,128.9,126.1,126.0,125.7,124.9$ (q, J $=280.2 \mathrm{~Hz}$ ), 123.7, 122.5, 121.6, 120.4, 118.1, 117.8, 113.4, 111.5, 111.3, 79.0 (q, J = 31.2 Hz), 16.8, 12.6, 7.9. ${ }^{19}$ F NMR (376 MHz, $\left.\mathrm{CDCl}_{3}\right)$ ס: -78.00 .

HRMS (ESI): $m / z$ calcd. for $\mathrm{C}_{25} \mathrm{H}_{19} \mathrm{NOF}_{3}[\mathrm{M}+\mathrm{H}]^{+}:$: 406.1413; found: 406.1405 .

HPLC analysis: The enantiomeric excess was determined by HPLC with Chiralpak IB column (hexane/i-PrOH $=90 / 10$, flow rate $\left.1 \mathrm{~mL} \cdot \mathrm{min}^{-1}, \lambda=280 \mathrm{~nm}\right): t_{\text {major }}=7.6 \mathrm{~min}, t_{\text {minor }}=7.3 \mathrm{~min}$. 


\section{The scale-up experiment}<smiles>OC(C#Cc1ccccc1)(C(F)(F)F)C(F)(F)C(F)(F)F</smiles>

$1.5 \mathrm{mmol}$<smiles>Oc1cccc2ccccc12</smiles>

2a

$1.8 \mathrm{mmol}$

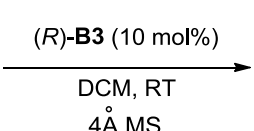

$4 \mathrm{~A} M S$

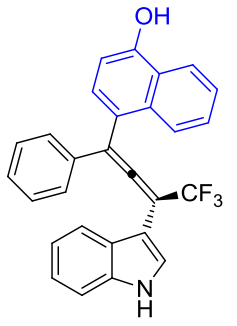

$3 a$

$602.6 \mathrm{mg}, 91 \%$ yield $96: 4 \mathrm{er}$

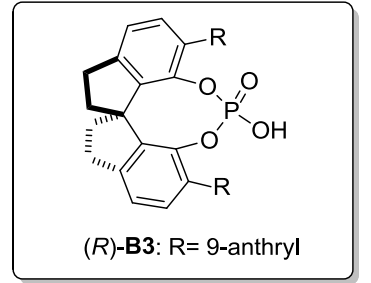

(R)-B3: R= 9-anthryl

To a solution of $\alpha$-indolyl propargylic alcohols $1 \mathrm{a}$ (1.50 mmol, 1.0 equiv.), $150 \mathrm{mg} 4 \AA$ molecular sieves, and catalyst $(\boldsymbol{R})$-B3 $(0.15 \mathrm{mmol}, 10 \mathrm{~mol} \%)$ in DCM $(20 \mathrm{~mL})$ was added phenols 2 a $(1.80 \mathrm{mmol}, 1.2$ equiv.). The mixture was stirred at room temperature until the reaction was completed (monitored by TLC analysis). The crude product was purified directly by flash column chromatography on silica gel (petroleum ether/ ethyl acetate $=8: 1 \sim 3: 1)$ to give the desired chiral products 3 a with $602.6 \mathrm{mg}, 91 \%$ yield and $96: 4$ er.

\section{Experimental Procedure and Characterization Data of Compounds 11-17.}

$\mathrm{Ph}$<smiles>CC#CC(O)(c1ccccc1)c1ccc(O)cc1</smiles>

10<smiles>Oc1cccc2ccccc12</smiles>

2a

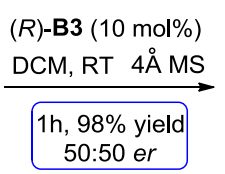

$50: 50$ er

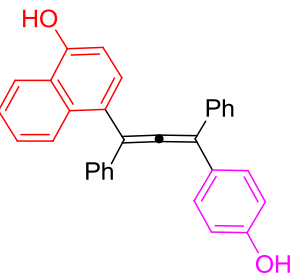

11

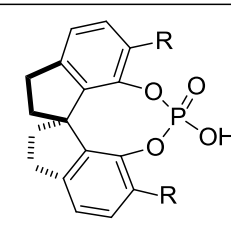

(R)-B3: $\mathrm{R}=9$-anthry

To a solution of 10 ( $0.10 \mathrm{mmol}, 1.0$ equiv. $), 50 \mathrm{mg} 4 \AA$ molecular sieves, and catalyst $(\boldsymbol{R})$-B3 $(0.01 \mathrm{mmol}, 10$ mol\%) in DCM (2 mL) was added phenols $2 \mathrm{a}(0.12 \mathrm{mmol}, 1.2$ equiv.). The mixture was stirred at room temperature until the reaction was completed (monitored by TLC analysis). The crude product was purified directly by flash column chromatography on silica gel (petroleum ether/ ethyl acetate $=10: 1 \sim 5: 1$ ) to give the desired chiral product 11.

4-(3-(4-hydroxyphenyl)-1,3-diphenylpropa-1,2-dien-1-yl)naphthalen-1-ol (11):

White foam, $41.8 \mathrm{mg}, 98 \%$ yield. purified by flash column chromatography on silica gel (petroleum ether/ ethyl acetate $=10: 1, \mathrm{Rf}=0.4)$.

${ }^{1} \mathrm{H}$ NMR $\left(400 \mathrm{MHz}, \mathrm{CDCl}_{3}\right) \delta: 8.13(\mathrm{~d}, J=8.4 \mathrm{~Hz}, 1 \mathrm{H}), 7.72(\mathrm{~d}, J=8.4 \mathrm{~Hz}, 1 \mathrm{H}), 7.38-7.27(\mathrm{~m}, 4 \mathrm{H}), 7.24-7.06$ $(\mathrm{m}, 11 \mathrm{H}), 6.75(\mathrm{~d}, J=7.6 \mathrm{~Hz}, 1 \mathrm{H}), 6.70(\mathrm{~d}, J=8.5 \mathrm{~Hz}, 2 \mathrm{H}), 5.57(\mathrm{~s}, 1 \mathrm{H}), 5.03(\mathrm{~s}, 1 \mathrm{H})$;

${ }^{13} \mathrm{C}$ NMR $\left(126 \mathrm{MHz}, \mathrm{CDCl}_{3}\right) \delta: 208.0,155.3,151.7,137.2,136.8,133.4,130.1,128.9,128.7,128.6,127.8$, 127.6, 127.2, 127.0, 126.7, 126.7, 126.6, 125.4, 124.9, 122.1, 115.5, 111.8, 109.6, 108.5.

HRMS (ESI): $m / z$ calcd. for $\mathrm{C}_{31} \mathrm{H}_{23} \mathrm{O}_{2}[\mathrm{M}+\mathrm{H}]^{+}:$427.1693; found: 427.1693 .<smiles>C#CC(O)(c1ccccc1)c1ccc(O)cc1</smiles>

10

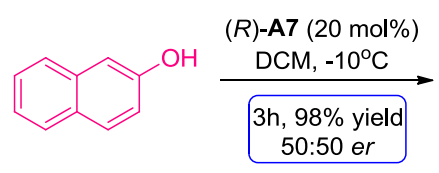

$4 a$
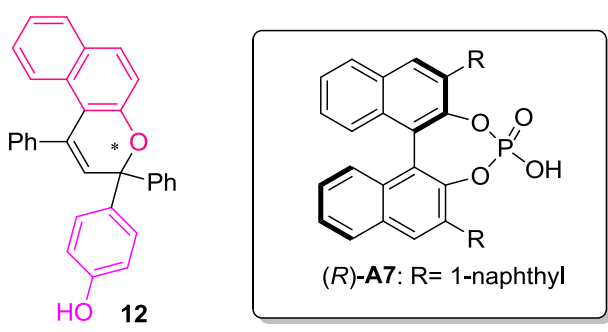

To a solution of 10 (0.05 mmol, 1.0 equiv.) and catalyst $(\boldsymbol{R})$-A7 (0.01 mmol, $20 \mathrm{~mol} \%)$ in DCM (0.5 mL) was 
added phenols $4 \mathrm{a}\left(0.05 \mathrm{mmol}, 1.0\right.$ equiv.). The mixture was stirred at $-10{ }^{\circ} \mathrm{C}$ until the reaction was completed (monitored by TLC analysis). The crude product was purified directly by flash column chromatography on silica gel (petroleum ether/ ethyl acetate = 10:1 5:1) to give the desired chiral product 12.

4-(1,3-diphenyl-3H-benzo[f]chromen-3-yl)phenol (12):

White foam, $20.9 \mathrm{mg}, 98 \%$ yield. purified by flash column chromatography on silica gel (petroleum ether/ ethyl acetate $=10: 1, \mathrm{Rf}=0.7)$.

${ }^{1}$ H NMR $\left(400 \mathrm{MHz}, \mathrm{CDCl}_{3}\right) \delta: 7.73(\mathrm{~d}, J=8.8 \mathrm{~Hz}, 1 \mathrm{H}), 7.70(\mathrm{~d}, J=8.2 \mathrm{~Hz}, 1 \mathrm{H}), 7.57(\mathrm{~d}, J=7.4 \mathrm{~Hz}, 2 \mathrm{H}), 7.43(\mathrm{~d}$, $J=8.6 \mathrm{~Hz}, 2 \mathrm{H}), 7.40-7.18(\mathrm{~m}, 10 \mathrm{H}), 7.13(\mathrm{~d}, J=8.6 \mathrm{~Hz}, 1 \mathrm{H}), 7.06-7.01(\mathrm{~m}, 1 \mathrm{H}), 6.74(\mathrm{~d}, J=8.6 \mathrm{~Hz}, 2 \mathrm{H})$, $6.20(\mathrm{~s}, 1 \mathrm{H}), 4.89(\mathrm{~s}, 1 \mathrm{H})$;

${ }^{13} \mathrm{C}$ NMR $\left(101 \mathrm{MHz}, \mathrm{CDCl}_{3}\right) \delta: 155.0,152.7,144.8,141.4,137.2,136.8,130.9,130.4,129.9,129.7,128.9$, 128.6, 128.5, 128.1, 127.8, 127.5, 127.1, 126.6, 125.2, 123.3, 118.9, 116.7, 114.9, 82.1.

HRMS (ESI): $\mathrm{m} / \mathrm{z}$ calcd. for $\mathrm{C}_{31} \mathrm{H}_{23} \mathrm{O}_{2}[\mathrm{M}+\mathrm{H}]^{+}:$427.1693; found: 427.1686 .

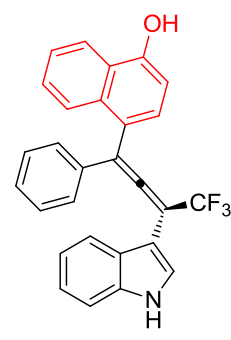

3a (0.1 mmol) 98:2 er

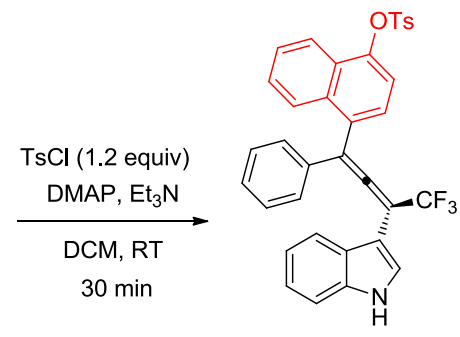

13

$86 \%$ yield, $98: 2$ er

To a solution of $3 \mathrm{a}\left(0.10 \mathrm{mmol}, 1.0\right.$ equiv), $\mathrm{Et}_{3} \mathrm{~N}(0.10 \mathrm{mmol}, 1.0$ equiv) and DMAP $(0.01 \mathrm{mmol}, 10 \mathrm{~mol} \%)$ in $\mathrm{DCM}(1 \mathrm{~mL})$ was added dropwise the solution of $\mathrm{TsCl}(0.12 \mathrm{mmol}, 1.2$ equiv) in DCM (1 mL). The mixture was further stirred at room temperature for $30 \mathrm{~min}$. The crude product was purified directly by flash column chromatography on silica gel (petroleum ether/ ethyl acetate $=10: 1 \sim 5: 1$ ) to give the desired compound 13.

(S)-4-(4,4,4-trifluoro-3-(1H-indol-3-yl)-1-phenylbuta-1,2-dien-1-yl)naphthalen-1-yl 4-methylbenzenesulfonate (13):

White foam, $51.2 \mathrm{mg}, 86 \%$ yield, $98: 2 \mathrm{er},[a]_{D}^{20}=+78.21$ (c $\left.=0.17, \mathrm{MeOH}\right)$. purified by flash column chromatography on silica gel (petroleum ether/ ethyl acetate $=5: 1, \mathrm{Rf}=0.4$ ).

${ }^{1}$ H NMR $\left(400 \mathrm{MHz}, \mathrm{CDCl}_{3}\right) \delta: 8.36(\mathrm{~s}, 1 \mathrm{H}), 8.01(\mathrm{~d}, J=8.5 \mathrm{~Hz}, 1 \mathrm{H}), 7.91(\mathrm{~d}, J=8.1 \mathrm{~Hz}, 1 \mathrm{H}), 7.83(\mathrm{~d}, J=8.3 \mathrm{~Hz}$, $2 \mathrm{H}), 7.78(\mathrm{~d}, J=8.1 \mathrm{~Hz}, 1 \mathrm{H}), 7.48(\mathrm{~d}, J=7.9 \mathrm{~Hz}, 1 \mathrm{H}), 7.46-7.36(\mathrm{~m}, 4 \mathrm{H}), 7.32-7.28(\mathrm{~m}, 6 \mathrm{H}), 7.26-7.14(\mathrm{~m}$, $3 \mathrm{H}), 7.05(\mathrm{t}, J=7.1 \mathrm{~Hz}, 1 \mathrm{H}), 2.42(\mathrm{~s}, 3 \mathrm{H})$;

${ }^{13} \mathrm{C}$ NMR $\left(126 \mathrm{MHz}, \mathrm{CDCl}_{3}\right) \delta: 205.0,146.3,145.7,136.3,135.2,133.1,132.9,132.0,130.0,129.0,128.7$, 128.6, 127.9, 127.6, 127.4, 127.3, 127.0, 126.4, 125.6, 123.8 (q, $J=279.9 \mathrm{~Hz}), 123.6$ (d, $J=2.4 \mathrm{~Hz}), 123.2$, 122.4, 120.9, 119.9, 118.2, 115.0, 111.6, 104.5, 99.2 (q, $J=35.3 \mathrm{~Hz}), 21.8$.

${ }^{19} \mathrm{~F}$ NMR $\left(376 \mathrm{MHz}, \mathrm{CDCl}_{3}\right) \delta:-61.32$.

HRMS (ESI): $m / z$ calcd. for $\mathrm{C}_{35} \mathrm{H}_{25} \mathrm{NO}_{3} \mathrm{~F}_{3} \mathrm{~S}[\mathrm{M}+\mathrm{H}]^{+}: 596.1502$; found: 596.1507.

HPLC analysis: The enantiomeric excess was determined by HPLC with Chiralpak IA column (hexane/i-PrOH $=90 / 10$, flow rate $\left.1.0 \mathrm{~mL} \cdot \mathrm{min}^{-1}, \lambda=254 \mathrm{~nm}\right): t_{\text {major }}=17.2 \mathrm{~min}, t_{\text {minor }}=32.6 \mathrm{~min}$. 


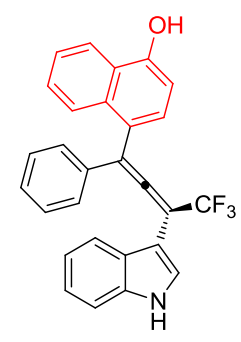

3 a $(0.1 \mathrm{mmol})$ 98:2 er

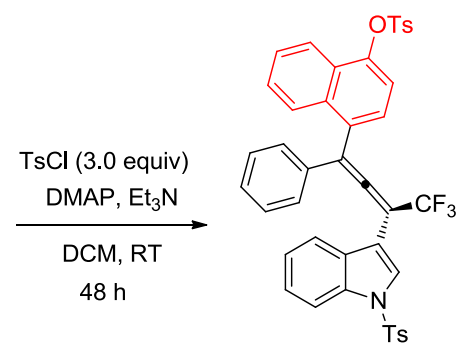

14

$52 \%$ yield, $98: 2$ er

To a solution of $3 \mathrm{a}\left(0.10 \mathrm{mmol}, 1.0\right.$ equiv), $\mathrm{Et}_{3} \mathrm{~N}(0.25 \mathrm{mmol}, 2.5$ equiv) and DMAP $(0.01 \mathrm{mmol}, 10 \mathrm{~mol} \%)$ in $\mathrm{DCM}(1 \mathrm{~mL})$ was added dropwise the solution of $\mathrm{TsCl}(0.30 \mathrm{mmol}, 3.0$ equiv) in DCM $(1 \mathrm{~mL})$. The mixture was further stirred at room temperature for $48 \mathrm{~h}$. The crude product was purified directly by flash column chromatography on silica gel (petroleum ether/ ethyl acetate $=10: 1$ ) to give the desired compound 14 .

(S)-4-(4,4,4-trifluoro-1-phenyl-3-(1-tosyl-1H-indol-3-yl)buta-1,2-dien-1-yl)naphthalen-1-yl 4-methylbenzenesulfonate. (14):

White foam, $39.0 \mathrm{mg}, 52 \%$ yield, $98: 2 \mathrm{er},[a]_{D}^{20}=+54.37$ (c $=0.13$, MeOH). purified by flash column chromatography on silica gel (petroleum ether/ ethyl acetate $=5: 1, \mathrm{Rf}=0.7$ ).

${ }^{1} \mathrm{H}$ NMR $\left(500 \mathrm{MHz}, \mathrm{CDCl}_{3}\right) \delta: 8.00(\mathrm{~d}, J=8.4 \mathrm{~Hz}, 2 \mathrm{H}), 7.85-7.76(\mathrm{~m}, 6 \mathrm{H}), 7.66(\mathrm{~d}, J=8.0 \mathrm{~Hz}, 1 \mathrm{H}), 7.47-7.43$ (m, 1H), $7.42(\mathrm{~d}, J=7.8 \mathrm{~Hz}, 1 \mathrm{H}), 7.38-7.30(\mathrm{~m}, 7 \mathrm{H}), 7.25-7.20(\mathrm{~m}, 5 \mathrm{H}), 7.10(\mathrm{t}, J=7.7 \mathrm{~Hz}, 1 \mathrm{H}), 2.43(\mathrm{~s}, 3 \mathrm{H})$, $2.34(\mathrm{~s}, 3 \mathrm{H})$;

${ }^{13} \mathrm{C}$ NMR $\left(126 \mathrm{MHz}, \mathrm{CDCl}_{3}\right) \delta: 205.8,146.6,145.8,145.5,135.0,134.9,134.2,133.0,132.9,131.0,130.2$, 130.1, 129.2, 129.0, 128.6, 128.5, 128.0, 127.7, 127.5, 127.4, 127.2, 127.1, 126.1, 125.5, 124.7 (d, J = 2.6 Hz), 123.9, 123.2 (q, $J=275.7 \mathrm{~Hz}$ ), 122.5, 120.4, 118.1, 115.9, 113.9, 110.5, 97.7 (q, $J=35.9 \mathrm{~Hz}$ ), 21.9, 21.7;

${ }^{19} \mathrm{~F}$ NMR $\left(376 \mathrm{MHz}, \mathrm{CDCl}_{3}\right) \delta:-61.38$

HRMS (ESI): $\mathrm{m} / z$ calcd. for $\mathrm{C}_{42} \mathrm{H}_{31} \mathrm{NO}_{5} \mathrm{~F}_{3} \mathrm{~S}_{2}[\mathrm{M}+\mathrm{H}]^{+}:$: 750.1590; found: 750.1597 .

HPLC analysis: The enantiomeric excess was determined by HPLC with Chiralpak IA column (hexane $/ \mathrm{i}-\mathrm{PrOH}=90 / 10$, flow rate $1.0 \mathrm{~mL} \cdot \mathrm{min}^{-1}, \lambda=254 \mathrm{~nm}$ ): $t_{\text {major }}=13.2 \mathrm{~min}, t_{\text {minor }}=11.2 \mathrm{~min}$.

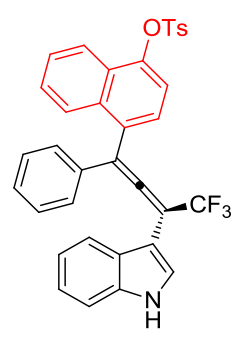

13

$(0.1 \mathrm{mmol})$

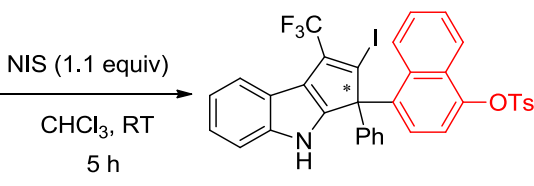

15 $47 \%$ yield, $95: 5$ er

To a solution of 13 (0.10 mmol, 1.0 equiv) in $\mathrm{CHCl}_{3}(1 \mathrm{~mL})$ was added dropwise the solution of NIS $(0.11$ mmol, 1.1 equiv) in $\mathrm{CHCl}_{3}(0.5 \mathrm{~mL})$. The mixture was further stirred at room temperature for $5 \mathrm{~h}$. The crude product was purified directly by flash column chromatography on silica gel (petroleum ether/ ethyl acetate = 10:1) to give the desired compound $\mathbf{1 5}$.

4-(2-iodo-3-phenyl-1-(trifluoromethyl)-3,4-dihydrocyclopenta[b]indol-3-yl)naphthalen-1-yl-4-methylbenzenesulf onate (15):

Light yellow solid, $33.9 \mathrm{mg}, 47 \%$ yield. 95:5 er, $[a]_{D}^{20}=+37.24(\mathrm{c}=0.18, \mathrm{MeOH})$. Mp.123.6-125.2 ${ }^{\circ} \mathrm{C}$. purified by flash column chromatography on silica gel (petroleum ether/ ethyl acetate $=5: 1, \mathrm{Rf}=0.6$ ).

${ }^{1}$ H NMR $\left(400 \mathrm{MHz}, \mathrm{CDCl}_{3}\right) \delta: 8.41(\mathrm{~s}, 1 \mathrm{H}), 8.23(\mathrm{~d}, J=8.2 \mathrm{~Hz}, 1 \mathrm{H}), 7.85(\mathrm{~d}, J=8.3 \mathrm{~Hz}, 2 \mathrm{H}), 7.79(\mathrm{~d}, J=7.9 \mathrm{~Hz}$, 
$2 \mathrm{H}), 7.63-7.49(\mathrm{~m}, 2 \mathrm{H}), 7.48-7.41(\mathrm{~m}, 1 \mathrm{H}), 7.40-7.28(\mathrm{~m}, 5 \mathrm{H}), 7.27-7.19(\mathrm{~m}, 3 \mathrm{H}), 7.09-6.93(\mathrm{~m}, 3 \mathrm{H})$, $2.48(\mathrm{~s}, 3 \mathrm{H})$.

${ }^{13} \mathrm{C}$ NMR $\left(126 \mathrm{MHz}, \mathrm{CDCl}_{3}\right) \delta: 148.8,146.5,145.8,139.4,139.1,133.4,133.1,132.9,130.8,130.1,129.5$, 128.7, 128.6, 128.5, 127.2, 126.5, 125.6, 125.5, 124.4, 124.3, 123.0, 122.4 (q, $J=271.1 \mathrm{~Hz}$ ), 121.7, 120.2, 120.0 (d, $J=2.4 \mathrm{~Hz}$ ), 117.8, 117.2, 112.4, 66.0, 21.9;

${ }^{19}$ F NMR $\left(376 \mathrm{MHz}, \mathrm{CDCl}_{3}\right) \delta:-60.27$.

HRMS (ESI): $\mathrm{m} / z$ calcd. for $\mathrm{C}_{35} \mathrm{H}_{24} \mathrm{NO}_{3} \mathrm{~F}_{3} \mathrm{IS}[\mathrm{M}+\mathrm{H}]^{+}:$722.1295; found: 722.1290 .

HPLC analysis: The enantiomeric excess was determined by HPLC with Chiralpak IA column (hexane $/ \mathrm{i}-\mathrm{PrOH}=95 / 5$, flow rate $1.0 \mathrm{~mL} \cdot \mathrm{min}^{-1}, \lambda=280 \mathrm{~nm}$ ): $t_{\text {major }}=16.1 \mathrm{~min}, t_{\text {minor }}=18.2 \mathrm{~min}$.

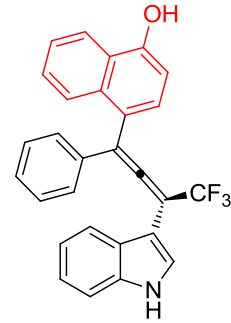

3a (0.1 $\mathrm{mmol})$

$98: 2$ er

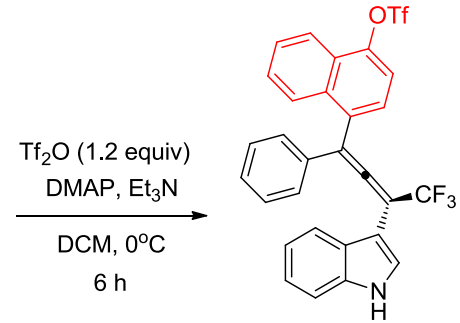

16

$92 \%$ yield, 98:2 er

To a solution of $3 \mathrm{a}\left(0.10 \mathrm{mmol}, 1.0\right.$ equiv), $\mathrm{Et}_{3} \mathrm{~N}(0.10 \mathrm{mmol}, 1.0$ equiv) and DMAP $(0.01 \mathrm{mmol}, 10 \mathrm{~mol} \%)$ in $\mathrm{DCM}(1 \mathrm{~mL})$ was added dropwise the solution of $\mathrm{Tf}_{2} \mathrm{O}\left(0.12 \mathrm{mmol}, 1.2\right.$ equiv) in $\mathrm{DCM}(1 \mathrm{~mL})$ at $0{ }^{\circ} \mathrm{C}$. The mixture was further stirred at $0{ }^{\circ} \mathrm{C}$ for $6 \mathrm{~h}$. The crude product was purified directly by flash column chromatography on silica gel (petroleum ether/ ethyl acetate $=10: 1 \sim 5: 1$ ) to give the desired compound 16 .

(S)-4-(4,4,4-trifluoro-3-(1H-indol-3-yl)-1-phenylbuta-1,2-dien-1-yl)naphthalen-1-yl $\quad$ trifluoromethanesulfonate. (16):

White foam, $52.8 \mathrm{mg}, 92 \%$ yield, $98: 2 \mathrm{er},[a]_{D}^{20}=+67.29(\mathrm{c}=0.13, \mathrm{MeOH})$. purified by flash column chromatography on silica gel (petroleum ether/ ethyl acetate $=5: 1, \mathrm{Rf}=0.6$ ).

${ }^{1} \mathrm{H}$ NMR $\left(400 \mathrm{MHz}, \mathrm{CDCl}_{3}\right) \delta: 8.33(\mathrm{~s}, 1 \mathrm{H}), 8.09(\mathrm{~d}, J=8.5 \mathrm{~Hz}, 1 \mathrm{H}), 7.97(\mathrm{~d}, J=8.5 \mathrm{~Hz}, 1 \mathrm{H}), 7.74(\mathrm{~d}, J=8.1 \mathrm{~Hz}$, $1 \mathrm{H}), 7.60(\mathrm{t}, J=7.4 \mathrm{~Hz}, 1 \mathrm{H}), 7.56(\mathrm{~d}, J=7.9 \mathrm{~Hz}, 1 \mathrm{H}), 7.50-7.43(\mathrm{~m}, 2 \mathrm{H}), 7.39(\mathrm{~s}, 1 \mathrm{H}), 7.36(\mathrm{~d}, J=8.2 \mathrm{~Hz}, 1 \mathrm{H})$, $7.30-7.26(\mathrm{~m}, 4 \mathrm{H}), 7.22-7.15(\mathrm{~m}, 2 \mathrm{H}), 7.03(\mathrm{t}, J=7.6 \mathrm{~Hz}, 1 \mathrm{H})$;

${ }^{13} \mathrm{C}$ NMR (126 MHz, $\mathrm{CDCl}_{3}$ ) $\delta: 205.0$ (q, $\left.J=3.5 \mathrm{~Hz}\right), 146.0,136.3,135.0,133.7,133.3,129.2,128.7,128.2$, 128.0, 127.6, 127.3, 126.9, 126.8, 125.5, 123.7 (q, $J=275.4 \mathrm{~Hz}$ ), 123.7 (d, $J=2.3 \mathrm{~Hz}$ ), 123.3, 121.4, 121.0, $119.8,118.9$ (q, $J=320.4 \mathrm{~Hz}), 117.8,114.7,111.7,104.2,99.7$ (q, $J=35.6 \mathrm{~Hz}$ );

${ }^{19} \mathrm{~F}$ NMR $\left(376 \mathrm{MHz}, \mathrm{CDCl}_{3}\right)$ ס: $-61.29,-73.27$.

HRMS (ESI): $m / z$ calcd. for $\mathrm{C}_{29} \mathrm{H}_{18} \mathrm{NO}_{3} \mathrm{~F}_{6} \mathrm{~S}[\mathrm{M}+\mathrm{H}]^{+}: 574.0906$; found: 574.0908 .

HPLC analysis: The enantiomeric excess was determined by HPLC with Chiralpak IA column (hexane $/ \mathrm{i}-\mathrm{PrOH}=90 / 10$, flow rate $1.0 \mathrm{~mL} \cdot \mathrm{min}^{-1}, \lambda=254 \mathrm{~nm}$ ): $t_{\text {major }}=6.9 \mathrm{~min}, t_{\text {minor }}=6.1 \mathrm{~min}$. 

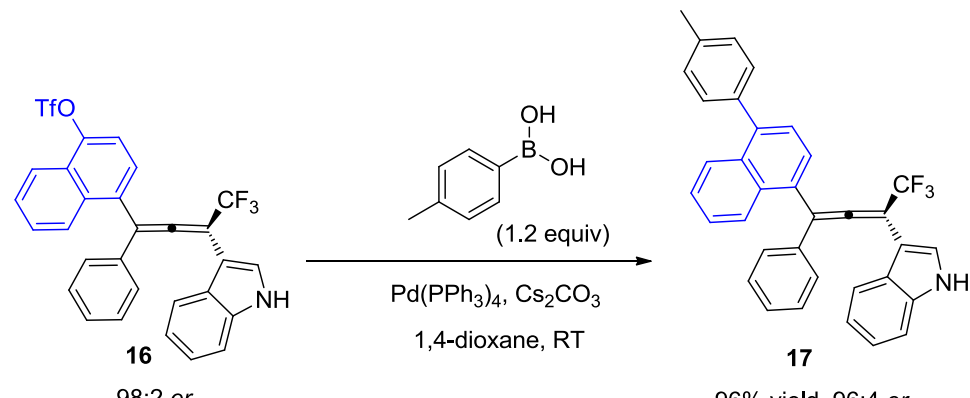

$96 \%$ yield, $96: 4$ er

To a solution of $16\left(0.10 \mathrm{mmol}, 1.0\right.$ equiv), $\mathrm{Pd}\left(\mathrm{PPh}_{3}\right)_{4}\left(0.010 \mathrm{mmol}, 0.1\right.$ equiv) and $\mathrm{Cs}_{2} \mathrm{CO}_{3}(0.12 \mathrm{mmol}$, 1.2equiv) in 1,4-dioxane (5 mL) was added the 4-Tolylboronic acid ( $0.12 \mathrm{mmol}, 1.2$ equiv) under nitrogen protection at room temperature. The mixture was further stirred at room temperature for $12 \mathrm{~h}$. The crude product was purified directly by flash column chromatography on silica gel (petroleum ether/ ethyl acetate = 20:1) to give the desired compound 17.

(S)-3-(1,1,1-trifluoro-4-phenyl-4-(4-(p-tolyl)naphthalen-1-yl)buta-2,3-dien-2-yl)-1H-indole. (17):

Light yellow foam, $49.5 \mathrm{mg}, 96 \%$ yield. $96: 4 \mathrm{er},[a]_{D}^{20}=+29.71(\mathrm{c}=0.23, \mathrm{MeOH})$. purified by flash column chromatography on silica gel (petroleum ether/ ethyl acetate $=10: 1, R f=0.5$ ).

${ }^{1} \mathrm{H}$ NMR $\left(400 \mathrm{MHz}, \mathrm{CDCl}_{3}\right) \delta: 8.31(\mathrm{~s}, 1 \mathrm{H}), 8.08-8.02(\mathrm{~m}, 1 \mathrm{H}), 8.00-7.95(\mathrm{~m}, 1 \mathrm{H}), 7.91(\mathrm{~d}, J=8.0 \mathrm{~Hz}, 1 \mathrm{H})$, $7.65(\mathrm{~d}, J=7.2 \mathrm{~Hz}, 1 \mathrm{H}), 7.49-7.36(\mathrm{~m}, 9 \mathrm{H}), 7.36-7.27(\mathrm{~m}, 5 \mathrm{H}), 7.26-7.22(\mathrm{~m}, 1 \mathrm{H}), 7.09(\mathrm{t}, J=7.2 \mathrm{~Hz}, 1 \mathrm{H})$, $2.48(\mathrm{~s}, 3 \mathrm{H})$.

${ }^{13}$ C NMR (126 MHz, $\left.\mathrm{CDCl}_{3}\right)$ ס: 205.1 (q, $\left.J=3.3 \mathrm{~Hz}\right), 141.3,137.7,137.3,136.3,135.6,132.4,132.1,131.9$, 130.1, 129.2, 129.0, 128.4, 127.8, 127.6, 126.8, 126.7, 126.7, 126.2, 126.2, 125.7, 124.0 (q, J = $275.5 \mathrm{~Hz}$ ), $123.4(\mathrm{~d}, J=2.1 \mathrm{~Hz}), 123.1,120.9,120.2,115.7,111.5,104.8,98.8$ (q, $J=35.1 \mathrm{~Hz}), 21.4$.

${ }^{19} \mathrm{~F}$ NMR $\left(376 \mathrm{MHz}^{\mathrm{CDCl}} 3\right)$ ס: -61.23.

HRMS (ESI): $\mathrm{m} / z$ calcd. for $\mathrm{C}_{35} \mathrm{H}_{25} \mathrm{~F}_{3} \mathrm{~N}[\mathrm{M}+\mathrm{H}]^{+}: 516.2761$; found: 516.2765 .

HPLC analysis: The enantiomeric excess was determined by HPLC with Chiralpak AD-H column (hexane $/ \mathrm{i}-\mathrm{PrOH}=99 / 1$, flow rate $0.8 \mathrm{~mL} \cdot \mathrm{min}^{-1}, \lambda=245 \mathrm{~nm}$ ): $t_{\text {major }}=21.7 \mathrm{~min}, t_{\operatorname{minor}}=23.9 \mathrm{~min}$.

\section{Proposed reaction pathways}

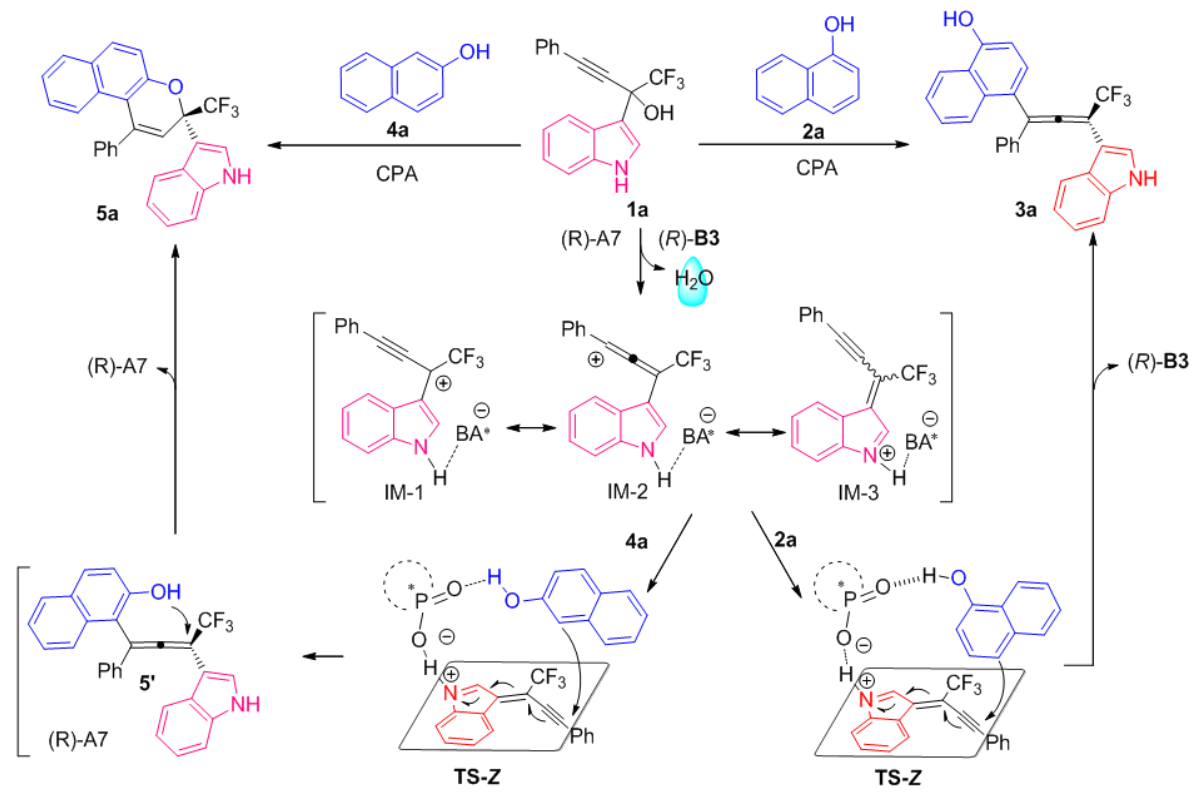

Figure S1. Proposed reaction pathways 
Based on the experiment results and previous reports, ${ }^{2}$ a plausible reaction mechanism is proposed (Figure S1). The carbocation IM-1 and IM-2, and vinyliminium IM-3 (Z/E mixed isomers) intermediates are firstly generated in situ from $\alpha$-indolyl propargylic alcohol 1a via dehydration under acidic conditions. In the presence of a chiral phosphoric acid catalyst, the vinyliminium intermediate IM-3 and $\alpha$-naphthol 2a are simultaneously activated through a dual hydrogen-bonding activated transition state TS-Z, enabling the stereocontrolled 1,6-conjugative addition to afford chiral tetrasubstituted allenes. The remote regioselective para-allenylation of phenol is owing to the strong electronic effects as well as the reduced steric hindrance of C4 relative to $\mathrm{C} 2$ of the phenol. ${ }^{3}$ When $\alpha$-naphthol $2 \mathbf{a}$ was replaced by $\beta$-naphthol $4 \mathbf{a}$, the transition state TS- $Z$ was underwent to afford the tetrasubstituted alkene intermediate 5', which subsequently underwent an intramolecular ring closure to give the naphthopyran 5 a bearing a tetrasubstituted stereogenic center.

\section{Determination of the absolute configuration of $5 d$}

A solution of compound $5 \mathbf{d}$ in acetonitrile $(0.08379 \mathrm{mM})$ was measured at room temperature and a path length of $10.00 \mathrm{~mm}$ on a Chirascan electronic circular dichroism (ECD) spectrometer. In order to determine the $\boldsymbol{R} / \boldsymbol{S}$ configuration of compound $\mathbf{5 d}$, ECD calculations of $\mathbf{5 d}-\boldsymbol{S}$ and its enantiomer $\mathbf{5 d} \boldsymbol{d} \boldsymbol{R}$ were performed in the Gaussian 09 program $^{4}$. Structures of $\mathbf{5 d} \mathbf{d} \boldsymbol{S}$ and $\mathbf{5 d} \mathbf{d} \boldsymbol{R}$ were obtained from the X-ray diffraction. First, the structures were optimized, then using Time-dependent Density functional theory (TD-DFT) to calculated the ECD at B3LYP/6-31G(d) theory level in acetonitrile solvent environment. The excited states were set to 30. Comparing the experimental ECD spectrum with the calculated ECD spectra ${ }^{5}$ (Figure S2), it indicated the absolute configuration of compound $\mathbf{5} \mathbf{d}$ was $\mathbf{S}$ configuration.

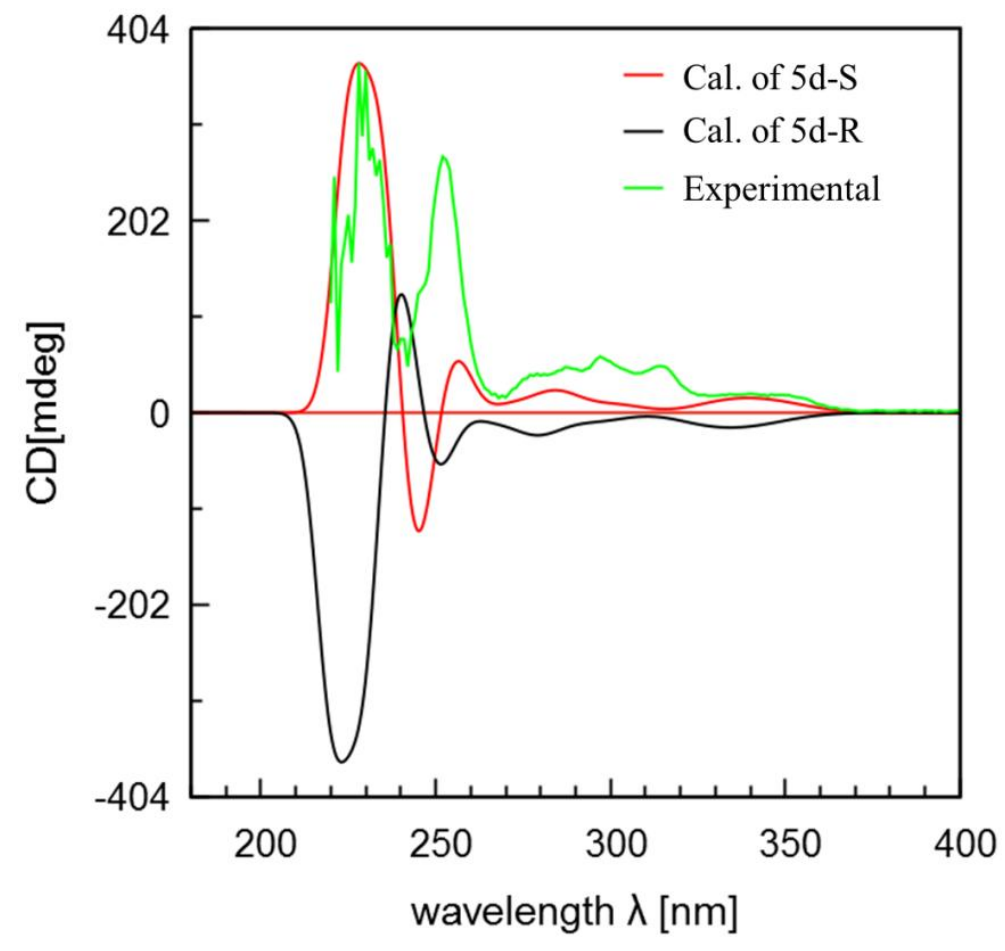

Figure S2. Comparison of the calculated ECD spectra of $\mathbf{5} \mathbf{d}-\boldsymbol{S}$ and its enantiomer $\mathbf{5} \mathbf{d}-\boldsymbol{R}$ with experimental ECD spectrum of compound 5d. (Note: $\sigma_{(5 d-S)}=0.16 \mathrm{ev}$, shift $_{(5 d-S)}=5 \mathrm{~nm} ; \sigma_{(5 d-R)}=0.16 \mathrm{ev}$, shift $(5 d-R)=0 \mathrm{~nm}$.)

\section{References}

(1) (a) Qian, D.; Wu, L.; Lin, Z.; Sun, J. Nat. Commun. 2017, 8, 567; (b) Li, H.; Wang, Y.-Q.; Deng L., Org. Lett. 2006, 8, 4063.

(2) (a) Liu, Y.; Zhang, H.-H.; Zhang, Y.-C.; Jiang, Y.; Shi, F.; Tu, S.-J. Chem. Commun. 2014, 50, 12054. (b) 
Zhang, H.-H.; Wang, C.-S.; Li, C.; Mei, G.-J.; Li, Y.; Shi, F. Angew. Chem., Int. Ed. 2017, 56, 116.

(3) (a) Ciana, C.-L.; Phipps, R. J.; Brandt, J. R.; Meyer, F.-M.; Gaunt, M. J. Angew. Chem., Int. Ed. 2011, 50, 458. (b) Yu, Z.; Ma, B.; Chen, M.; Wu, H.-H.; Liu, L.; Zhang, J. J. Am. Chem. Soc. 2014, 136, 6904. (c) Liu, L.; Chen, K.; Wu, W.-Z.; Wang, P.-F.; Song, H.-Y.; Sun, H.; Wen, X.; Xu, Q.-L. Org. Lett. 2017, 19, 3823. (d) Sagadevan, A.; Charpe, V. P.; Ragupathi, A.; Hwang, K. C. J. Am. Chem. Soc. 2017, 139, 2896. (e) Xiao, P.; Li, C.-X.; Fang, W.-H.; Cui, G.; Thiel, W. J. Am. Chem. Soc. 2018, 140, 15099.

(4) Frisch, M. J.; Trucks, G. W.; Schlegel, H. B.; Scuseria, G. E.; Robb, M. A.; Cheeseman, J. R.; Scalmani, G.; Barone, V.; Petersson, G. A.; Nakatsuji, H.; Li, X.; Caricato, M.; Marenich, A.; Bloino, J.; Janesko, B. G.; Gomperts, R.; Mennucci, B.; Hratchian, H. P.; Ortiz, J. V.; Izmaylov, A. F.; Sonnenberg, J. L.; Williams-Young, D.; Ding, F.; Lipparini, F.; Egidi, F.; Goings, J.; Peng, B.; Petrone, A.; Henderson, T.; Ranasinghe, D.; Zakrzewski, V. G.; Gao, J.; Rega, N.; Zheng, G.; Liang, W.; Hada, M.; Ehara, M.; Toyota, K.; Fukuda, R.; Hasegawa, J.; Ishida, M.; Nakajima, T.; Honda, Y.; Kitao, O.; Nakai, H.; Vreven, T.; Throssell, K.; Montgomery, J. A., Jr., Peralta, J. E.; Ogliaro, F.; Bearpark, M.; Heyd, J. J.; Brothers, E.; Kudin, K. N.; Staroverov, V. N.; Keith, T.; Kobayashi, R.; Normand, J.; Raghavachari, K.; Rendell, A.; Burant, J. C.; lyengar, S. S.; Tomasi, J.; Cossi, M.; Millam, J. M.; Klene, M.; Adamo, C.; Cammi, R.; Ochterski, J. W.; Martin, R. L.; Morokuma, K.; Farkas, O.; Foresman, J. B.; Fox, D. J. Gaussian 09, Revision A.02; Gaussian, Inc.,: Wallingford, CT, 2016.

(5) Bruhn, Torsten, et al. "SpecDis: Quantifying the Comparison of Calculated and Experimental Electronic Circular Dichroism Spectra." Chirality 25.4(2013):243-249. 


\section{Data for X-Ray Crystal Structure of $3 z$ and $5 d$}

Procedure for the recrystallization of $\mathbf{3 z :}$ To a $10 \mathrm{~mL}$ vial containing $\mathbf{3 z}(25 \mathrm{mg})$, was added a 1:9 mixture of isopropanol and $n$-hexane until it formed a clear solution. The mixture was kept aside 2 days at room temperature to obtain crystals by slow evaporation. These crystals were subjected for single crystal XRD to determine the absolute configuration of $\mathbf{3 z}$.
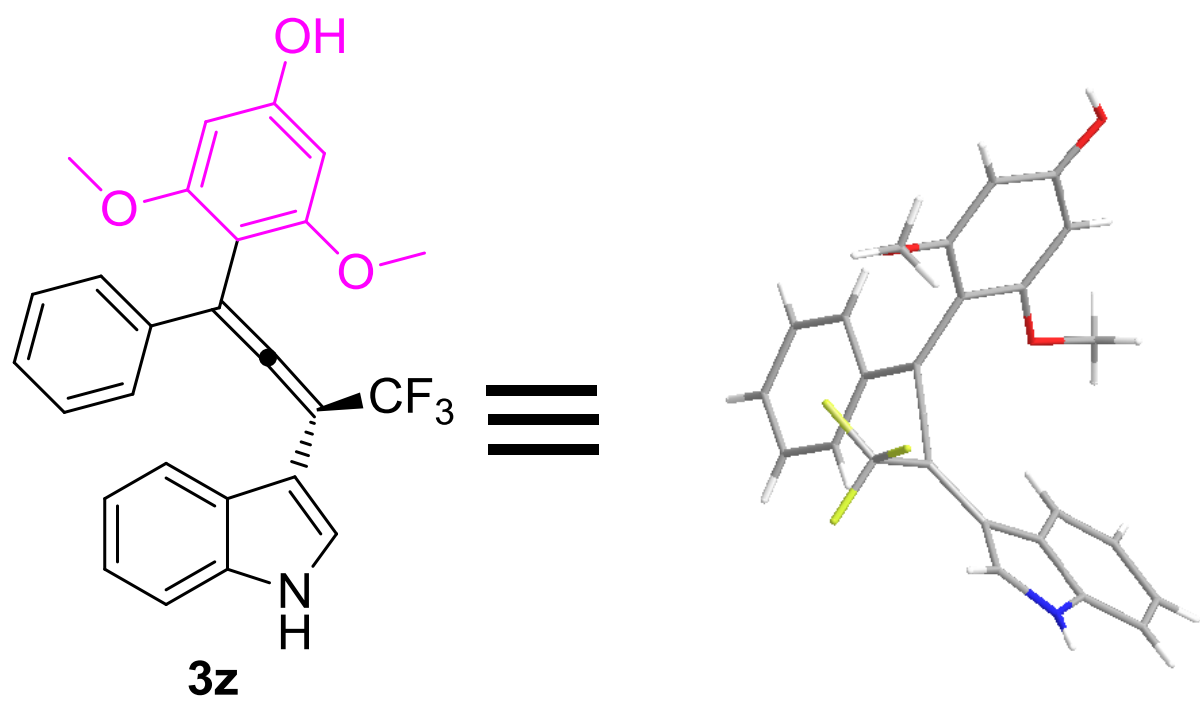

Figure S3. X-ray crystal structure of $\mathbf{3 z}$ (ellipsoids: $50 \%$ probability). 
Table 1 Crystal data and structure refinement for $\mathbf{3 z}$.

\begin{tabular}{|c|c|}
\hline Empirical formula & $\mathrm{C}_{29} \mathrm{H}_{27} \mathrm{~F}_{3} \mathrm{NO}_{4}$ \\
\hline Formula weight & 510.51 \\
\hline Temperature/K & $100.00(10)$ \\
\hline Crystal system & orthorhombic \\
\hline Space group & $\mathrm{P} 2{ }_{1} 2_{1} 2_{1}$ \\
\hline $\mathrm{a} / \AA$ & $8.55460(10)$ \\
\hline $\mathrm{b} / \AA$ & $10.8585(2)$ \\
\hline$c / \AA$ & $28.2459(5)$ \\
\hline$\alpha /^{\circ}$ & 90 \\
\hline$\beta /{ }^{\circ}$ & 90 \\
\hline$\gamma /{ }^{\circ}$ & 90 \\
\hline Volume $/ \AA^{3}$ & $2623.77(7)$ \\
\hline $\mathrm{Z}$ & 4 \\
\hline$\rho_{\text {calc }} \mathrm{g} / \mathrm{cm}^{3}$ & 1.292 \\
\hline$\mu / \mathrm{mm}^{-1}$ & 0.840 \\
\hline $\mathrm{F}(000)$ & 1068.0 \\
\hline Crystal size $/ \mathrm{mm}^{3}$ & $0.2 \times 0.1 \times 0.1$ \\
\hline Radiation & $\operatorname{CuK} \alpha(\lambda=1.54184)$ \\
\hline $2 \Theta$ range for data collection ${ }^{\circ}$ & 6.258 to 144.224 \\
\hline Index ranges & $-10 \leq \mathrm{h} \leq 8,-13 \leq \mathrm{k} \leq 12,-34 \leq 1 \leq 34$ \\
\hline Reflections collected & 25854 \\
\hline Independent reflections & $5170\left[R_{\text {int }}=0.0781, R_{\text {sigma }}=0.0509\right]$ \\
\hline Data/restraints/parameters & $5170 / 0 / 339$ \\
\hline Goodness-of-fit on $\mathrm{F}^{2}$ & 1.050 \\
\hline Final $\mathrm{R}$ indexes $[\mathrm{I}>=2 \sigma(\mathrm{I})]$ & $\mathrm{R}_{1}=0.0505, \mathrm{wR}_{2}=0.1250$ \\
\hline Final $\mathrm{R}$ indexes [all data] & $\mathrm{R}_{1}=0.0531, \mathrm{w} \mathrm{R}_{2}=0.1276$ \\
\hline Largest diff. peak/hole / e $\AA^{-3}$ & $0.48 /-0.24$ \\
\hline Flack parameter & $0.04(8)$ \\
\hline
\end{tabular}


Procedure for the recrystallization of $5 \mathrm{~d}$ : To a $10 \mathrm{~mL}$ vial containing $5 \mathbf{d}(35 \mathrm{mg})$, was added a 1:3 mixture of DCM and $n$-hexane until it formed a clear solution. The mixture was kept aside 3 days at room temperature to obtain crystals by slow evaporation. These crystals were subjected for single crystal XRD to determine the configuration of $\mathbf{5 d}$.

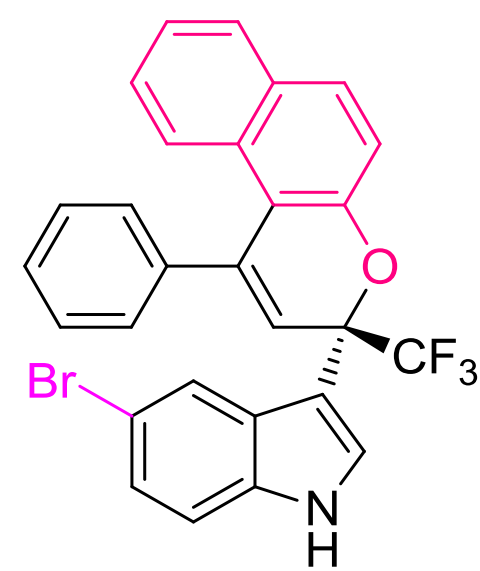

5d

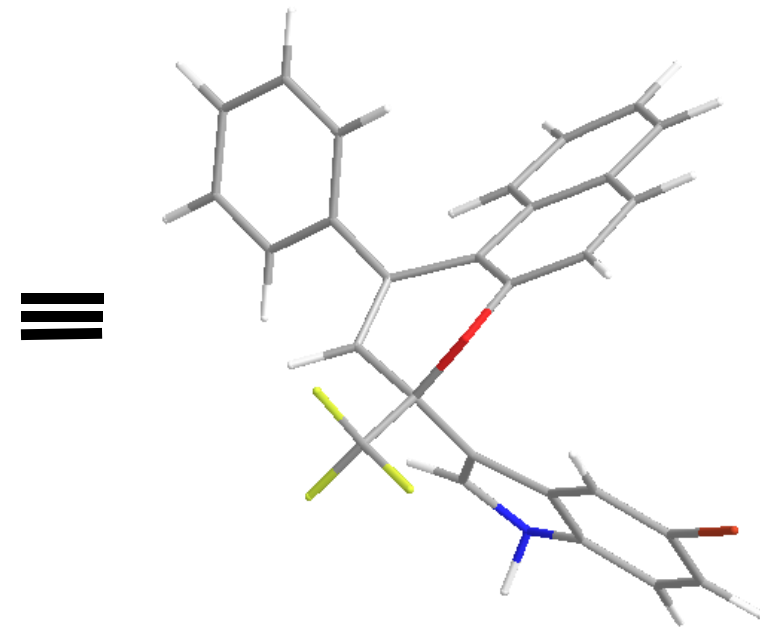

Figure S4. X-ray crystal structure of $\mathbf{5 d}$ (ellipsoids: $50 \%$ probability). 
Table 2 Crystal data and structure refinement for $\mathbf{5 d}$ (ellipsoids: 50\% probability).

\begin{tabular}{|c|c|}
\hline Empirical formula & $\mathrm{C}_{28} \mathrm{H}_{17} \mathrm{NOBrF}_{3}$ \\
\hline Formula weight & 520.33 \\
\hline Temperature/K & $100.00(10)$ \\
\hline Crystal system & triclinic \\
\hline Space group & $\mathrm{P}-1$ \\
\hline $\mathrm{a} / \AA ̊$ & $12.92853(6)$ \\
\hline $\mathrm{b} / \AA$ & $13.68174(7)$ \\
\hline $\mathrm{c} / \AA$ & $26.70654(10)$ \\
\hline$\alpha /^{\circ}$ & $77.9244(4)$ \\
\hline$\beta /{ }^{\circ}$ & $89.7066(4)$ \\
\hline$\gamma /{ }^{\circ}$ & $75.5701(4)$ \\
\hline Volume $/ \AA^{3}$ & $4468.21(4)$ \\
\hline $\mathrm{Z}$ & 8 \\
\hline$\rho_{\text {calc }} \mathrm{g} / \mathrm{cm}^{3}$ & 1.547 \\
\hline$\mu / \mathrm{mm}^{-1}$ & 2.918 \\
\hline $\mathrm{F}(000)$ & 2096.0 \\
\hline Crystal size $/ \mathrm{mm}^{3}$ & $0.1 \times 0.1 \times 0.1$ \\
\hline Radiation & $\mathrm{CuK} \alpha(\lambda=1.54184)$ \\
\hline $2 \Theta$ range for data collection $/^{\circ}$ & 6.778 to 153.89 \\
\hline Index ranges & $-16 \leq \mathrm{h} \leq 16,-17 \leq \mathrm{k} \leq 17,-33 \leq 1 \leq 30$ \\
\hline Reflections collected & 185177 \\
\hline Independent reflections & $18509\left[\mathrm{R}_{\mathrm{int}}=0.0500, \mathrm{R}_{\mathrm{sigma}}=0.0217\right]$ \\
\hline Data/restraints/parameters & $18509 / 0 / 1225$ \\
\hline Goodness-of-fit on $\mathrm{F}^{2}$ & 1.039 \\
\hline Final $R$ indexes $[\mathrm{I}>=2 \sigma(\mathrm{I})]$ & $\mathrm{R}_{1}=0.0438, \mathrm{wR}_{2}=0.1210$ \\
\hline Final $\mathrm{R}$ indexes [all data] & $\mathrm{R}_{1}=0.0462, \mathrm{wR}_{2}=0.1230$ \\
\hline Largest diff. peak/hole / e $\AA^{-3}$ & $1.49 /-0.95$ \\
\hline
\end{tabular}


11. Copies of NMR Spectra

${ }^{1} \mathrm{H}$ NMR of compound $1 \mathrm{a}\left(400 \mathrm{MHz}\right.$ in $\mathrm{CDCl}_{3}$ )

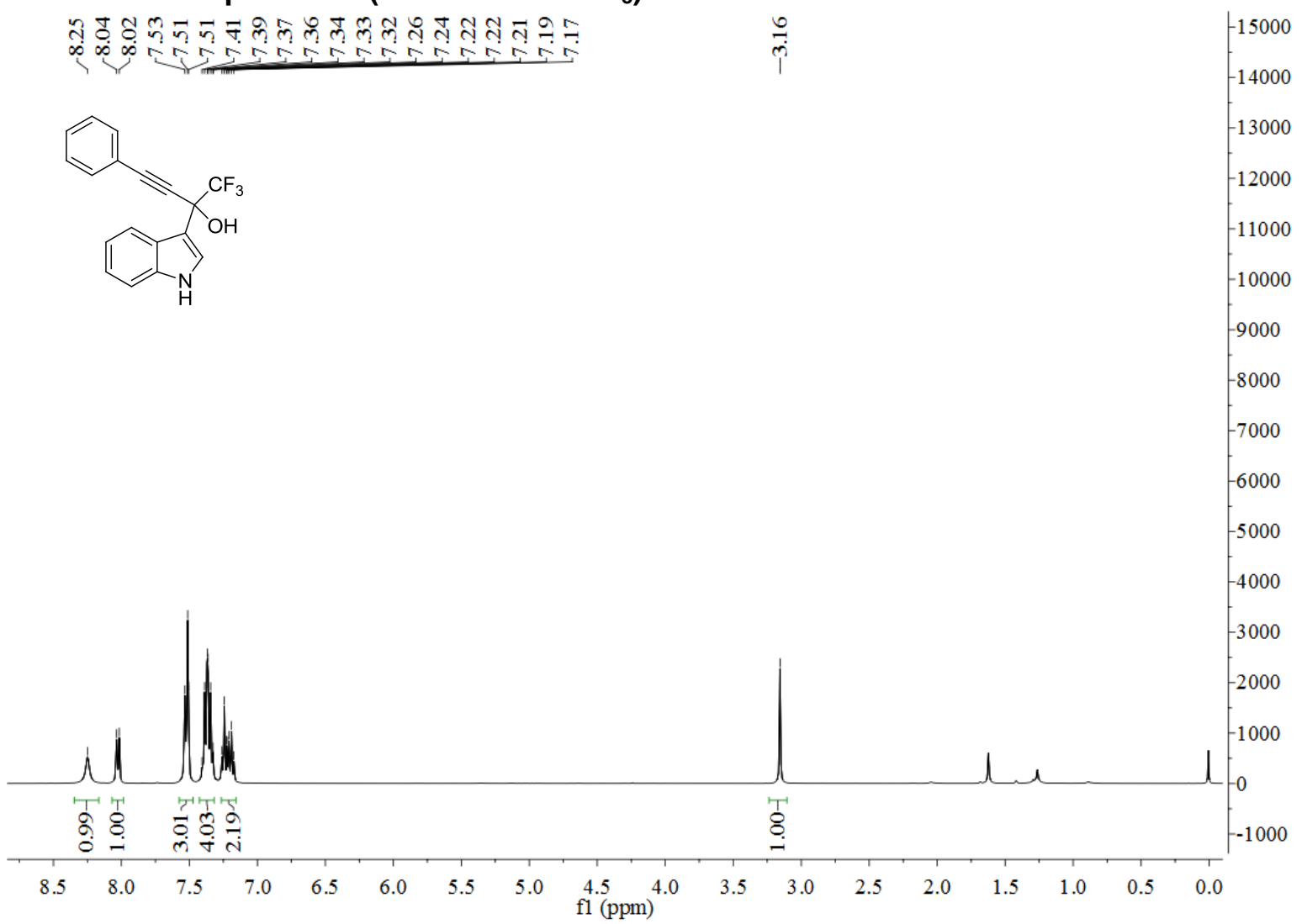

${ }^{13} \mathrm{C}$ NMR of compound $1 \mathrm{a}\left(101 \mathrm{MHz}\right.$ in $\mathrm{CDCl}_{3}$ )

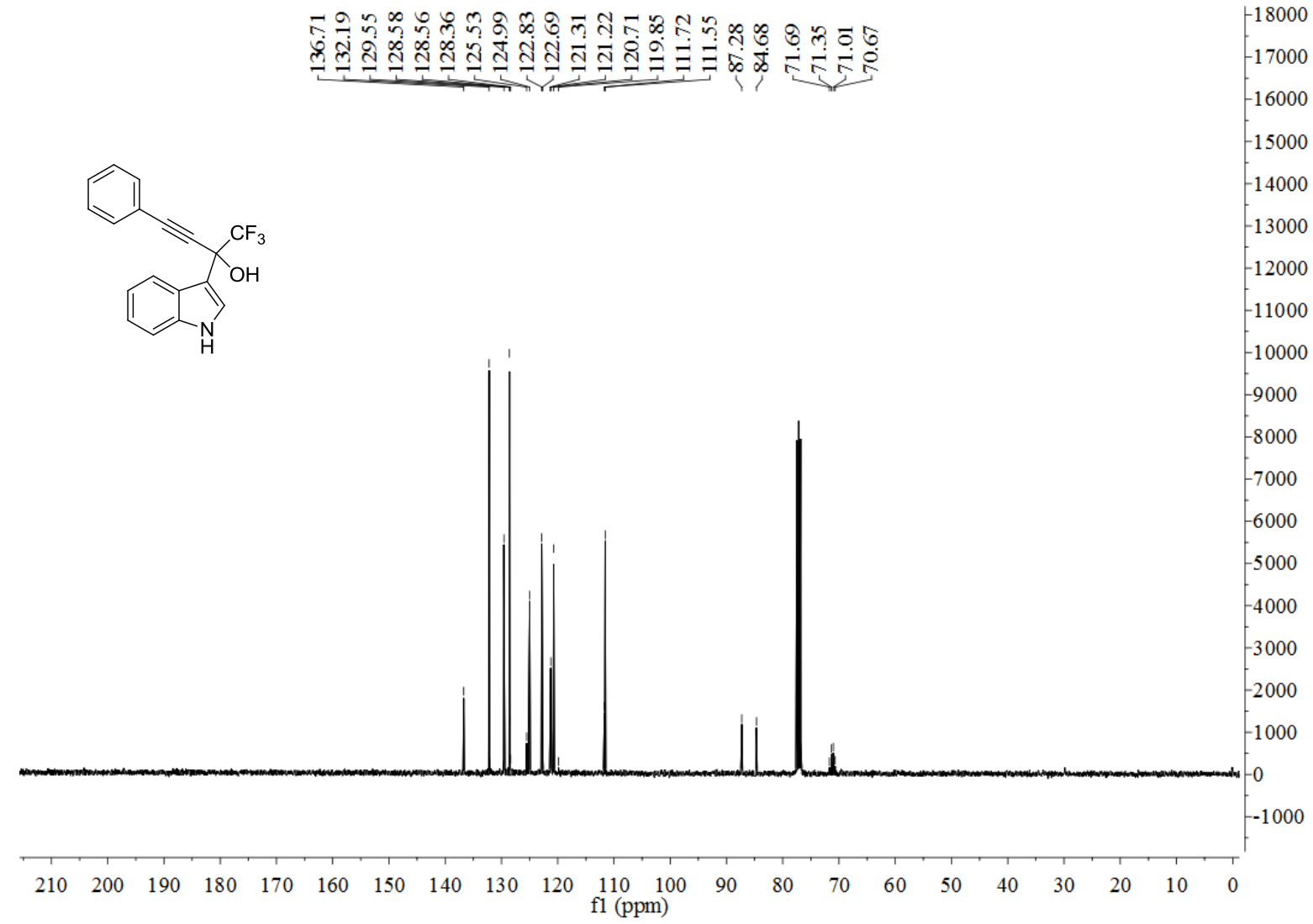


${ }^{19} \mathrm{~F}$ NMR of compound $1 \mathrm{a}\left(376 \mathrm{MHz}\right.$ in $\left.\mathrm{CDCl}_{3}\right)$

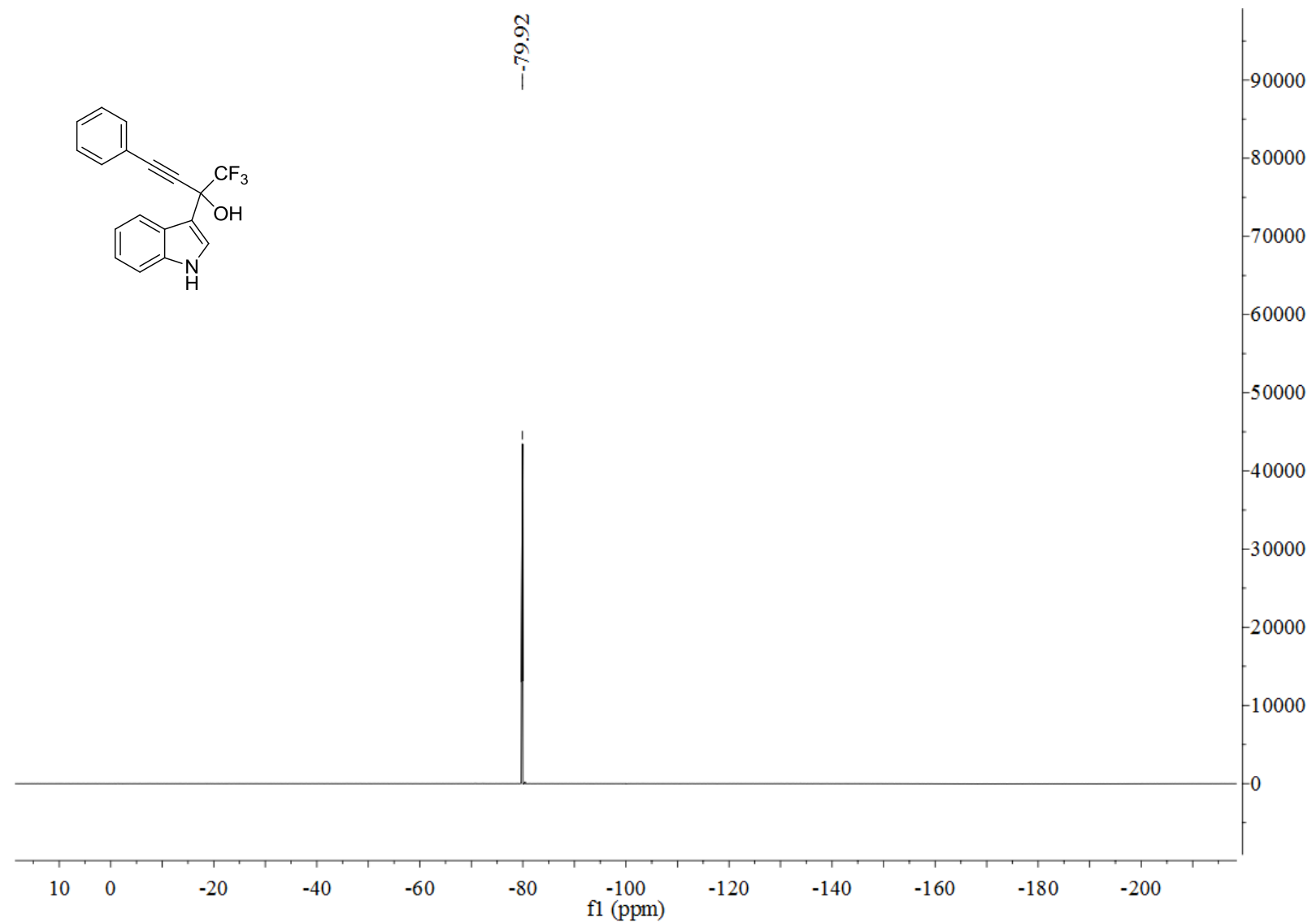

${ }^{1} \mathrm{H}$ NMR of compound $1 \mathrm{~b}\left(400 \mathrm{MHz}\right.$ in $\left.\mathrm{CDCl}_{3}\right)$

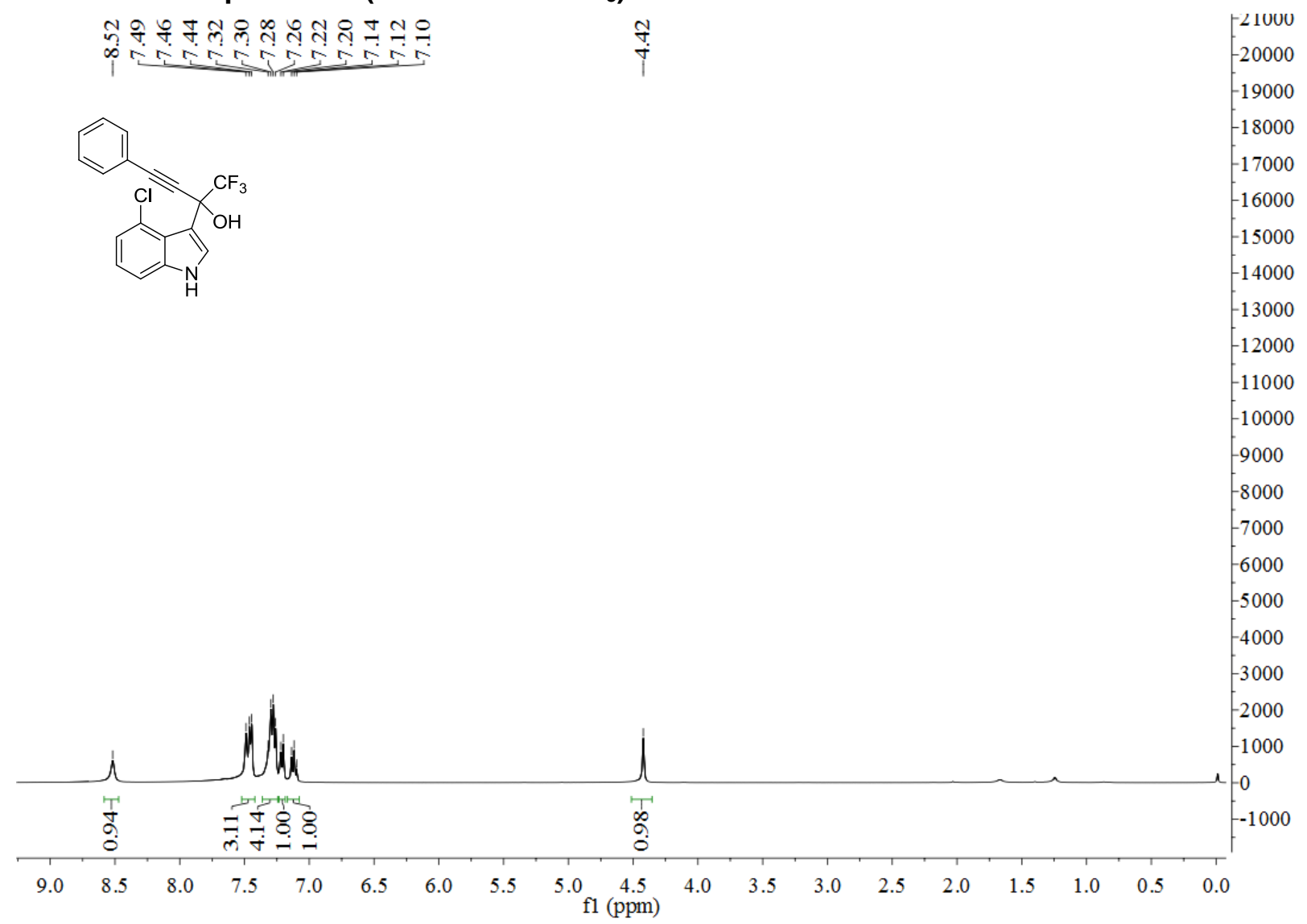


${ }^{13} \mathrm{C}$ NMR of compound $1 \mathrm{~b}\left(101 \mathrm{MHz}\right.$ in $\mathrm{CDCl}_{3}$ )

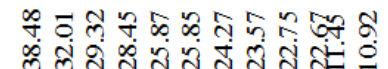

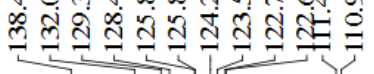

कह ह

लिख्र<smiles>CC(O)(C1CNc2cccc(Cl)c21)C(F)(F)F</smiles>

$\infty$

14000

13000

2000

11000

$-10000$

9000

8000

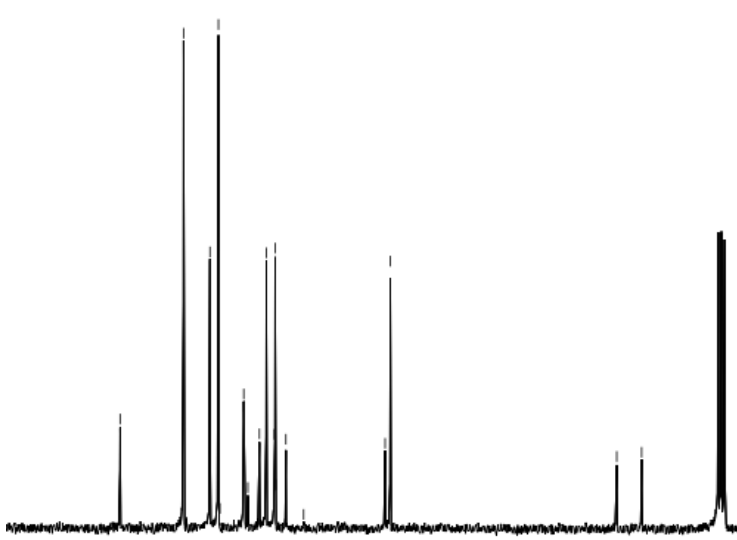

$-7000$

6000

5000

4000

3000

2000

1000

$50 \quad 140 \quad 130 \quad 120$

$\begin{array}{rr}80 & 70 \\ \mathrm{fl}(\mathrm{ppm})\end{array}$

${ }^{19} \mathrm{~F} \mathrm{NMR}$ of compound $1 \mathrm{~b}\left(376 \mathrm{MHz}\right.$ in $\mathrm{CDCl}_{3}$ )

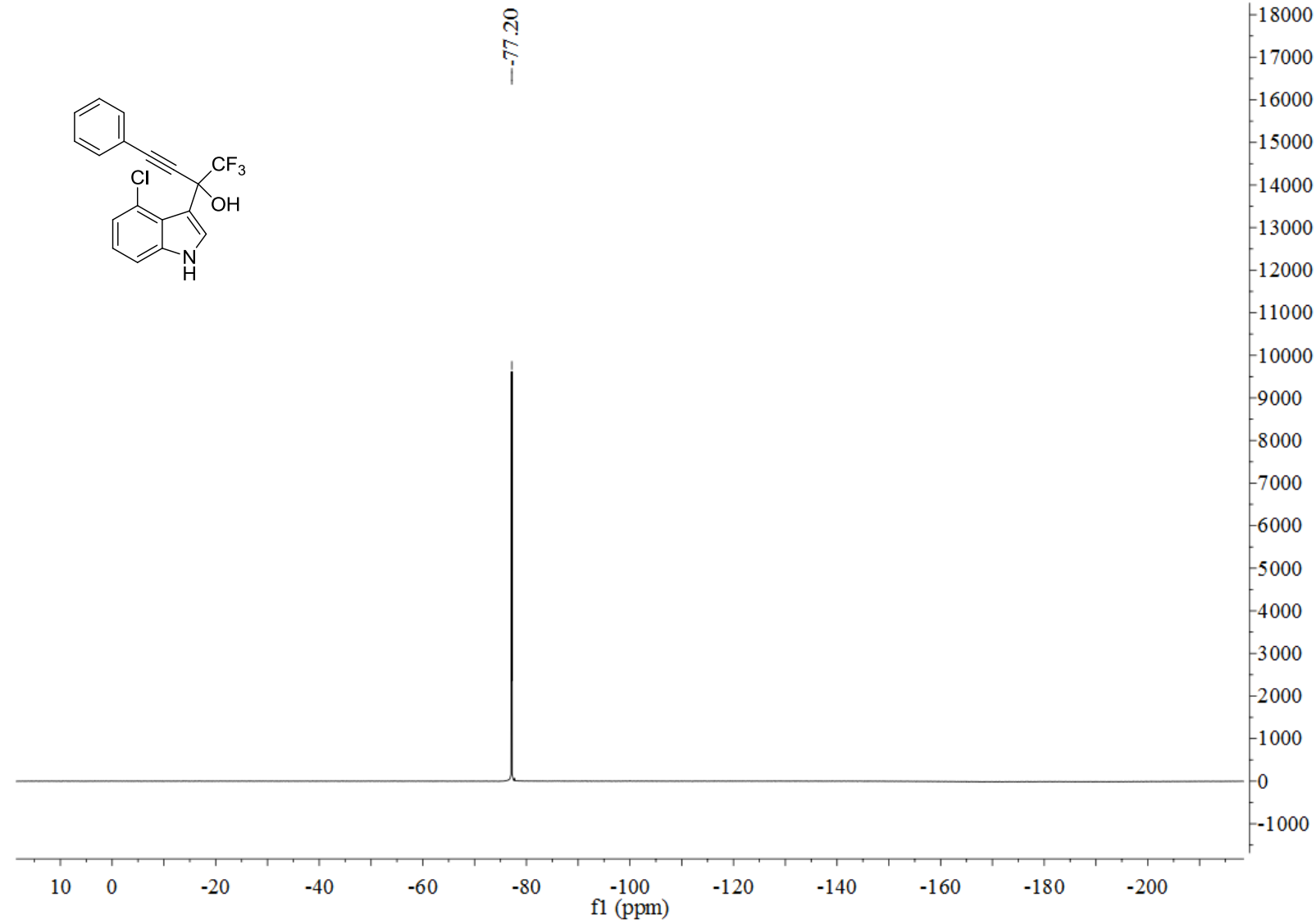


${ }^{1} \mathrm{H}$ NMR of compound $1 \mathrm{c}\left(400 \mathrm{MHz}\right.$ in $\left.\mathrm{CDCl}_{3}\right)$

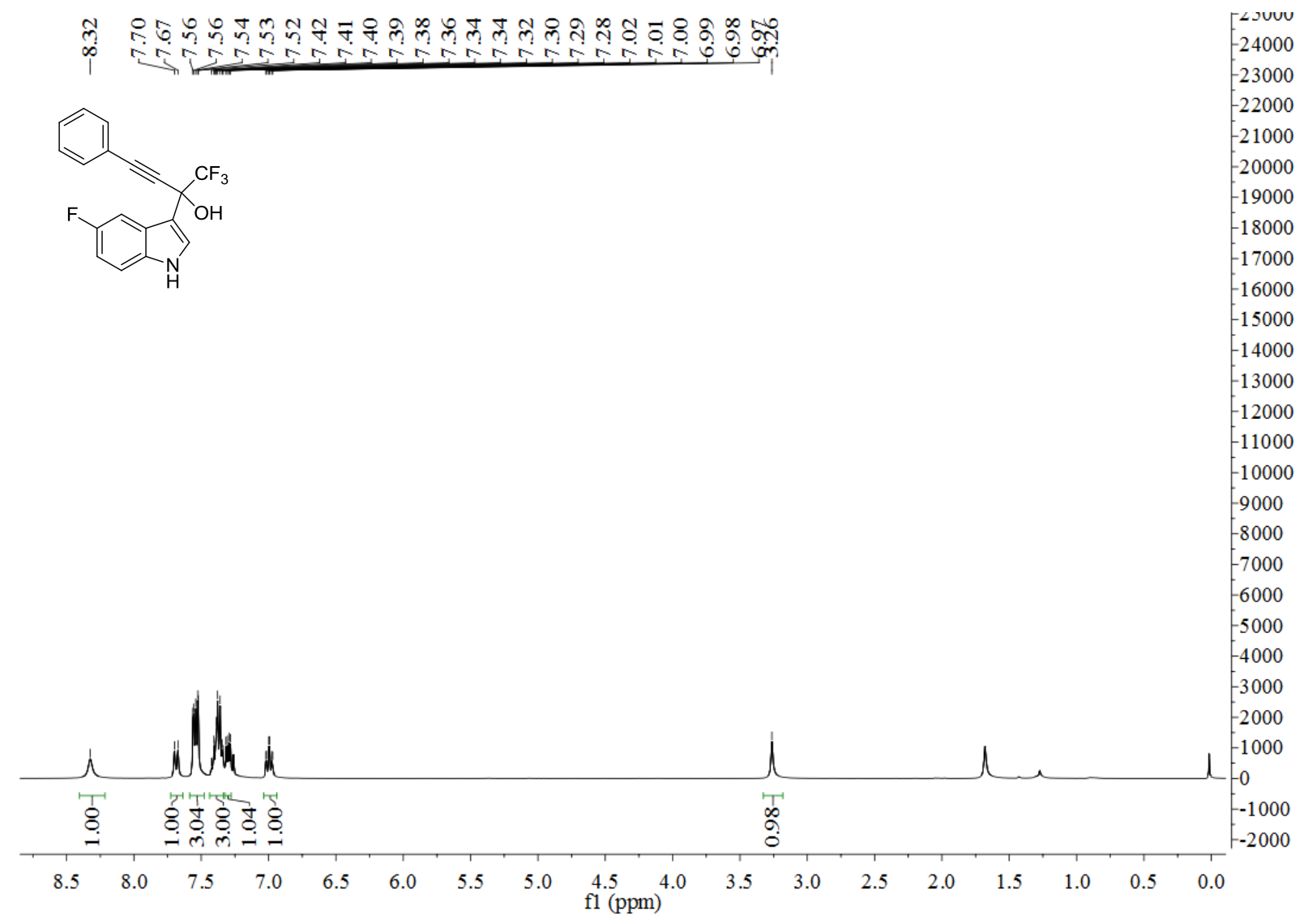

${ }^{13} \mathrm{C}$ NMR of compound $1 \mathrm{c}\left(101 \mathrm{MHz}\right.$ in $\left.\mathrm{CDCl}_{3}\right)$

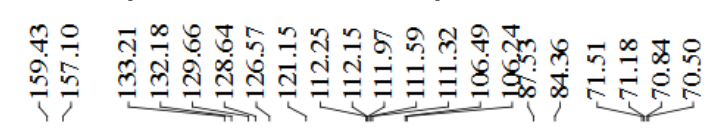

(c)

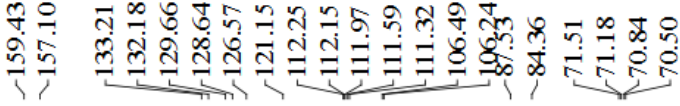

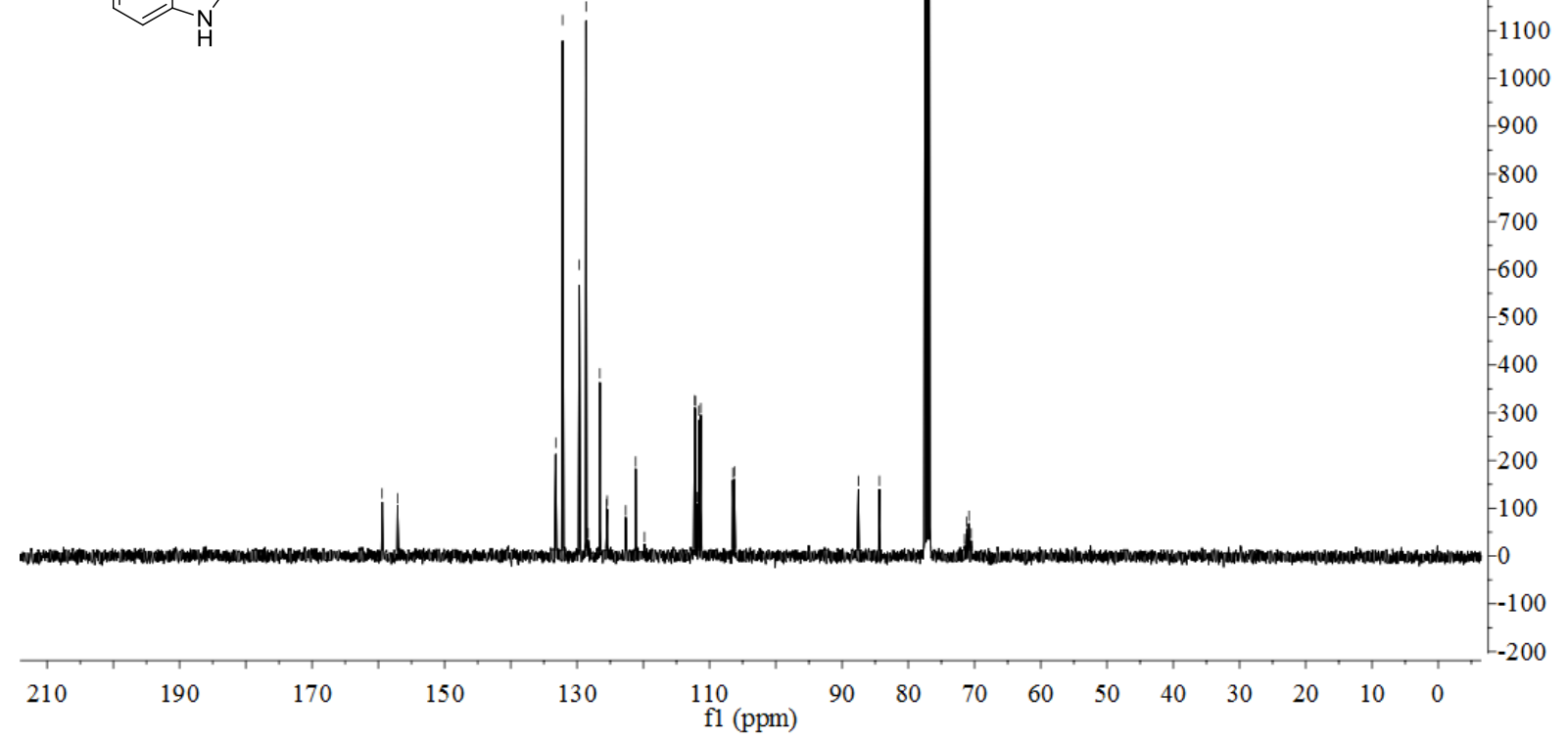


${ }^{19} \mathrm{~F}$ NMR of compound $1 \mathrm{c}\left(376 \mathrm{MHz}\right.$ in $\left.\mathrm{CDCl}_{3}\right)$

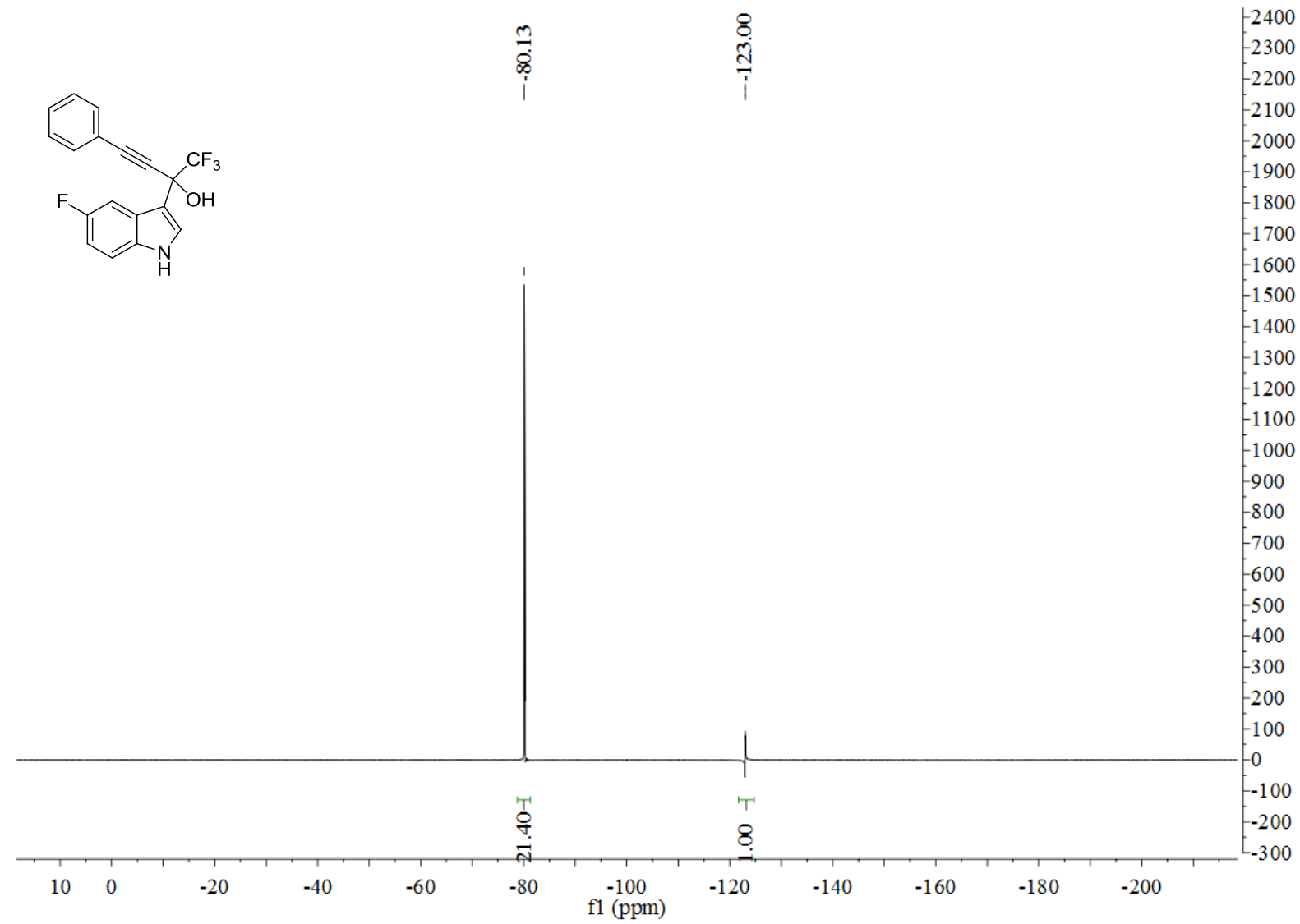

${ }^{1} \mathrm{H}$ NMR of compound $1 \mathrm{~d}\left(400 \mathrm{MHz}\right.$ in $\left.\mathrm{CDCl}_{3}\right)$

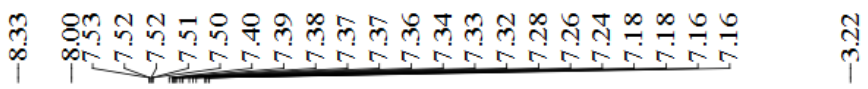

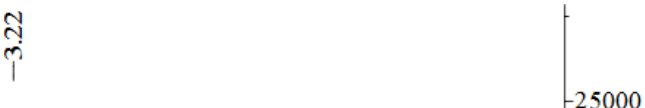

(c)

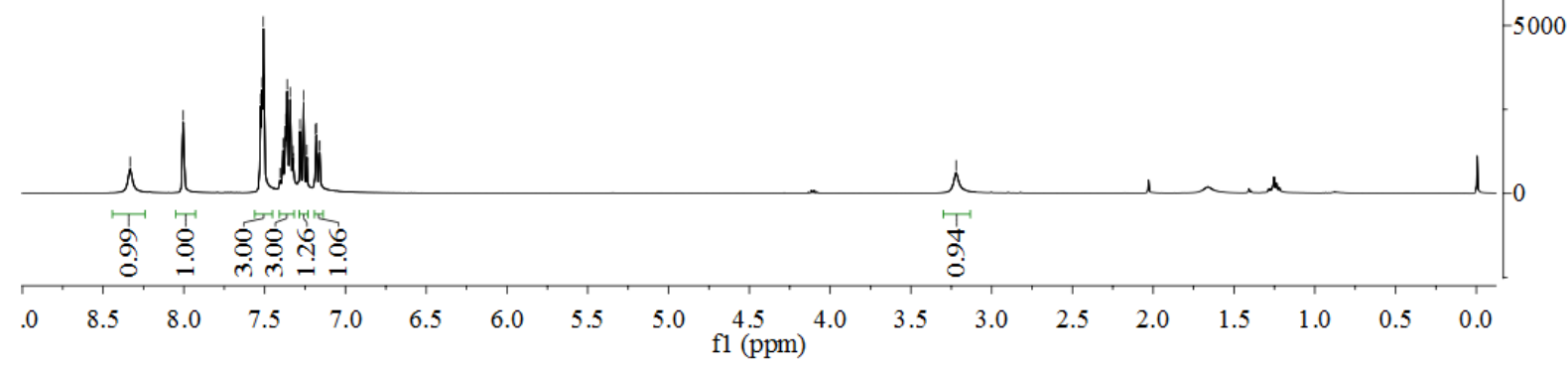


${ }^{13} \mathrm{C}$ NMR of compound $1 \mathrm{~d}\left(101 \mathrm{MHz}\right.$ in $\left.\mathrm{CDCl}_{3}\right)$

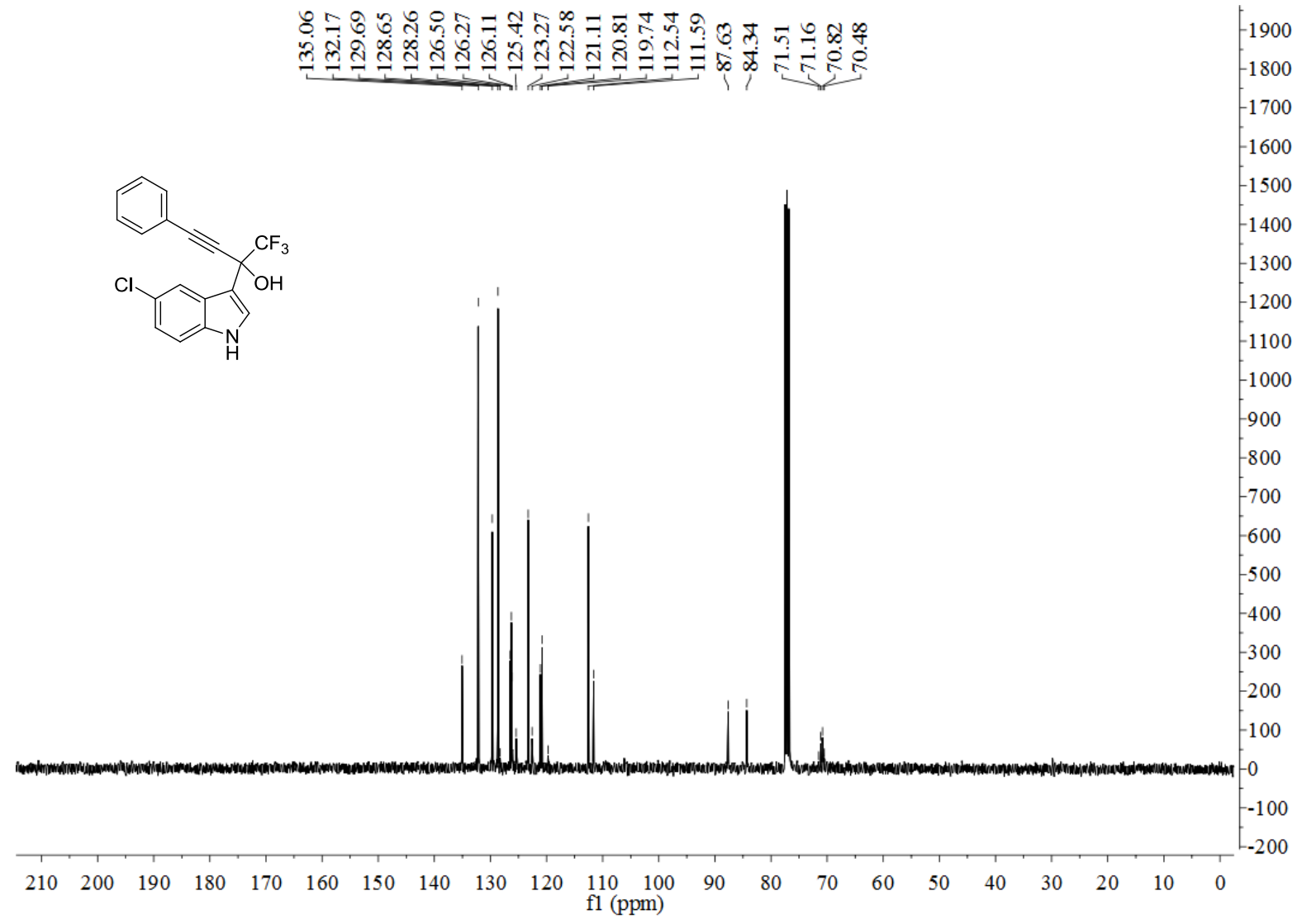

${ }^{19} \mathrm{~F} \mathrm{NMR}$ of compound $1 \mathrm{~d}\left(376 \mathrm{MHz}\right.$ in $\left.\mathrm{CDCl}_{3}\right)$

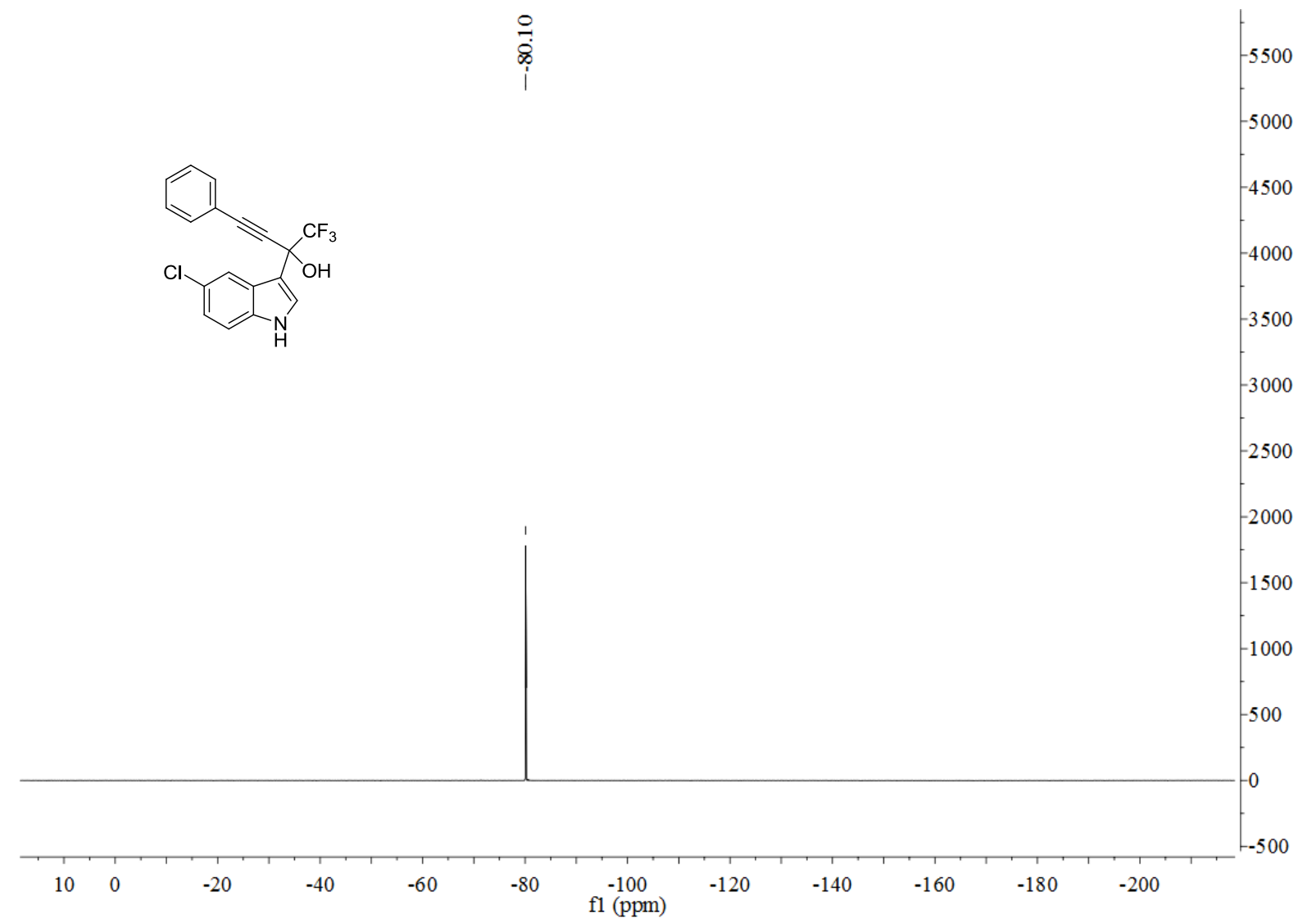


${ }^{1} \mathrm{H}$ NMR of compound $1 \mathrm{e}\left(400 \mathrm{MHz}\right.$ in $\left.\mathrm{CDCl}_{3}\right)$

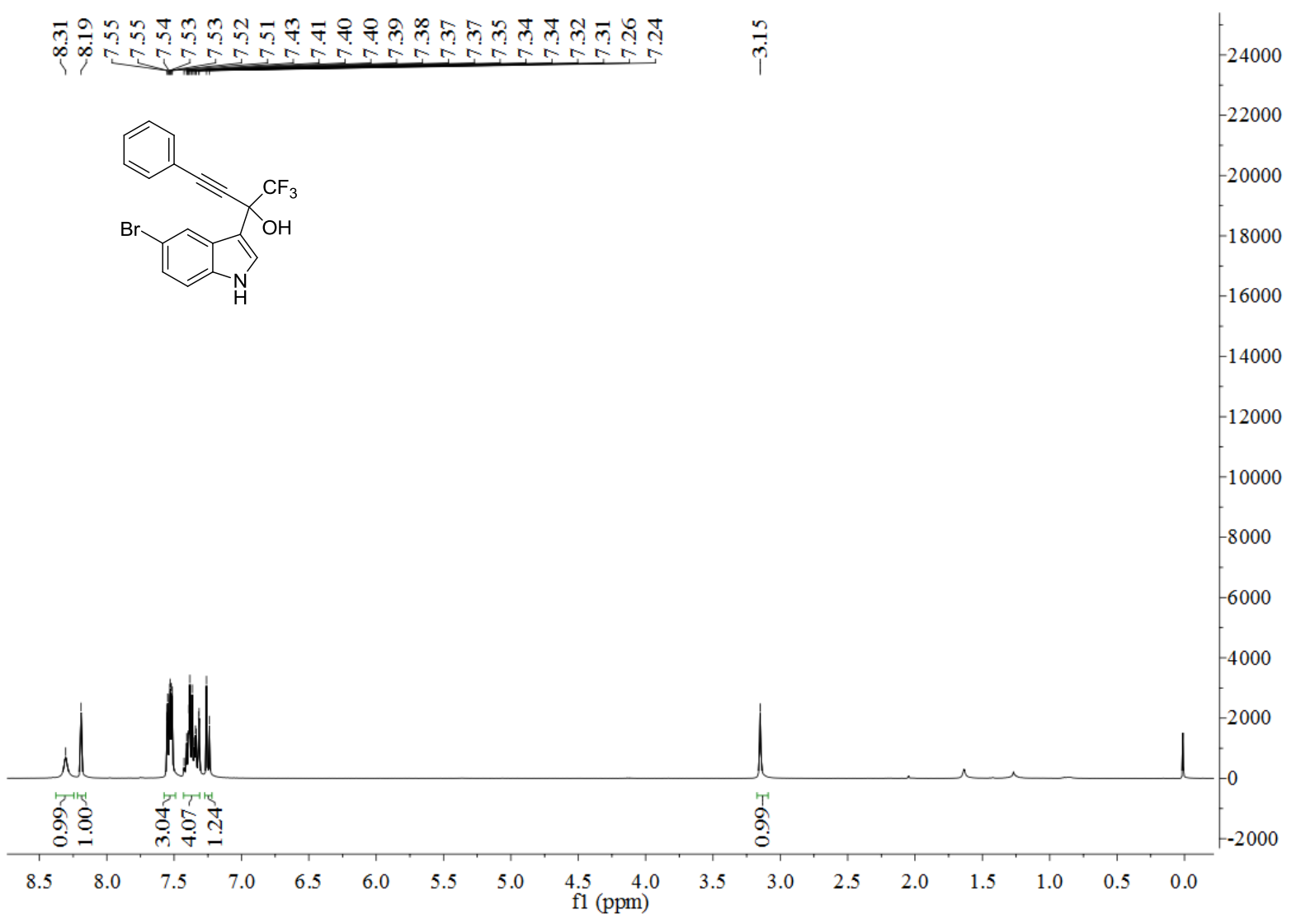

${ }^{13} \mathrm{C} \mathrm{NMR}$ of compound $1 \mathrm{e}$ (101 $\mathrm{MHz}$ in $\mathrm{CDCl}_{3}$ )

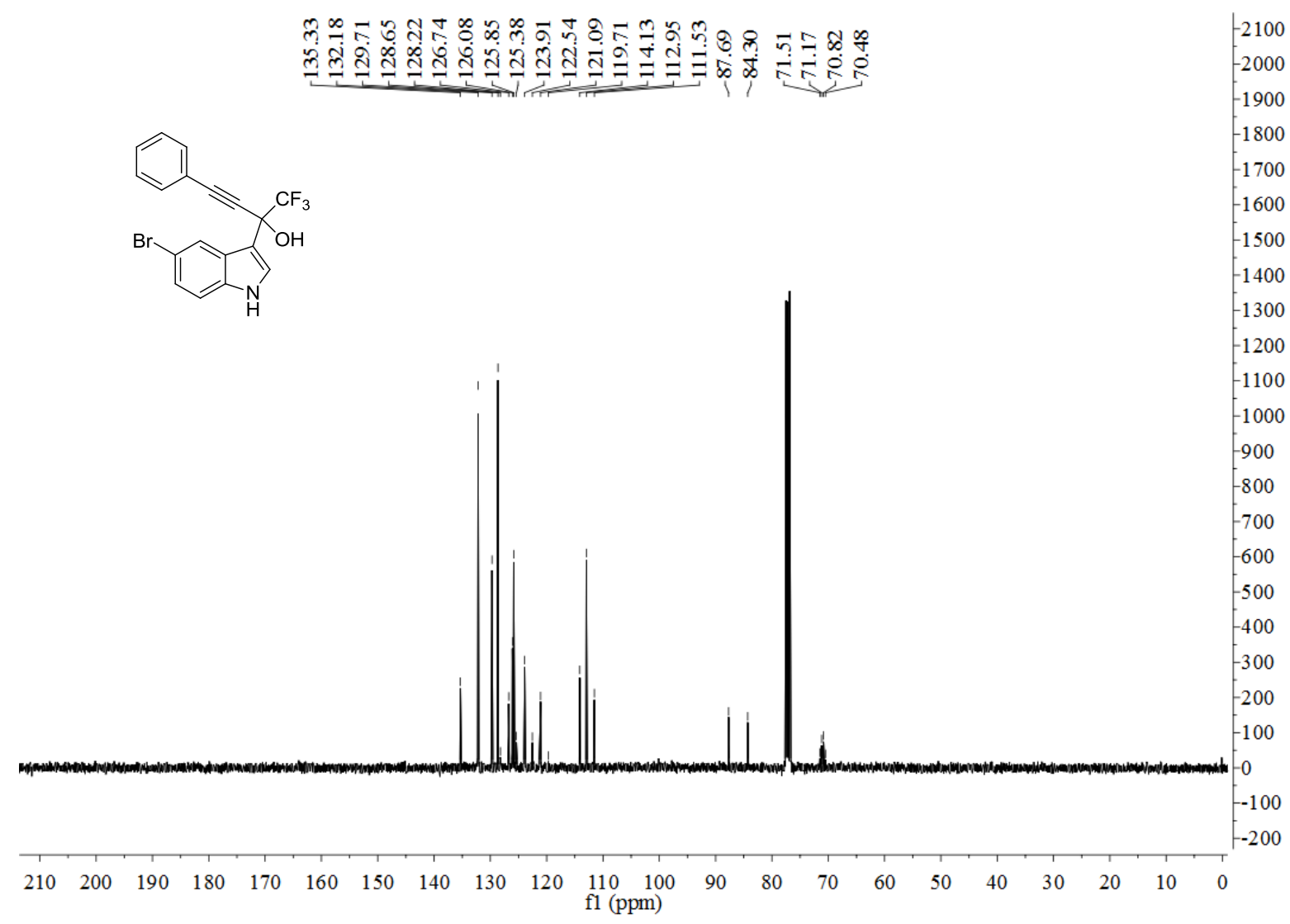


${ }^{19} \mathrm{~F} \mathrm{NMR}$ of compound $1 \mathrm{e}\left(376 \mathrm{MHz}\right.$ in $\left.\mathrm{CDCl}_{3}\right)$

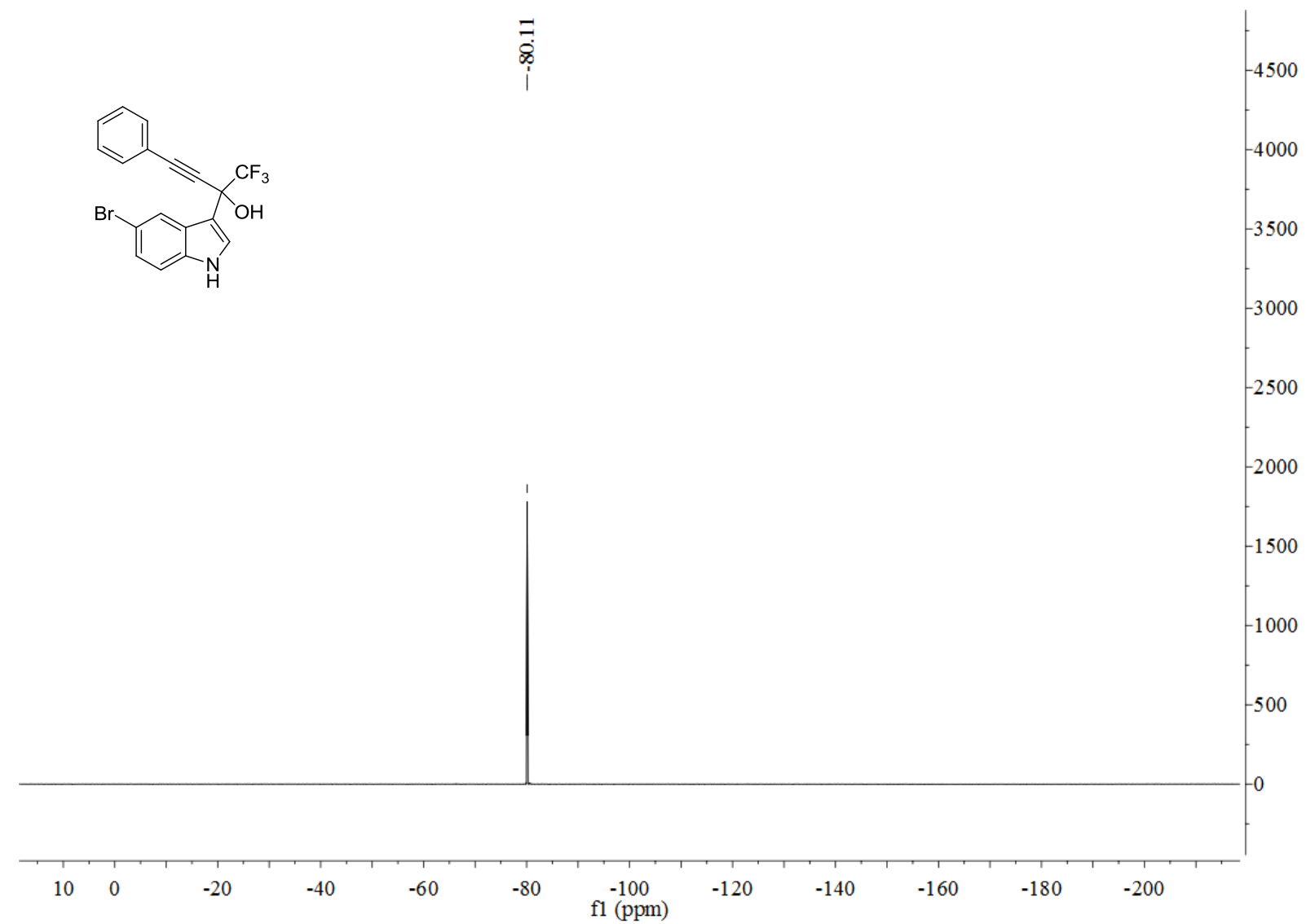

${ }^{1} \mathrm{H}$ NMR of compound $1 \mathrm{f}\left(400 \mathrm{MHz}\right.$ in $\mathrm{CDCl}_{3}$ )

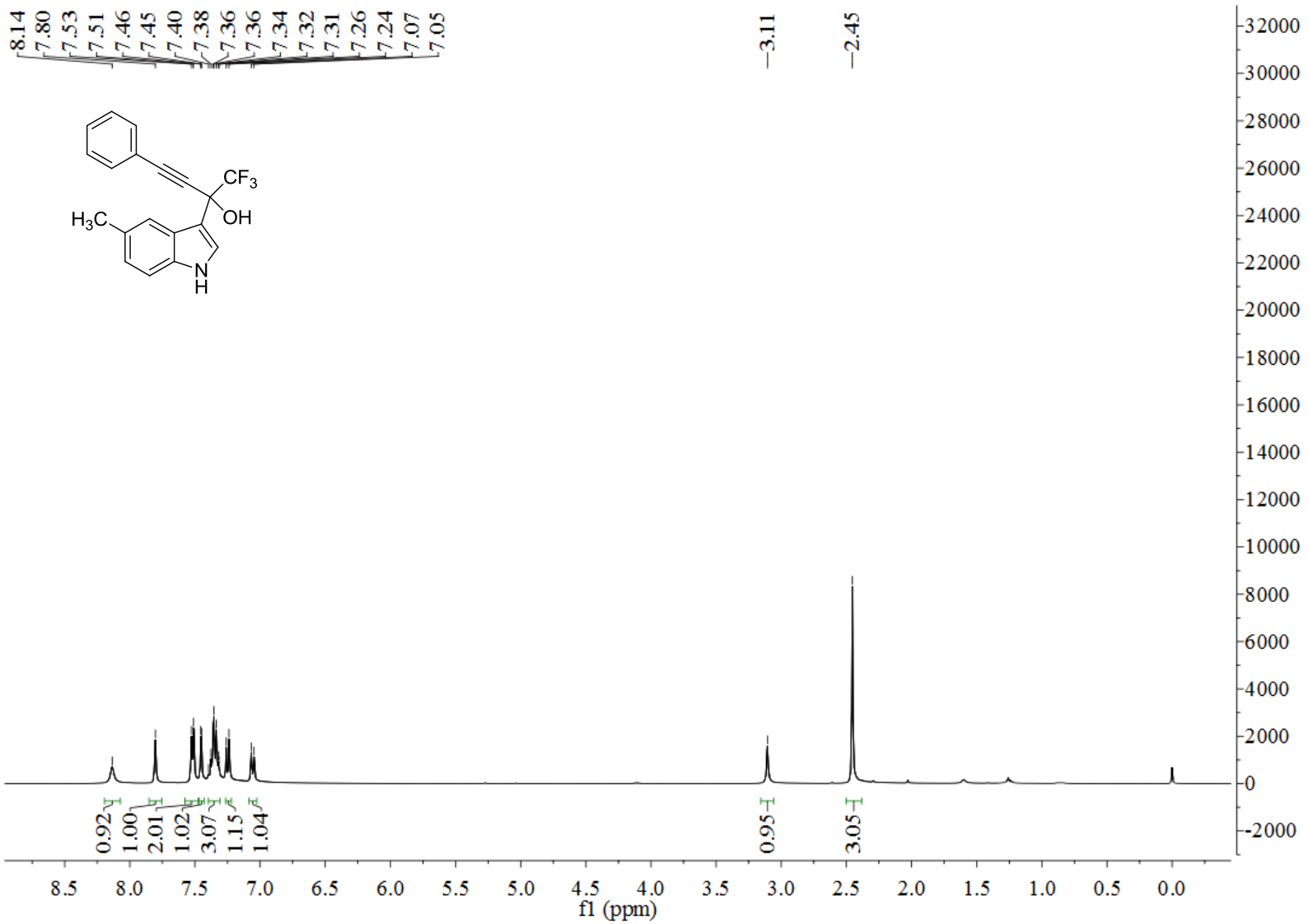


${ }^{13} \mathrm{C}$ NMR of compound $1 \mathrm{f}\left(101 \mathrm{MHz}\right.$ in $\mathrm{CDCl}_{3}$ )

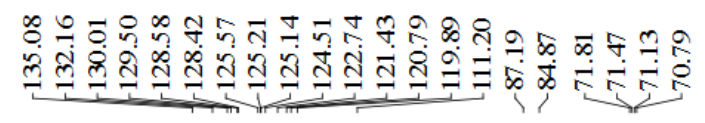<smiles>Cc1ccc2[nH]cc(C(O)(C#Cc3ccccc3)C(F)(F)F)c2c1</smiles>

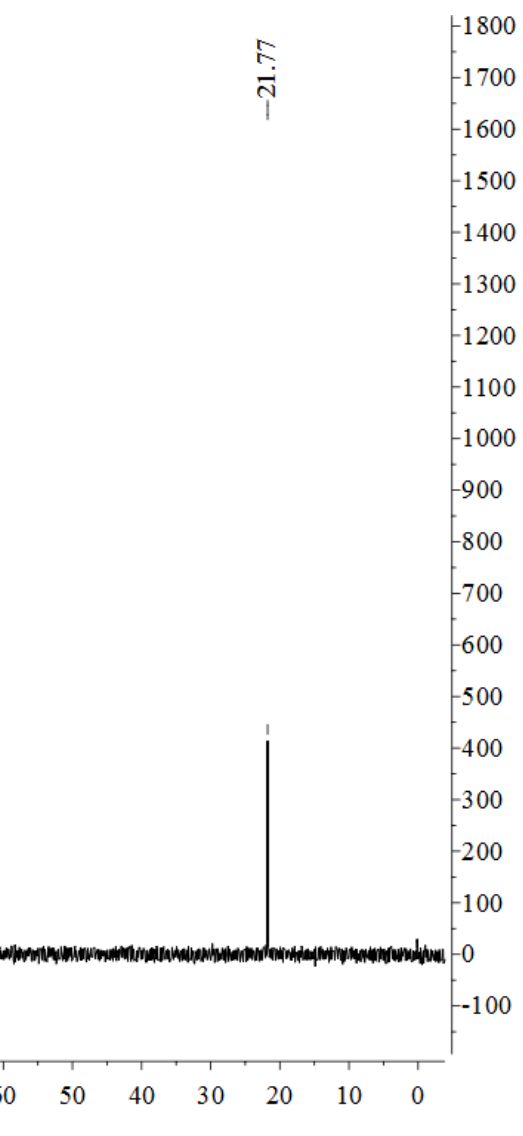

$\begin{array}{lllllllllll}210 & 200 & 190 & 180 & 170 & 160 & 150 & 140 & 130 & 120 & 110 \\ \mathrm{fl}(\mathrm{ppm})\end{array}$

${ }^{19} \mathrm{~F}$ NMR of compound $1 f\left(376 \mathrm{MHz}\right.$ in $\left.\mathrm{CDCl}_{3}\right)$

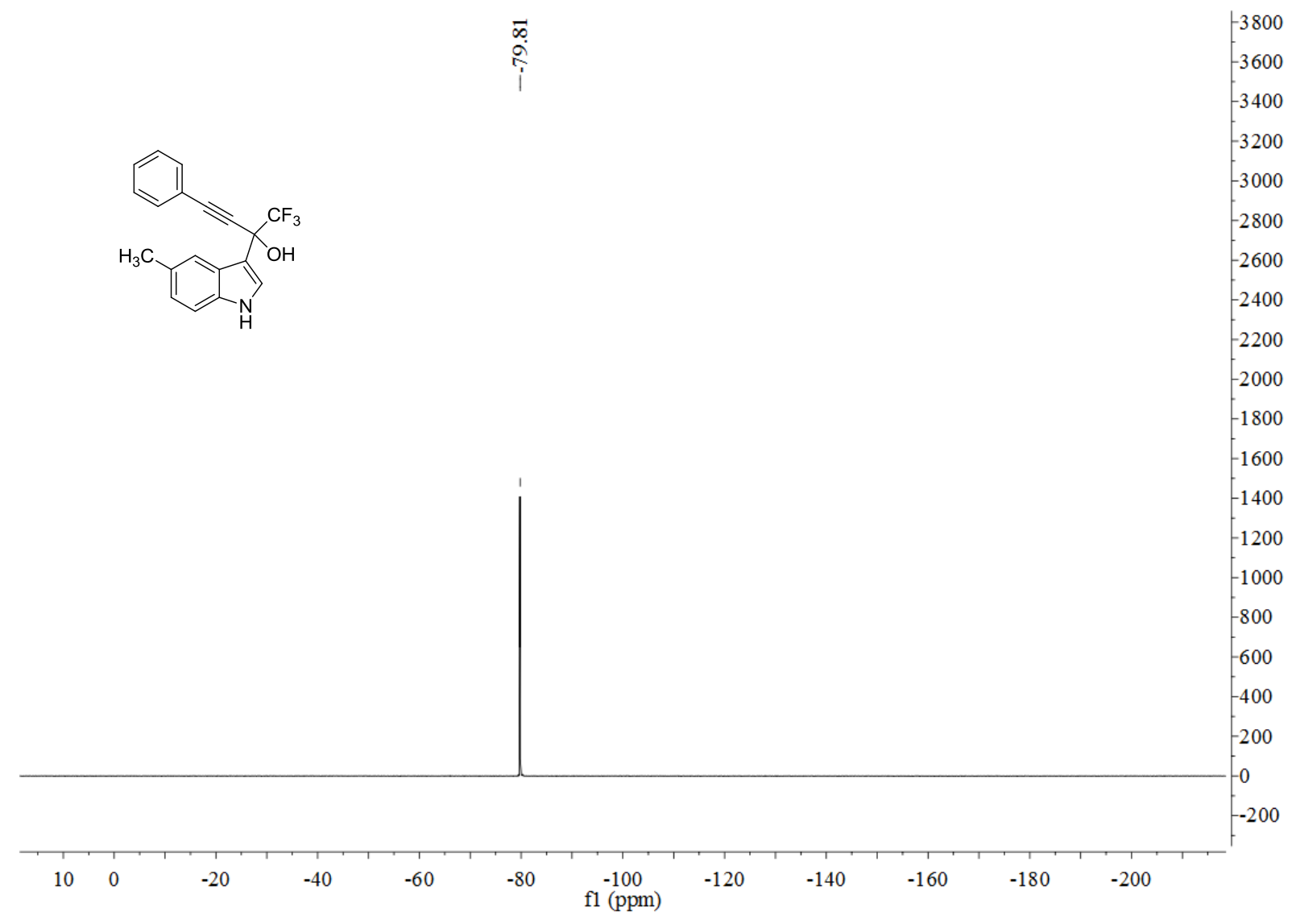


${ }^{1} \mathrm{H}$ NMR of compound $1 \mathrm{~g}(400 \mathrm{MHz}$ in DMSO-d 6 )

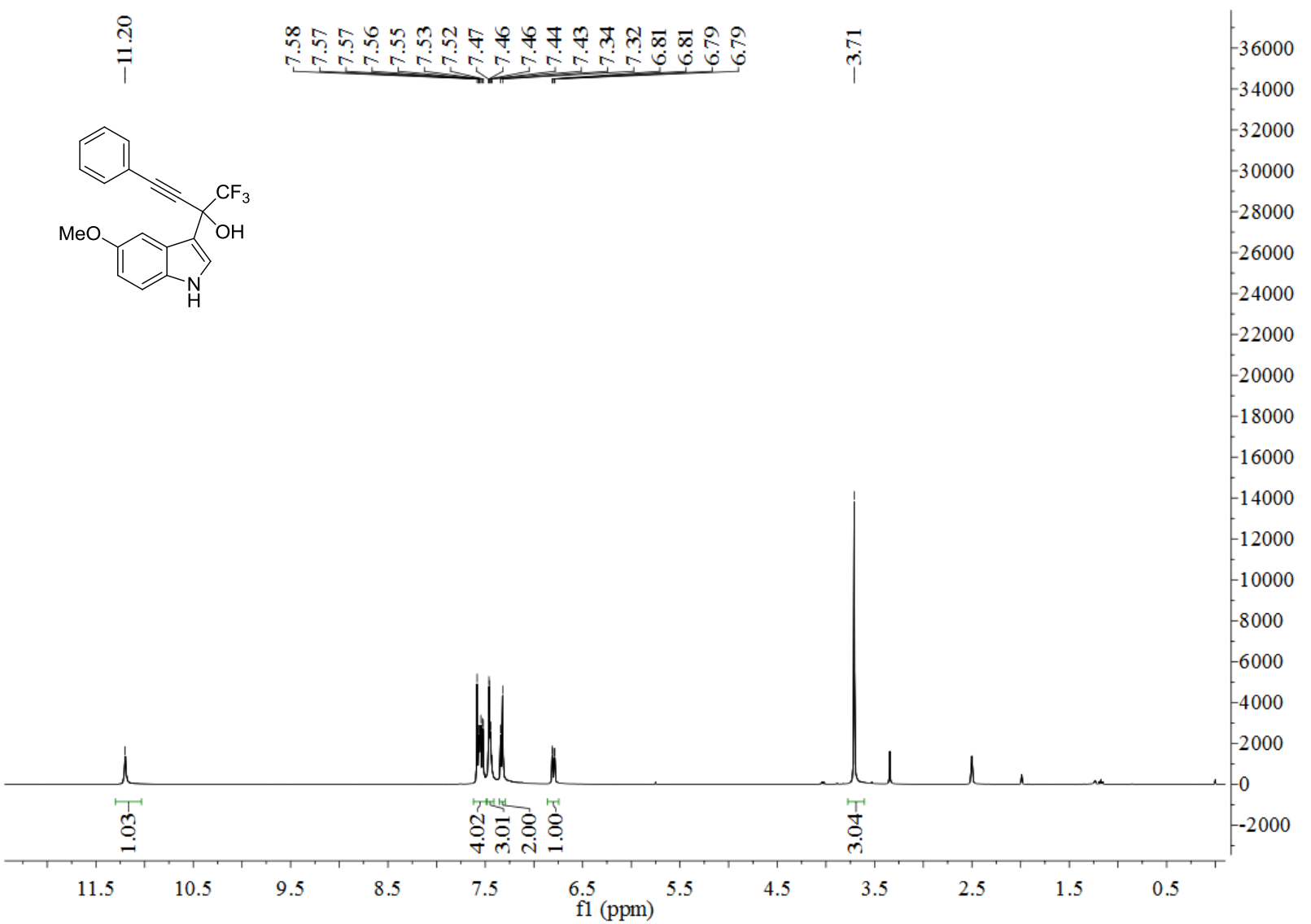

${ }^{13} \mathrm{C}$ NMR of compound $1 \mathrm{~g}$ (101 MHz in DMSO-d $\mathrm{d}_{6}$ )

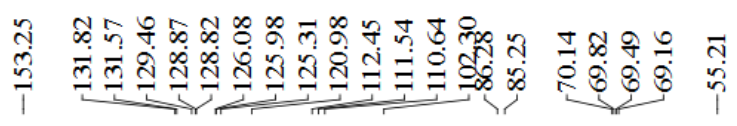<smiles>COc1ccc2[nH]cc(C(O)(C#Cc3ccccc3)C(F)(F)F)c2c1</smiles>

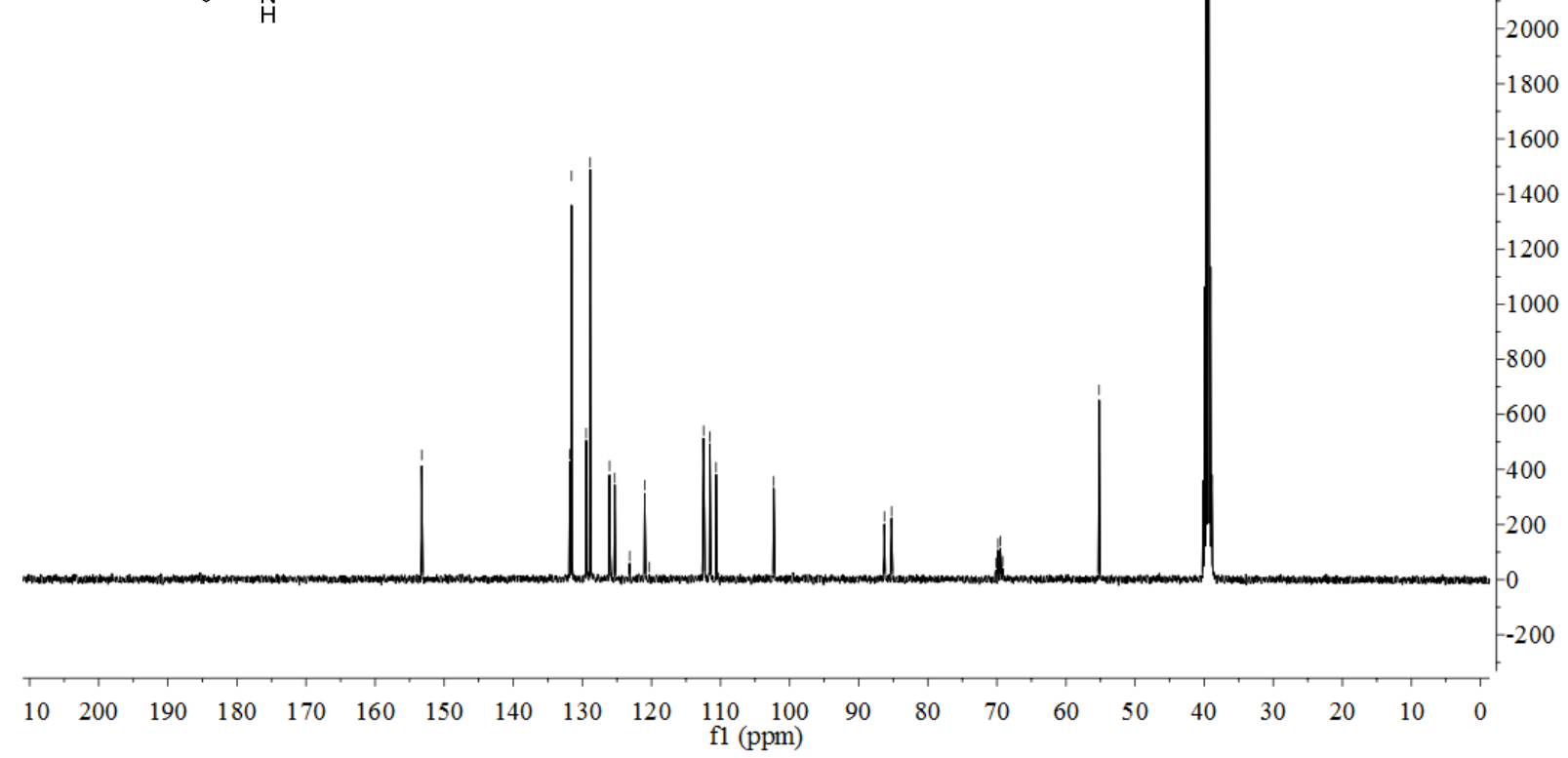


${ }^{19} \mathrm{~F}$ NMR of compound $1 \mathrm{~g}$ (376 MHz in DMSO-d $\mathrm{d}_{6}$ )

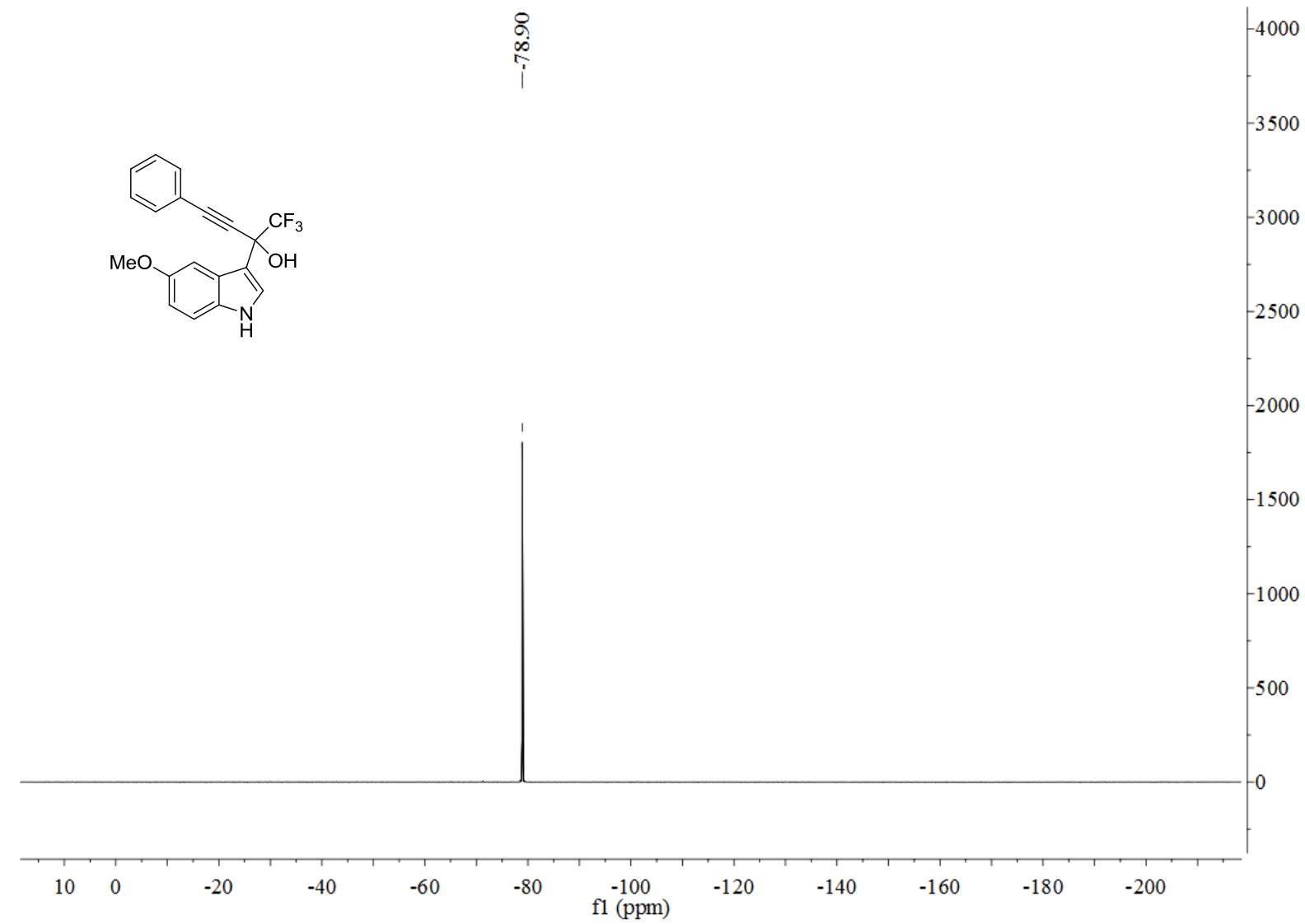

${ }^{1} \mathrm{H}$ NMR of compound $1 \mathrm{~h}\left(400 \mathrm{MHz}\right.$ in $\left.\mathrm{CDCl}_{3}\right)$

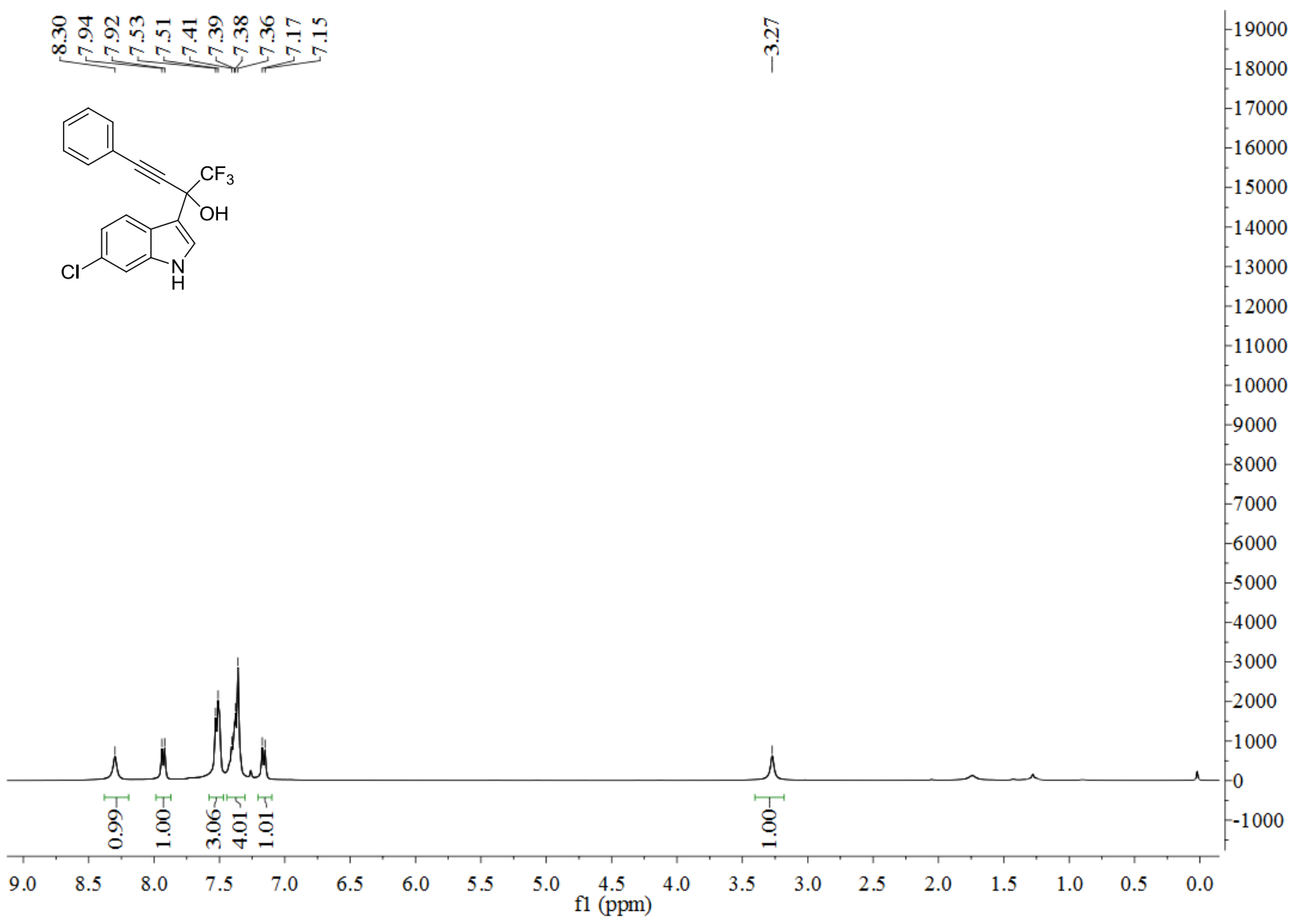


${ }^{13} \mathrm{C}$ NMR of compound $1 \mathrm{~h}\left(101 \mathrm{MHz}\right.$ in $\left.\mathrm{CDCl}_{3}\right)$

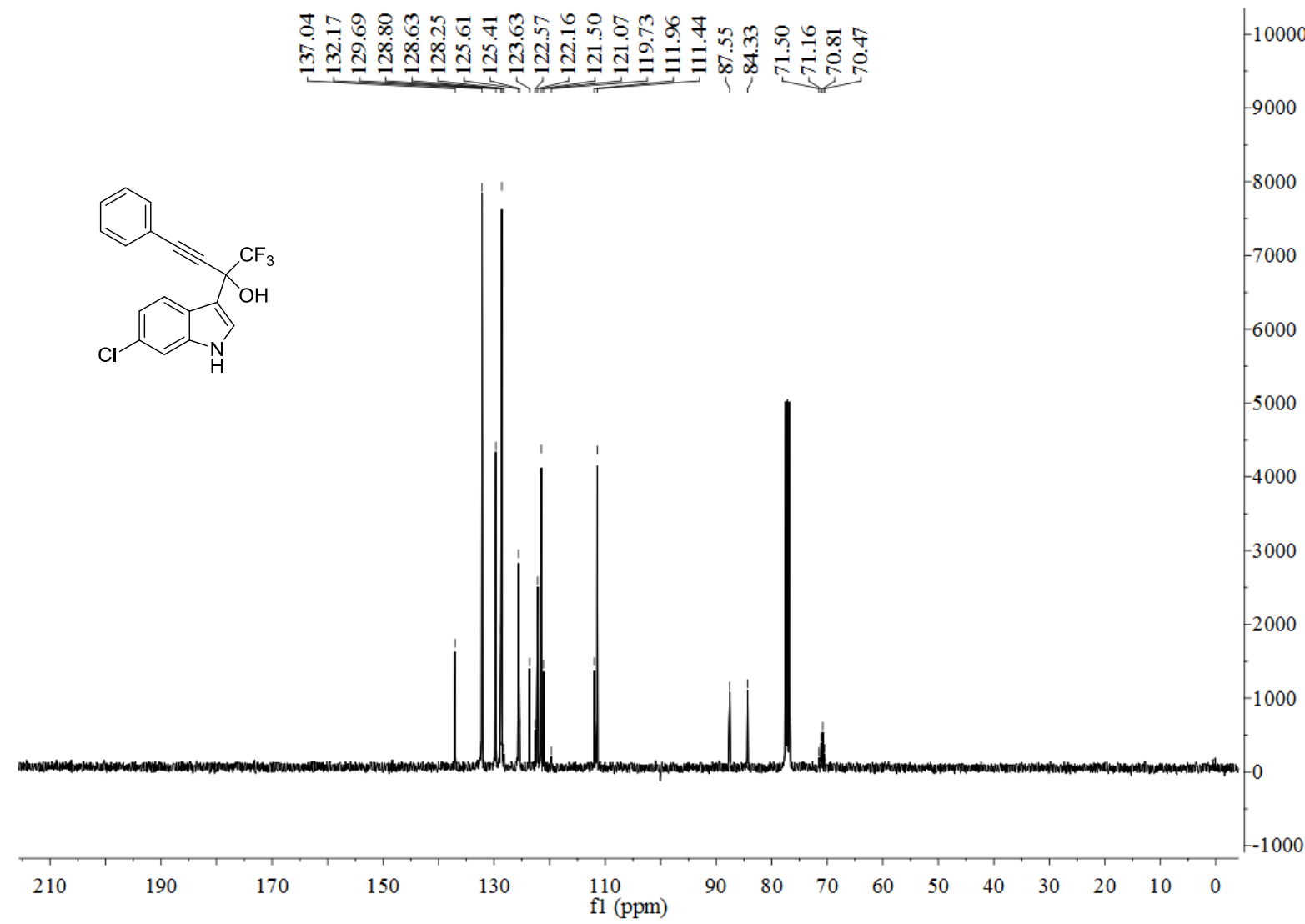

${ }^{19} \mathrm{~F} \mathrm{NMR}$ of compound $1 \mathrm{~h}\left(376 \mathrm{MHz}\right.$ in $\left.\mathrm{CDCl}_{3}\right)$

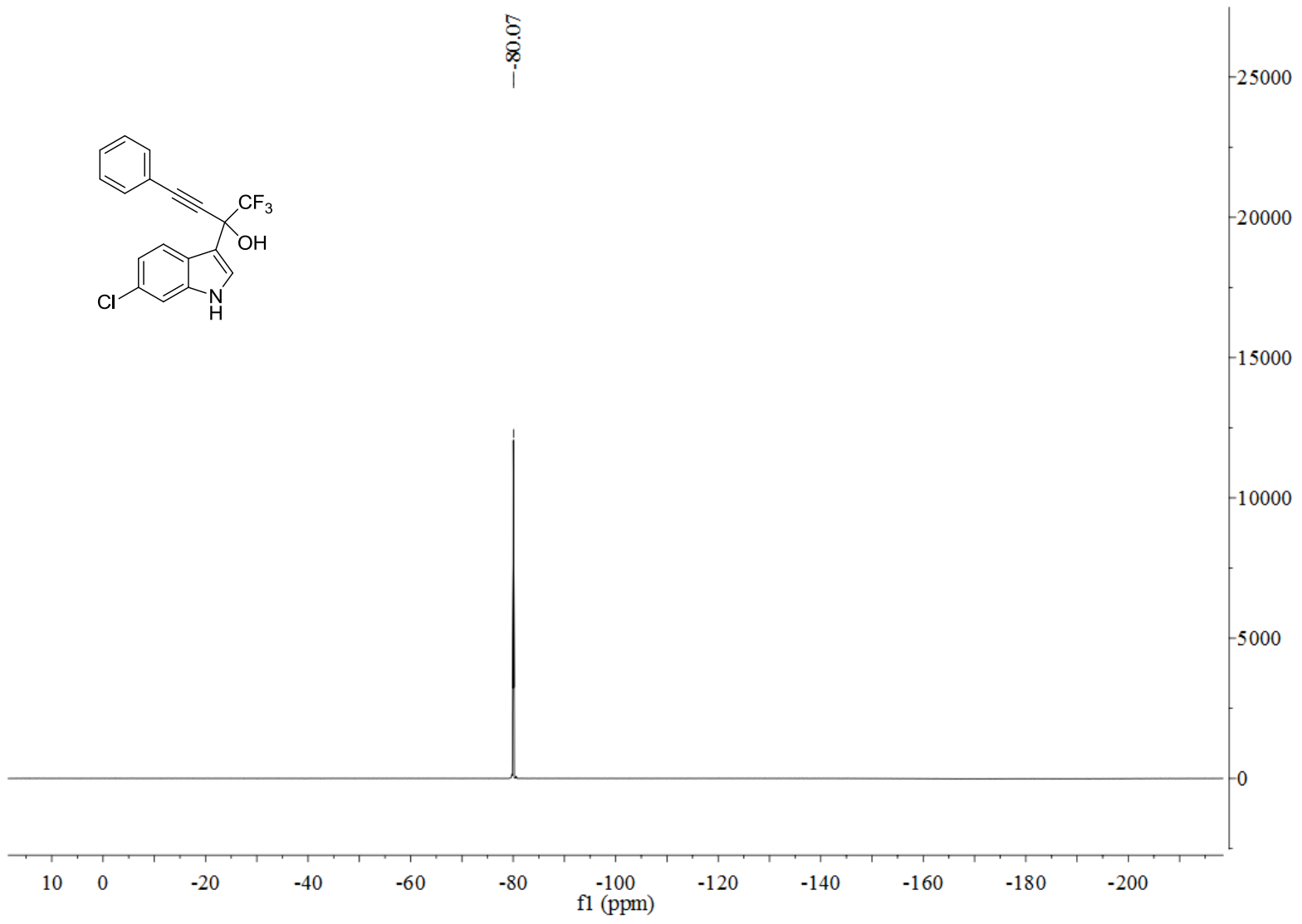


${ }^{1} \mathrm{H}$ NMR of compound $1 \mathrm{i}$ (400 $\mathrm{MHz}$ in $\mathrm{CDCl}_{3}$ )

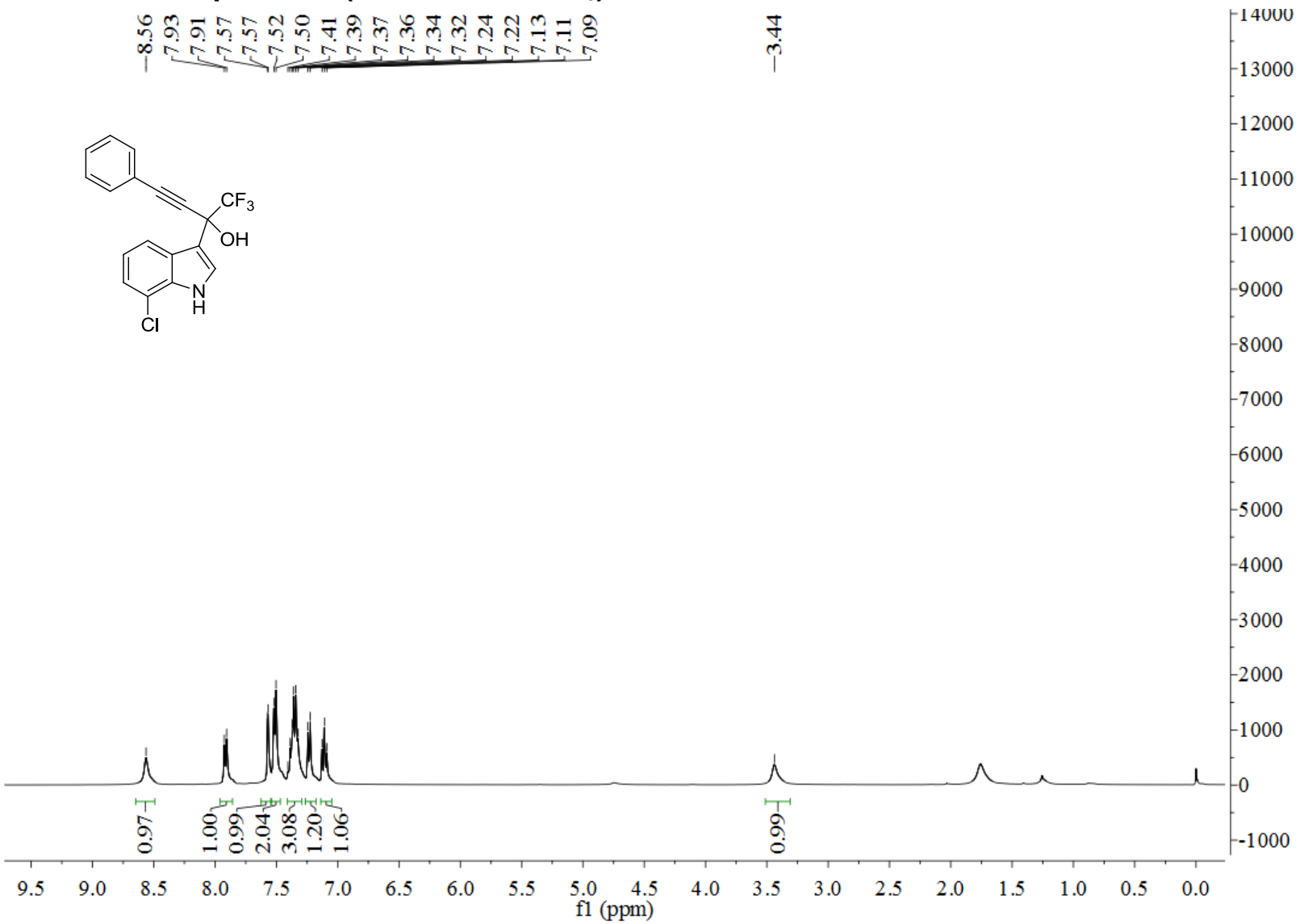

${ }^{13} \mathrm{C}$ NMR of compound $1 \mathrm{i}\left(101 \mathrm{MHz}\right.$ in $\mathrm{CDCl}_{3}$ )

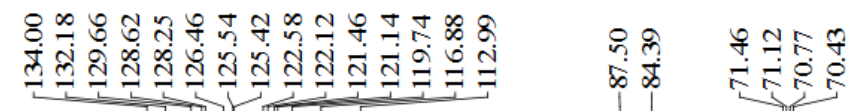

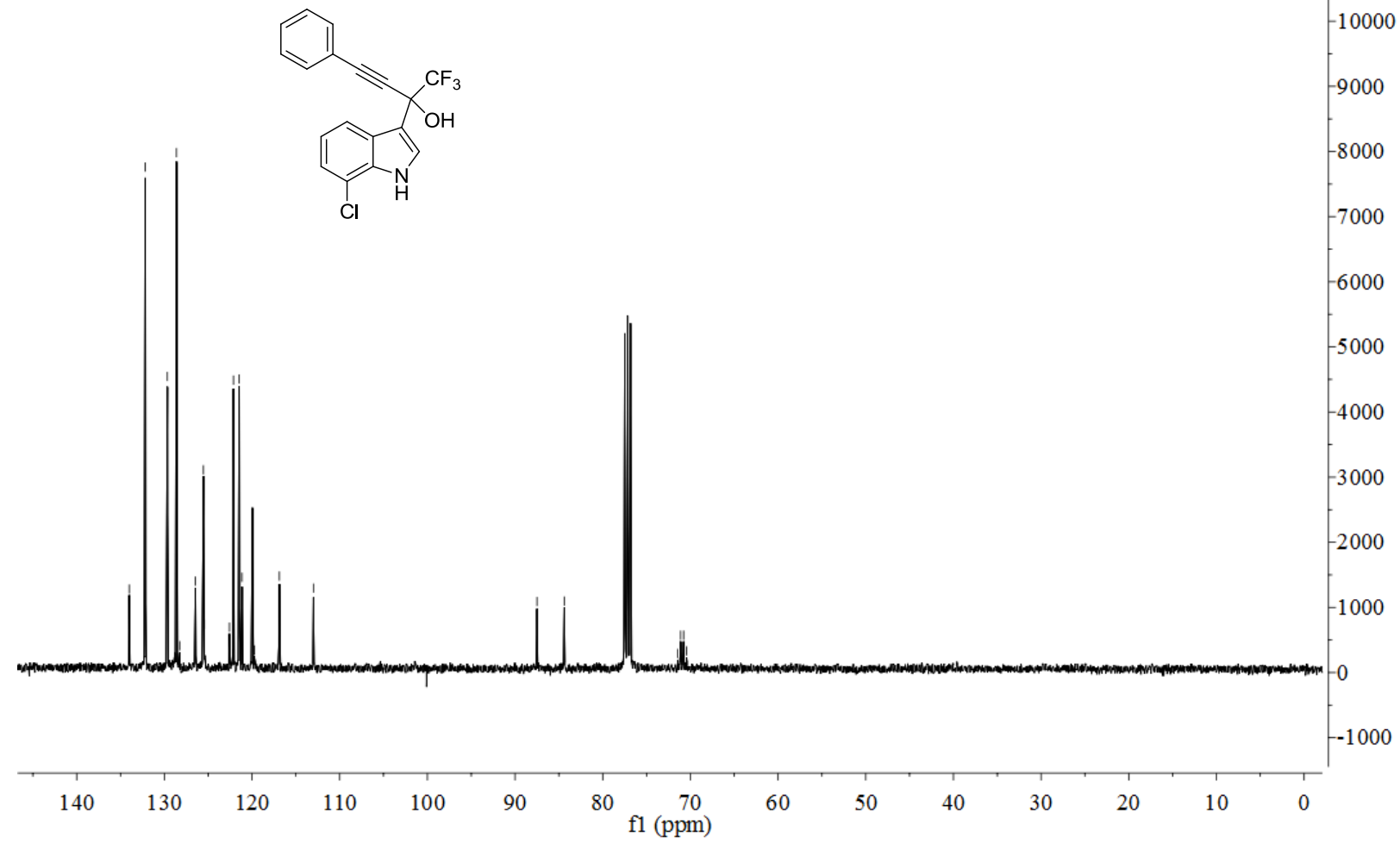


${ }^{19} \mathrm{~F} \mathrm{NMR}$ of compound $1 \mathrm{i}\left(376 \mathrm{MHz}\right.$ in $\left.\mathrm{CDCl}_{3}\right)$

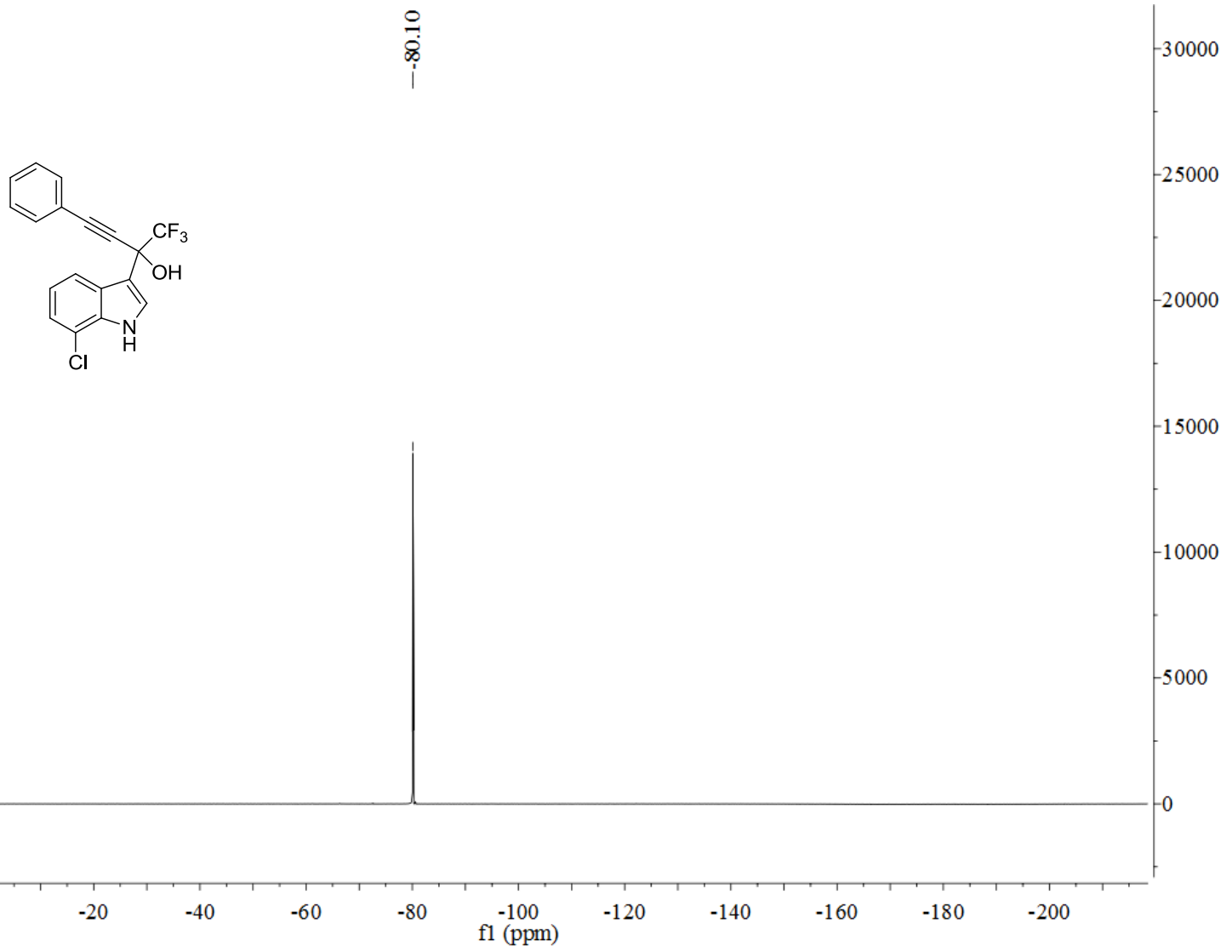

${ }^{1} \mathrm{H}$ NMR of compound $1 \mathrm{j}$ (400 MHz in DMSO-d $\mathrm{d}_{6}$ )

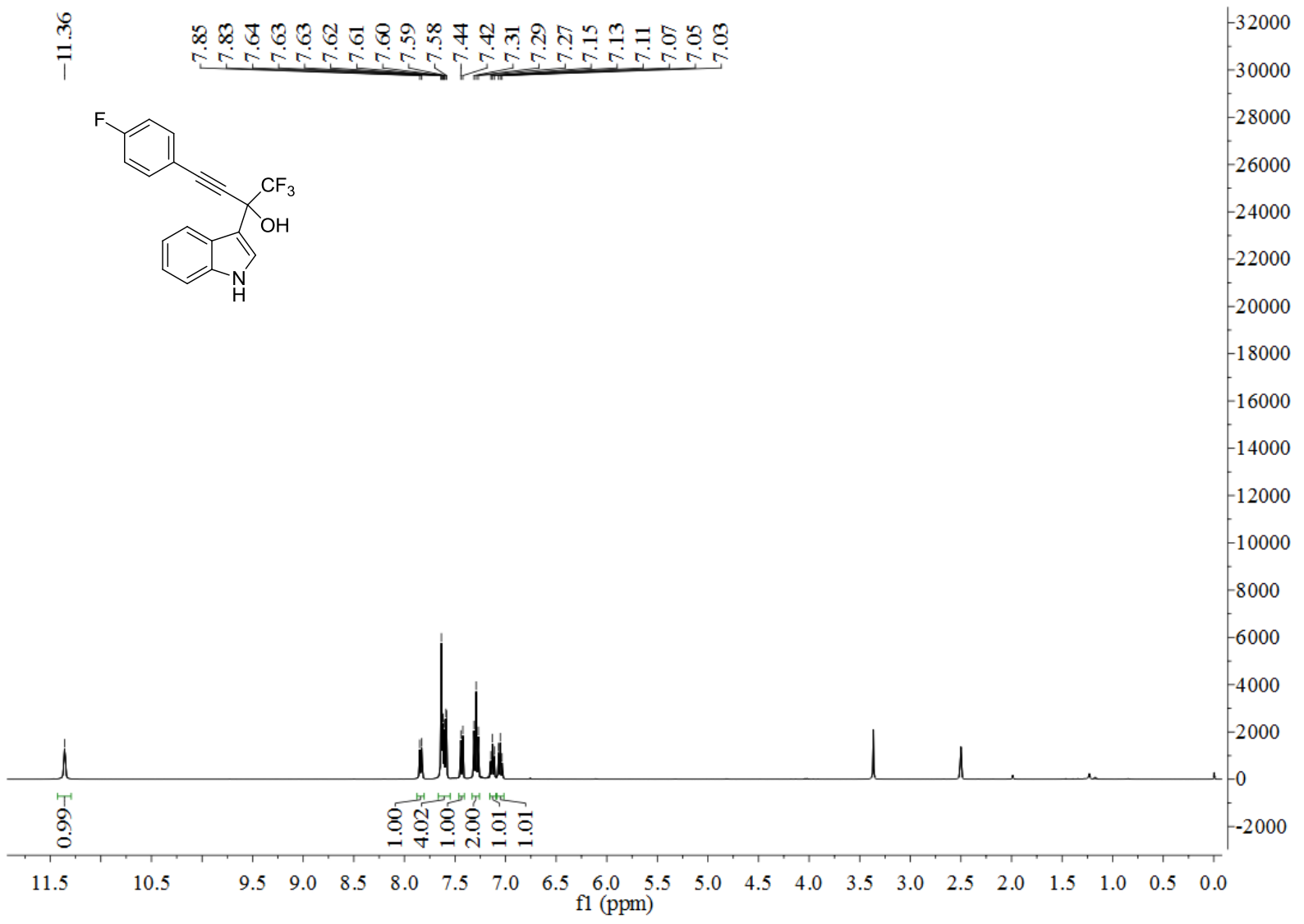


${ }^{13} \mathrm{C}$ NMR of compound $1 \mathrm{j}$ (101 MHz in DMSO- $\mathrm{d}_{6}$ )

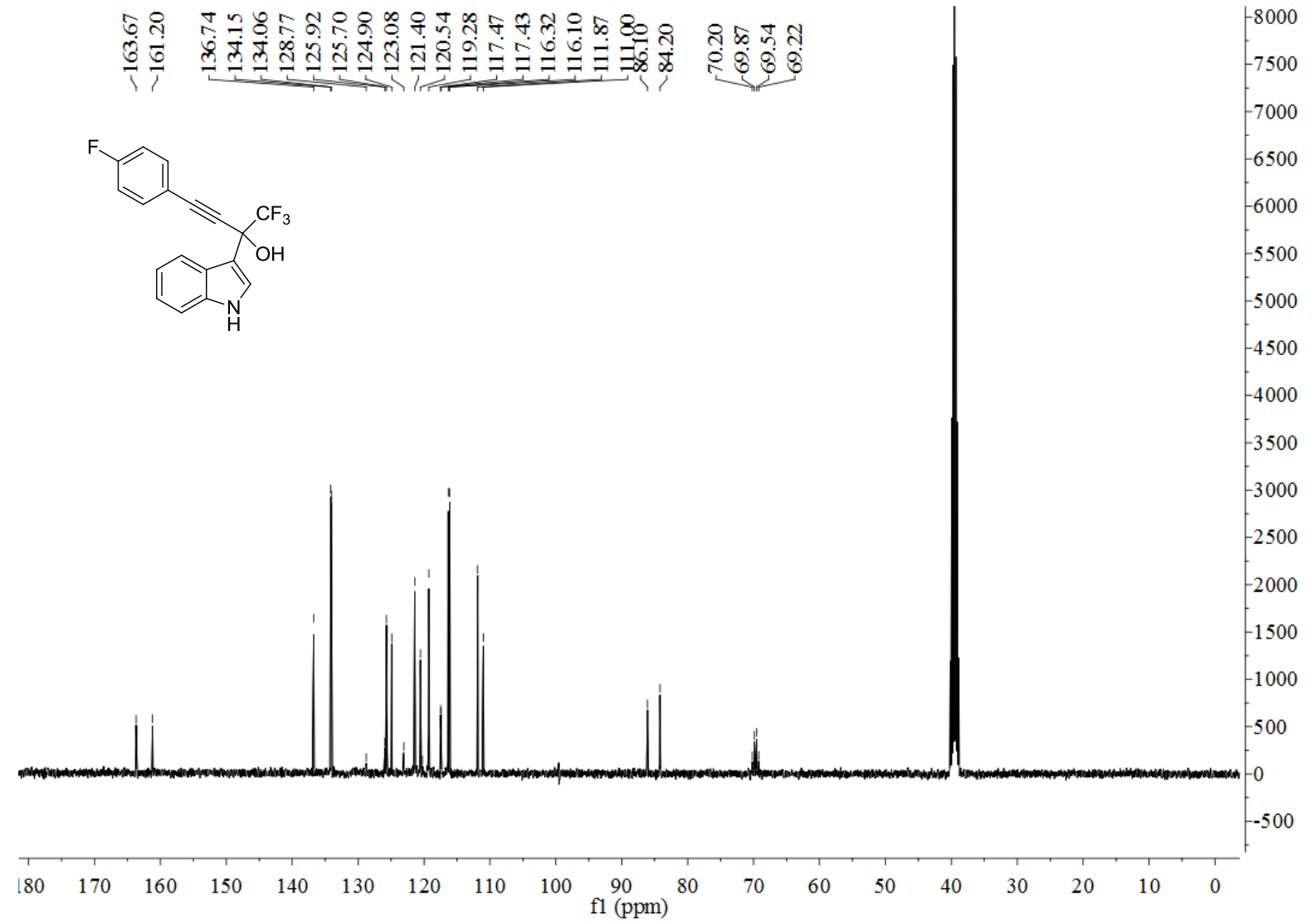

${ }^{19} \mathrm{~F}$ NMR of compound $1 \mathrm{j}\left(376 \mathrm{MHz}\right.$ in $\left.\mathrm{CDCl}_{3}\right)$ ?

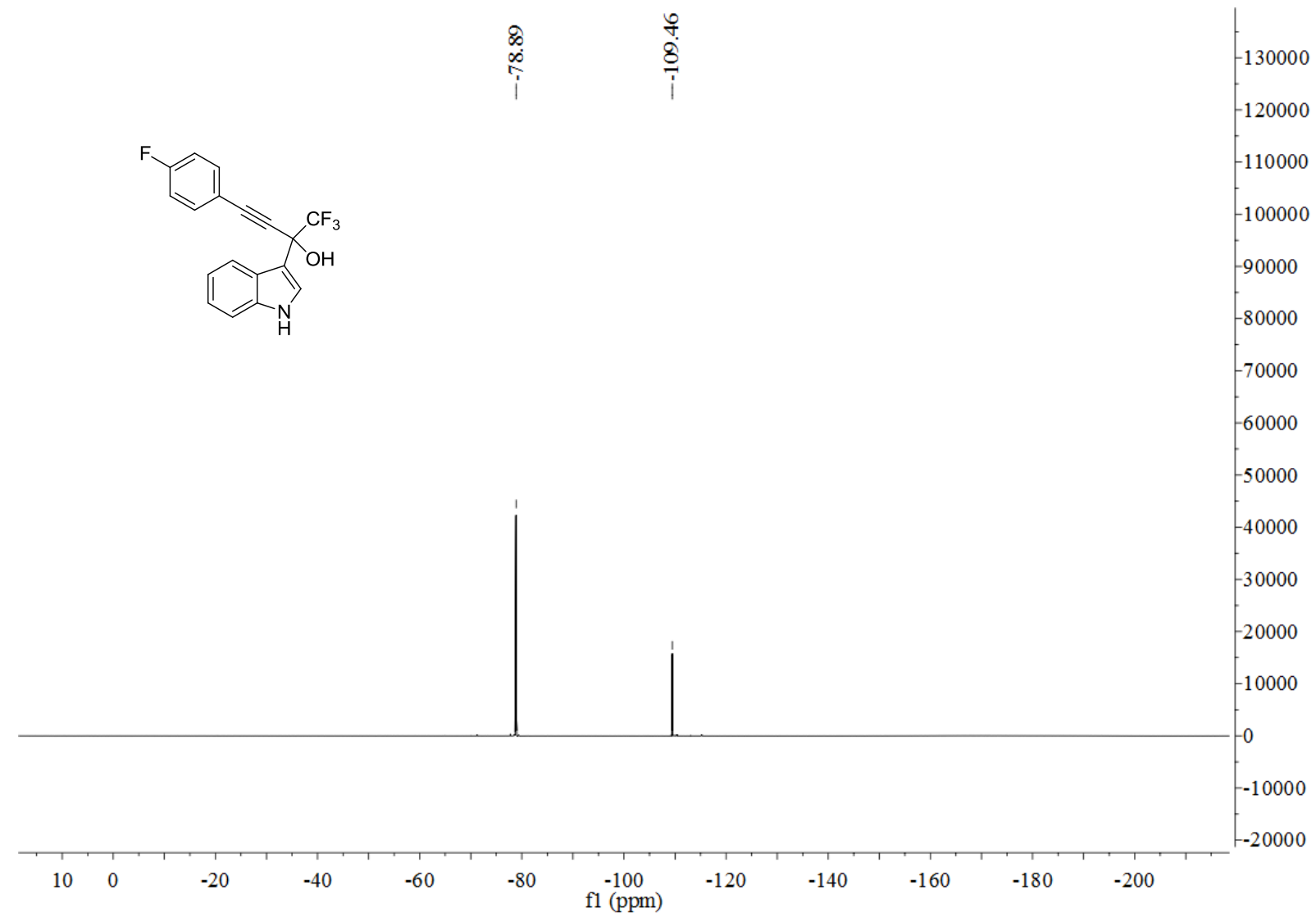


${ }^{1} \mathrm{H}$ NMR of compound $1 \mathrm{k}\left(400 \mathrm{MHz}\right.$ in $\left.\mathrm{CDCl}_{3}\right)$

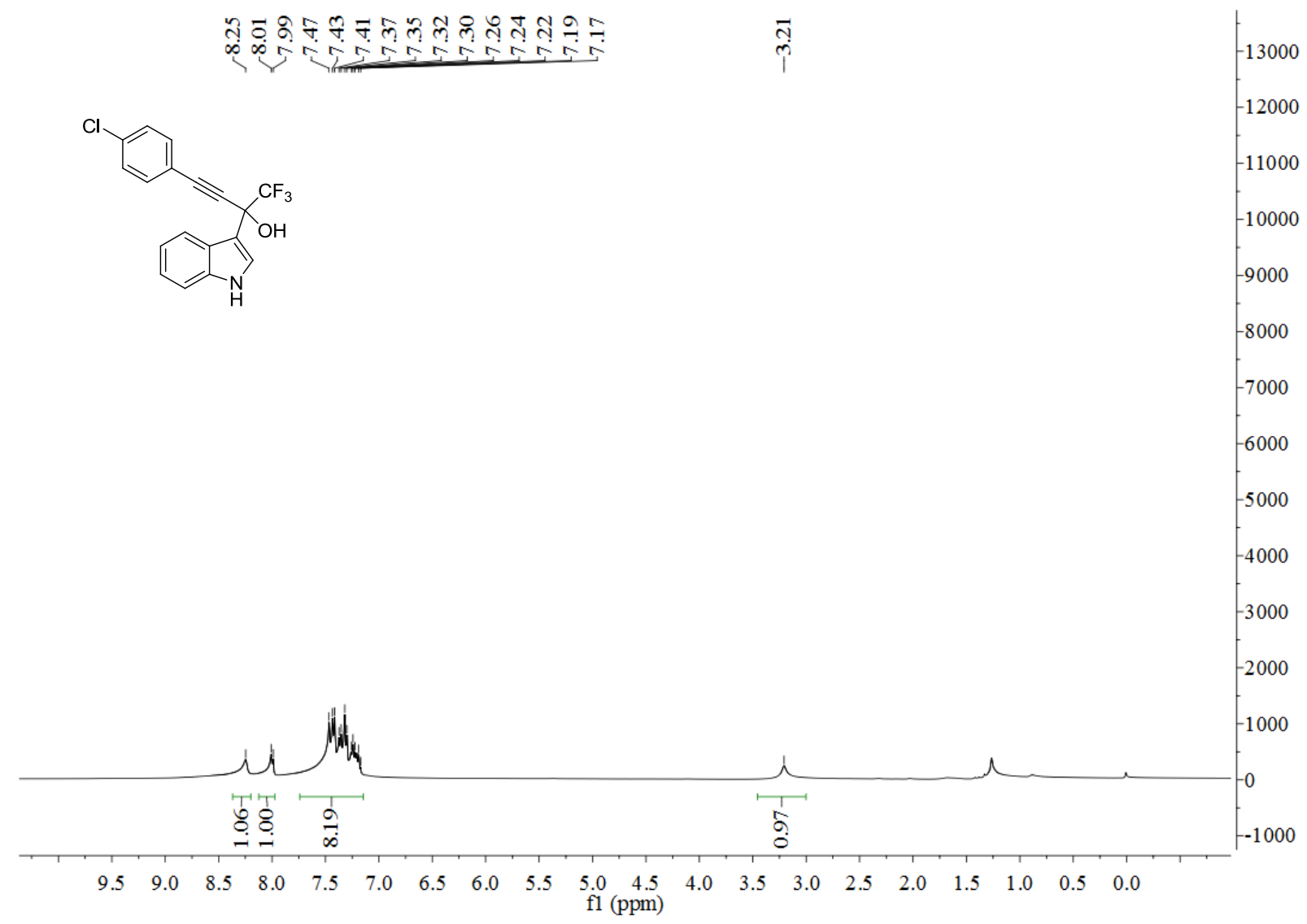

${ }^{13} \mathrm{C}$ NMR of compound $1 \mathrm{k}\left(101 \mathrm{MHz}\right.$ in $\mathrm{CDCl}_{3}$ )
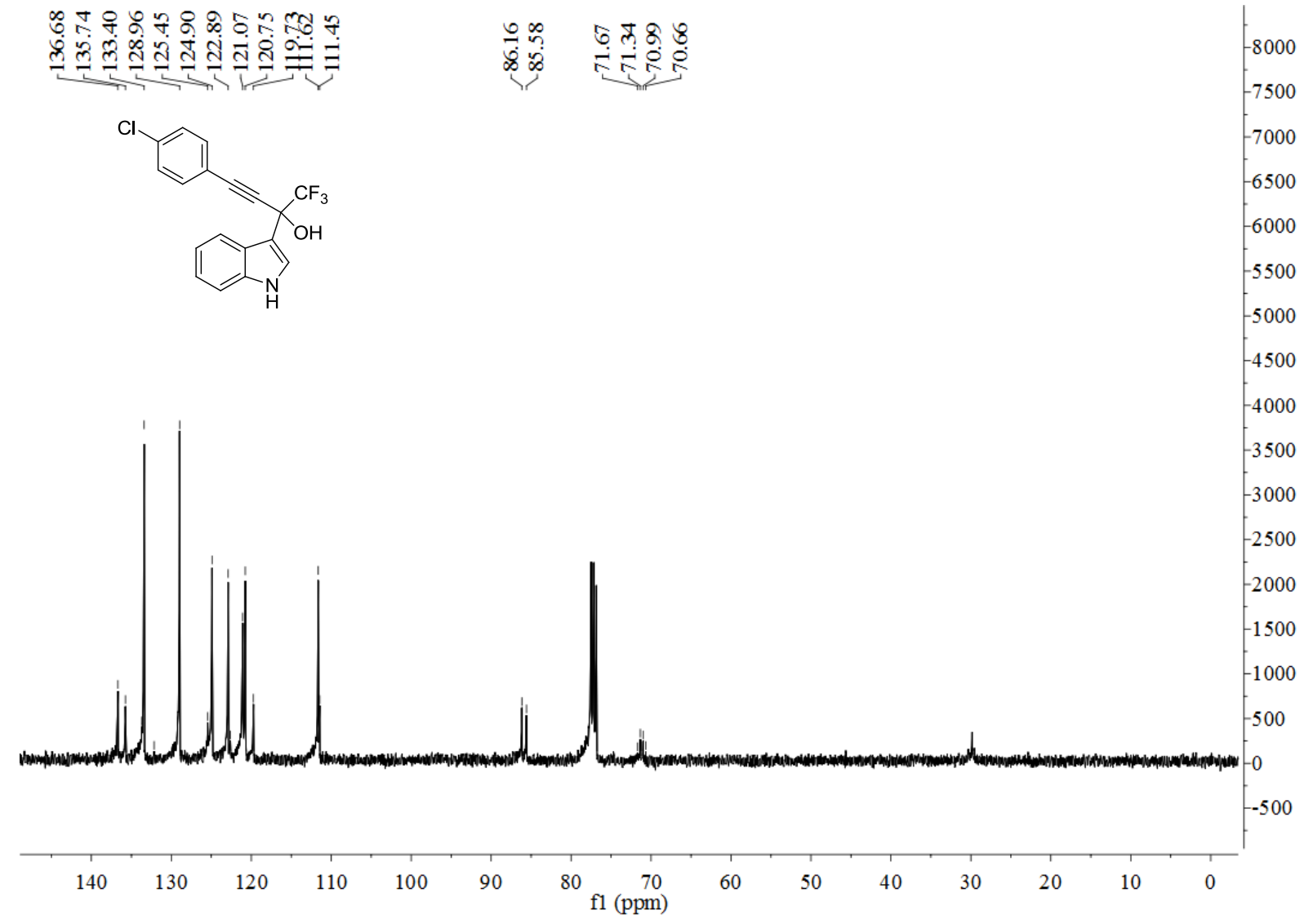


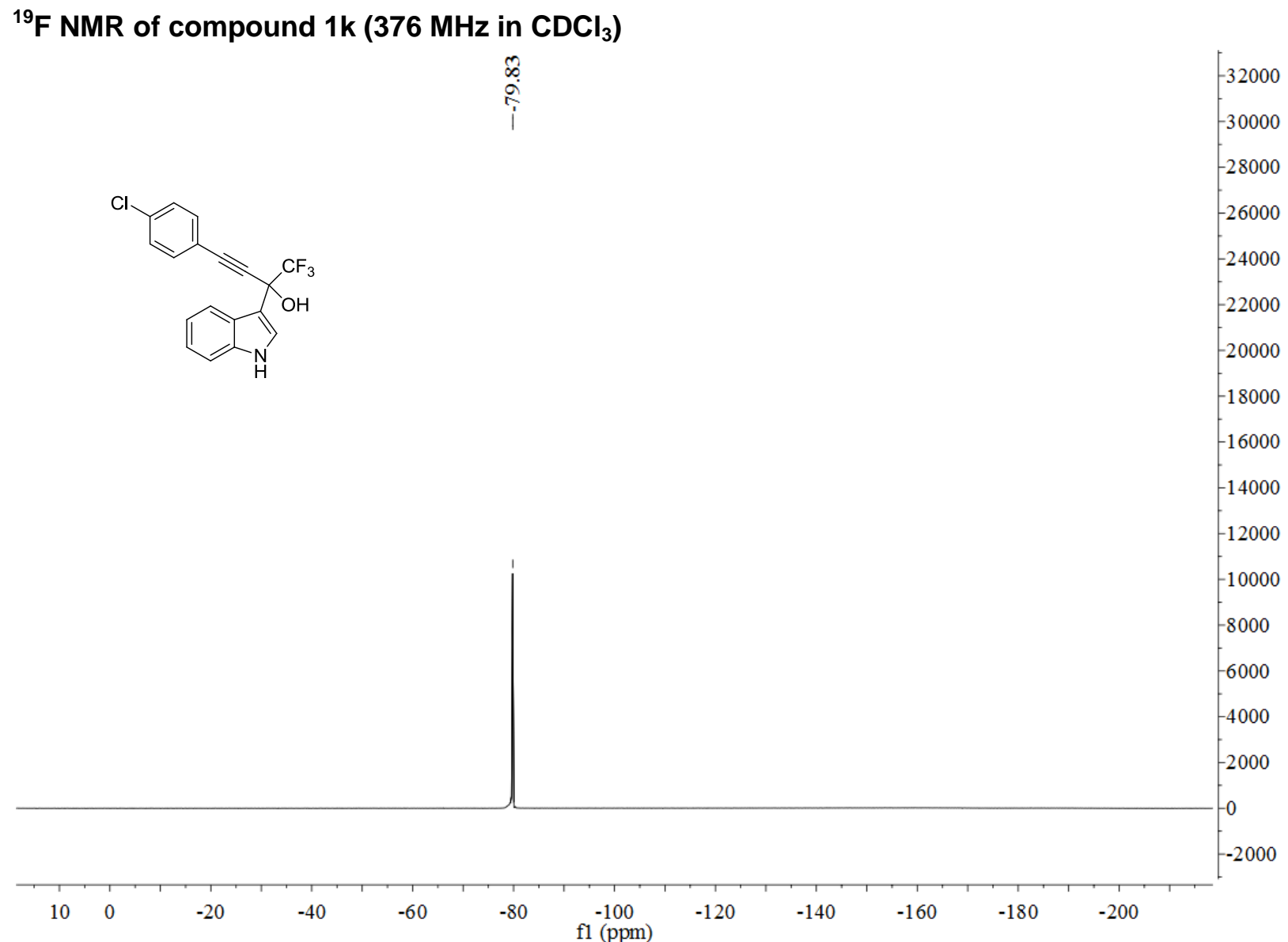

${ }^{1} \mathrm{H}$ NMR of compound $1 \mathrm{l}\left(400 \mathrm{MHz}\right.$ in $\mathrm{CDCl}_{3}$ )

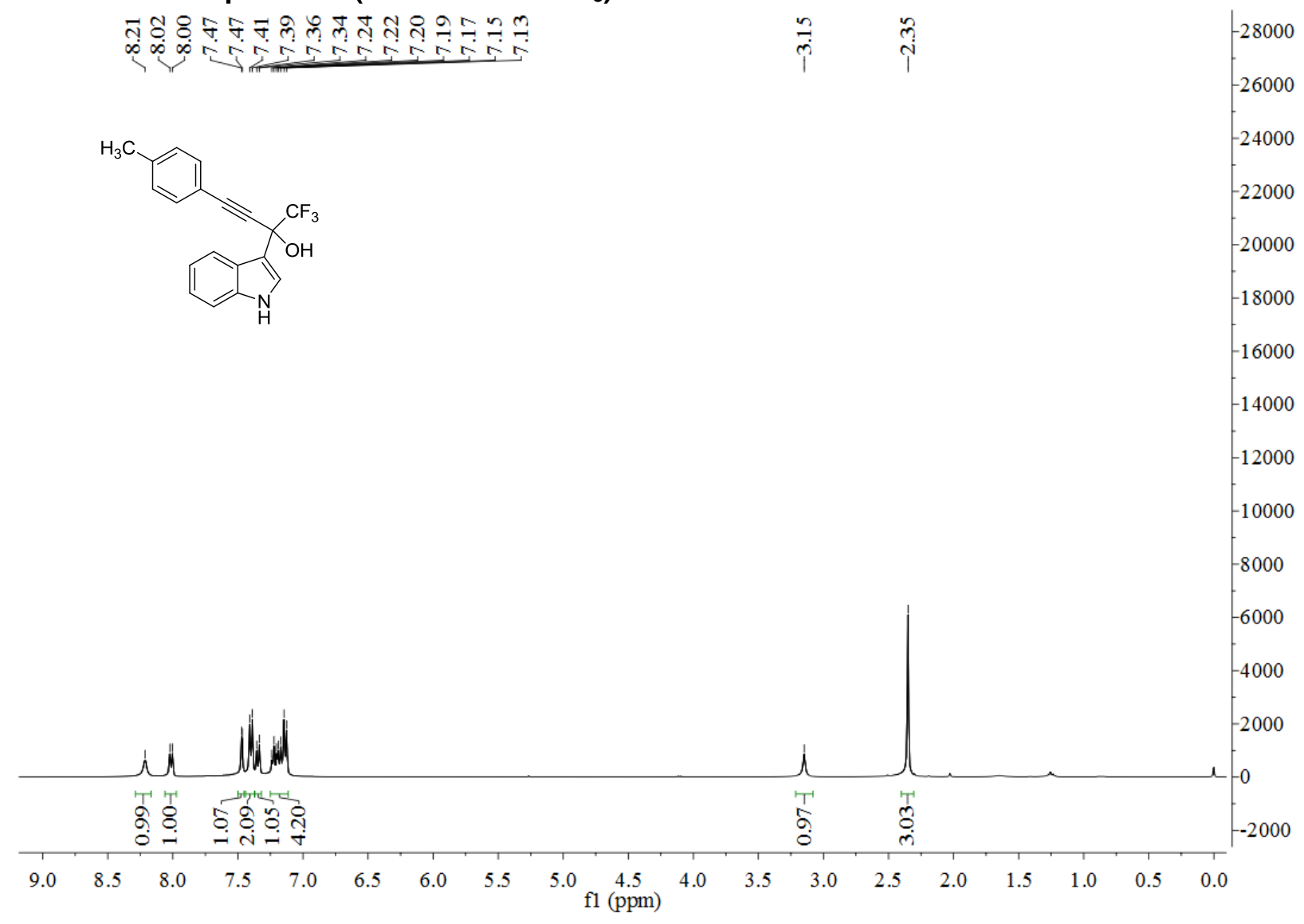


${ }^{13} \mathrm{C}$ NMR of compound $1 \mathrm{l}\left(101 \mathrm{MHz}\right.$ in $\mathrm{CDCl}_{3}$ )

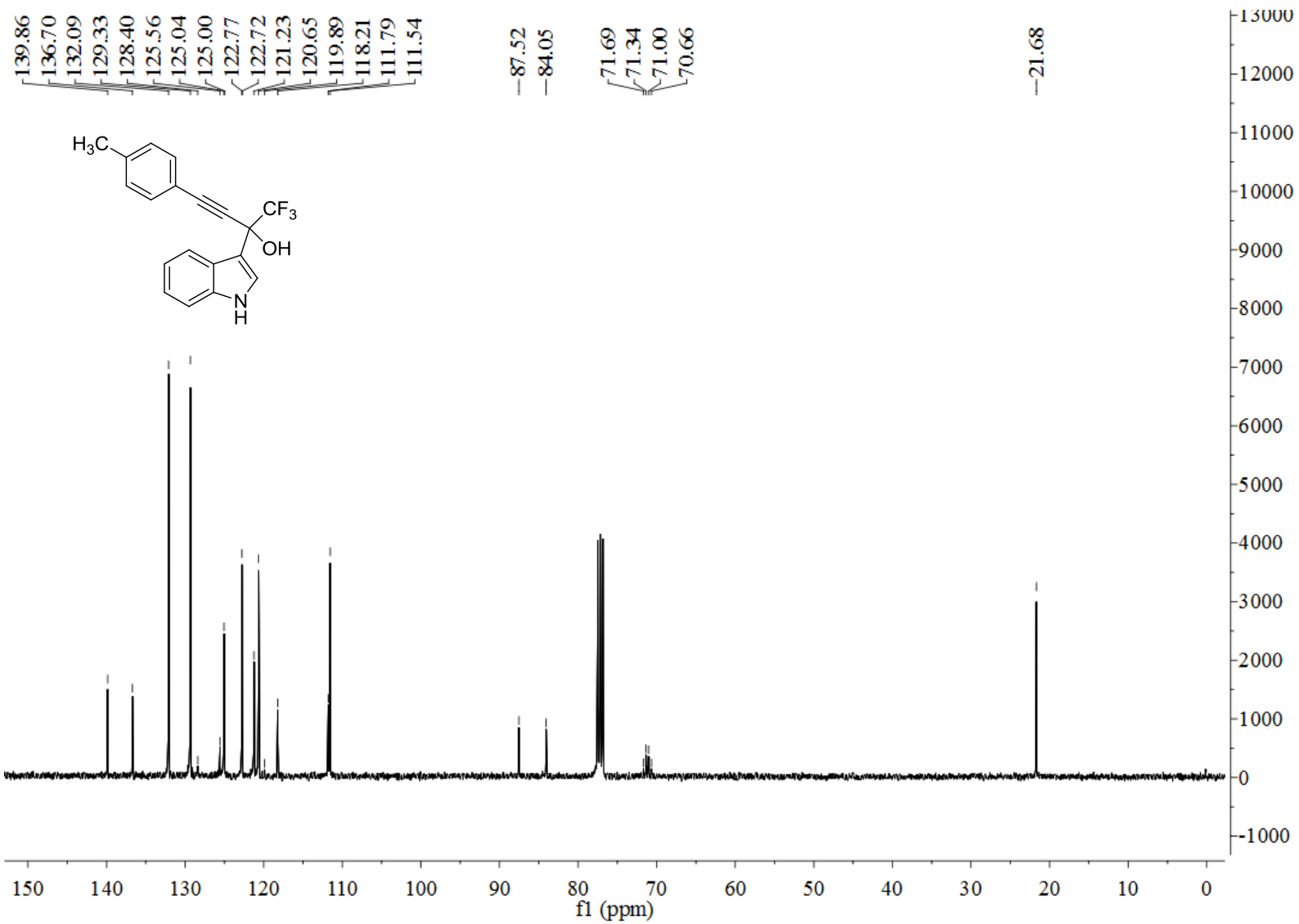

${ }^{19} \mathrm{~F} \mathrm{NMR}$ of compound 1 ( $\left(376 \mathrm{MHz}\right.$ in $\mathrm{CDCl}_{3}$ )

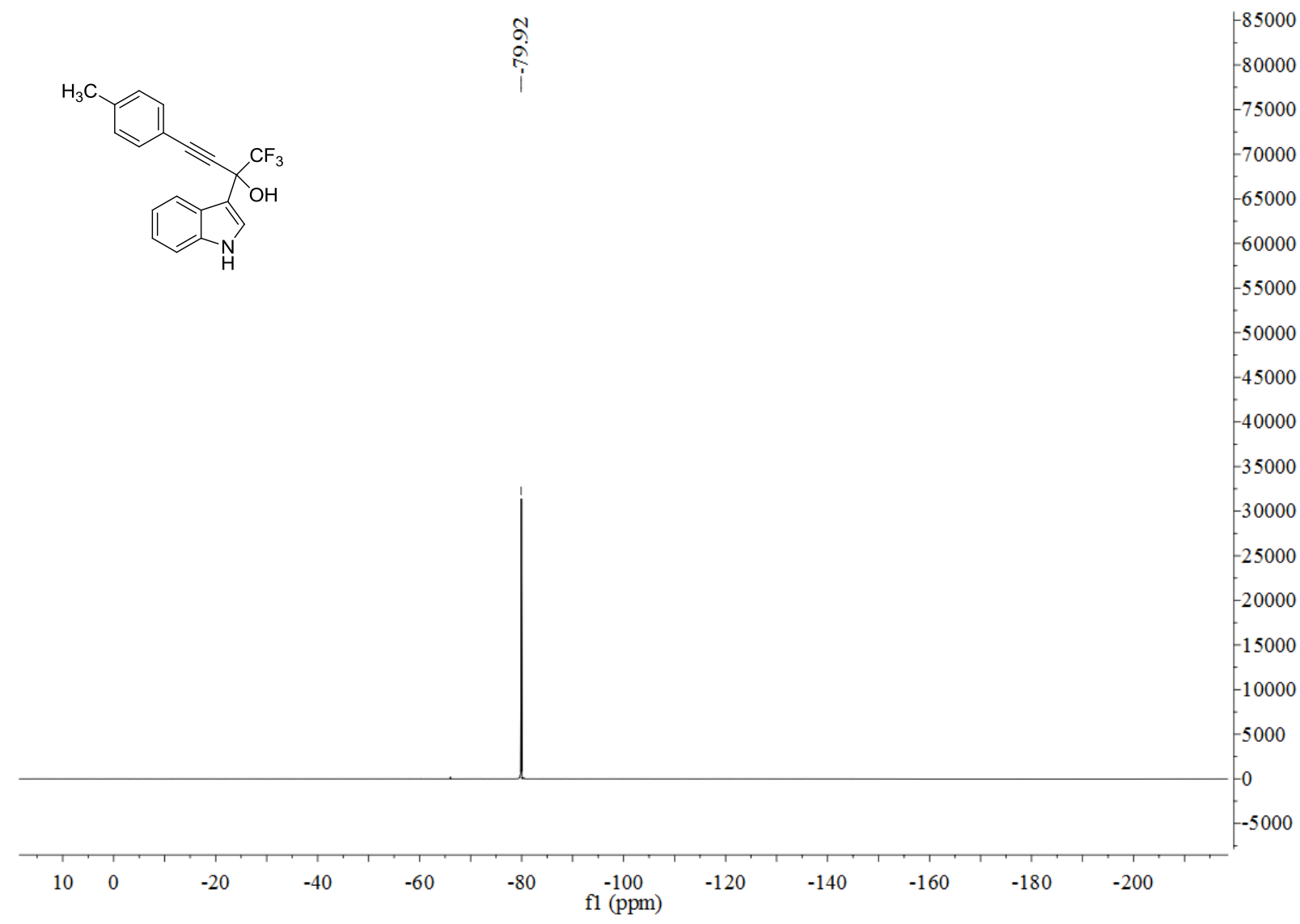


${ }^{1} \mathrm{H}$ NMR of compound $1 \mathrm{~m}\left(400 \mathrm{MHz}\right.$ in DMSO-d $\left.\mathrm{d}_{6}\right)$

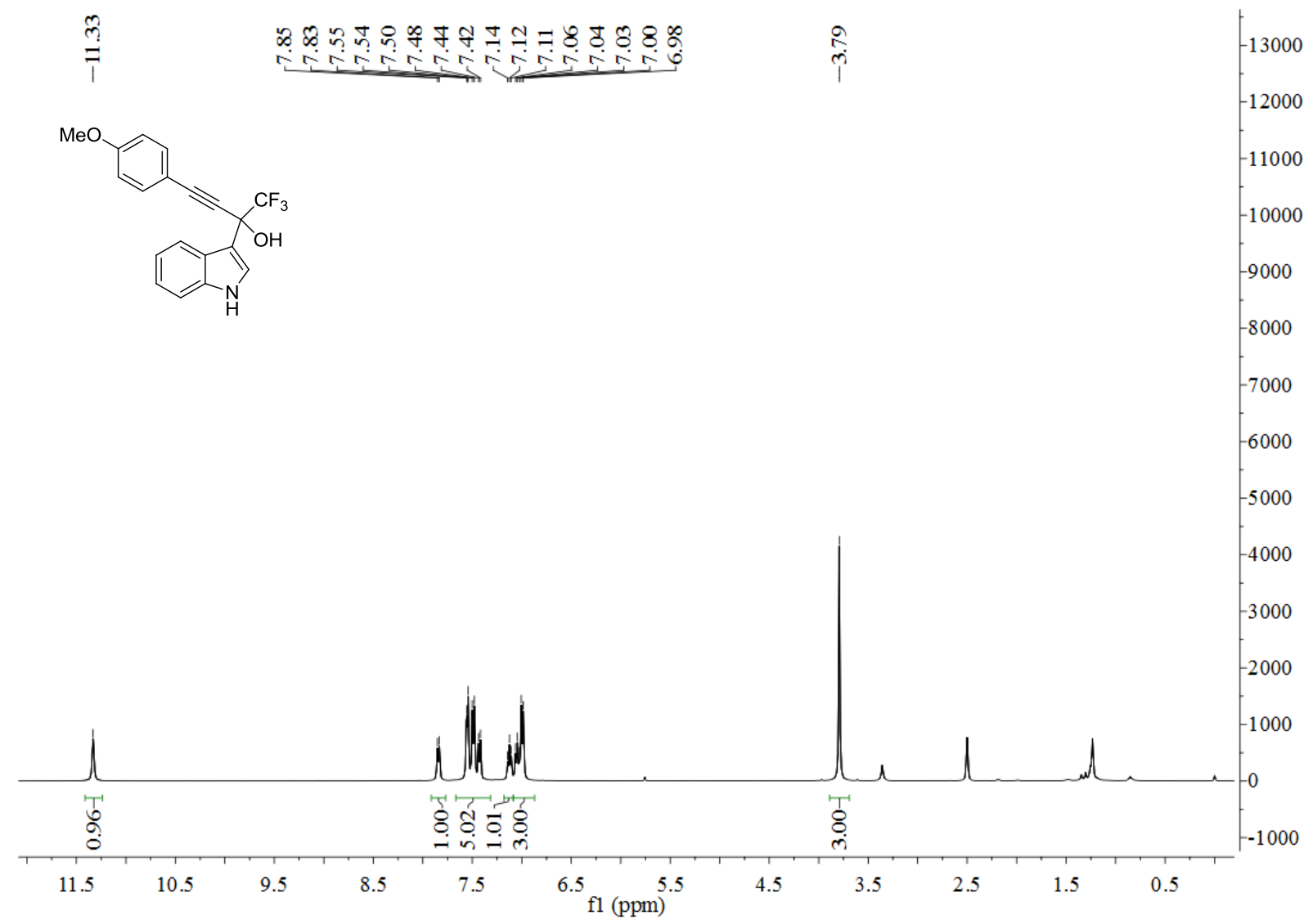

${ }^{13} \mathrm{C}$ NMR of compound $1 \mathrm{~m}$ (101 MHz in DMSO-d $\mathrm{d}_{6}$ )

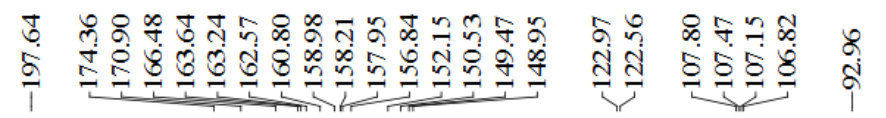<smiles>CC(=Cc1ccc(C)cc1)C(C)(C)C</smiles>

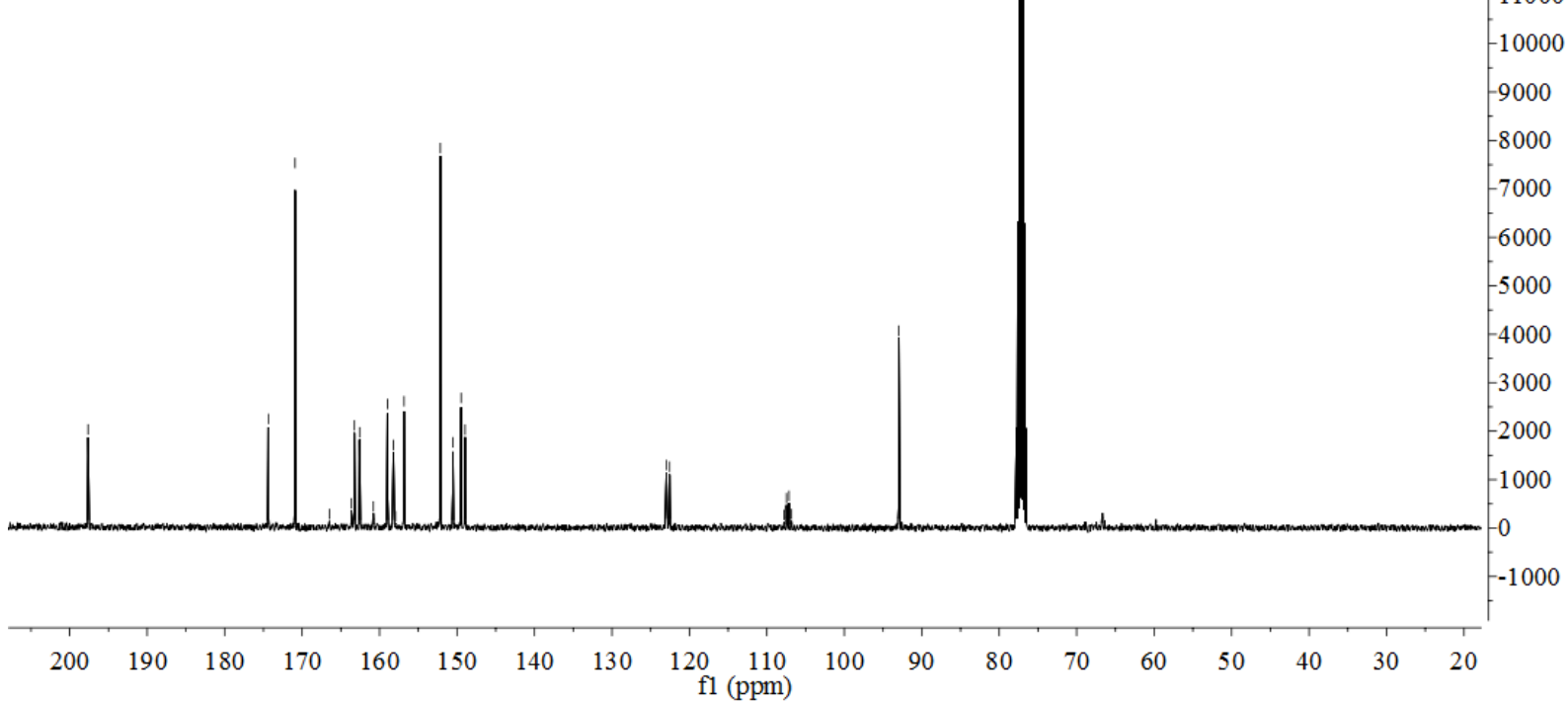


${ }^{19} \mathrm{~F}$ NMR of compound $1 \mathrm{~m}$ (376 MHz in DMSO-d $\left.\mathrm{d}_{6}\right)$

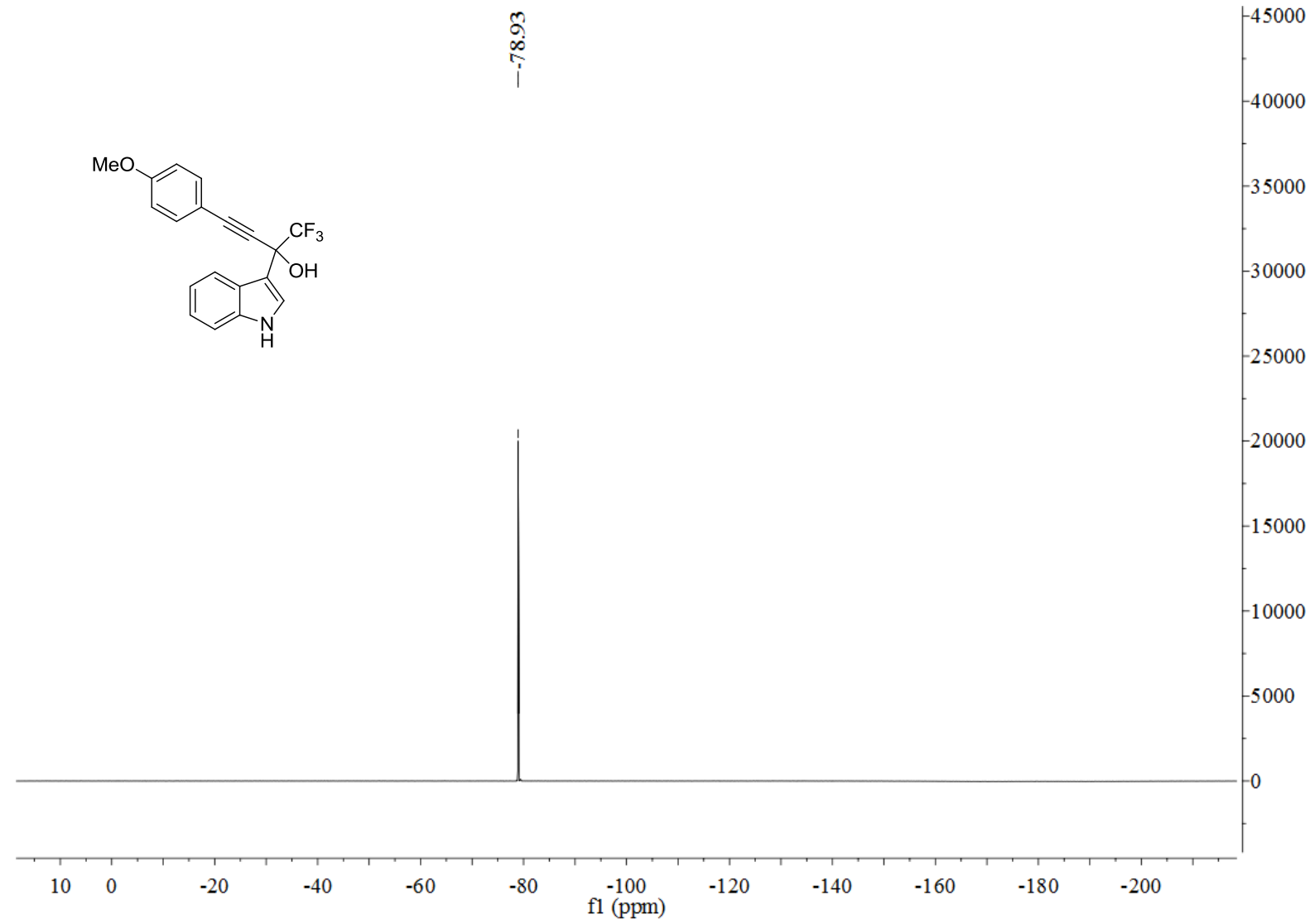

${ }^{1} \mathrm{H}$ NMR of compound $1 \mathrm{n}\left(400 \mathrm{MHz}\right.$ in $\left.\mathrm{CDCl}_{3}\right)$

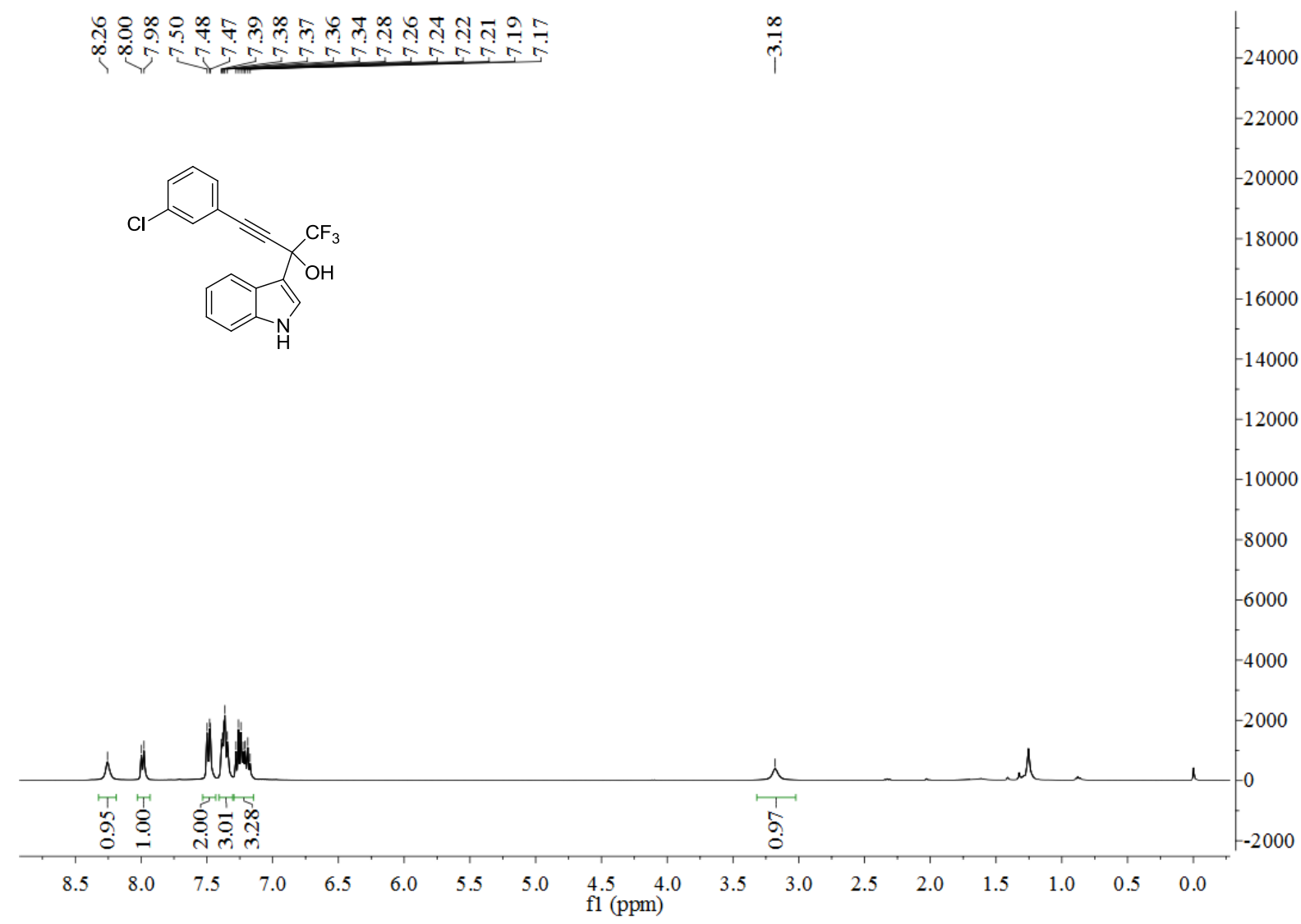


${ }^{13} \mathrm{C}$ NMR of compound 1n (101 $\mathrm{MHz}$ in $\mathrm{CDCl}_{3}$ )

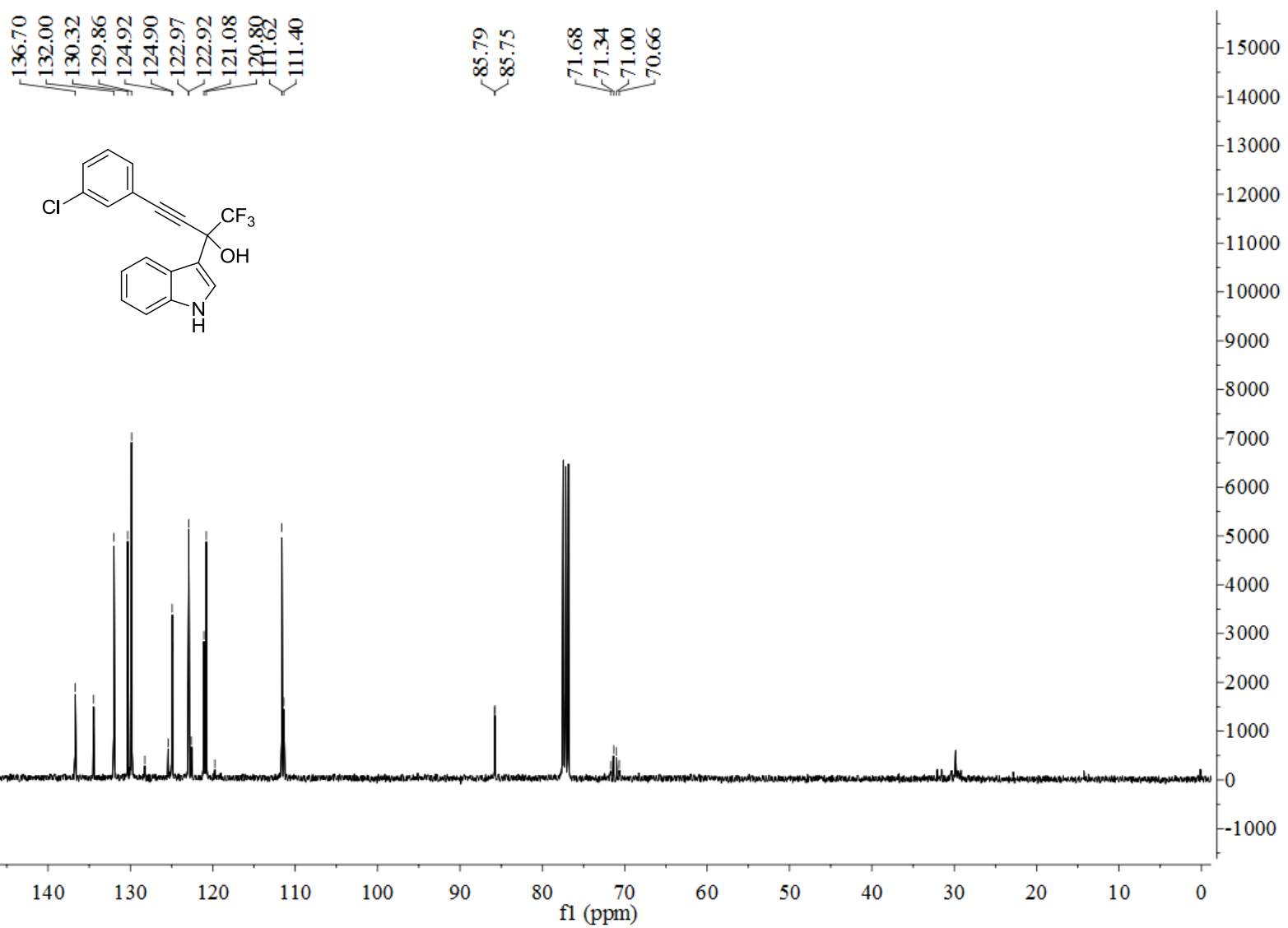

${ }^{19} \mathrm{~F} \mathrm{NMR}$ of compound $1 \mathrm{n}\left(376 \mathrm{MHz}\right.$ in $\mathrm{CDCl}_{3}$ )

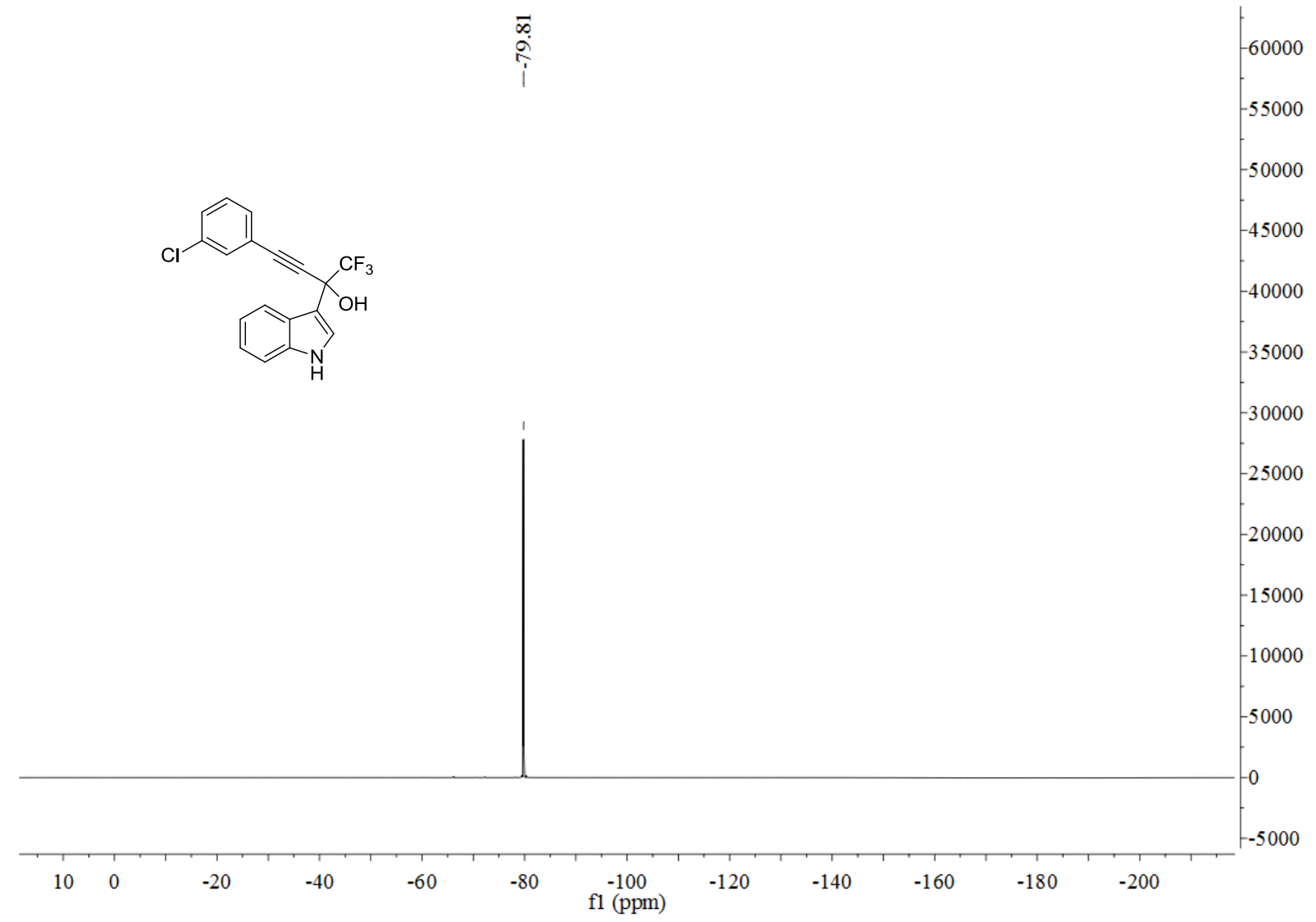


${ }^{1} \mathrm{H}$ NMR of compound $10\left(400 \mathrm{MHz}\right.$ in $\left.\mathrm{CDCl}_{3}\right)$

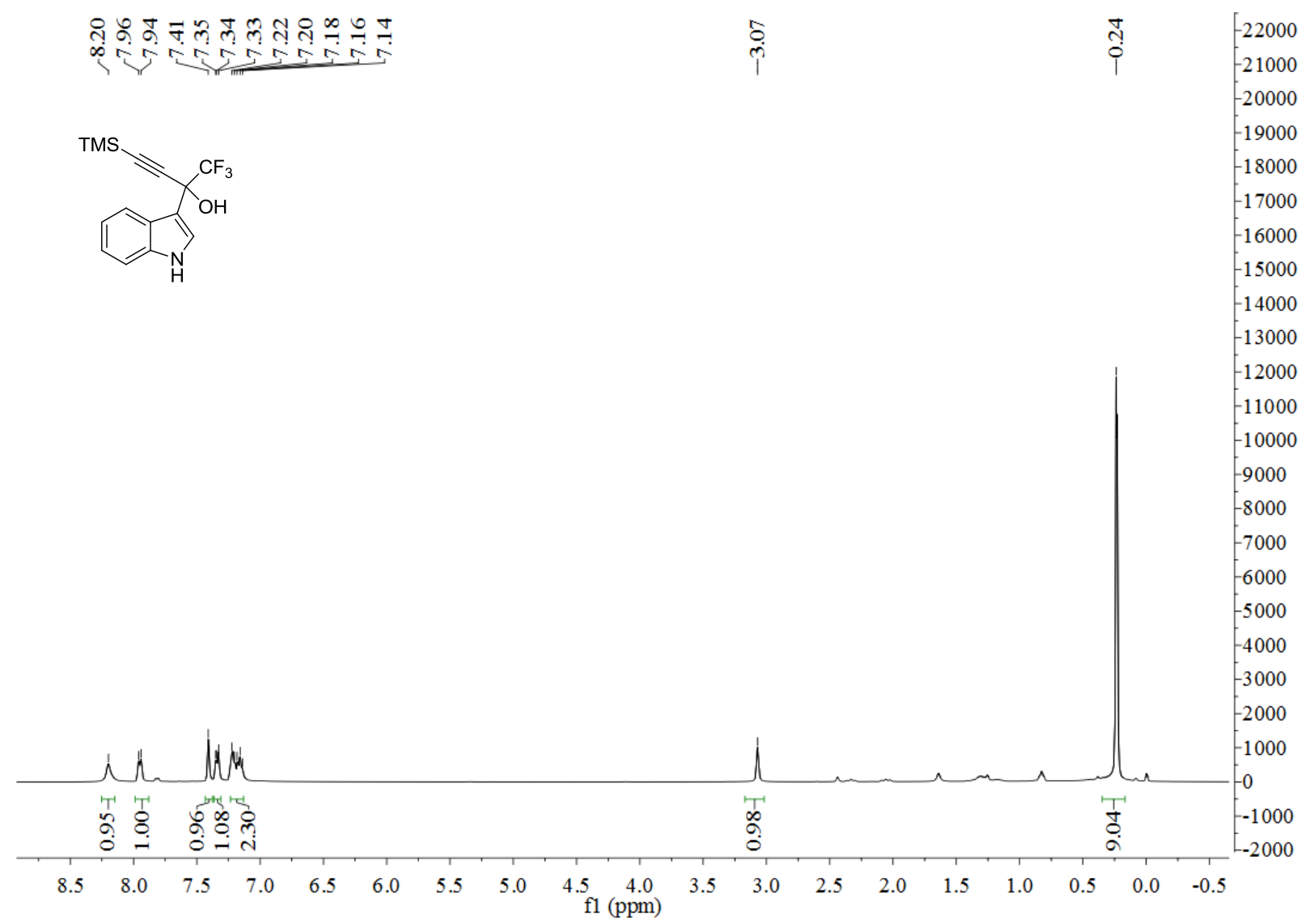

${ }^{13} \mathrm{C}$ NMR of compound $10\left(101 \mathrm{MHz}\right.$ in $\left.\mathrm{CDCl}_{3}\right)$
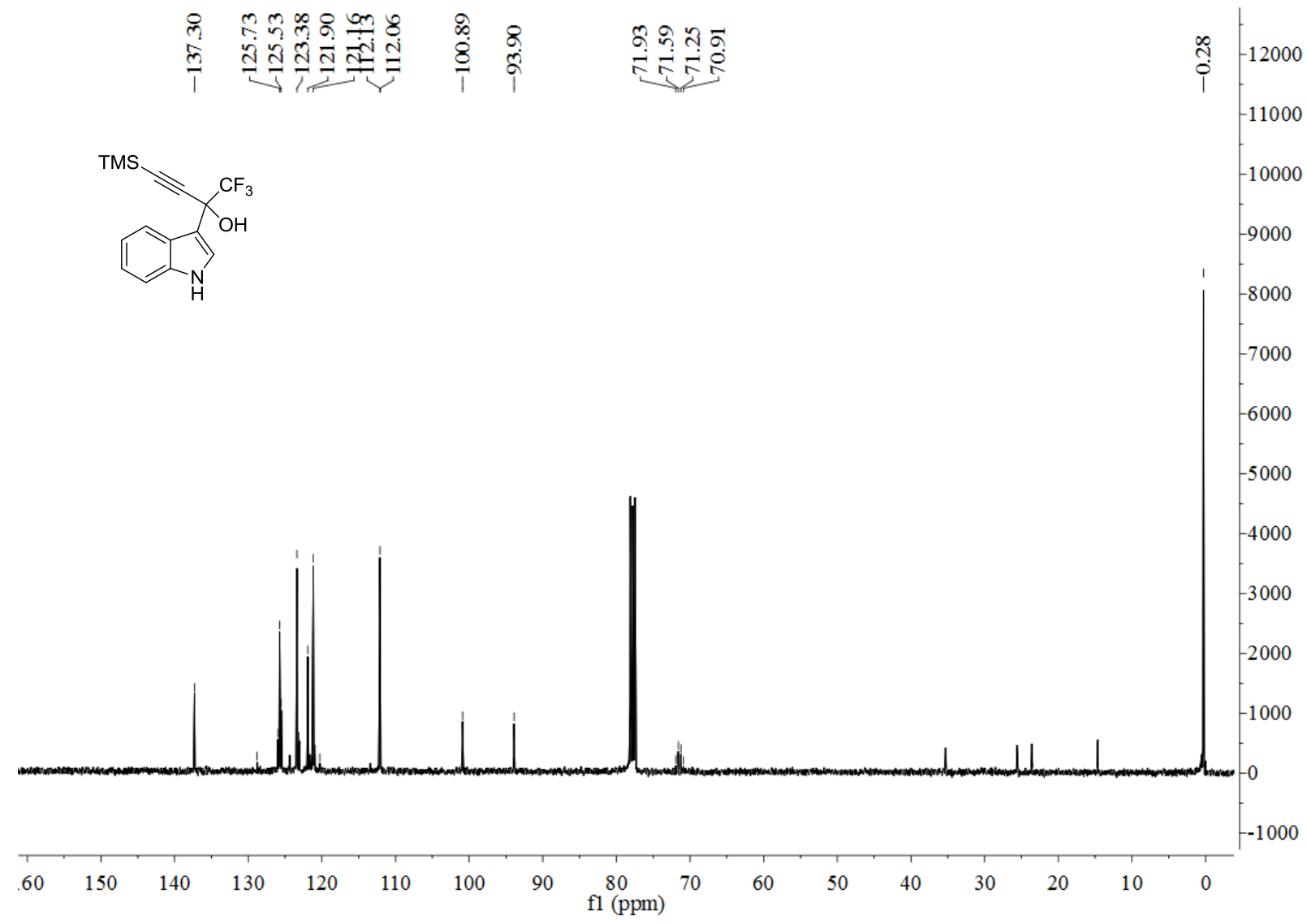
${ }^{19} \mathrm{~F}$ NMR of compound 10 (376 $\mathrm{MHz}$ in $\mathrm{CDCl}_{3}$ )

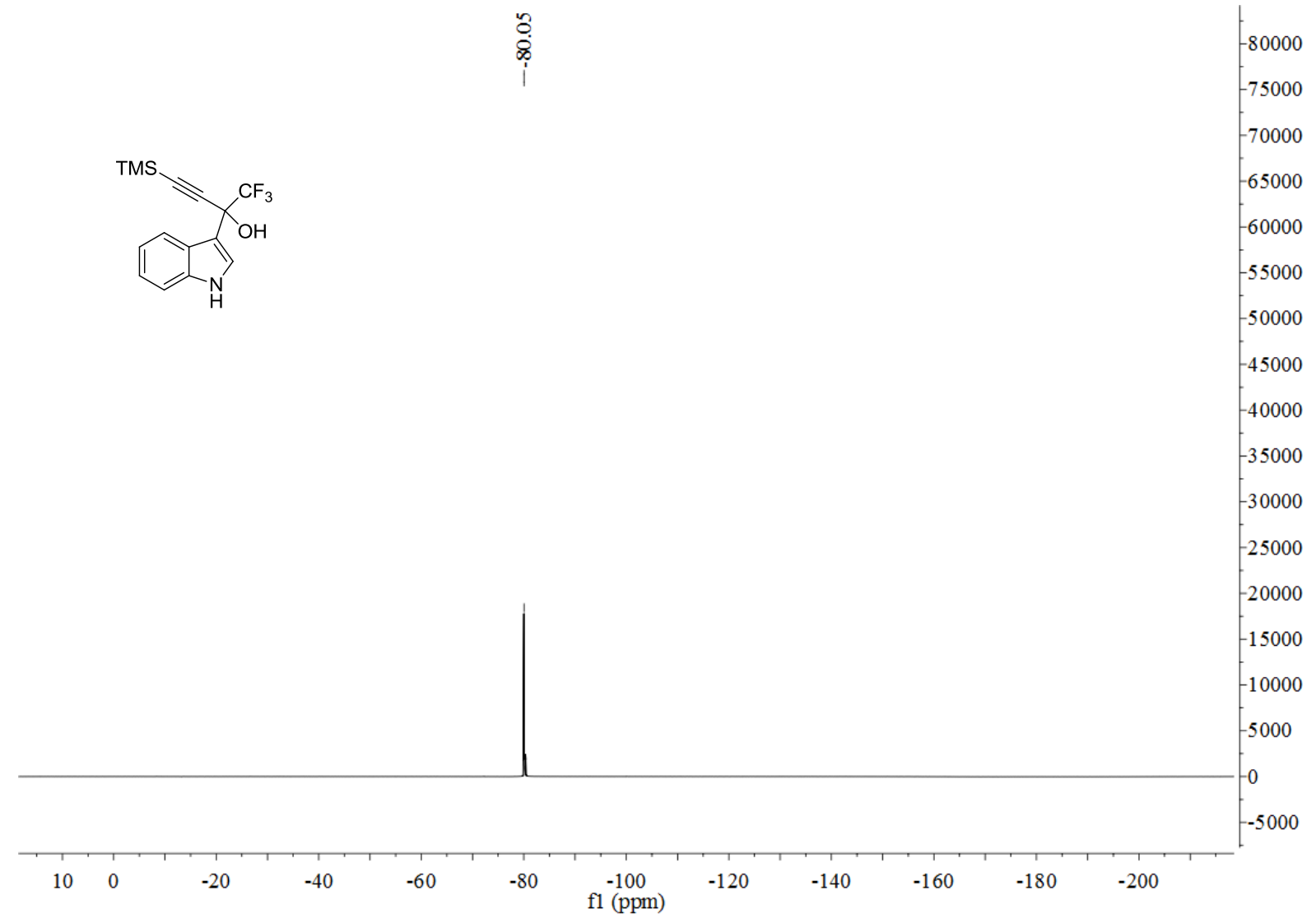

${ }^{1} \mathrm{H}$ NMR of compound $1 \mathrm{p}\left(400 \mathrm{MHz}\right.$ in $\mathrm{CDCl}_{3}$ )

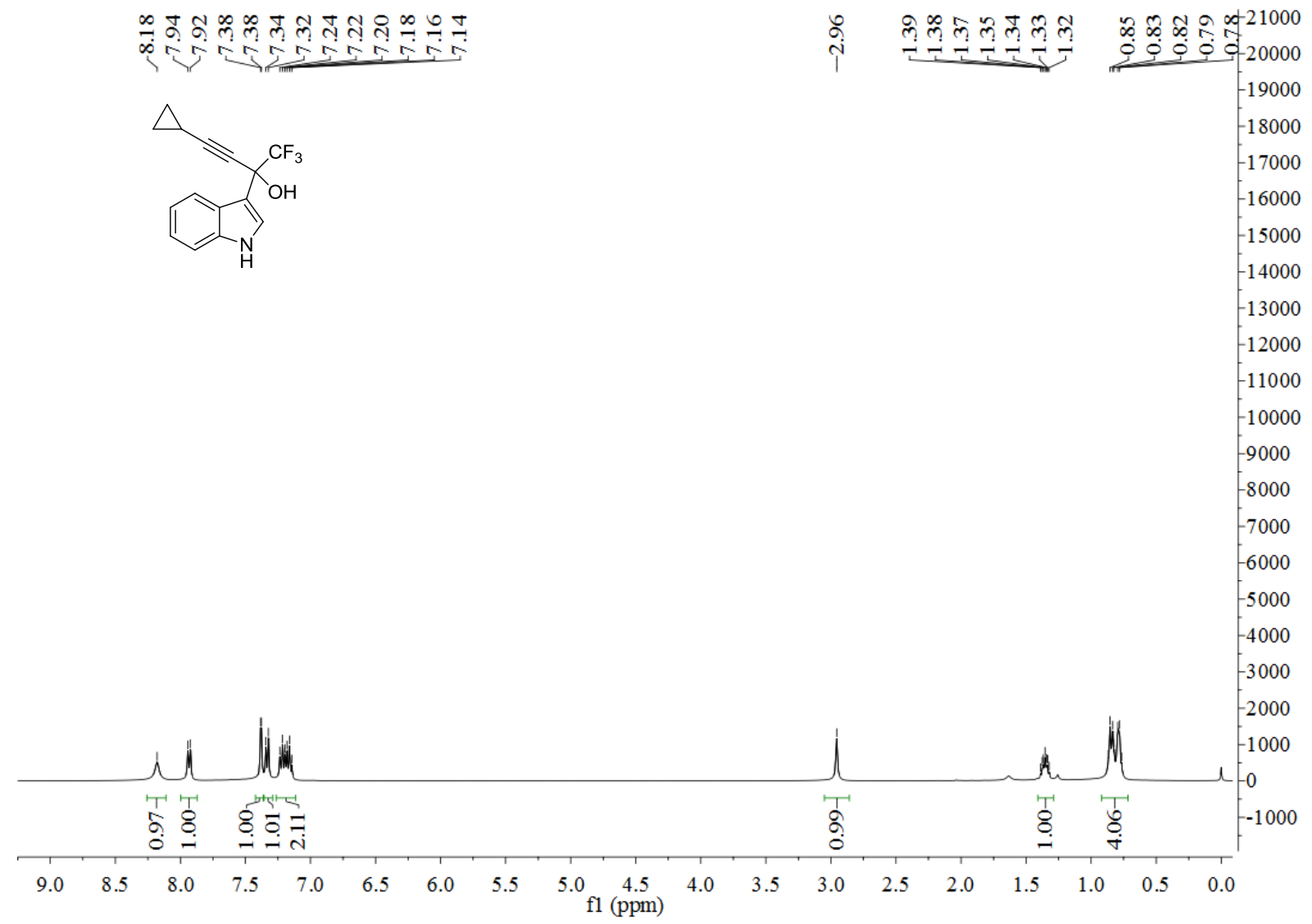


${ }^{13} \mathrm{C}$ NMR of compound $1 \mathrm{p}\left(101 \mathrm{MHz}\right.$ in $\mathrm{CDCl}_{3}$ )

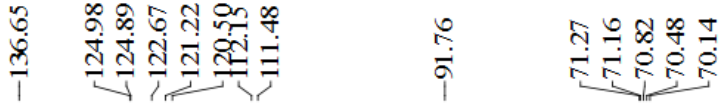

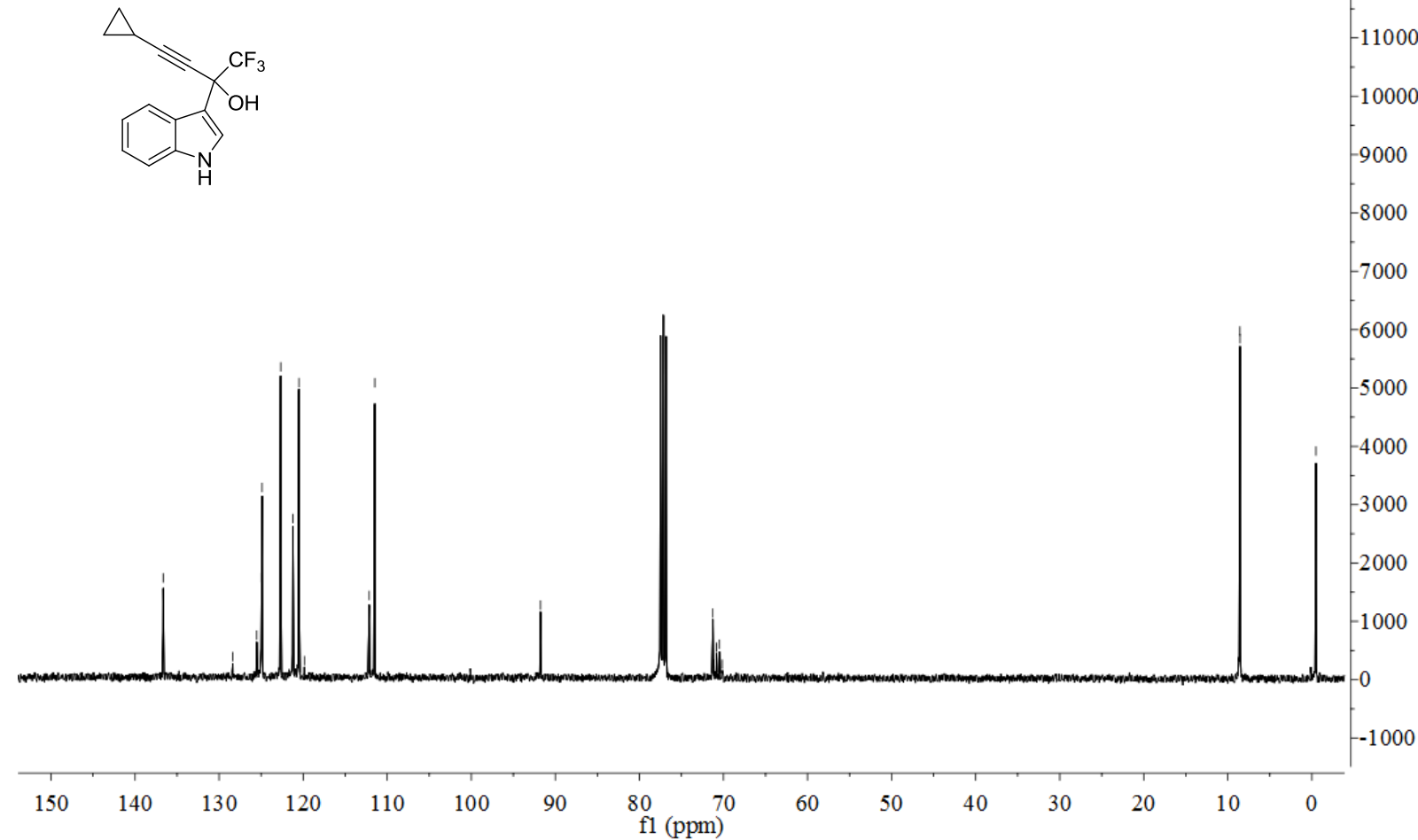

${ }^{19} \mathrm{~F} \mathrm{NMR}$ of compound $1 \mathrm{p}$ (376 $\mathrm{MHz}$ in $\mathrm{CDCl}_{3}$ )

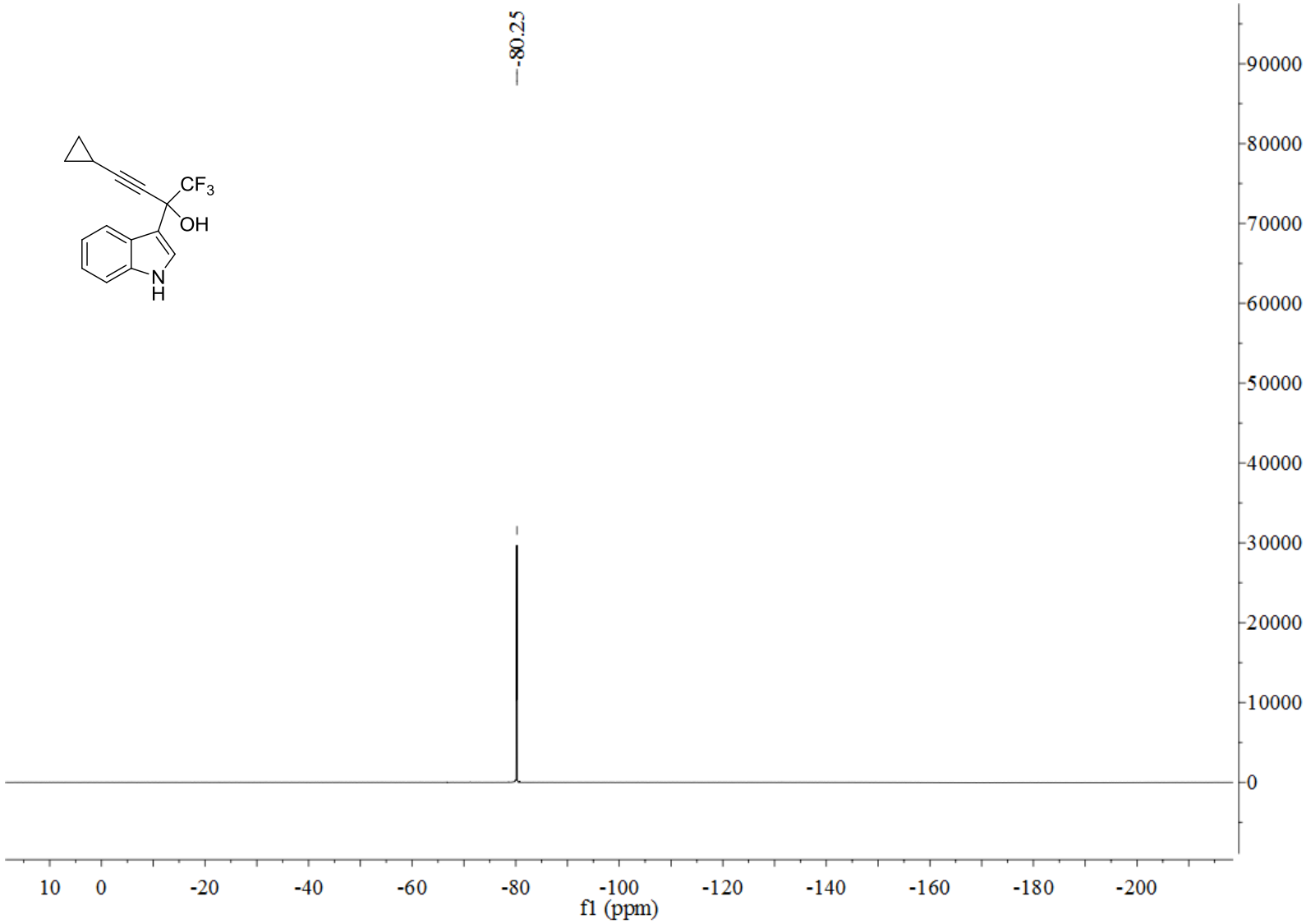


${ }^{1} \mathrm{H}$ NMR of compound $1 \mathrm{q}\left(400 \mathrm{MHz}\right.$ in DMSO-d $\left.{ }_{6}\right)$

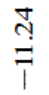

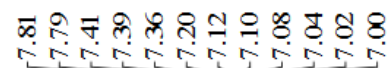

CHe

पivinivitis

$\begin{array}{ll}\text { T) } & -21000 \\ \top & -20000 \\ & -19000 \\ & -18000\end{array}$

17000

16000

$-15000$

$-14000$

$-13000$

$-12000$

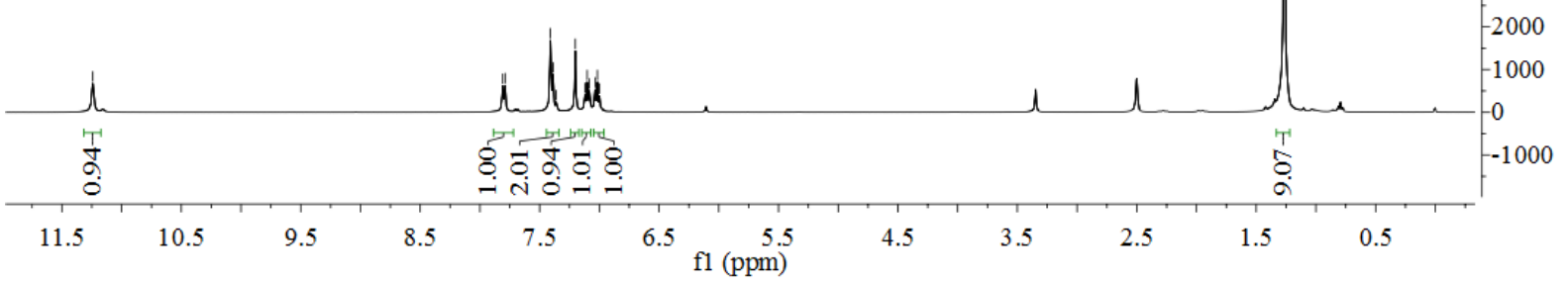

${ }^{13} \mathrm{C}$ NMR of compound 1q (101 MHz in DMSO-d 6 )

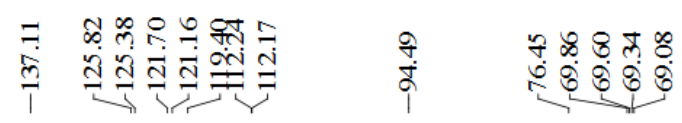<smiles></smiles>

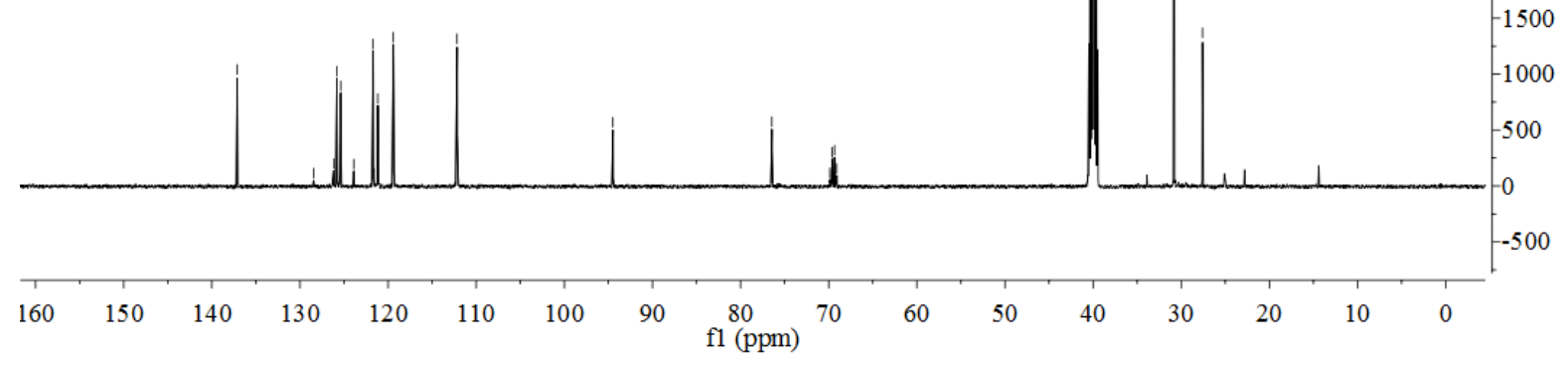


${ }^{19} \mathrm{~F}$ NMR of compound 1q (376 MHz in DMSO-d ( $\left._{6}\right)$

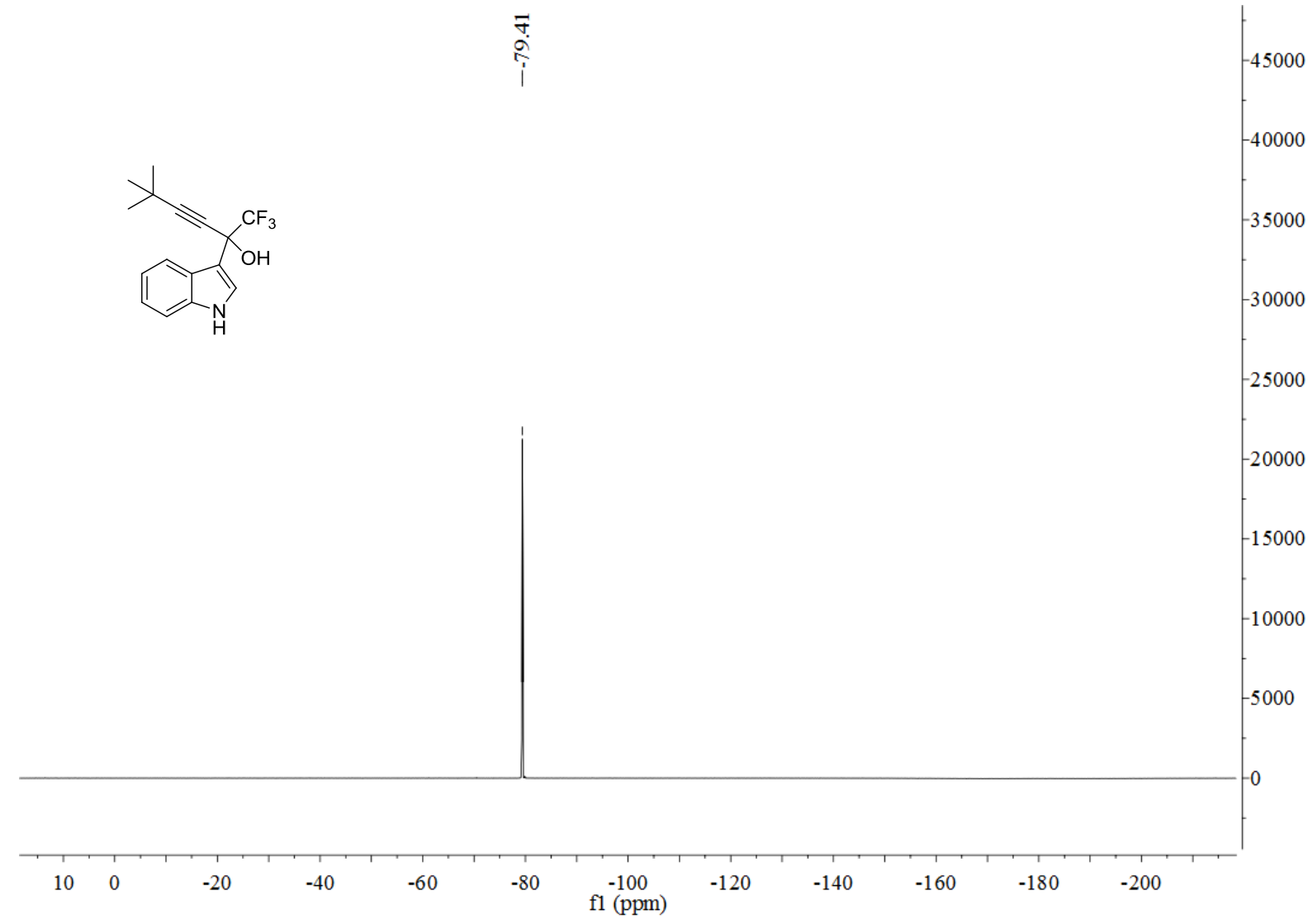

${ }^{1} \mathrm{H}$ NMR of compound $1 \mathrm{r}\left(400 \mathrm{MHz}\right.$ in $\left.\mathrm{CDCl}_{3}\right)$

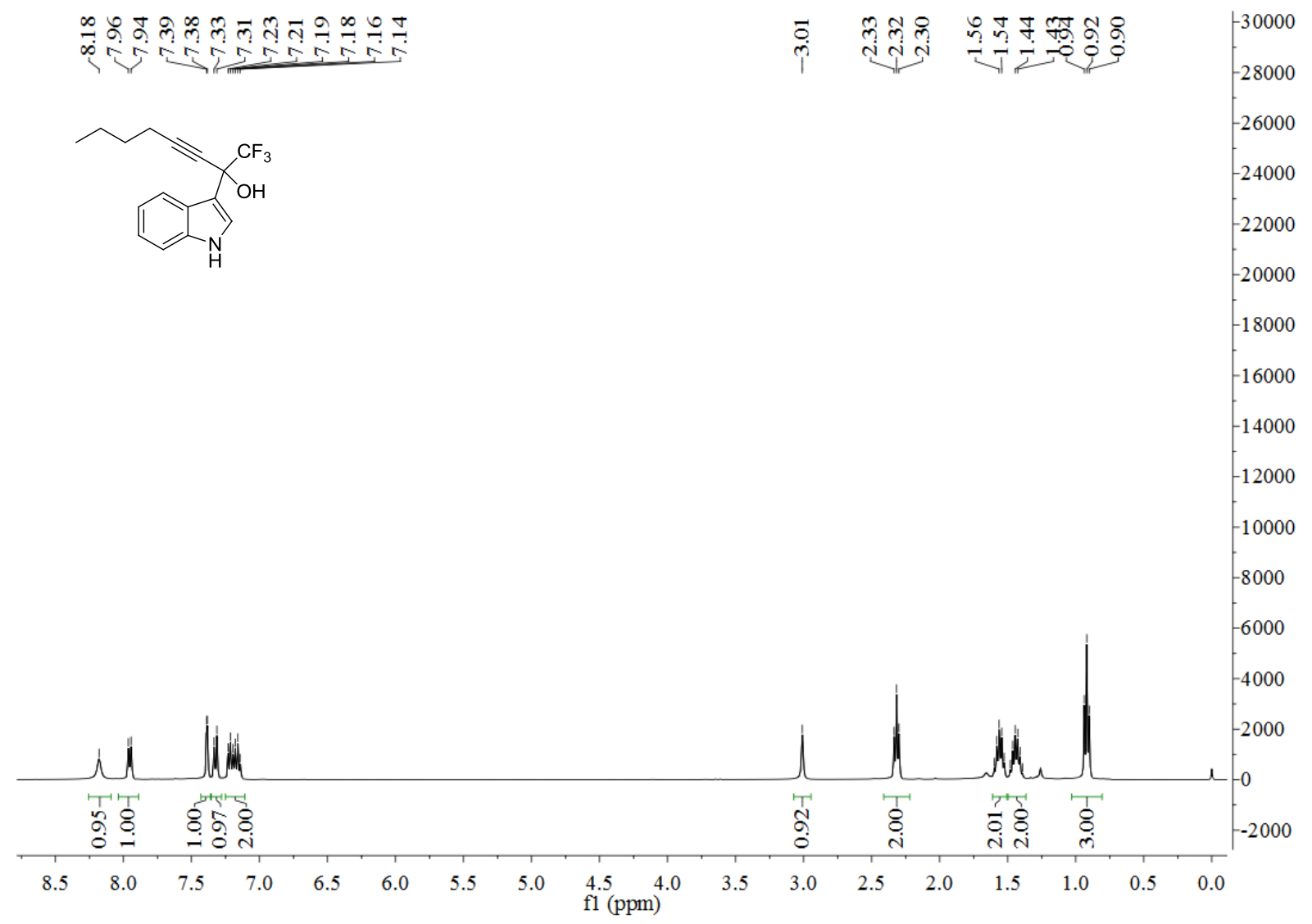


${ }^{13} \mathrm{C}$ NMR of compound $1 \mathrm{r}\left(101 \mathrm{MHz}\right.$ in $\left.\mathrm{CDCl}_{3}\right)$

我

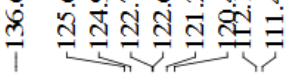

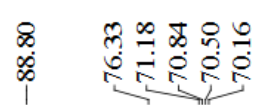

के
के

16000

15000

14000

$\sim \mathrm{CF}_{3}$

$1 \mathrm{OH}^{\mathrm{OH}}$

$\sim \mathrm{N}$

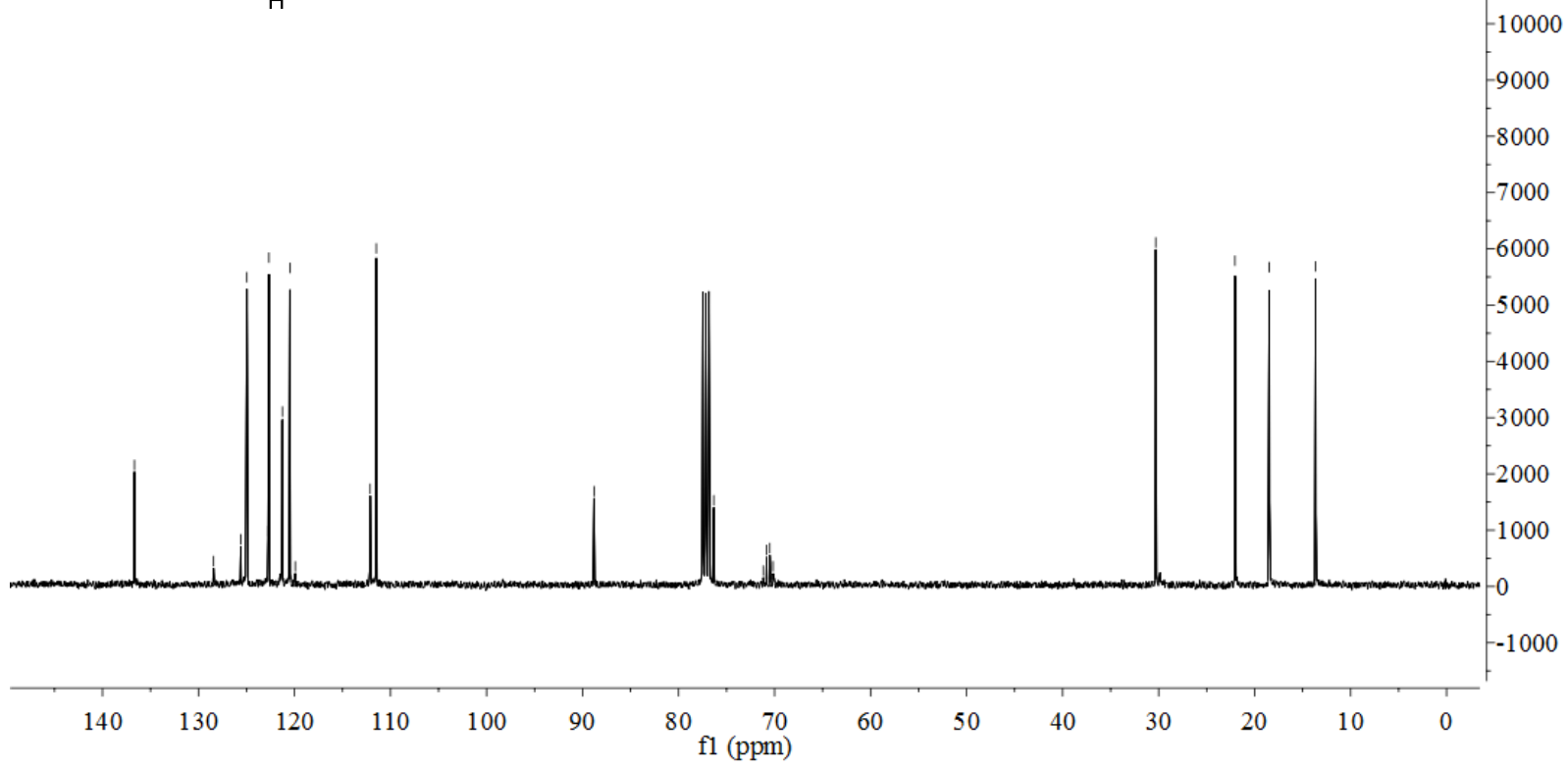

${ }^{19} \mathrm{~F} \mathrm{NMR}$ of compound $\operatorname{1r}\left(376 \mathrm{MHz}\right.$ in $\left.\mathrm{CDCl}_{3}\right)$

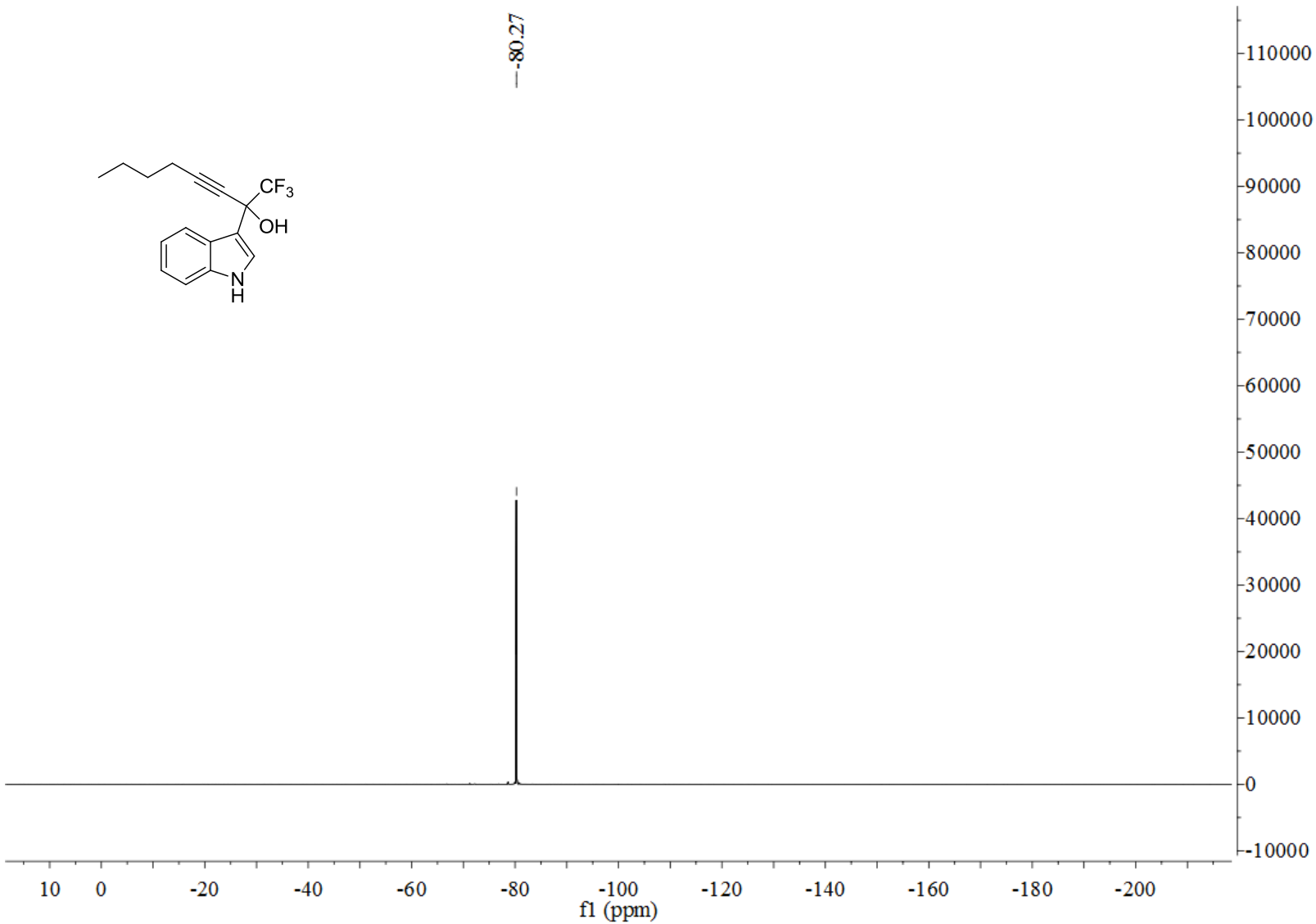


${ }^{1} \mathrm{H}$ NMR of compound $8\left(400 \mathrm{MHz}\right.$ in $\mathrm{CDCl}_{3}$ )

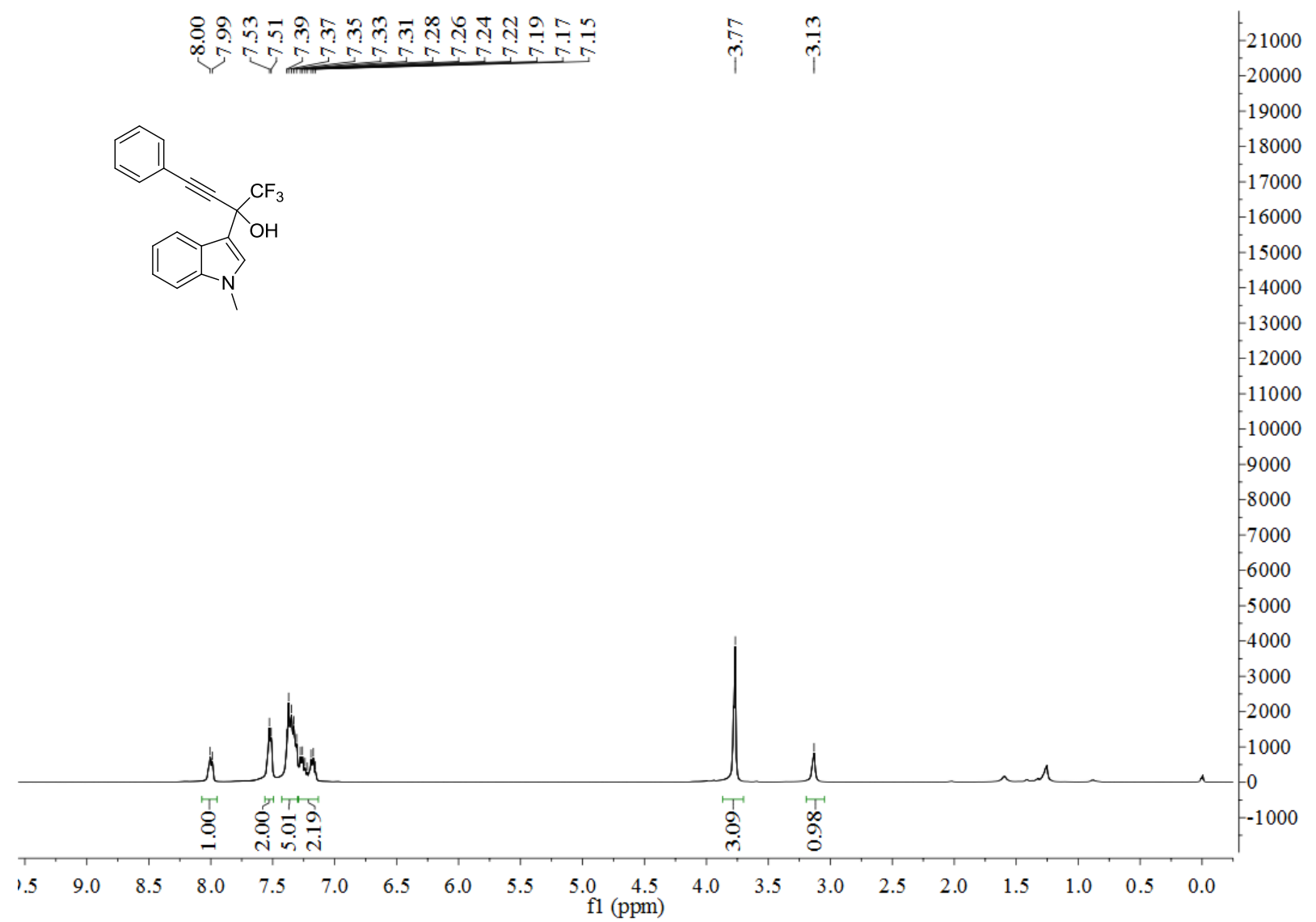

${ }^{13} \mathrm{C}$ NMR of compound 8 (101 $\mathrm{MHz}$ in $\mathrm{CDCl}_{3}$ )

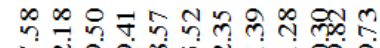

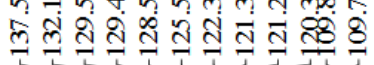

(n)

$\begin{array}{ll}9 \infty & 0 \\ 0 & 1\end{array}$

$\stackrel{\text { กิ }}{1}$

$-9000$

800

$\mid$

5000

4000

$-3000$

$-2000$

$-1000$

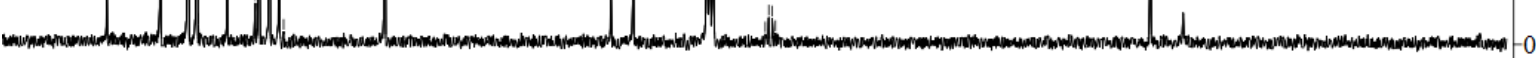

140

$130 \quad 120$

110

100

90

80

f1 (ppm)

60

$50 \quad 40$

30

20 


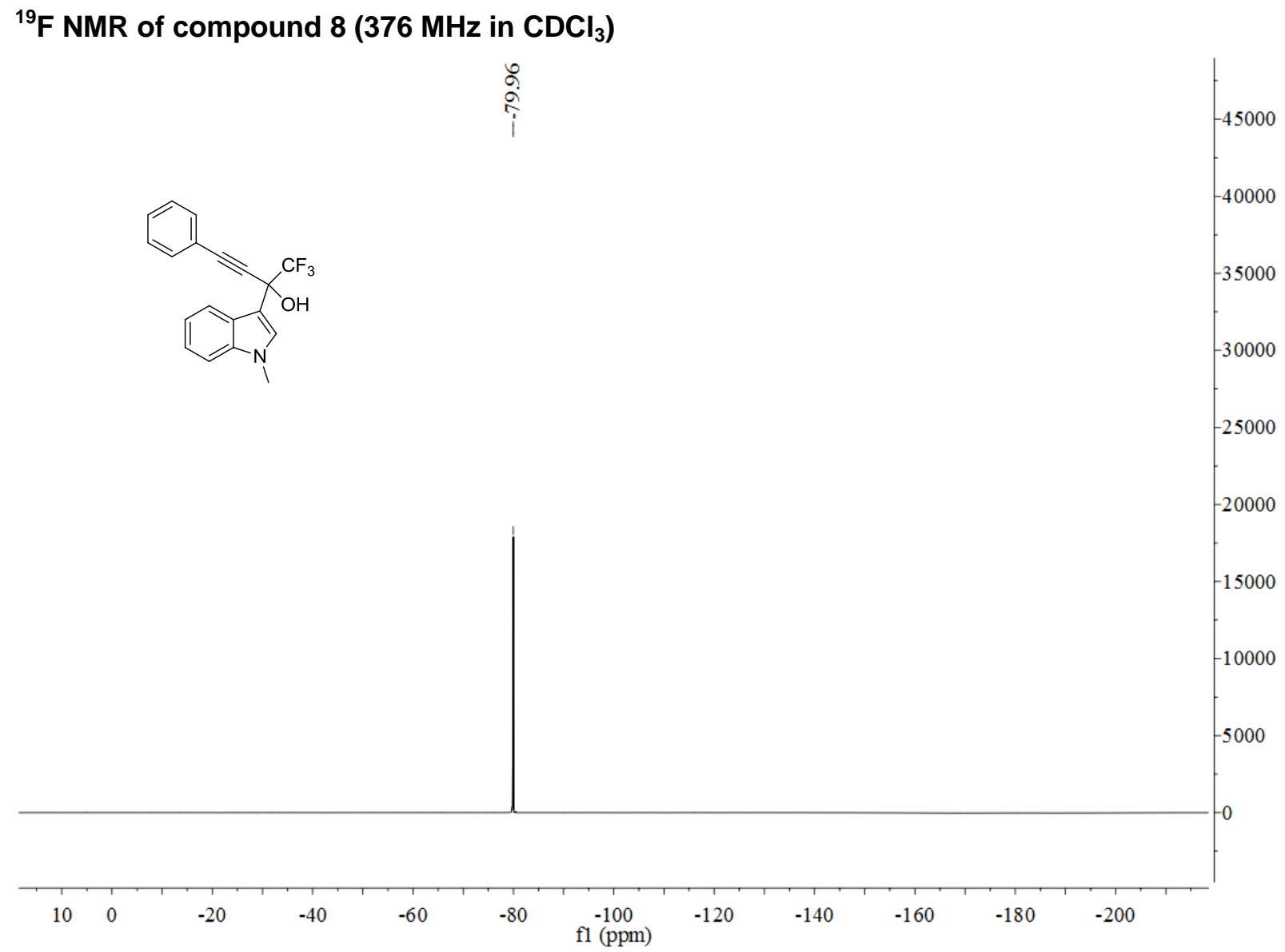

${ }^{1} \mathrm{H}$ NMR of compound $1 \mathrm{aa}\left(400 \mathrm{MHz}\right.$ in $\left.\mathrm{CDCl}_{3}\right)$

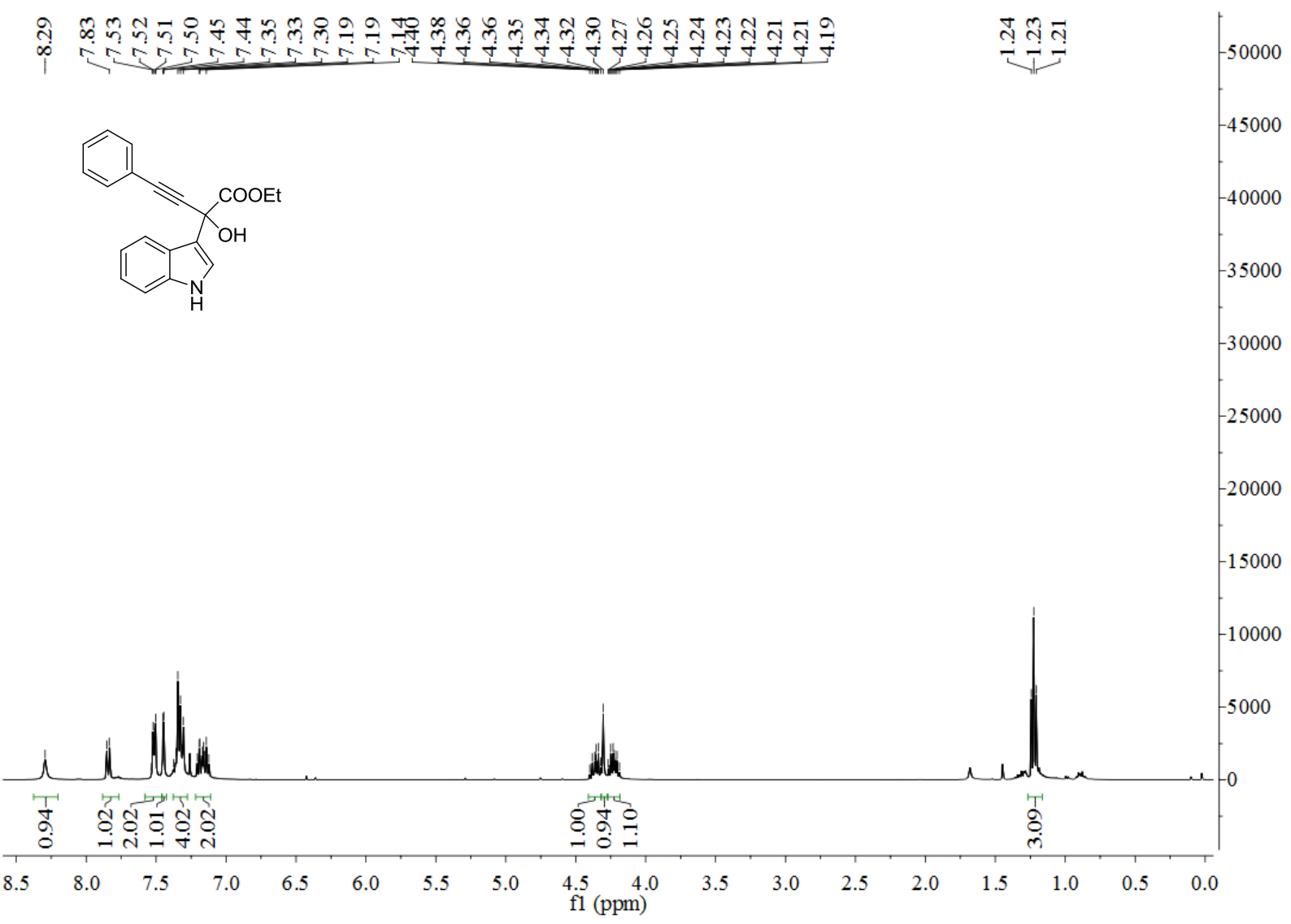


${ }^{13} \mathrm{C}$ NMR of compound $1 \mathrm{aa}\left(126 \mathrm{MHz}\right.$ in $\mathrm{CDCl}_{3}$ )

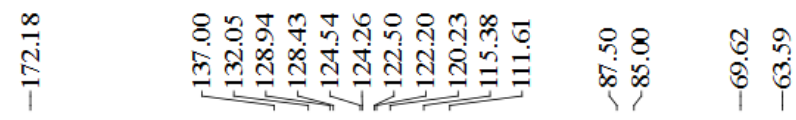

के

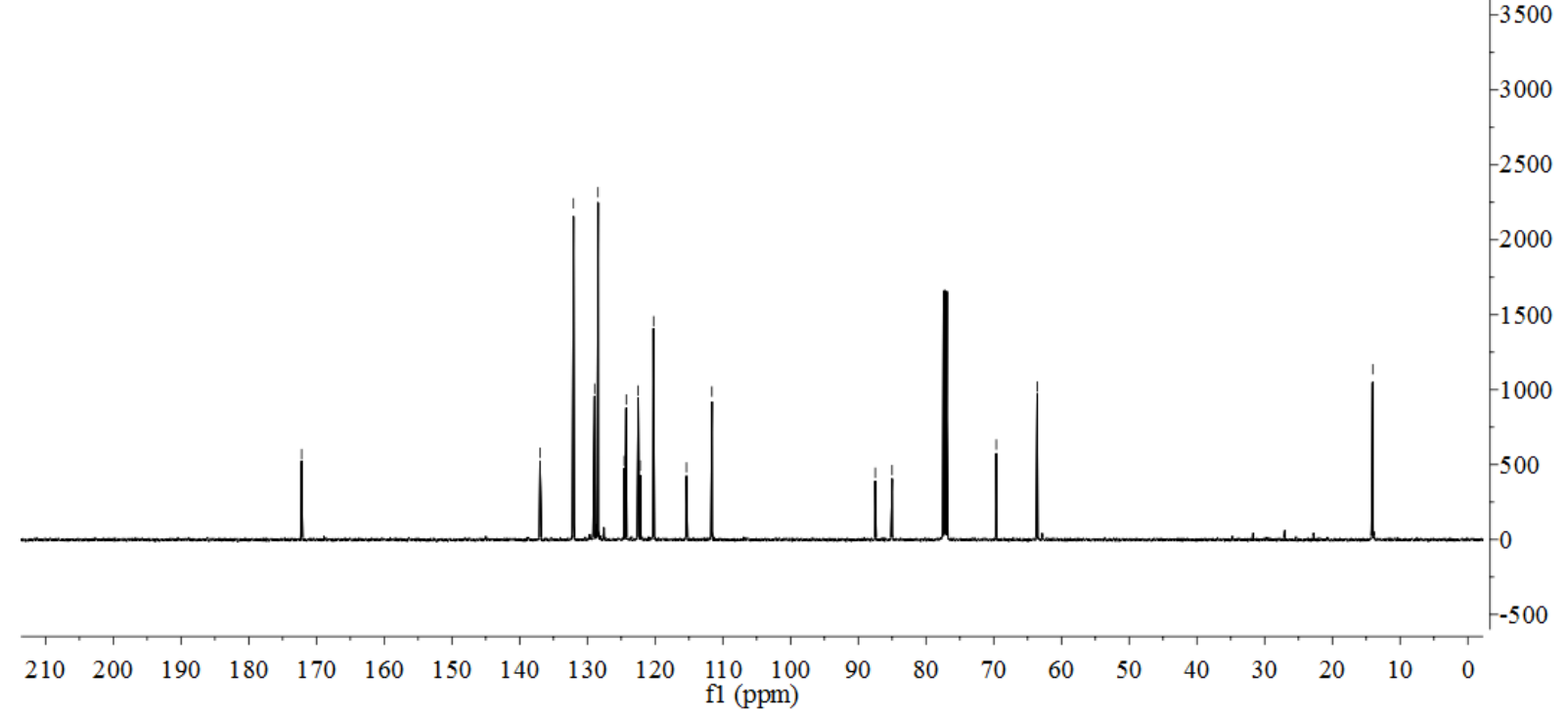

\section{${ }^{1} \mathrm{H}$ NMR of compound $3 \mathrm{a}\left(500 \mathrm{MHz}\right.$ in $\left.\mathrm{CDCl}_{3}\right)$}

लि ते त्र<smiles>Oc1ccc(C(=CC(c2ccccc2)C(F)(F)F)c2c[nH]c3ccccc23)c2ccccc12</smiles>

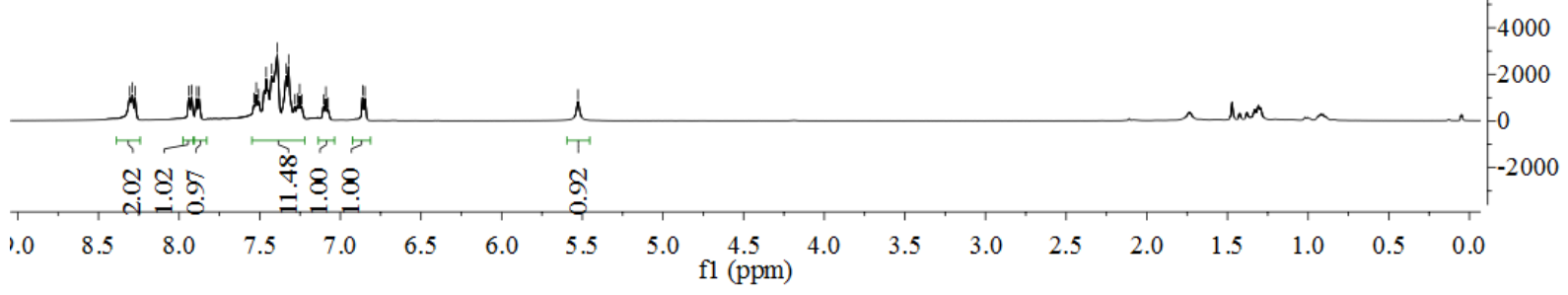


${ }^{13} \mathrm{C}$ NMR of compound $3 \mathrm{a}\left(126 \mathrm{MHz}\right.$ in $\mathrm{CDCl}_{3}$ )

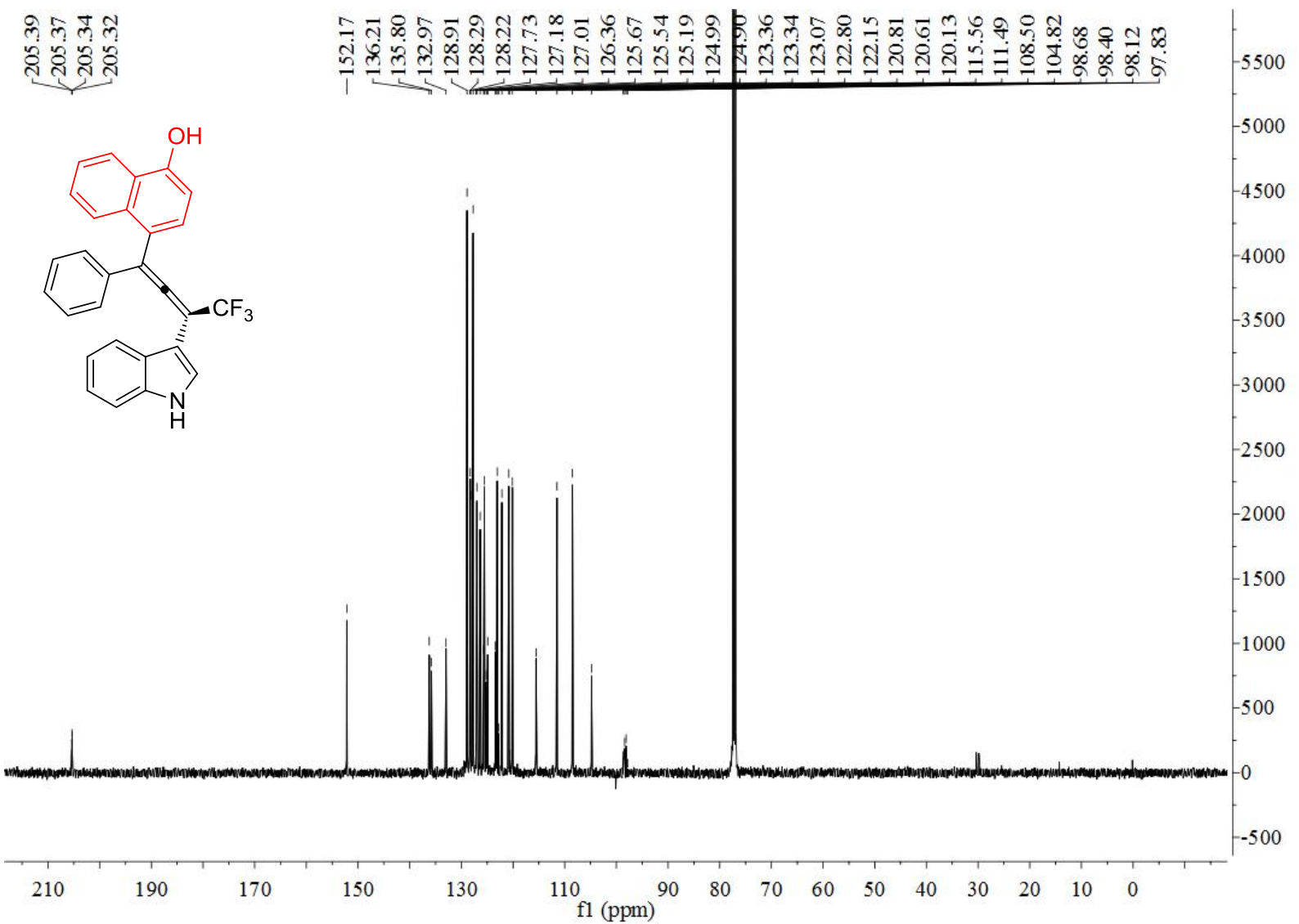

${ }^{19} \mathrm{~F} \mathrm{NMR}$ of compound $3 \mathrm{a}\left(376 \mathrm{MHz}\right.$ in $\mathrm{CDCl}_{3}$ )

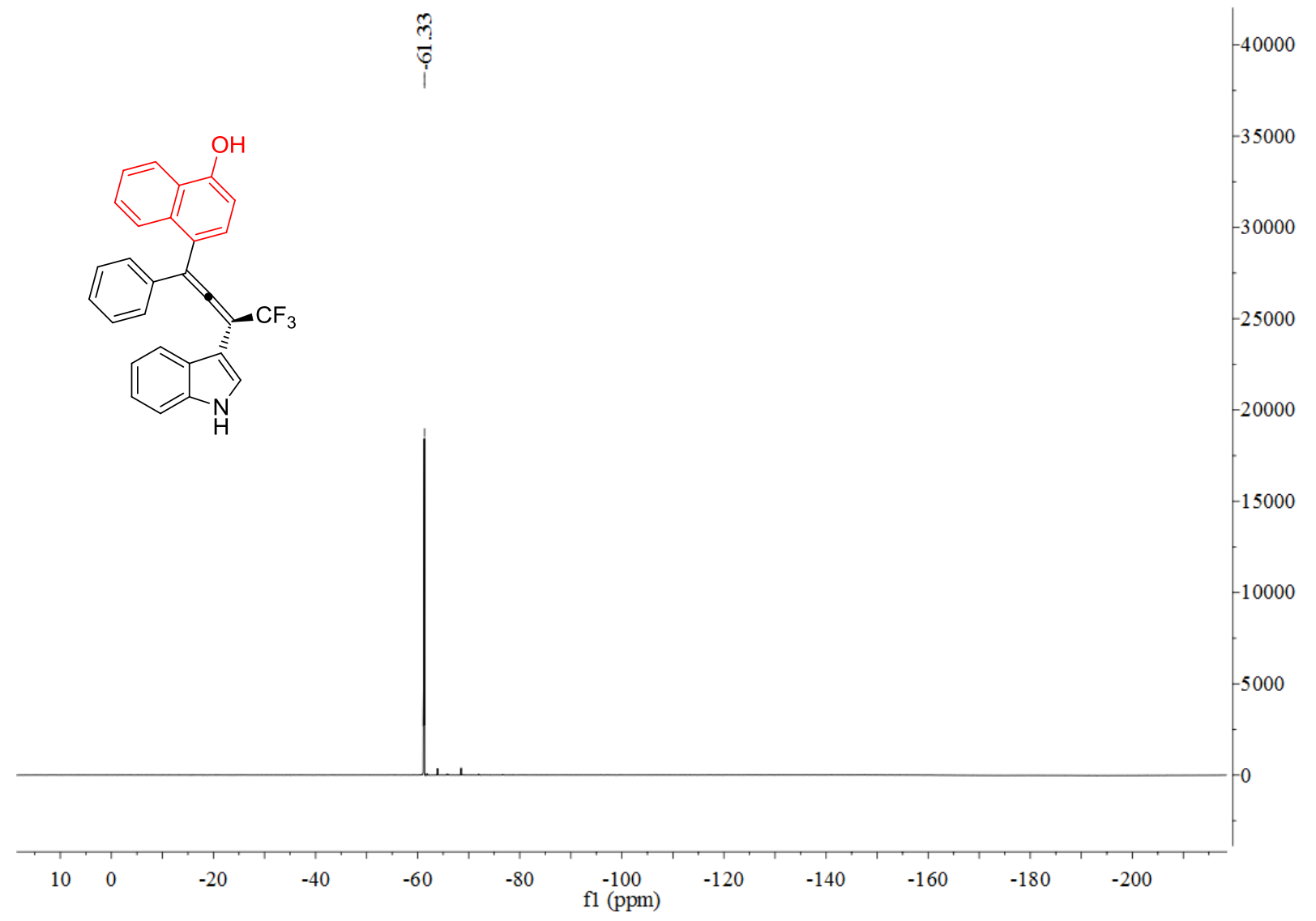


${ }^{1} \mathrm{H}$ NMR of compound $3 \mathrm{~b}\left(400 \mathrm{MHz}\right.$ in $\left.\mathrm{CDCl}_{3}\right)$

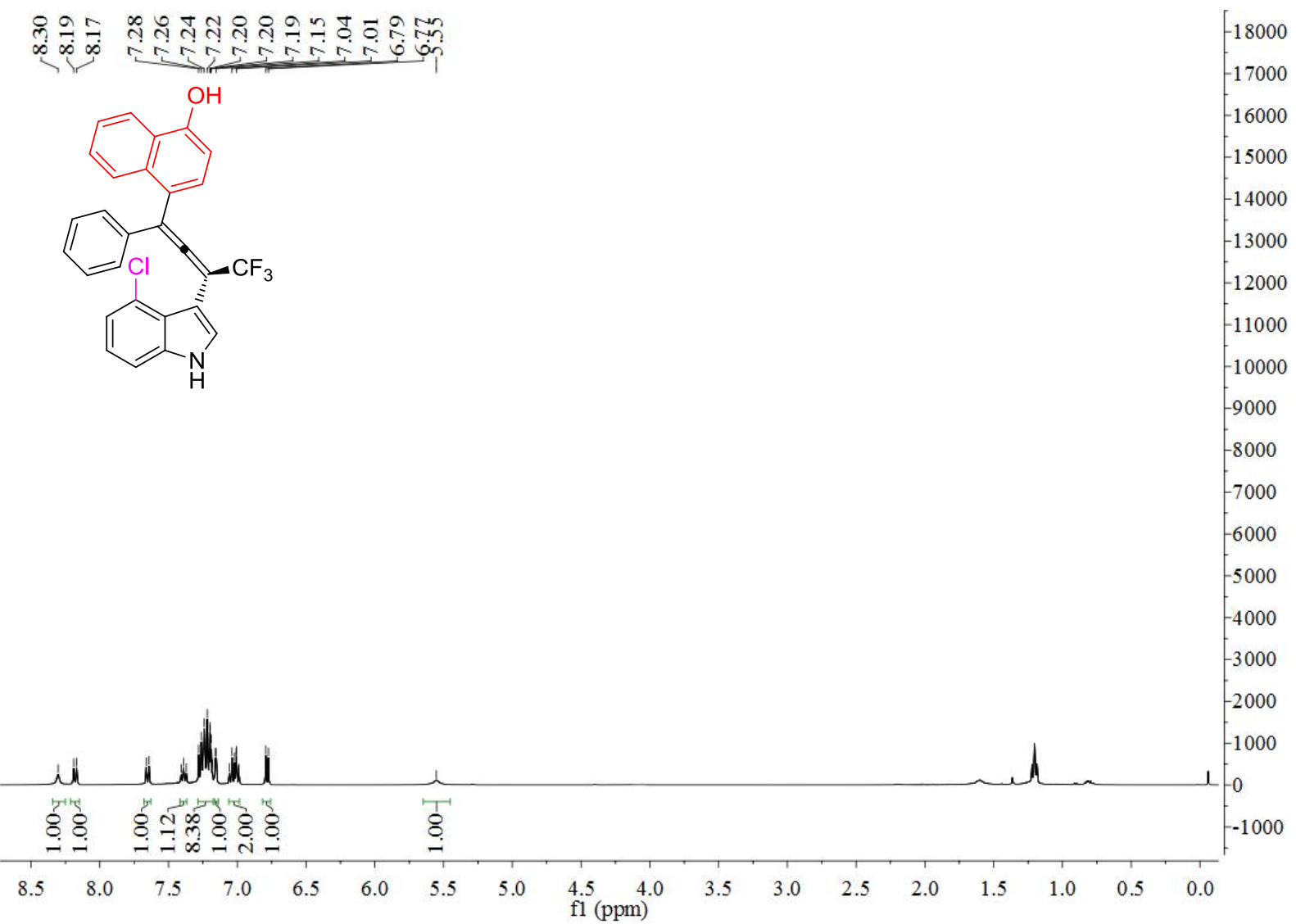

${ }^{13} \mathrm{C}$ NMR of compound $3 \mathrm{~b}\left(126 \mathrm{MHz}\right.$ in $\left.\mathrm{CDCl}_{3}\right)$

है

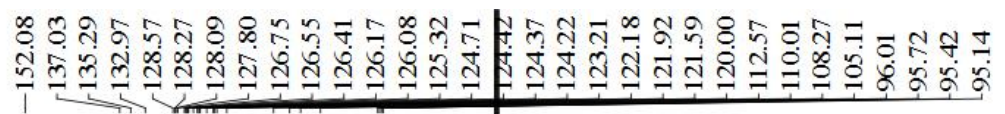<smiles>Oc1ccc(C(=CC(=C(c2ccccc2)c2c[nH]c3cccc(Cl)c23)C(F)(F)F)C(F)(F)F)c2ccccc12</smiles>

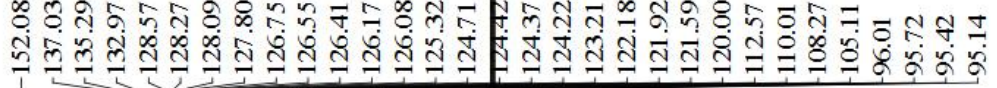

$-3400$

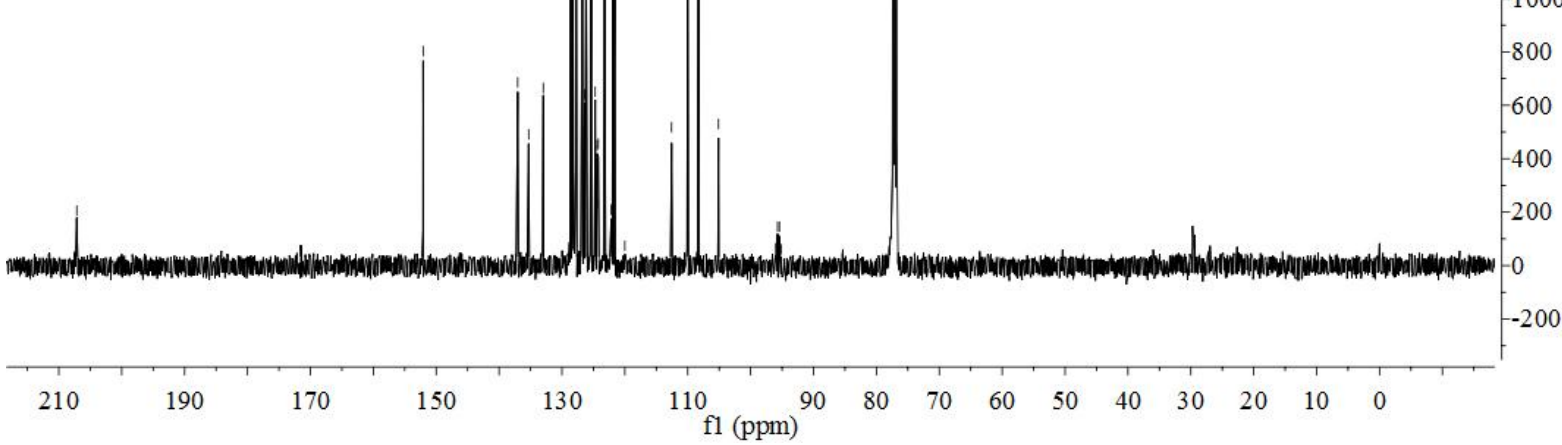


${ }^{19} \mathrm{~F} \mathrm{NMR}$ of compound $3 \mathrm{~b}$ (376 $\mathrm{MHz}$ in $\mathrm{CDCl}_{3}$ )<smiles>Oc1ccc(C(=CC(=C(c2c[nH]c3ccccc23)C(F)(F)F)c2ccccc2)c2ccccc2)c2ccccc12</smiles>

ํํํ

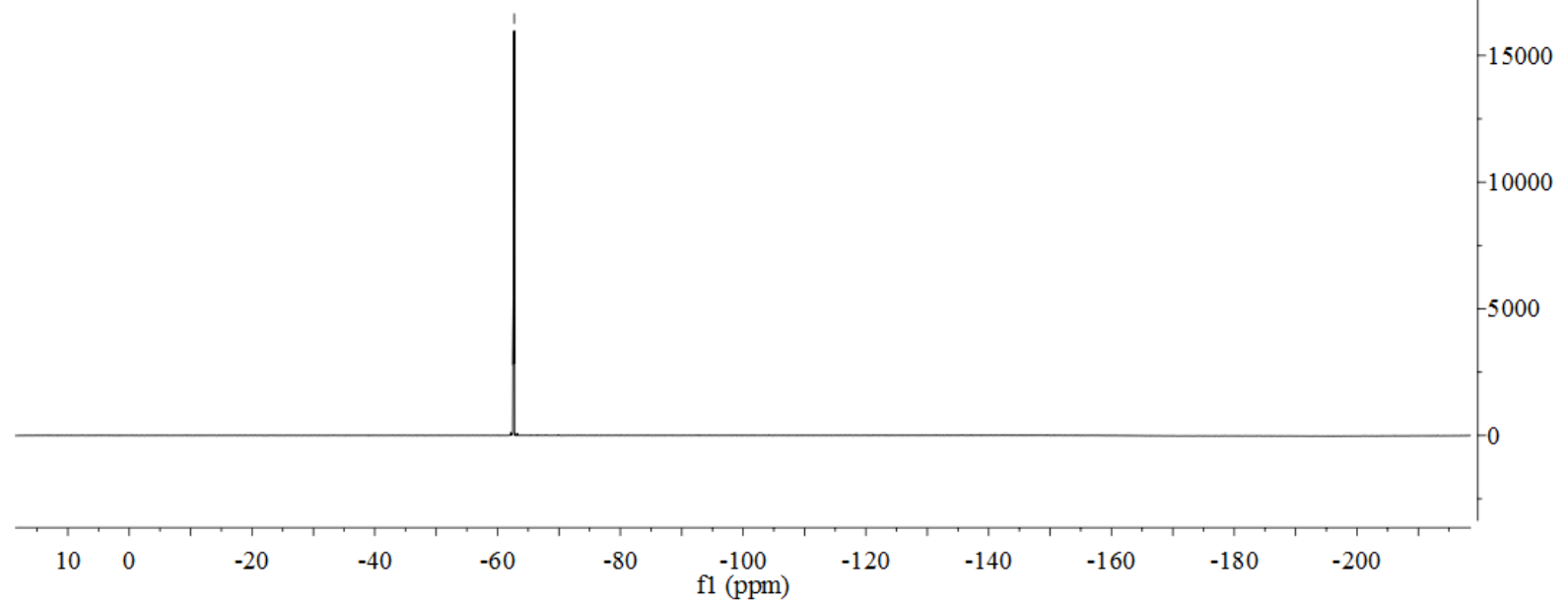

${ }^{1} \mathrm{H}$ NMR of compound $3 \mathrm{c}\left(400 \mathrm{MHz}\right.$ in $\left.\mathrm{CDCl}_{3}\right)$

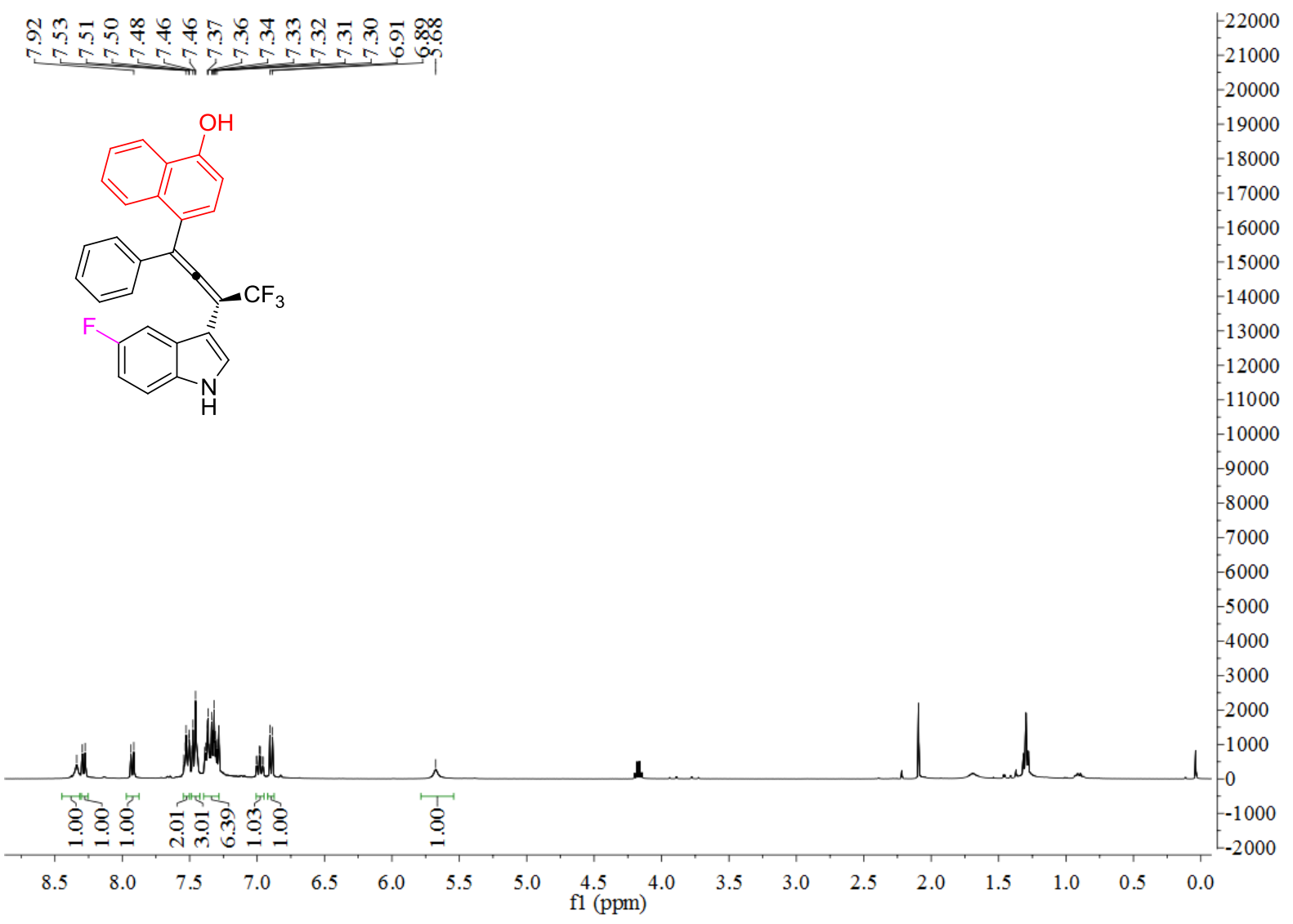


${ }^{13} \mathrm{C}$ NMR of compound $3 \mathrm{c}\left(126 \mathrm{MHz}\right.$ in $\left.\mathrm{CDCl}_{3}\right)$

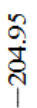

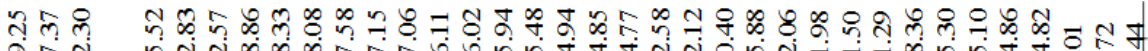

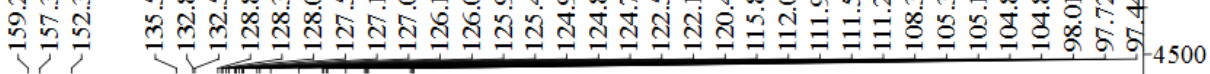<smiles></smiles>

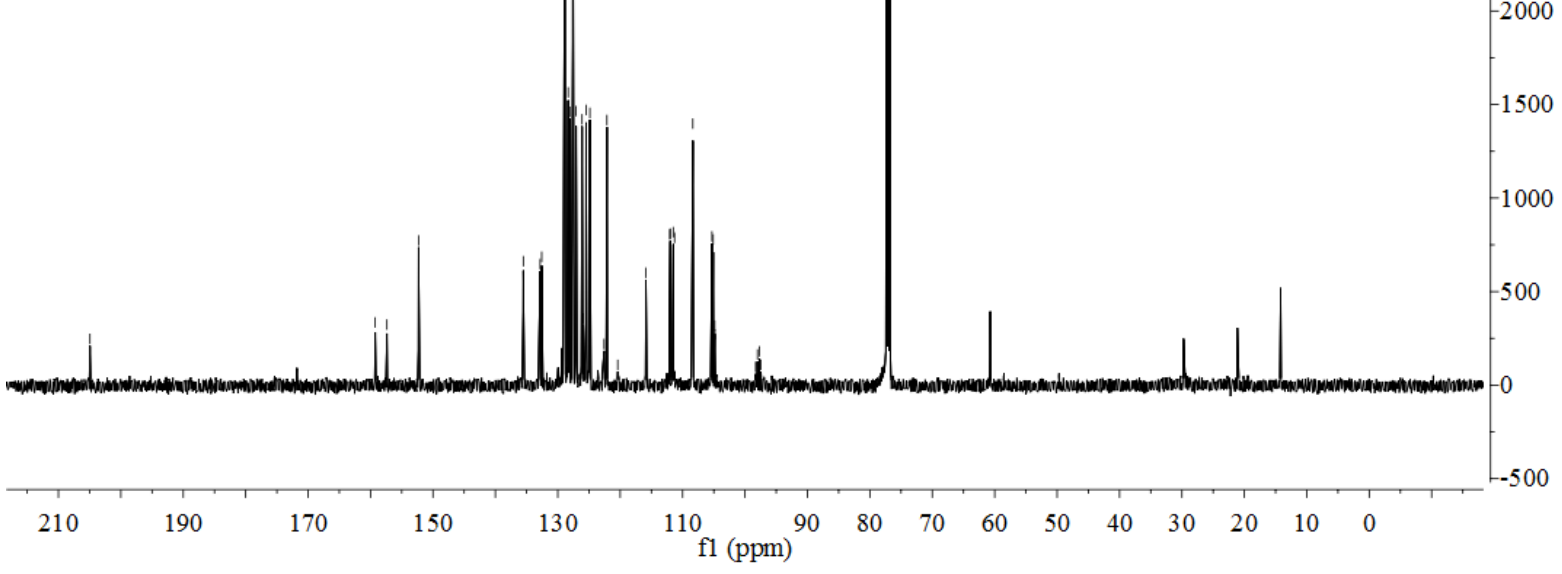

${ }^{19} \mathrm{~F} \mathrm{NMR}$ of compound $3 \mathrm{c}\left(471 \mathrm{MHz}\right.$ in $\left.\mathrm{CDCl}_{3}\right)$

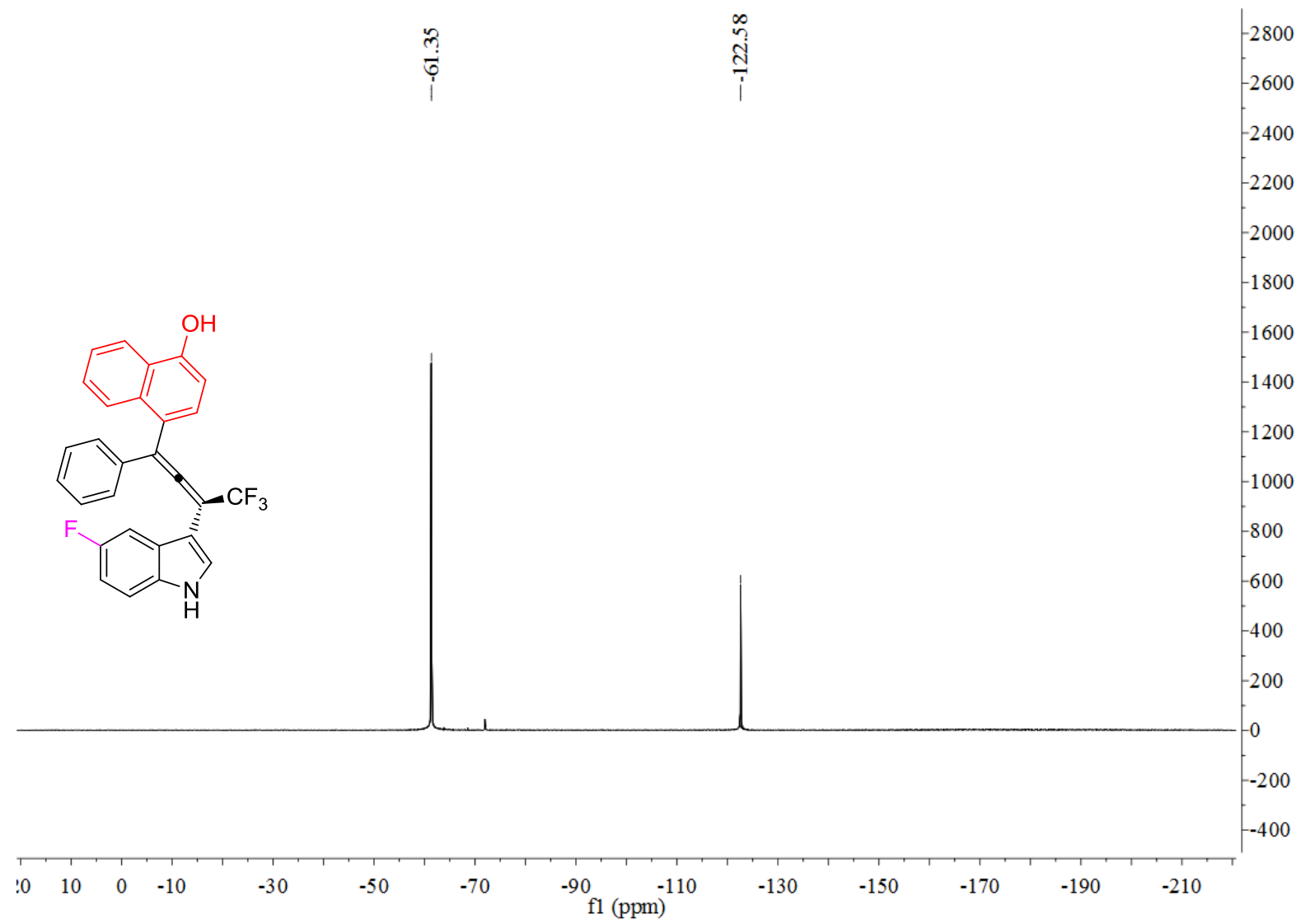




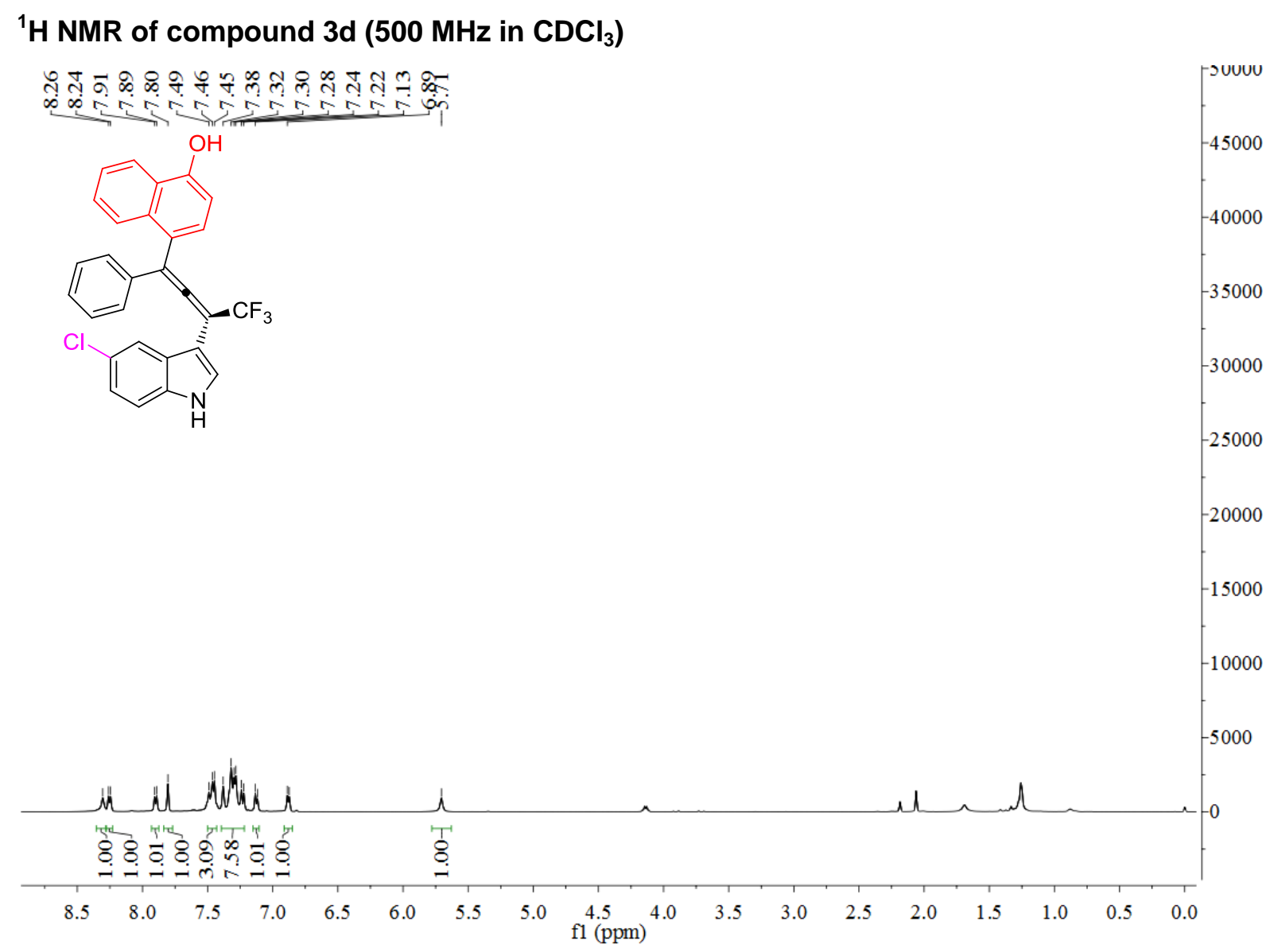

${ }^{13} \mathrm{C}$ NMR of compound $3 \mathrm{~d}\left(126 \mathrm{MHz}\right.$ in $\mathrm{CDCl}_{3}$ )

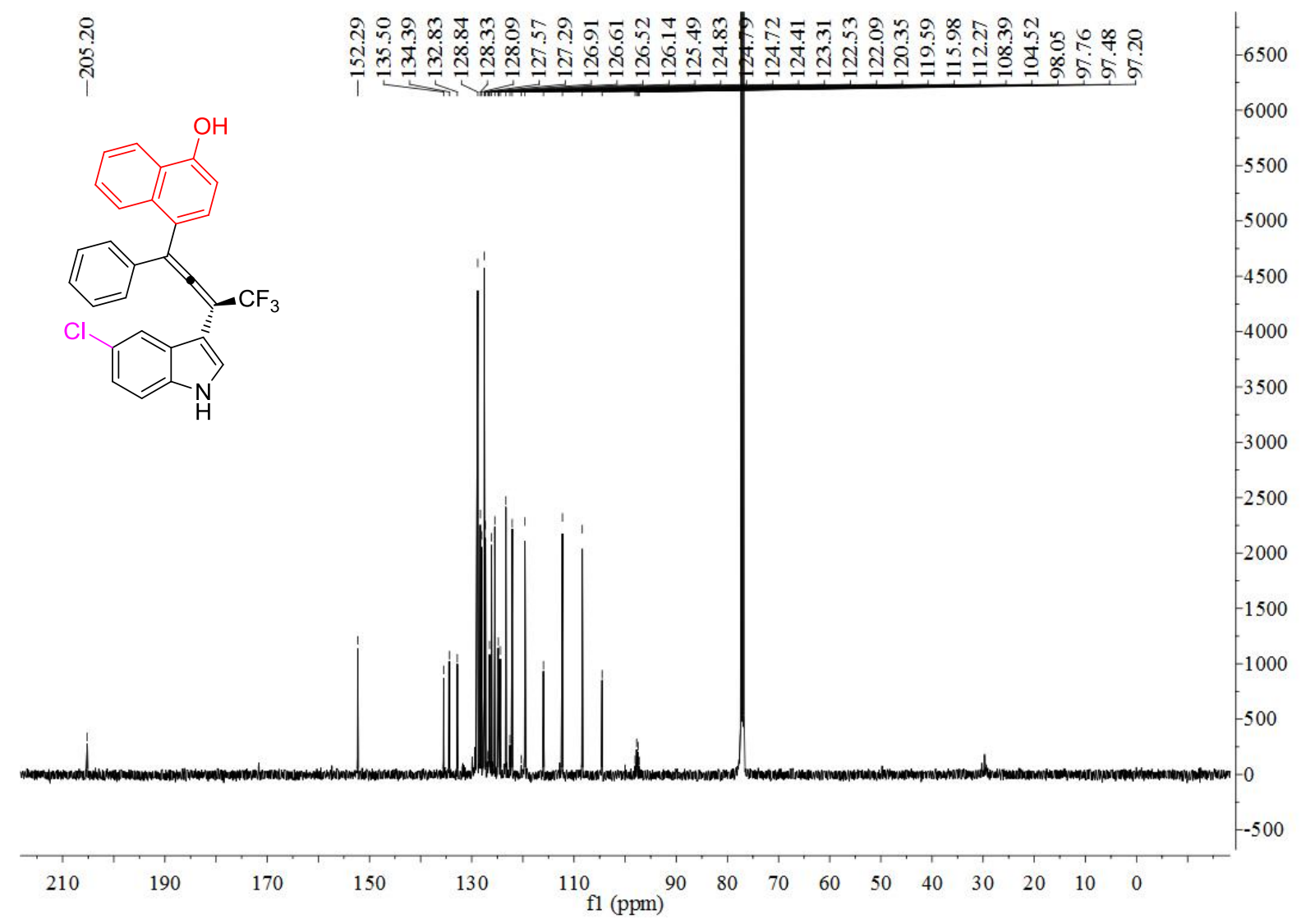


${ }^{19} \mathrm{~F} \mathrm{NMR}$ of compound $3 \mathrm{~d}\left(376 \mathrm{MHz}\right.$ in $\left.\mathrm{CDCl}_{3}\right)$

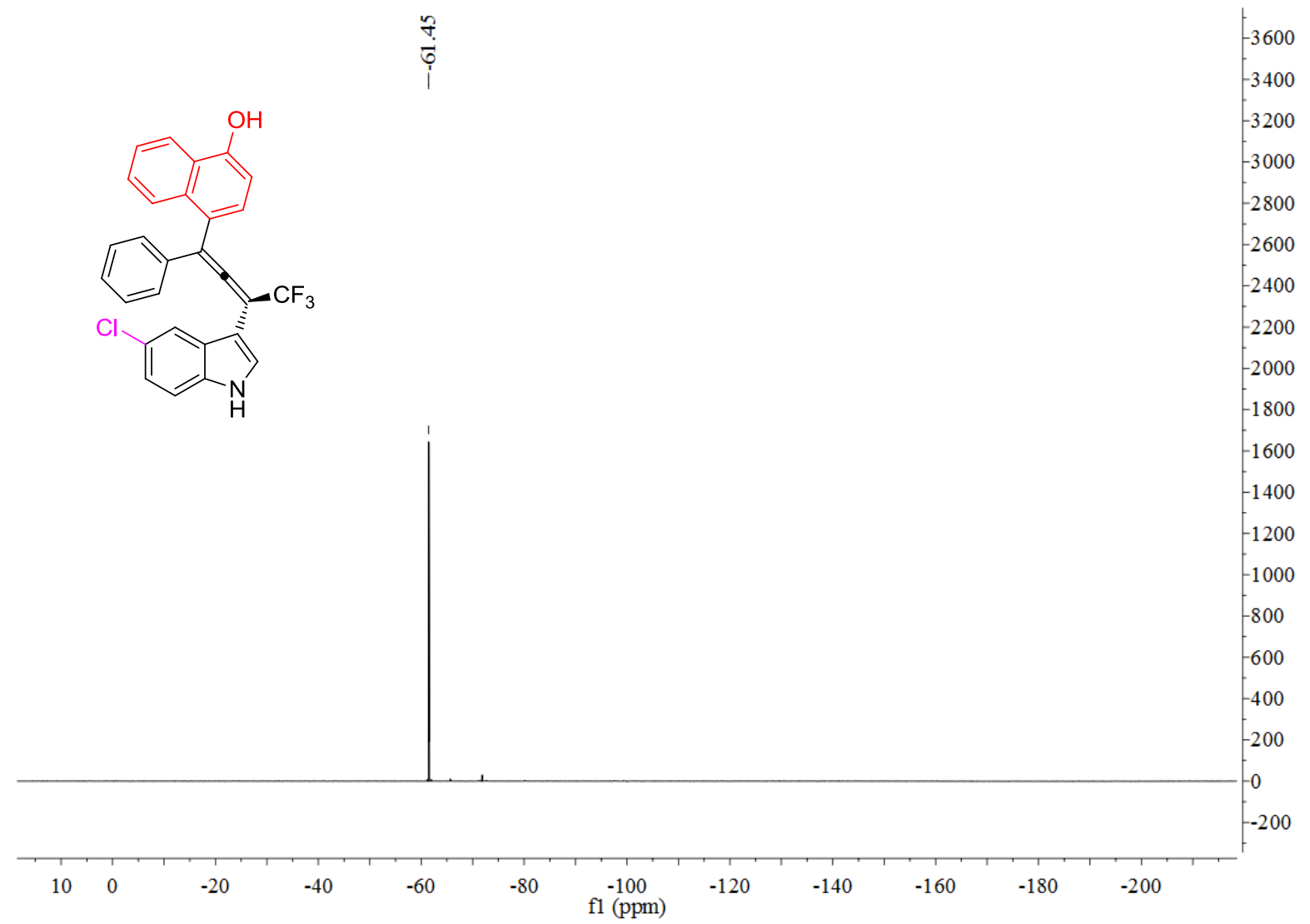

${ }^{1} \mathrm{H}$ NMR of compound $3 \mathrm{e}\left(500 \mathrm{MHz}\right.$ in $\left.\mathrm{CDCl}_{3}\right)$

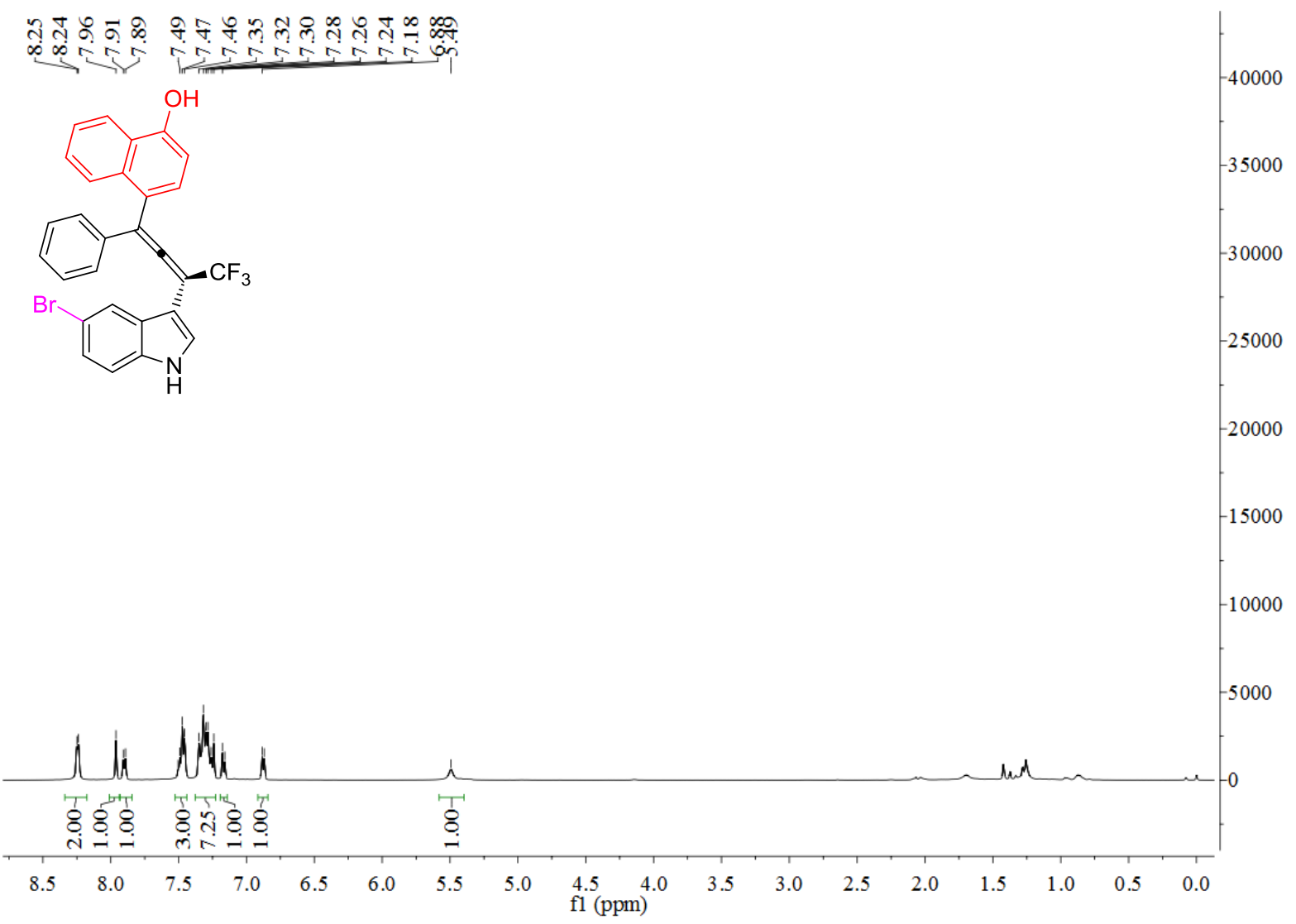


${ }^{13} \mathrm{C}$ NMR of compound $3 \mathrm{e}\left(126 \mathrm{MHz}\right.$ in $\mathrm{CDCl}_{3}$ )

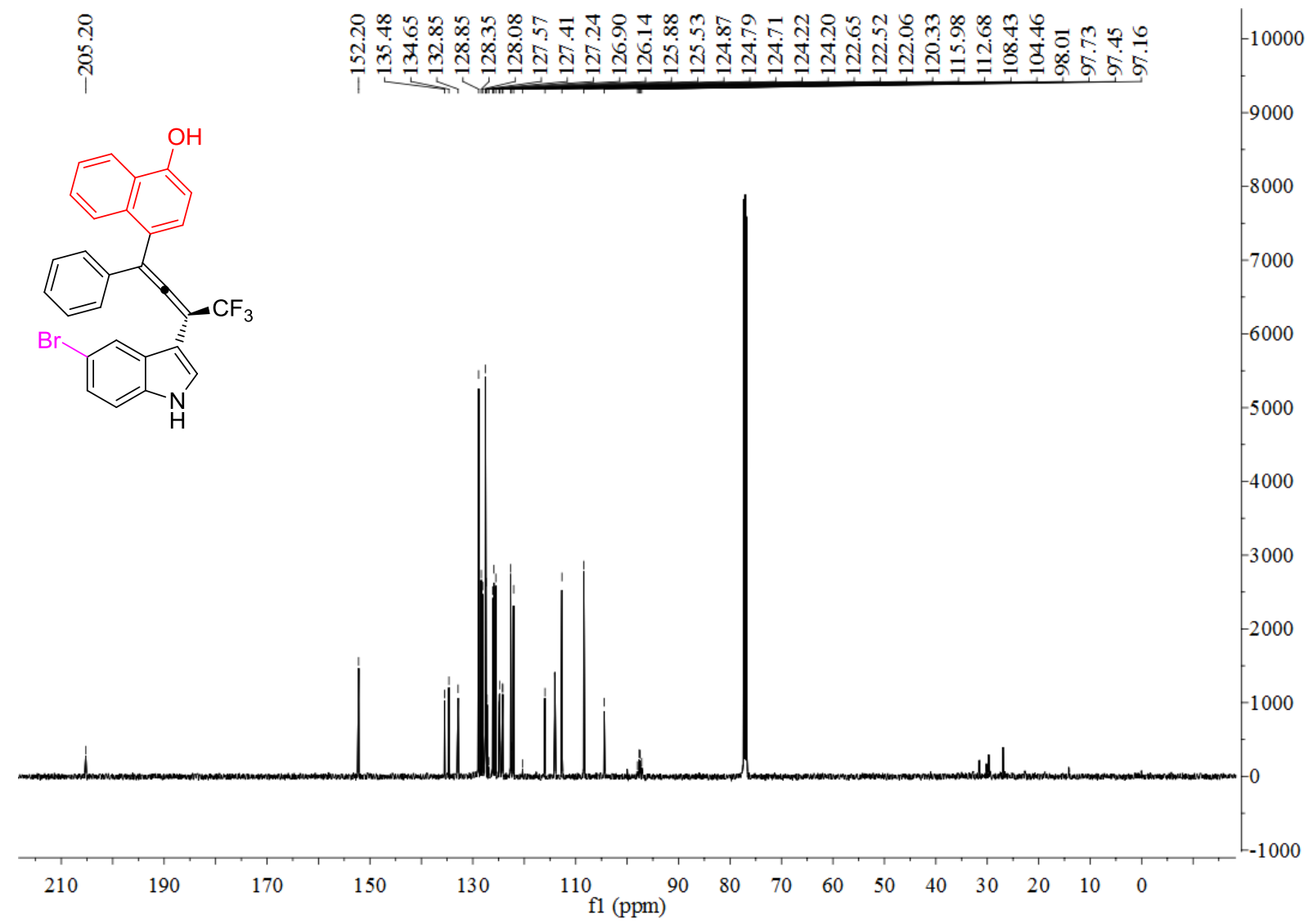

${ }^{19} \mathrm{~F}$ NMR of compound $3 \mathrm{e}\left(471 \mathrm{MHz}\right.$ in $\left.\mathrm{CDCl}_{3}\right)$

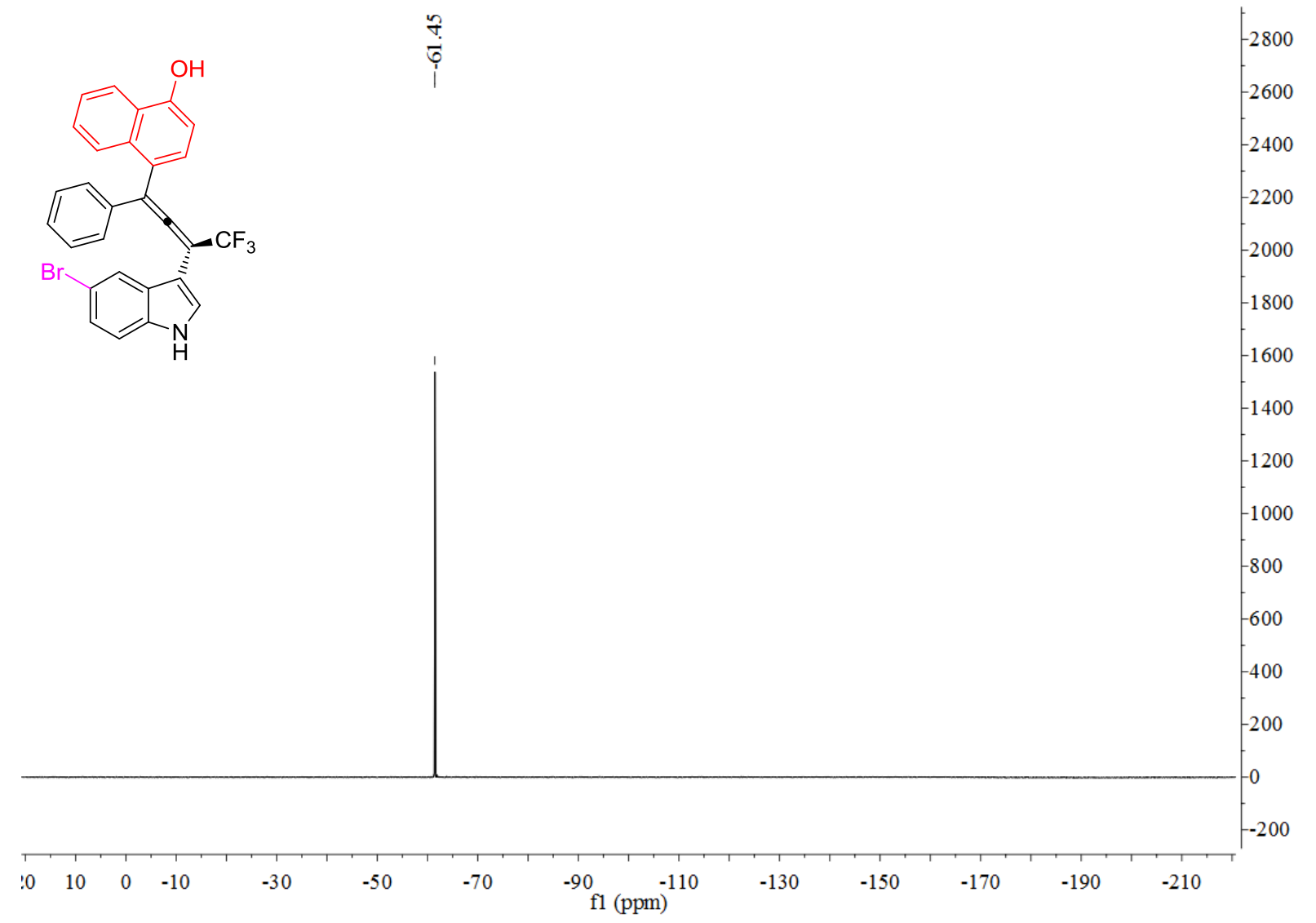


${ }^{1} \mathrm{H}$ NMR of compound $3 f\left(400 \mathrm{MHz}\right.$ in $\mathrm{CDCl}_{3}$ )

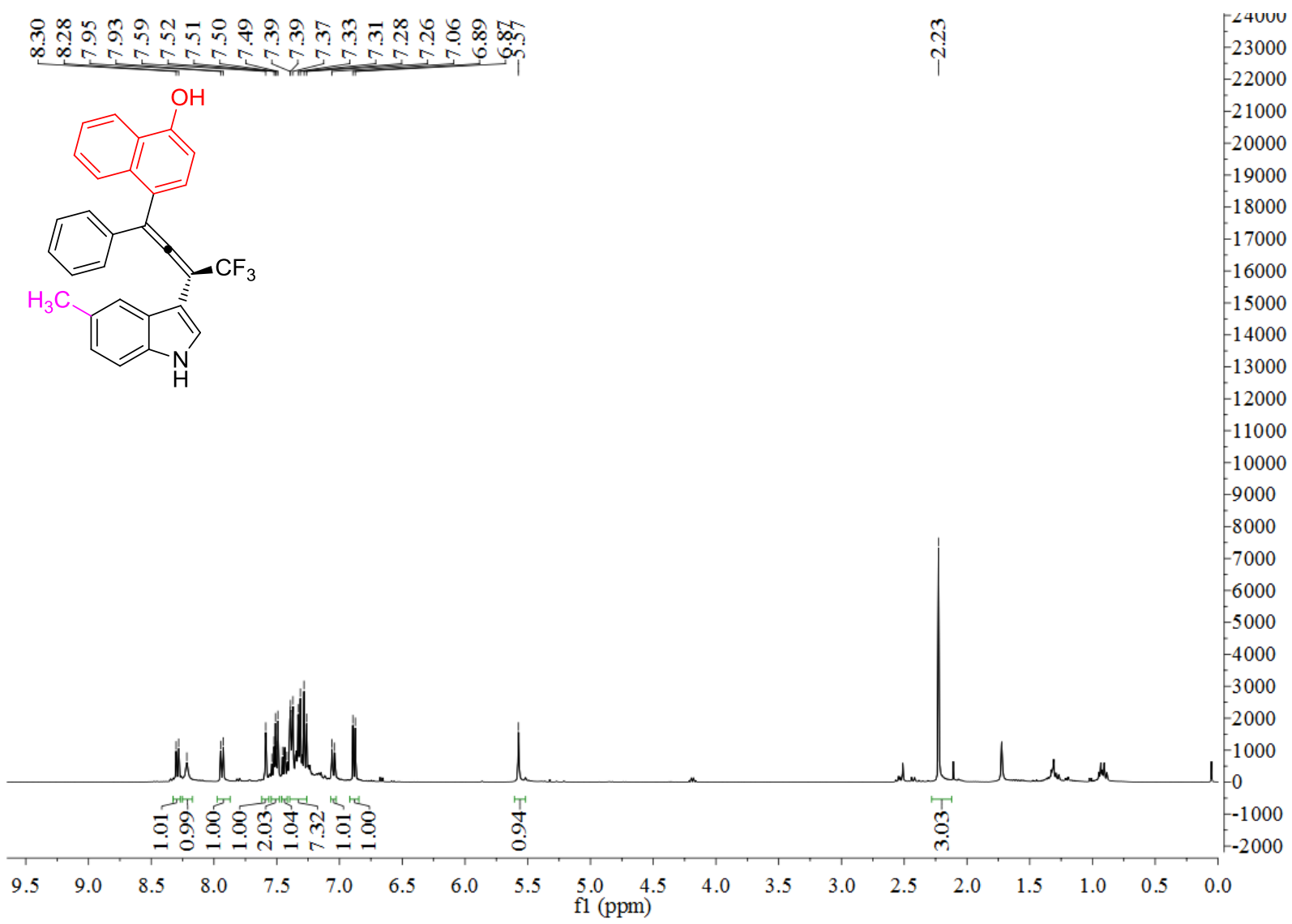

${ }^{13} \mathrm{C}$ NMR of compound $3 f\left(126 \mathrm{MHz}\right.$ in $\mathrm{CDCl}_{3}$ )

हैं

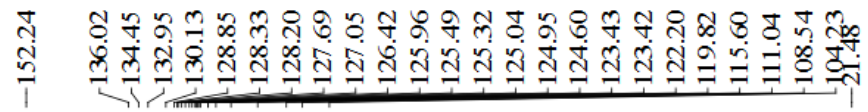<smiles>Cc1ccc2[nH]cc(C(C(F)(F)F)C(F)(F)F)c2c1C(=C(c1ccccc1)c1ccc(O)c2ccccc12)c1ccccc1</smiles>

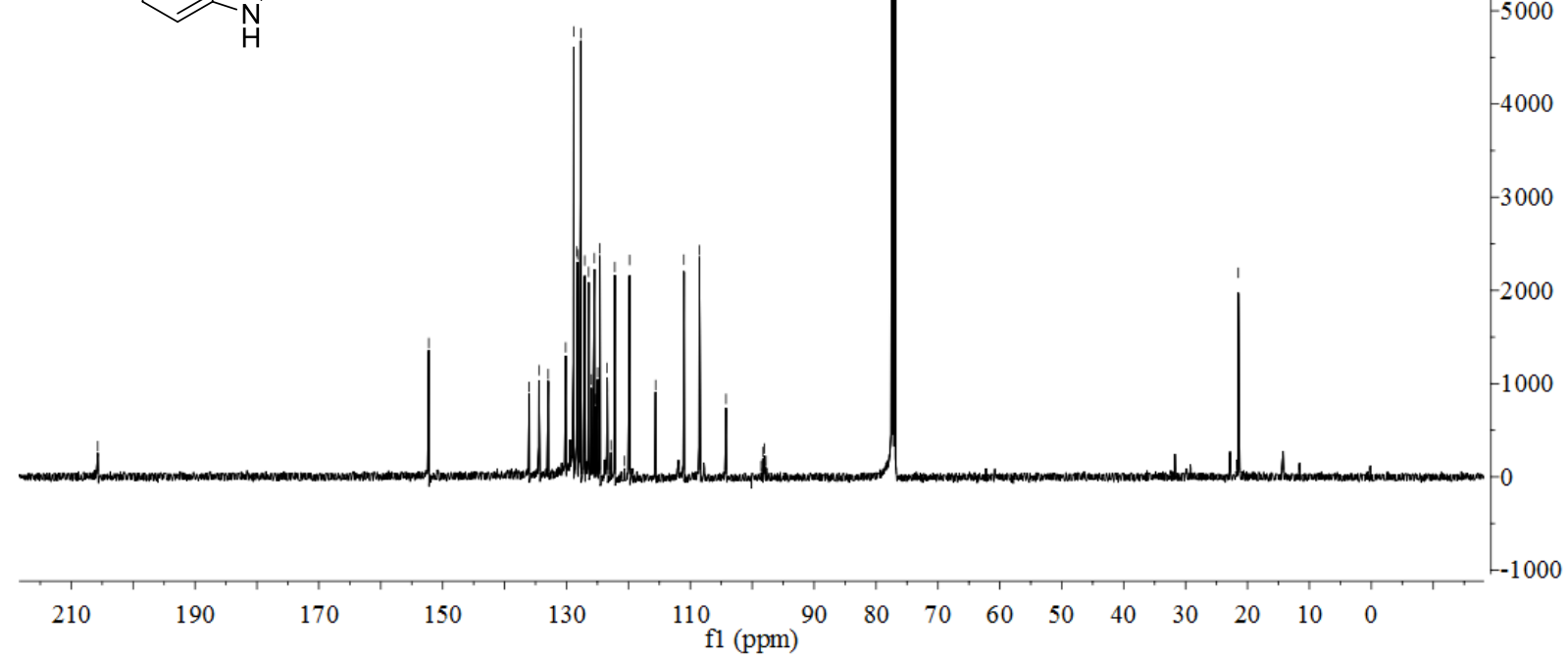


${ }^{19} \mathrm{~F} \mathrm{NMR}$ of compound $3 \mathrm{f}\left(376 \mathrm{MHz}\right.$ in $\left.\mathrm{CDCl}_{3}\right)$

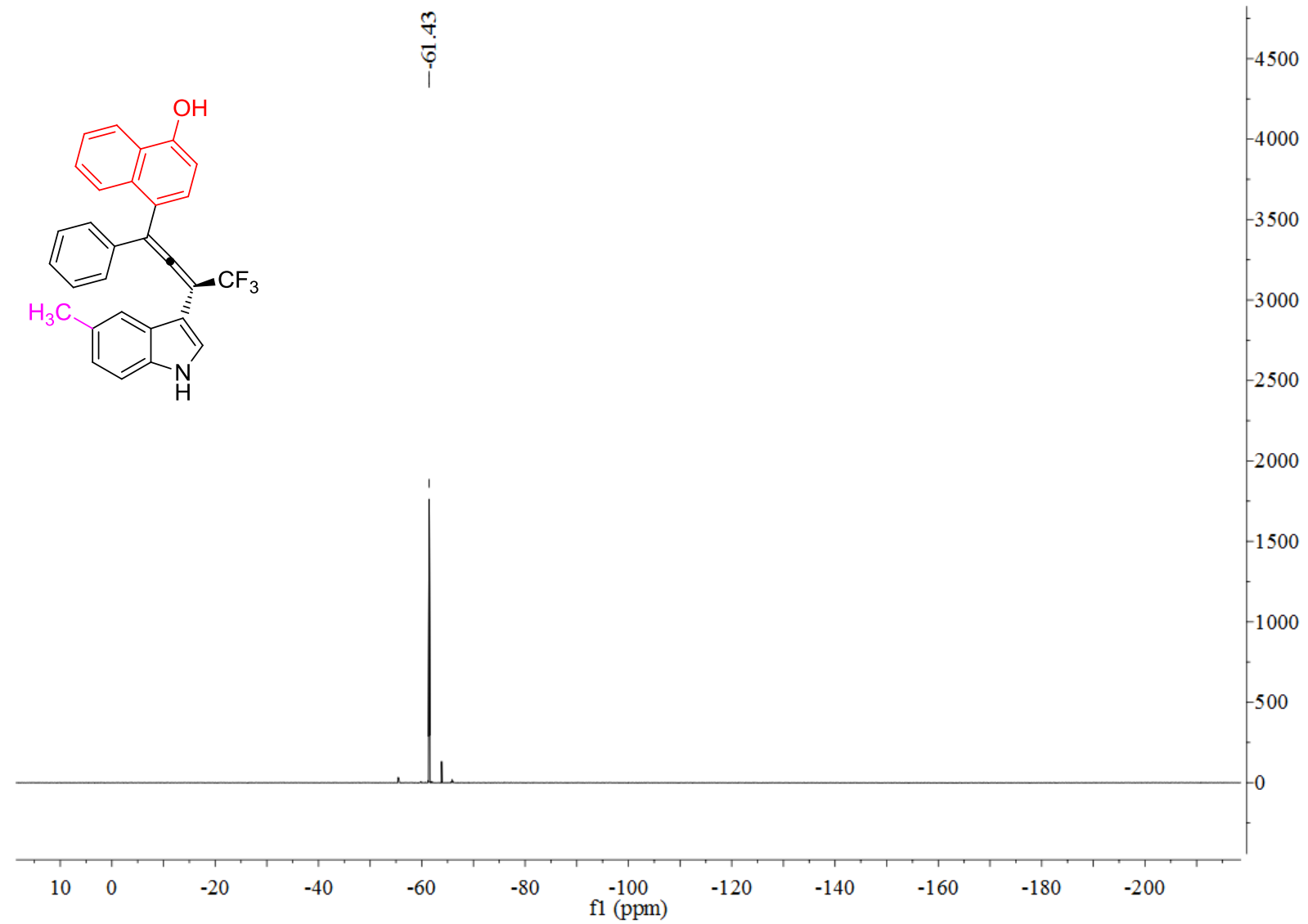

${ }^{1} \mathrm{H}$ NMR of compound $3 \mathrm{~g}\left(400 \mathrm{MHz}\right.$ in $\left.\mathrm{CDCl}_{3}\right)$

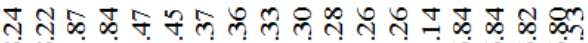

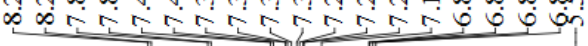<smiles>COc1ccc2[nH]cc(C(=CC(c3ccccc3)c3ccc(O)c4ccccc34)C(F)(F)F)c2c1</smiles>

กे

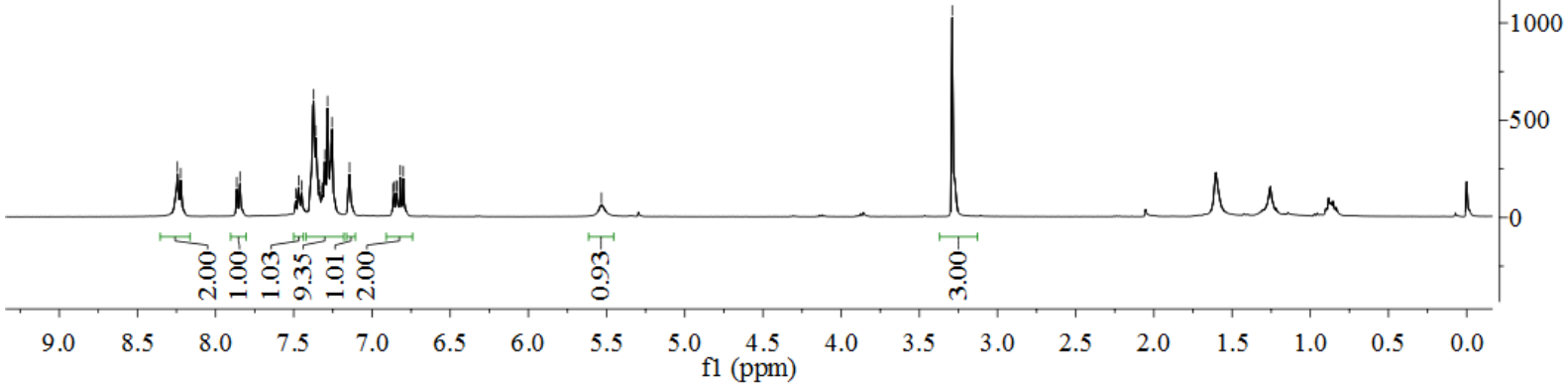


${ }^{13} \mathrm{C}$ NMR of compound $3 \mathrm{~g}\left(126 \mathrm{MHz}\right.$ in $\left.\mathrm{CDCl}_{3}\right)$

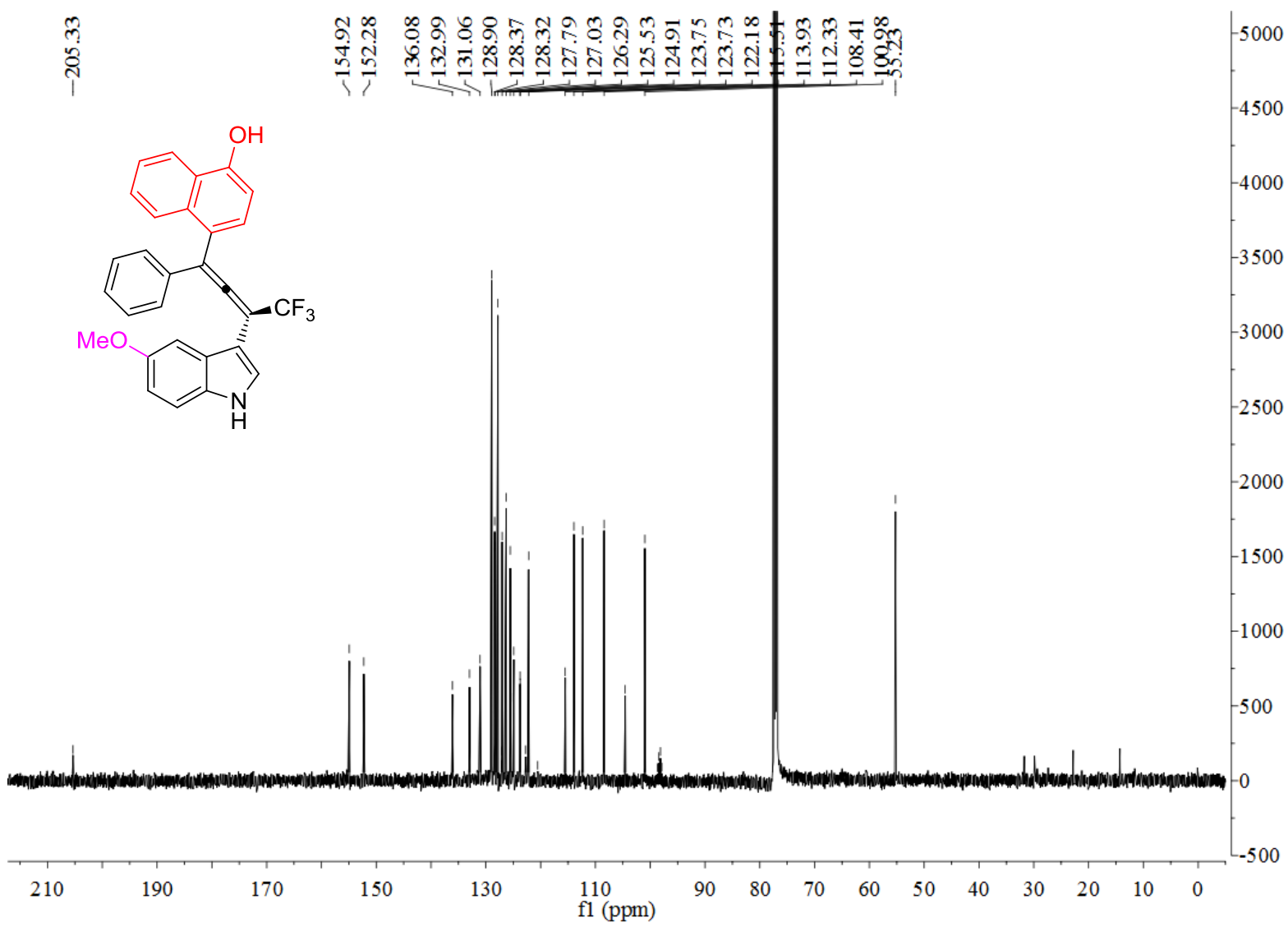

${ }^{19} \mathrm{~F} \mathrm{NMR}$ of compound $3 \mathrm{~g}$ (376 $\mathrm{MHz}$ in $\mathrm{CDCl}_{3}$ )

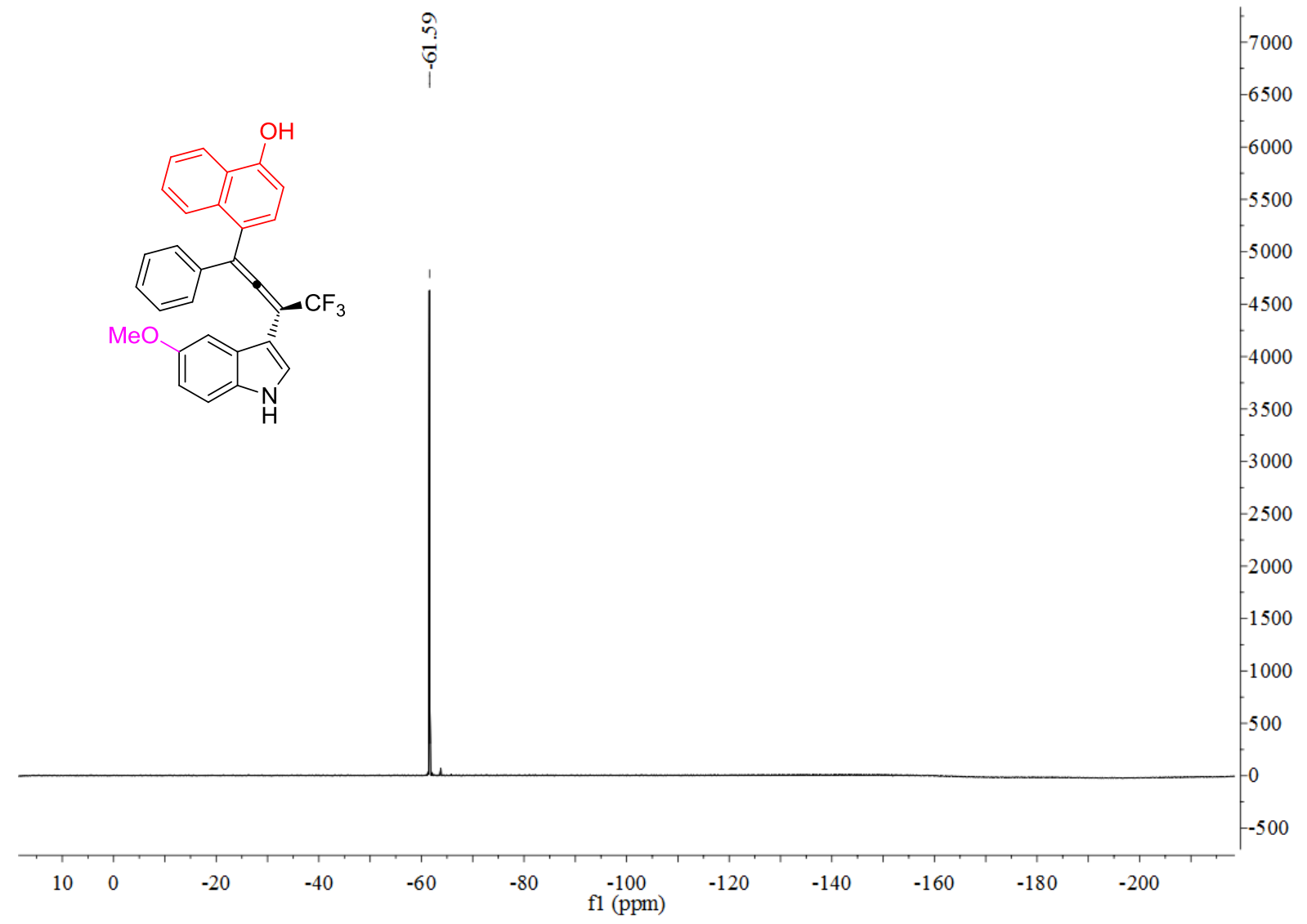


${ }^{1} \mathrm{H}$ NMR of compound $3 \mathrm{~h}\left(400 \mathrm{MHz}\right.$ in $\left.\mathrm{CDCl}_{3}\right)$

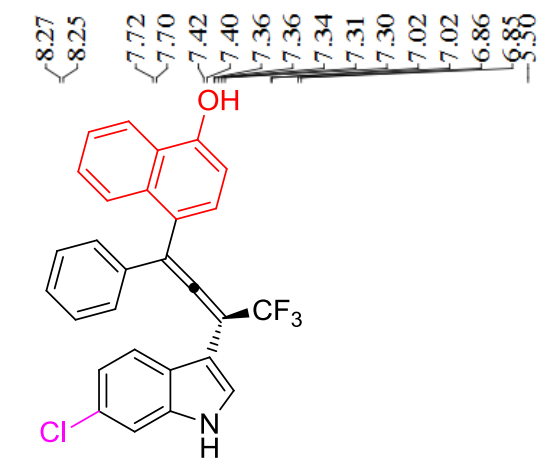

38000

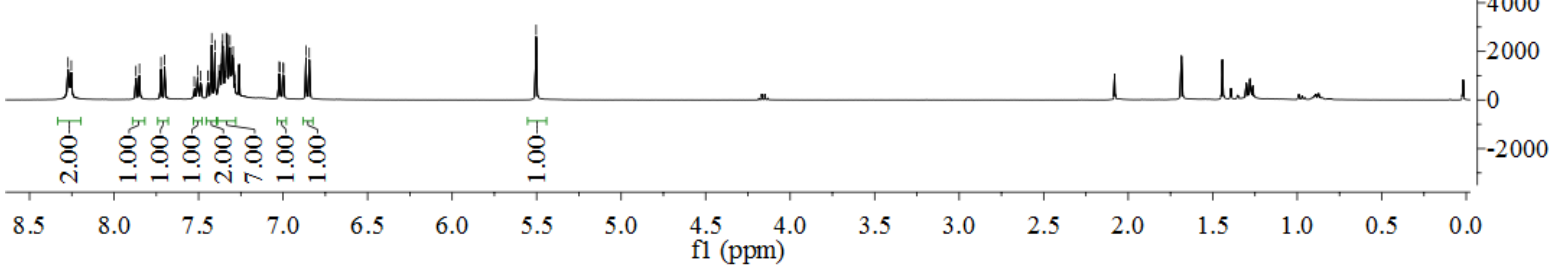

${ }^{13} \mathrm{C}$ NMR of compound $3 \mathrm{~h}\left(126 \mathrm{MHz}\right.$ in $\mathrm{CDCl}_{3}$ )

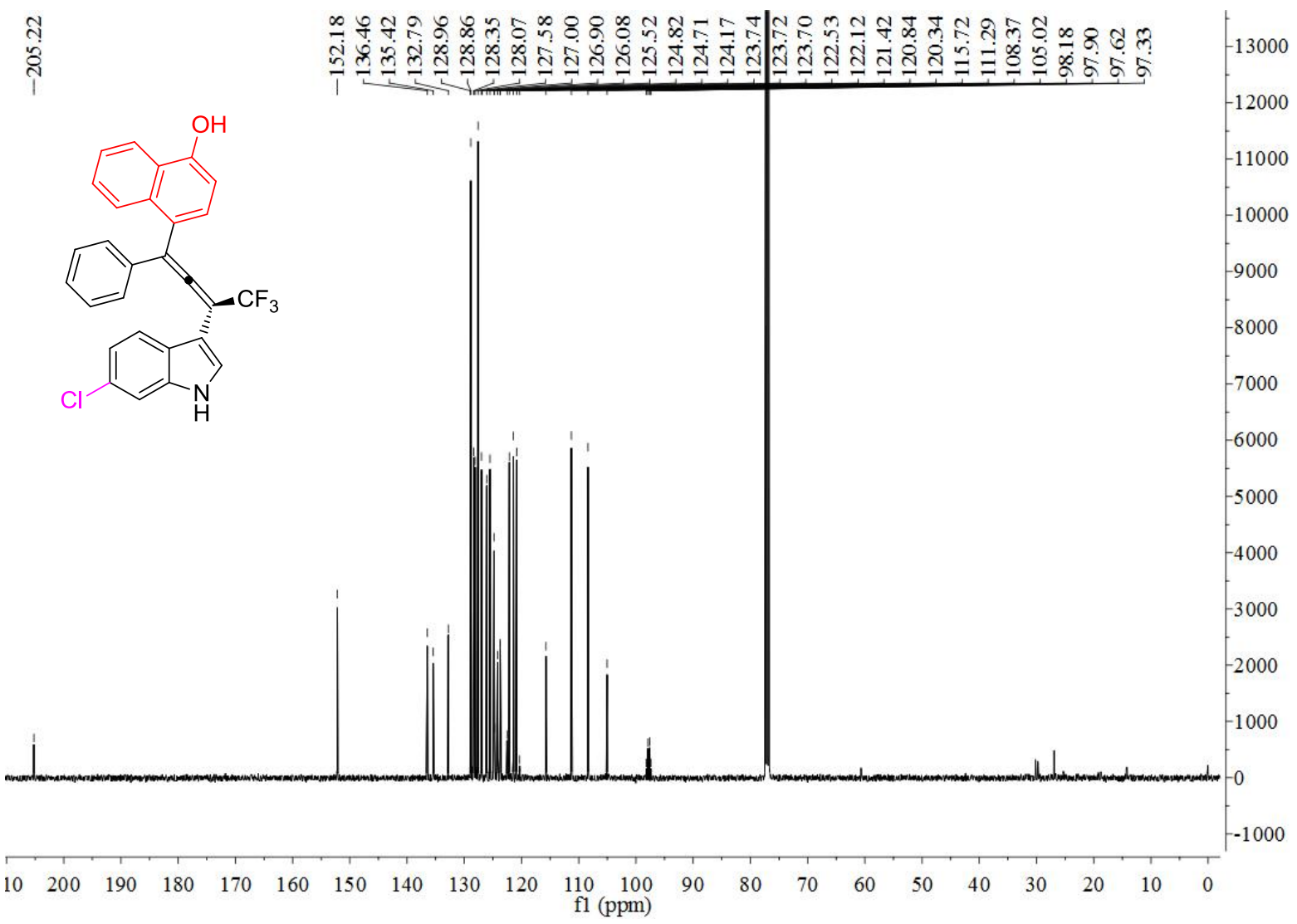


${ }^{19} \mathrm{~F}$ NMR of compound $3 \mathrm{~h}\left(376 \mathrm{MHz}\right.$ in $\mathrm{CDCl}_{3}$ )

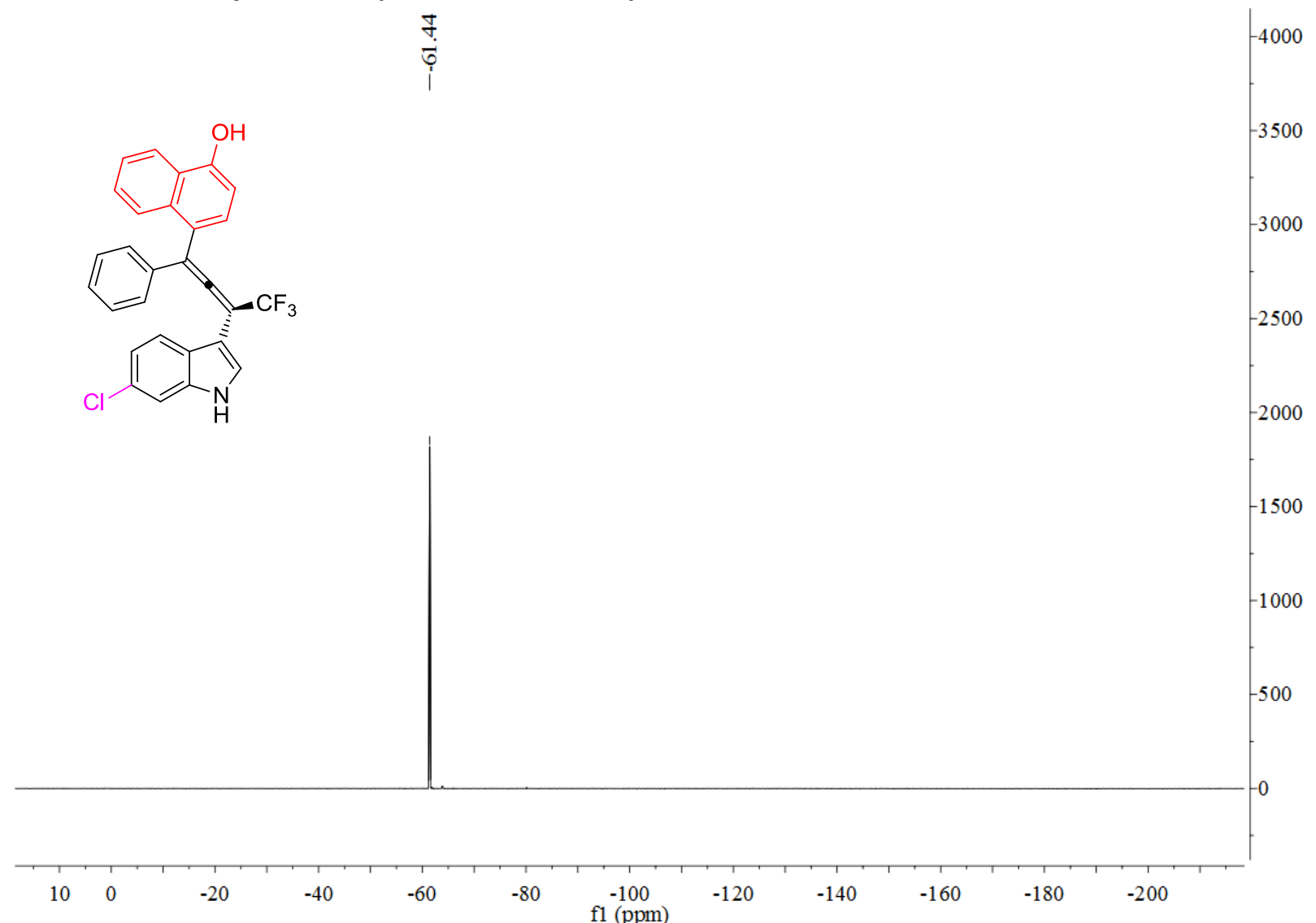

${ }^{1} \mathrm{H}$ NMR of compound $3 \mathrm{i}\left(500 \mathrm{MHz}\right.$ in $\mathrm{CDCl}_{3}$ )

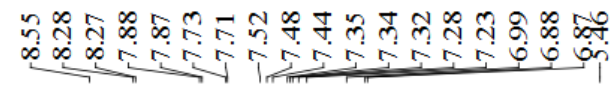

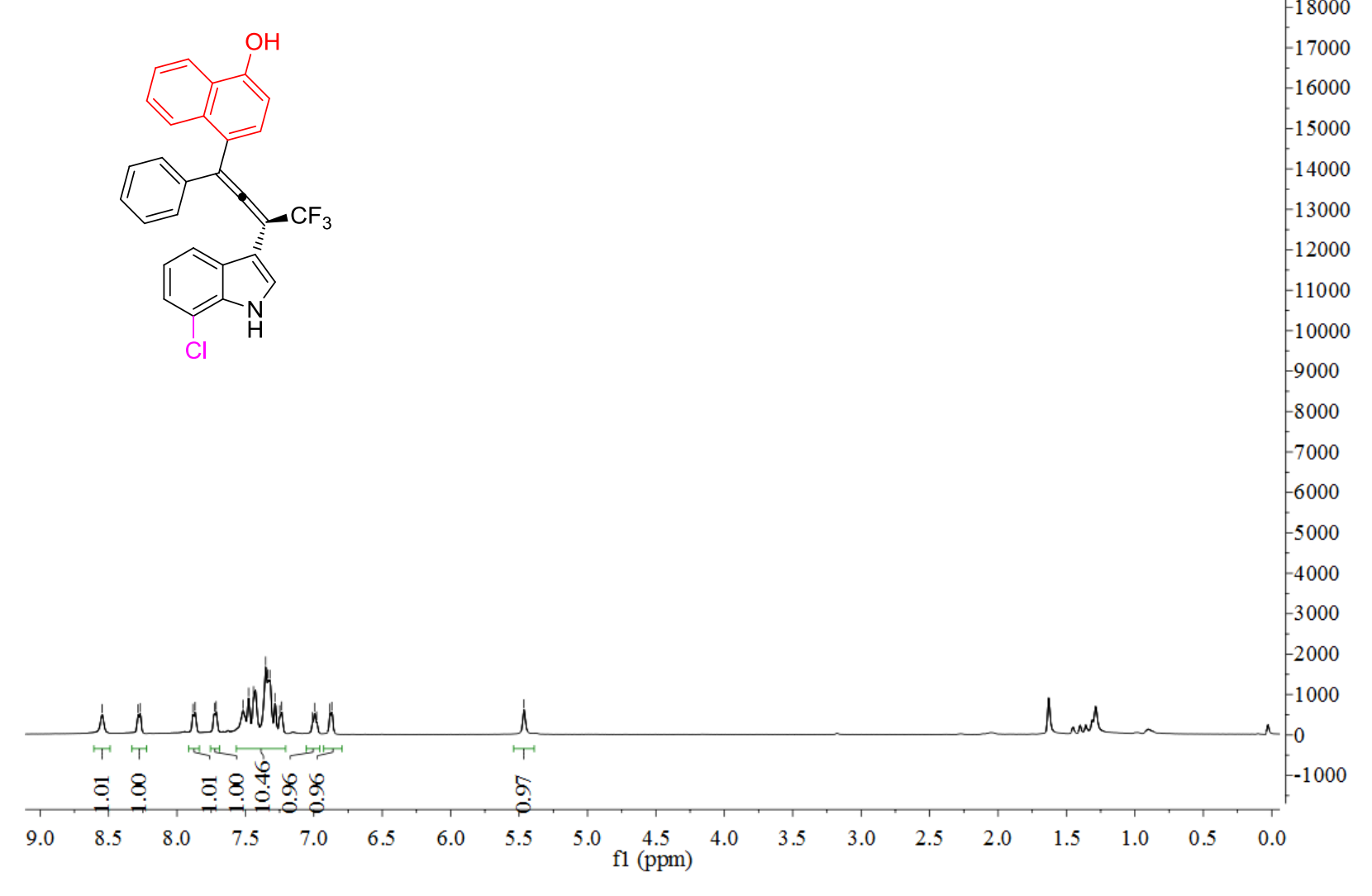


${ }^{13} \mathrm{C}$ NMR of compound $3 \mathrm{i}\left(126 \mathrm{MHz}\right.$ in $\mathrm{CDCl}_{3}$ )

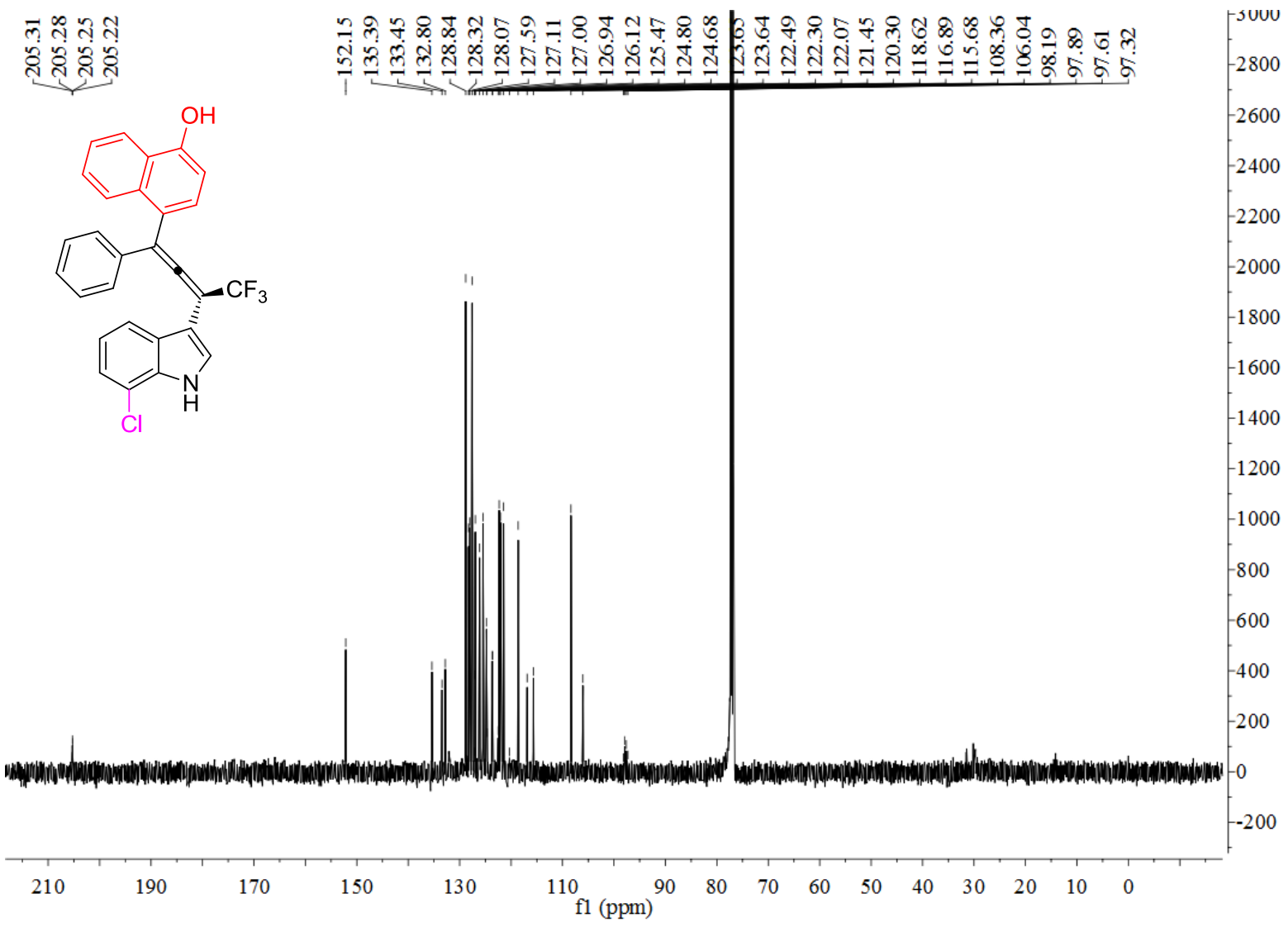

${ }^{19} \mathrm{~F} \mathrm{NMR}$ of compound $3 \mathrm{i}\left(471 \mathrm{MHz}\right.$ in $\left.\mathrm{CDCl}_{3}\right)$

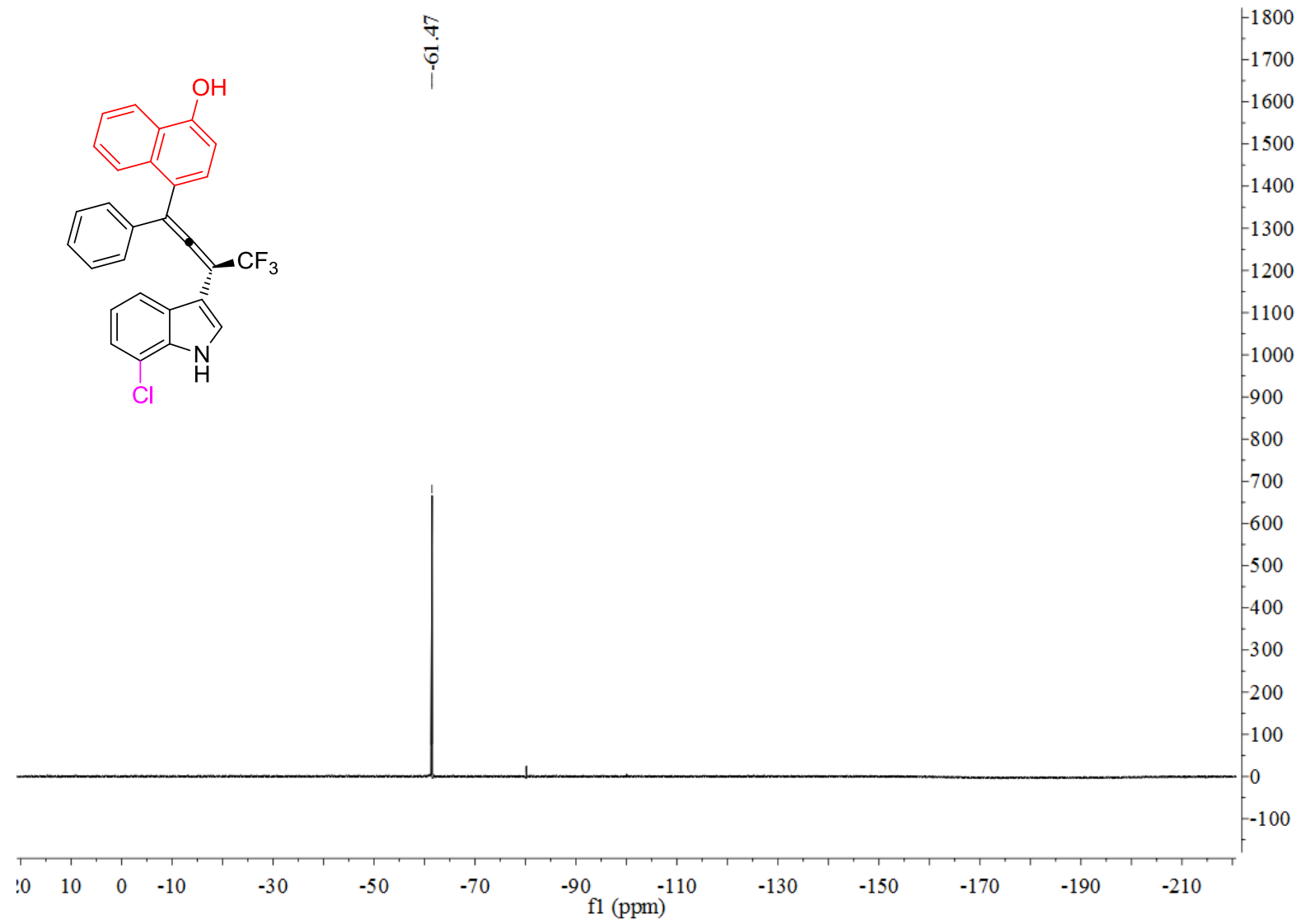


${ }^{1} \mathrm{H}$ NMR of compound $3 \mathrm{j}$ (400 $\mathrm{MHz}$ in $\mathrm{CDCl}_{3}$ )

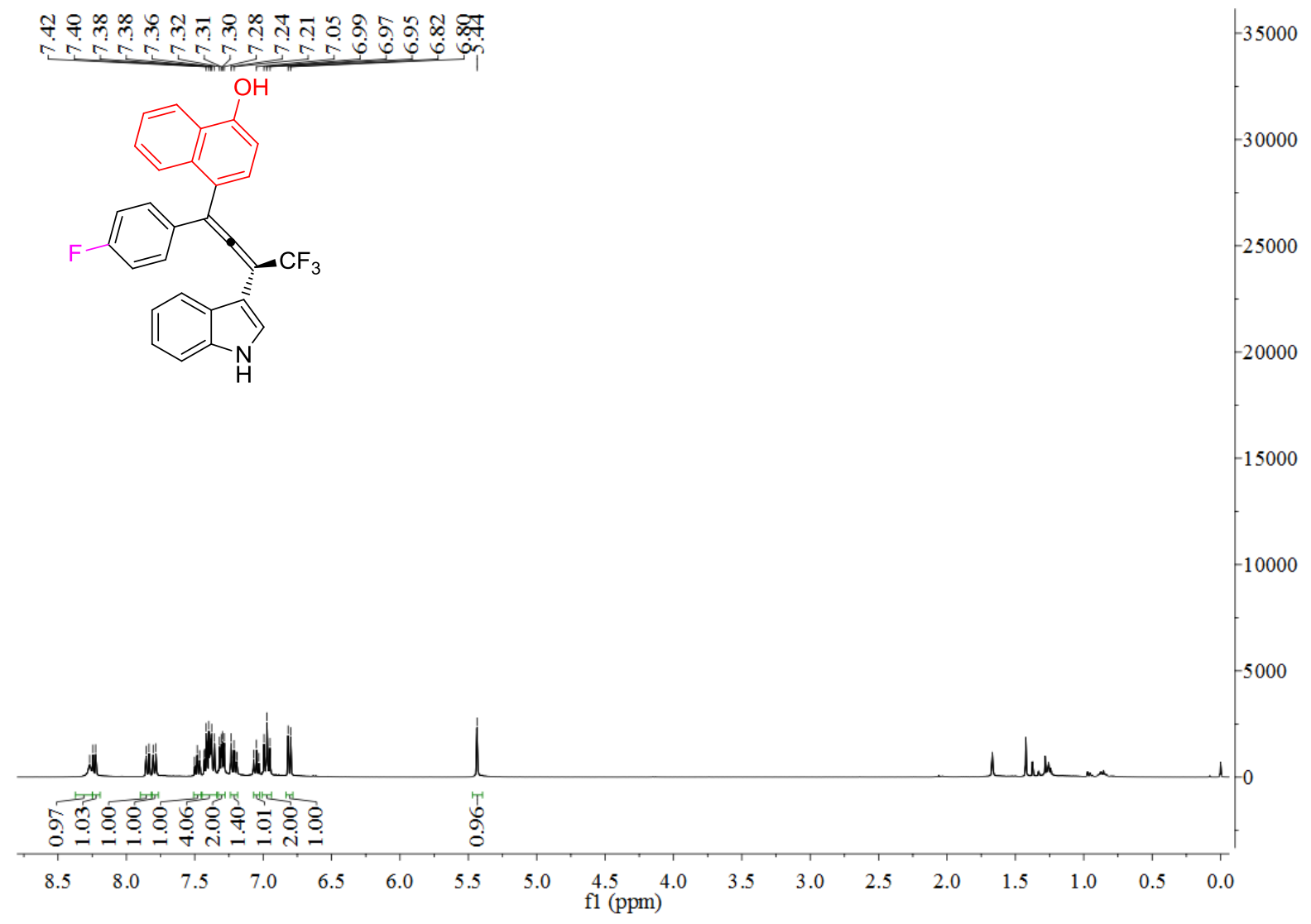

${ }^{13} \mathrm{C}$ NMR of compound $3 \mathrm{j}$ (126 MHz in $\mathrm{CDCl}_{3}$ )

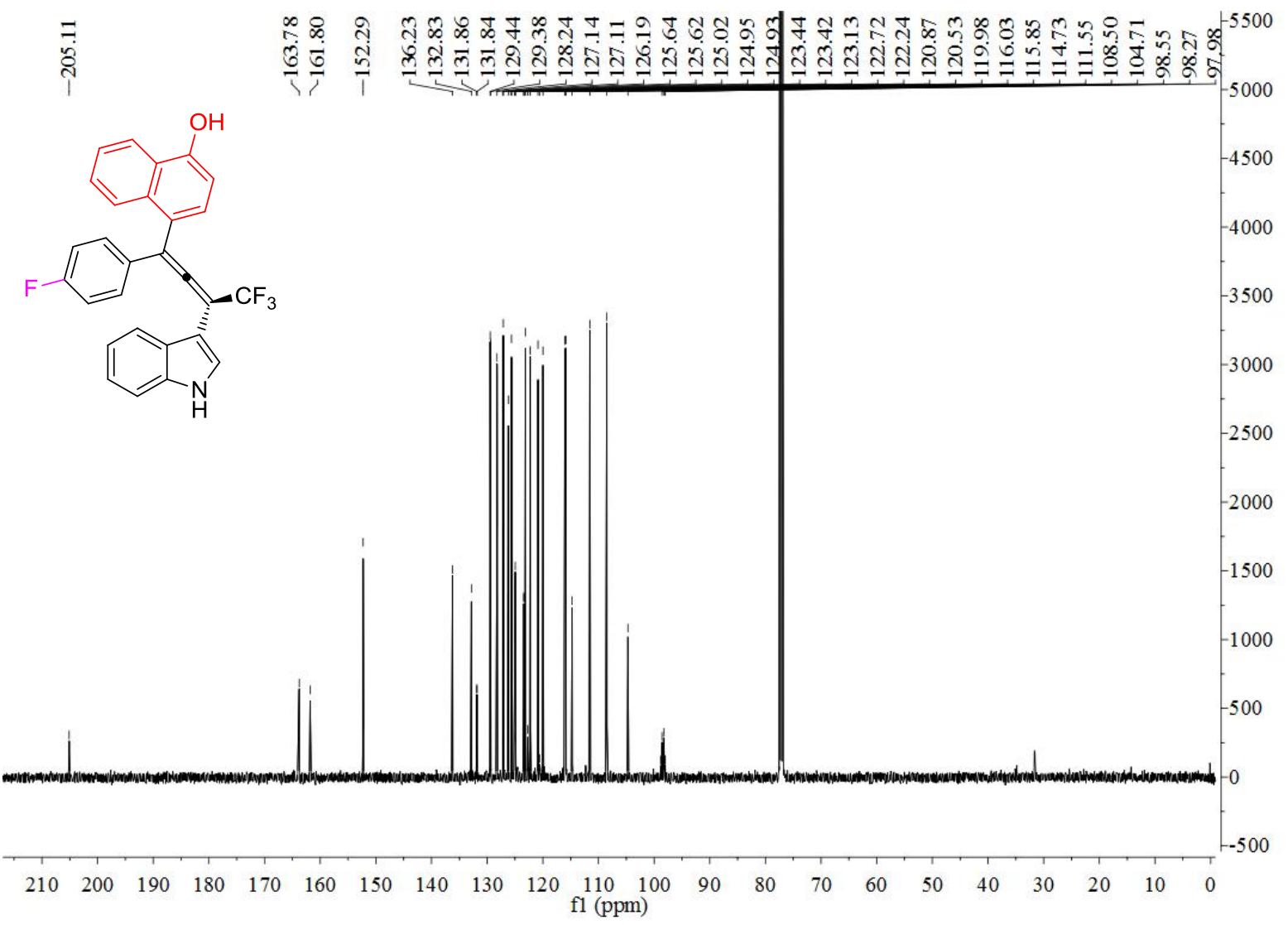


${ }^{19} \mathrm{~F} \mathrm{NMR}$ of compound $3 \mathrm{j}$ (376 $\mathrm{MHz}$ in $\mathrm{CDCl}_{3}$ )

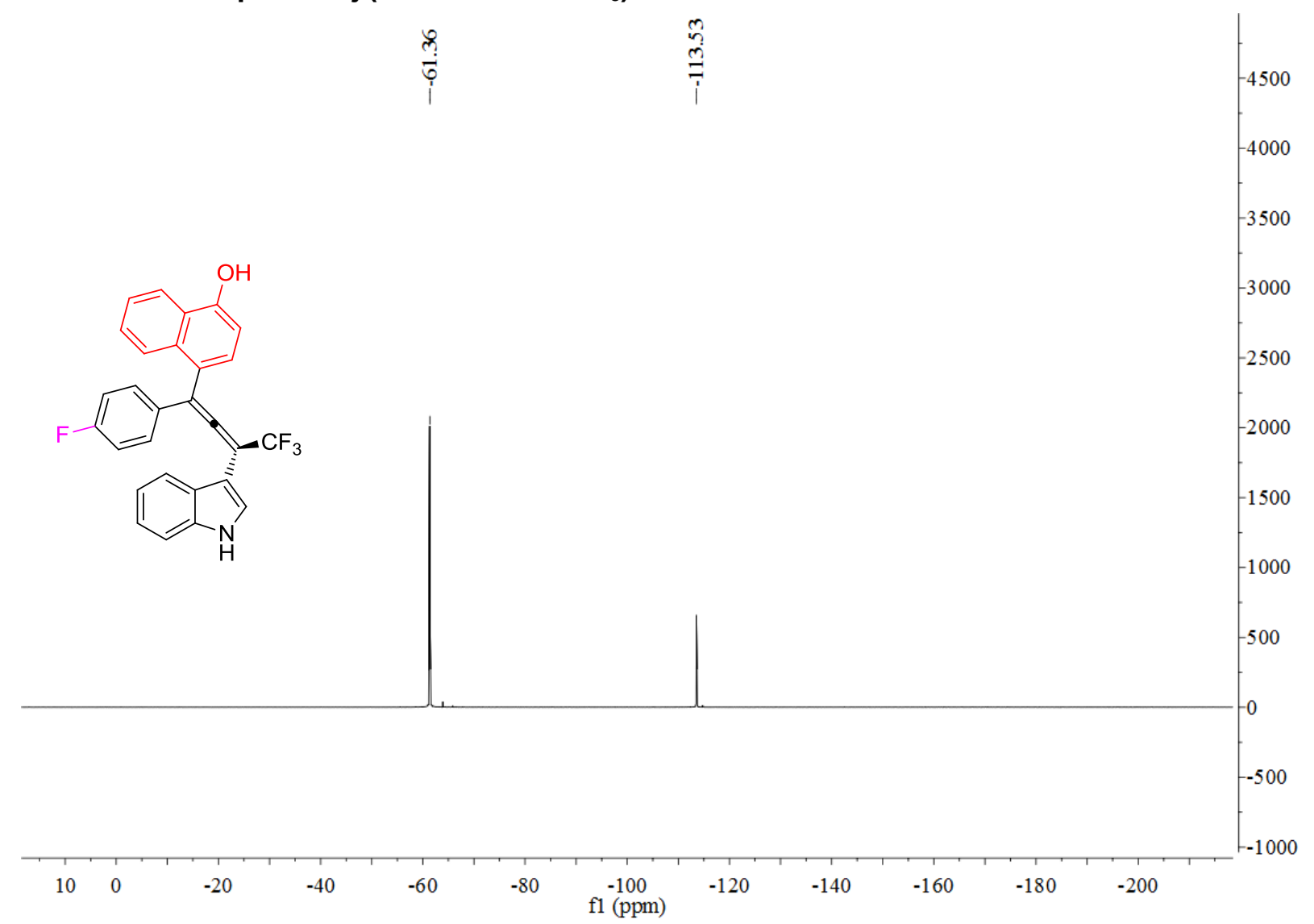

${ }^{1} \mathrm{H}$ NMR of compound $3 \mathrm{k}$ (400 $\mathrm{MHz}$ in $\mathrm{CDCl}_{3}$ )

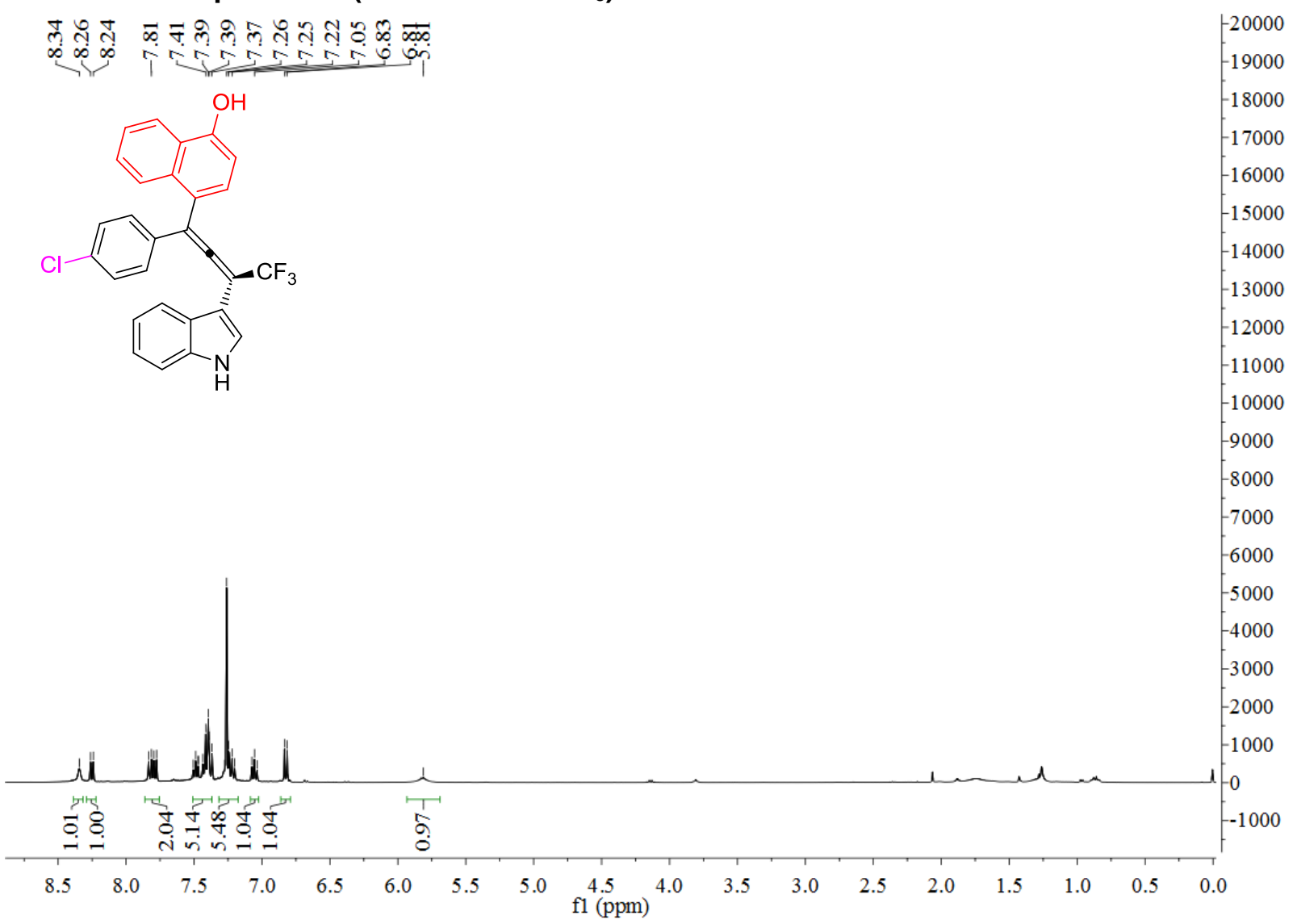


${ }^{13} \mathrm{C}$ NMR of compound 3k (101 $\mathrm{MHz}$ in $\mathrm{CDCl}_{3}$ )

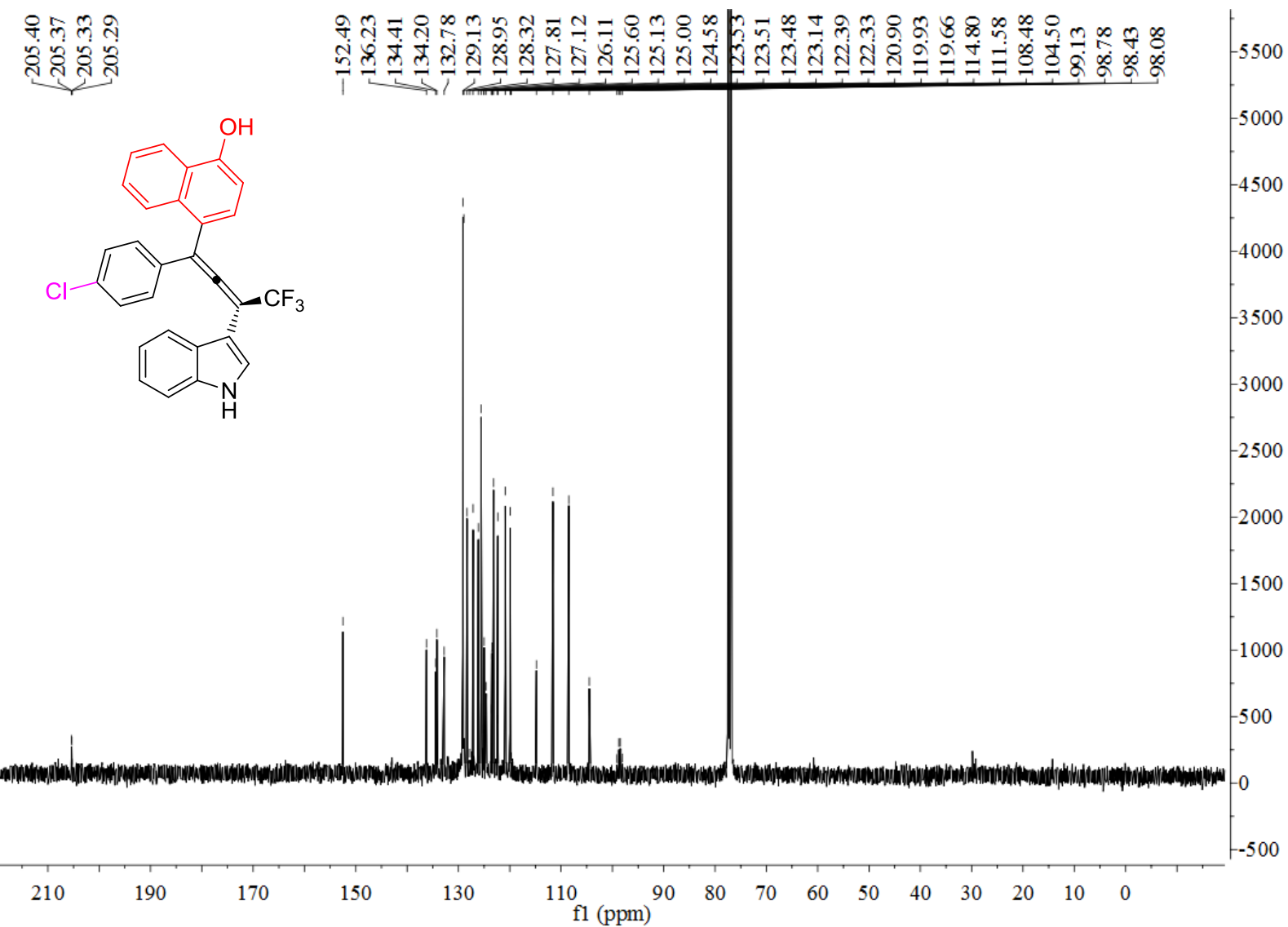

${ }^{19} \mathrm{~F} \mathrm{NMR}$ of compound $3 \mathrm{k}$ (376 $\mathrm{MHz}$ in $\mathrm{CDCl}_{3}$ )

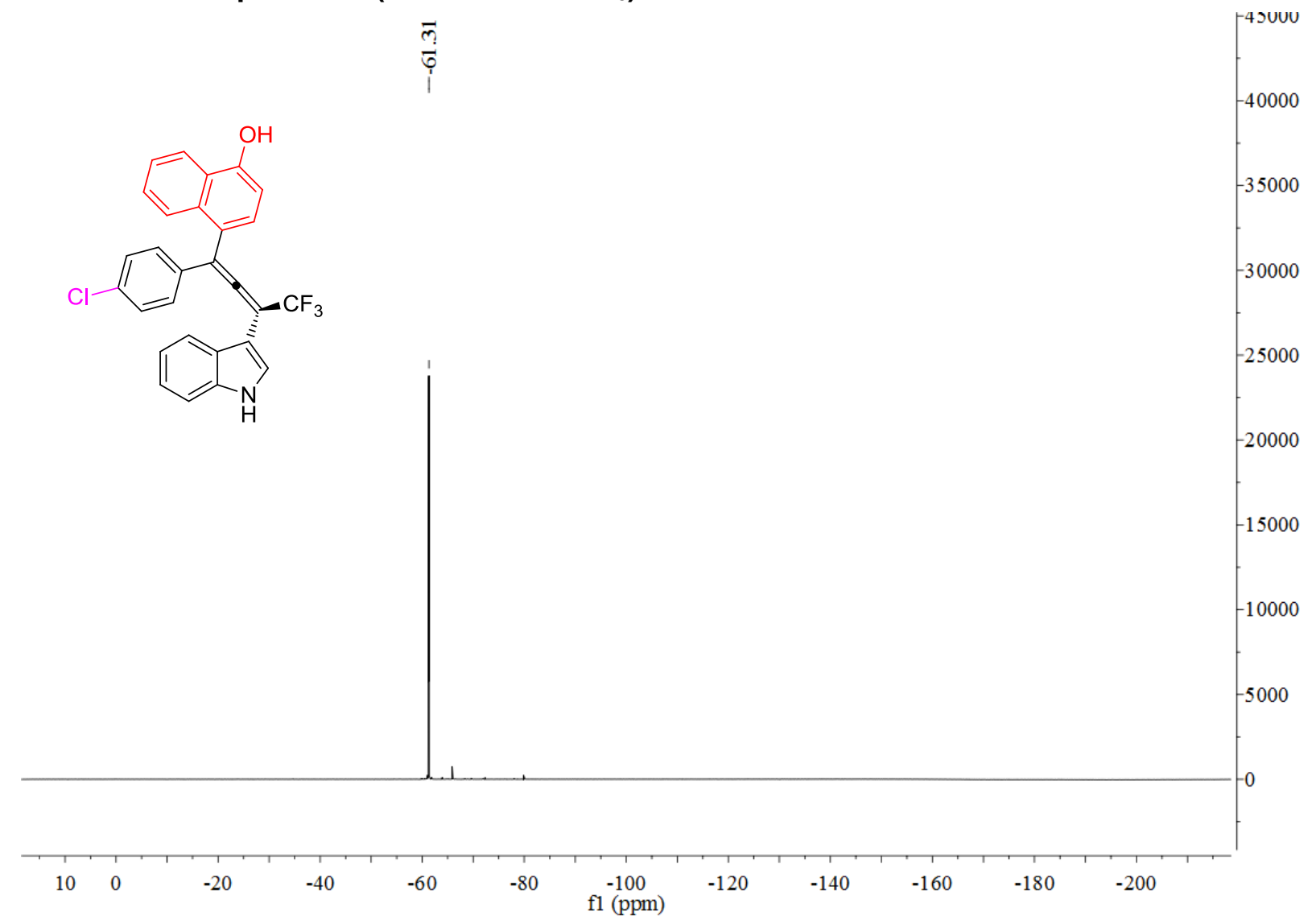


${ }^{1} \mathrm{H}$ NMR of compound $3 \mathrm{I}\left(400 \mathrm{MHz}\right.$ in $\mathrm{CDCl}_{3}$ )

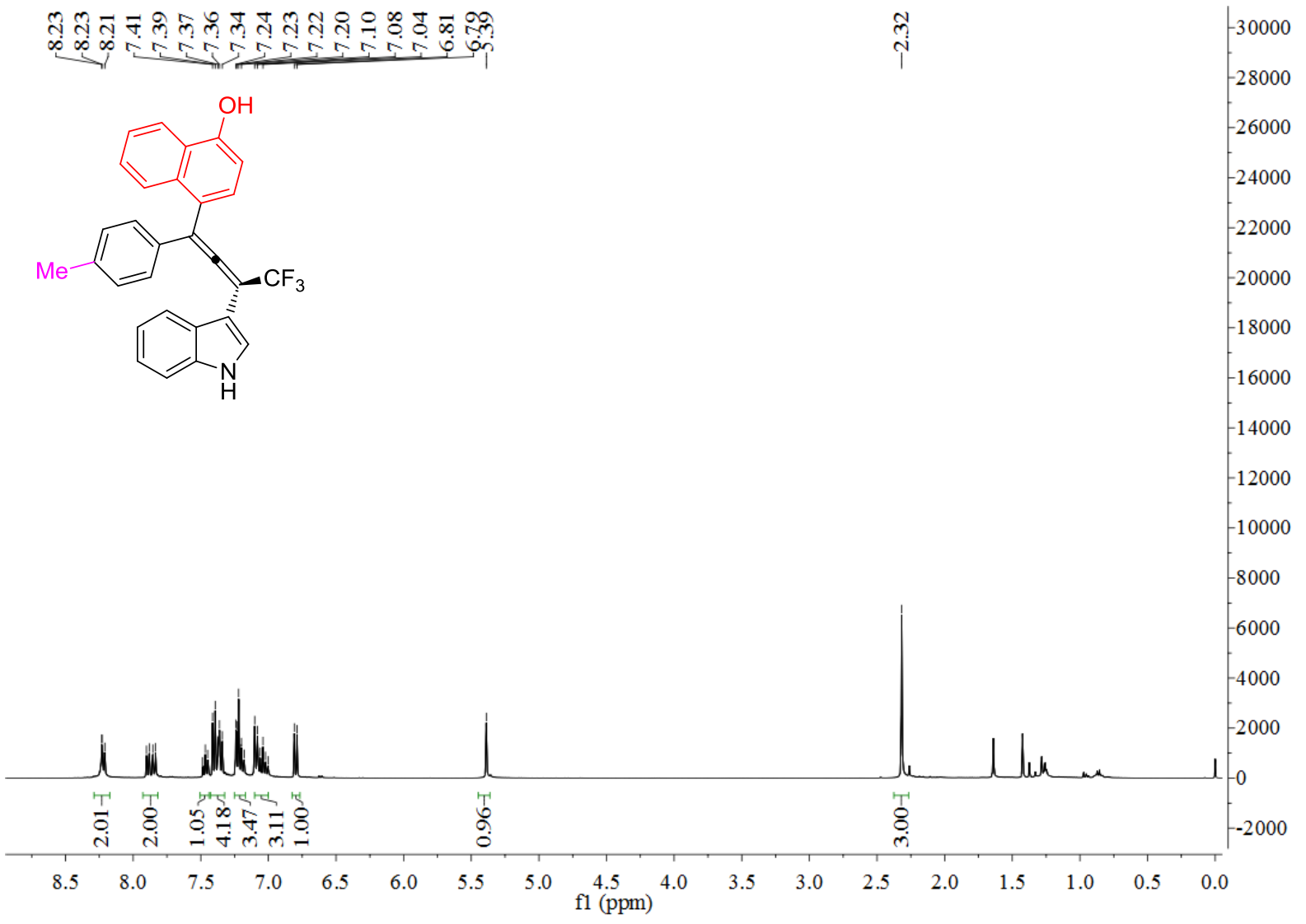

${ }^{13} \mathrm{C}$ NMR of compound 3I (126 MHz in $\mathrm{CDCl}_{3}$ )

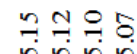

สู่ ถู่

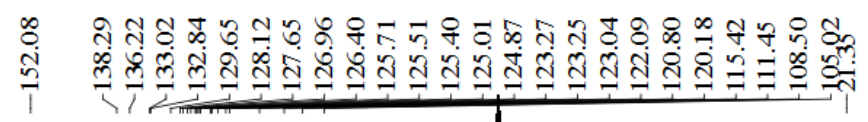<smiles>Cc1ccc(C(=CC(=C(C(F)(F)F)C(F)(F)F)c2c[nH]c3ccccc23)c2ccc(O)c3ccccc23)cc1</smiles>

o ouv

8000

7500

$-7000$

6500

$-6000$

5500

5000

$-4500$

$-4000$

3500

$-3000$

$-2500$

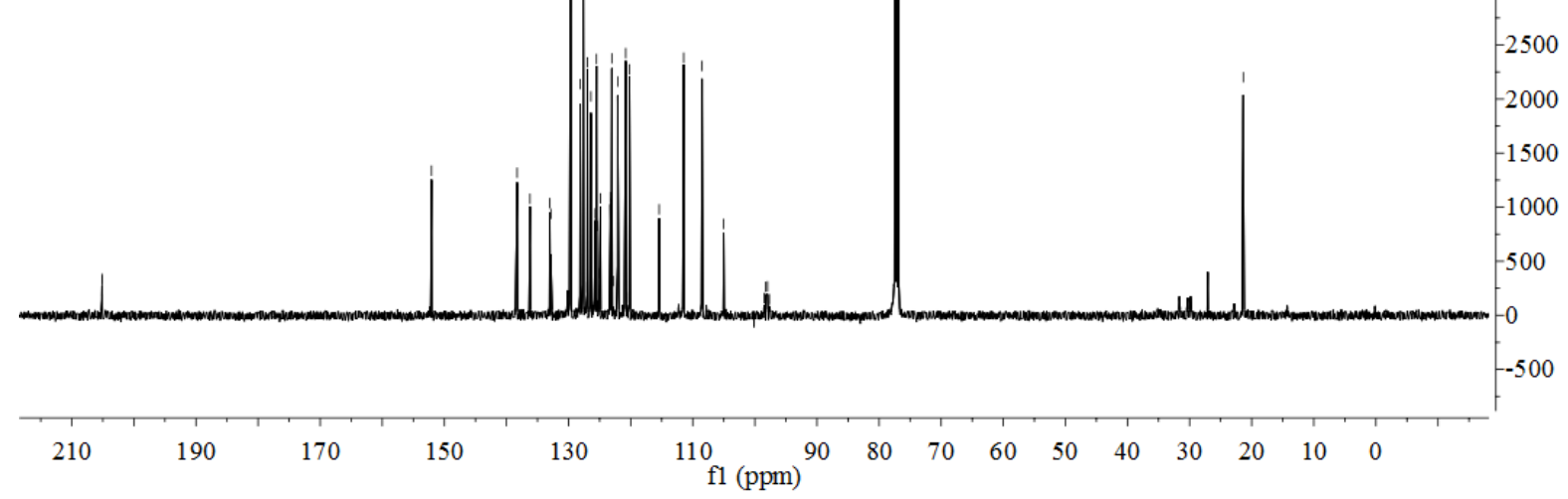




\section{${ }^{19} \mathrm{~F} \mathrm{NMR}$ of compound $3 \mathrm{l}\left(376 \mathrm{MHz}\right.$ in $\mathrm{CDCl}_{3}$ )}

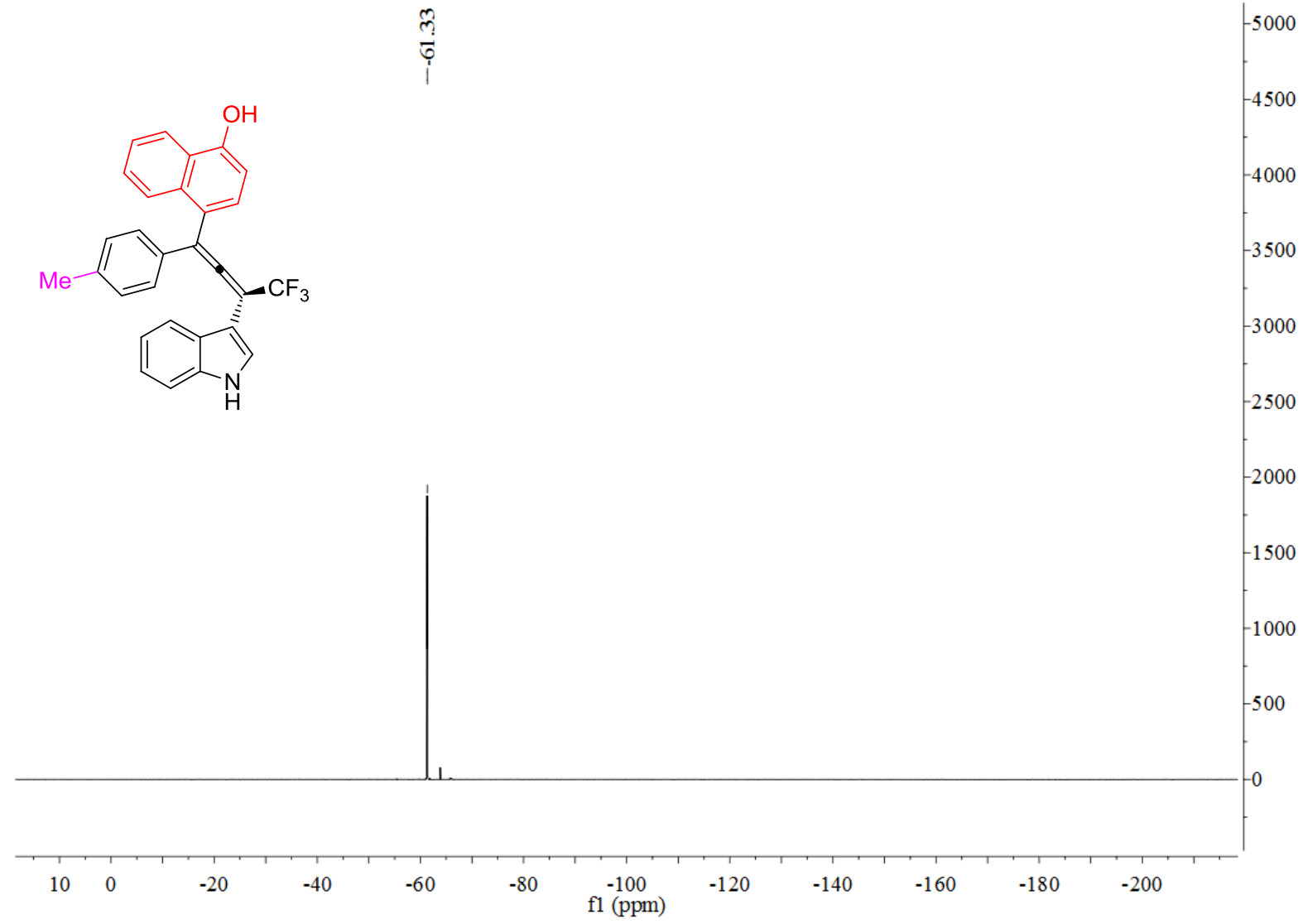

${ }^{1} \mathrm{H}$ NMR of compound $3 \mathrm{~m}\left(400 \mathrm{MHz}\right.$ in $\left.\mathrm{CDCl}_{3}\right)$

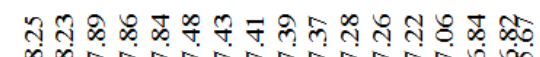

क

$\infty$<smiles>COc1ccc(C(=CC(=C(C(F)(F)F)C(F)(F)F)c2c[nH]c3ccccc23)c2ccc(O)c3ccccc23)cc1</smiles> 
${ }^{13} \mathrm{C}$ NMR of compound $3 \mathrm{~m}$ (126 $\mathrm{MHz}$ in $\mathrm{CDCl}_{3}$ )

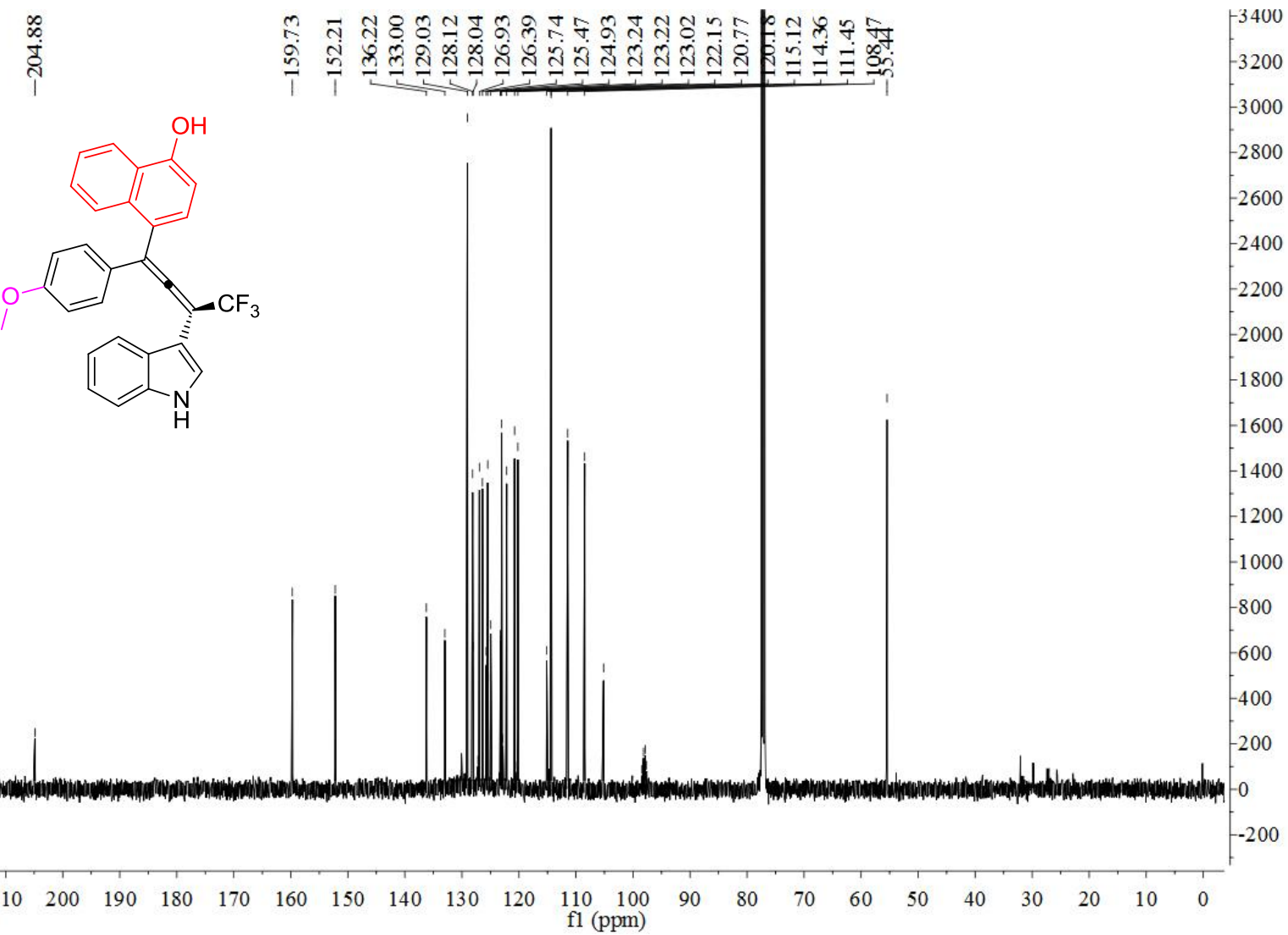

${ }^{19} \mathrm{~F} \mathrm{NMR}$ of compound $3 \mathrm{~m}\left(376 \mathrm{MHz}\right.$ in $\left.\mathrm{CDCl}_{3}\right)$

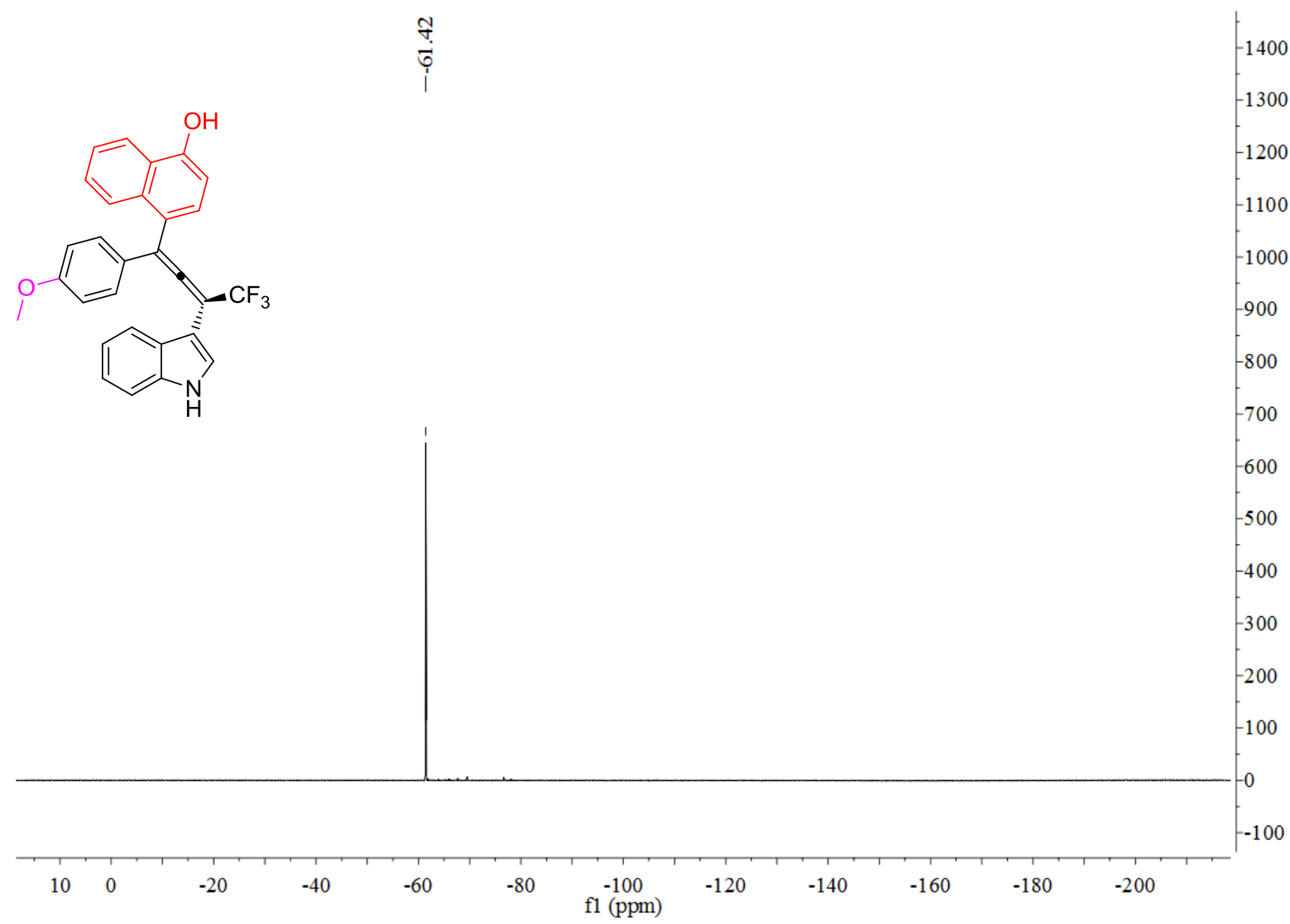


${ }^{1} \mathrm{H}$ NMR of compound $3 \mathrm{n}\left(400 \mathrm{MHz}\right.$ in $\left.\mathrm{CDCl}_{3}\right)$

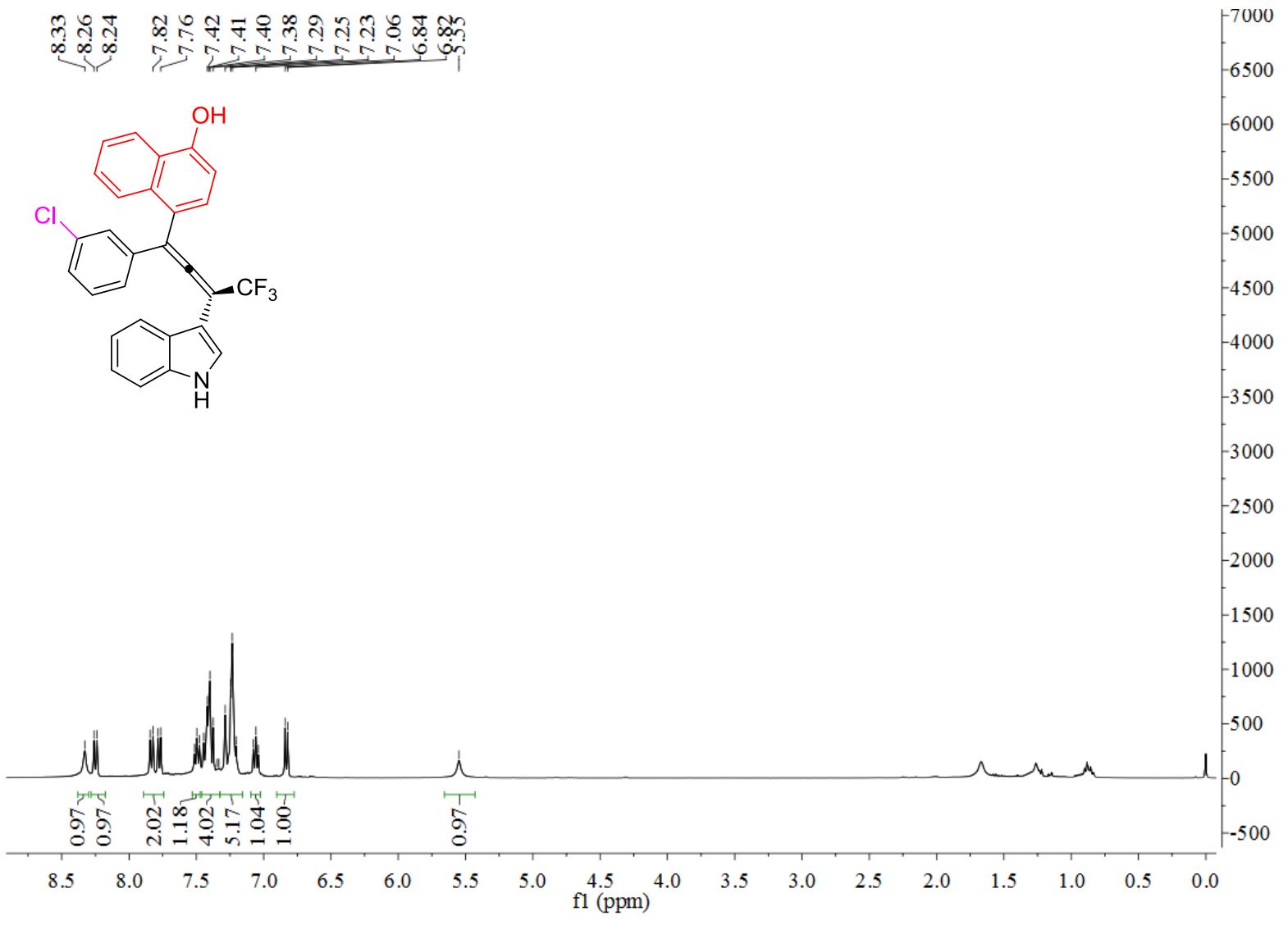

${ }^{13} \mathrm{C}$ NMR of compound 3n (126 $\mathrm{MHz}$ in $\left.\mathrm{CDCl}_{3}\right)$

กับ

ஸे

ก<smiles>Oc1ccc(C(=CC(=C(c2cccc(Cl)c2)c2c[nH]c3ccccc23)C(F)(F)F)C(F)(F)F)c2ccccc12</smiles>

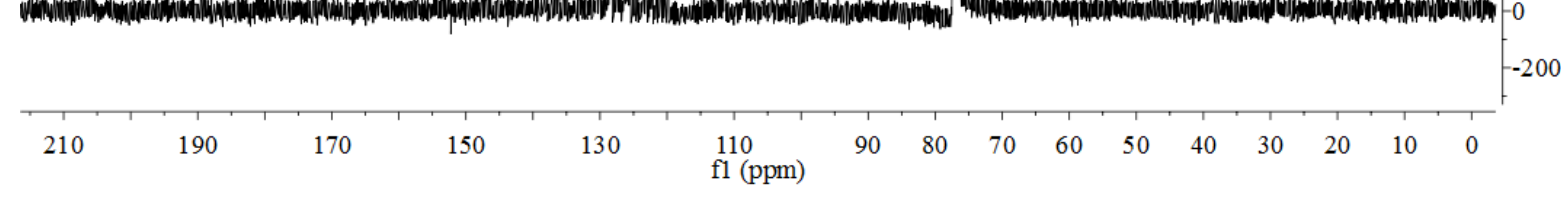


${ }^{19} \mathrm{~F}$ NMR of compound 3n (376 $\mathrm{MHz}$ in $\mathrm{CDCl}_{3}$ )

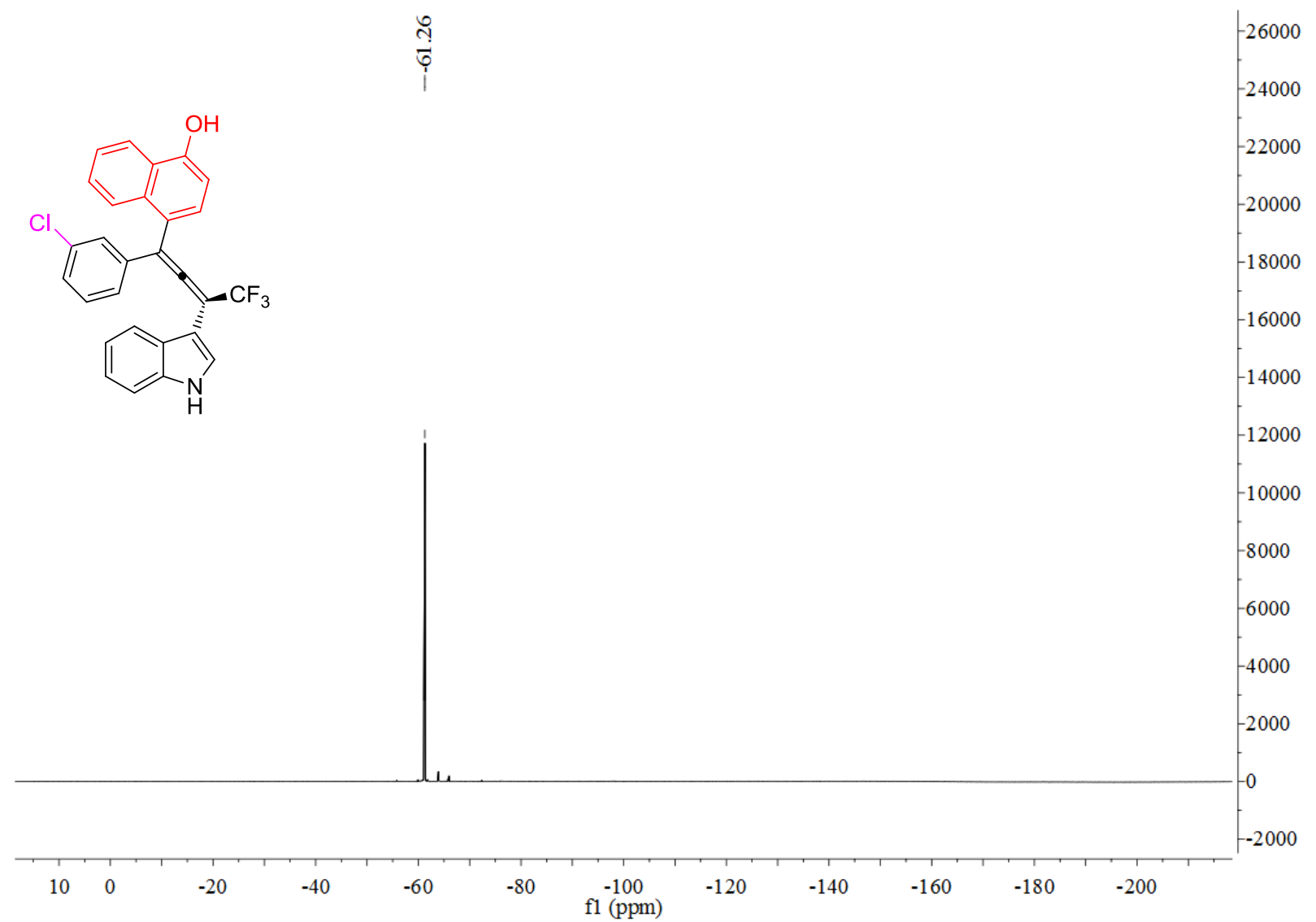

${ }^{1} \mathrm{H}$ NMR of compound $30\left(400 \mathrm{MHz}\right.$ in $\left.\mathrm{CDCl}_{3}\right)$

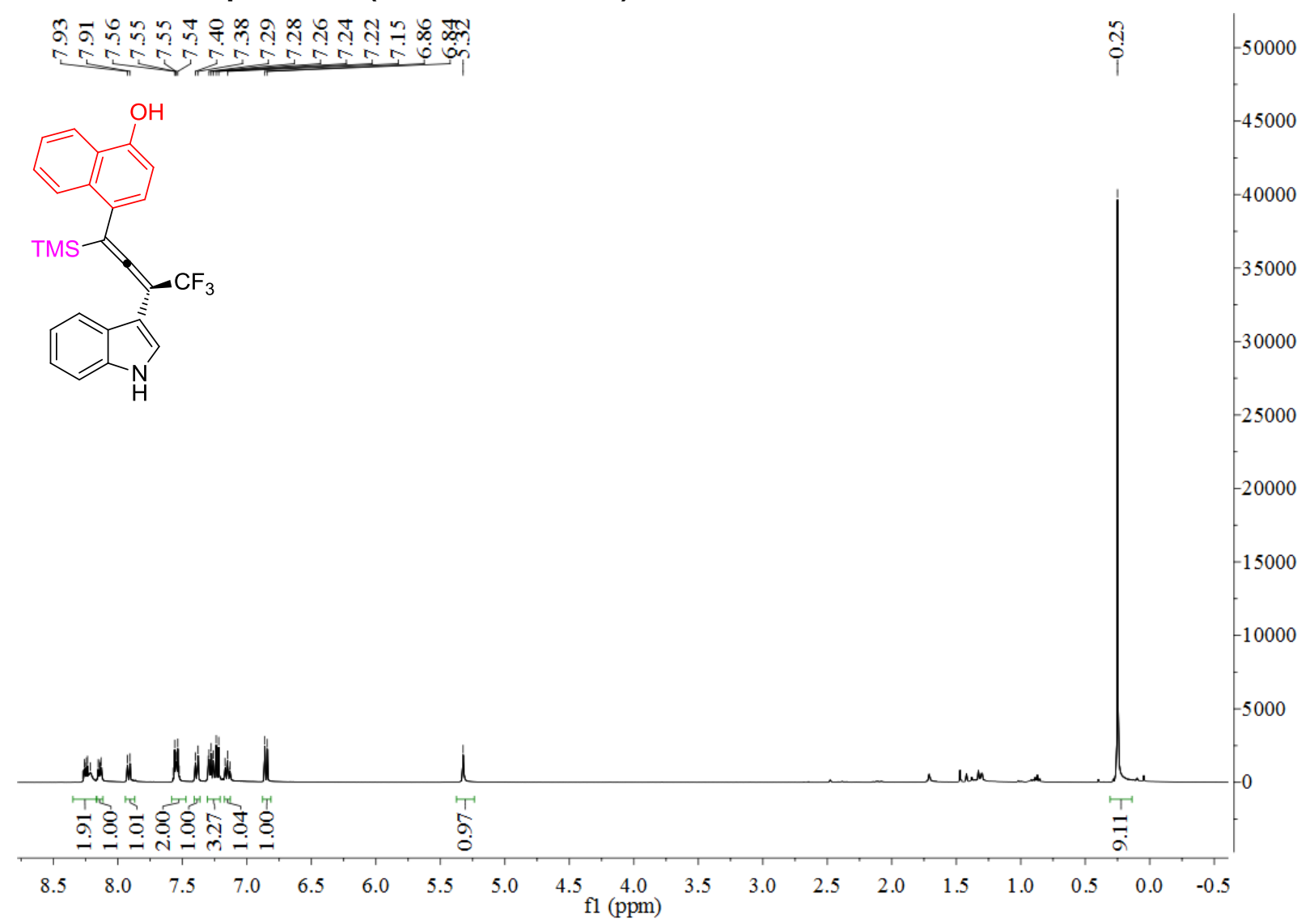


${ }^{13} \mathrm{C}$ NMR of compound 30 (126 $\mathrm{MHz}$ in $\mathrm{CDCl}_{3}$ )

ถे

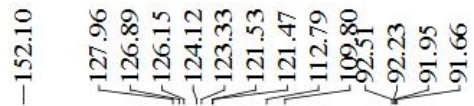<smiles>CS(=O)(=O)c1ccc(O)c2ccccc12</smiles>

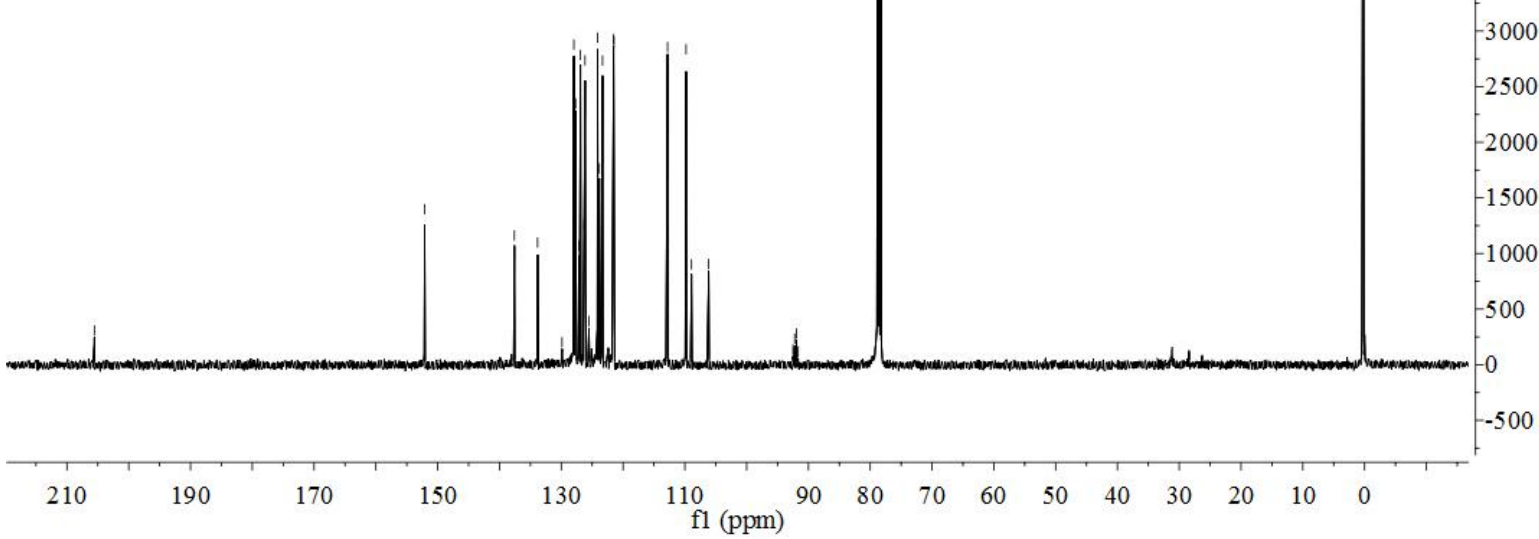

${ }^{19} \mathrm{~F} \mathrm{NMR}$ of compound 30 (376 $\mathrm{MHz}$ in $\mathrm{CDCl}_{3}$ )

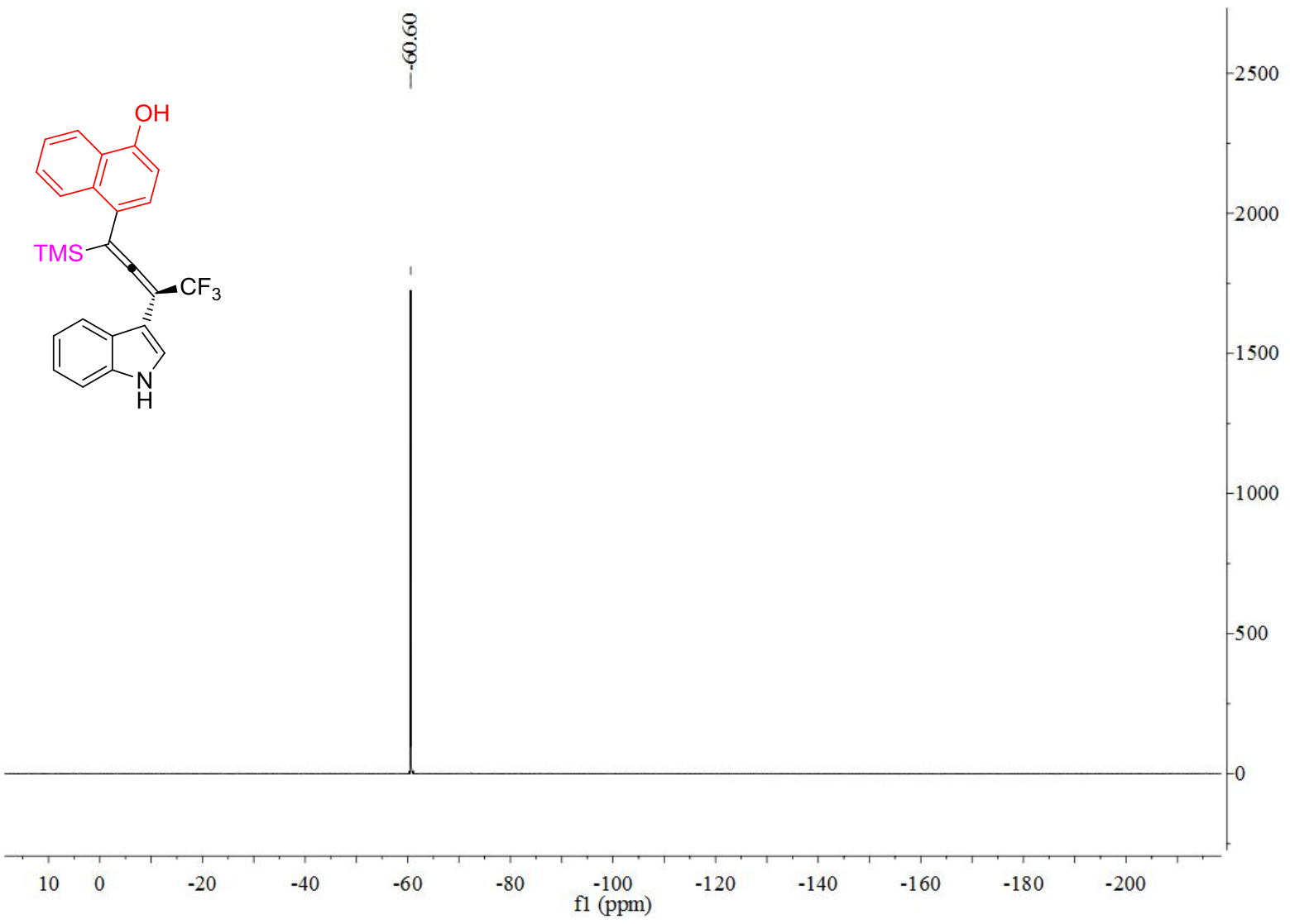




\section{${ }^{1} \mathrm{H}$ NMR of compound 3p (400 $\mathrm{MHz}$ in $\mathrm{CDCl}_{3}$ )}

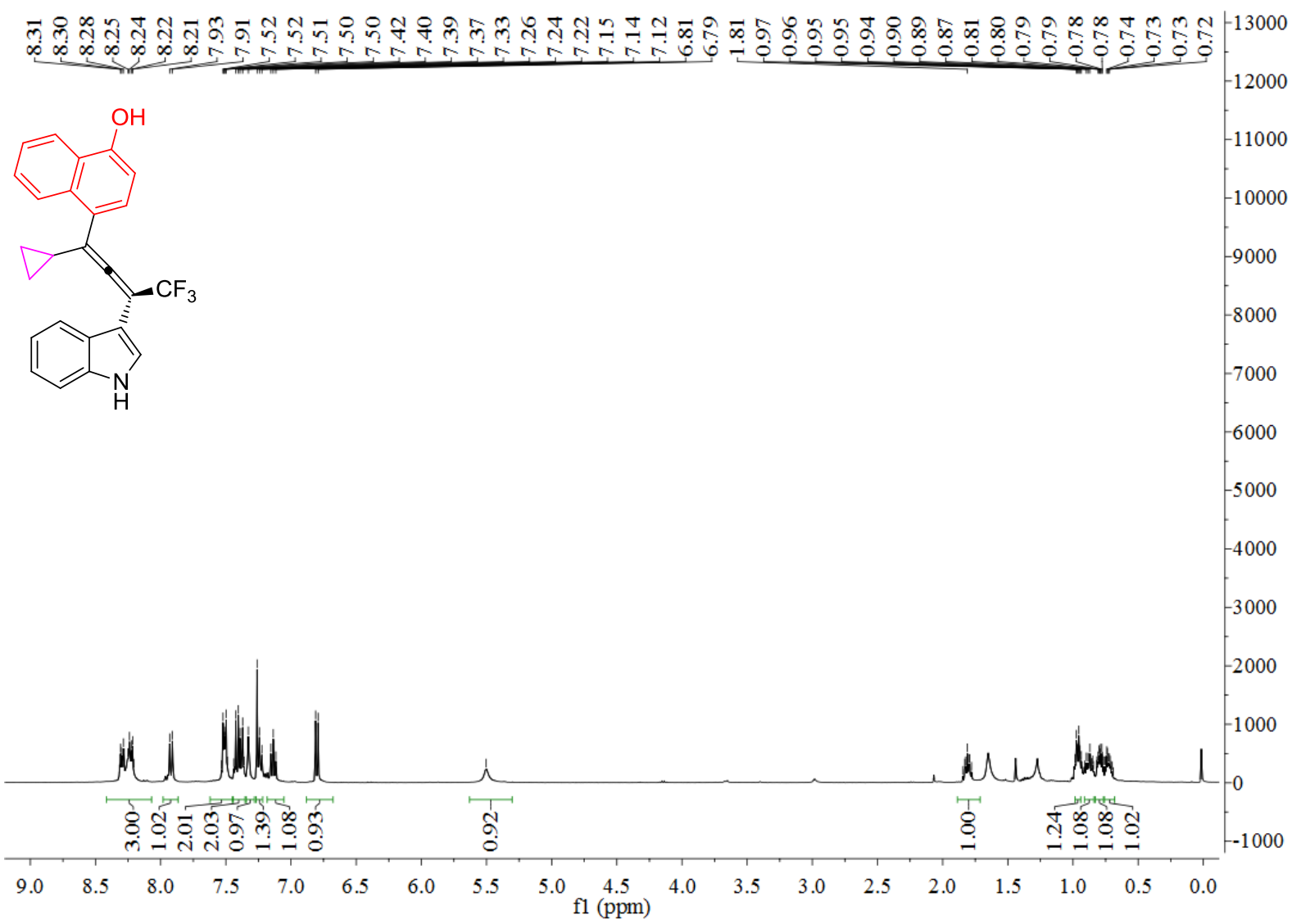

${ }^{13} \mathrm{C}$ NMR of compound 3p (126 $\mathrm{MHz}$ in $\mathrm{CDCl}_{3}$ )

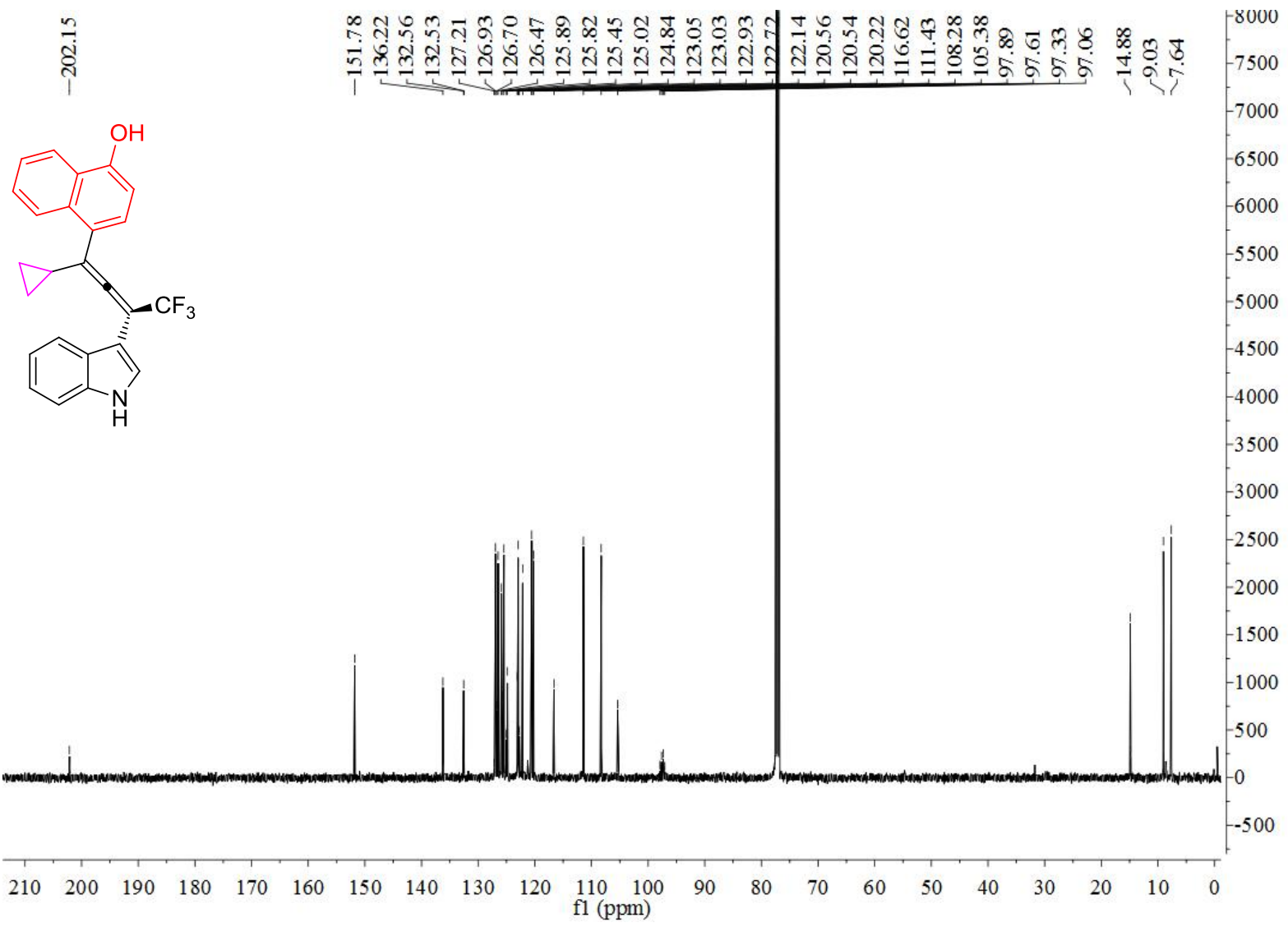


${ }^{19} \mathrm{~F}$ NMR of compound 3p (376 $\mathrm{MHz}$ in $\mathrm{CDCl}_{3}$ )

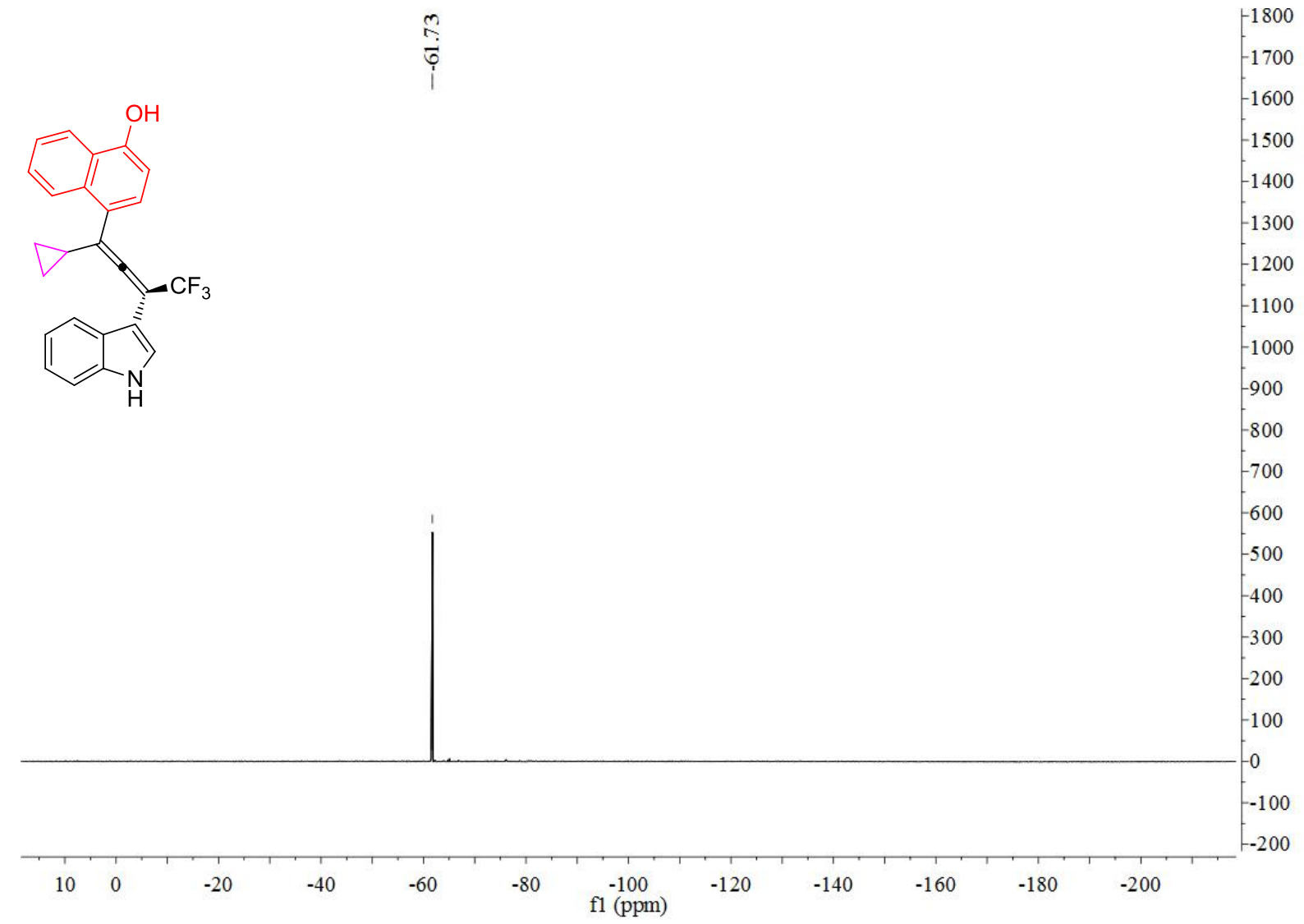

${ }^{1} \mathrm{H}$ NMR of compound $3 q\left(400 \mathrm{MHz}\right.$ in $\left.\mathrm{CDCl}_{3}\right)$

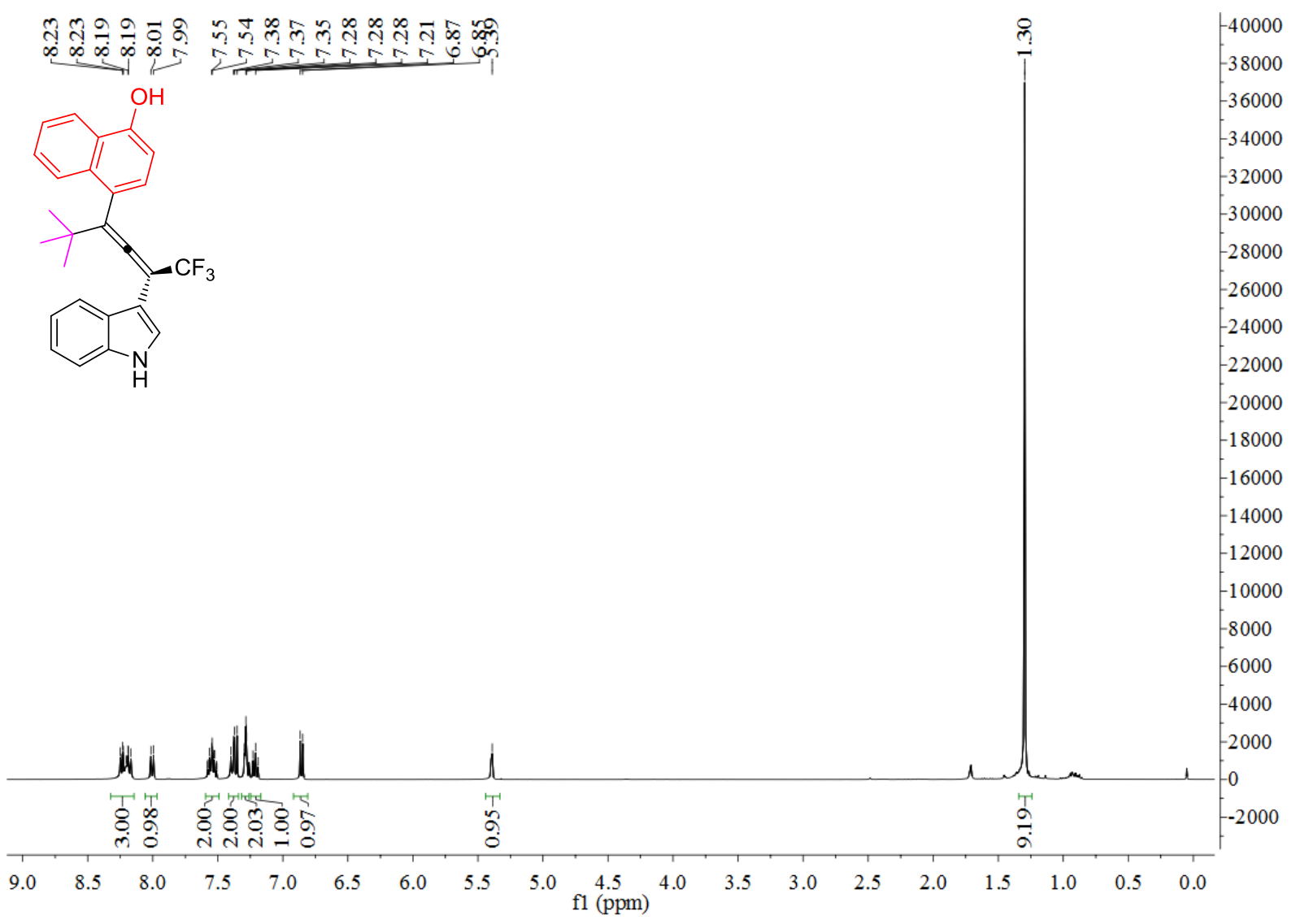


${ }^{13} \mathrm{C}$ NMR of compound $3 q\left(126 \mathrm{MHz}\right.$ in $\mathrm{CDCl}_{3}$ )

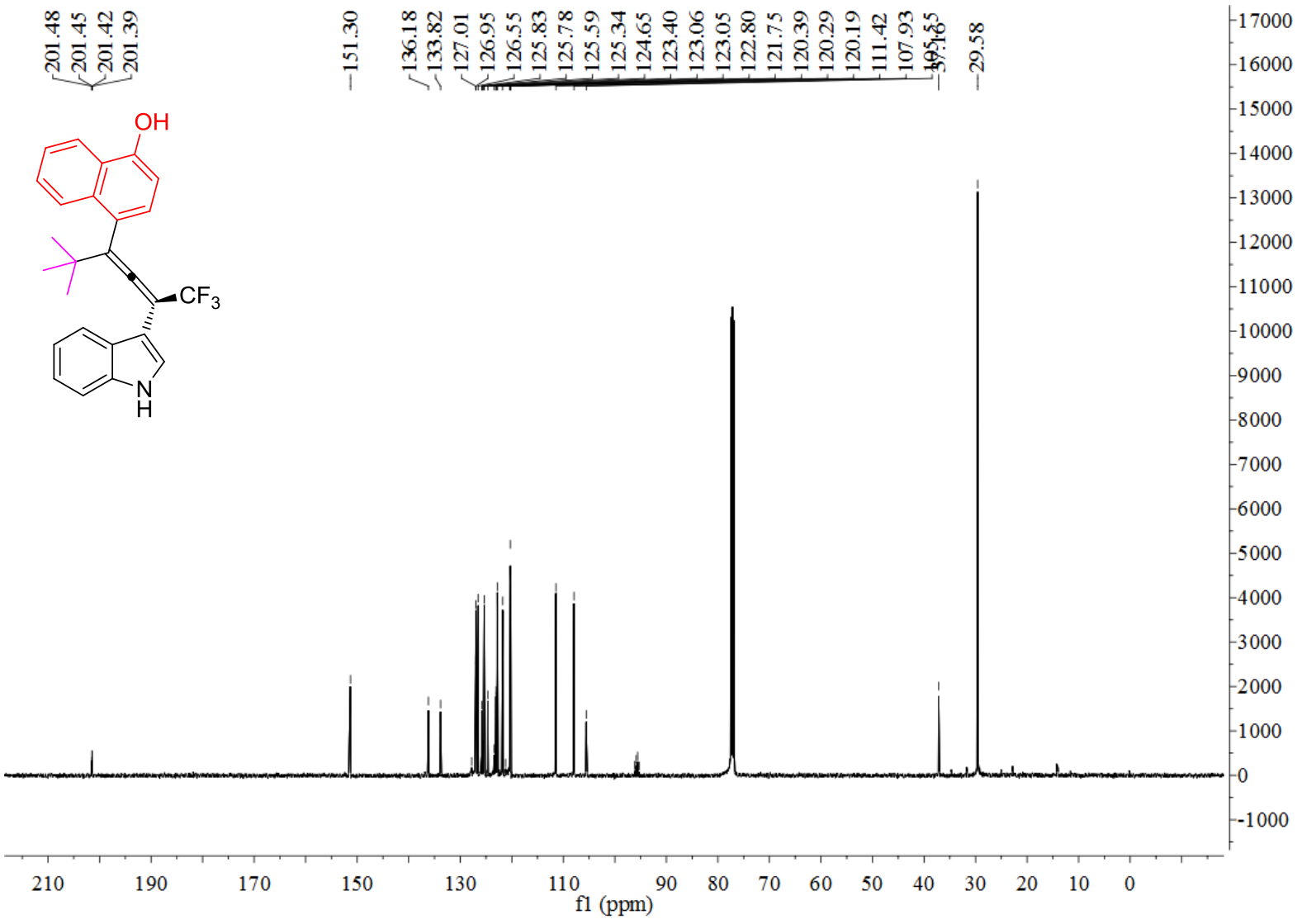

${ }^{19} \mathrm{~F} \mathrm{NMR}$ of compound $3 \mathrm{q}\left(376 \mathrm{MHz}\right.$ in $\mathrm{CDCl}_{3}$ )

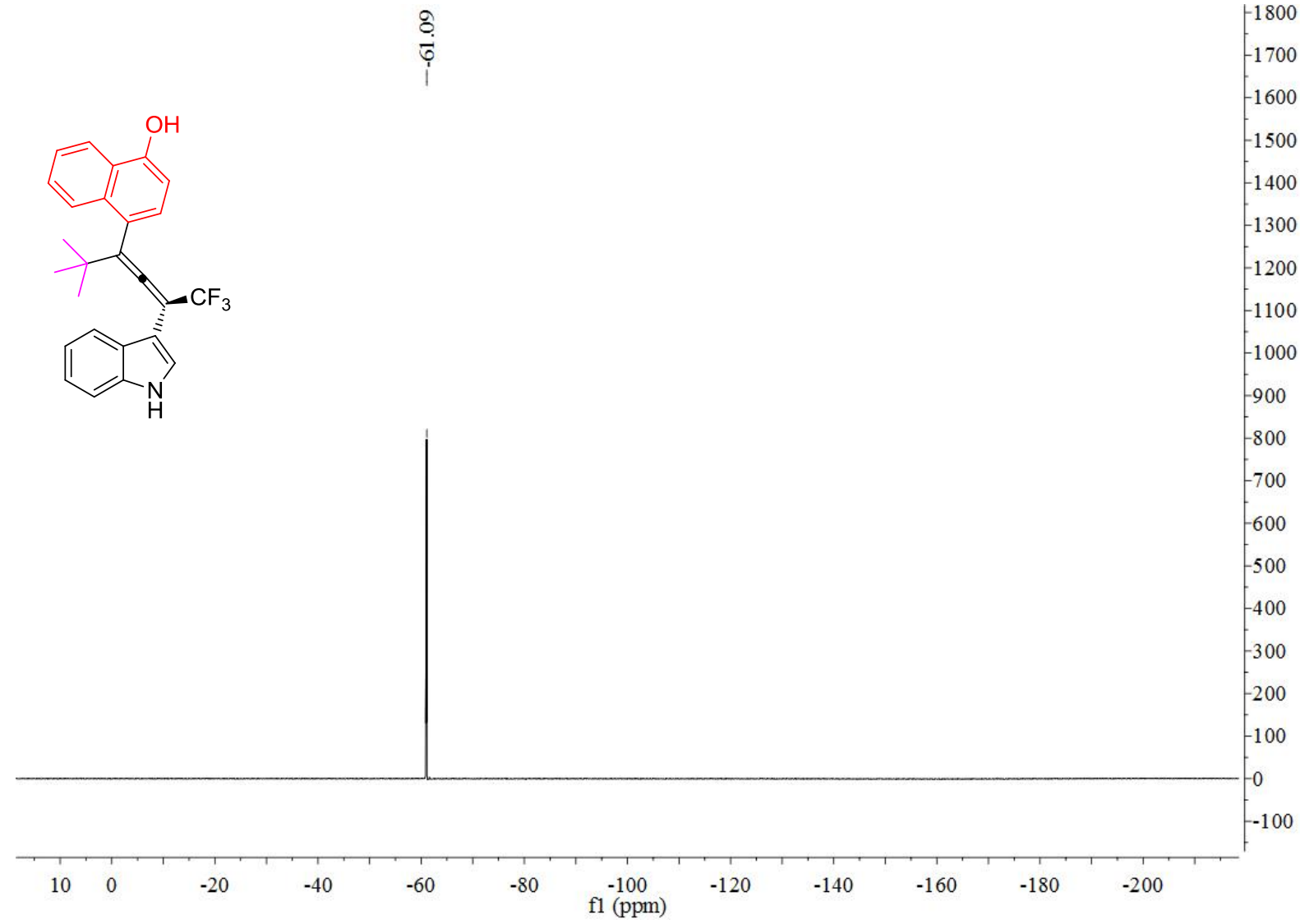




\section{${ }^{1} \mathrm{H}$ NMR of compound $3 \mathrm{r}\left(400 \mathrm{MHz}\right.$ in $\left.\mathrm{CDCl}_{3}\right)$}

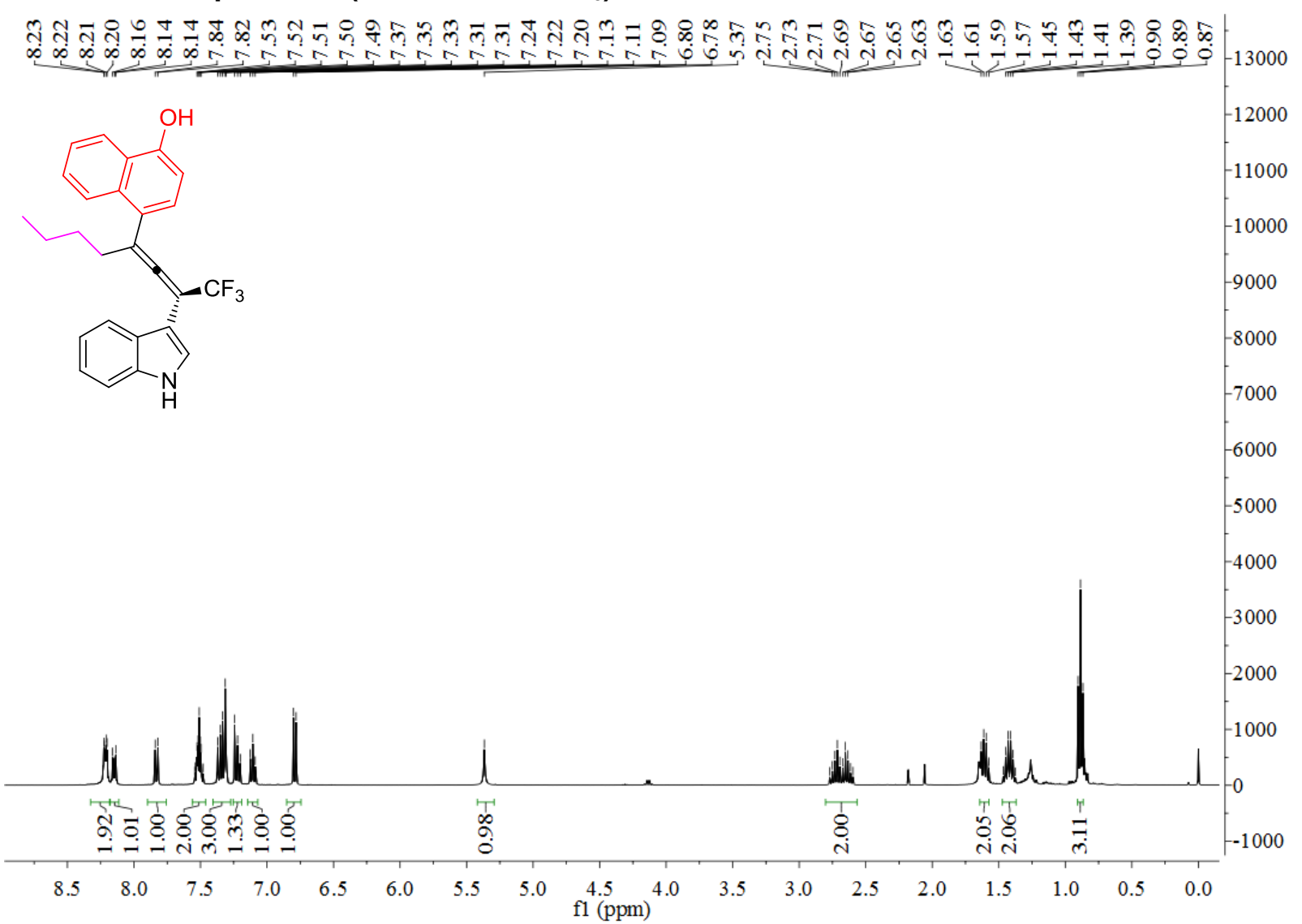

${ }^{13} \mathrm{C} \mathrm{NMR}$ of compound $3 \mathrm{r}\left(126 \mathrm{MHz}\right.$ in $\mathrm{CDCl}_{3}$ )

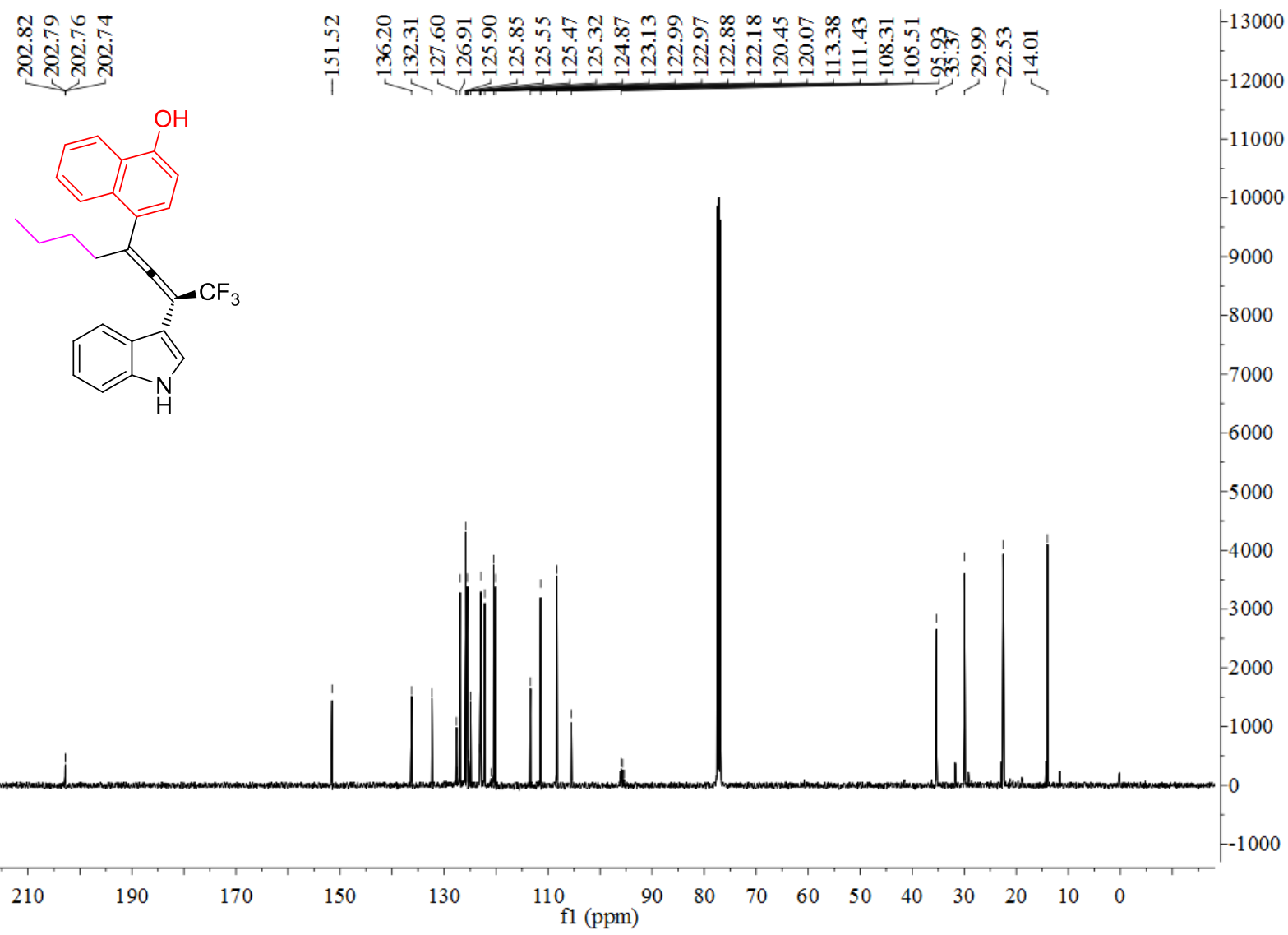


${ }^{19} \mathrm{~F}$ NMR of compound $3 \mathrm{r}\left(376 \mathrm{MHz}\right.$ in $\left.\mathrm{CDCl}_{3}\right)$

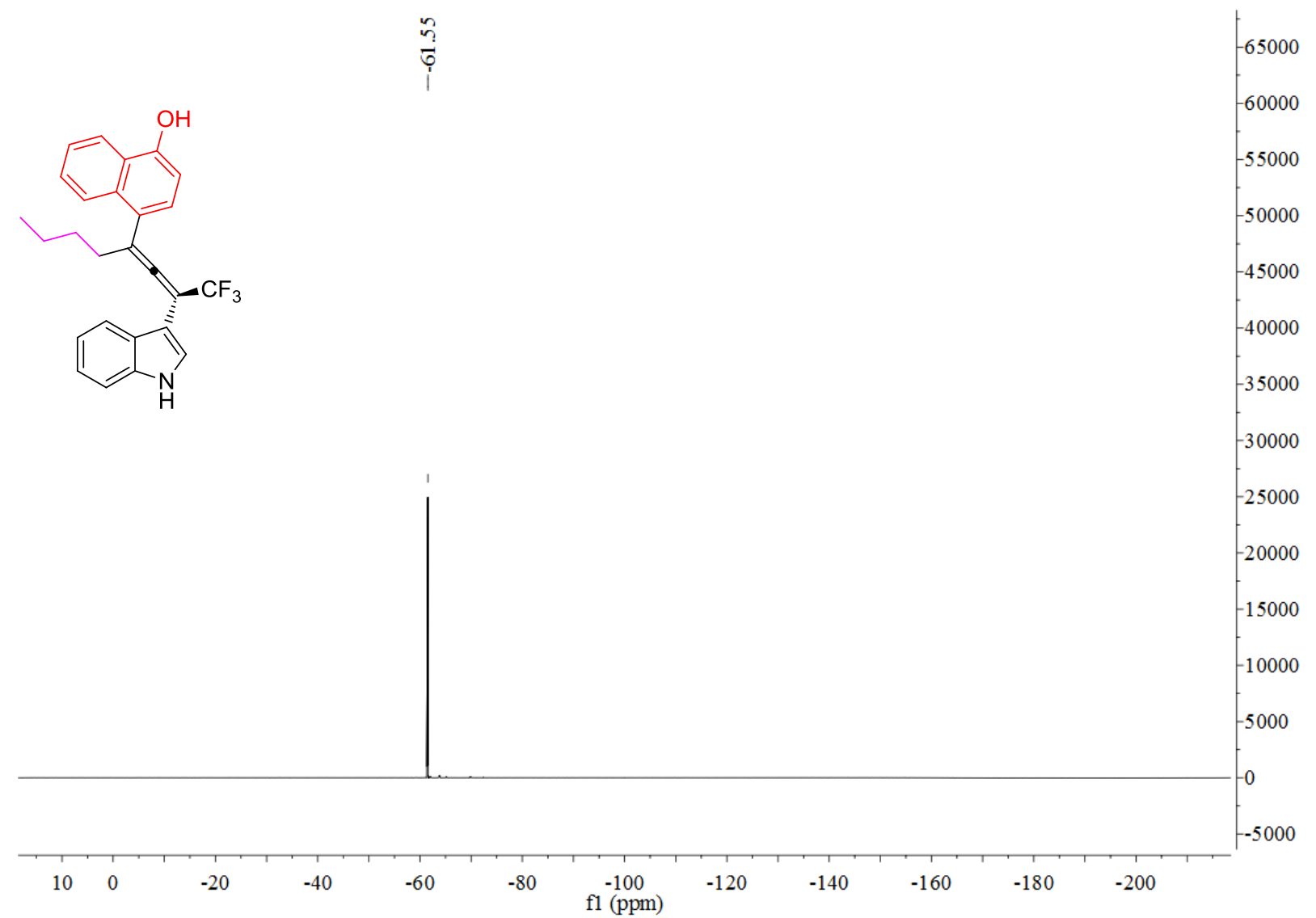

${ }^{1} \mathrm{H}$ NMR of compound $3 \mathrm{~s}$ (400 $\mathrm{MHz}$ in $\mathrm{CDCl}_{3}$ )

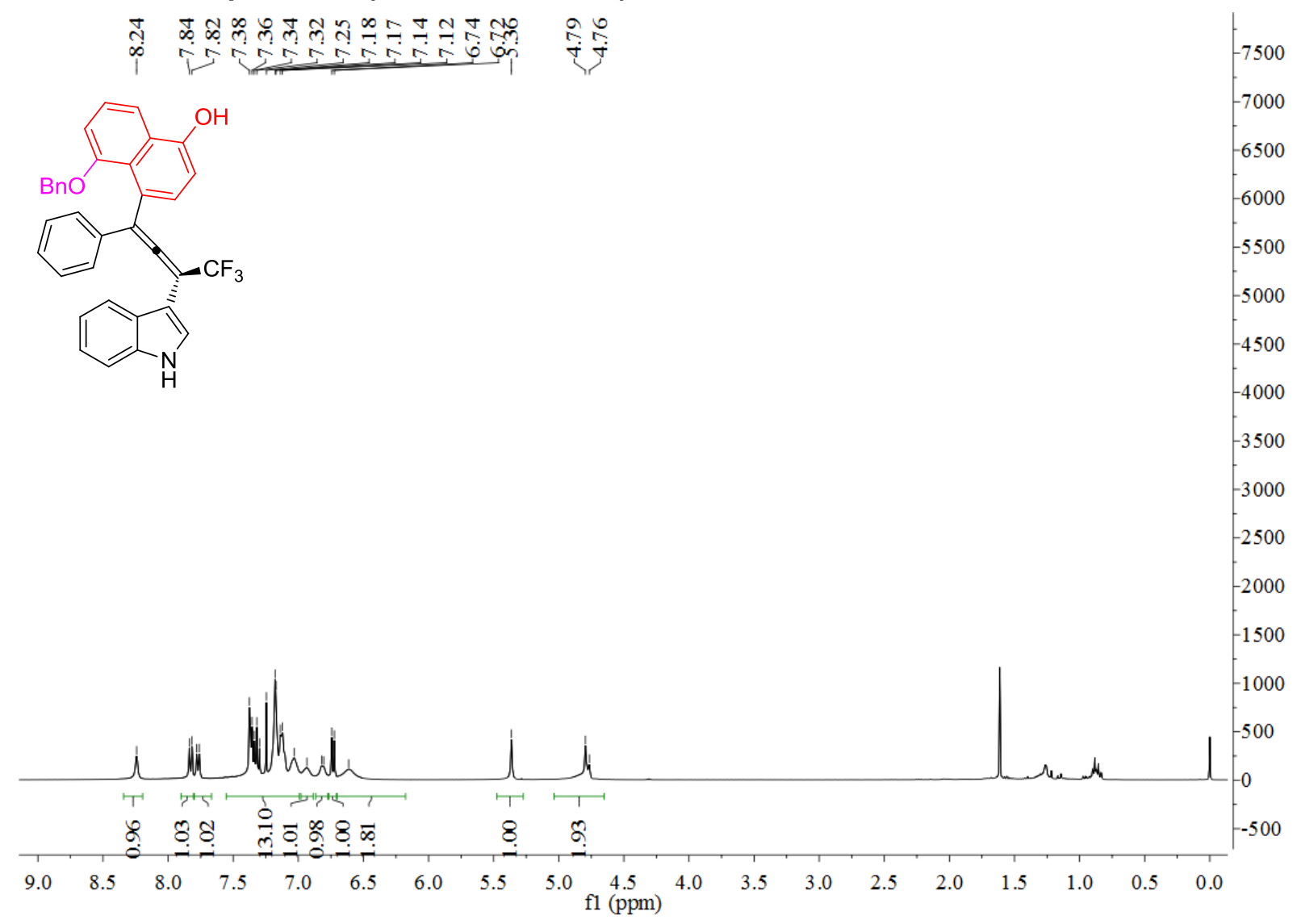


${ }^{13} \mathrm{C}$ NMR of compound $3 \mathrm{~s}$ (126 $\mathrm{MHz}$ in $\mathrm{CDCl}_{3}$ )

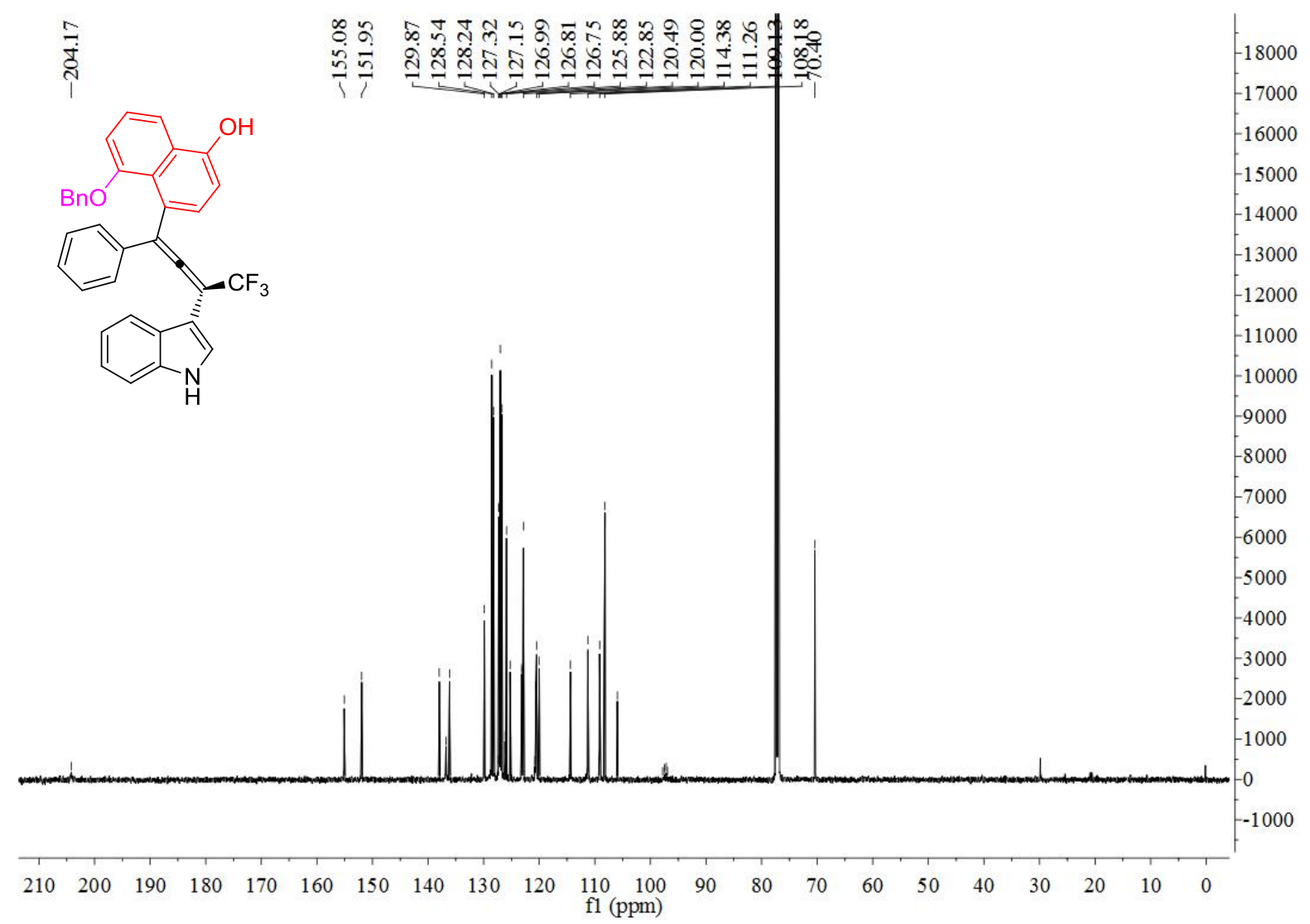

${ }^{19} \mathrm{~F}$ NMR of compound $3 \mathrm{~s}$ (376 $\mathrm{MHz}$ in $\mathrm{CDCl}_{3}$ )

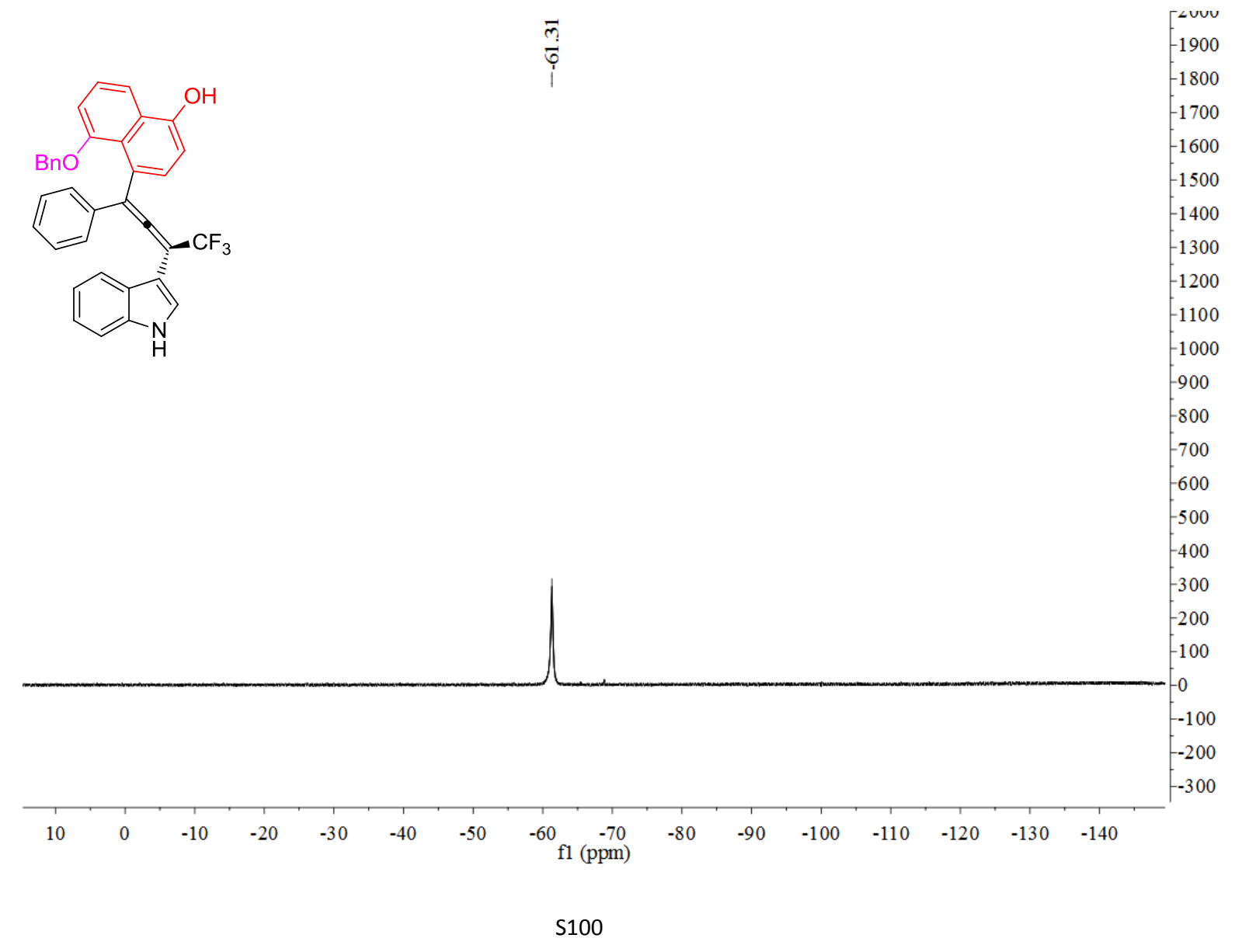


${ }^{1} \mathrm{H}$ NMR of compound $3 \mathrm{t}\left(400 \mathrm{MHz}\right.$ in $\mathrm{CDCl}_{3}$ )

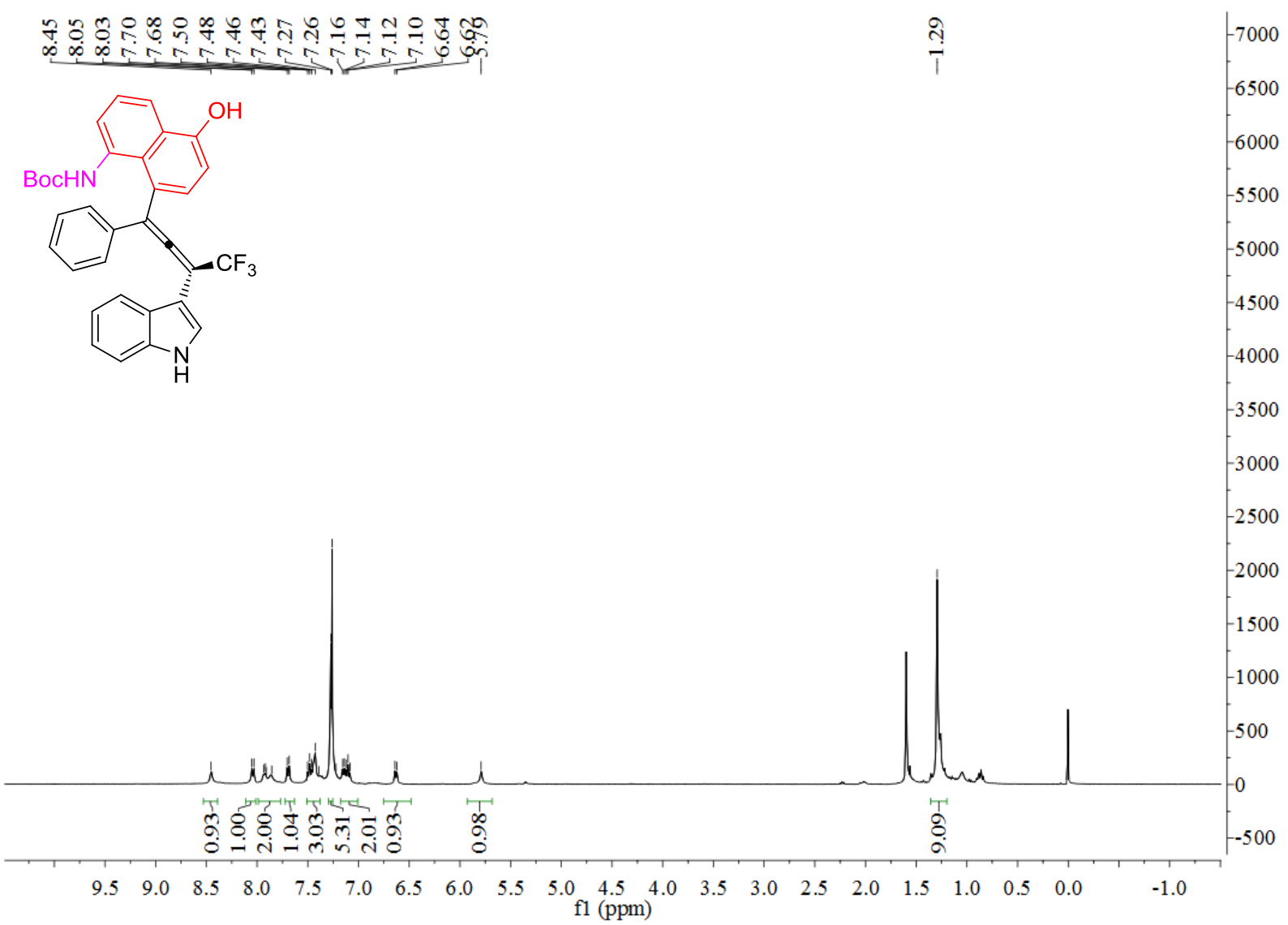

${ }^{13} \mathrm{C}$ NMR of compound 3t (126 $\mathrm{MHz}$ in $\mathrm{CDCl}_{3}$ )

है.

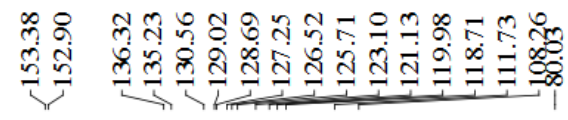<smiles>CC(C)(C)OC(=O)Nc1cccc2c(O)ccc(C(=C=C(c3ccccc3)C(F)(F)F)c3c[nH]c4ccccc34)c12</smiles>
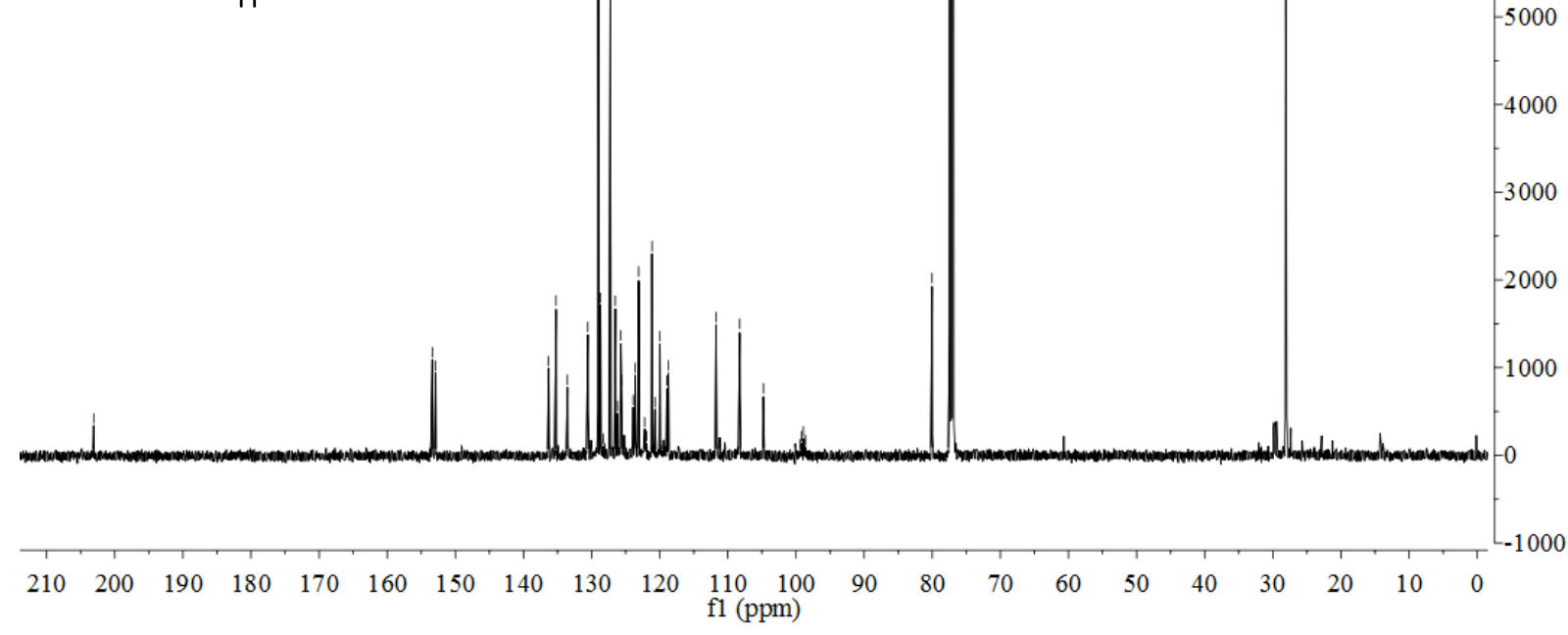
${ }^{19} \mathrm{~F} \mathrm{NMR}$ of compound $3 \mathrm{t}$ (376 $\mathrm{MHz}$ in $\mathrm{CDCl}_{3}$ )

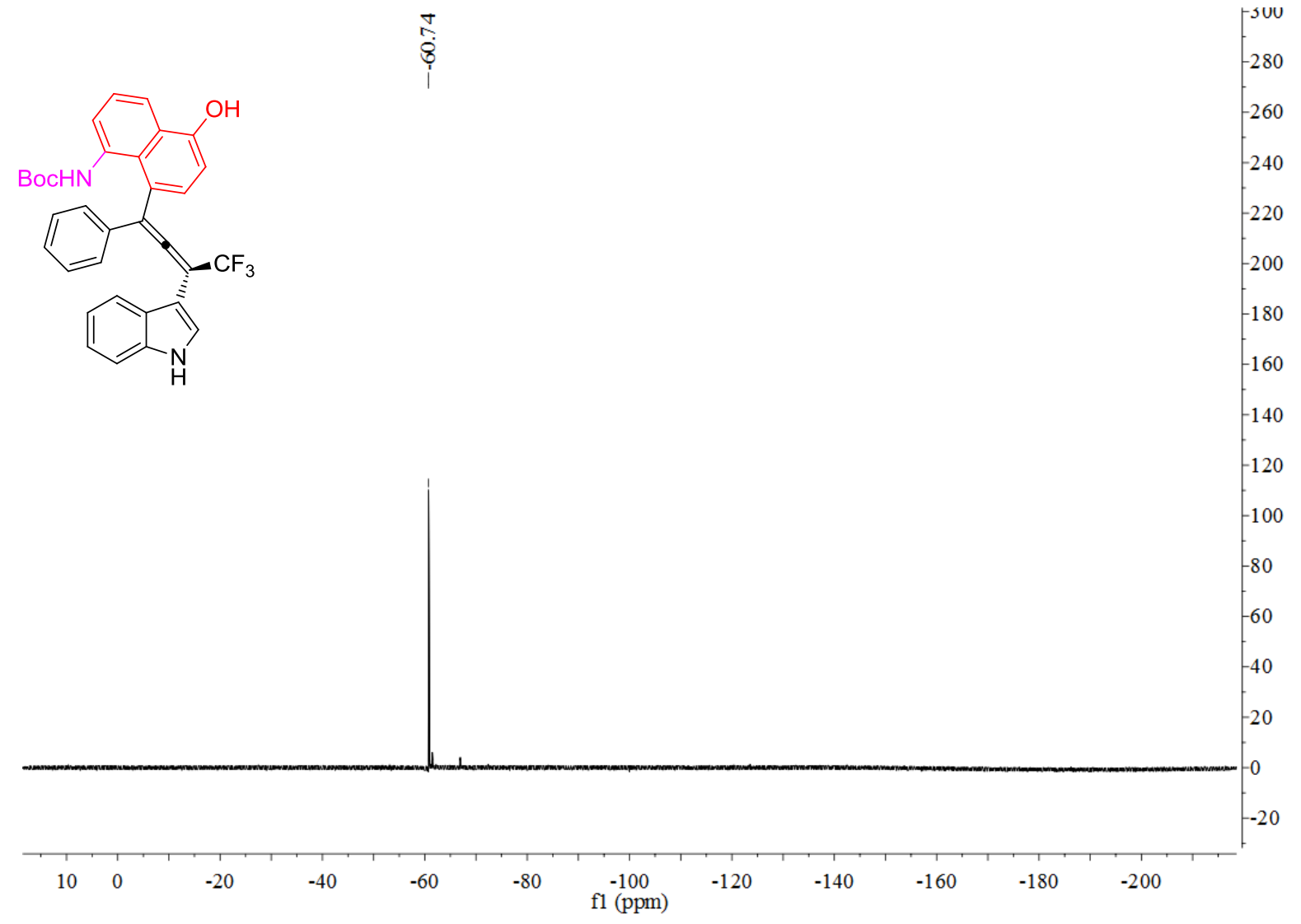

${ }^{1} \mathrm{H}$ NMR of compound $3 \mathrm{u}\left(400 \mathrm{MHz}\right.$ in $\mathrm{CDCl}_{3}$ )
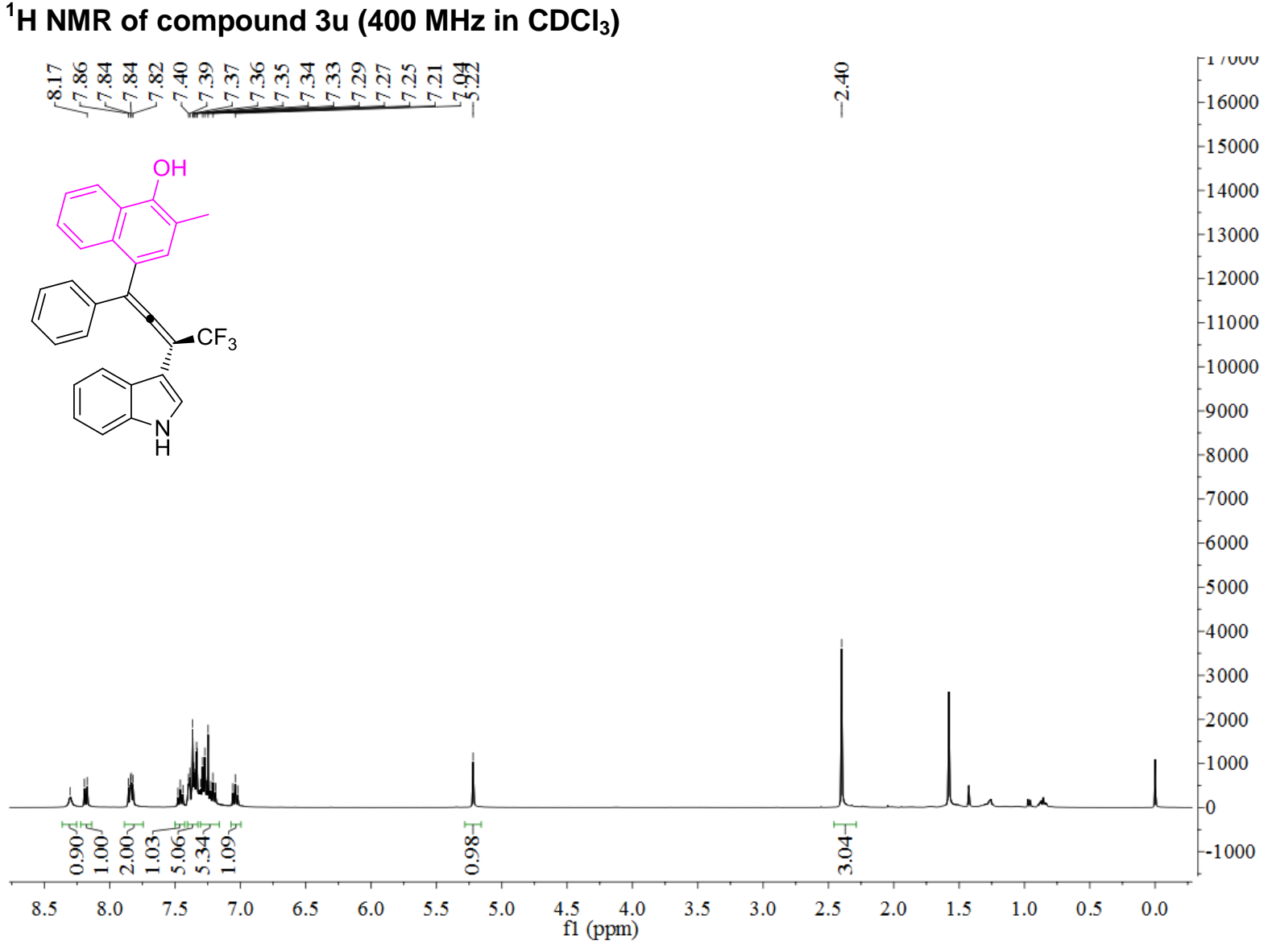


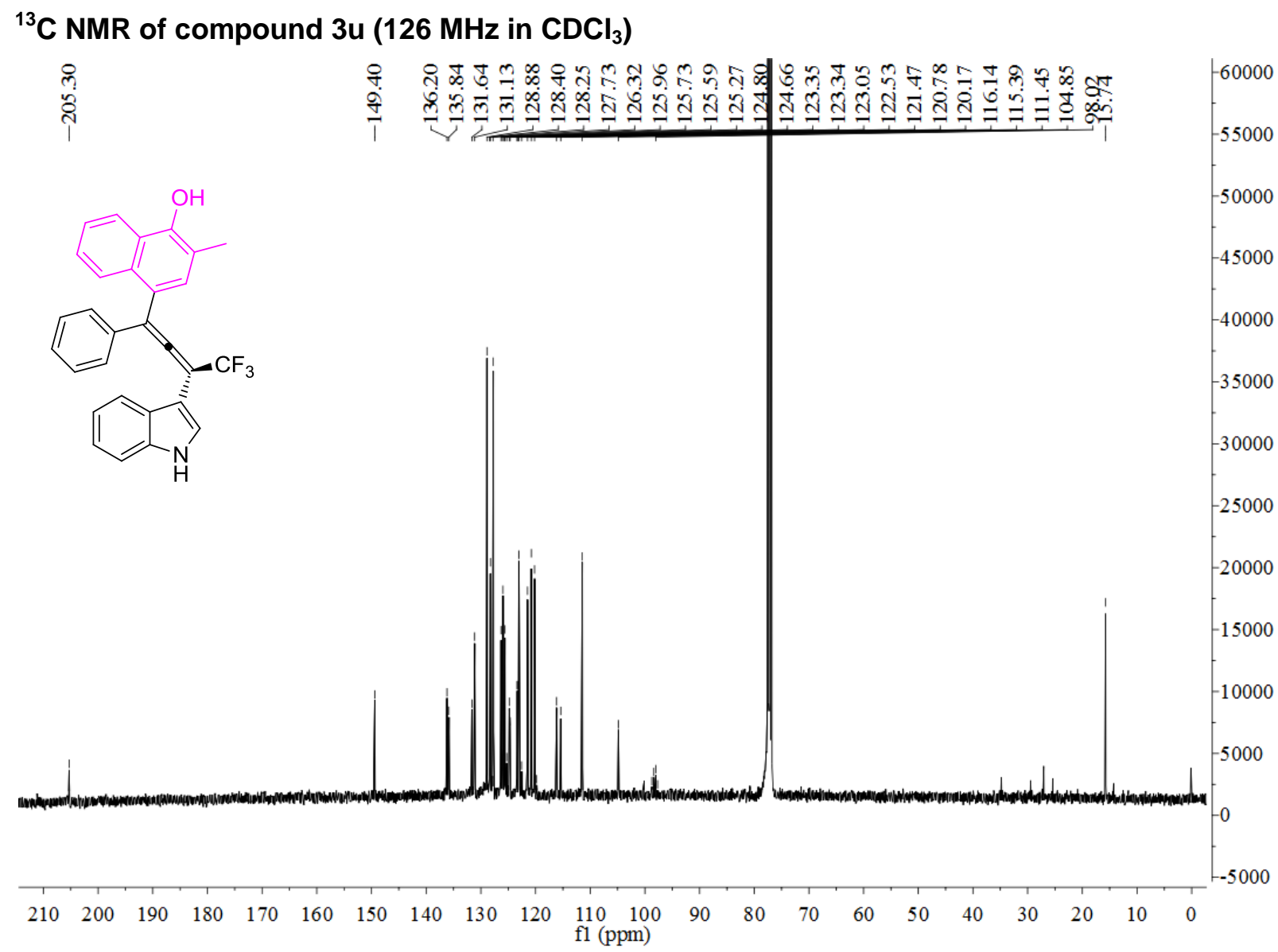

${ }^{19} \mathrm{~F} \mathrm{NMR}$ of compound $3 \mathrm{u}\left(376 \mathrm{MHz}\right.$ in $\mathrm{CDCl}_{3}$ )

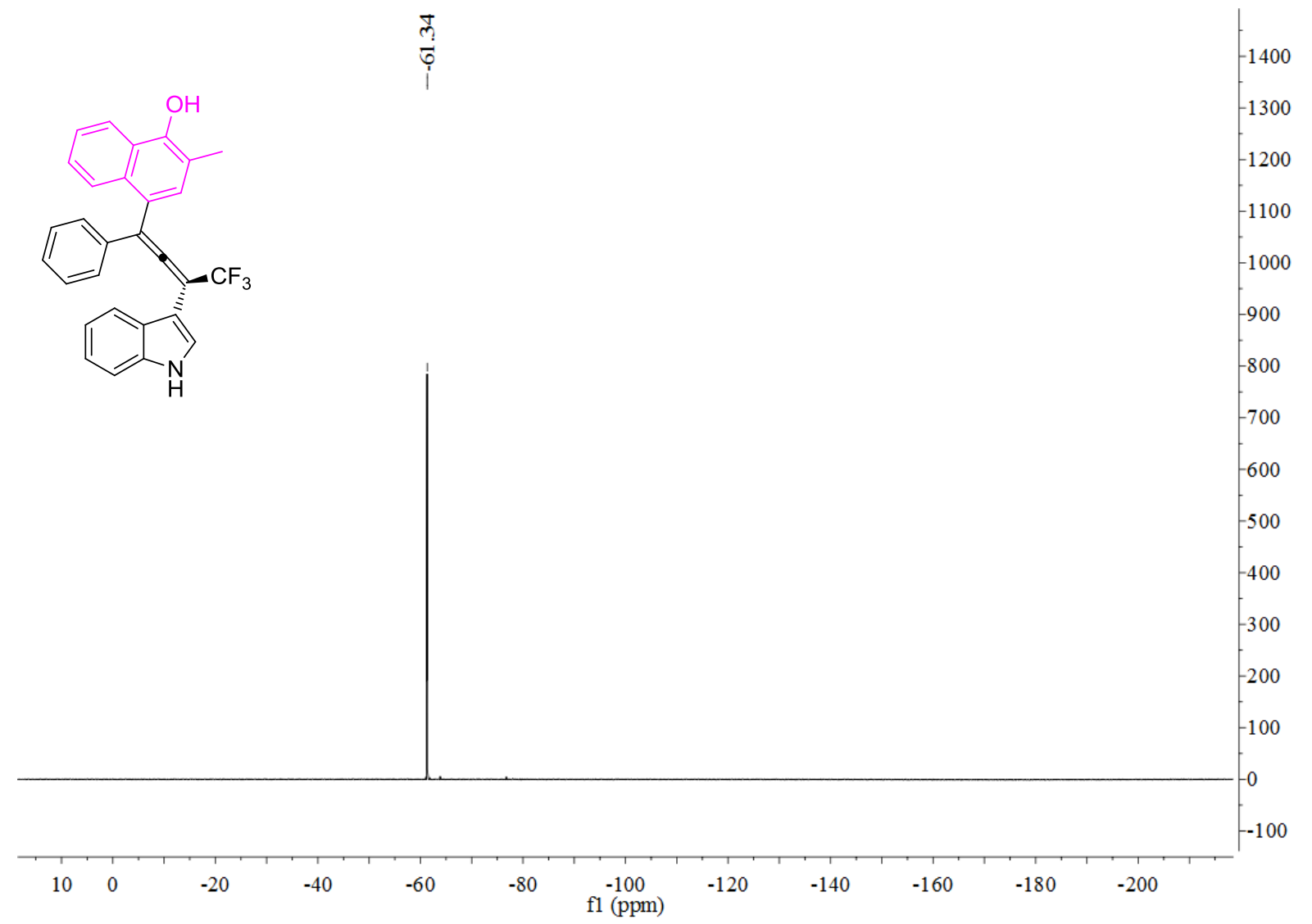


${ }^{1} \mathrm{H}$ NMR of compound $3 \mathrm{v}\left(400 \mathrm{MHz}\right.$ in $\left.\mathrm{CDCl}_{3}\right)$

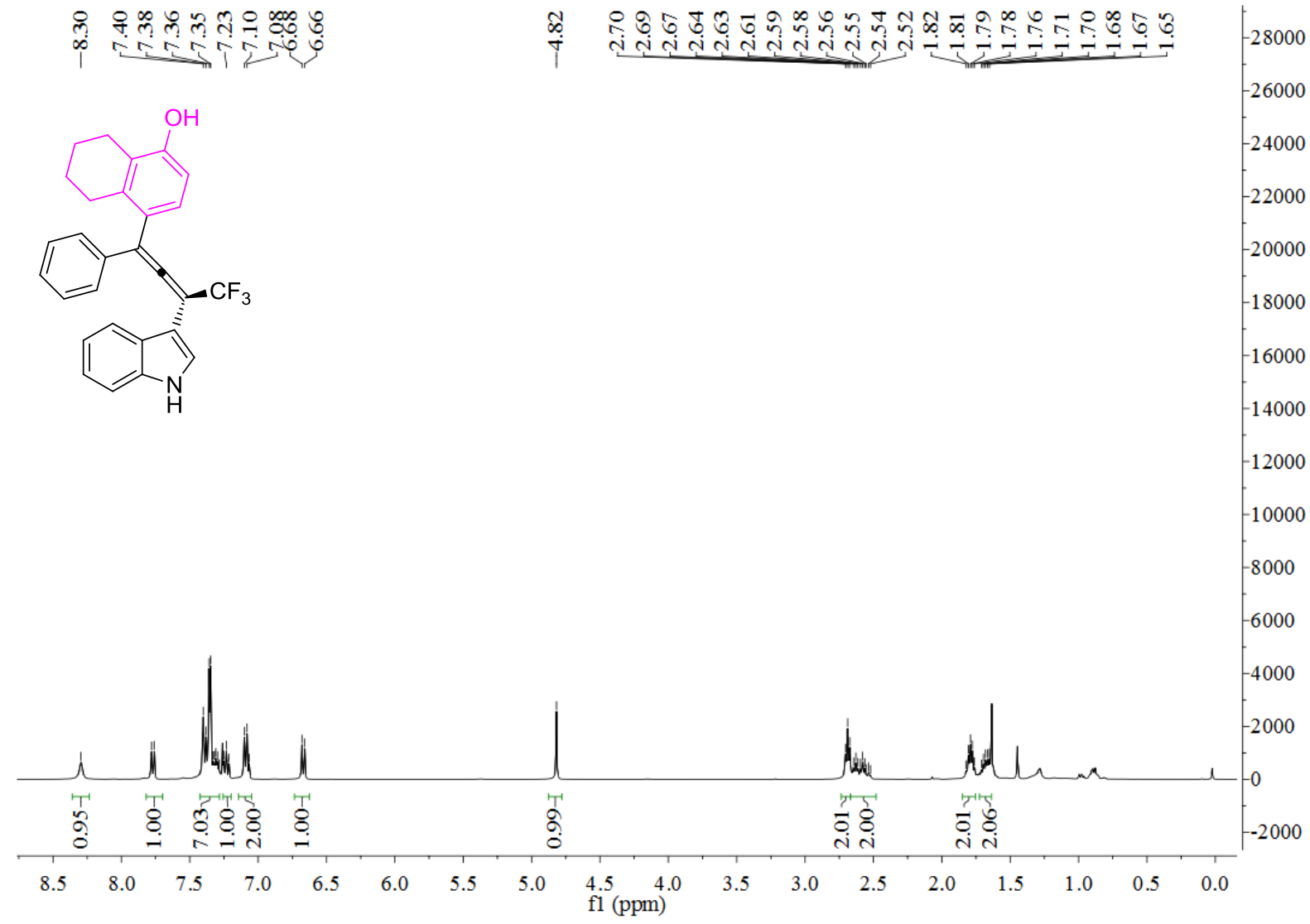

${ }^{13} \mathrm{C}$ NMR of compound $3 \mathrm{v}\left(101 \mathrm{MHz}\right.$ in $\left.\mathrm{CDCl}_{3}\right)$

กั่

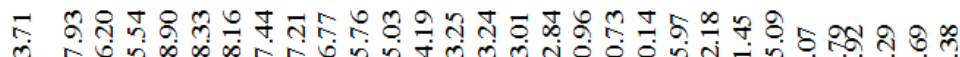

m
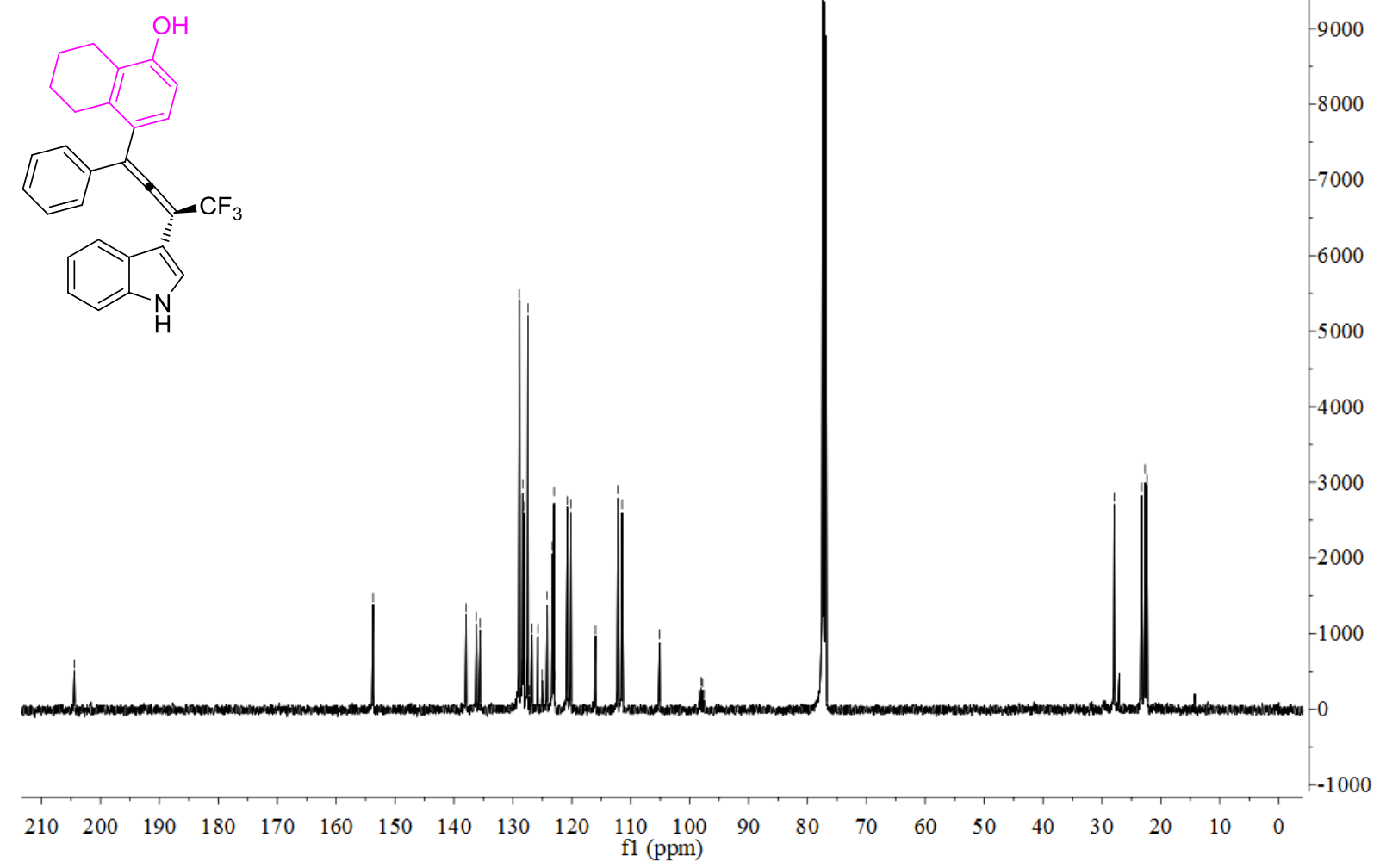
${ }^{19} \mathrm{~F}$ NMR of compound 3v (376 $\mathrm{MHz}$ in $\left.\mathrm{CDCl}_{3}\right)$

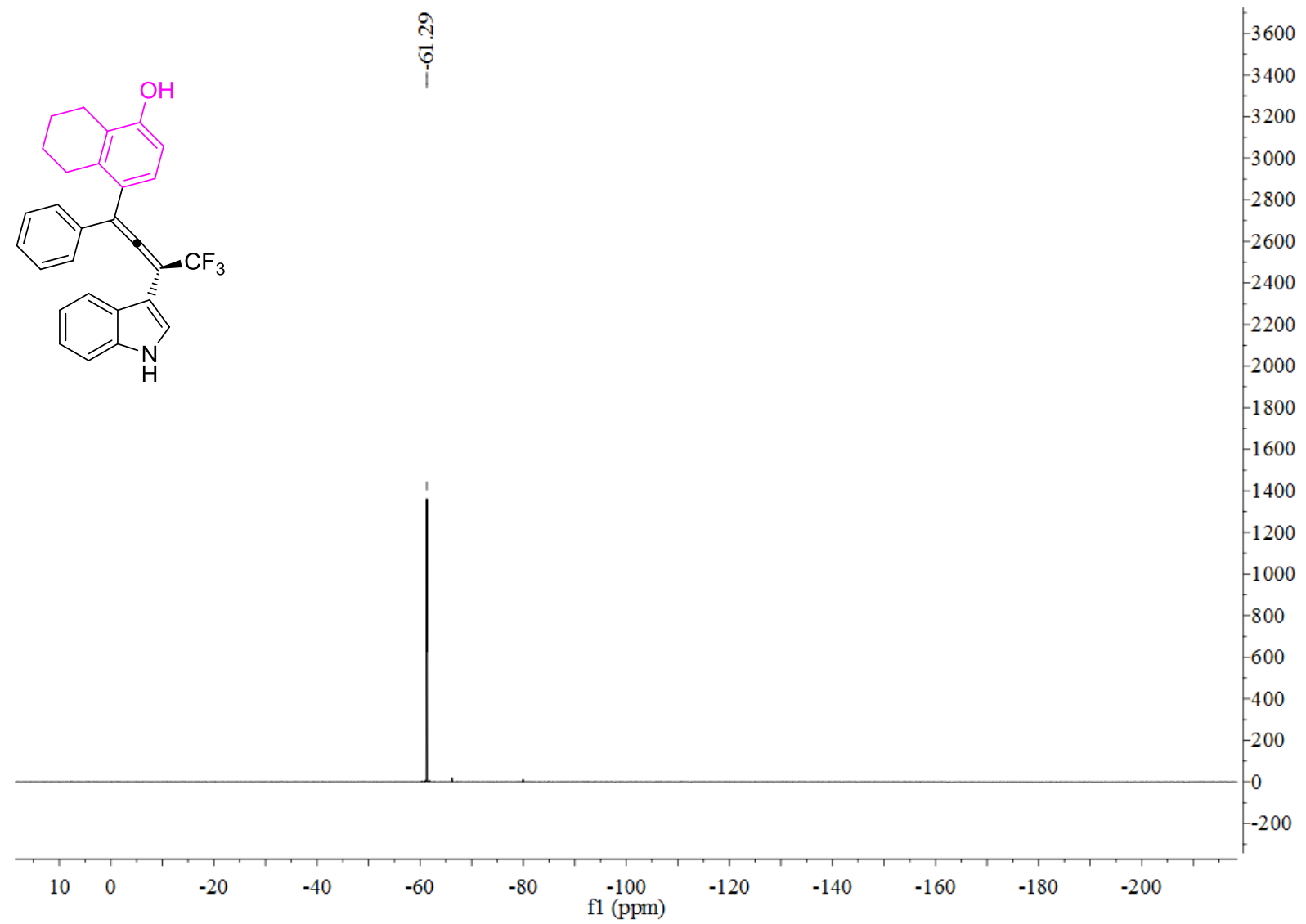

${ }^{1} \mathrm{H}$ NMR of compound 3w (400 $\mathrm{MHz}$ in $\mathrm{CDCl}_{3}$ )

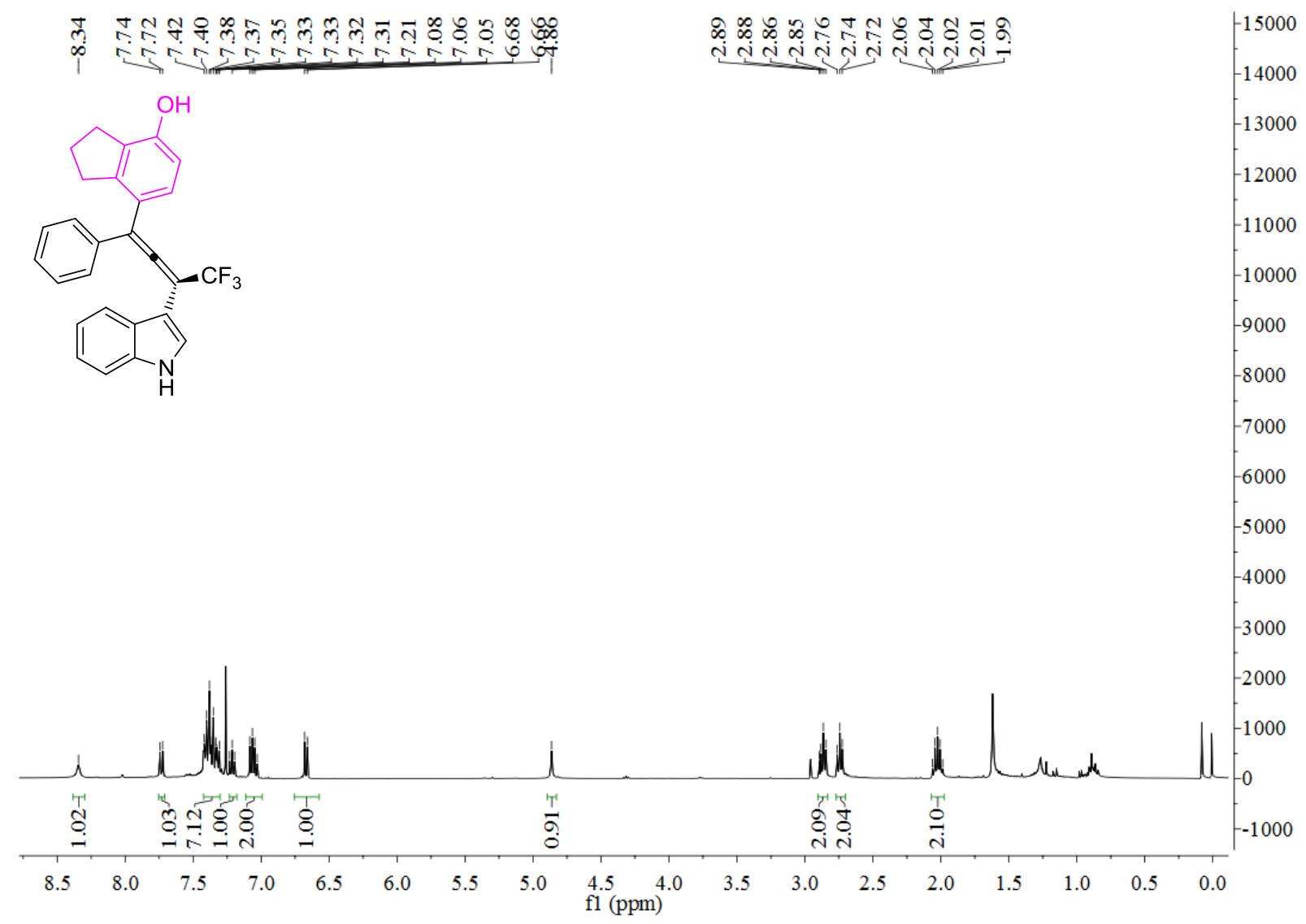




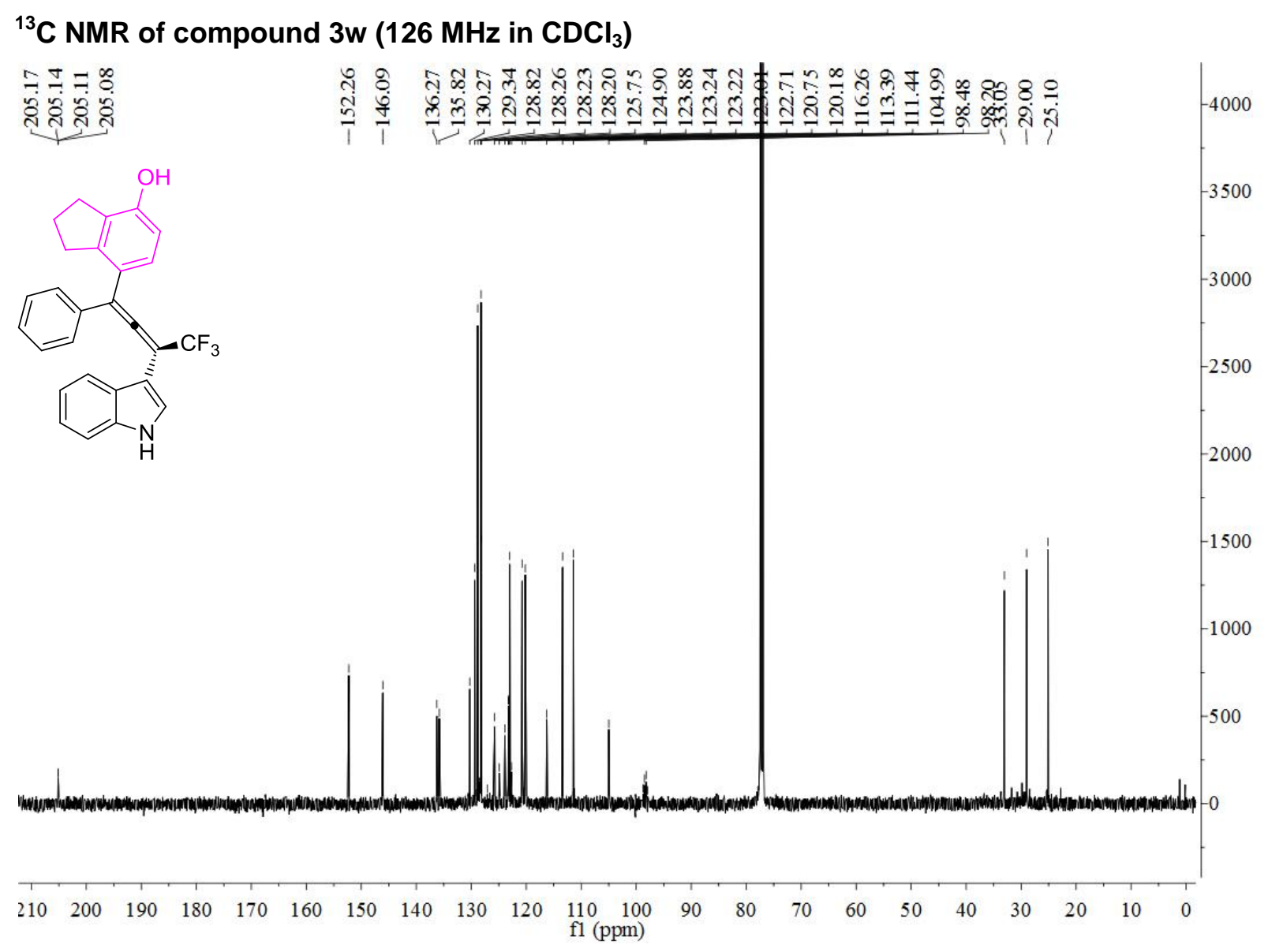

${ }^{19} \mathrm{~F}$ NMR of compound $3 \mathrm{w}\left(376 \mathrm{MHz}\right.$ in $\mathrm{CDCl}_{3}$ )

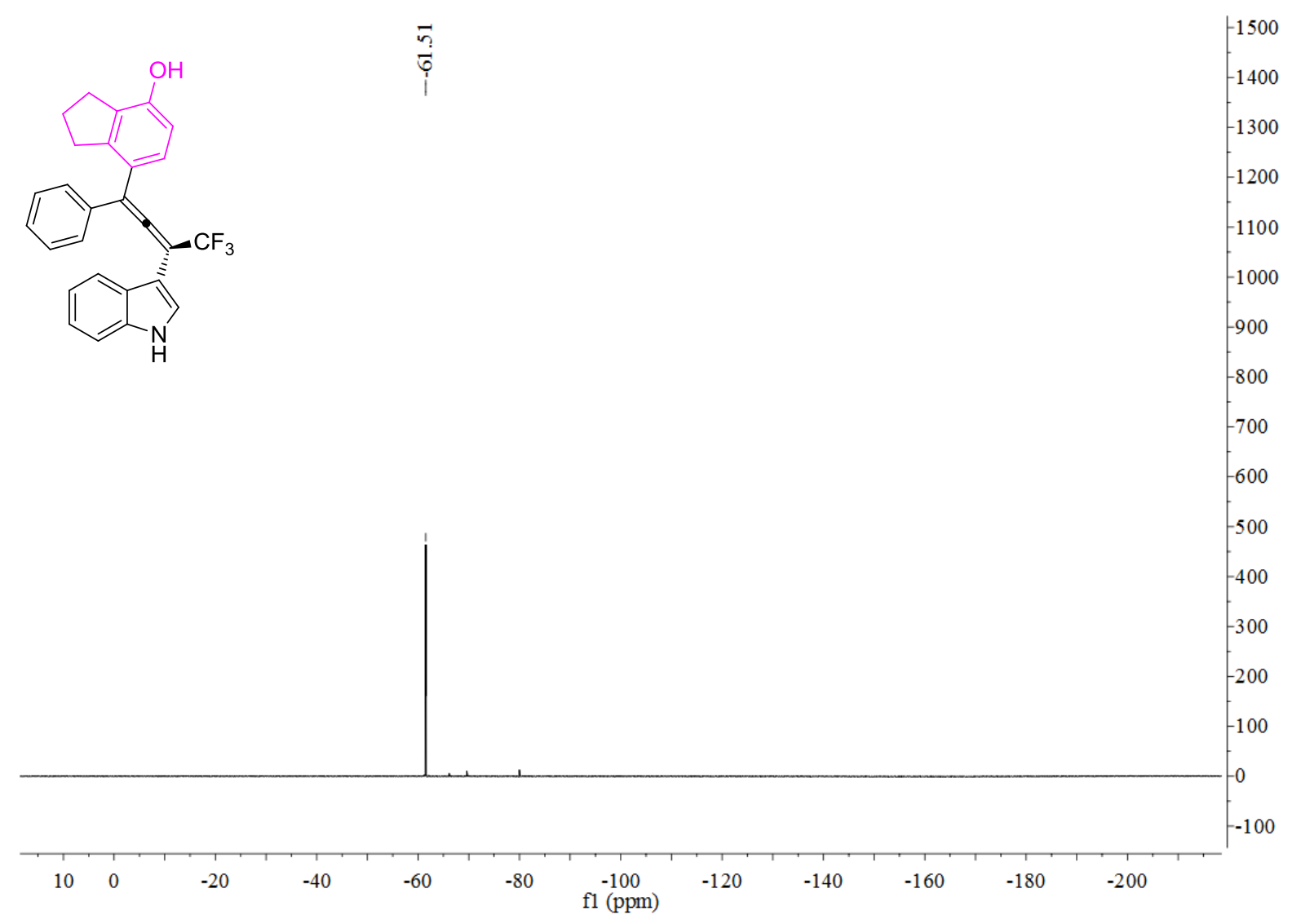


${ }^{1} \mathrm{H}$ NMR of compound $3 \times\left(400 \mathrm{MHz}\right.$ in $\left.\mathrm{CDCl}_{3}\right)$

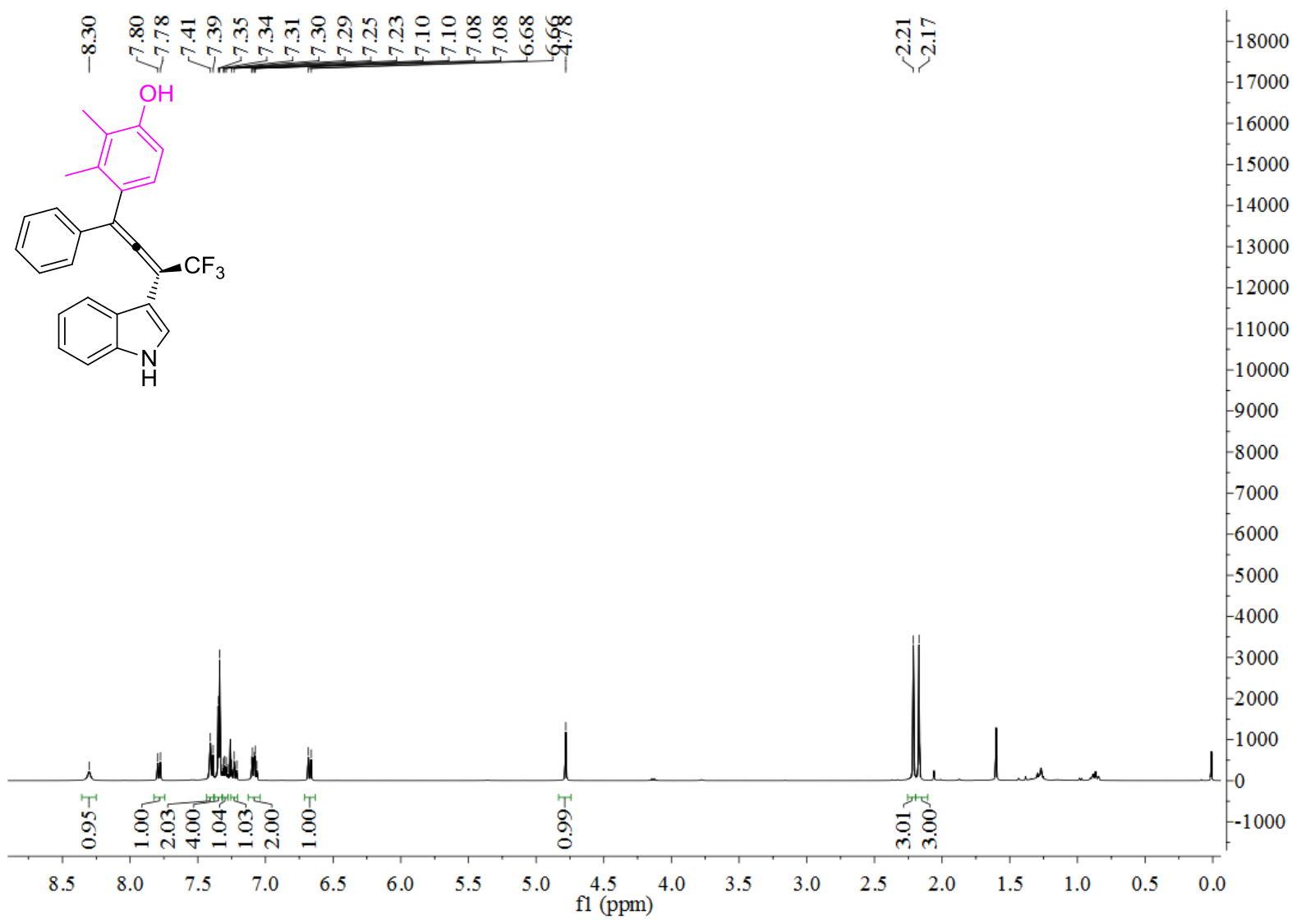

${ }^{13} \mathrm{C}$ NMR of compound $3 x\left(126 \mathrm{MHz}\right.$ in $\left.\mathrm{CDCl}_{3}\right)$

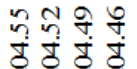

तुํํำ

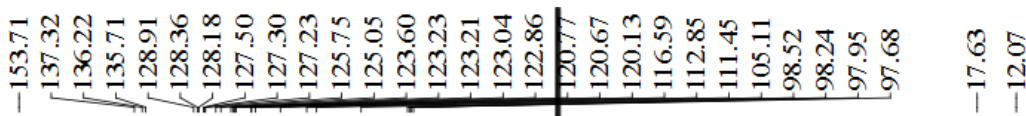<smiles>Cc1c(O)ccc(C(=CC(c2c[nH]c3ccccc23)(C(F)(F)F)C(F)(F)F)c2ccccc2)c1C</smiles>

\section{$\stackrel{1}{8}$}

$-3400$

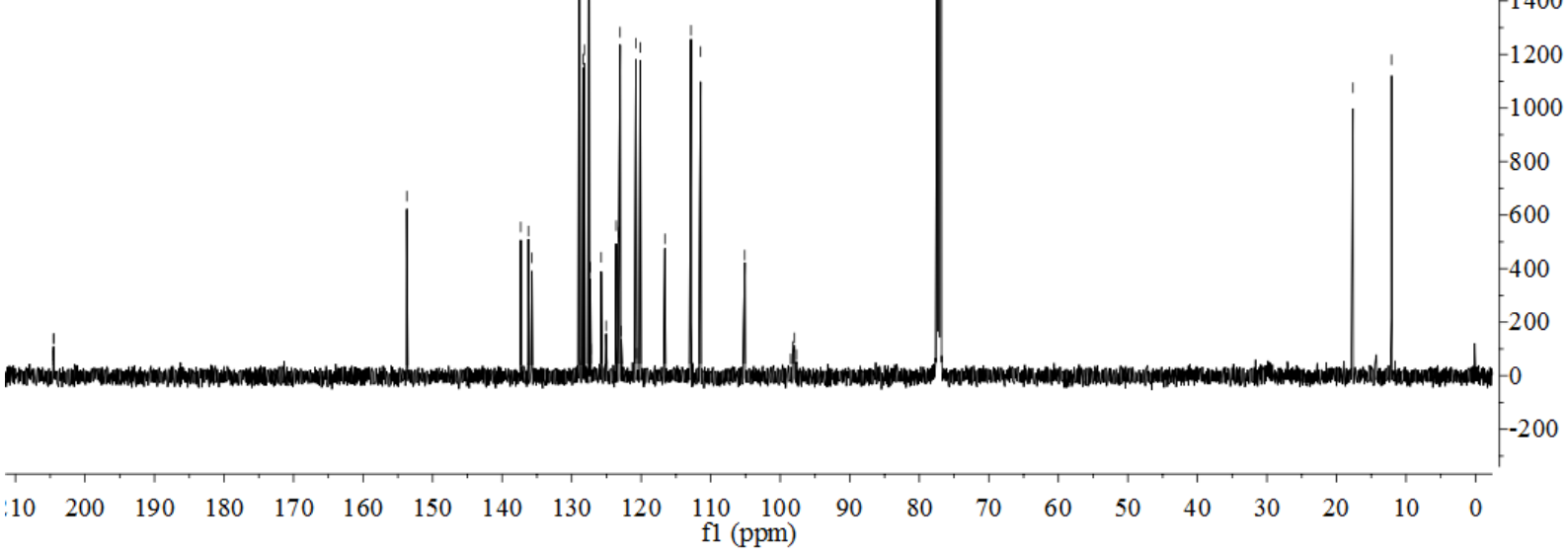


${ }^{19} \mathrm{~F}$ NMR of compound $3 \times\left(376 \mathrm{MHz}\right.$ in $\left.\mathrm{CDCl}_{3}\right)$

iे<smiles>Cc1c(O)ccc(C(=CC(c2ccccc2)C(F)(F)F)c2c[nH]c3ccccc23)c1C</smiles>

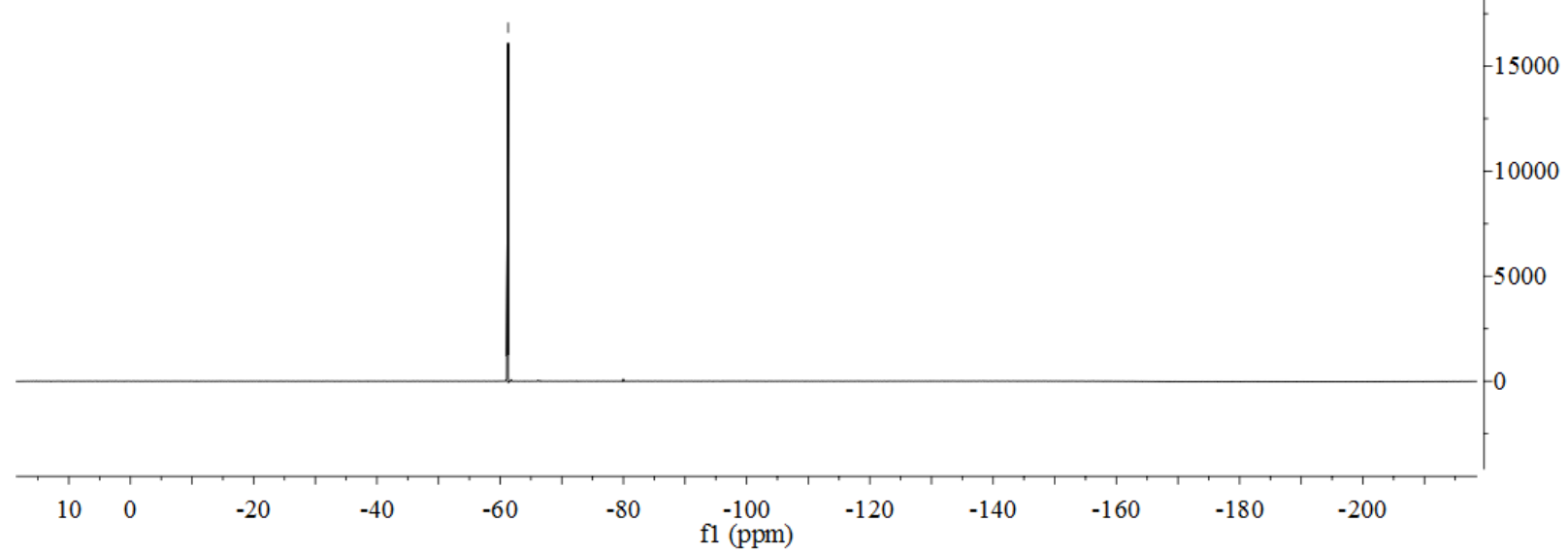

${ }^{1} \mathrm{H}$ NMR of compound $3 \mathrm{y}\left(400 \mathrm{MHz}\right.$ in $\left.\mathrm{CDCl}_{3}\right)$

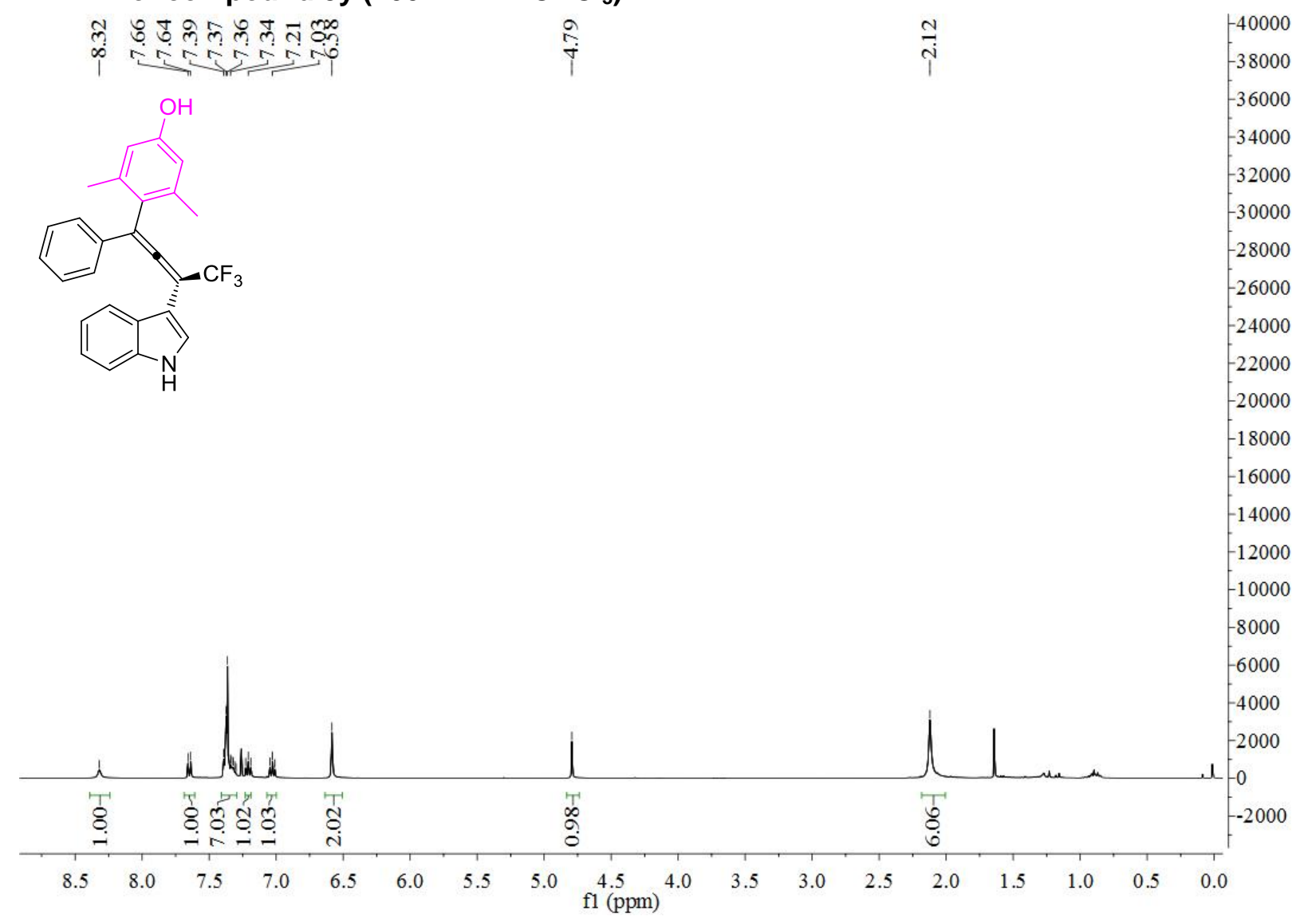


${ }^{13} \mathrm{C}$ NMR of compound $3 y\left(126 \mathrm{MHz}\right.$ in $\mathrm{CDCl}_{3}$ )

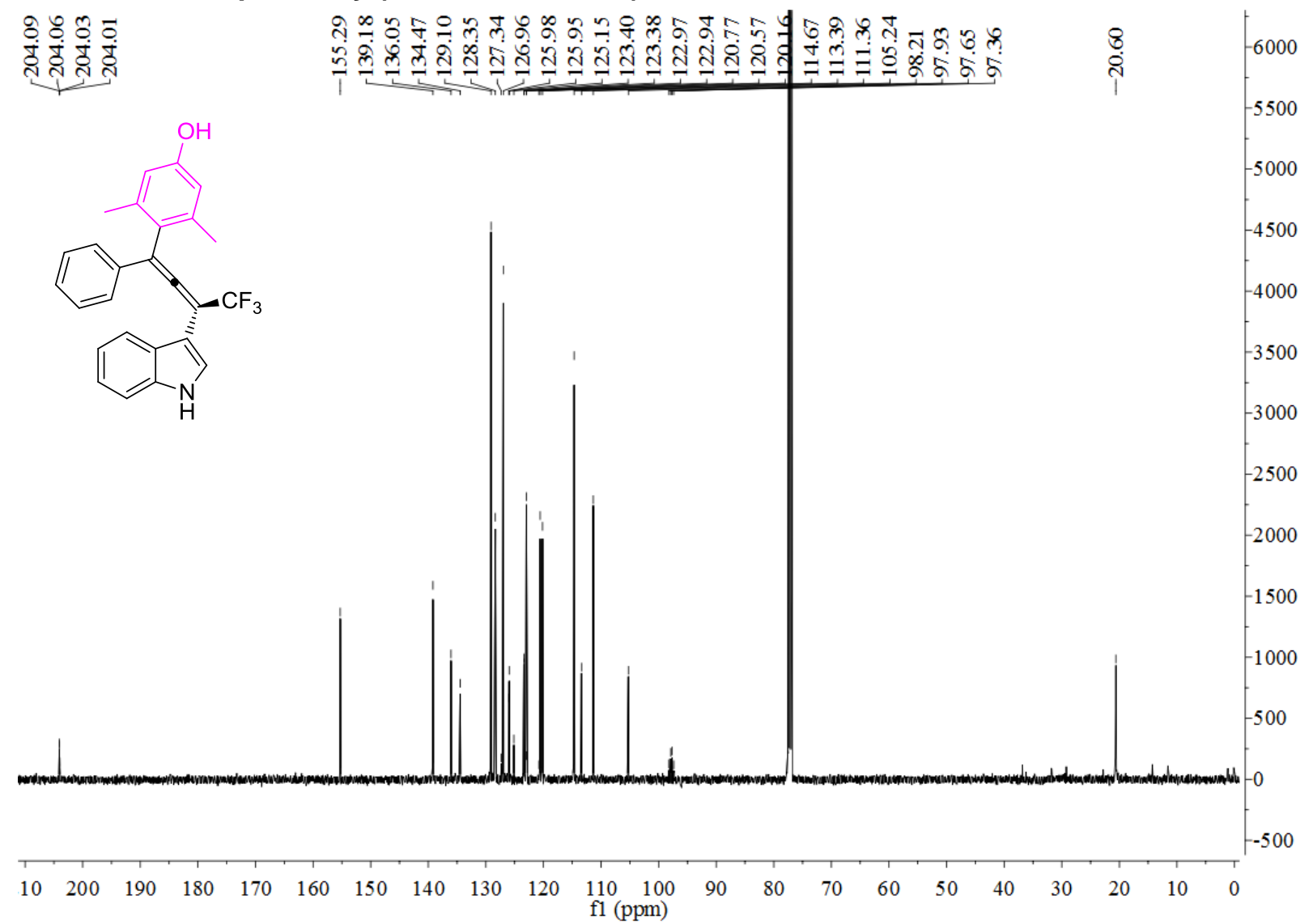

${ }^{19} \mathrm{~F} \mathrm{NMR}$ of compound $3 \mathrm{y}\left(376 \mathrm{MHz}\right.$ in $\mathrm{CDCl}_{3}$ )

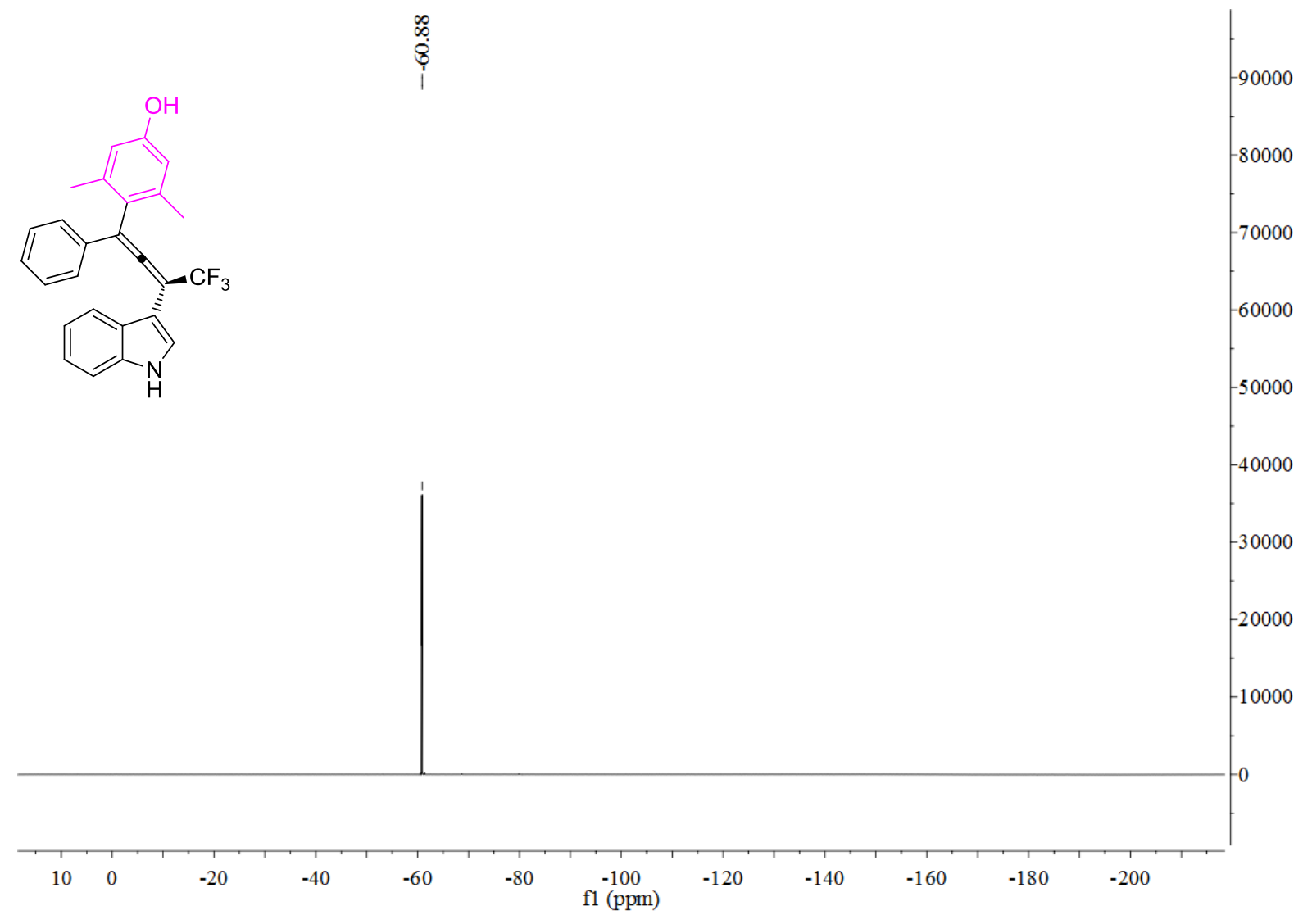


${ }^{1} \mathrm{H}$ NMR of compound $3 \mathrm{z}\left(400 \mathrm{MHz}\right.$ in $\left.\mathrm{CDCl}_{3}\right)$

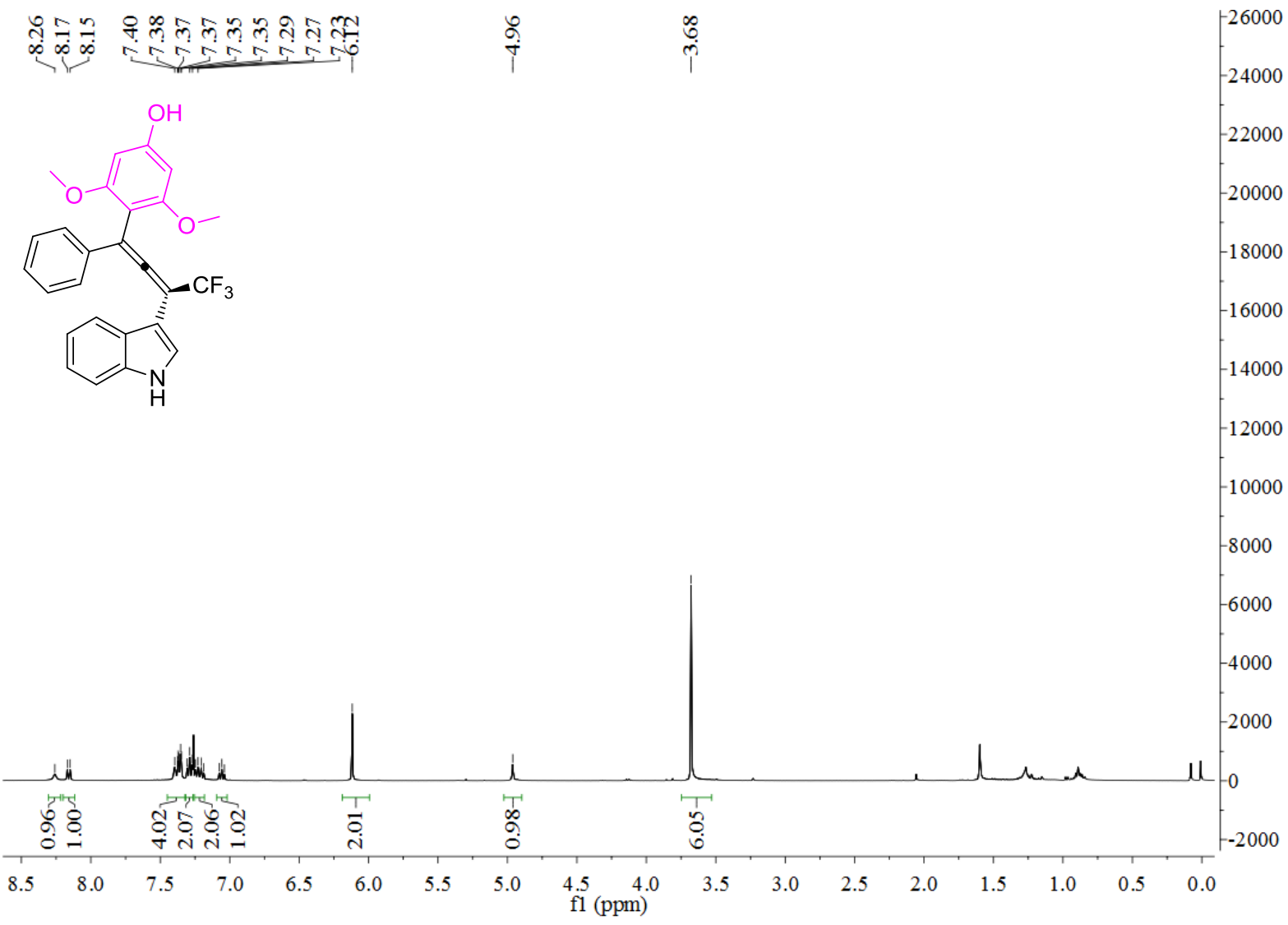

${ }^{13} \mathrm{C}$ NMR of compound $3 z$ (126 MHz in $\mathrm{CDCl}_{3}$ )

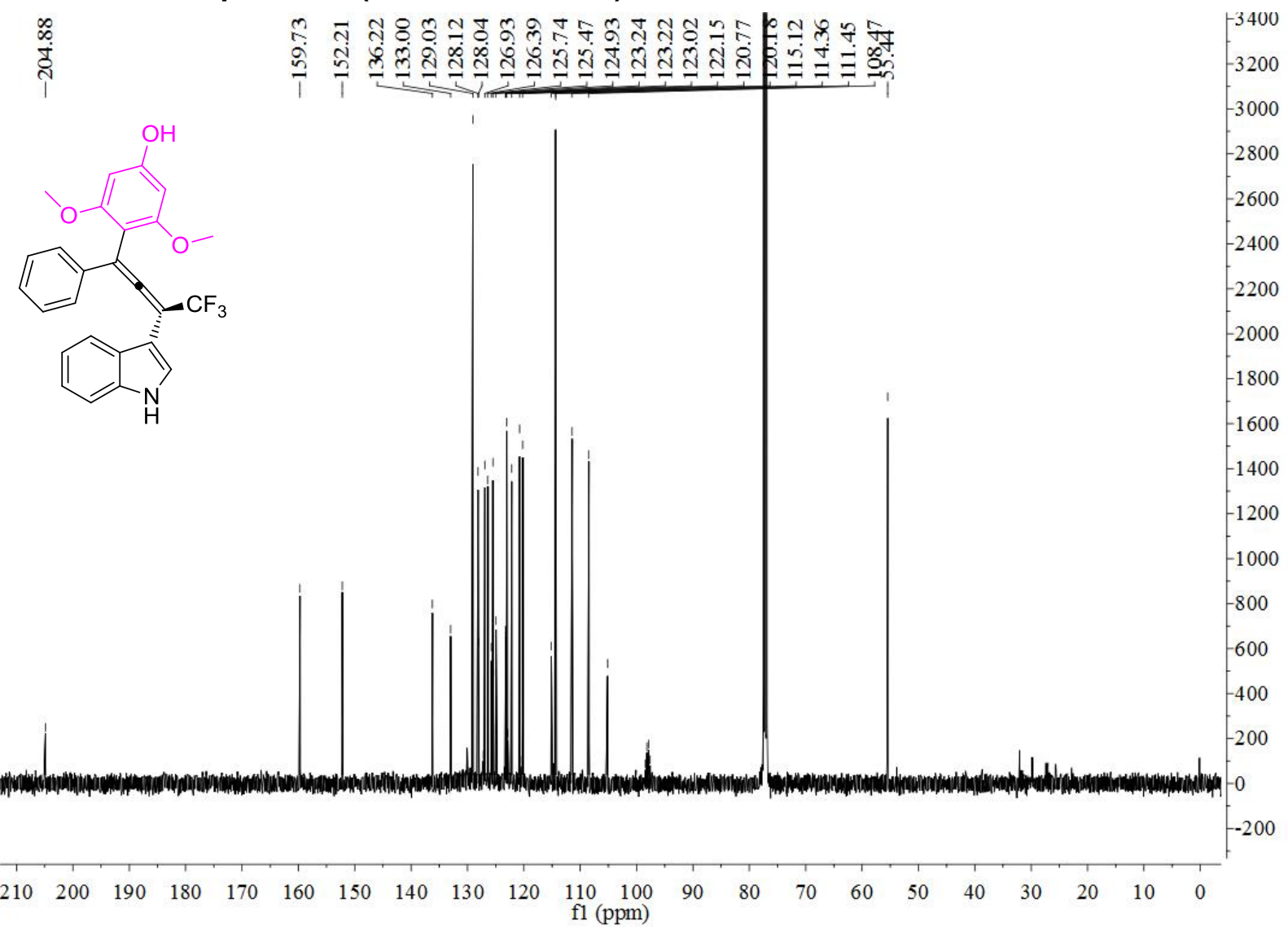




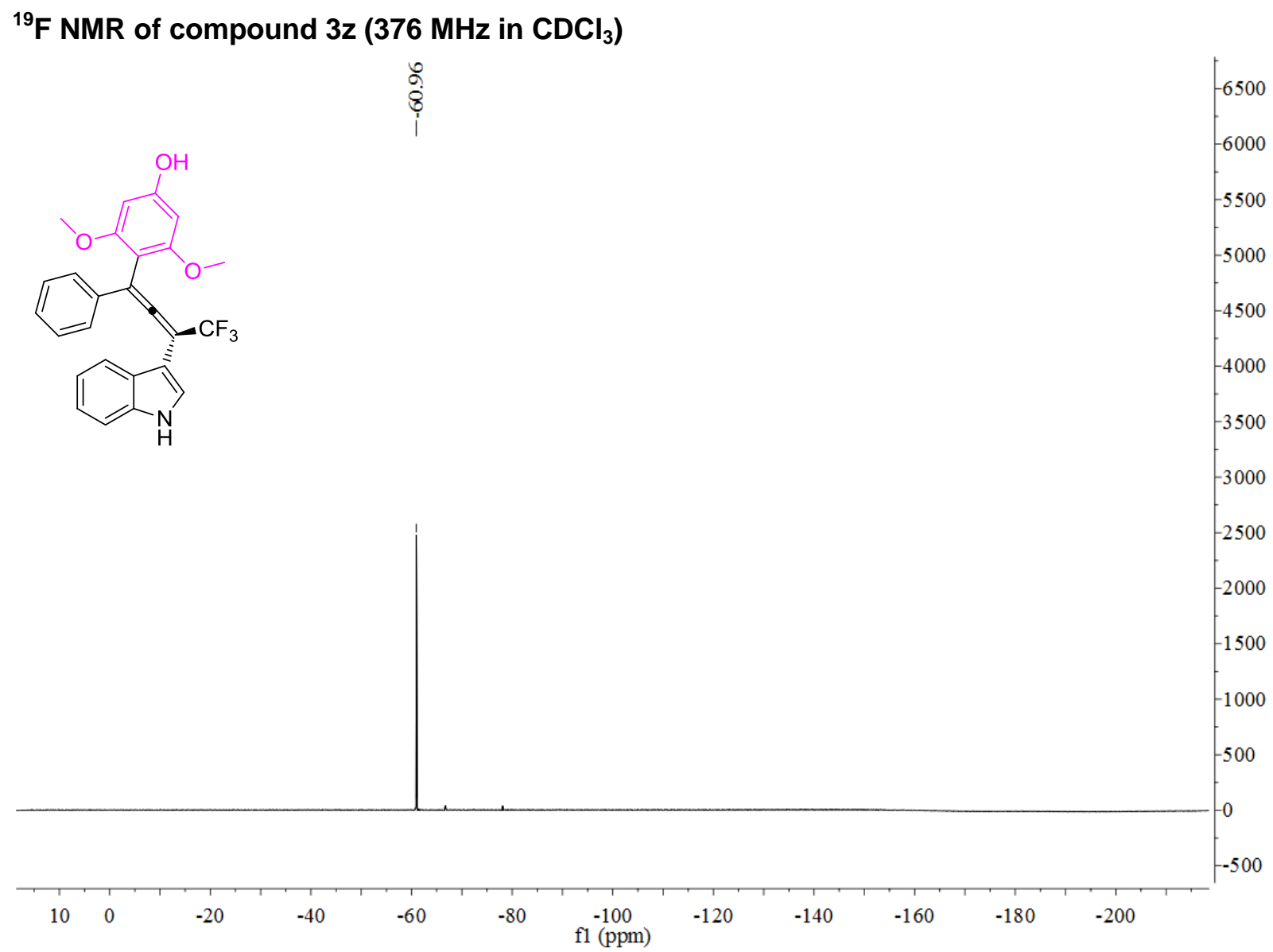

${ }^{1} \mathrm{H}$ NMR of compound 3aa (400 $\mathrm{MHz}$ in $\mathrm{CDCl}_{3}$ )

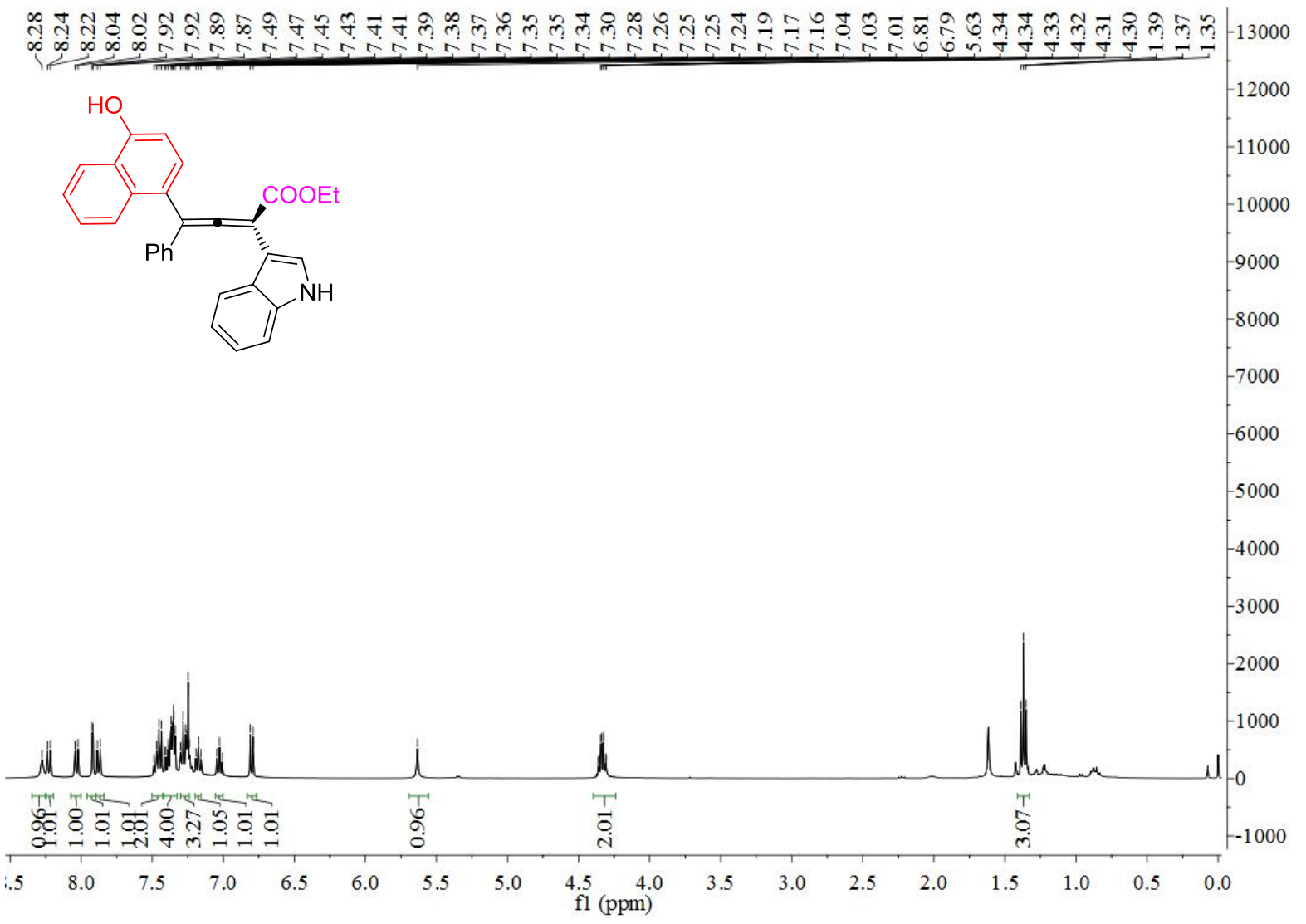




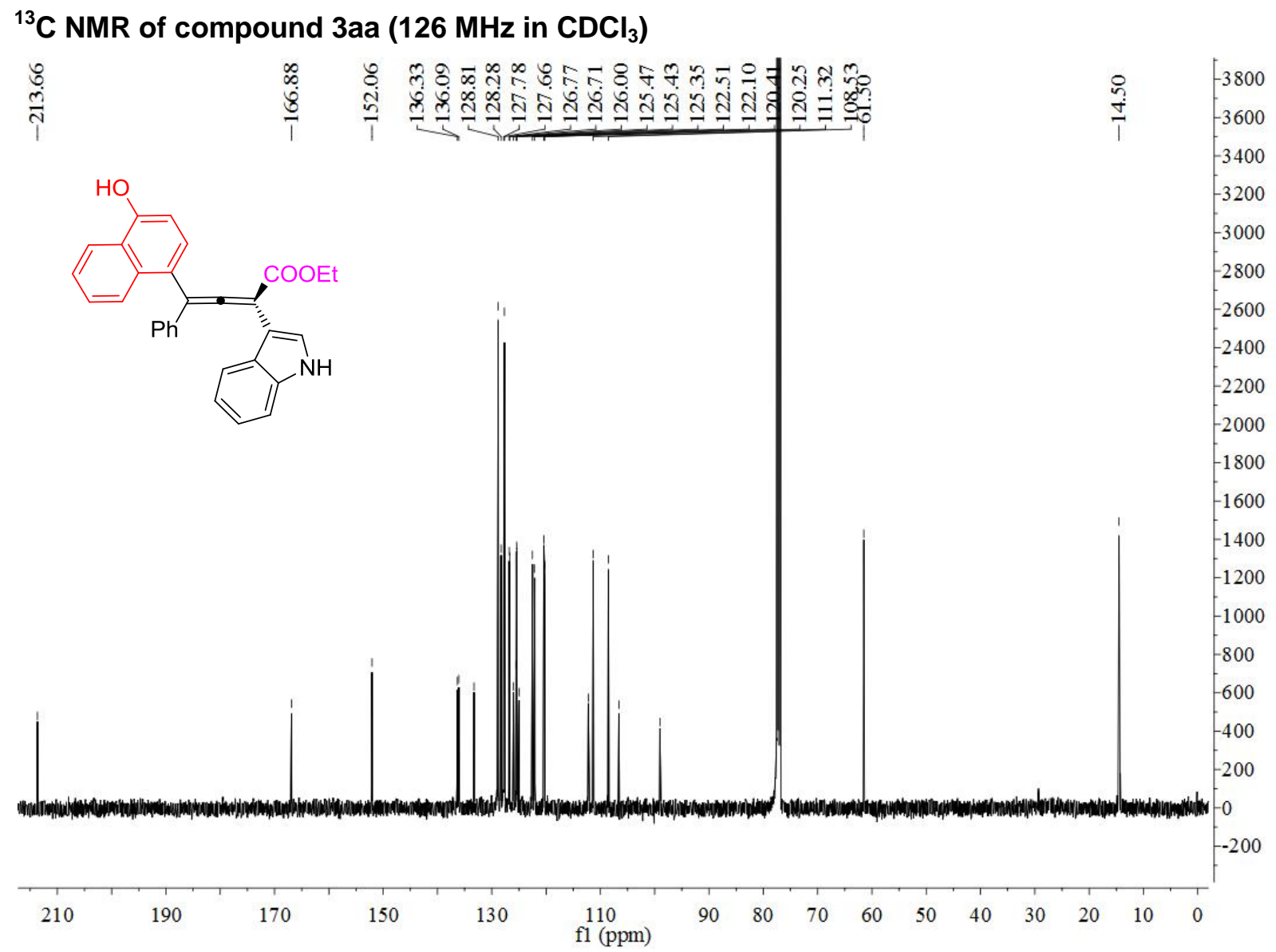

${ }^{1} \mathrm{H}$ NMR of compound $5 \mathrm{a}\left(400 \mathrm{MHz}\right.$ in $\left.\mathrm{CDCl}_{3}\right)$

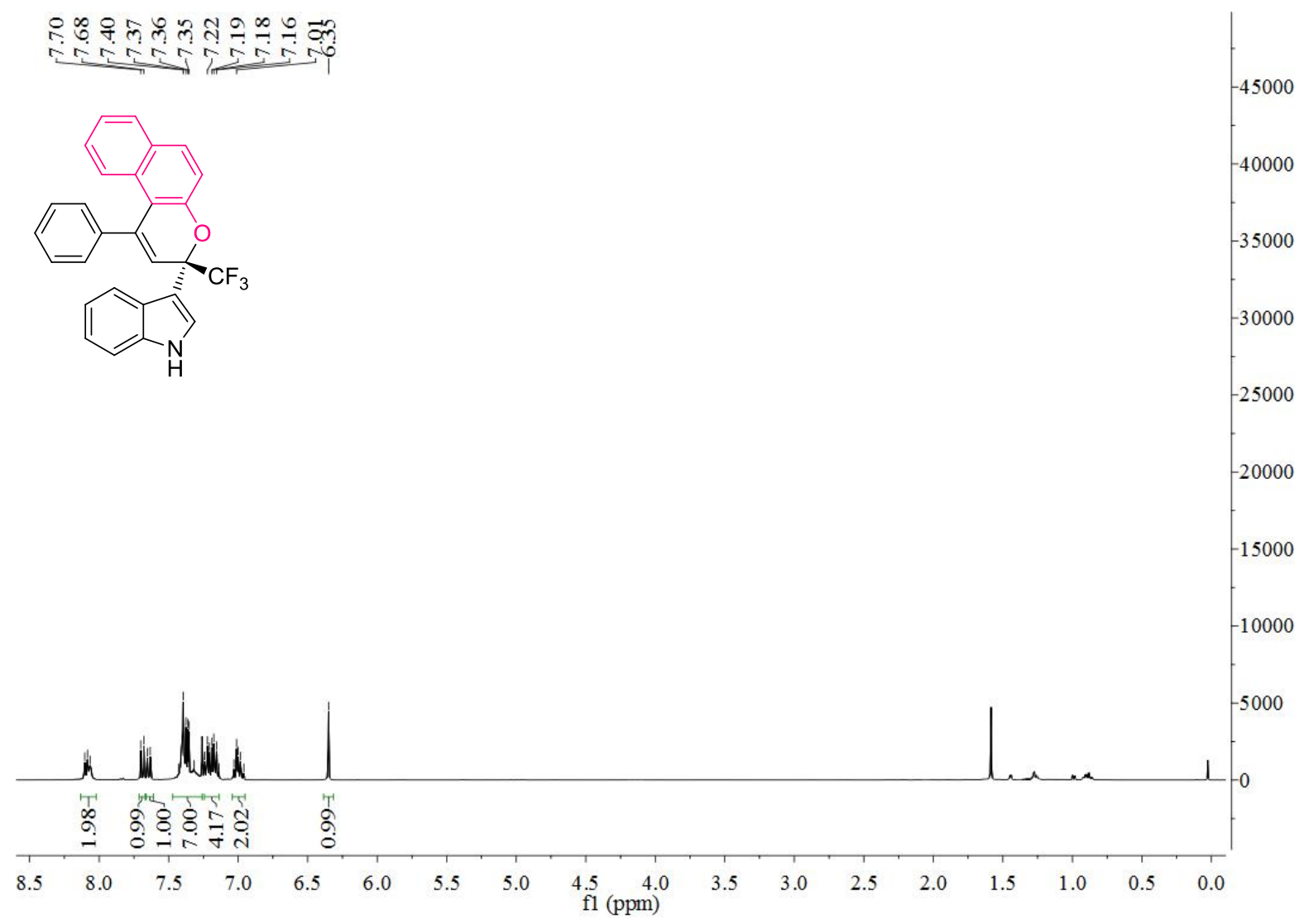




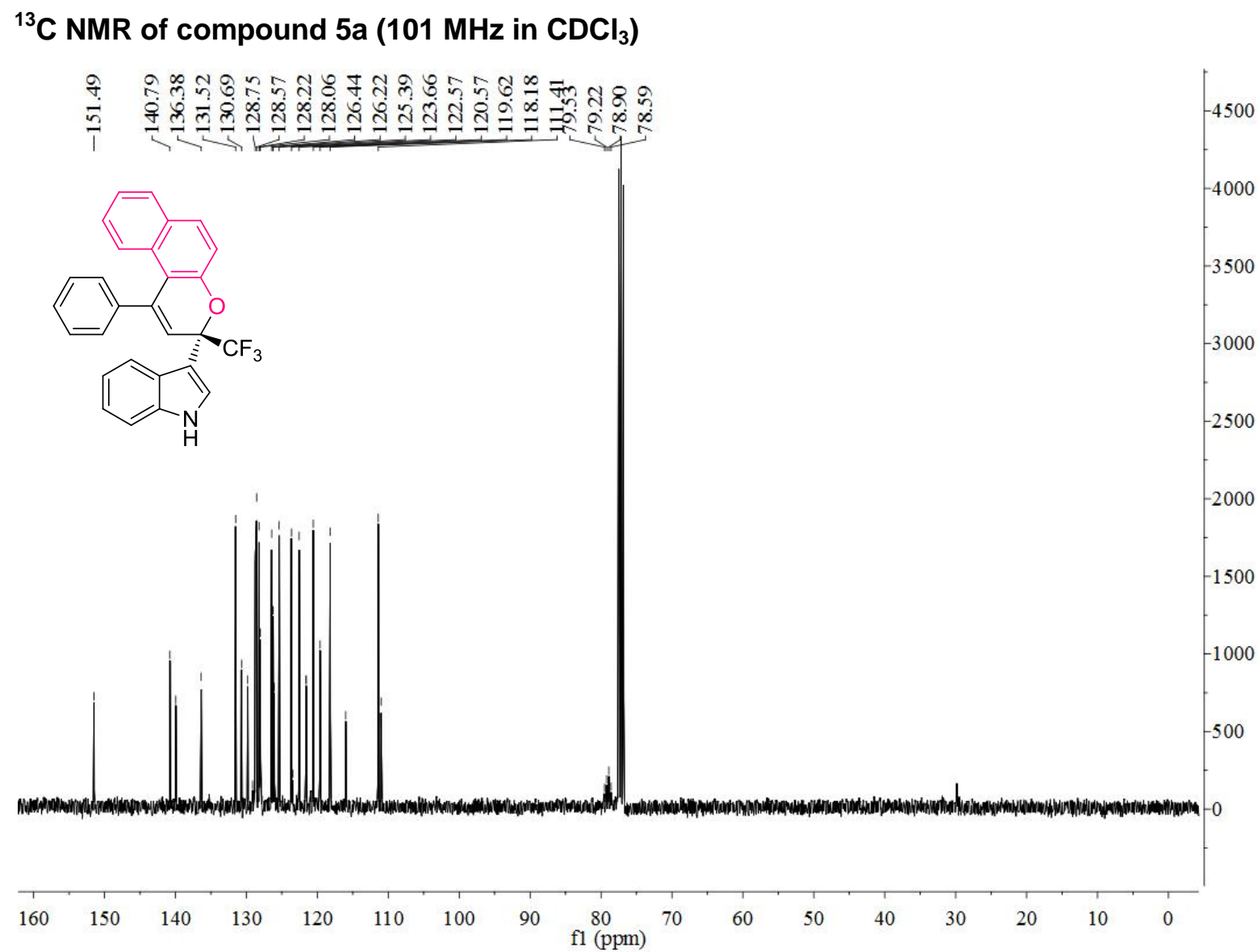

${ }^{19} \mathrm{~F} \mathrm{NMR}$ of compound $5 \mathrm{a}\left(376 \mathrm{MHz}\right.$ in $\mathrm{CDCl}_{3}$ )

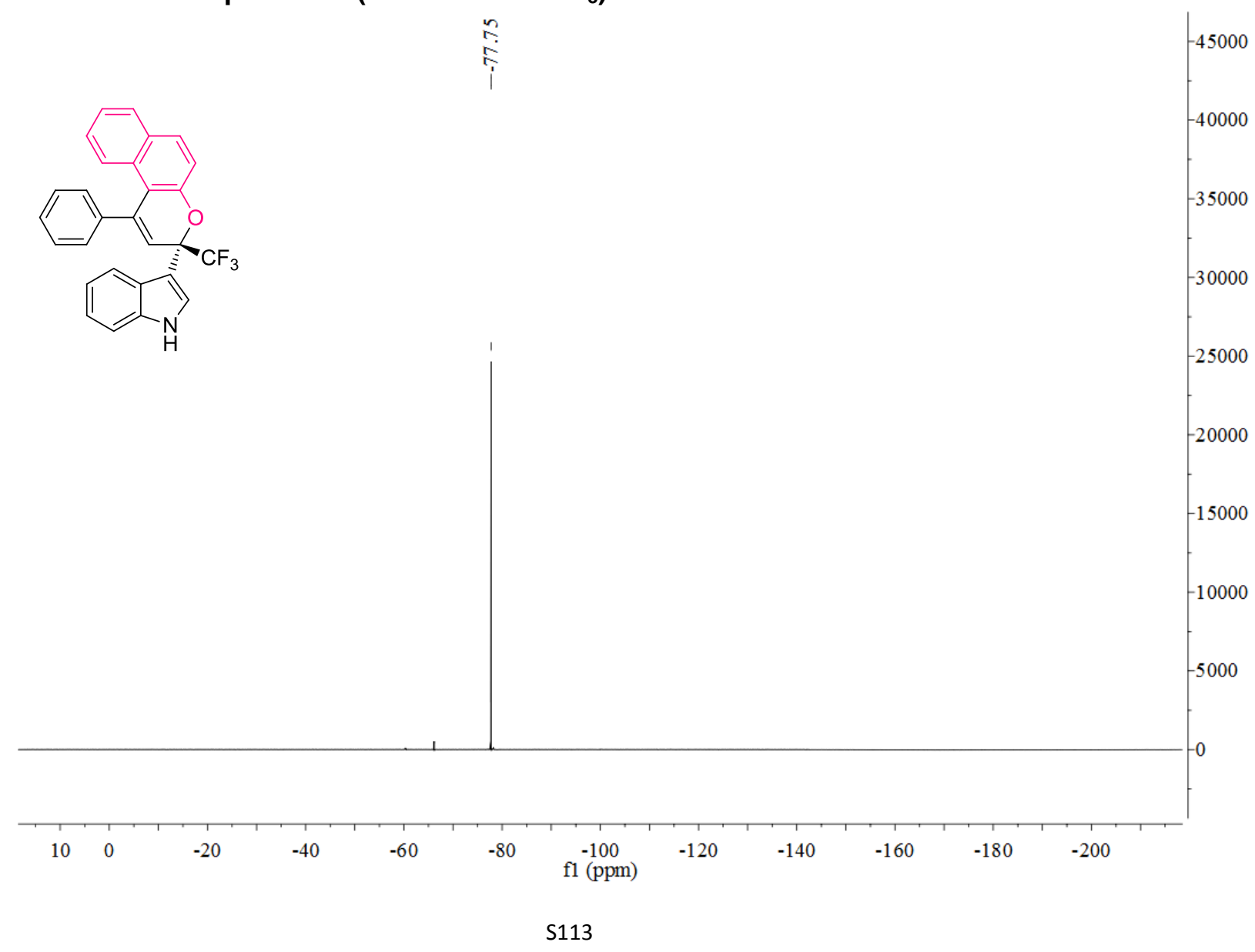


${ }^{1} \mathrm{H}$ NMR of compound $5 \mathrm{~b}\left(400 \mathrm{MHz}\right.$ in $\left.\mathrm{CDCl}_{3}\right)$

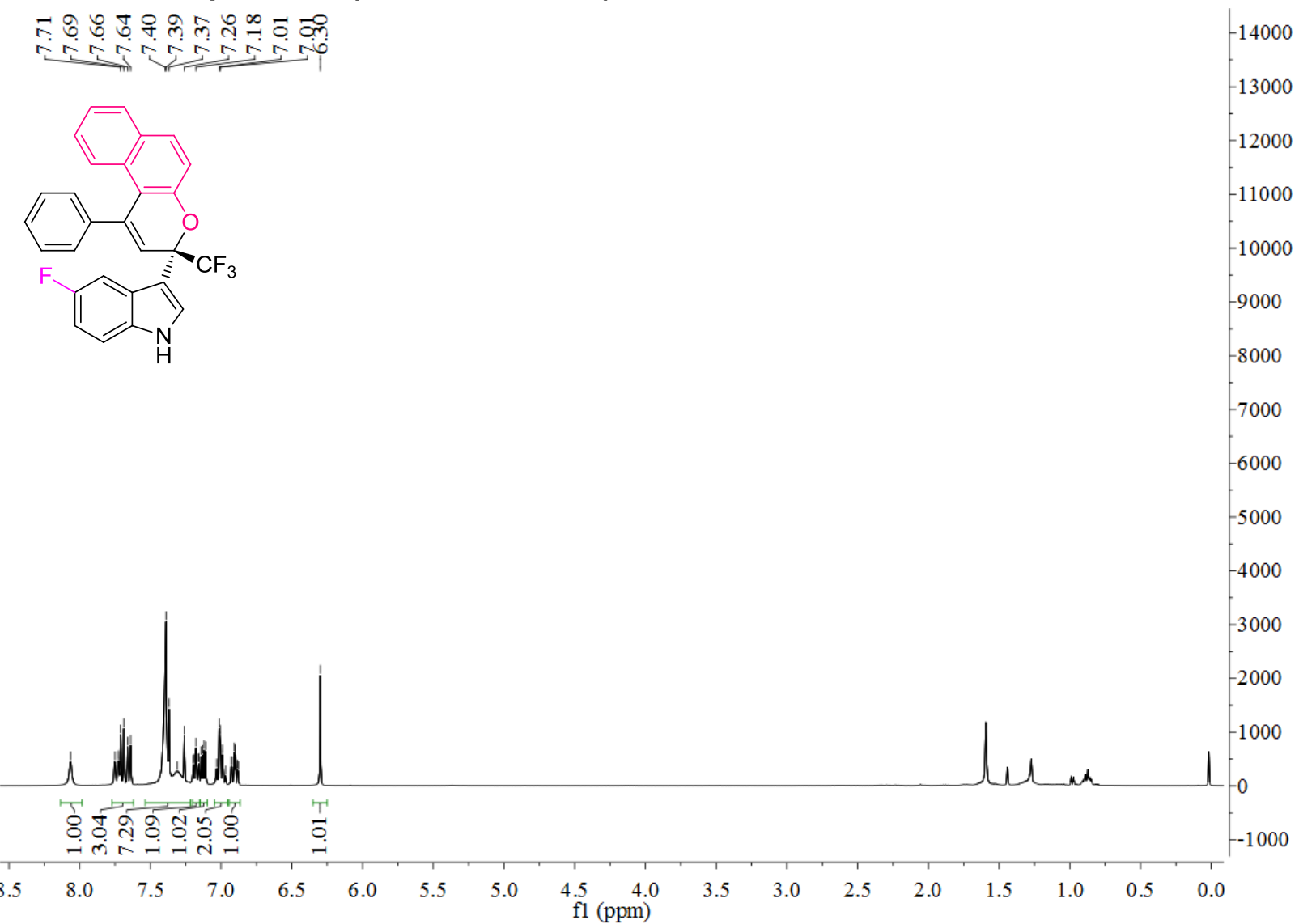

${ }^{13} \mathrm{C}$ NMR of compound $5 \mathrm{~b}\left(101 \mathrm{MHz}\right.$ in $\left.\mathrm{CDCl}_{3}\right)$

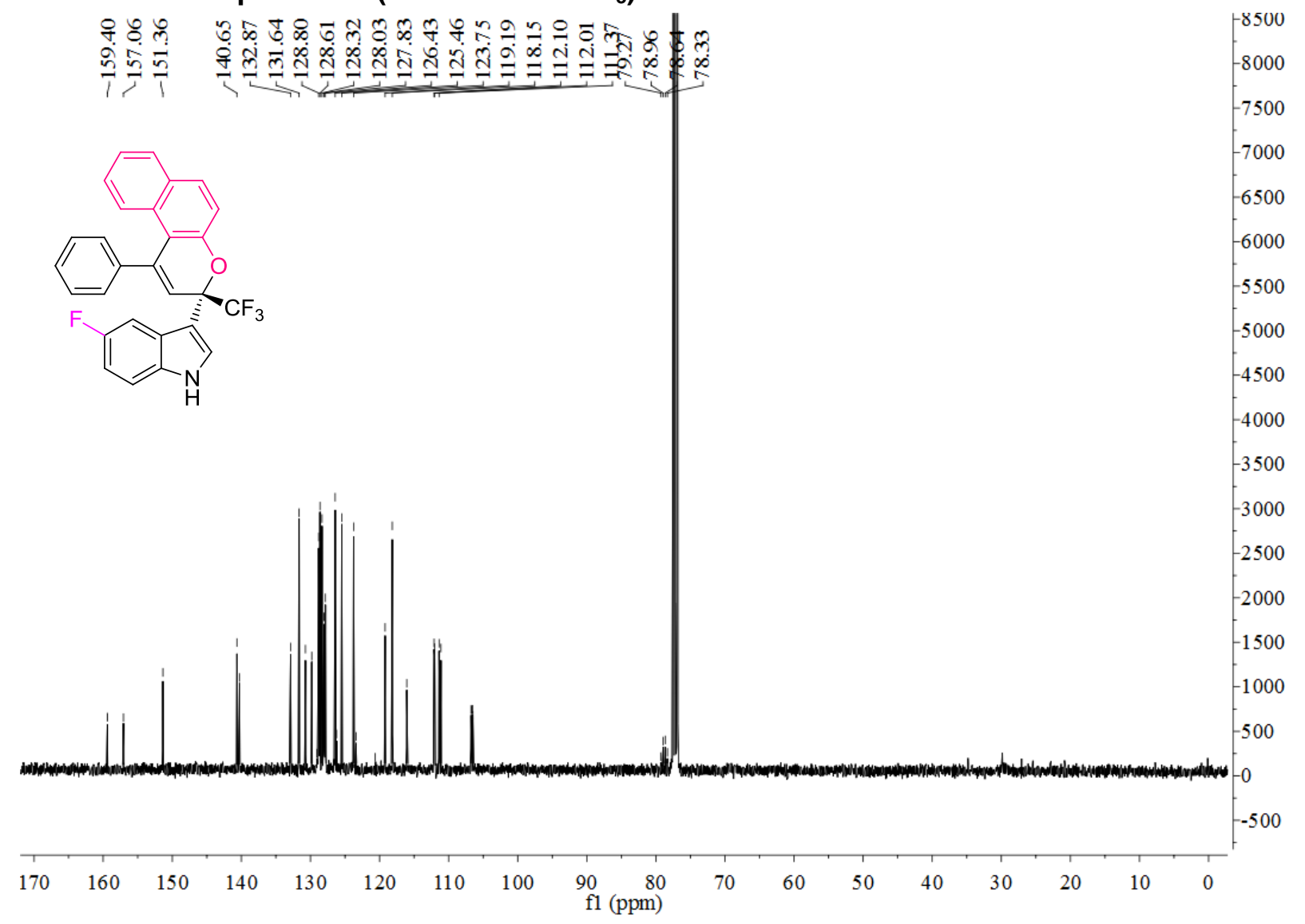


${ }^{19} \mathrm{~F}$ NMR of compound $5 \mathrm{~b}\left(376 \mathrm{MHz}\right.$ in $\left.\mathrm{CDCl}_{3}\right)$

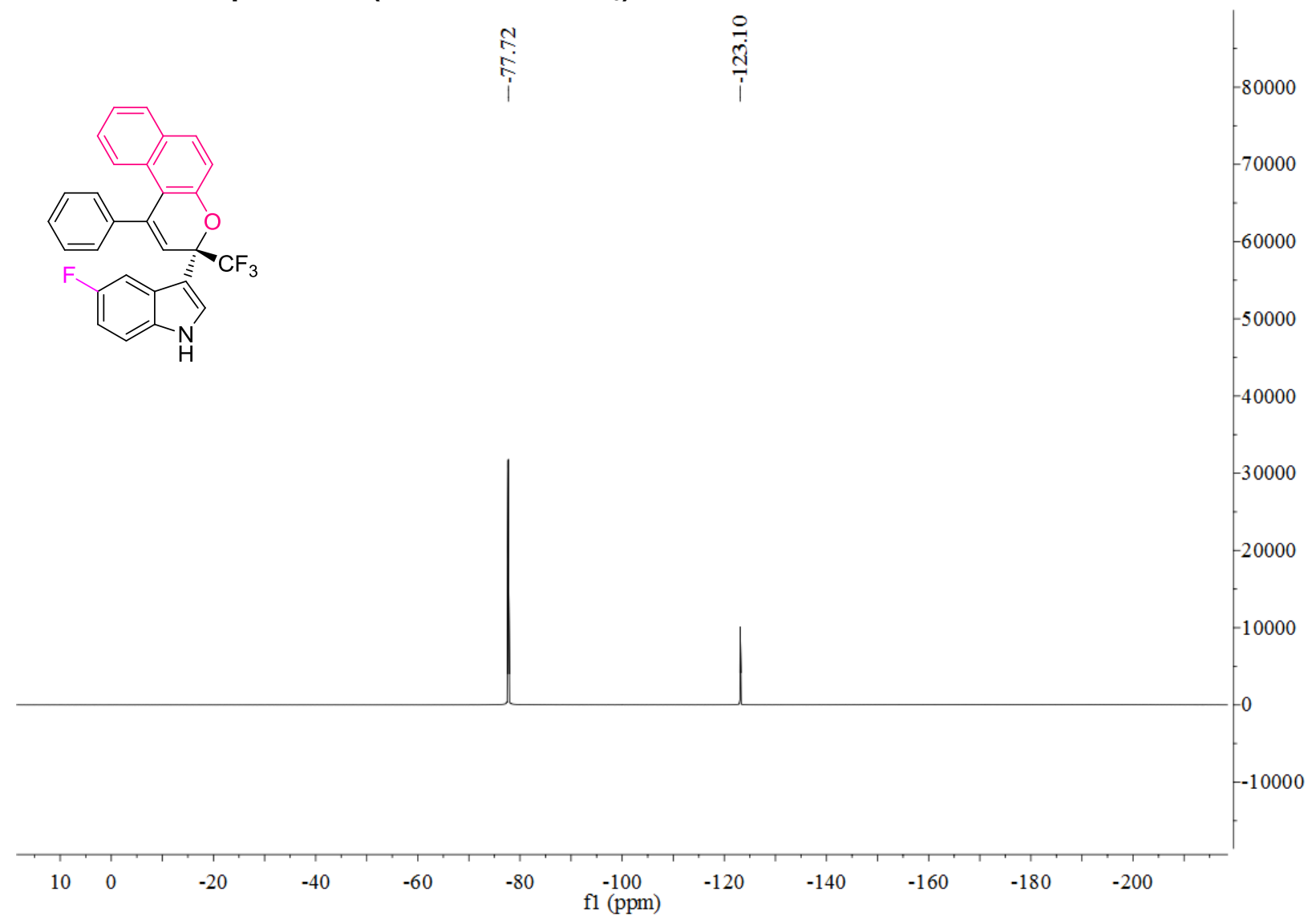

${ }^{1} \mathrm{H}$ NMR of compound $5 \mathrm{c}\left(400 \mathrm{MHz}\right.$ in $\left.\mathrm{CDCl}_{3}\right)$

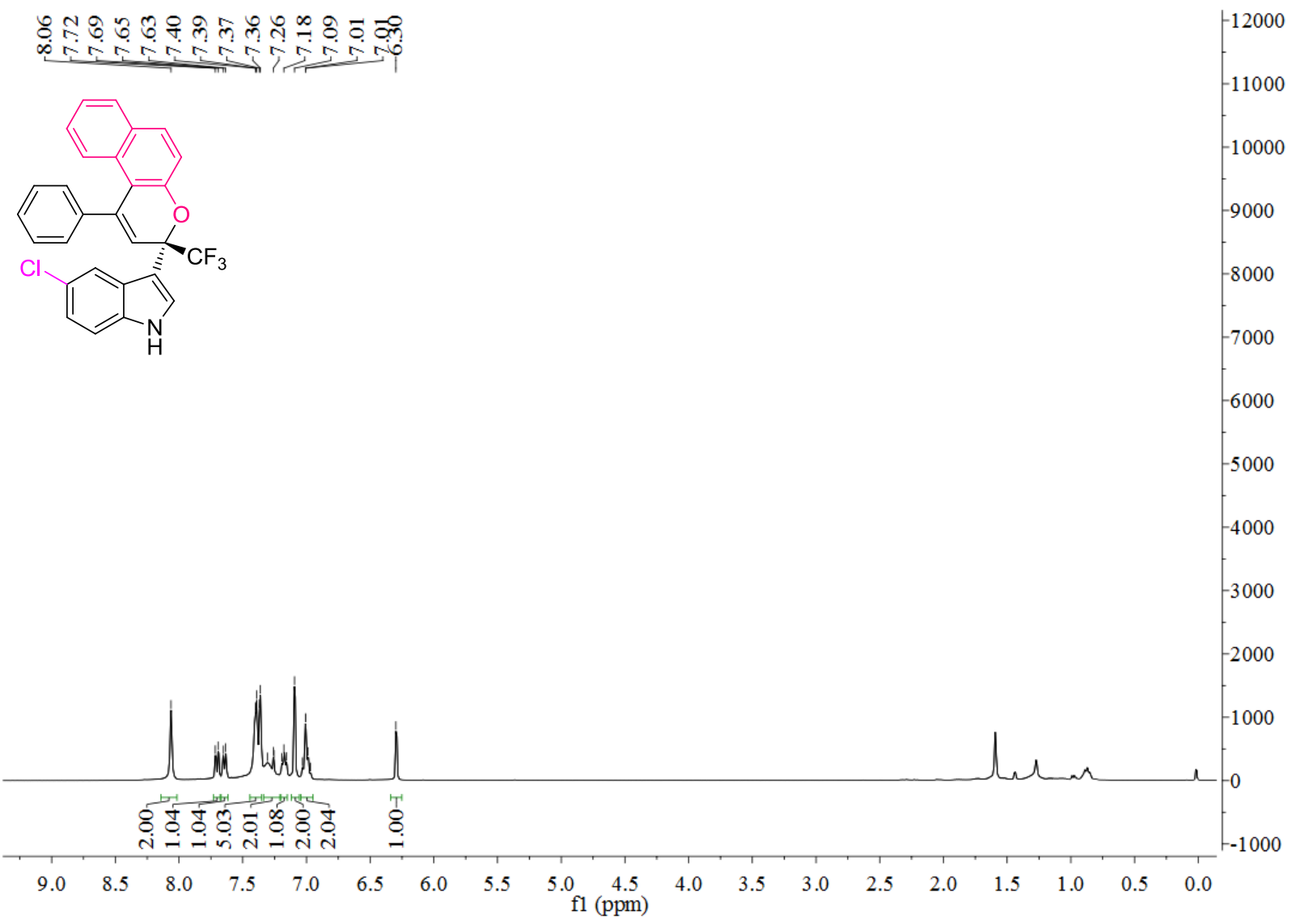




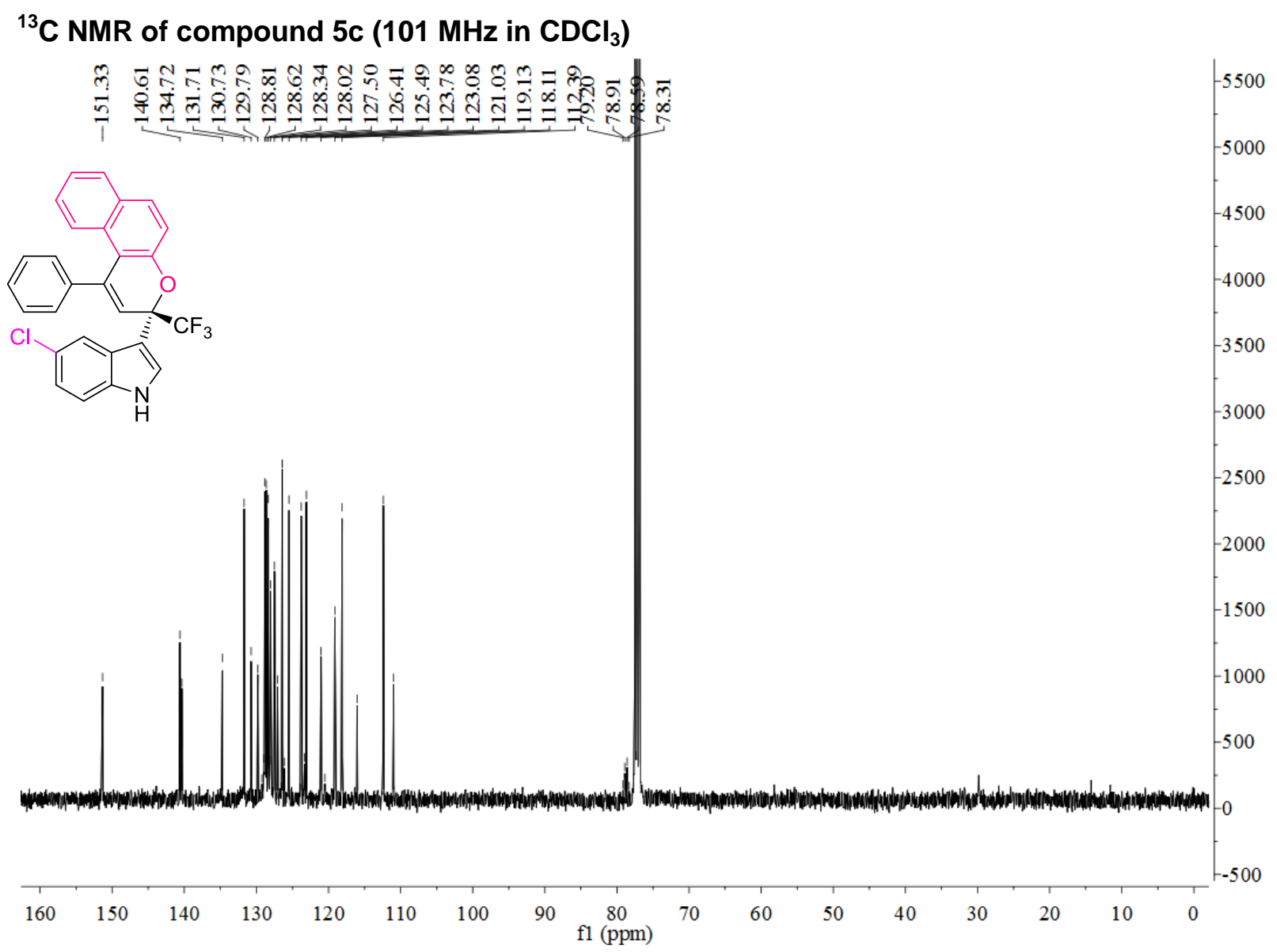

${ }^{19} \mathrm{~F}$ NMR of compound $5 \mathrm{c}\left(376 \mathrm{MHz}\right.$ in $\left.\mathrm{CDCl}_{3}\right)$

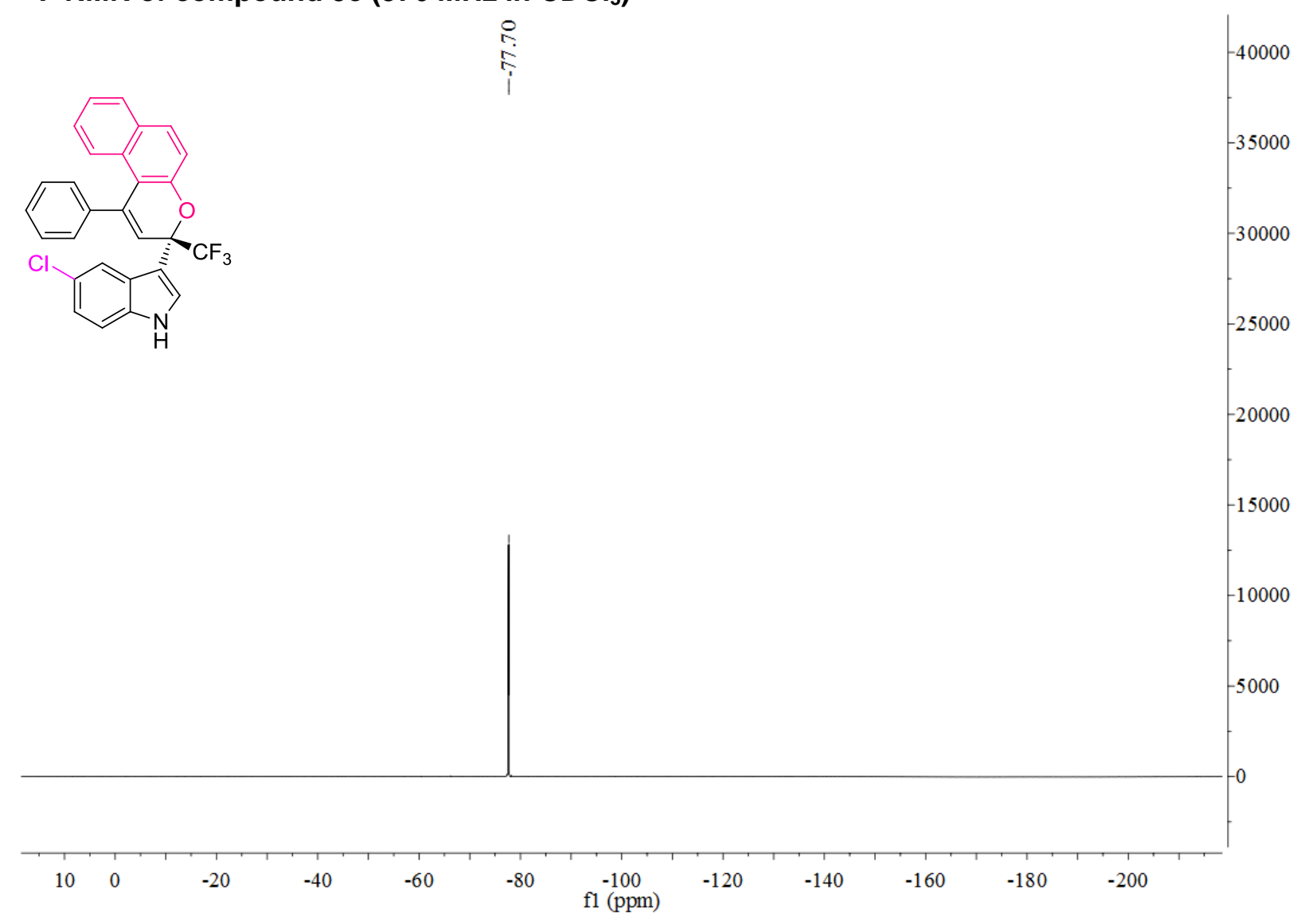


${ }^{1} \mathrm{H}$ NMR of compound $5 \mathrm{~d}\left(400 \mathrm{MHz}\right.$ in $\left.\mathrm{CDCl}_{3}\right)$

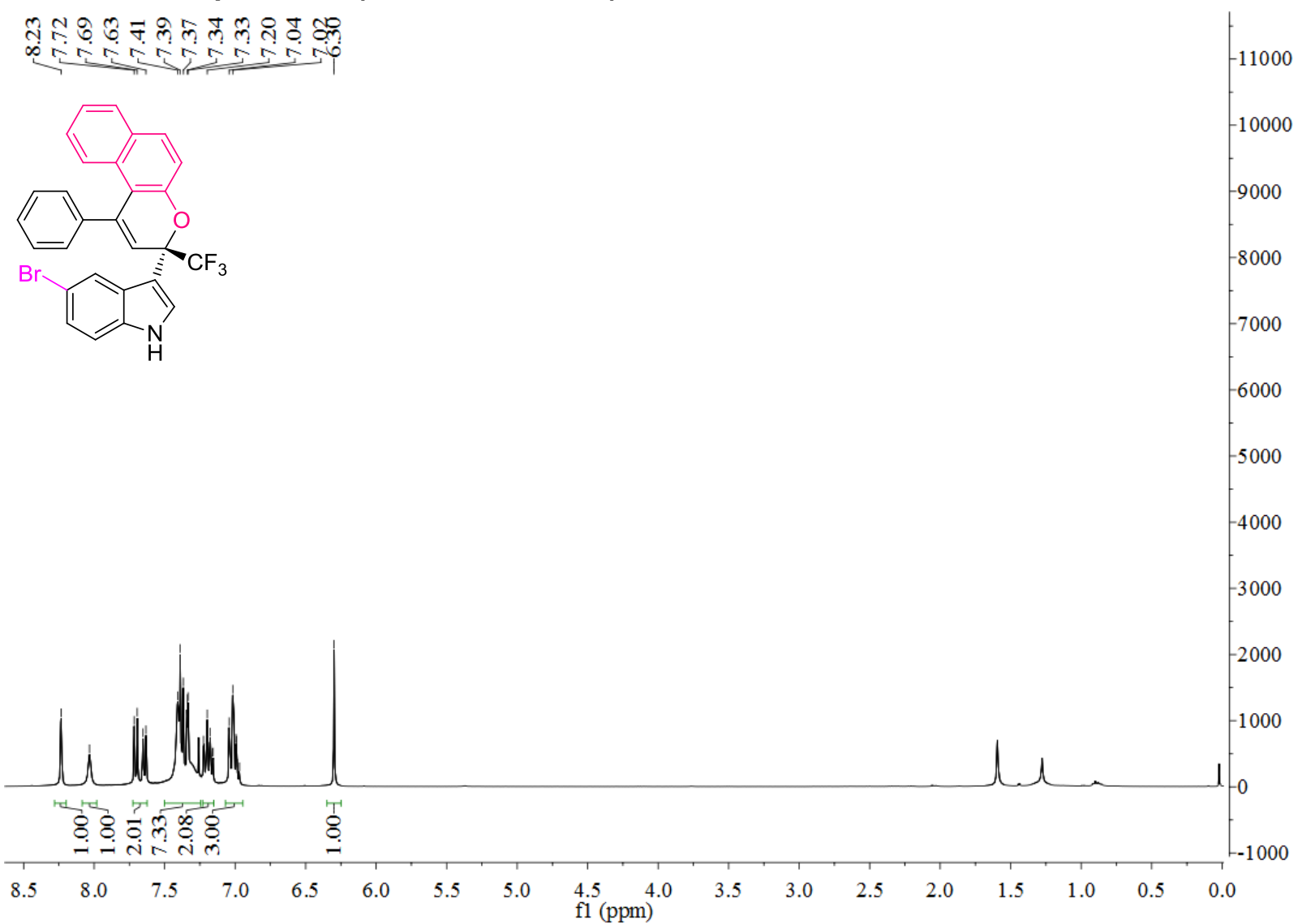

${ }^{13} \mathrm{C}$ NMR of compound $5 \mathrm{~d}\left(101 \mathrm{MHz}\right.$ in $\left.\mathrm{CDCl}_{3}\right)$

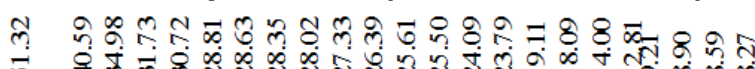

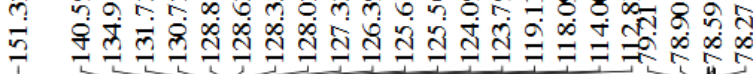<smiles>CC(C)(F)C1(c2c[nH]c3ccc(Br)cc23)C=C(c2ccccc2)c2c(ccc3ccccc23)O1</smiles>

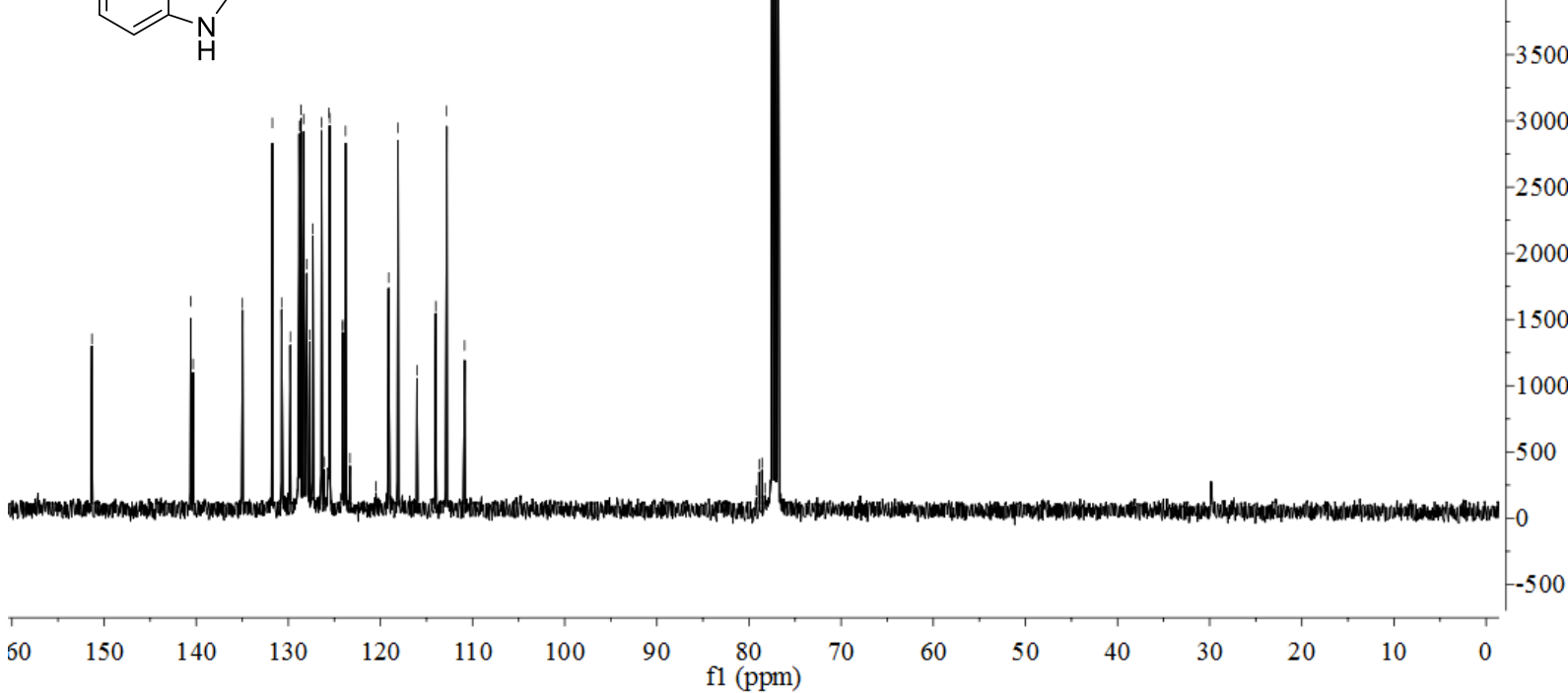




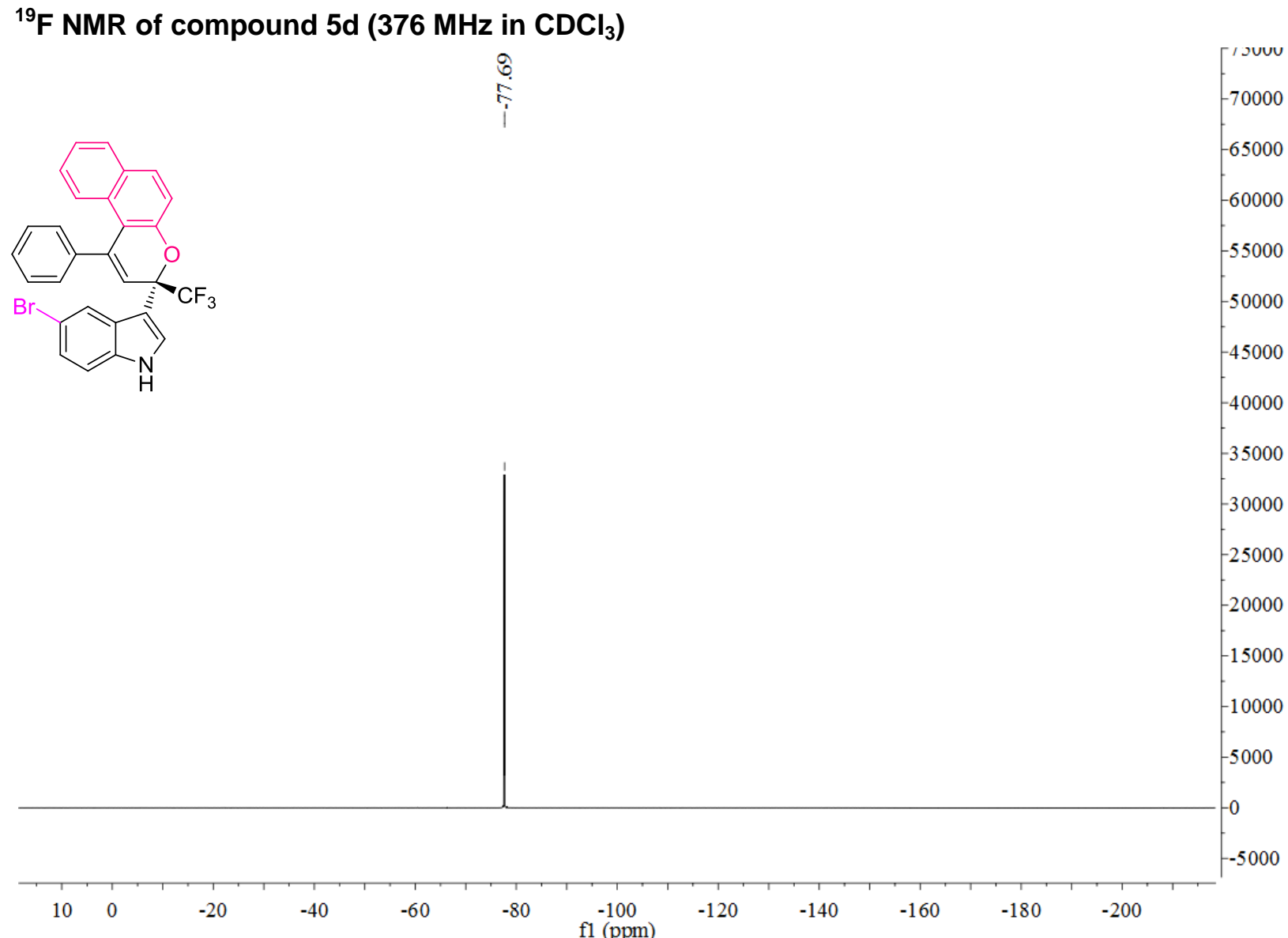

${ }^{1} \mathrm{H}$ NMR of compound $5 \mathrm{e}\left(400 \mathrm{MHz}\right.$ in $\left.\mathrm{CDCl}_{3}\right)$

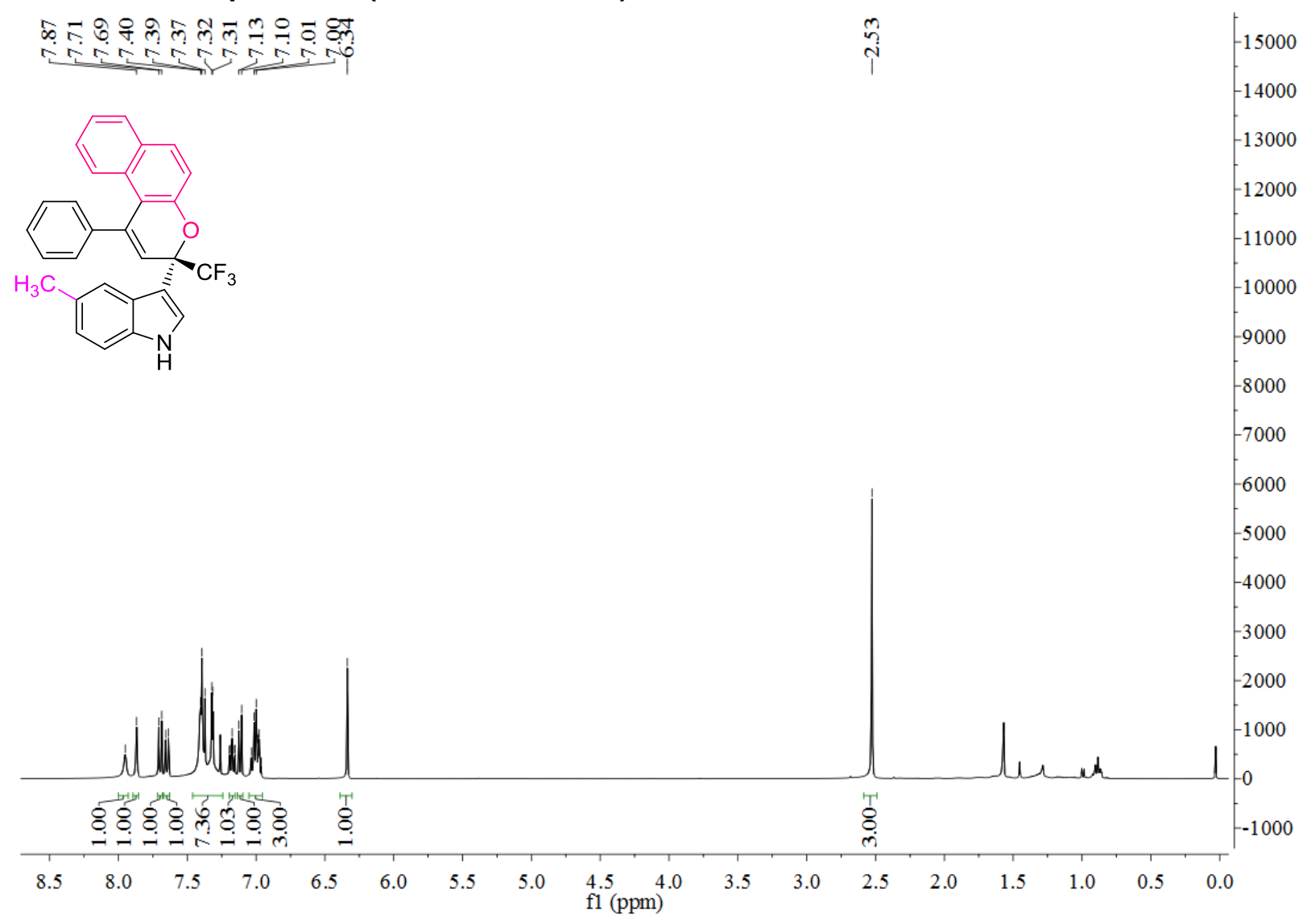


${ }^{13} \mathrm{C}$ NMR of compound $5 \mathrm{e}\left(101 \mathrm{MHz}\right.$ in $\mathrm{CDCl}_{3}$ )

m

万<smiles>Cc1ccc2[nH]cc([C@]3(C(F)(F)F)C=C(c4ccccc4)c4c(ccc5ccccc45)O3)c2c1</smiles>

$\frac{8}{1}$

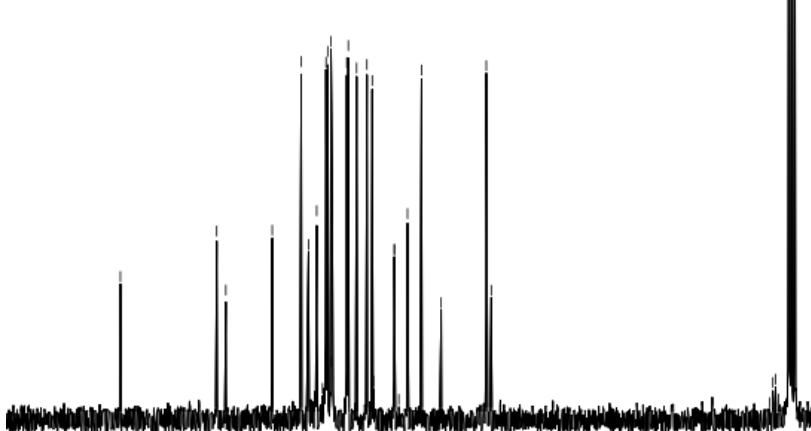

\section{${ }^{19} \mathrm{~F}$ NMR of compound $5 \mathrm{e}\left(376 \mathrm{MHz}\right.$ in $\left.\mathrm{CDCl}_{3}\right)$}

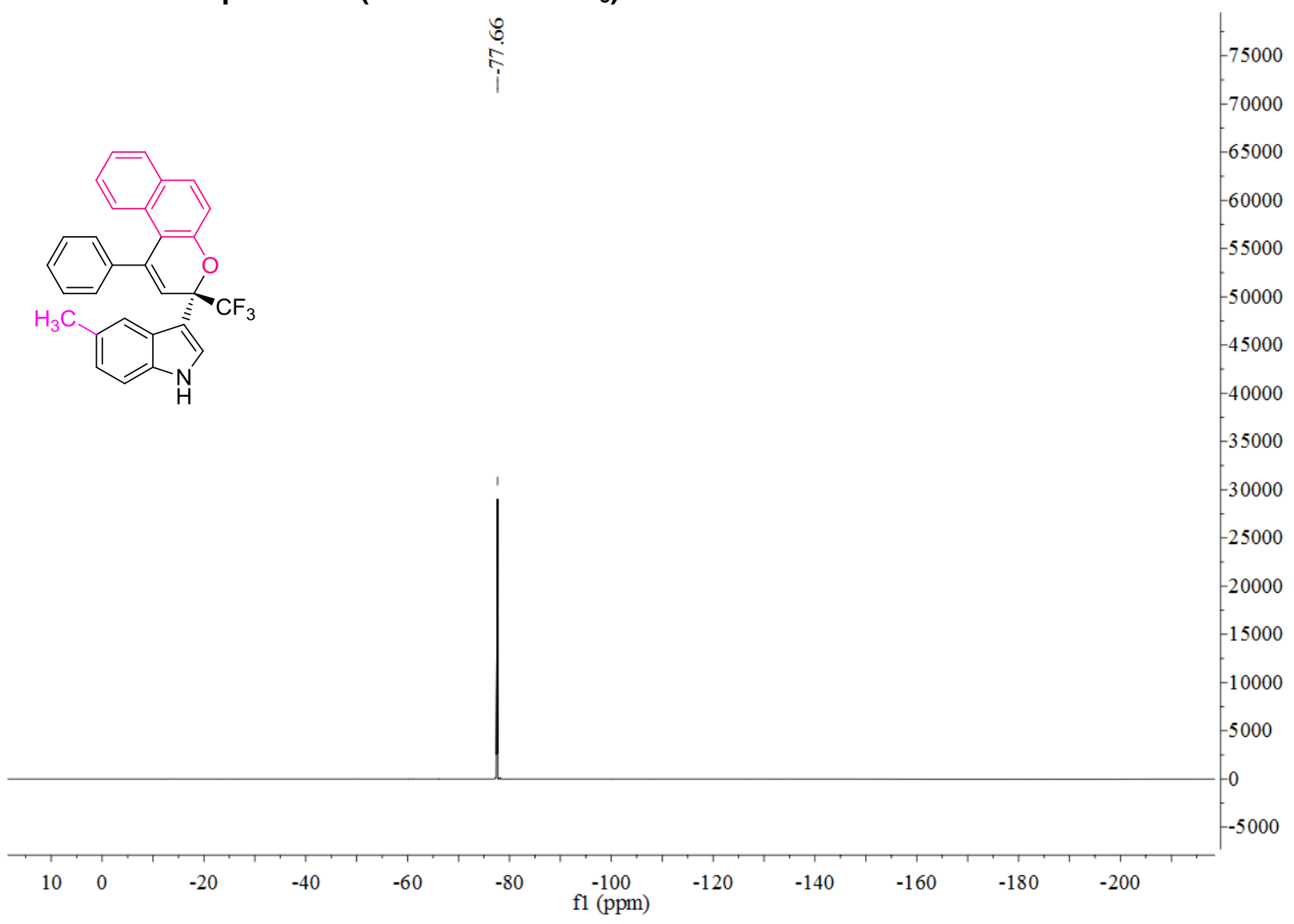


${ }^{1} \mathrm{H}$ NMR of compound $5 f\left(400 \mathrm{MHz}\right.$ in $\left.\mathrm{CDCl}_{3}\right)$

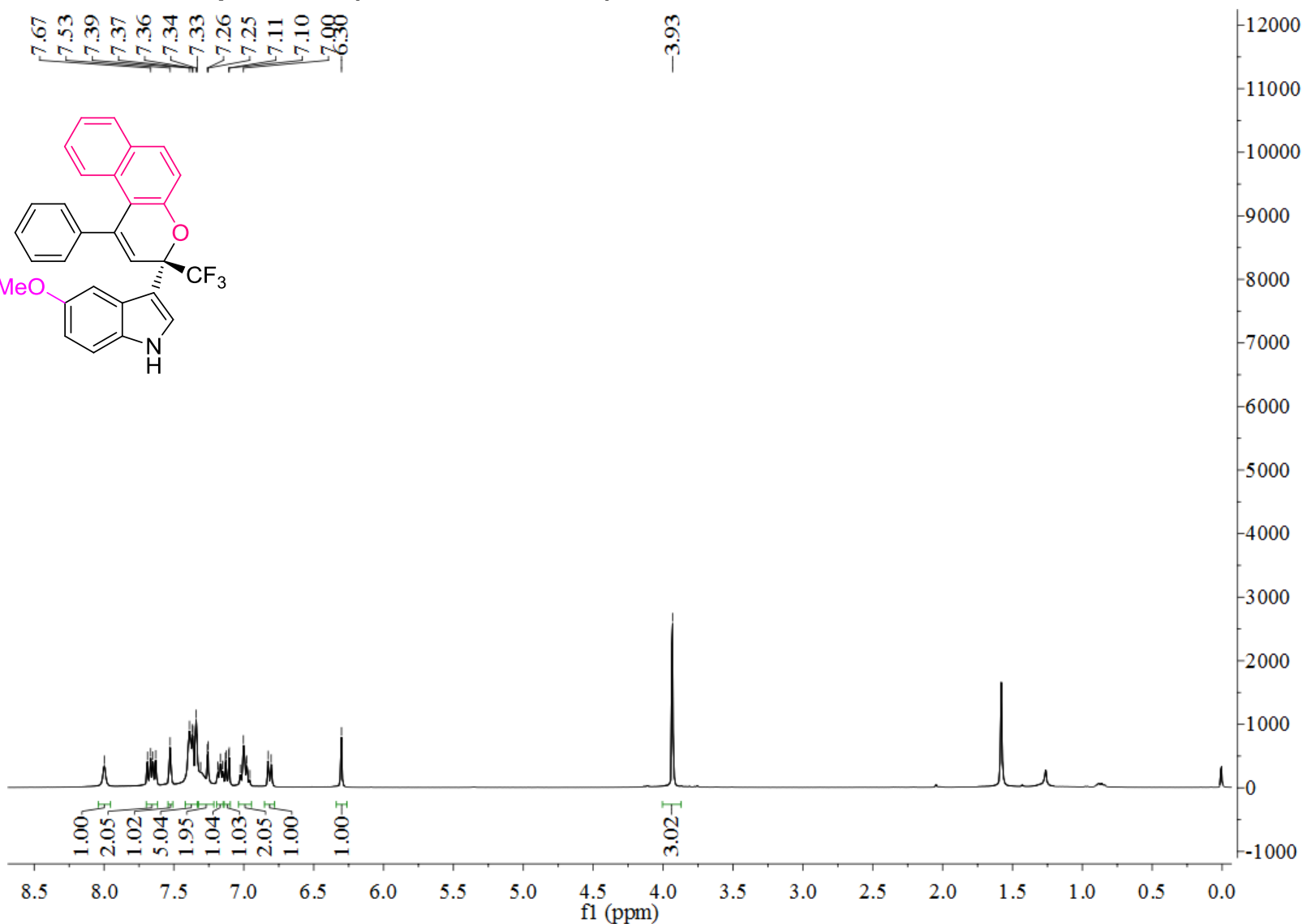

${ }^{13} \mathrm{C}$ NMR of compound $5 f\left(101 \mathrm{MHz}\right.$ in $\mathrm{CDCl}_{3}$ )

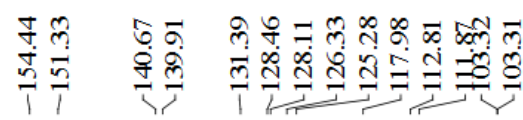<smiles>COc1ccc2[nH]cc(C3(C(F)(F)F)C=C(c4ccccc4)c4c(ccc5ccccc45)O3)c2c1</smiles>

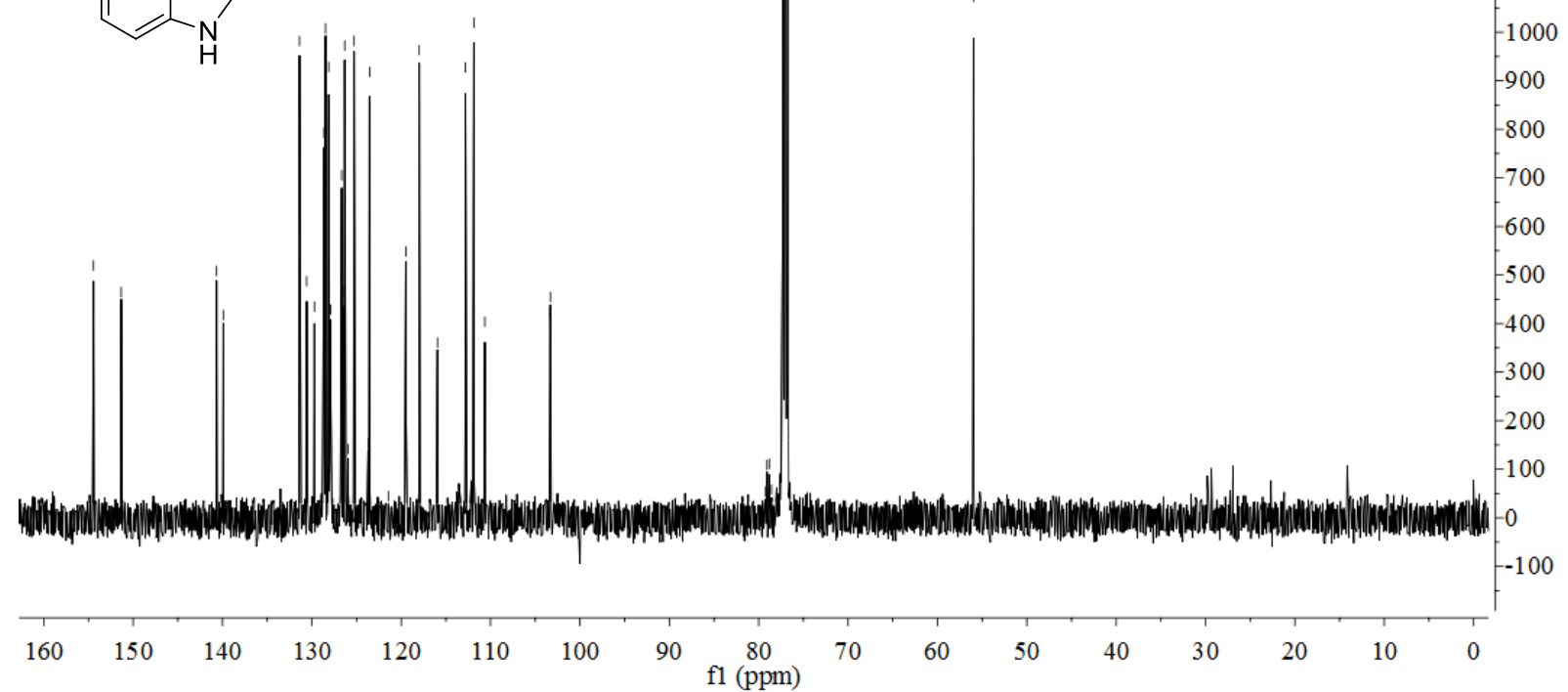


${ }^{19} \mathrm{~F} \mathrm{NMR}$ of compound $5 \mathrm{f}\left(376 \mathrm{MHz}\right.$ in $\left.\mathrm{CDCl}_{3}\right)$

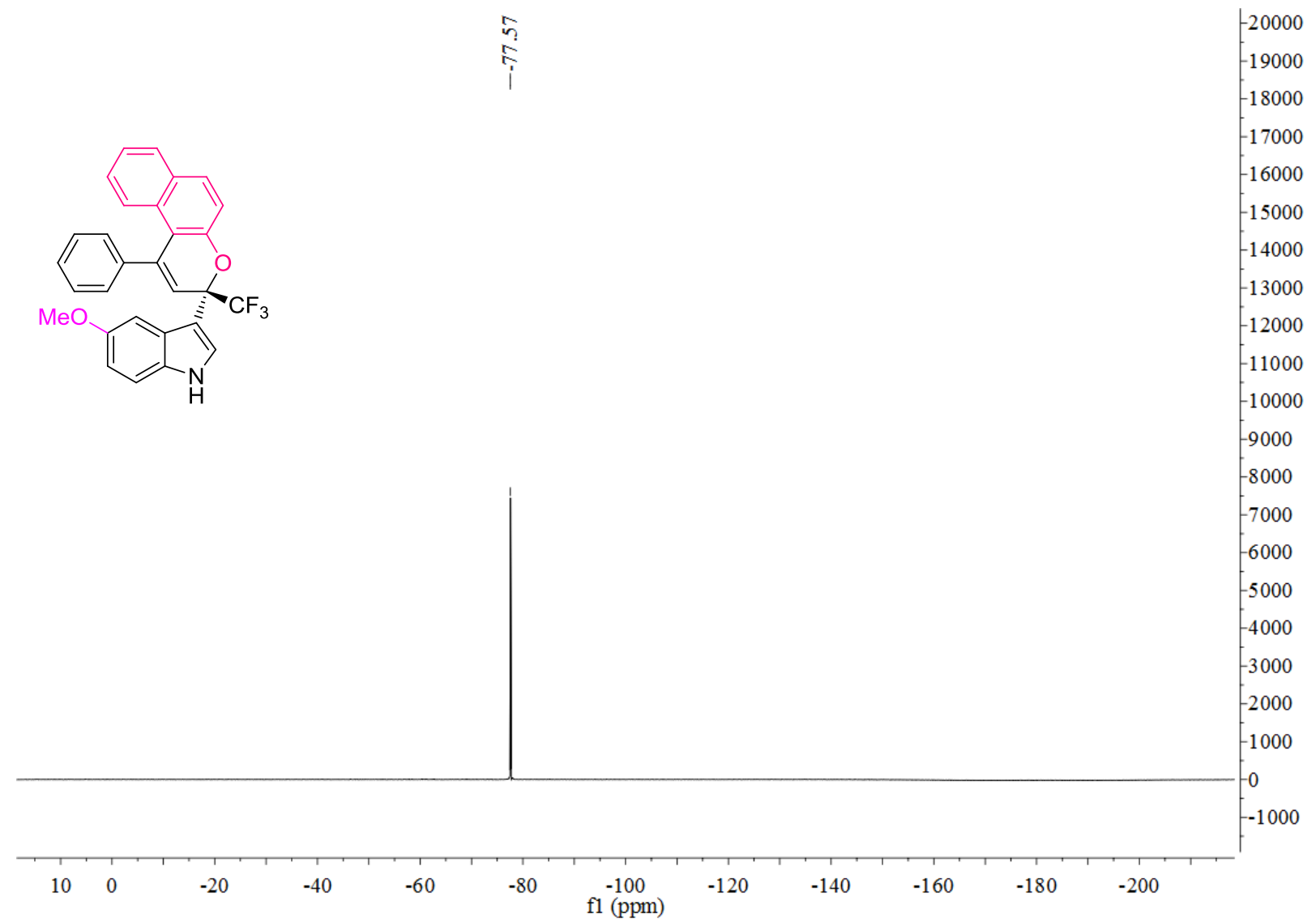

${ }^{1} \mathrm{H}$ NMR of compound $5 \mathrm{~g}\left(400 \mathrm{MHz}\right.$ in $\left.\mathrm{CDCl}_{3}\right)$

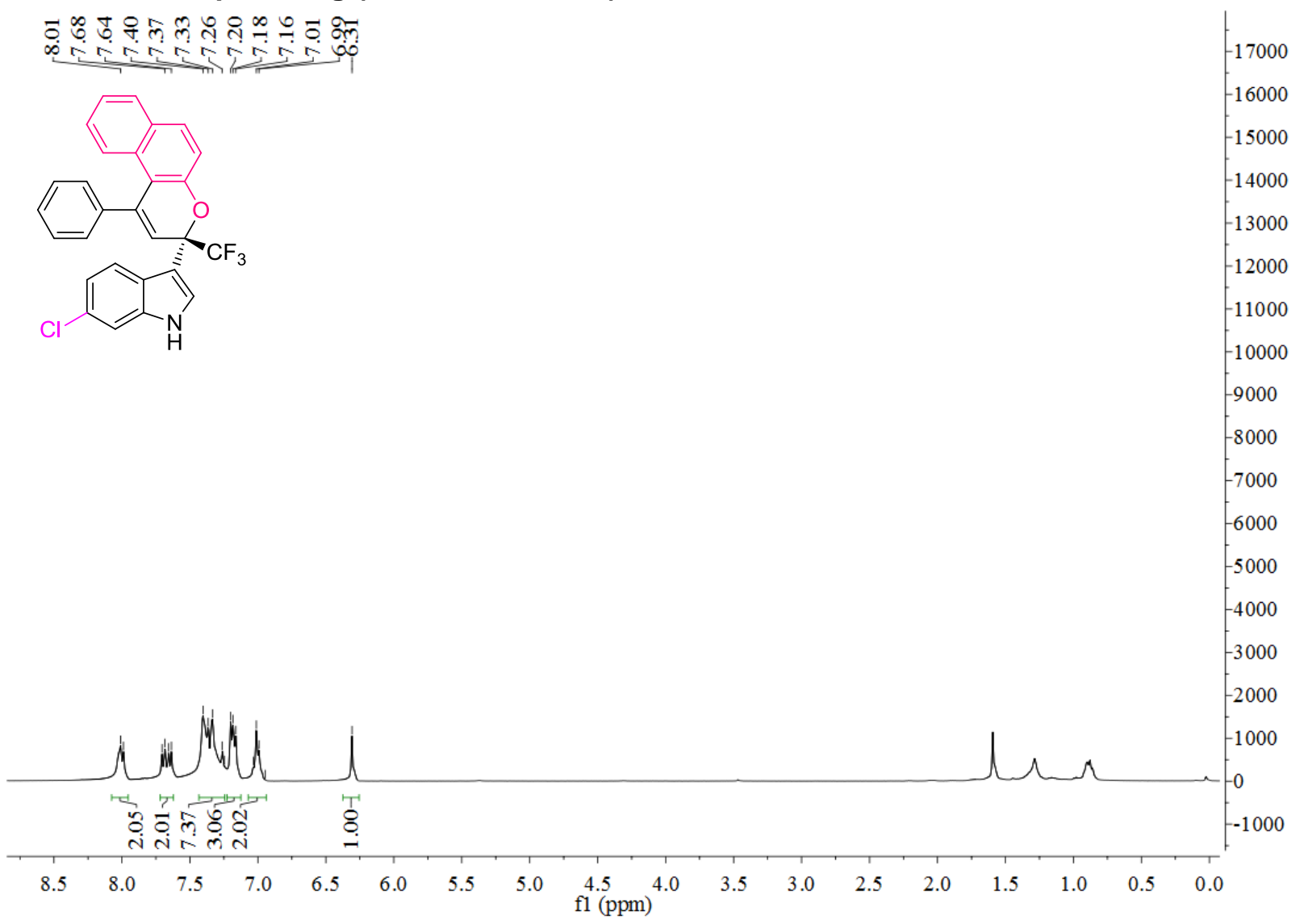




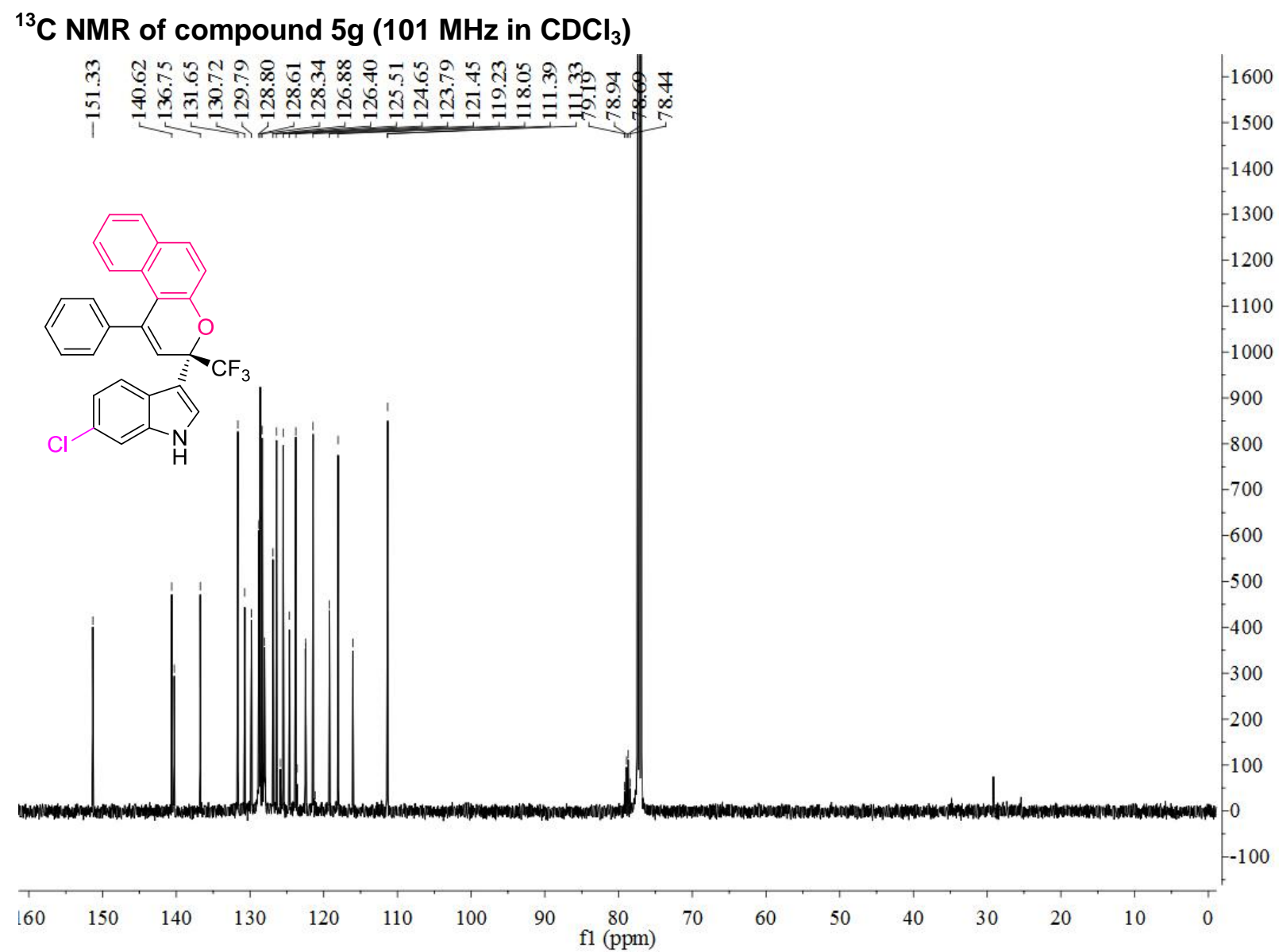

${ }^{19} \mathrm{~F}$ NMR of compound $5 \mathrm{~g}\left(376 \mathrm{MHz}\right.$ in $\mathrm{CDCl}_{3}$ )

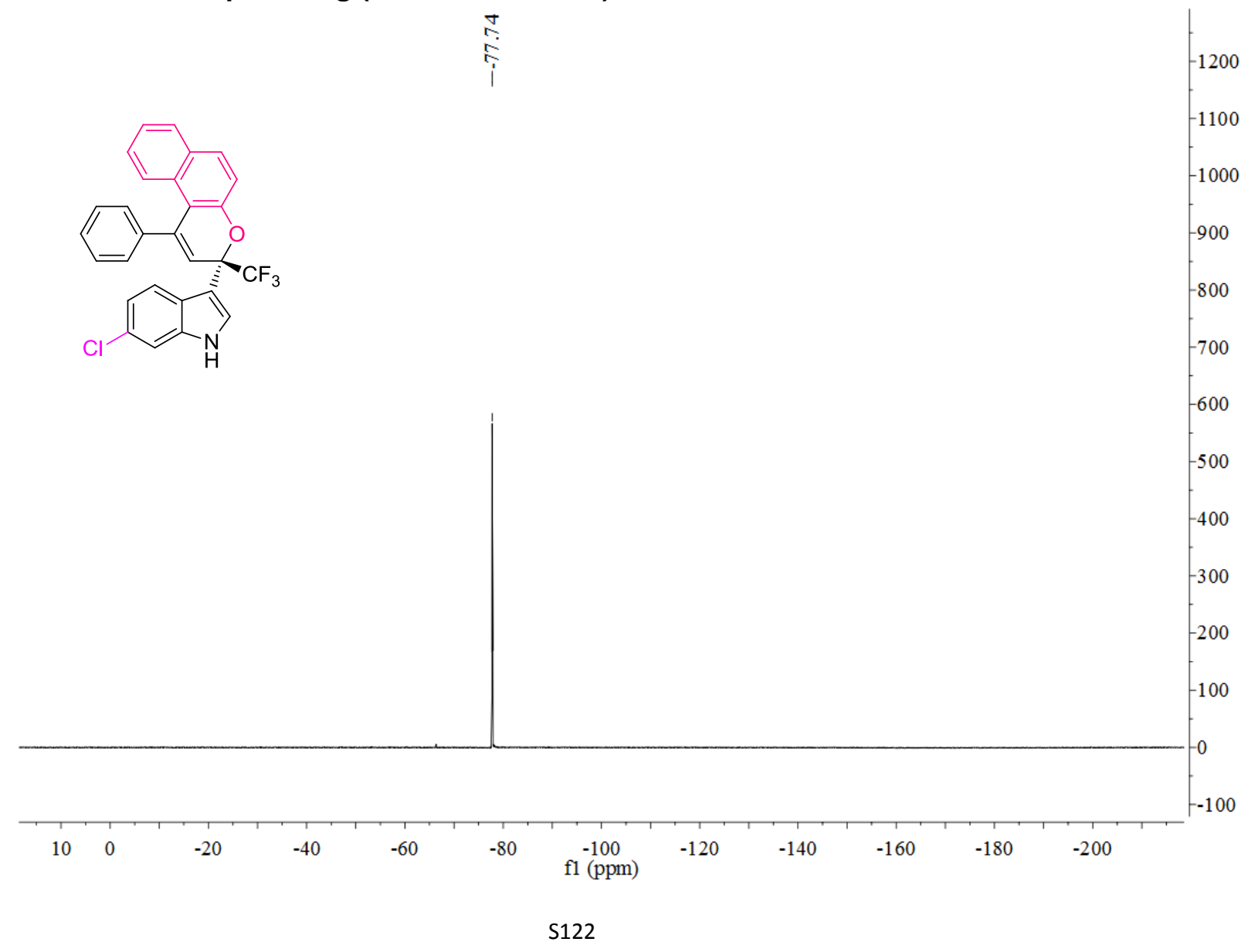


${ }^{1} \mathrm{H}$ NMR of compound $5 \mathrm{~h}\left(400 \mathrm{MHz}\right.$ in $\left.\mathrm{CDCl}_{3}\right)$

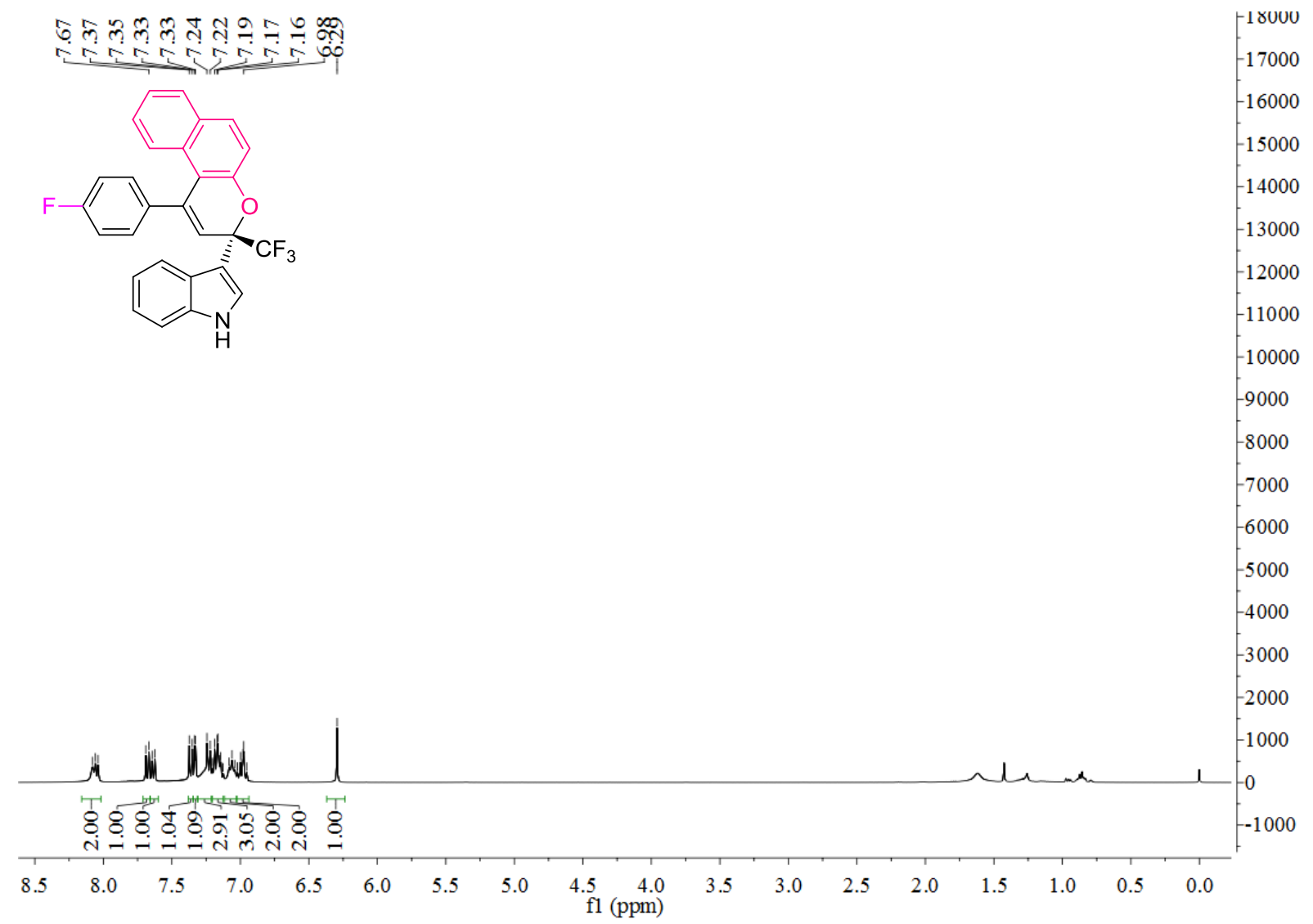

${ }^{13} \mathrm{C}$ NMR of compound $5 \mathrm{~h}\left(101 \mathrm{MHz}\right.$ in $\mathrm{CDCl}_{3}$ )

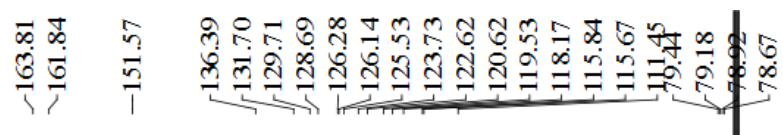<smiles>Fc1ccc(C2=C[C@](c3c[nH]c4ccccc34)(C(F)(F)F)Oc3ccc4ccccc4c32)cc1</smiles>

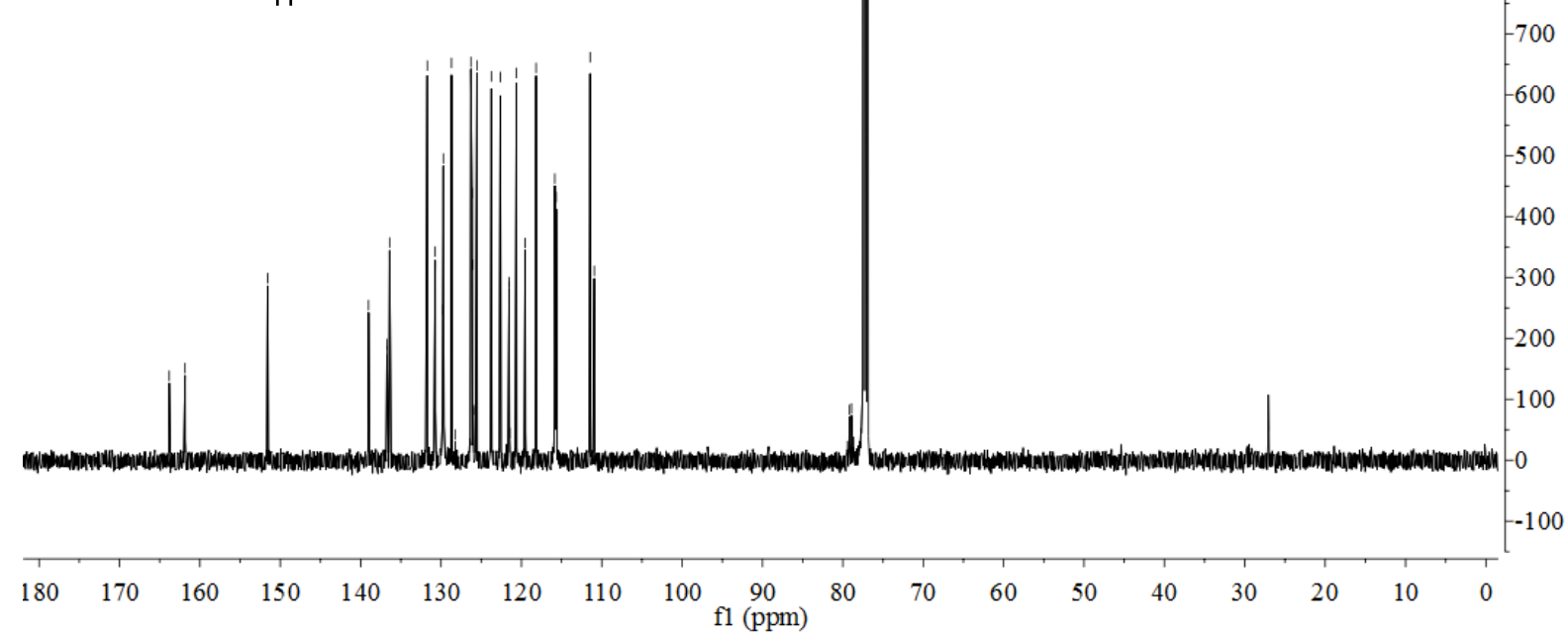


${ }^{19} \mathrm{~F} \mathrm{NMR}$ of compound $5 \mathrm{~h}\left(376 \mathrm{MHz}\right.$ in $\left.\mathrm{CDCl}_{3}\right)$

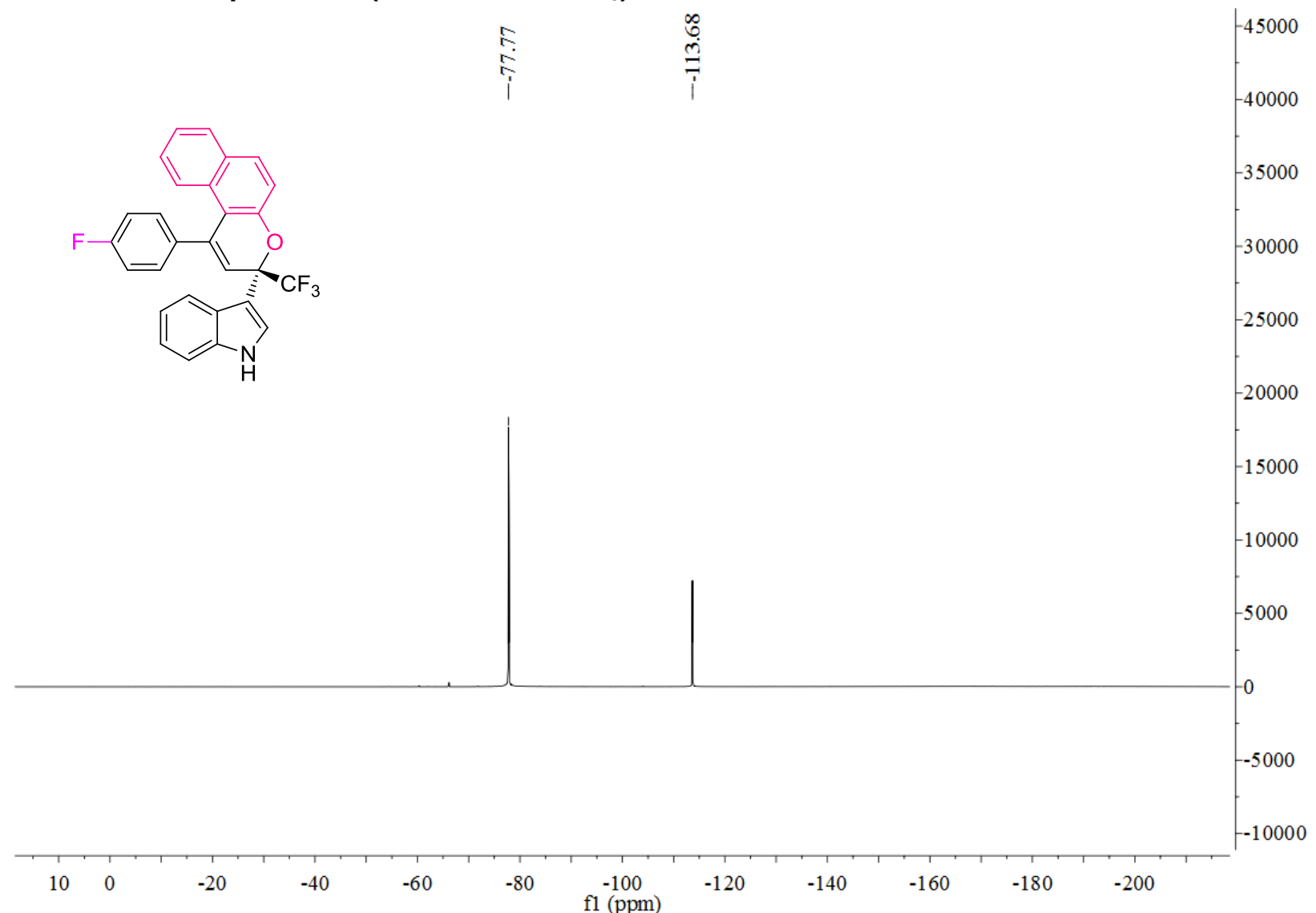

${ }^{1} \mathrm{H}$ NMR of compound $5 \mathrm{i}\left(400 \mathrm{MHz}\right.$ in $\mathrm{CDCl}_{3}$ )

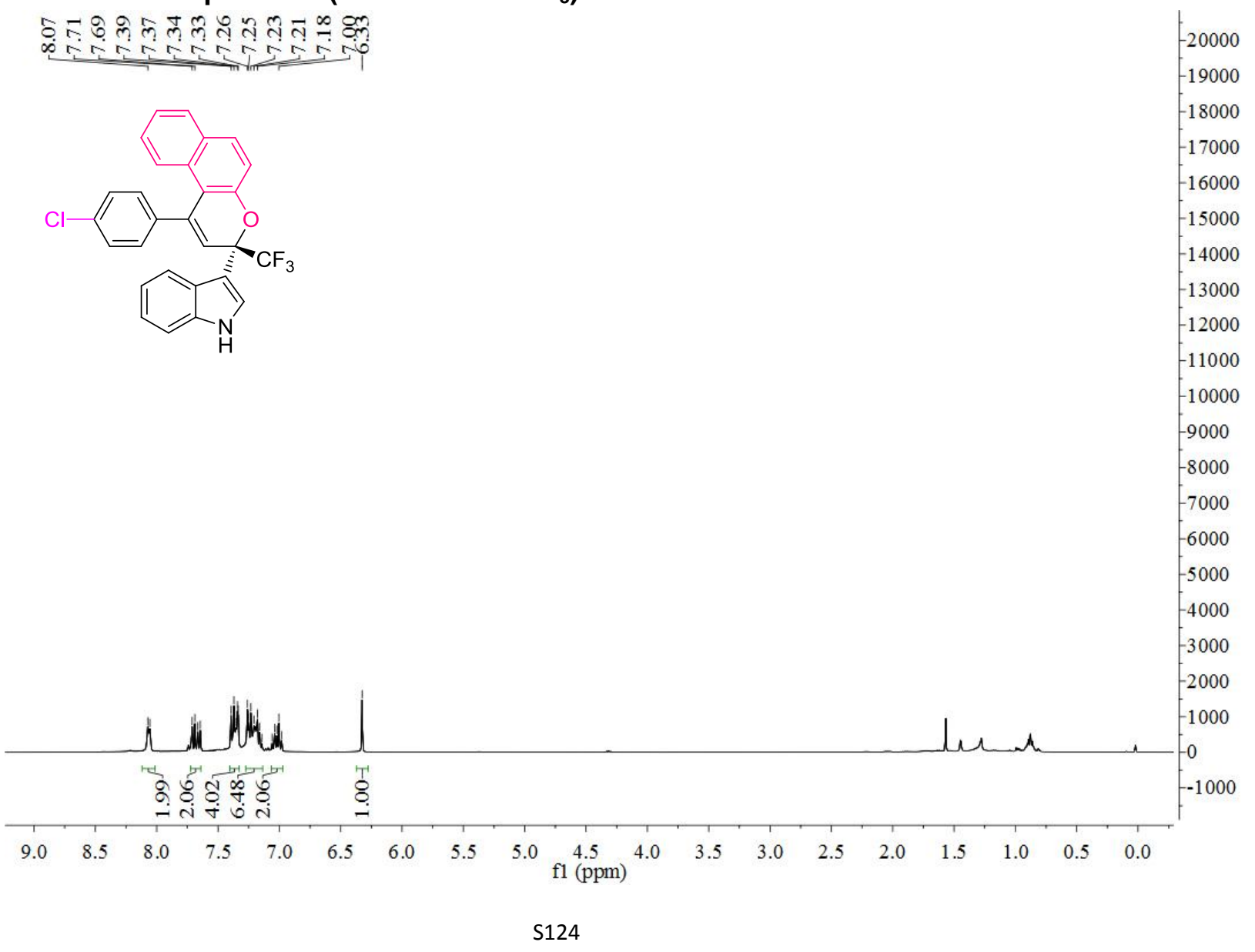




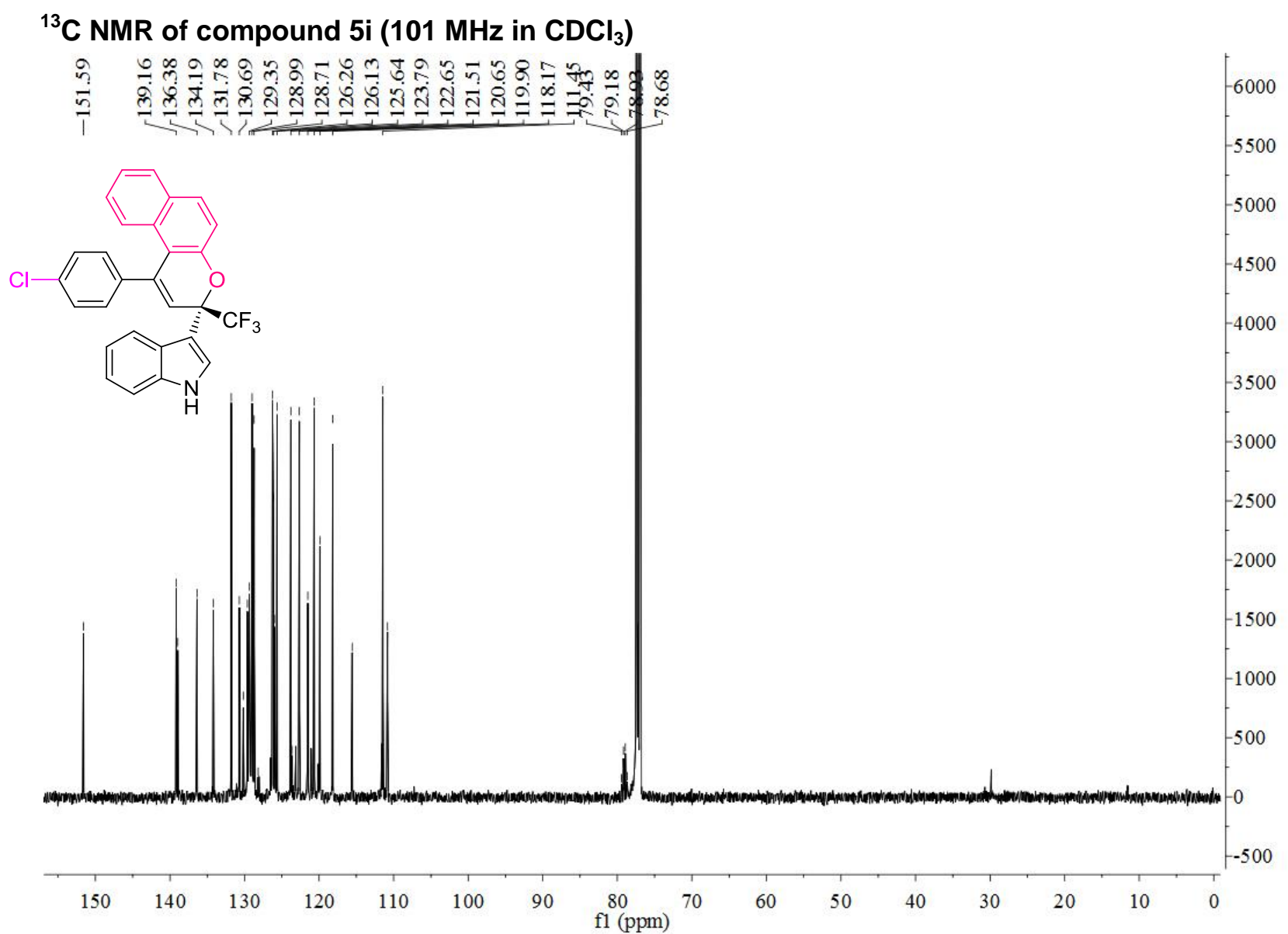

${ }^{19} \mathrm{~F}$ NMR of compound $5 \mathrm{i}\left(376 \mathrm{MHz}\right.$ in $\mathrm{CDCl}_{3}$ )

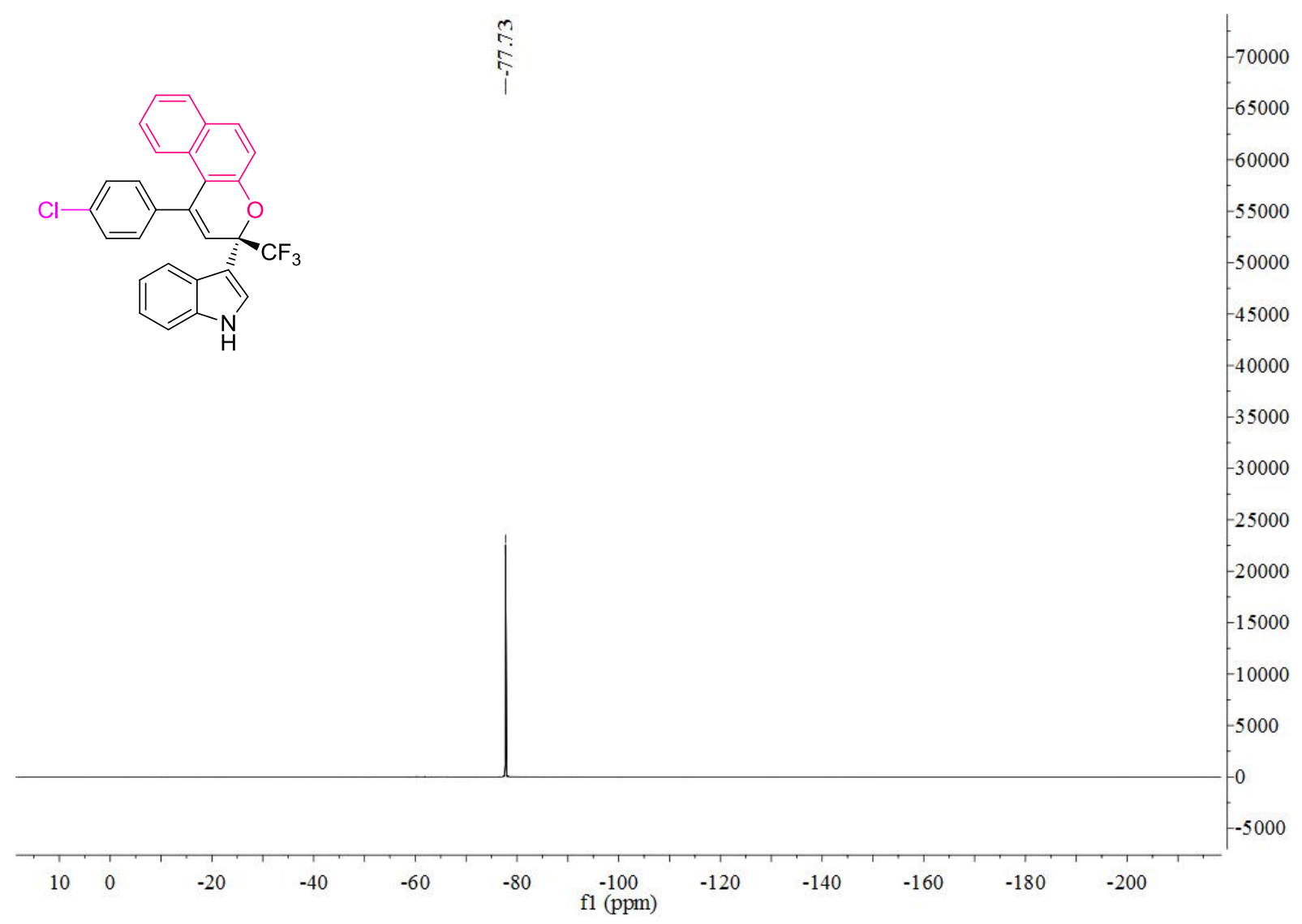


${ }^{1} \mathrm{H}$ NMR of compound $5 \mathrm{j}$ (400 $\mathrm{MHz}$ in $\mathrm{CDCl}_{3}$ )

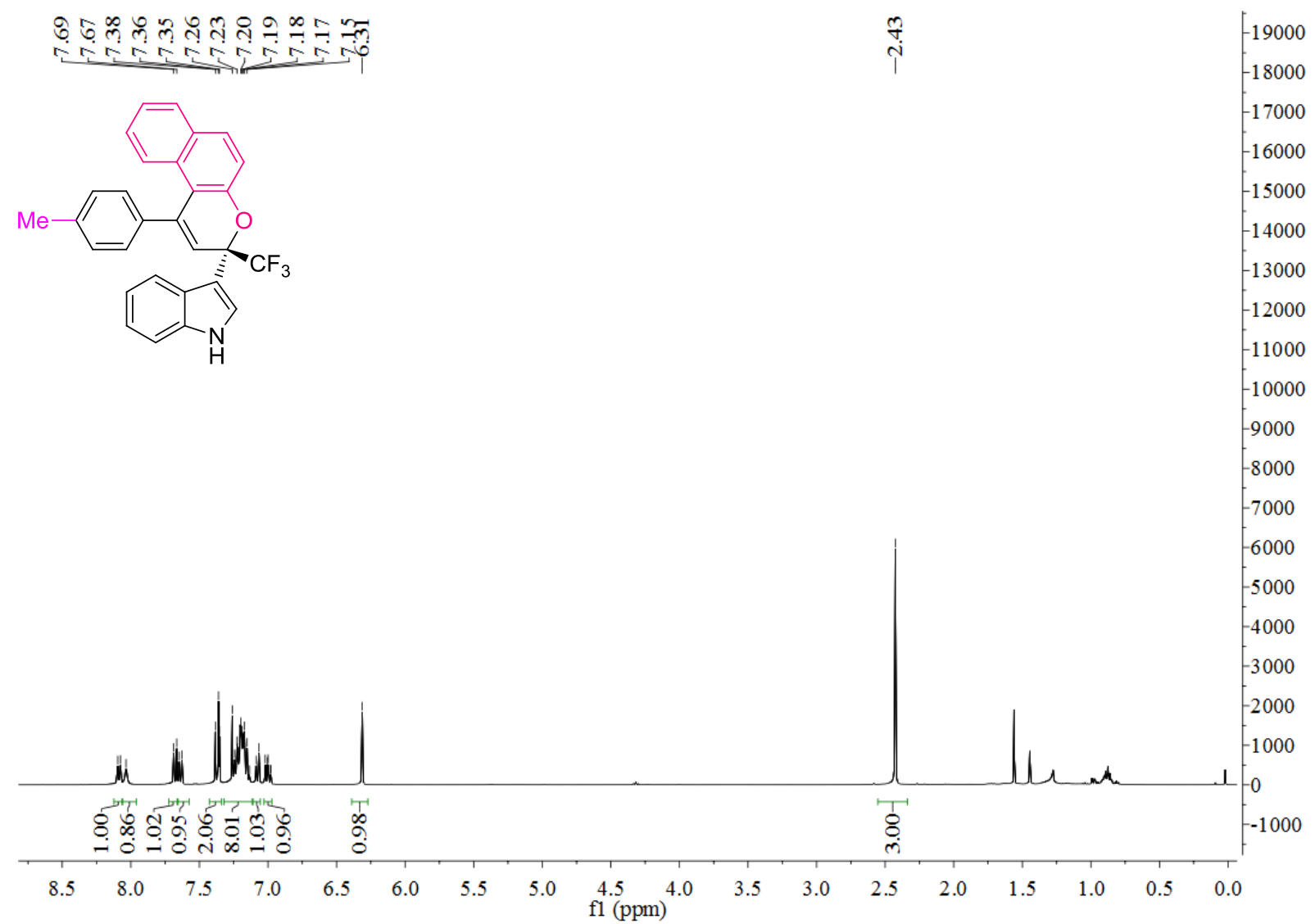

${ }^{13} \mathrm{C}$ NMR of compound $5 \mathrm{j}$ (101 $\mathrm{MHz}$ in $\mathrm{CDCl}_{3}$ )

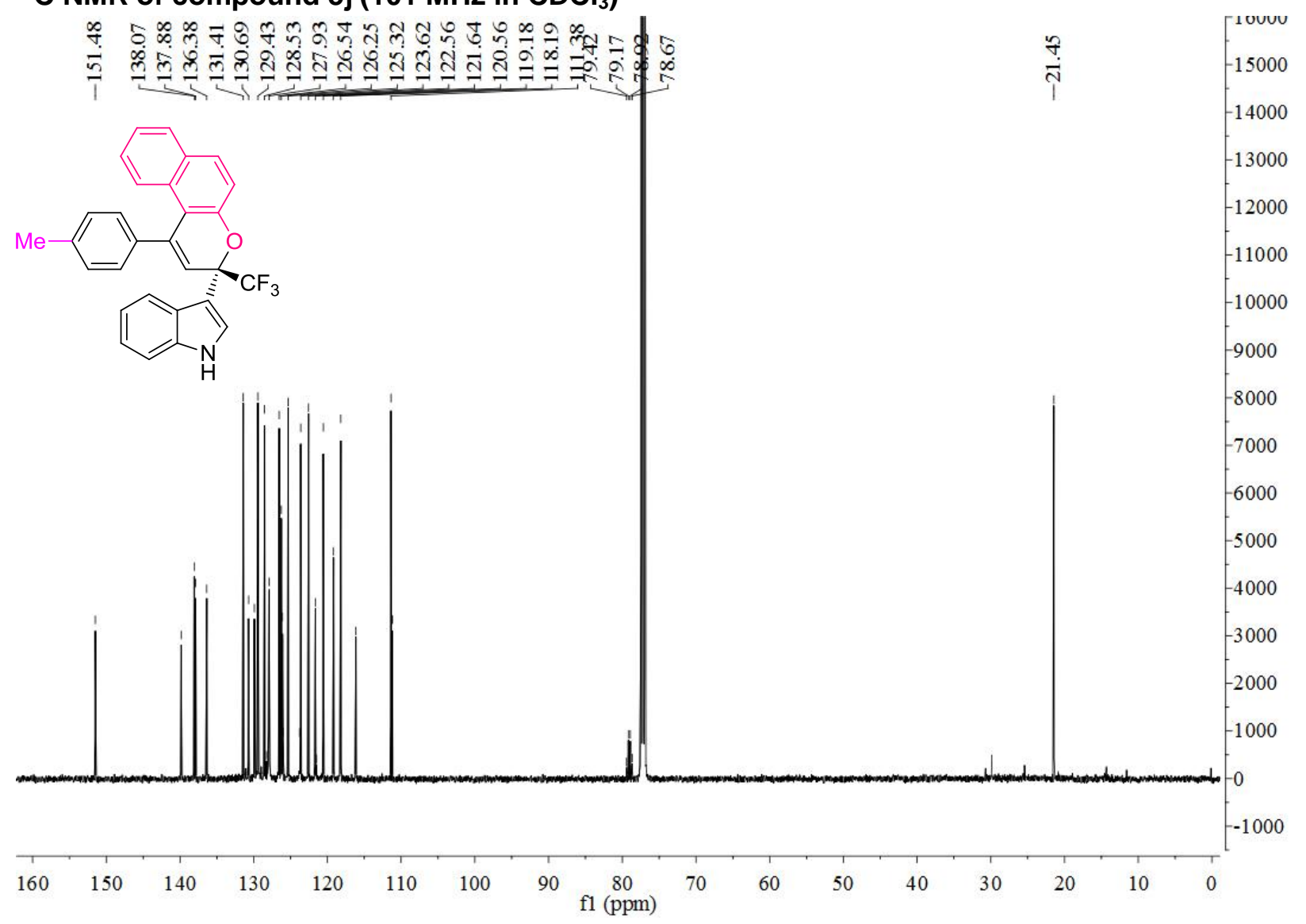




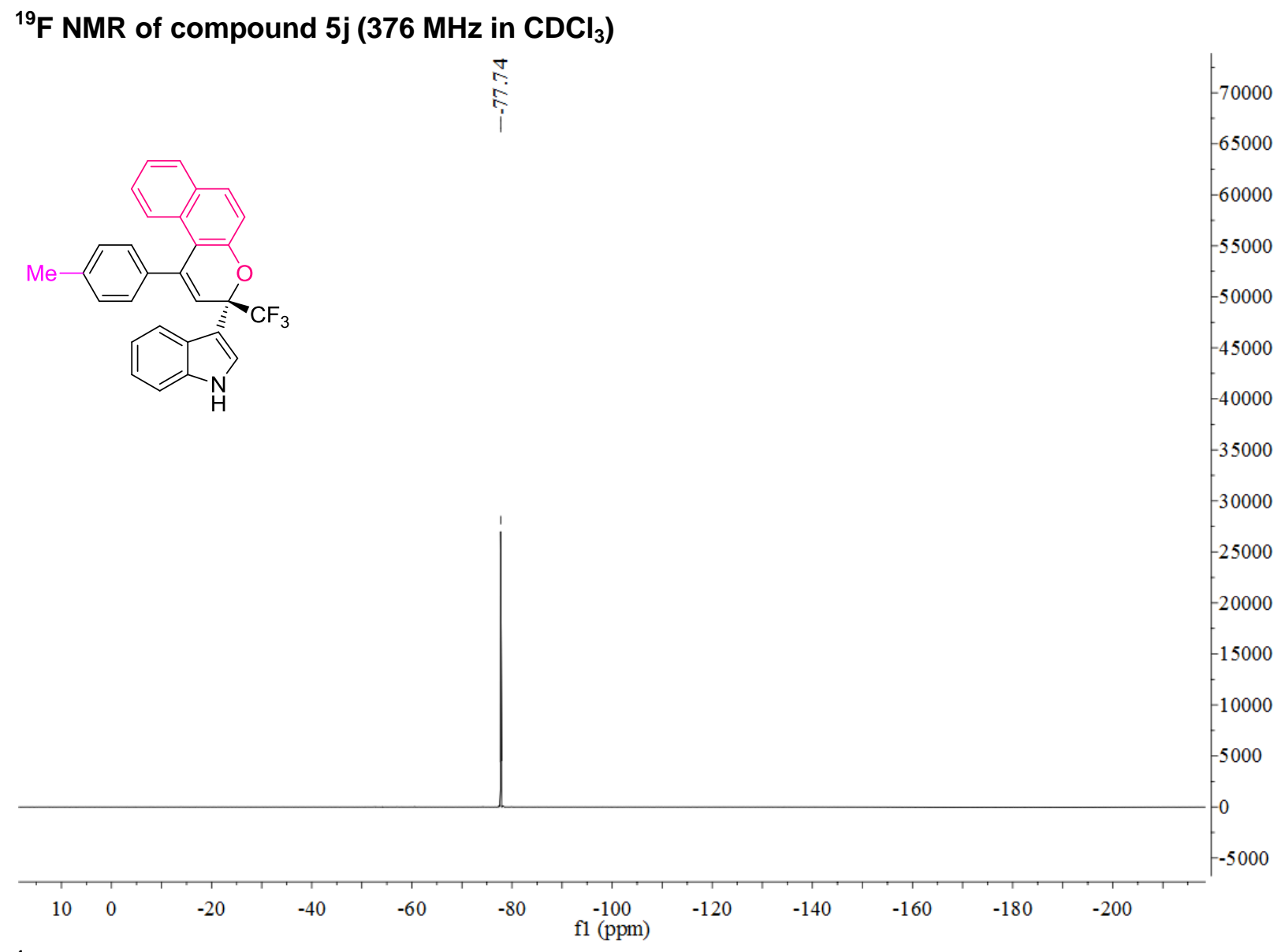

${ }^{1} \mathrm{H}$ NMR of compound $5 \mathrm{k}\left(400 \mathrm{MHz}\right.$ in $\mathrm{CDCl}_{3}$ )

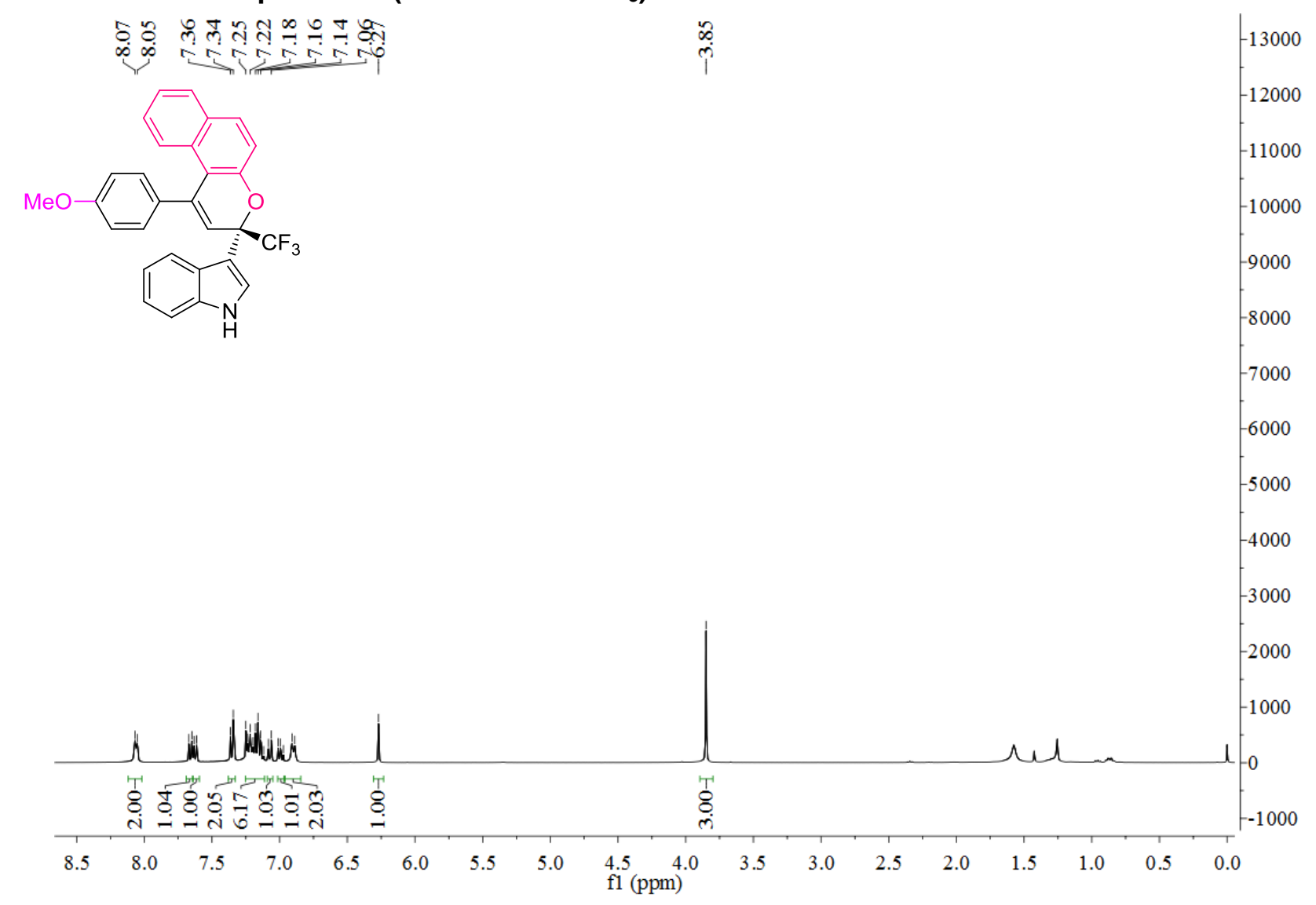




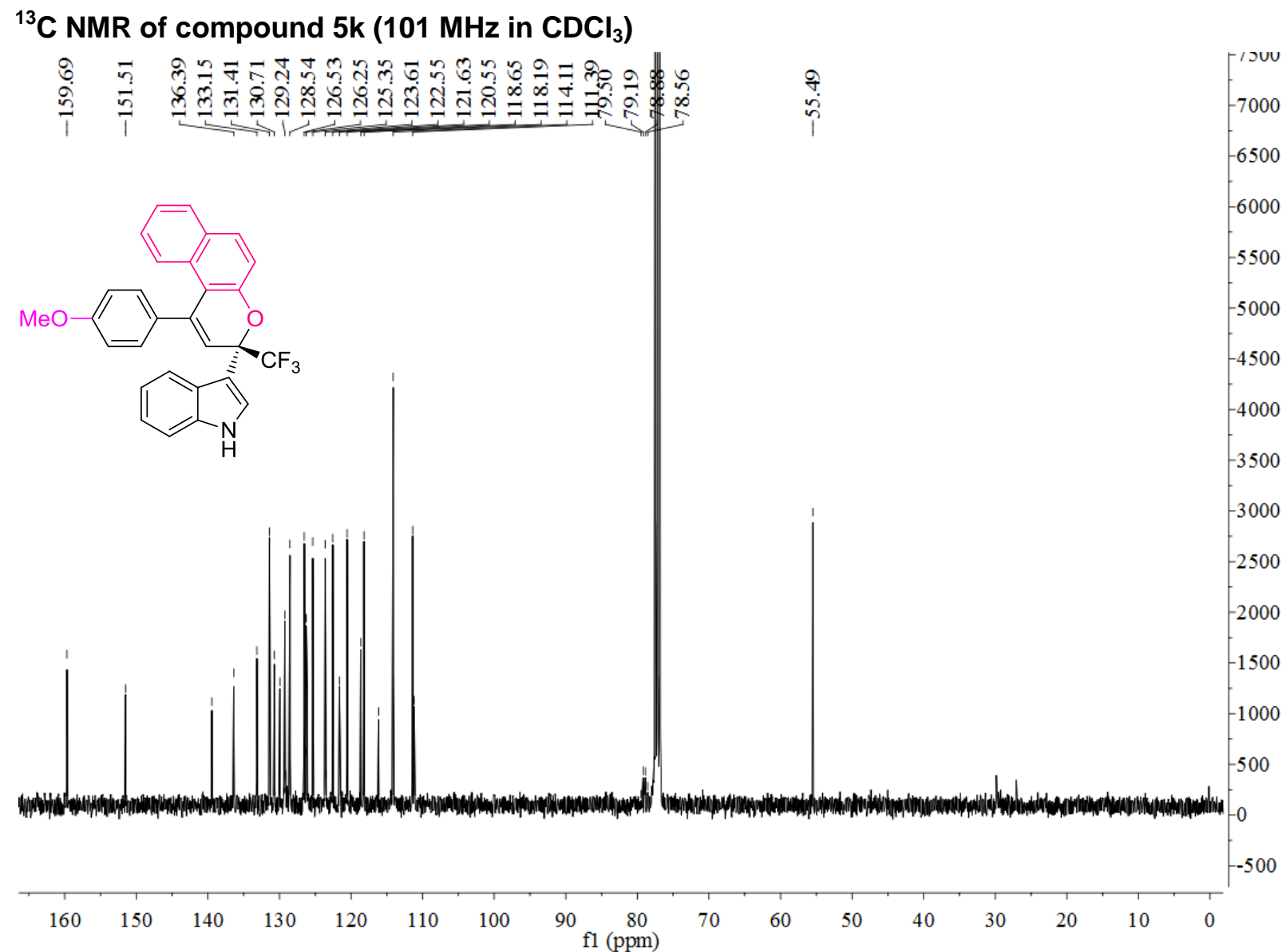

${ }^{19} \mathrm{~F} \mathrm{NMR}$ of compound $5 \mathrm{k}$ (376 $\mathrm{MHz}$ in $\mathrm{CDCl}_{3}$ )

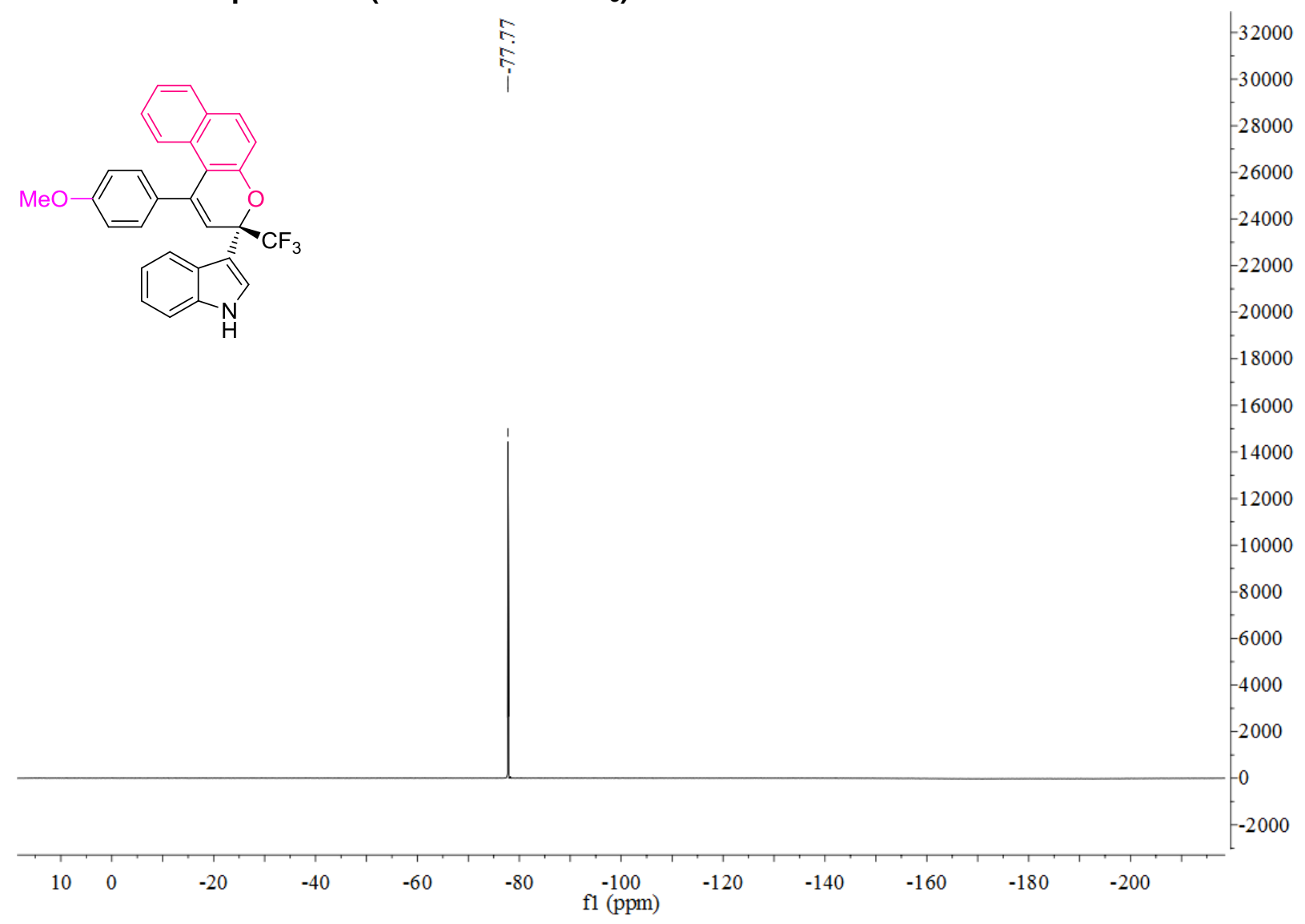


${ }^{1} \mathrm{H}$ NMR of compound $5 \mathrm{I}\left(400 \mathrm{MHz}\right.$ in $\left.\mathrm{CDCl}_{3}\right)$

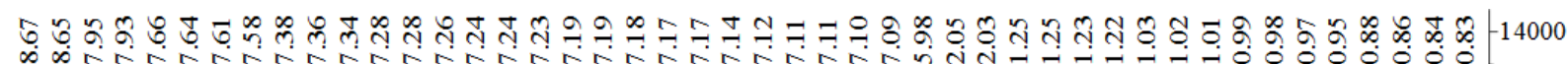

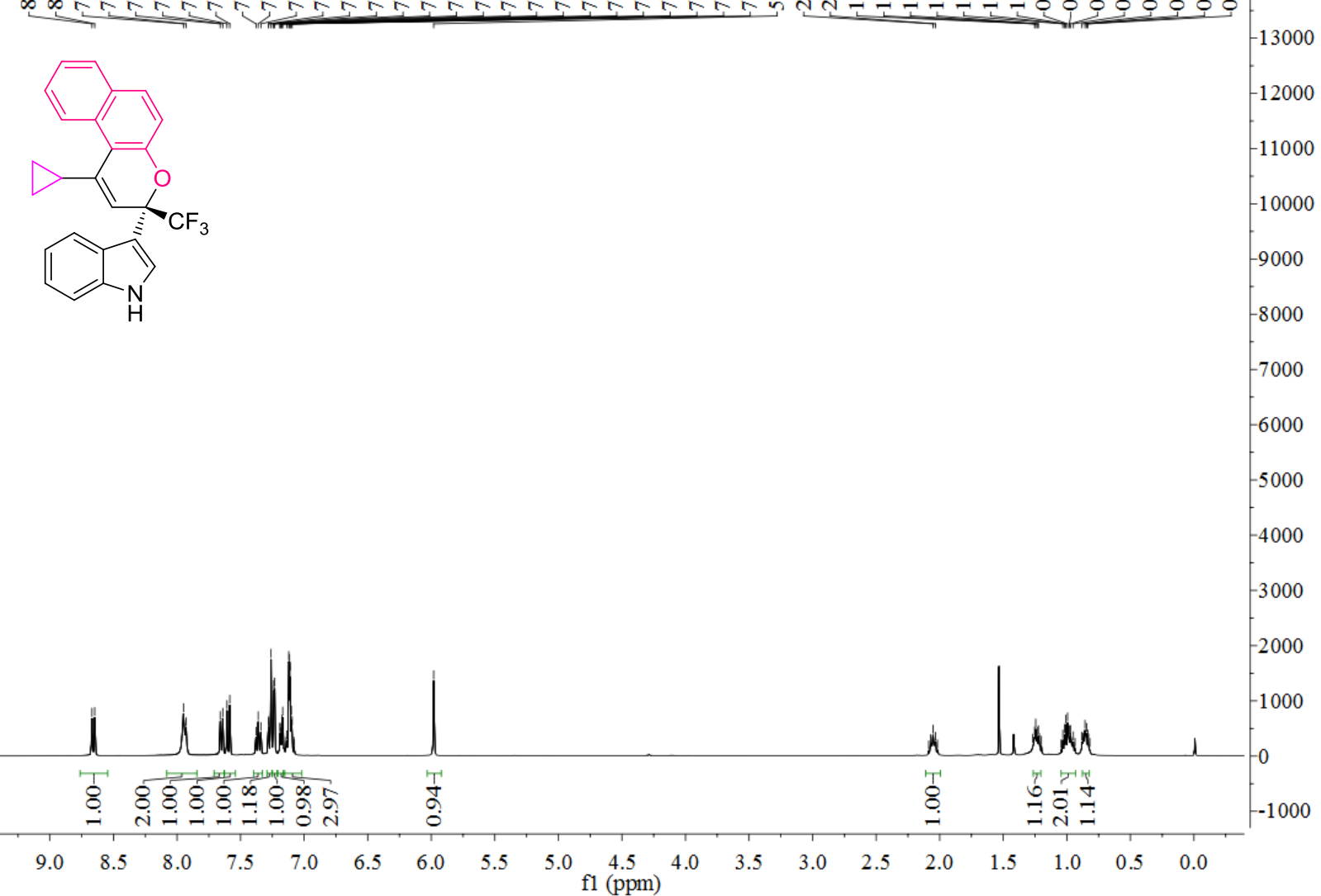

${ }^{13} \mathrm{C}$ NMR of compound $5 \mathrm{I}\left(101 \mathrm{MHz}\right.$ in $\left.\mathrm{CDCl}_{3}\right)$

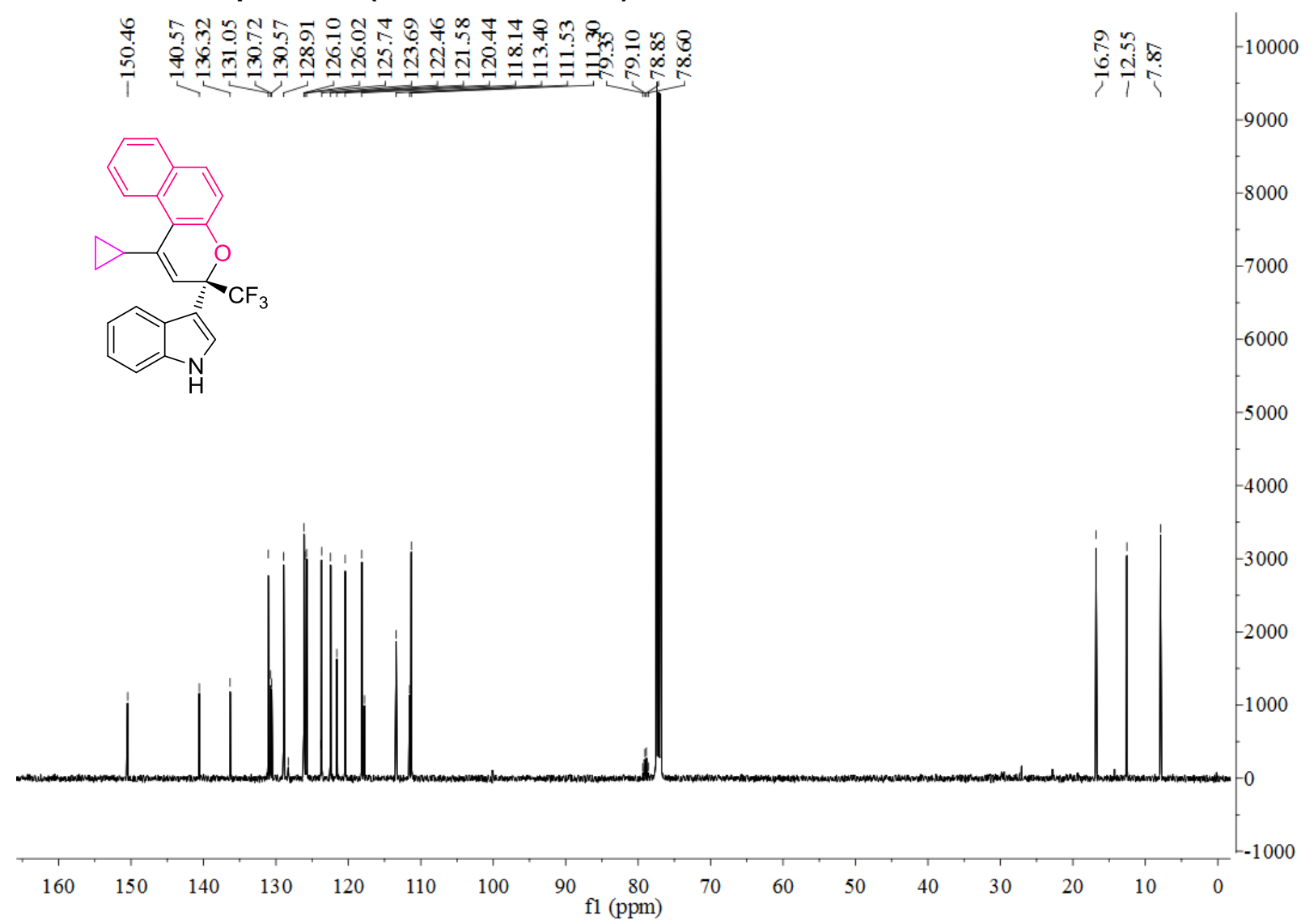


${ }^{19} \mathrm{~F} \mathrm{NMR}$ of compound $5 \mathrm{I}\left(376 \mathrm{MHz}\right.$ in $\mathrm{CDCl}_{3}$ )

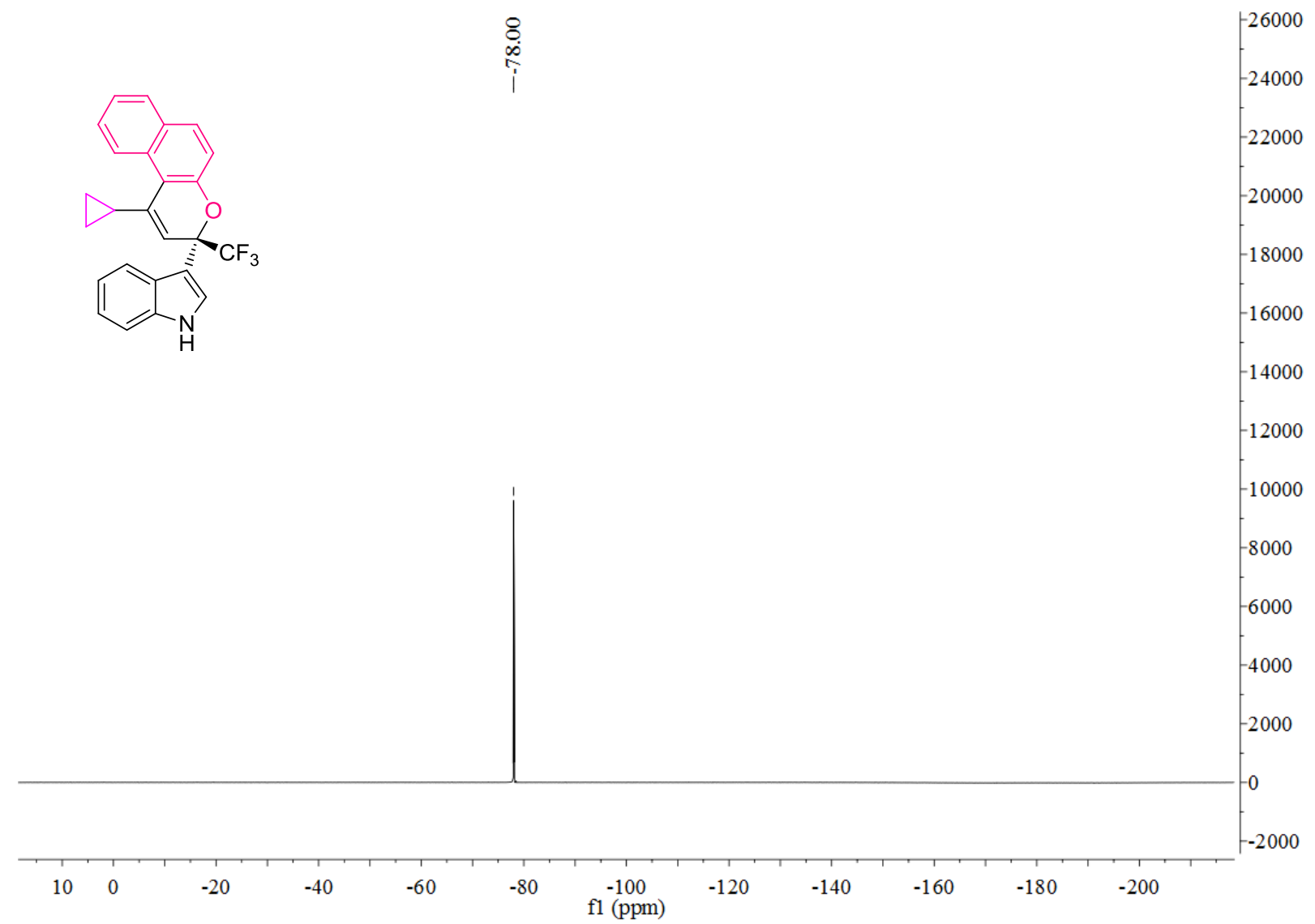

${ }^{1} \mathrm{H}$ NMR of compound 11 (400 $\mathrm{MHz}$ in $\mathrm{CDCl}_{3}$ )

东<smiles>Oc1ccc(C(=Cc2ccc(O)c3ccccc23)c2ccccc2)cc1</smiles>

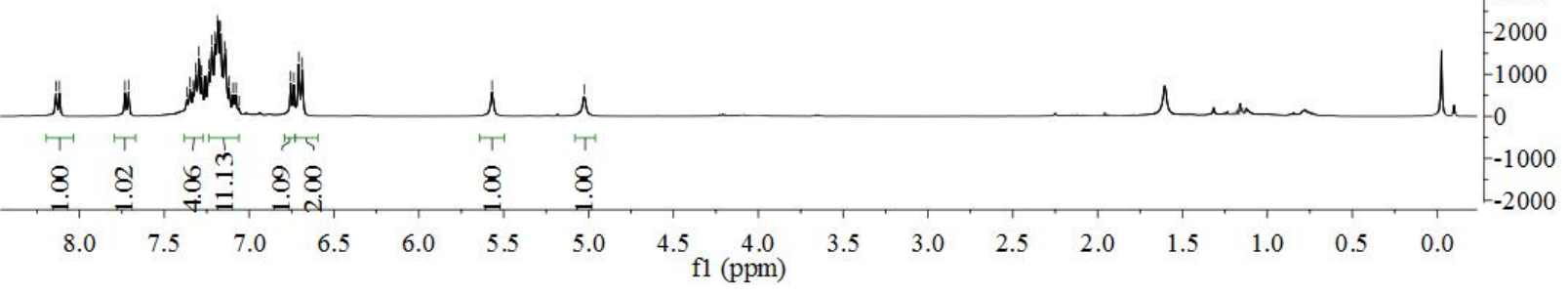


${ }^{13} \mathrm{C}$ NMR of compound 11 (126 MHz in $\mathrm{CDCl}_{3}$ )

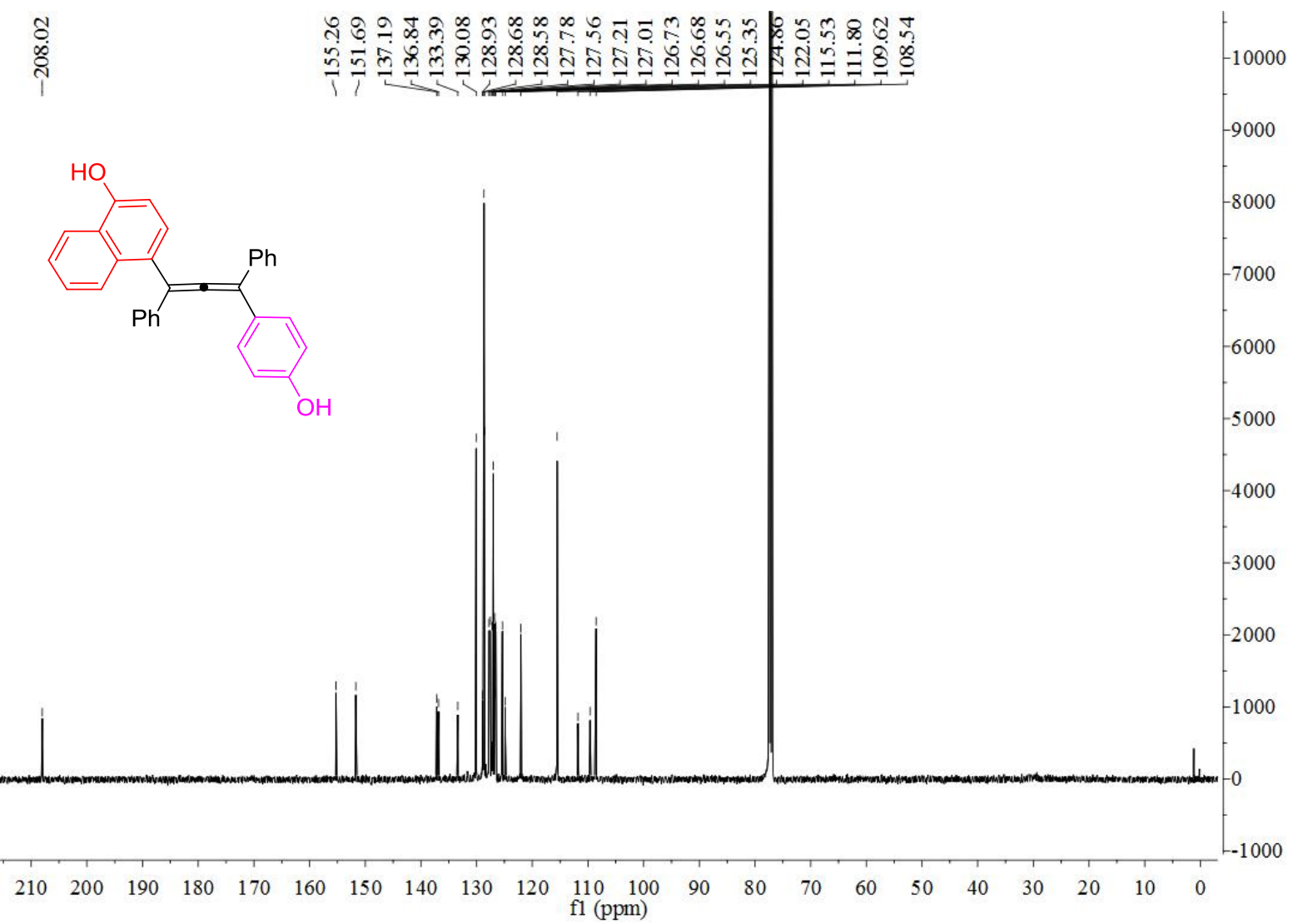

${ }^{1} \mathrm{H}$ NMR of compound $12\left(400 \mathrm{MHz}\right.$ in $\left.\mathrm{CDCl}_{3}\right)$

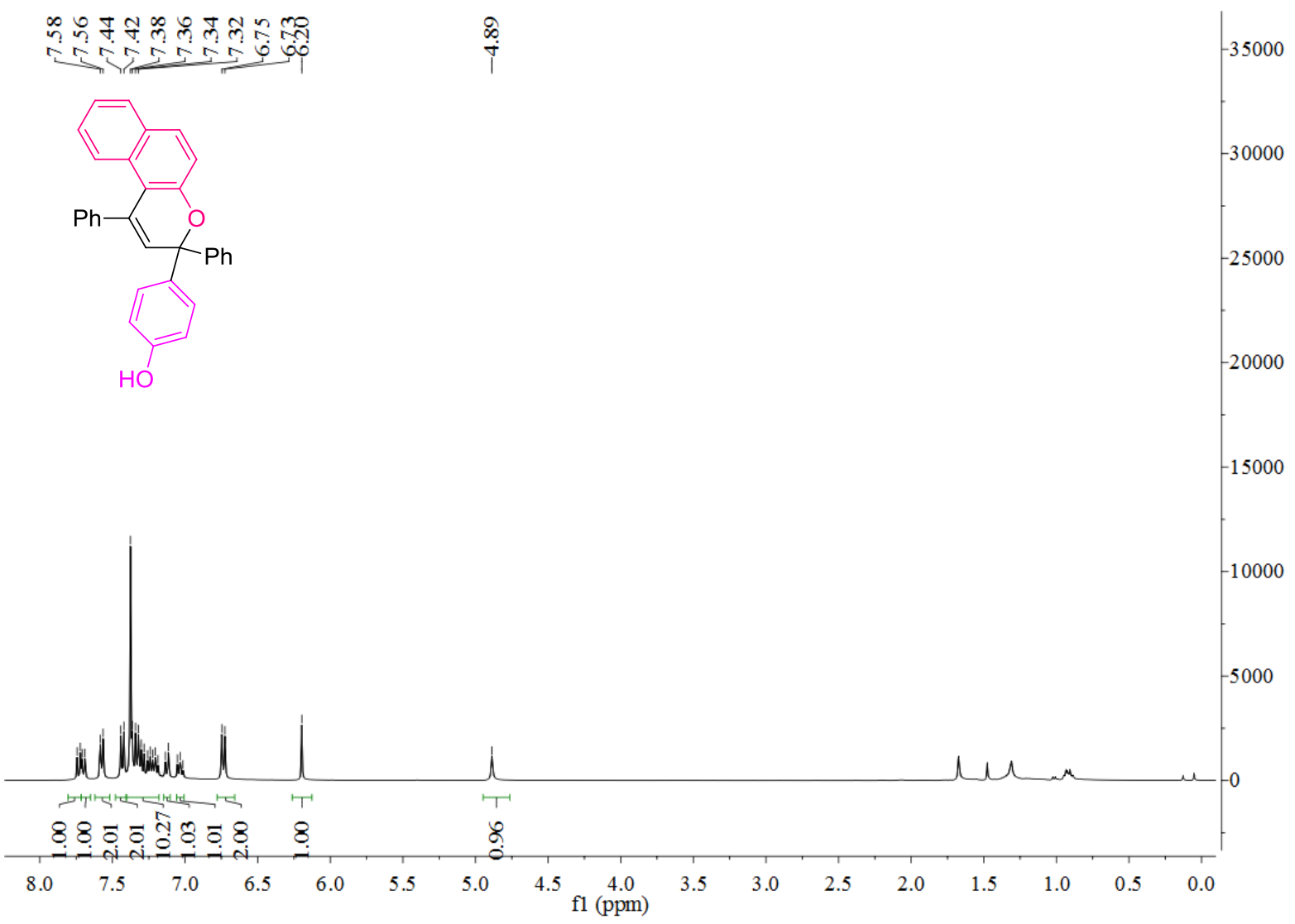


${ }^{13} \mathrm{C}$ NMR of compound $12\left(101 \mathrm{MHz}\right.$ in $\left.\mathrm{CDCl}_{3}\right)$

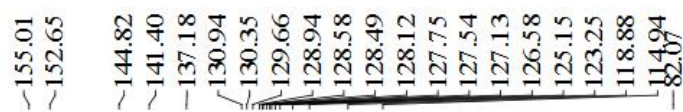

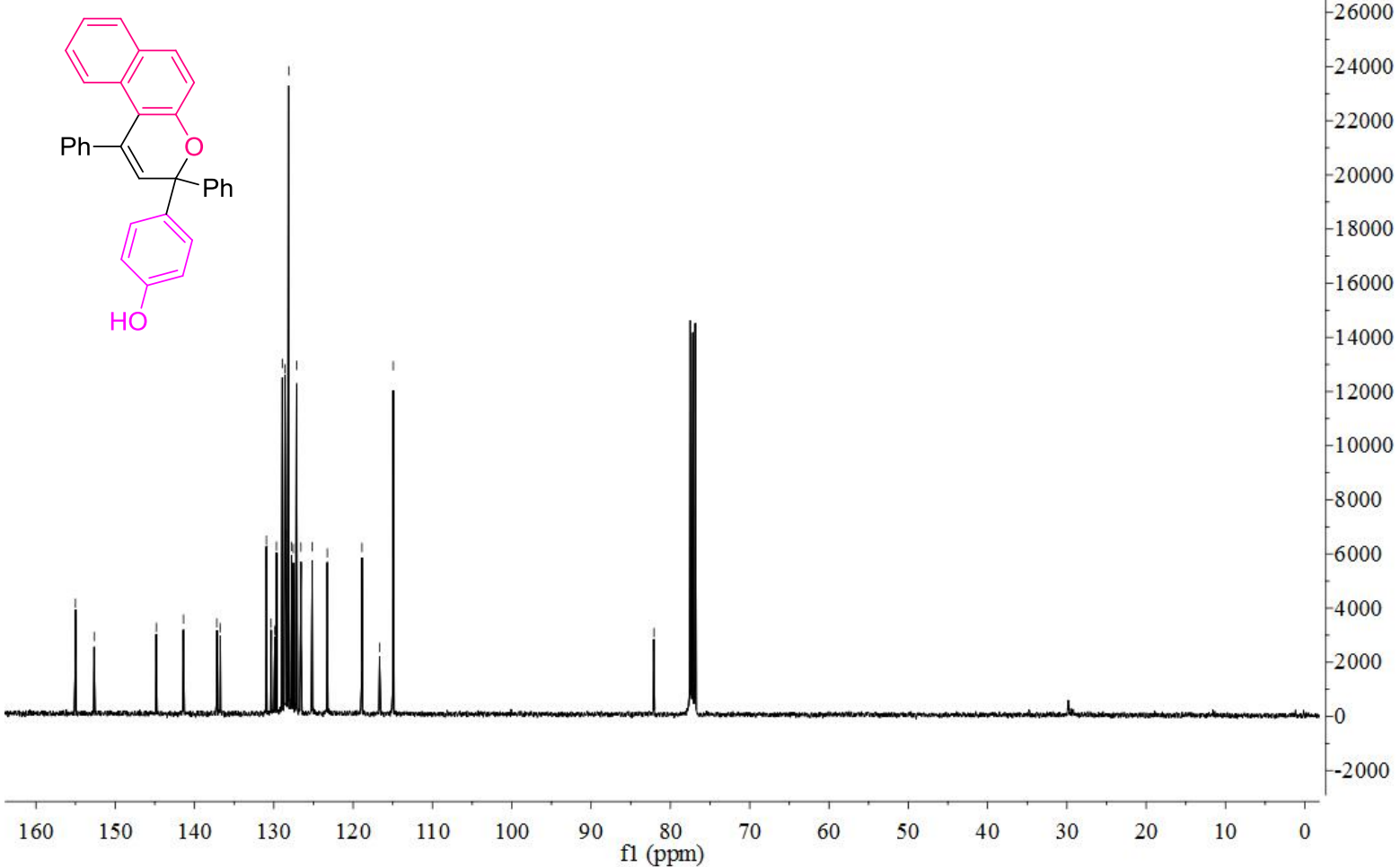

${ }^{1} \mathrm{H}$ NMR of compound $13\left(400 \mathrm{MHz}\right.$ in $\left.\mathrm{CDCl}_{3}\right)$

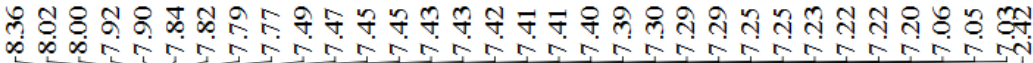
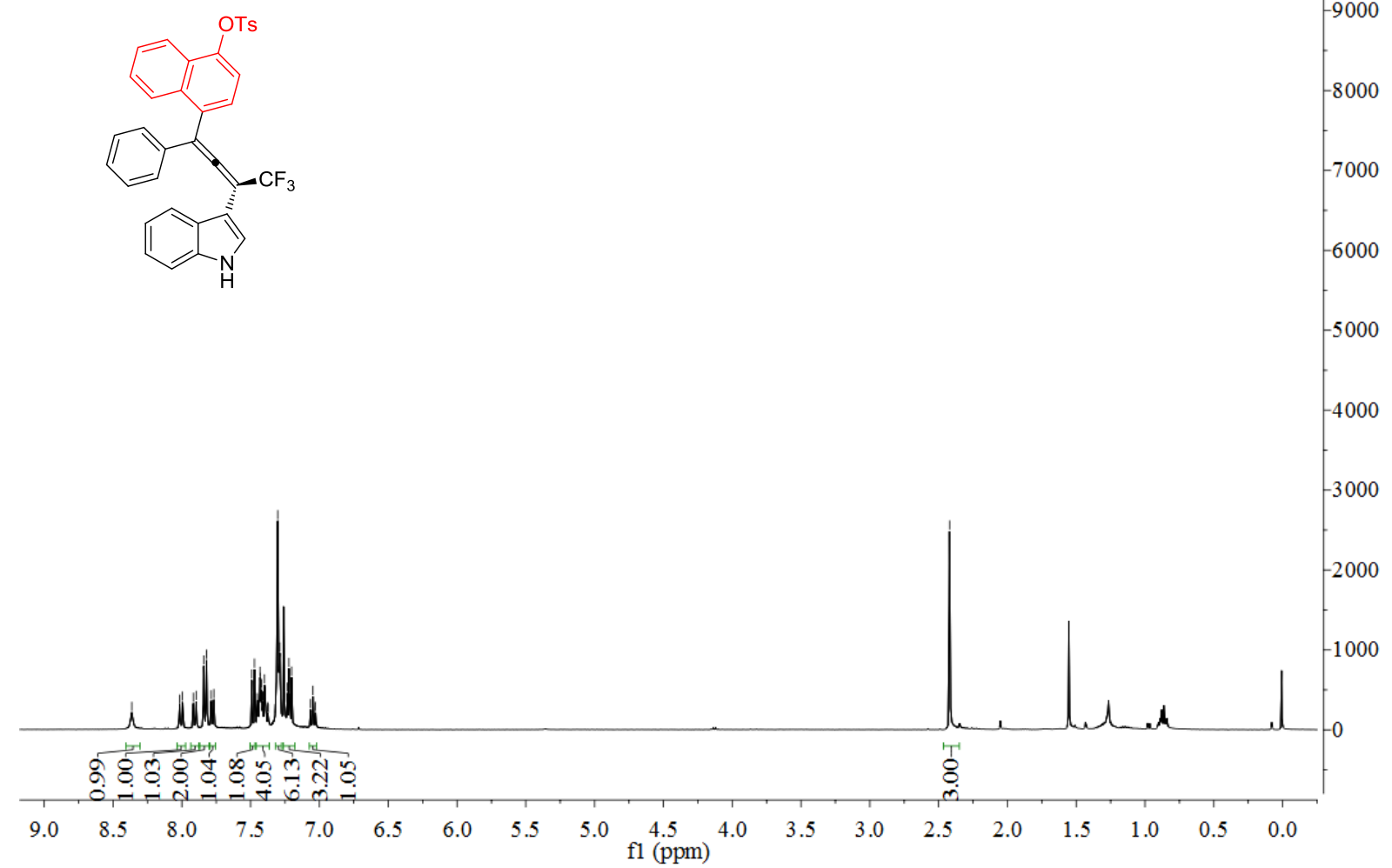
${ }^{13} \mathrm{C}$ NMR of compound $13\left(126 \mathrm{MHz}\right.$ in $\left.\mathrm{CDCl}_{3}\right)$

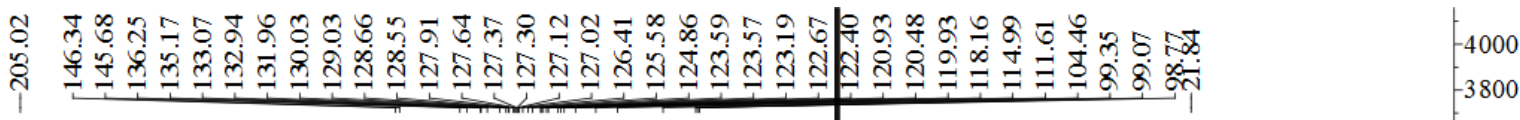<smiles>Oc1ccc(C(=Cc2c[nH]c3ccccc23)C(F)(F)F)c2ccccc12</smiles>

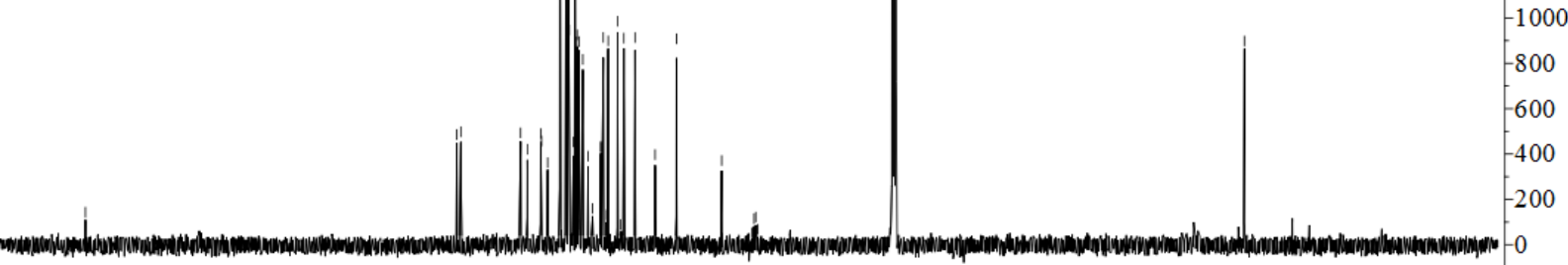

(n)
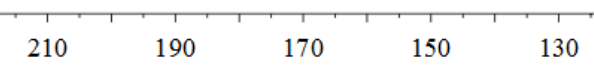

110 f1 (ppm) 90

$\begin{array}{lllllllll}80 & 70 & 60 & 50 & 40 & 30 & 20 & 10 & 0\end{array}$

${ }^{19} \mathrm{~F} \mathrm{NMR}$ of compound $13\left(376 \mathrm{MHz}\right.$ in $\left.\mathrm{CDCl}_{3}\right)$

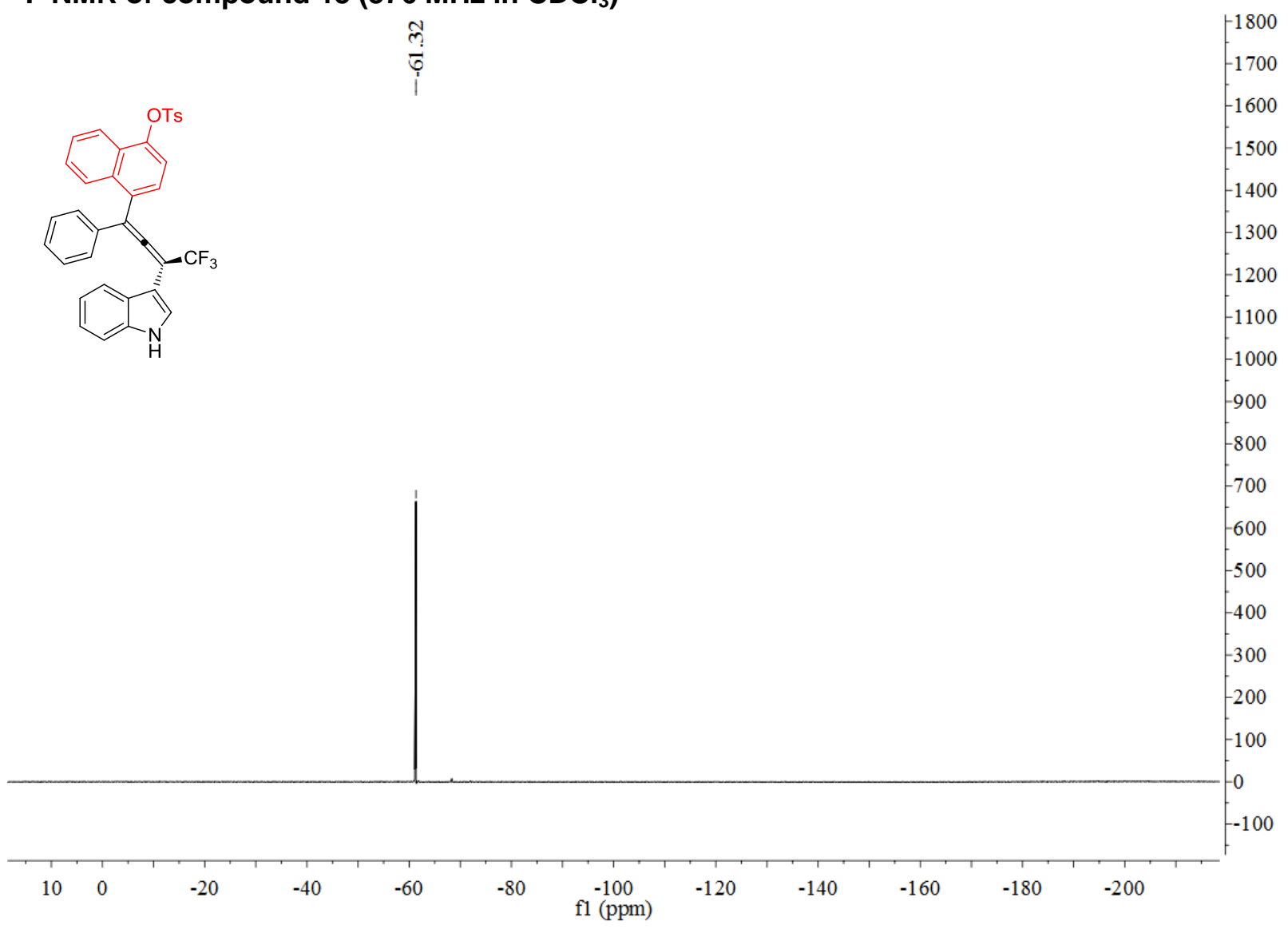


${ }^{1} \mathrm{H}$ NMR of compound $14\left(500 \mathrm{MHz}\right.$ in $\mathrm{CDCl}_{3}$ )

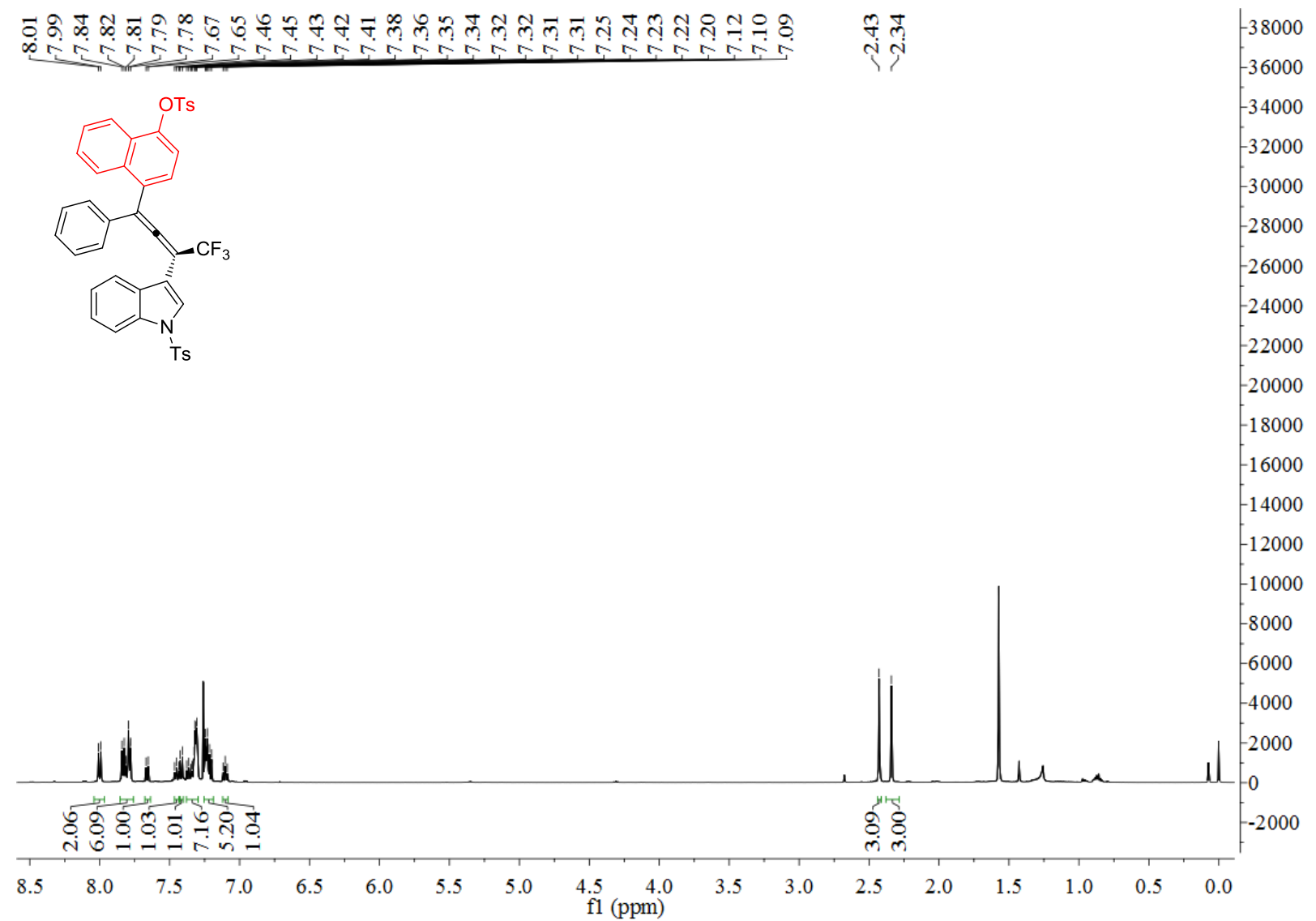

\section{${ }^{13} \mathrm{C}$ NMR of compound 14 (126 MHz in $\left.\mathrm{CDCl}_{3}\right)$}

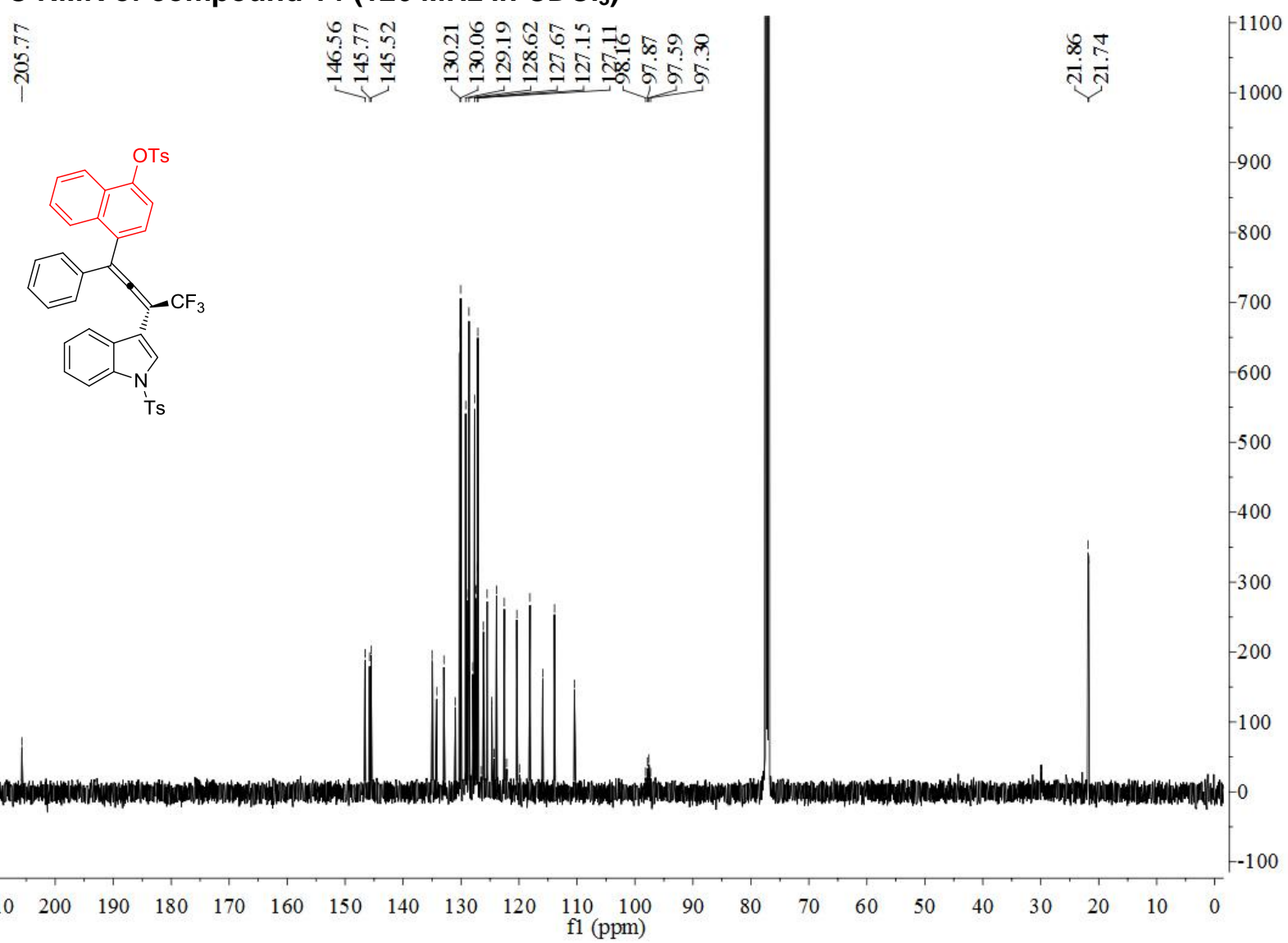


${ }^{19} \mathrm{~F}$ NMR of compound $14\left(376 \mathrm{MHz}\right.$ in $\left.\mathrm{CDCl}_{3}\right)$

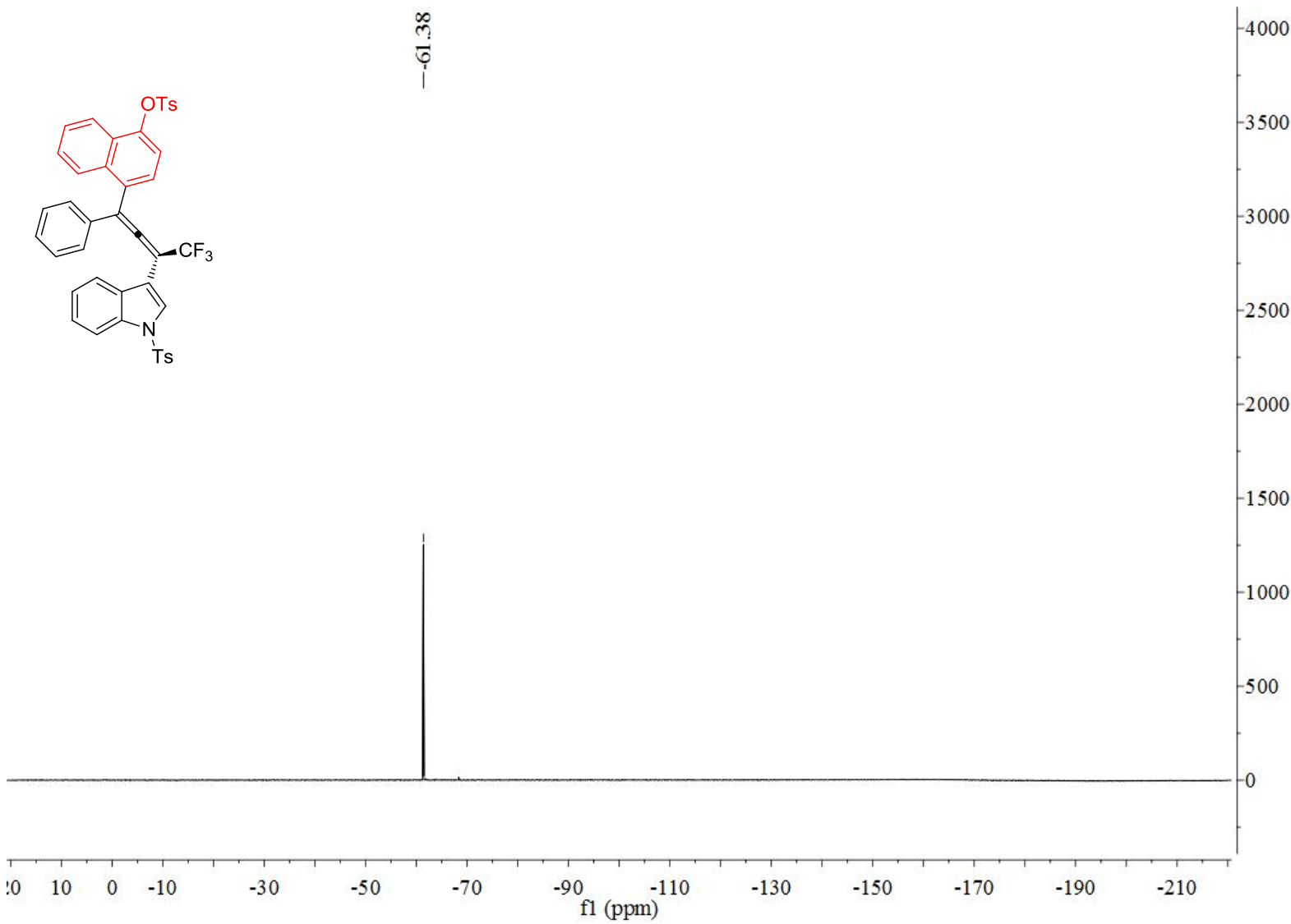

${ }^{1} \mathrm{H}$ NMR of compound 15 (400 $\mathrm{MHz}$ in $\mathrm{CDCl}_{3}$ )

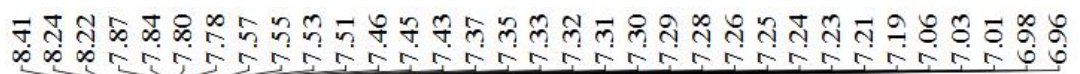
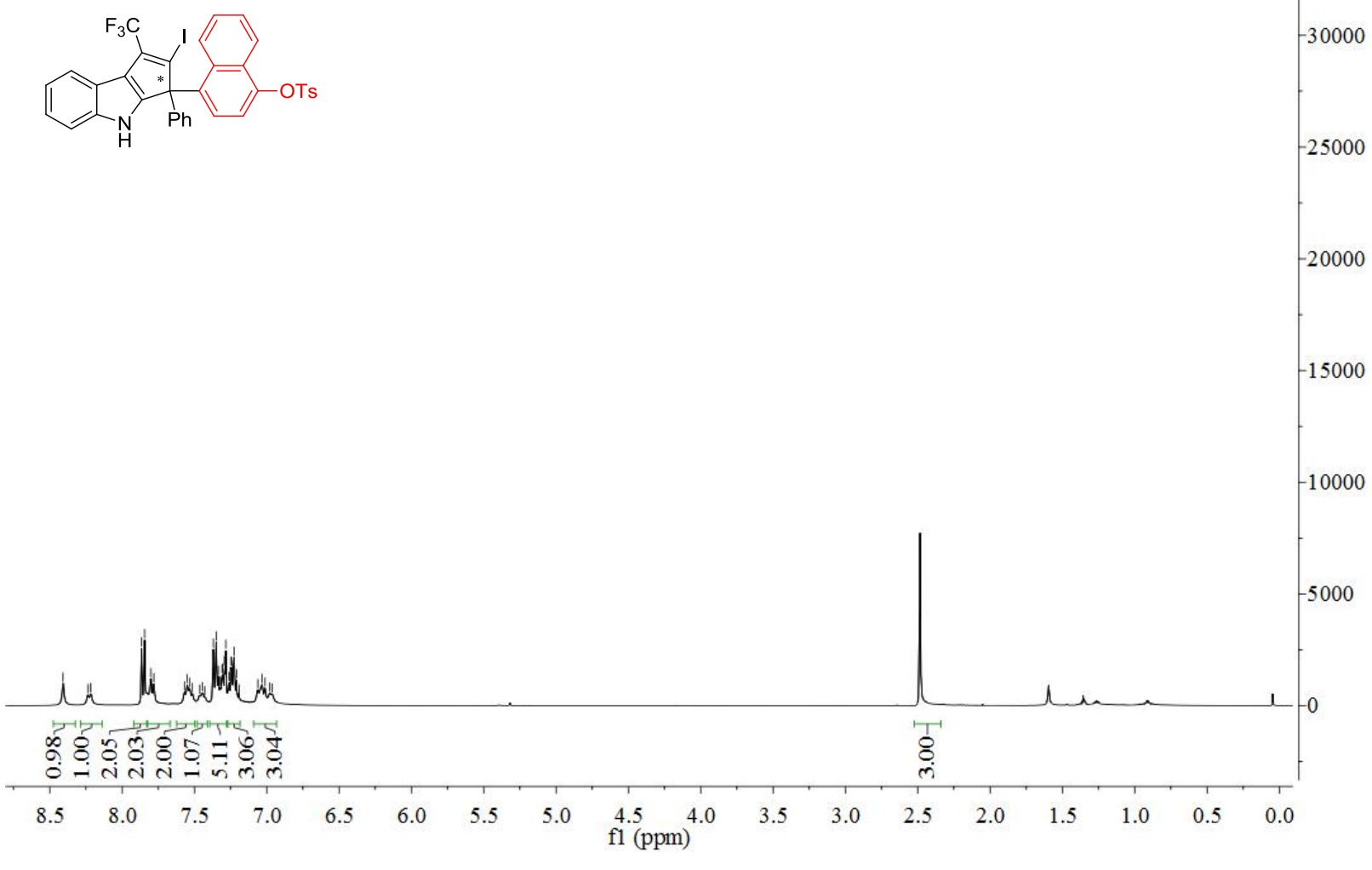


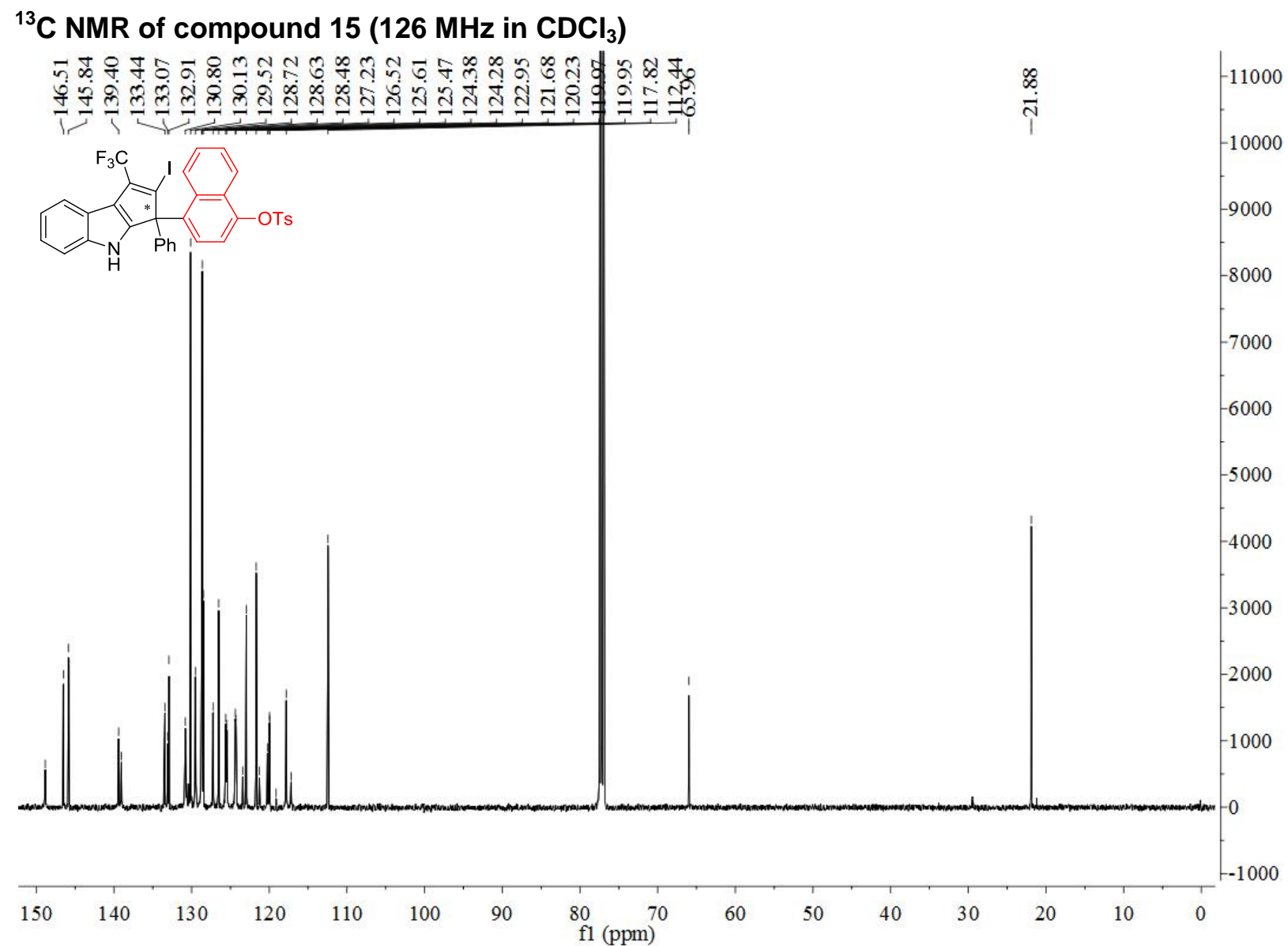

${ }^{19} \mathrm{~F}$ NMR of compound 15 (376 $\mathrm{MHz}$ in $\mathrm{CDCl}_{3}$ )

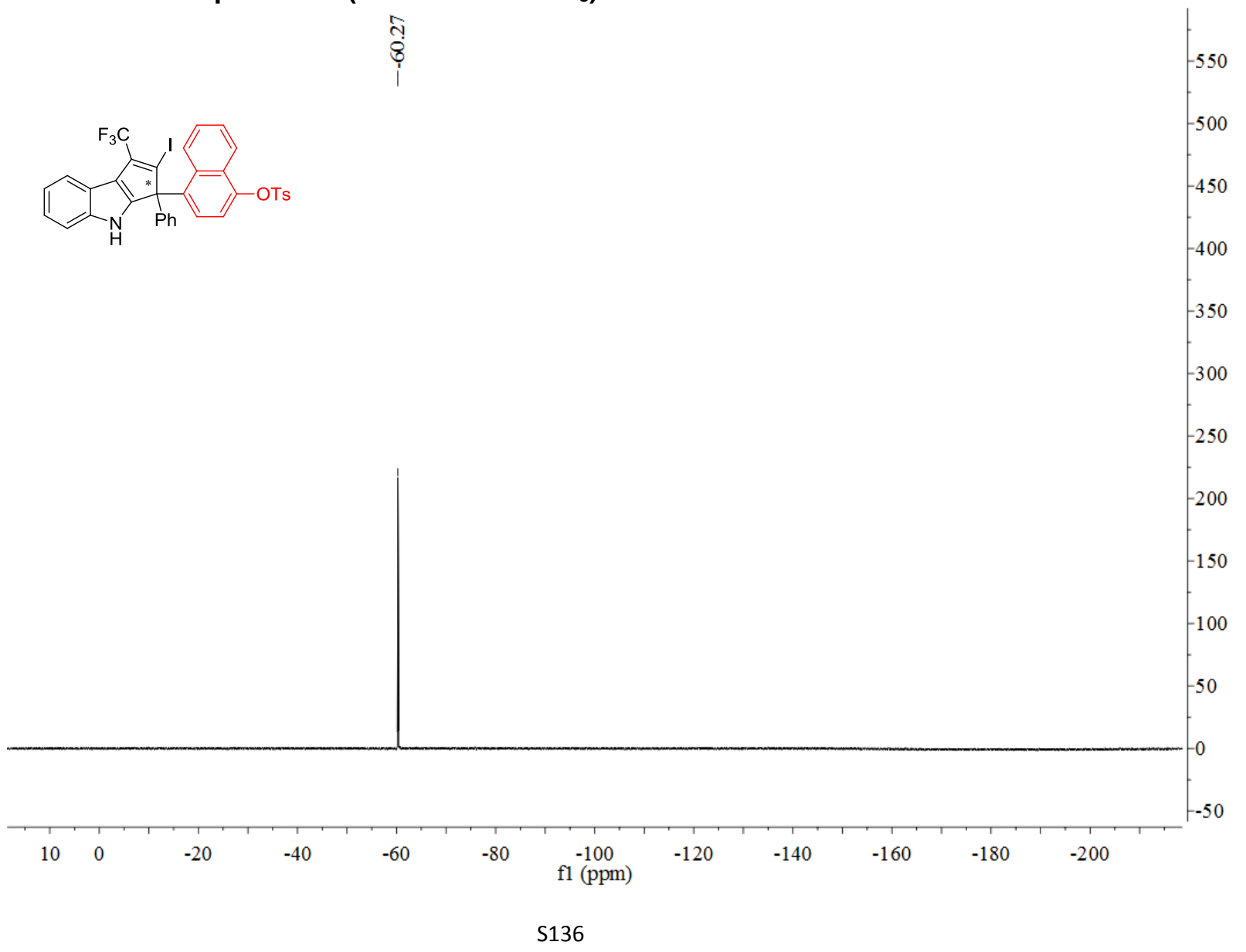




\section{${ }^{1} \mathrm{H}$ NMR of compound $16\left(400 \mathrm{MHz}\right.$ in $\left.\mathrm{CDCl}_{3}\right)$}

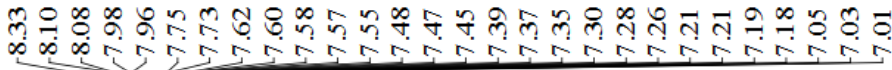<smiles>Oc1ccc(C(=C(c2ccccc2)c2c[nH]c3ccccc23)C(F)(F)F)c2ccccc12</smiles>

\section{${ }^{13} \mathrm{C}$ NMR of compound $16\left(126 \mathrm{MHz}\right.$ in $\left.\mathrm{CDCl}_{3}\right)$}

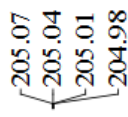

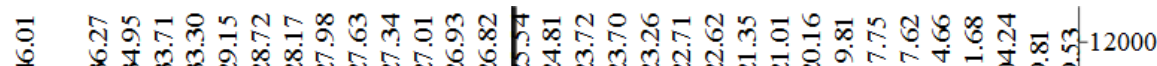
更

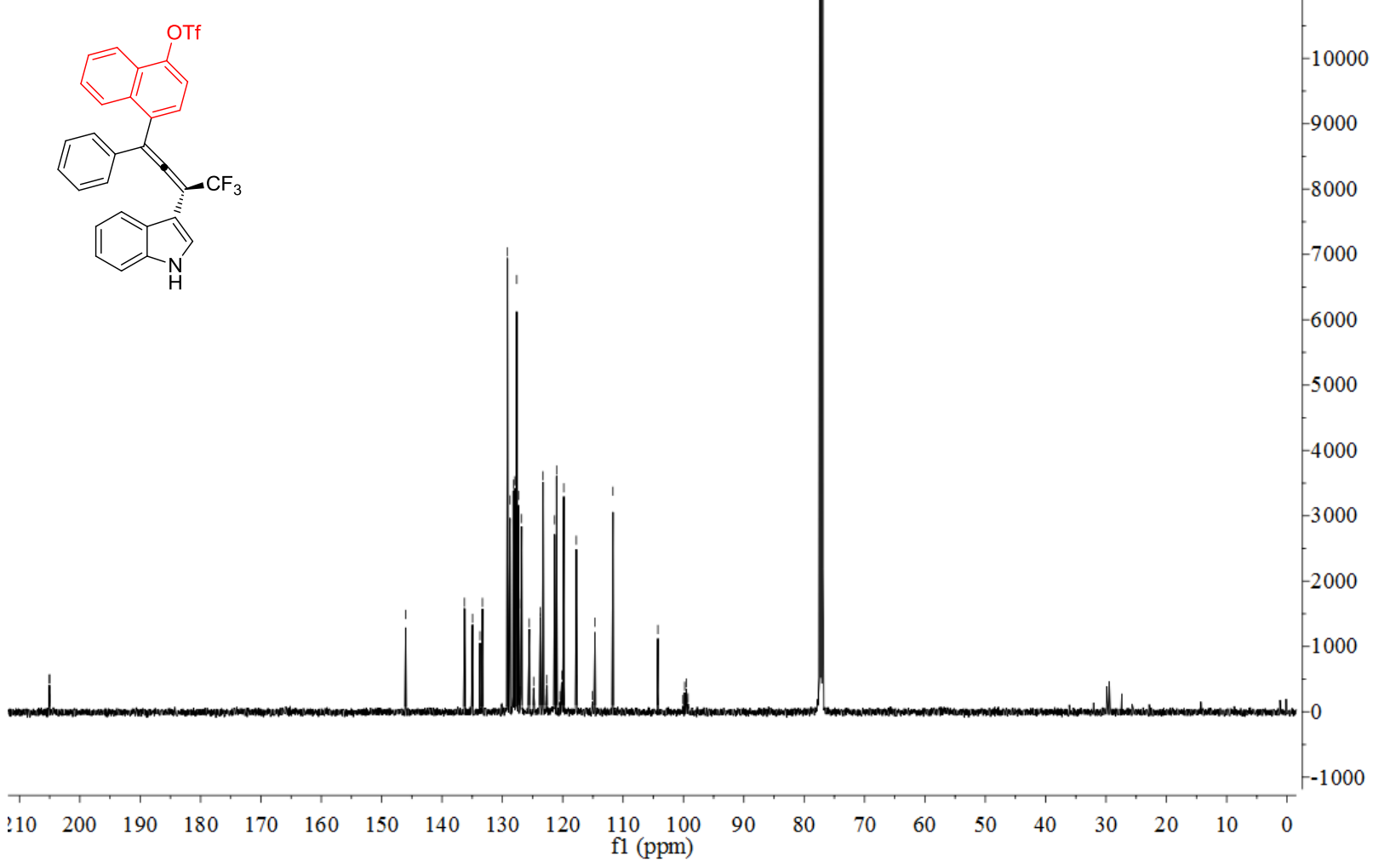




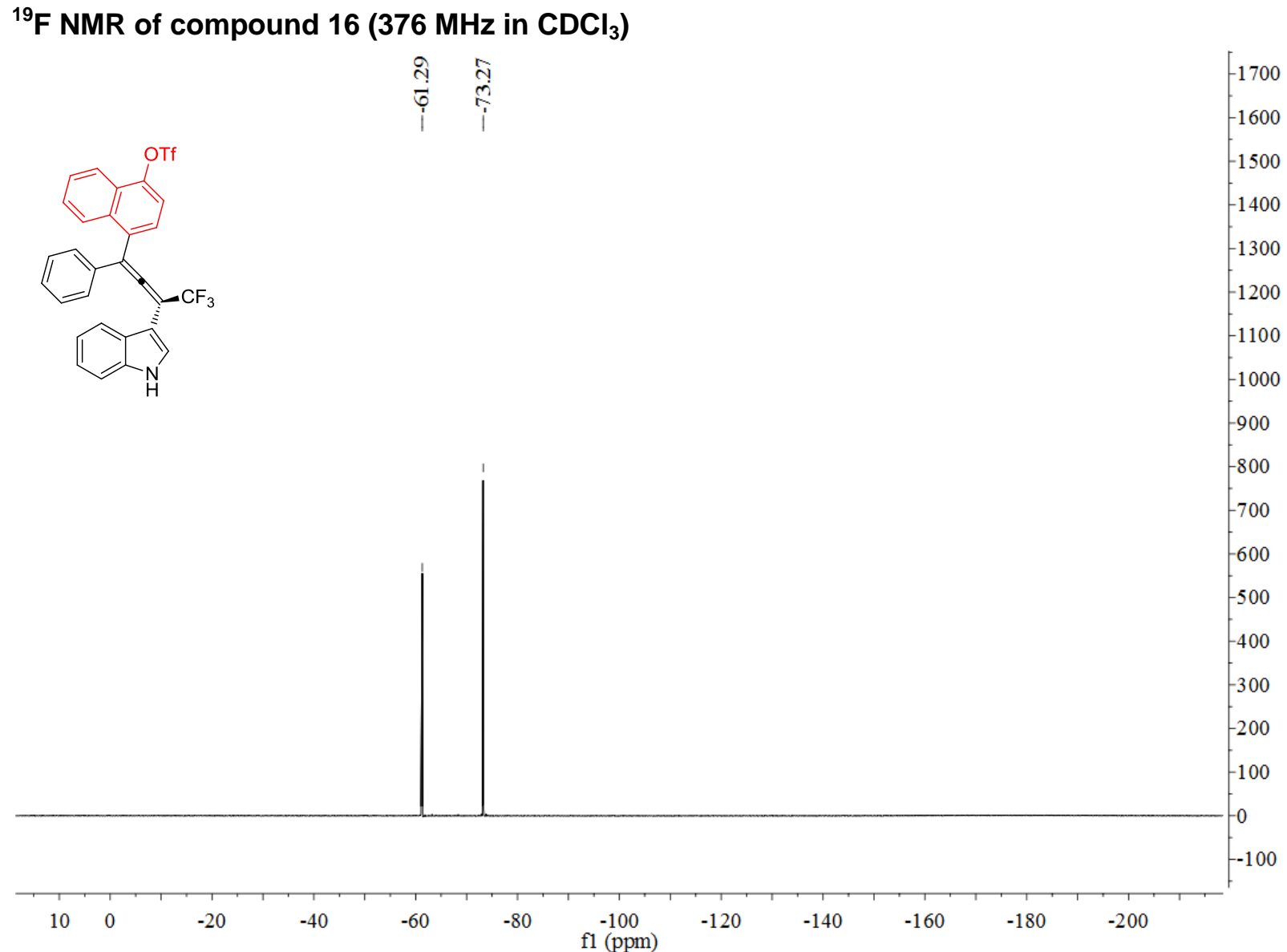

${ }^{1} \mathrm{H}$ NMR of compound $17\left(400 \mathrm{MHz}\right.$ in $\mathrm{CDCl}_{3}$ )

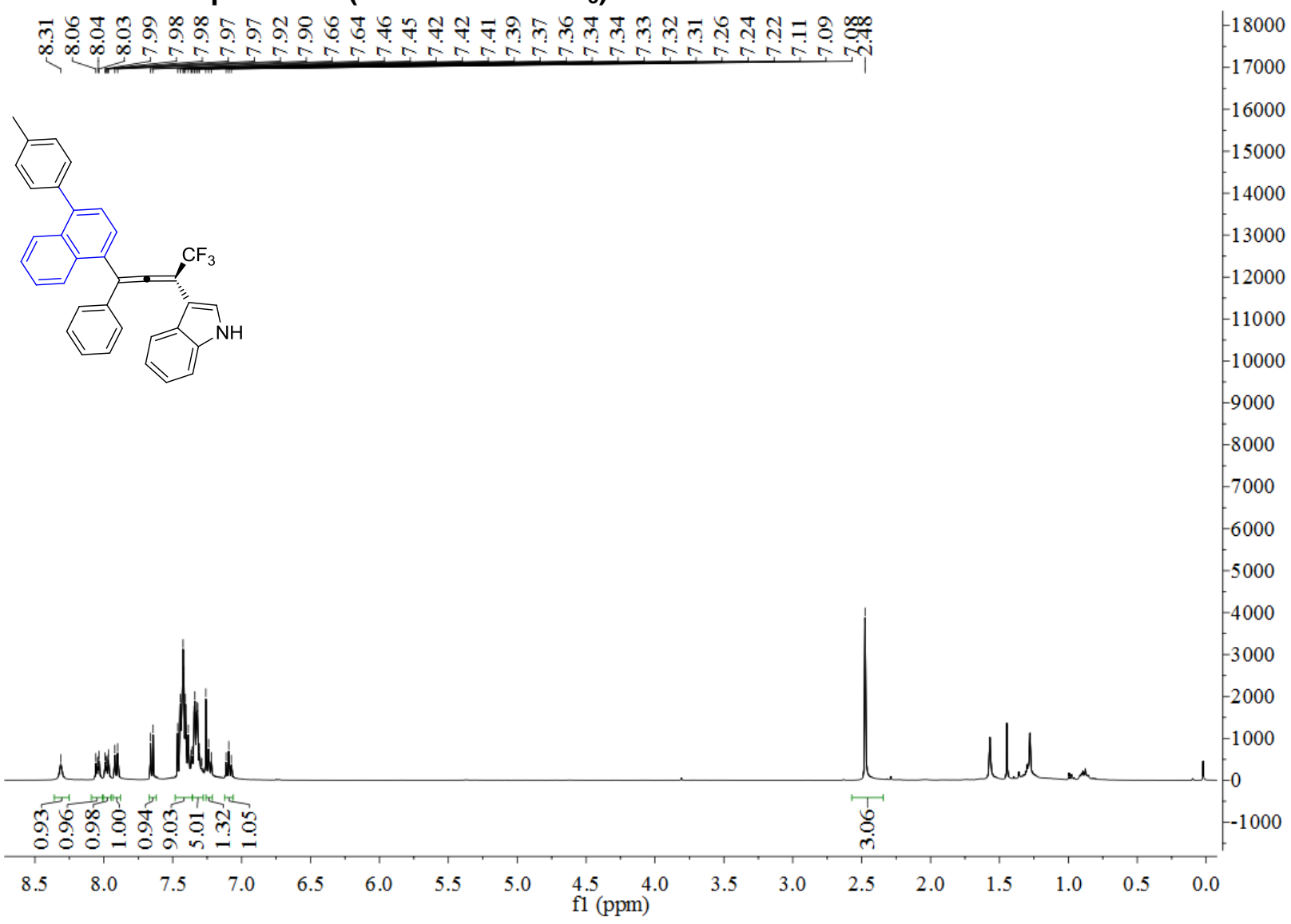




\section{${ }^{13} \mathrm{C}$ NMR of compound $17\left(126 \mathrm{MHz}\right.$ in $\mathrm{CDCl}_{3}$ )}

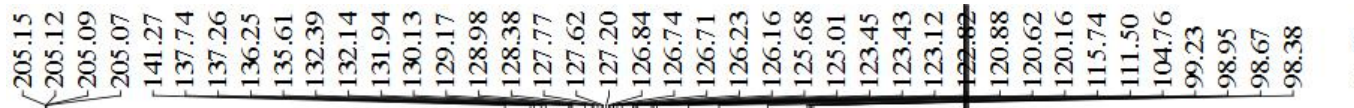

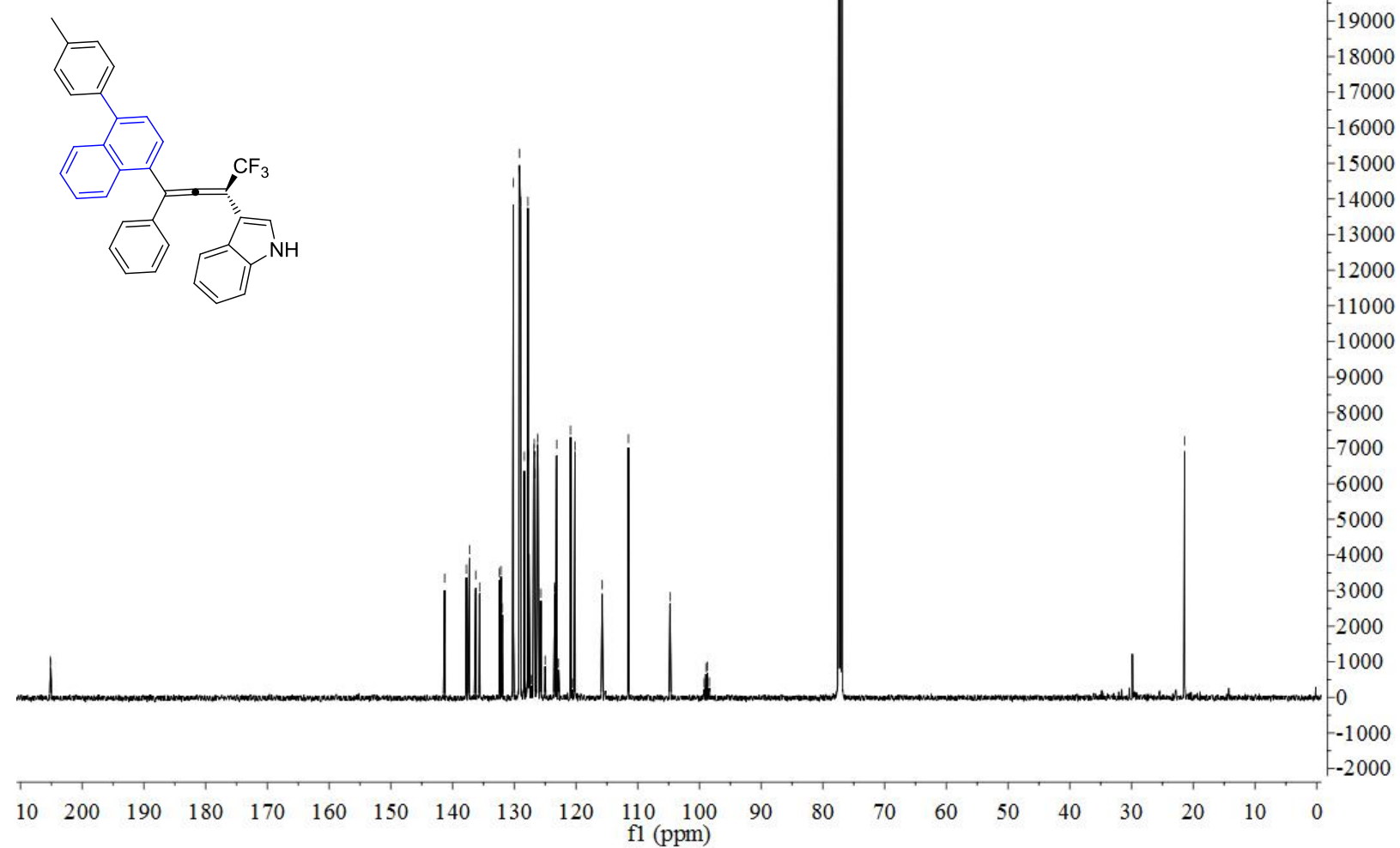

${ }^{19} \mathrm{~F}$ NMR of compound $17\left(376 \mathrm{MHz}\right.$ in $\left.\mathrm{CDCl}_{3}\right)$

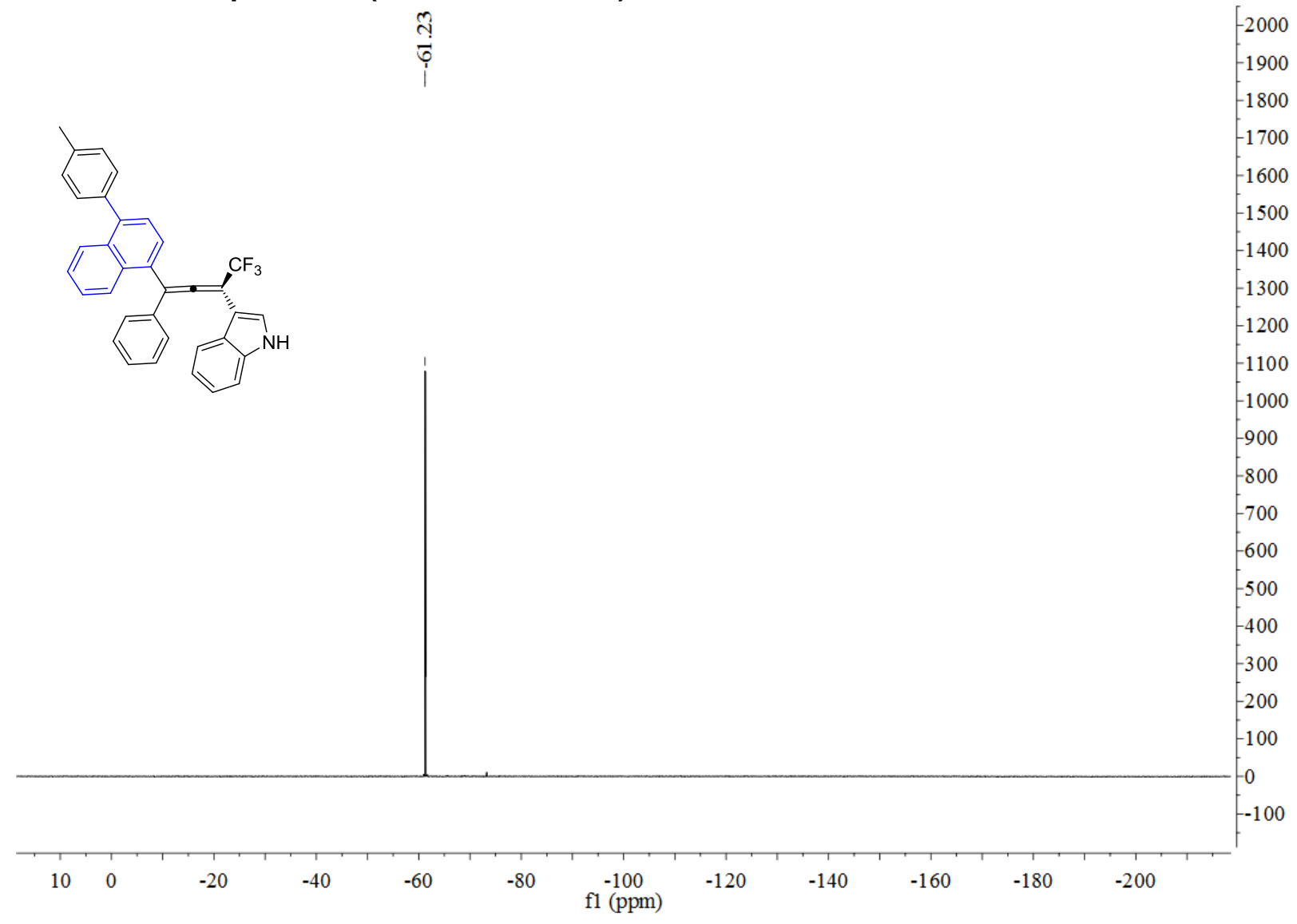


12. Copies of HPLC Spectra<smiles>Oc1ccc(C(=C(c2ccccc2)C(F)(F)F)c2c[nH]c3ccccc23)c2ccccc12</smiles>

$3 a$

HPLC spectrum of the racemate

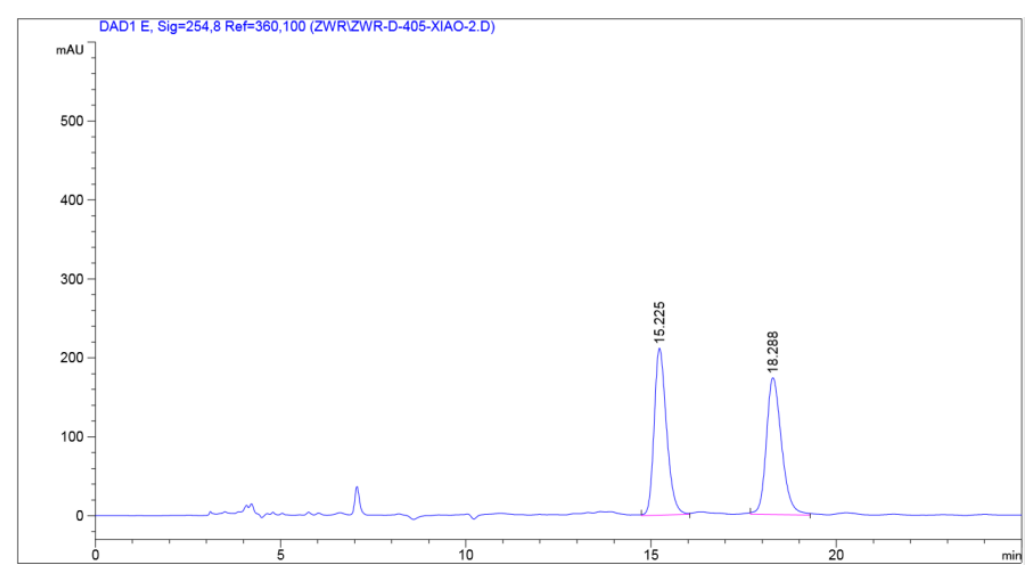

信号 1: DAD1 E， Sig=254,8 Ref=360,100

\begin{tabular}{|c|c|c|c|c|c|c|c|}
\hline 峰 & 保留时间 类型 & 峰宽 & & 峰面积 & 峰高 & \multicolumn{2}{|l|}{ 峰面积 } \\
\hline \# & {$[\mathrm{min}]$} & & [min ] & {$\left[\mathrm{mAU}{ }^{\star} \mathrm{s}\right]$} & & [mAU ] & 응 \\
\hline 1 & 15 & & 3886 & $6 \quad 4932.446$ & & 211.5 & \\
\hline 2 & $18.288 \mathrm{MN}$ & . & 0.4776 & 64966.258 & 30 & 173.31647 & 50.1708 \\
\hline
\end{tabular}

\section{HPLC spectrum of the chiral compound}

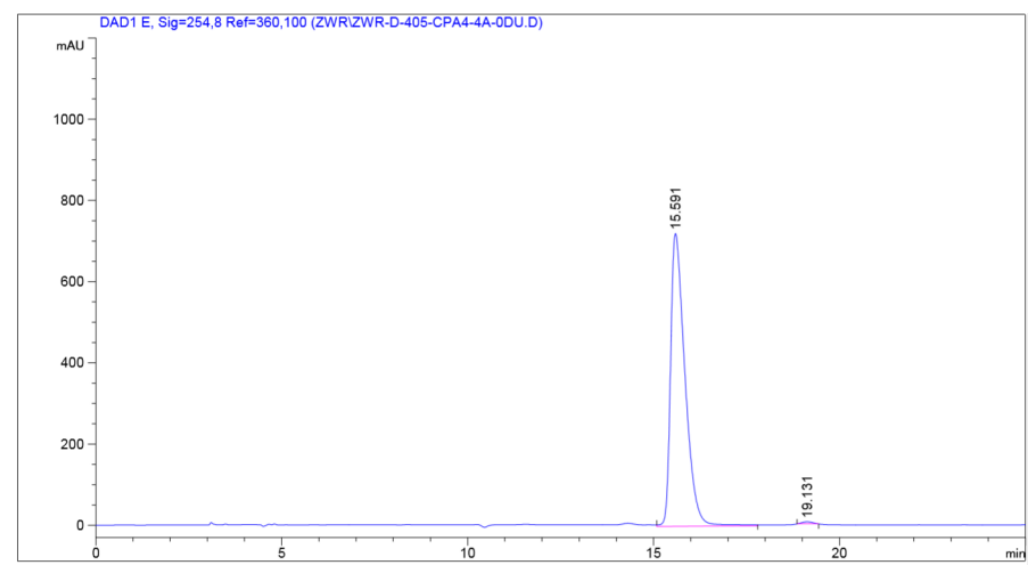

信号 1: DAD1 E, Sig=254,8 Ref=360,100

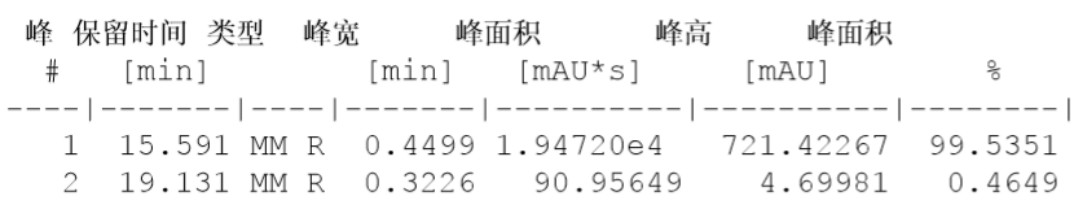




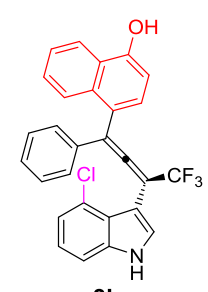

3b

HPLC spectrum of the racemate

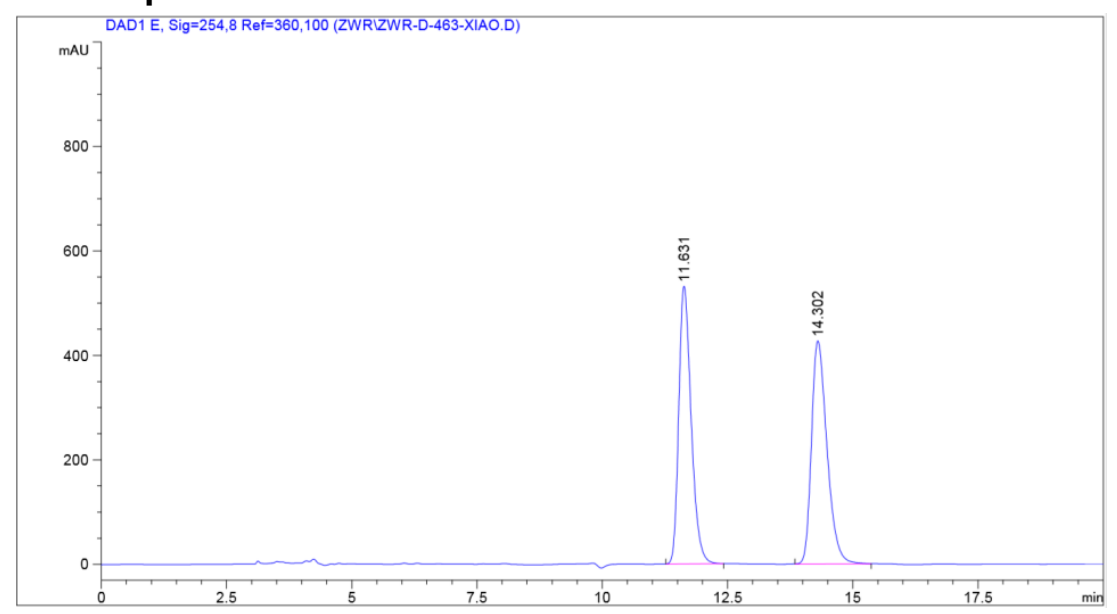

信号 1: DAD1 E， Sig=254, 8 Ref=360, 100

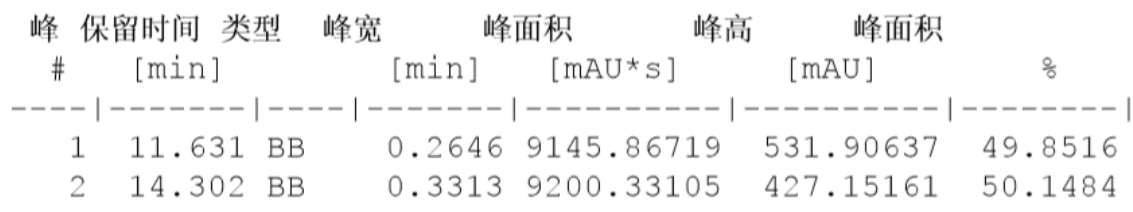

\section{HPLC spectrum of the chiral compound}

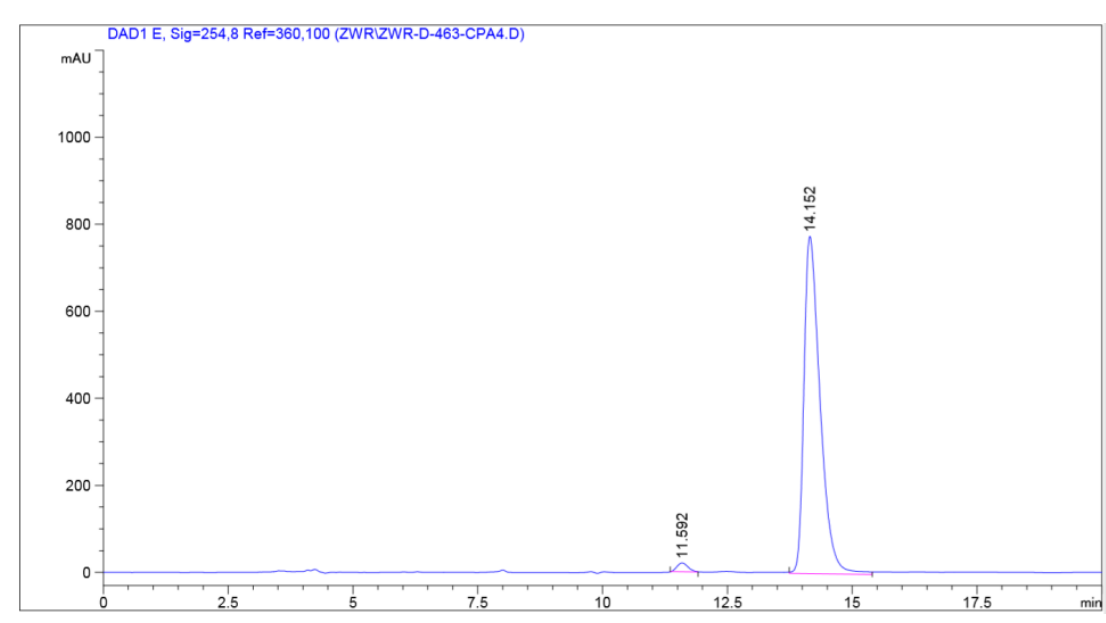

信号 1: DAD1 E， Sig=254,8 Ref=360,100

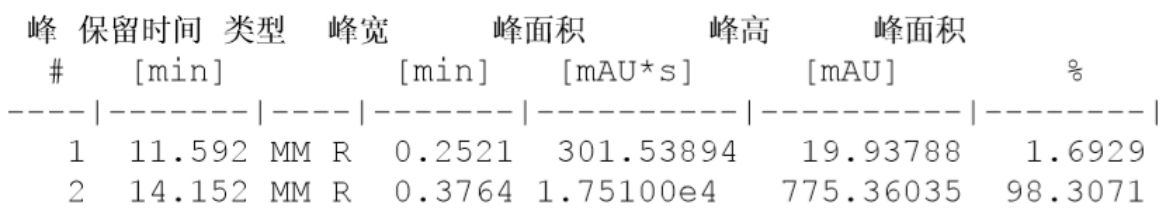




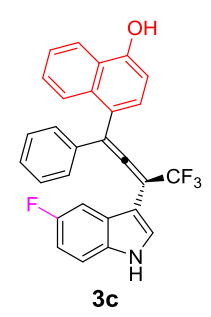

HPLC spectrum of the racemate

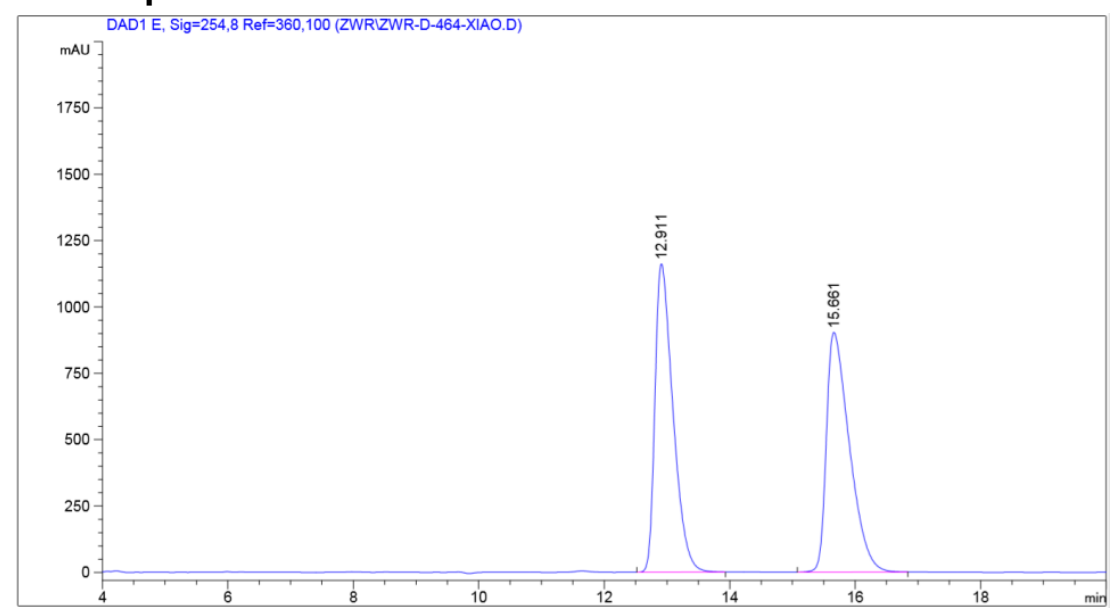

信号 1: DAD1 E， Sig=254,8 $\operatorname{Ref}=360,100$

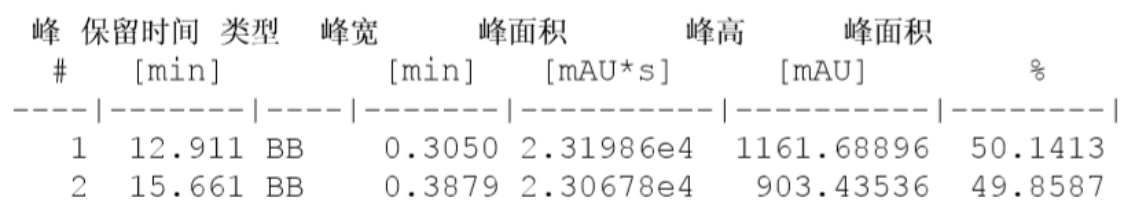

\section{HPLC spectrum of the chiral compound}

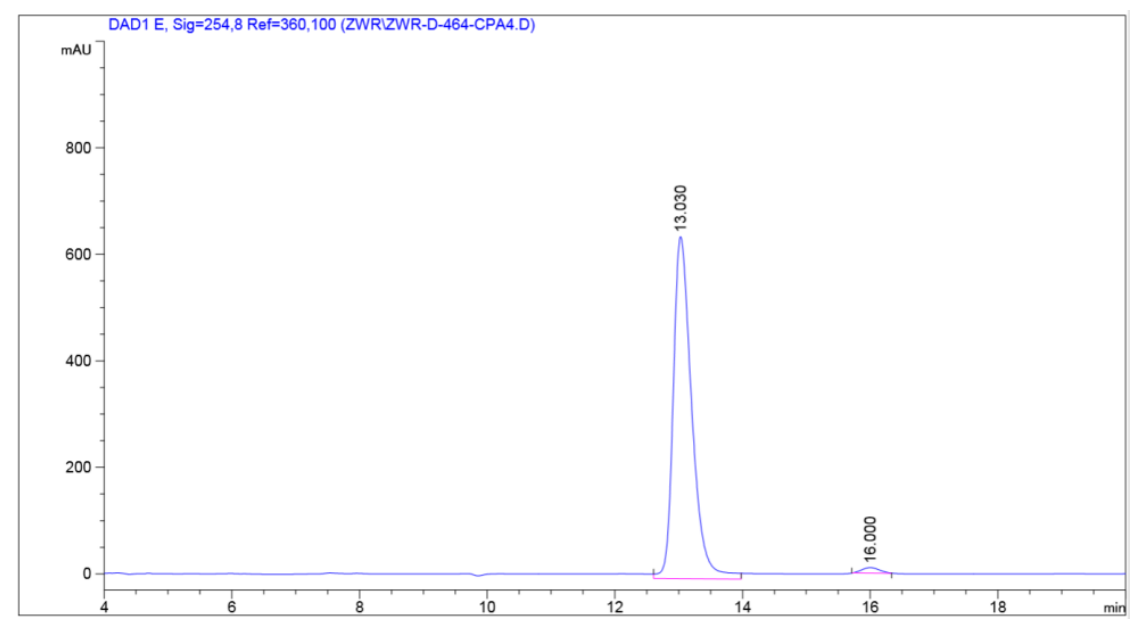

信号 1: DAD1 E, Sig=254, 8 Ref=360,100

\begin{tabular}{|c|c|c|c|c|c|c|c|}
\hline 峰 & 呆留时间 类型 & 峰宽 & & 面积 & 峰高 & \multicolumn{2}{|l|}{ 峰面积 } \\
\hline \# & [min] & & [min] & {$\left[\mathrm{mAU}^{*} \mathrm{~s}\right]$} & & [mAU] & $\frac{\circ}{0}$ \\
\hline & & & & & & ca & \\
\hline 1 & $13.030 \mathrm{M}$ & $\mathrm{R}$ & 0.3370 & $1.29761 €$ & & 641.78198 & 98.5581 \\
\hline 2 & $000 \mathrm{M}$ & $\mathrm{R}$ & 0.3149 & 89.846 & 66 & 10.04663 & 1.4419 \\
\hline
\end{tabular}




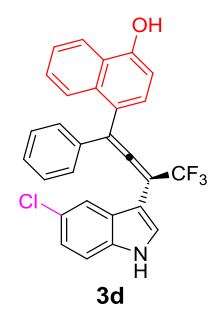

\section{HPLC spectrum of the racemate}

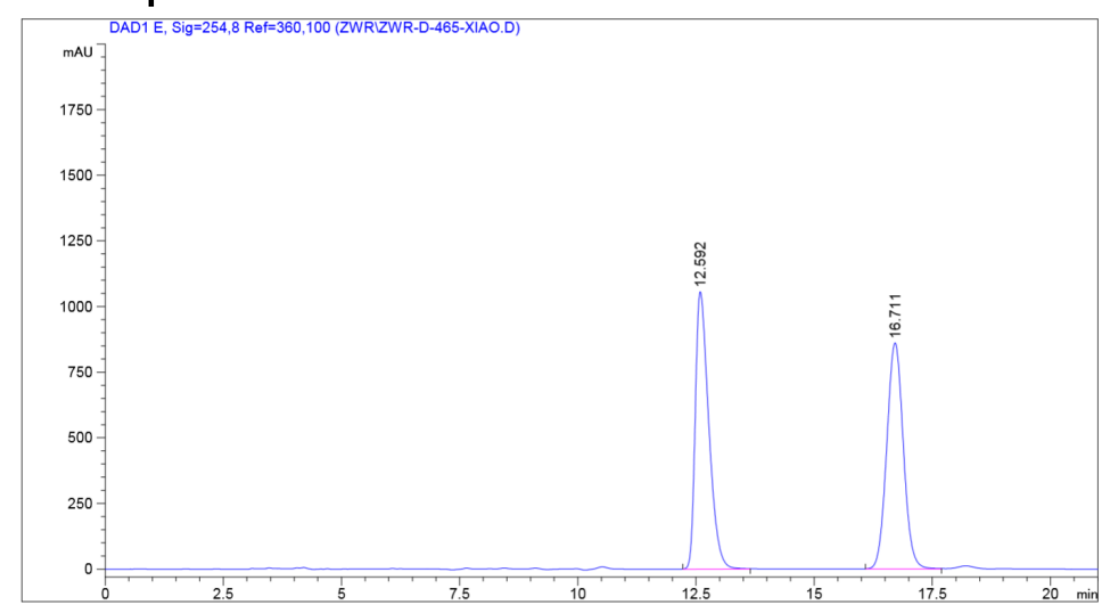

信号 1: DAD1 E, Sig=254,8 $\operatorname{Ref}=360,100$

\begin{tabular}{|c|c|c|c|c|c|}
\hline 峰 & 保留时间 类型 & 峰宽 & 峰面积 & \multicolumn{2}{|l|}{ 峰面积 } \\
\hline$\#$ & [min] & [min] & {$\left[\mathrm{mAU}{ }^{\star} \mathrm{s}\right]$} & [mAU] & 응 \\
\hline & -1 & -------- & $-\mid--------$ & | -------- & --------1 \\
\hline 1 & $12.592 \mathrm{BE}$ & 0.3010 & $02.09102 \mathrm{e}$ & 1056.27295 & 49.8934 \\
\hline 2 & $16.711 \mathrm{BE}$ & 0.3805 & $52.09996 \mathrm{e}$ & 861.00201 & 50.1066 \\
\hline
\end{tabular}

\section{HPLC spectrum of the chiral compound}

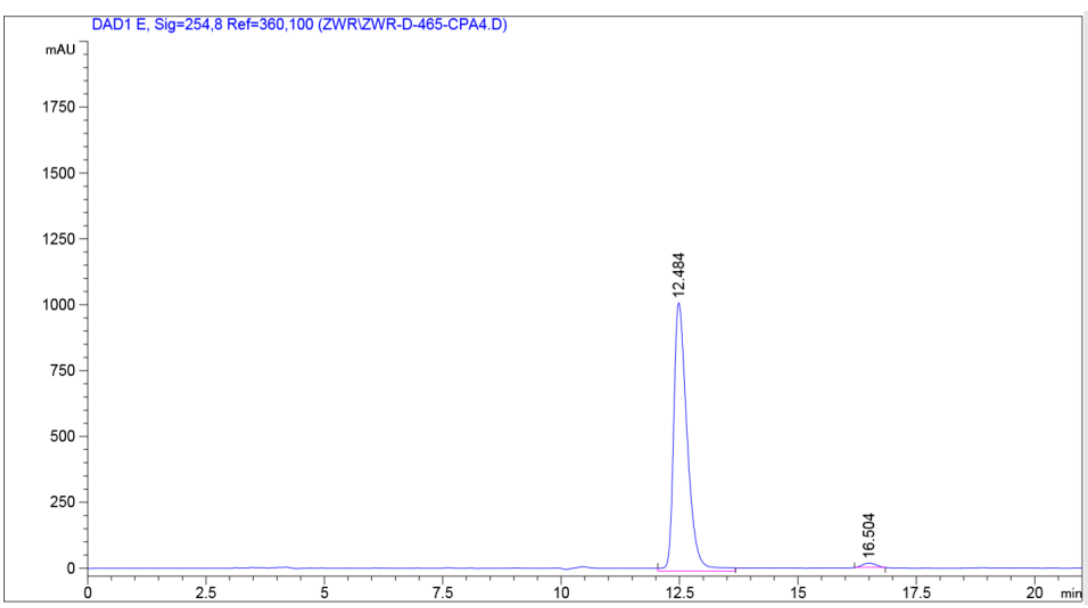

信号 1：DAD1 E， Sig=254,8 Ref=360,100

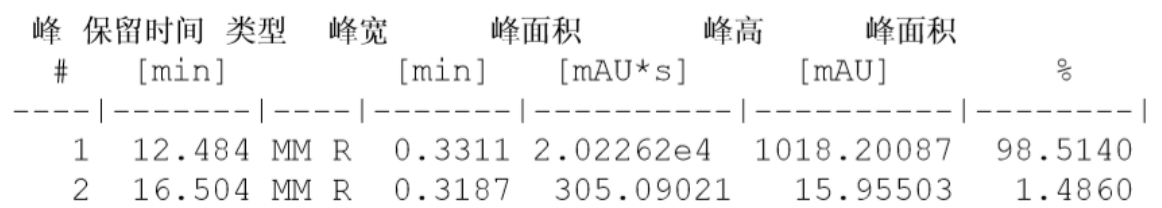




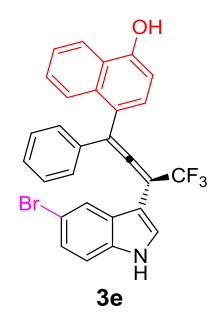

HPLC spectrum of the racemate

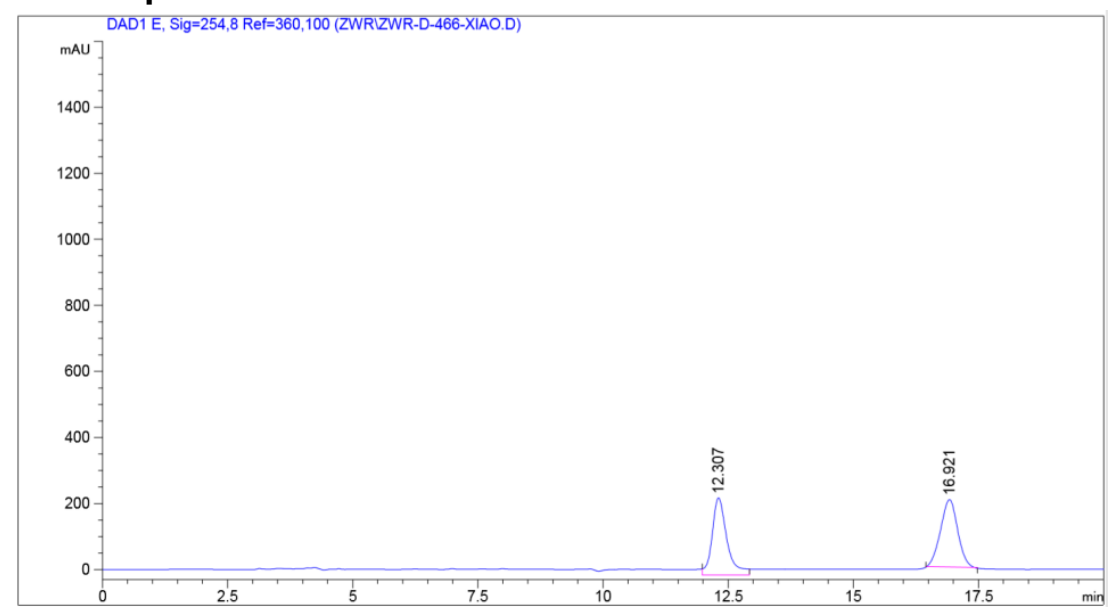

信号 1: DAD1 E， $\mathrm{Sig}=254,8$ Ref $=360,100$

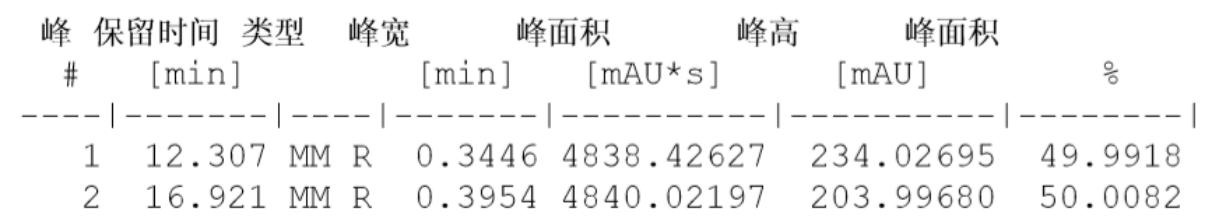

\section{HPLC spectrum of the chiral compound}

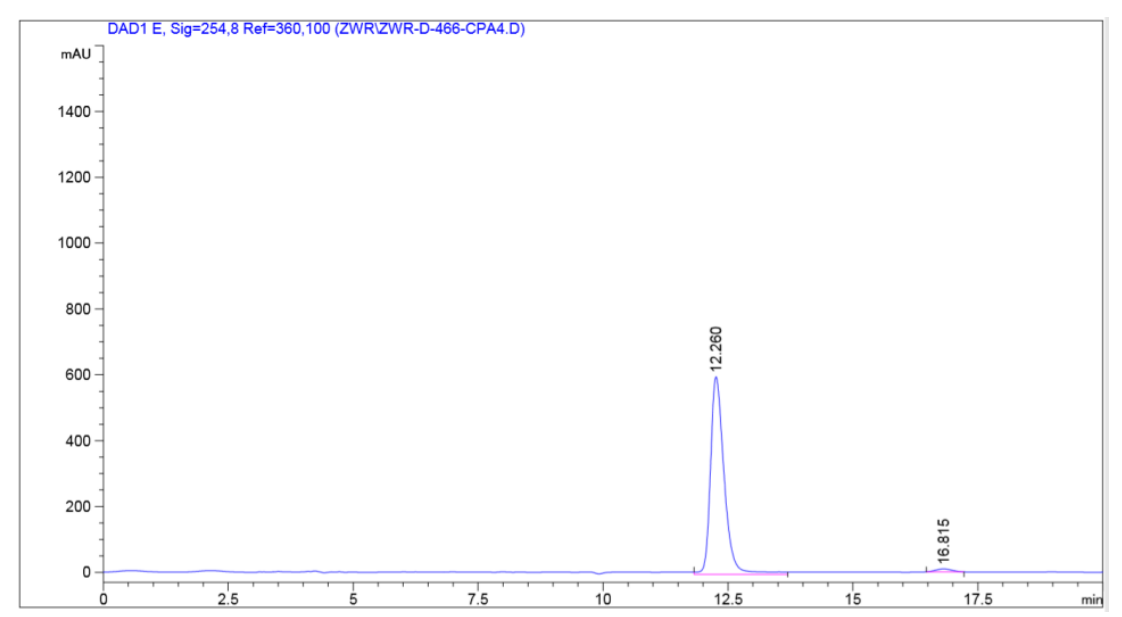

信号 1: DAD1 E， Sig=254,8 Ref=360, 100

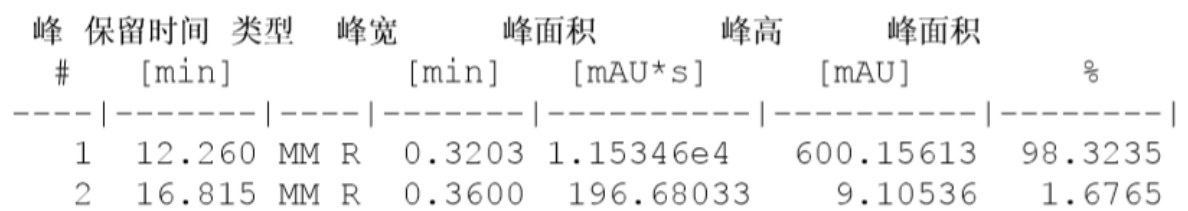




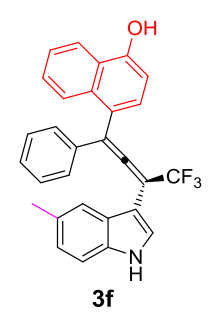

HPLC spectrum of the racemate

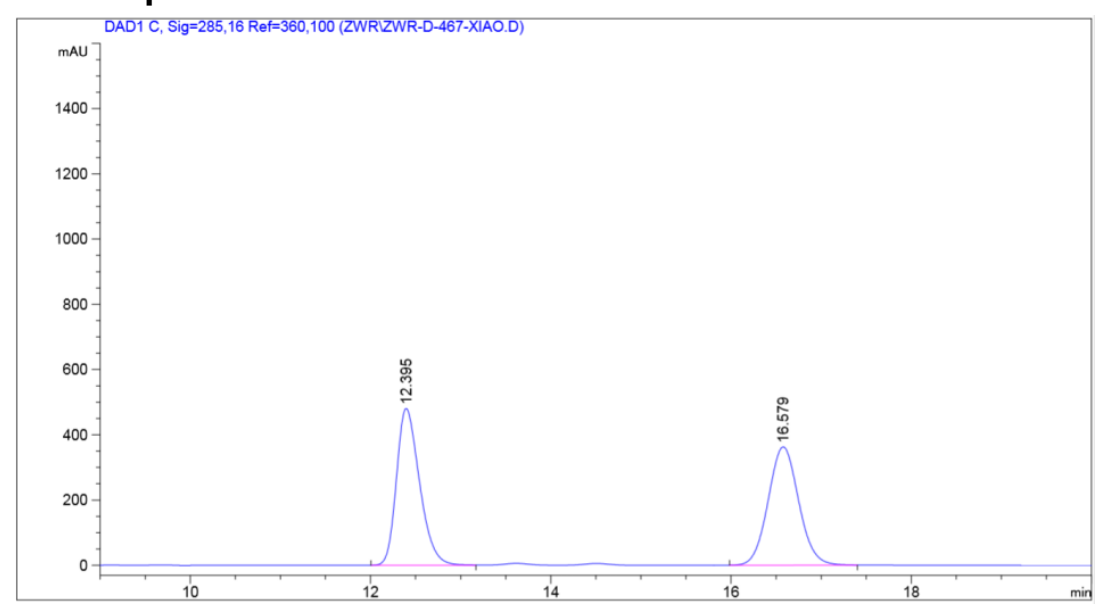

信号 1: DAD1 C, Sig=285,16 Ref=360,100

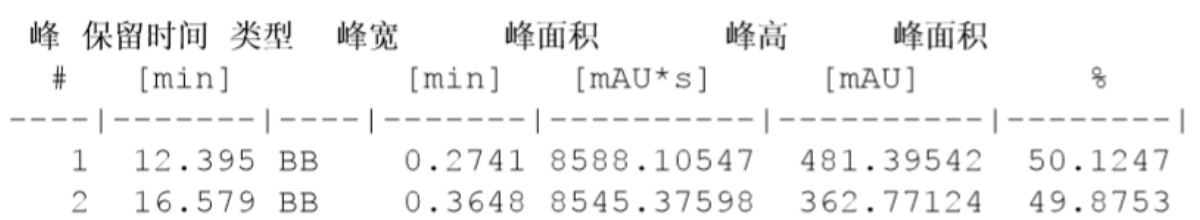

\section{HPLC spectrum of the chiral compound}

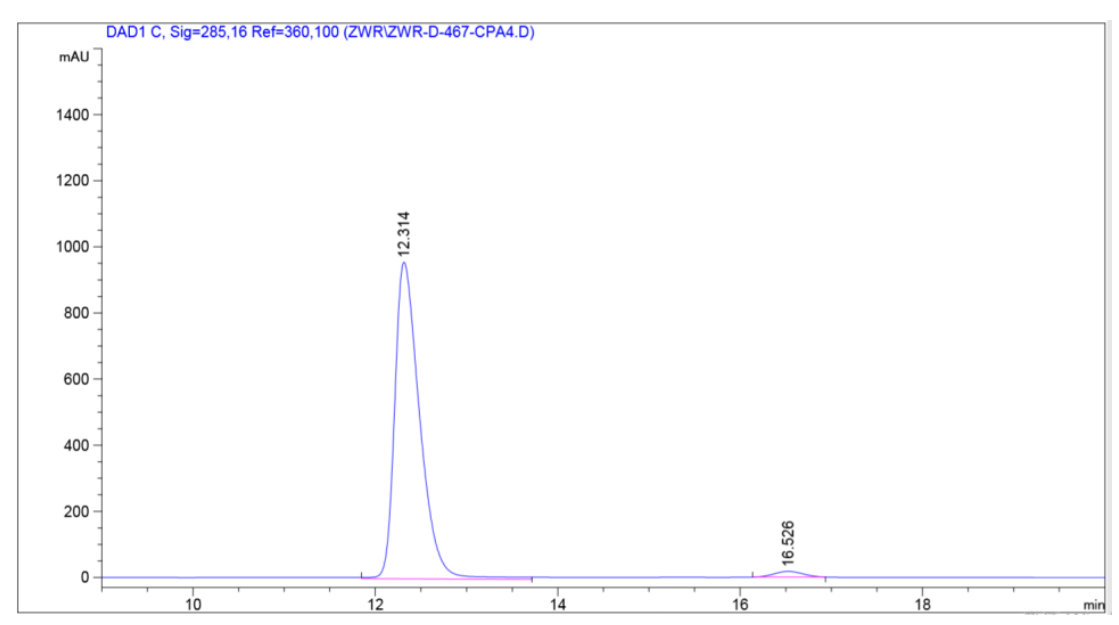

信号 1: DAD1 C, Sig=285,16 Ref=360,100

\begin{tabular}{|c|c|c|c|c|c|c|c|}
\hline 峰 位 & 留时间 类型 & 峰宽 & & 面积 & 峰高 & \multicolumn{2}{|l|}{ 峰面积 } \\
\hline$\#$ & [min] & & {$[\min ]$} & {$\left[\mathrm{mAU}{ }^{*} \mathrm{~s}\right]$} & & [mAU ] & $\%$ \\
\hline & & & ----- & -------- & & ---1 & $8-------1$ \\
\hline 1 & 12.31 & & .3177 & 1825870 & & 957.915 & 98.0977 \\
\hline 2 & $16.526 \mathrm{MI}$ & $\mathrm{R}$ & 0.3516 & 54.061 & 77 & 16.78 & 1.9023 \\
\hline
\end{tabular}




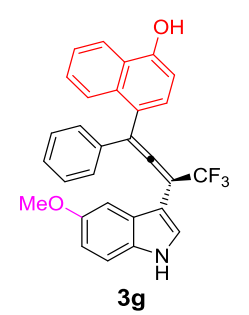

\section{HPLC spectrum of the racemate}

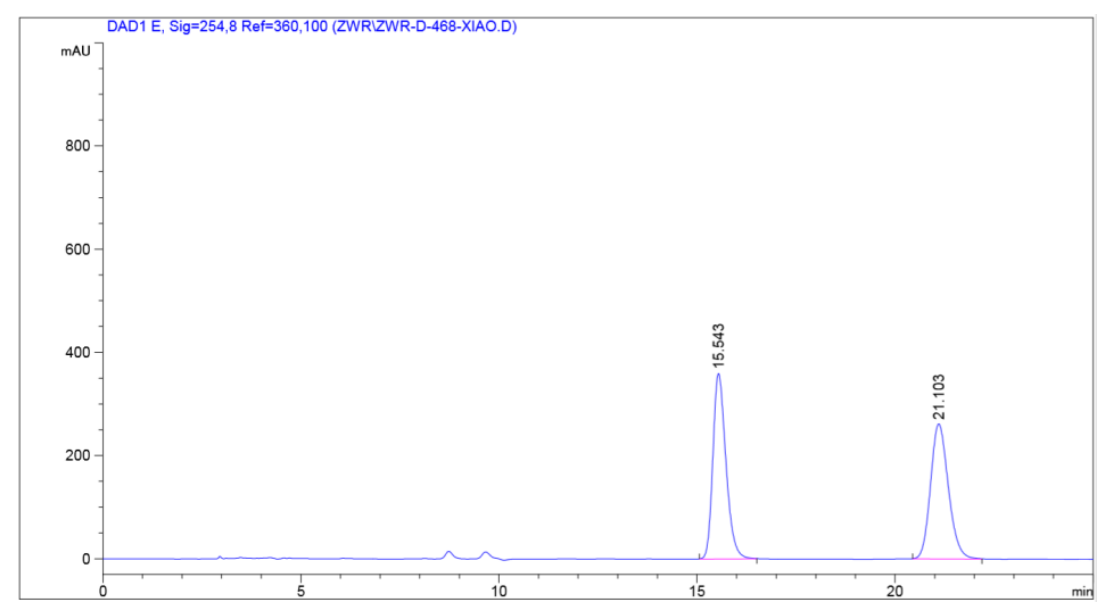

信号 1: DAD1 E， Sig=254,8 Ref=360, 100

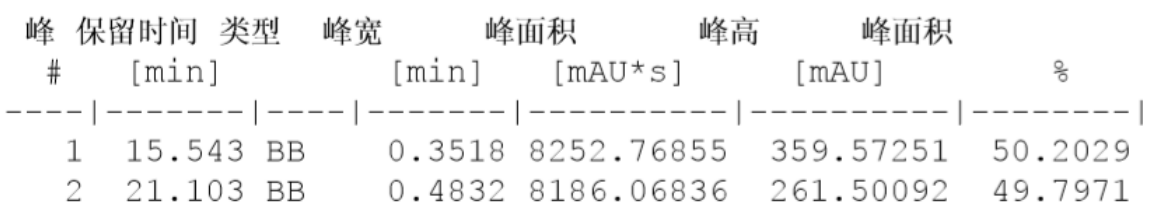

\section{HPLC spectrum of the chiral compound}

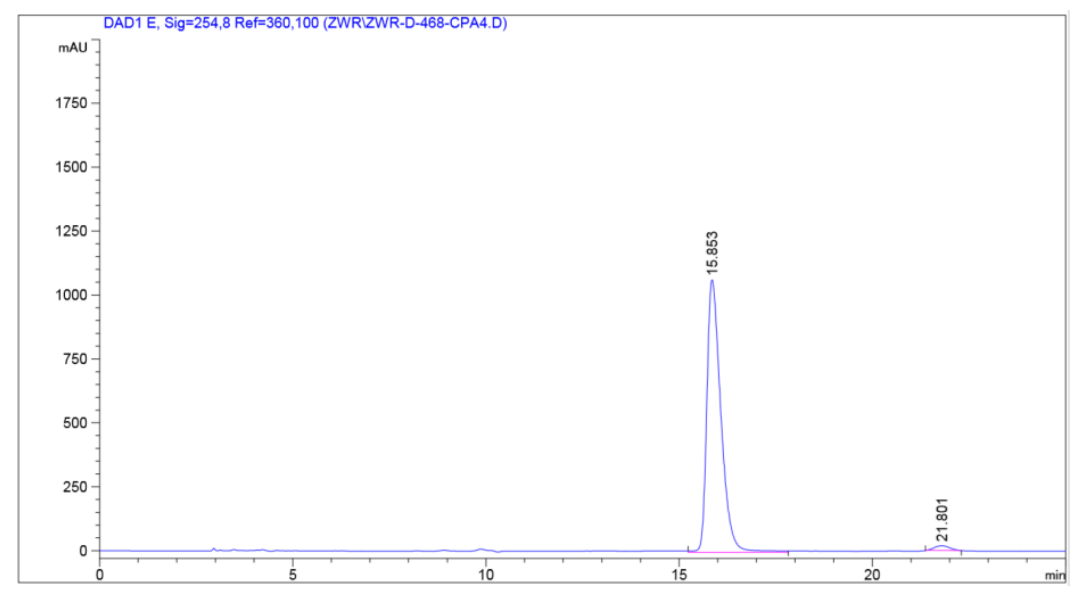

信号 1: DAD1 E， Sig=254, 8 Ref=360,100

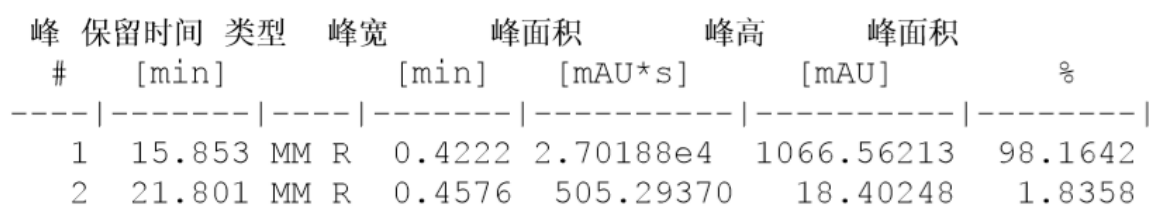




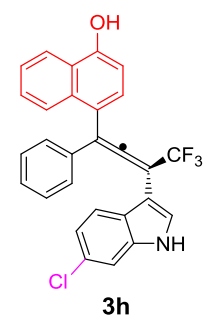

\section{HPLC spectrum of the racemate}

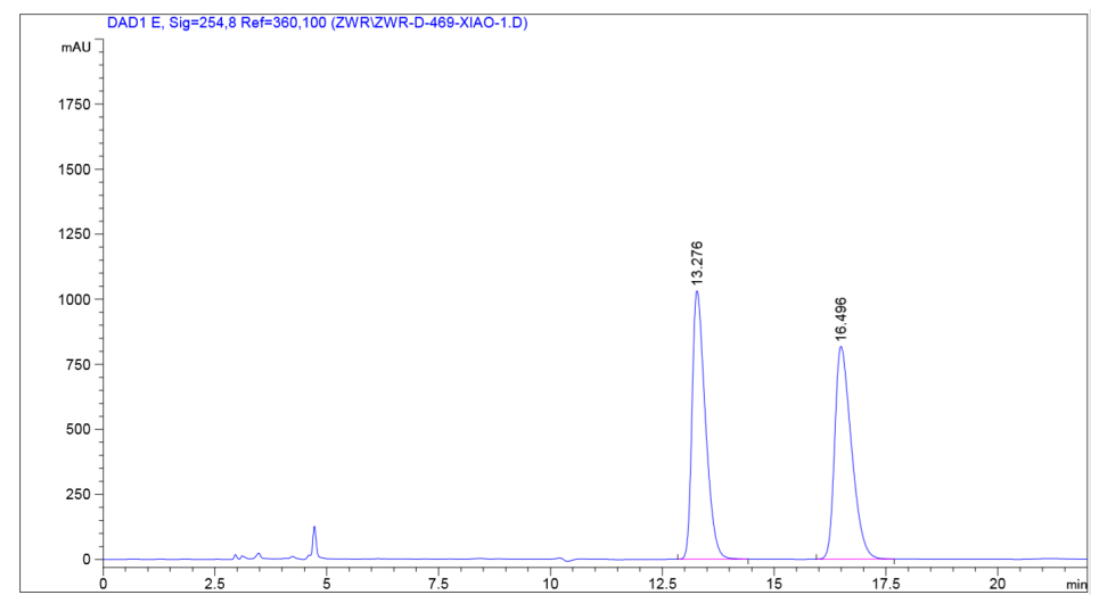

信号 1: DAD1 E， Sig=254,8 $\operatorname{Ref=360,100~}$

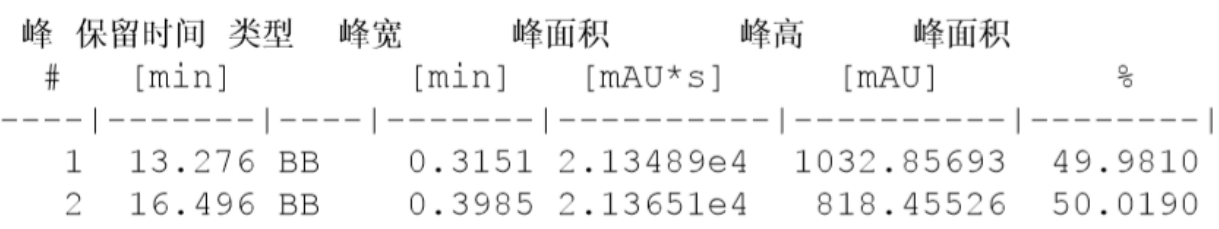

\section{HPLC spectrum of the chiral compound}

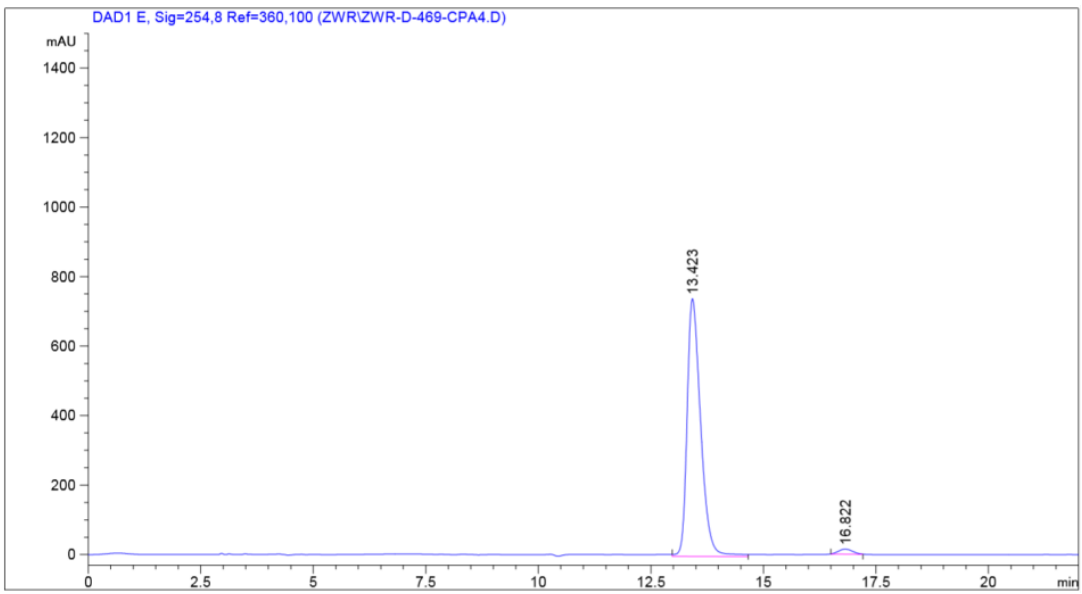

信号 1：DAD1 E， Sig=254,8 Ref=360,100

\begin{tabular}{|c|c|c|c|c|c|c|c|}
\hline 峰 侣 & 留时间 类型 & 峰宽 & & 面积 & 峰高 & \multicolumn{2}{|l|}{ 峰面积 } \\
\hline \# & {$[\mathrm{min}]$} & & [min] & {$\left[\mathrm{mAU}^{\star} \mathrm{s}\right]$} & & [mAU] & $\frac{\circ}{0}$ \\
\hline & & & $a$ & 5 & & 7 & \\
\hline 2 & $16.822 \mathrm{MN}$ & $\mathrm{R}$ & 0.3589 & 309.262 & & 14.36300 & 1.9538 \\
\hline
\end{tabular}




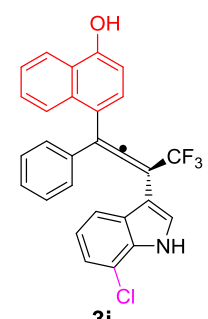

$3 \mathbf{i}$

\section{HPLC spectrum of the racemate}

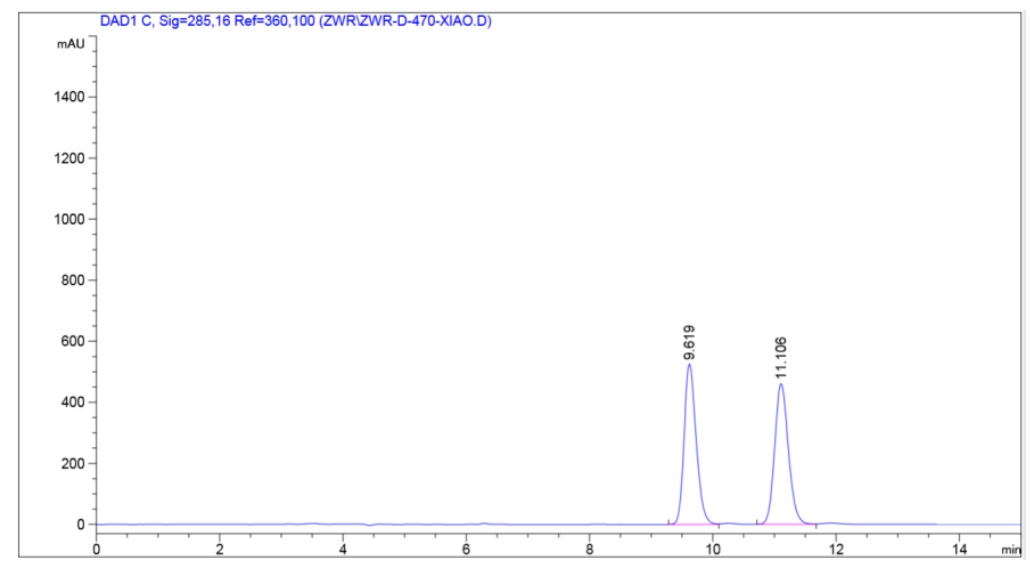

信号 1: DAD1 C, Sig=285,16 $\operatorname{Ref}=360,100$

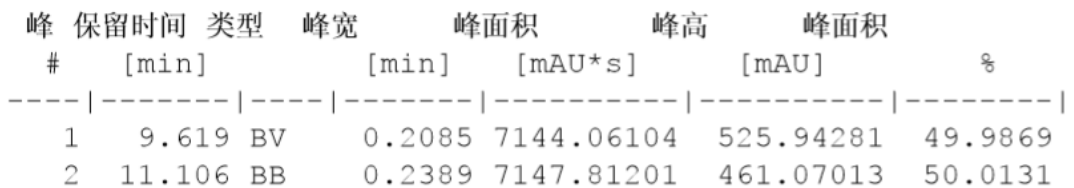

\section{HPLC spectrum of the chiral compound}

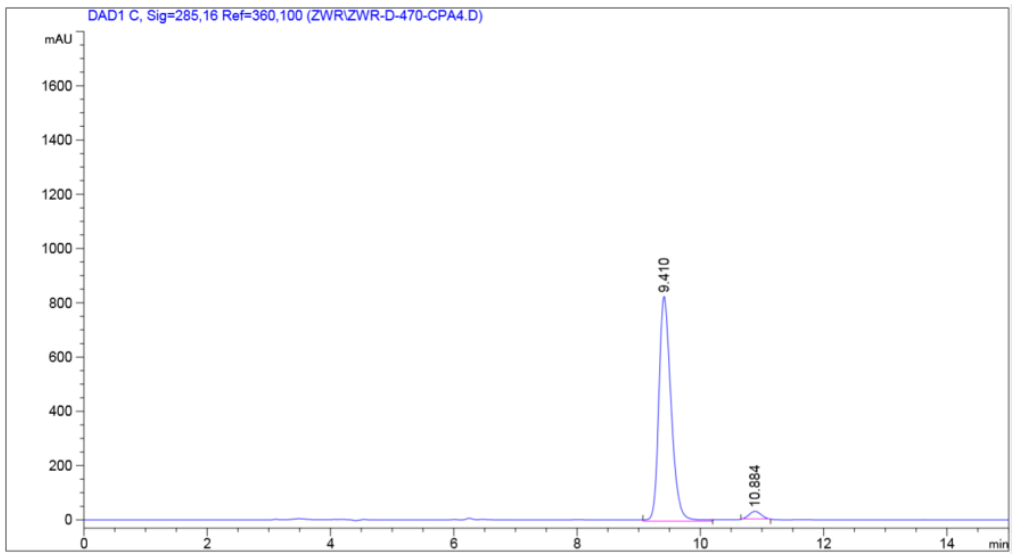

信号 1: DAD1 C, Sig=285,16 Ref=360,100

\begin{tabular}{|c|c|c|c|c|c|c|c|}
\hline 峰 & 保留时间 类型 & 峰宽 & & 面积 & 峰高 & \multicolumn{2}{|l|}{ 峰面积 } \\
\hline \# & [min ] & & {$[\mathrm{min}]$} & {$\left[\mathrm{mAU}^{*} \mathrm{~s}\right]$} & & [mAU] & 8 \\
\hline & $9.410 \mathrm{MN}$ & $\mathrm{R}$ & 0.2310 & $1.14781 \mathrm{e}$ & & 828.32025 & 96.9767 \\
\hline & $10.884 \mathrm{MM}$ & $\mathrm{R}$ & 0.2176 & 357.836 & & 27.40185 & 3.0233 \\
\hline
\end{tabular}




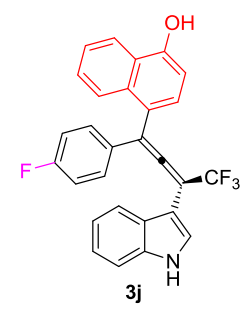

\section{HPLC spectrum of the racemate}

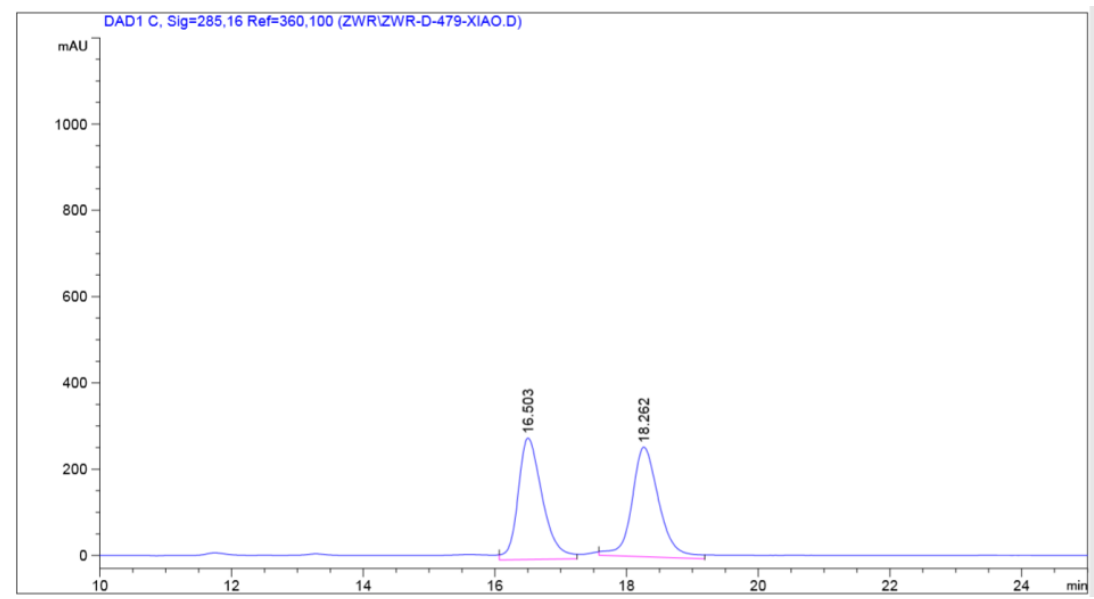

信号 1: DAD1 C, Sig=285,16 Ref=360,100

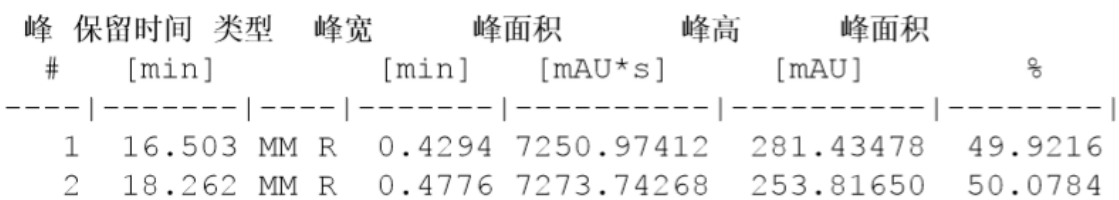

\section{HPLC spectrum of the chiral compound}

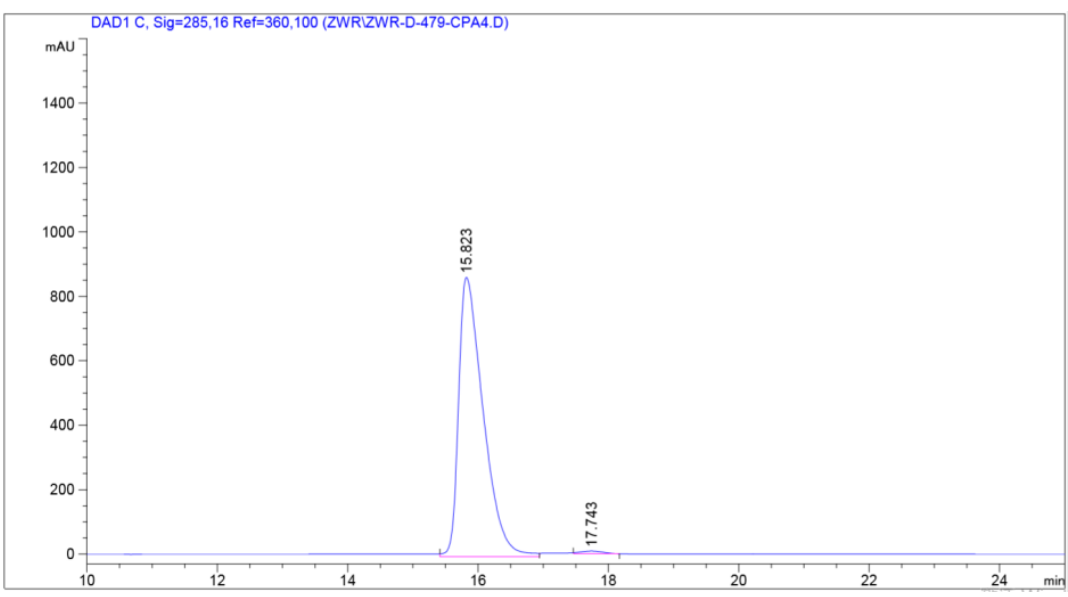

信号 1: DAD1 C, Sig=285,16 Ref=360,100

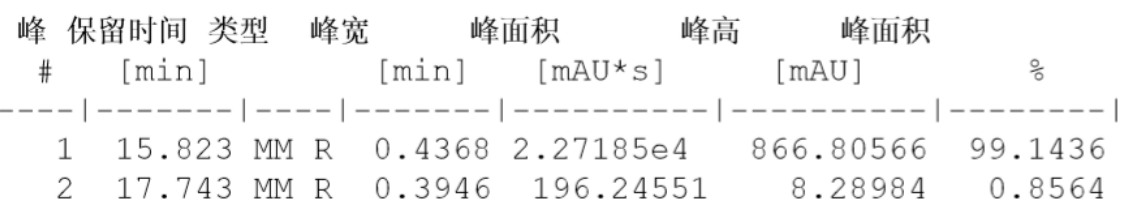




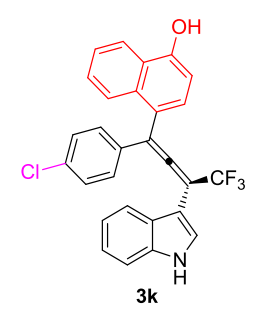

\section{HPLC spectrum of the racemate}

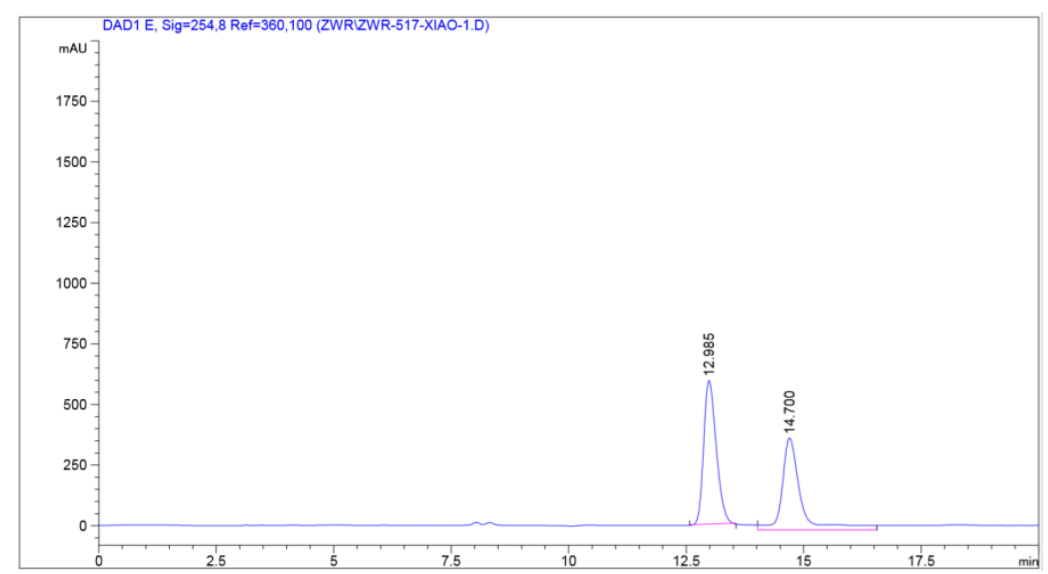

信号 1: DAD1 E， Sig=254, 8 Ref=360,100

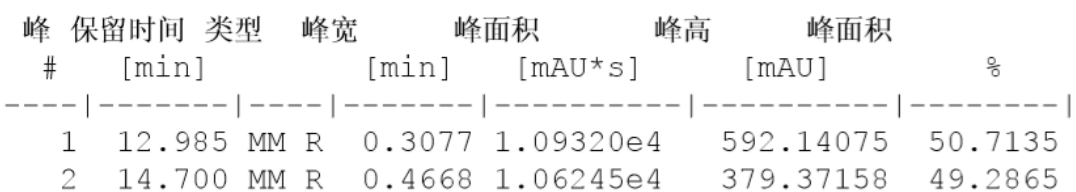

\section{HPLC spectrum of the chiral compound}

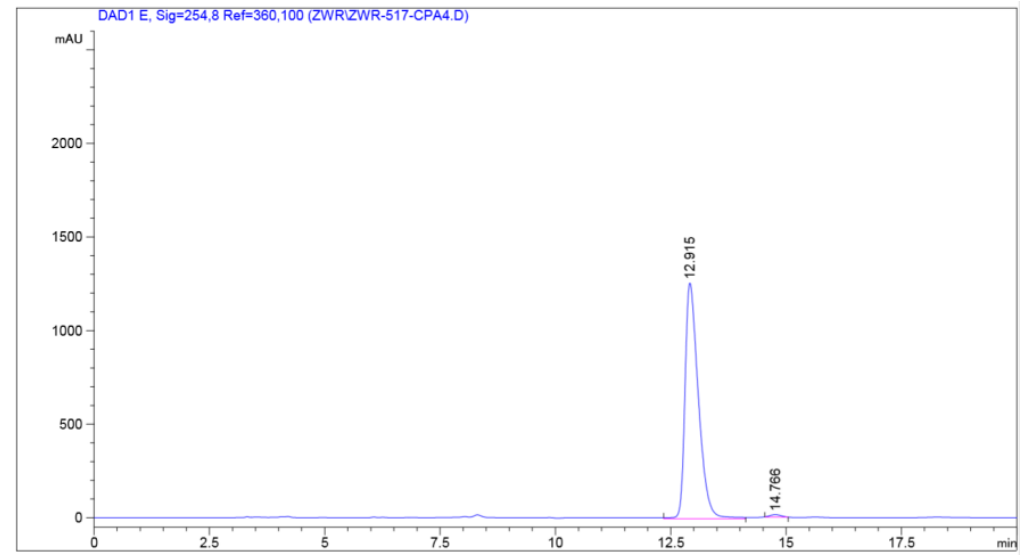

信号 1: DAD1 E， Sig=254,8 Ref=360,100

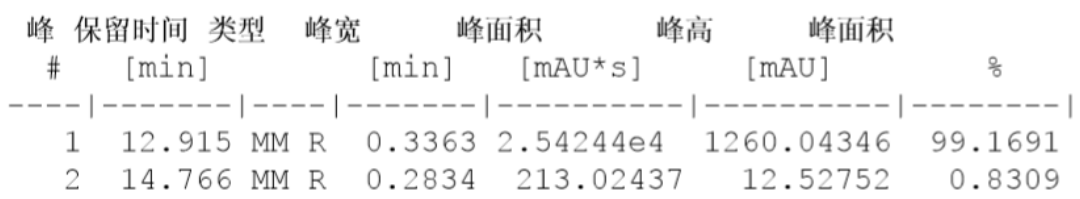




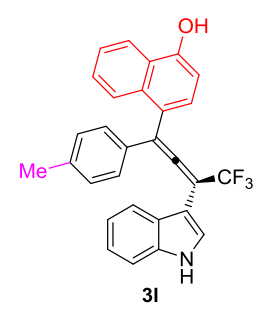

\section{HPLC spectrum of the racemate}

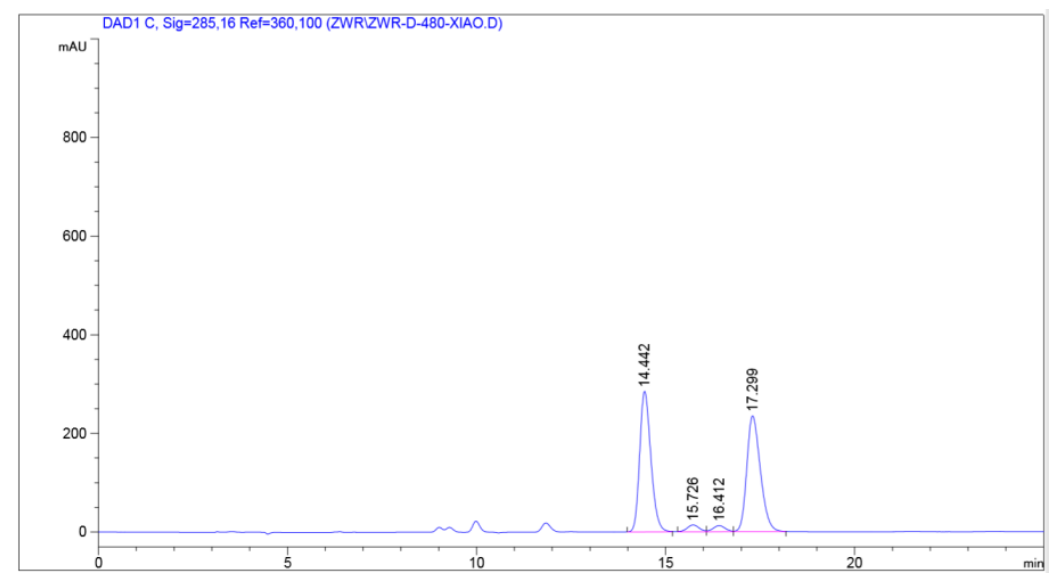

信号 1: DAD1 C, Sig=285,16 Ref=360,100

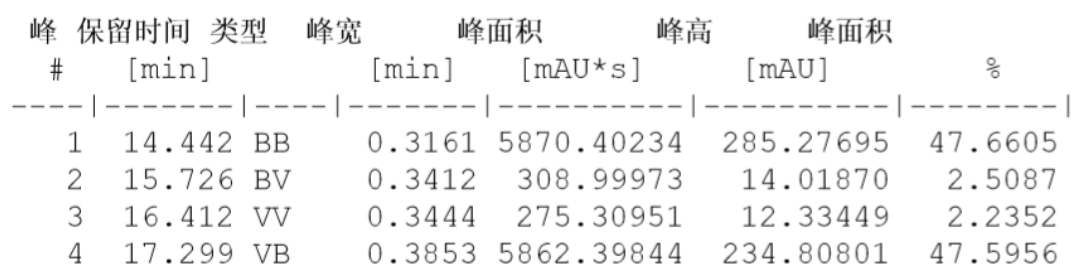

\section{HPLC spectrum of the chiral compound}

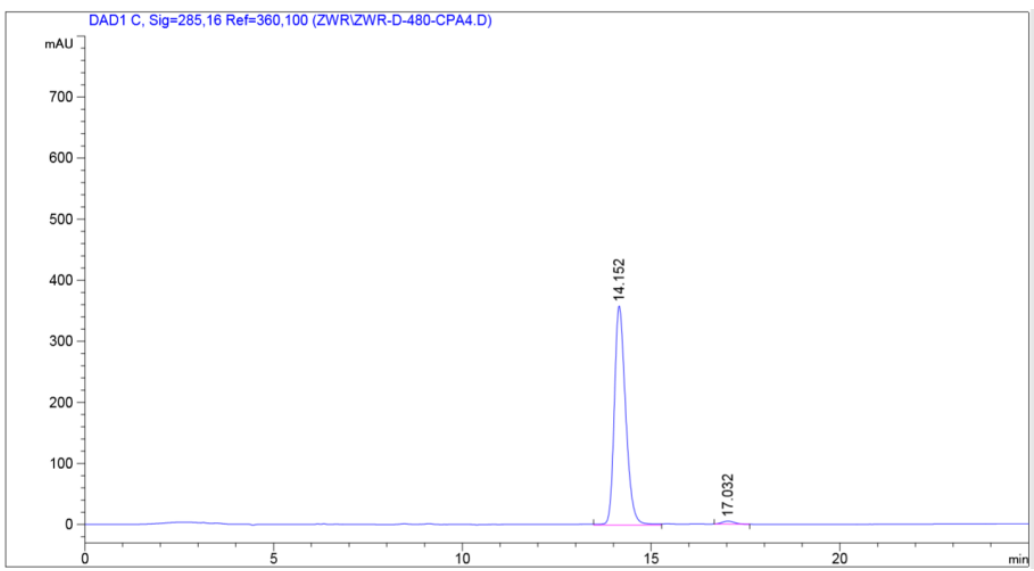

信号 1: DAD1 C， Sig=285,16 Ref=360,100

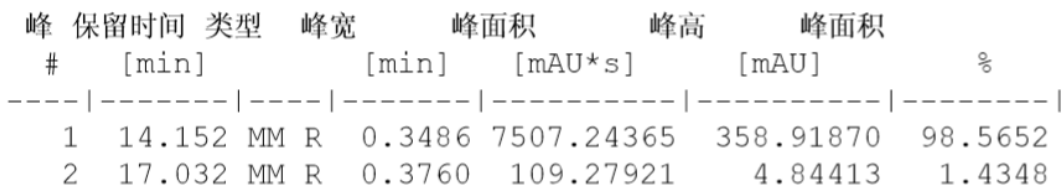


<smiles>COc1ccc(C(=C(C)c2ccccc2)c2cccc3c(O)cccc23)cc1</smiles>

\section{HPLC spectrum of the racemate}

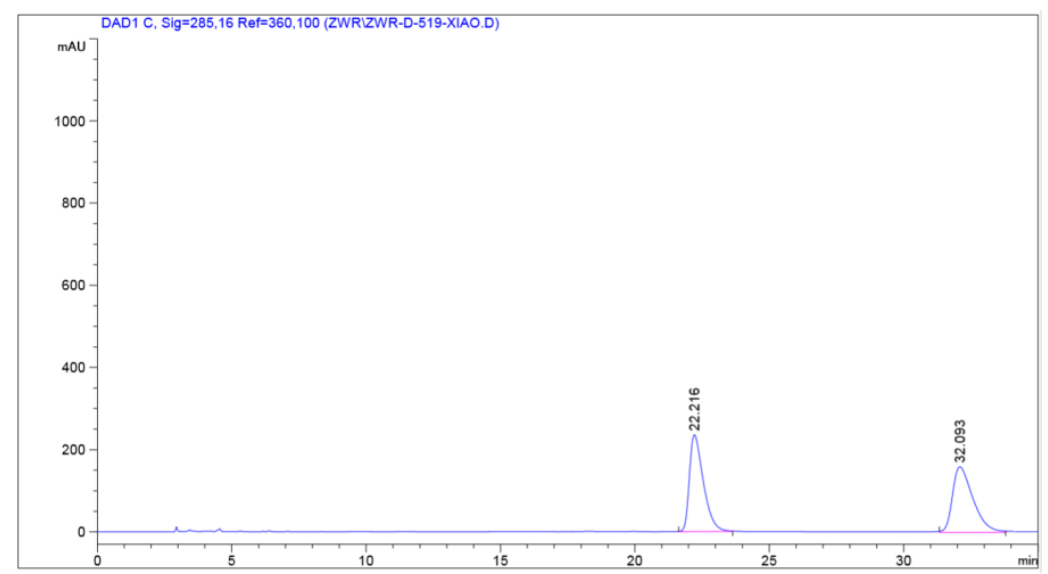

信号 1: DAD1 C, Sig=285,16 Ref=360,100

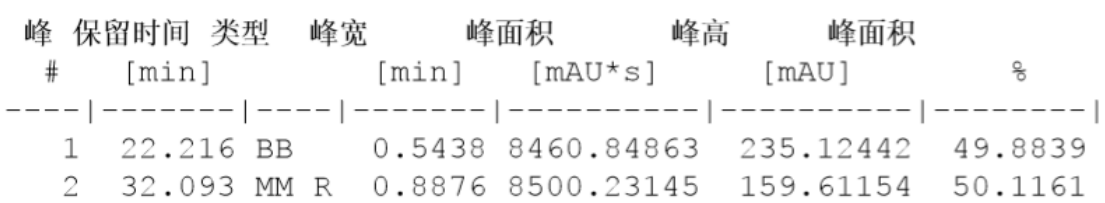

\section{HPLC spectrum of the chiral compound}

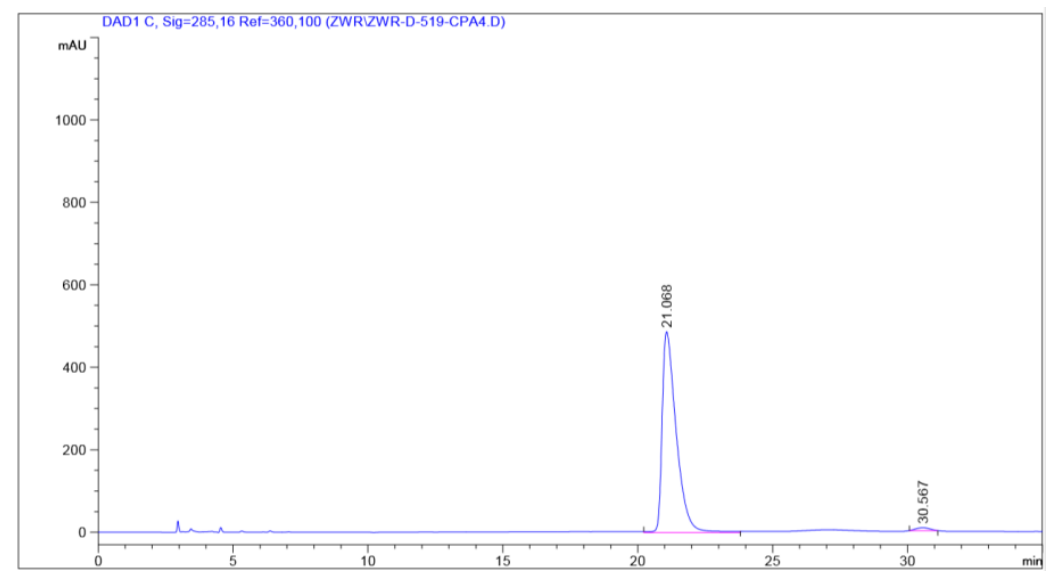

信号 1: DAD1 C， Sig=285,16 Ref=360,100

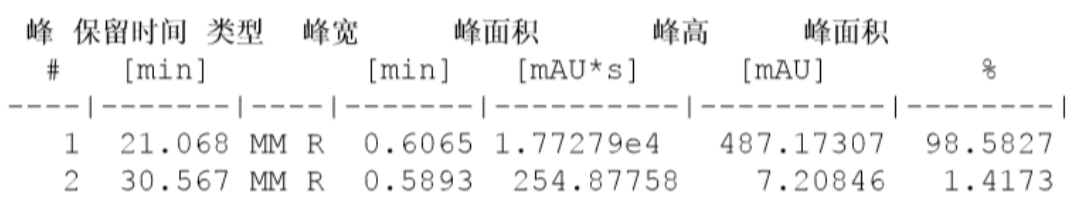




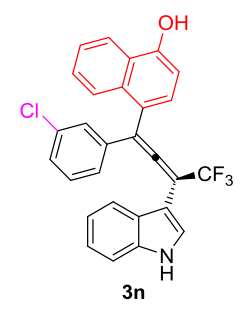

\section{HPLC spectrum of the racemate}

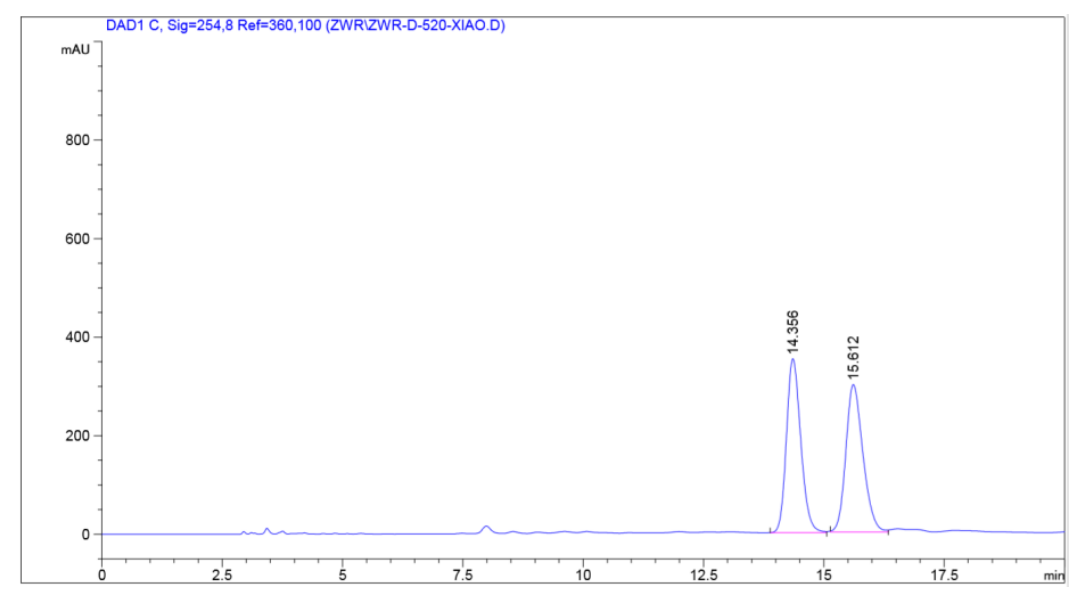

信号 1: DAD1 C, Sig=254,8 Ref=360,100

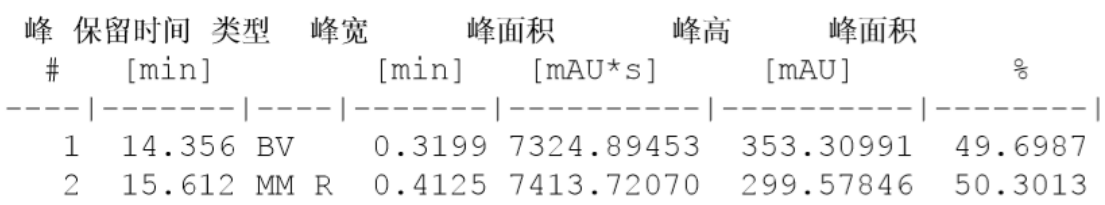

\section{HPLC spectrum of the chiral compound}

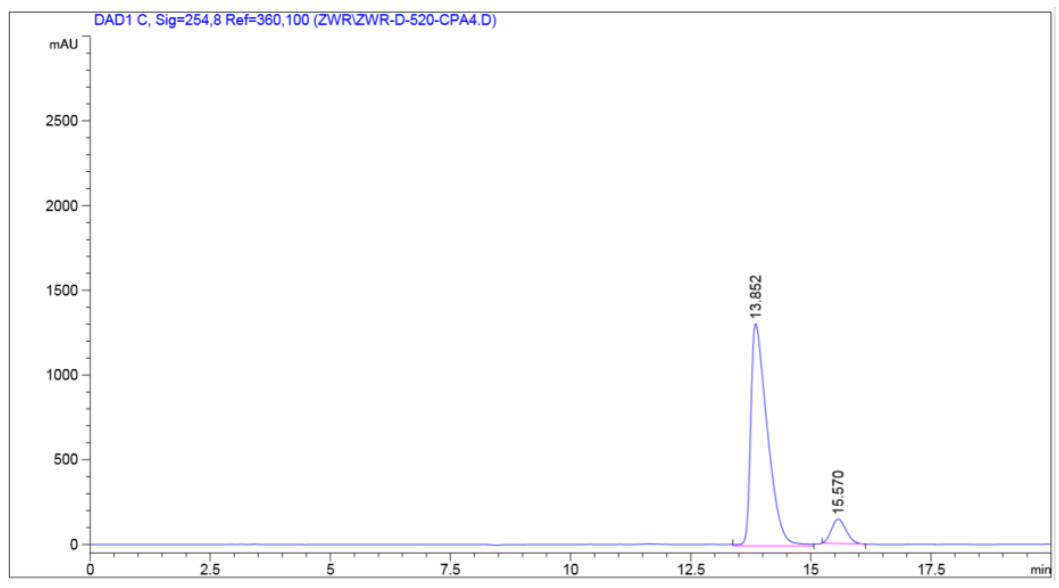

信号 1: DAD1 C, Sig=254,8 Ref=360, 100

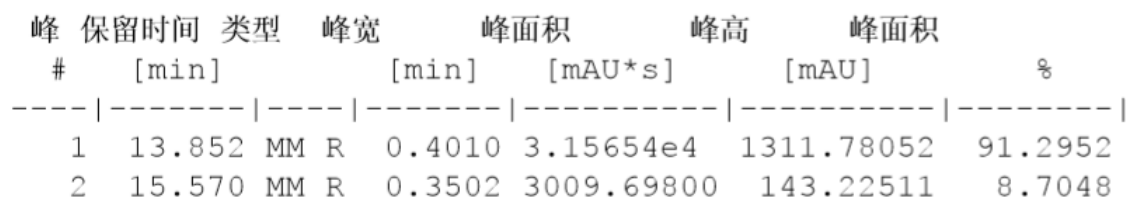




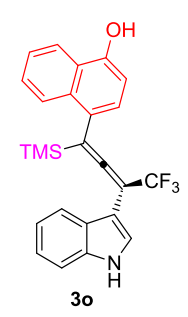

\section{HPLC spectrum of the racemate}

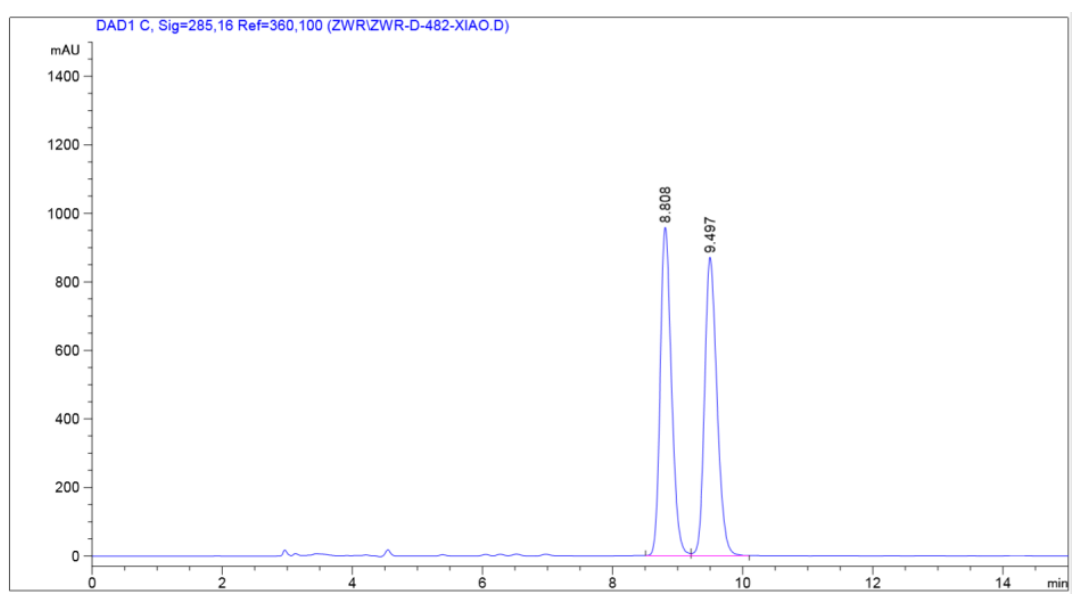

信号 1: DAD1 C, Sig=285,16 Ref=360,100

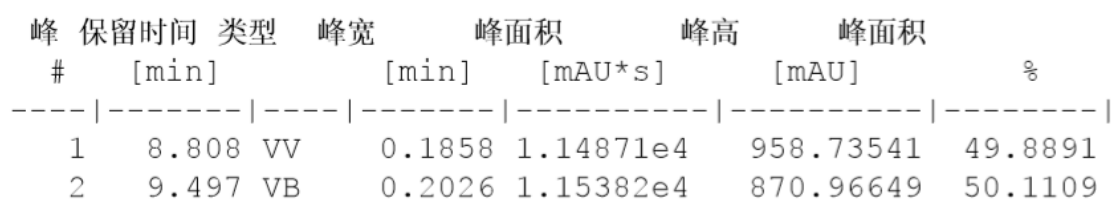

HPLC spectrum of the chiral compound

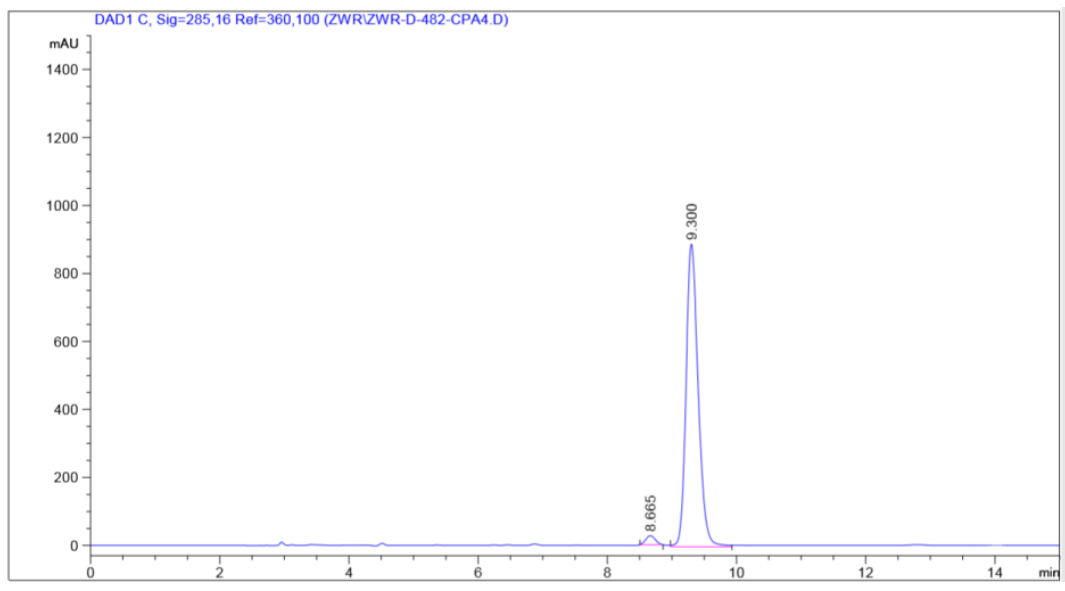

信号 1: DAD1 C, Sig=285, 16 Ref=360, 100

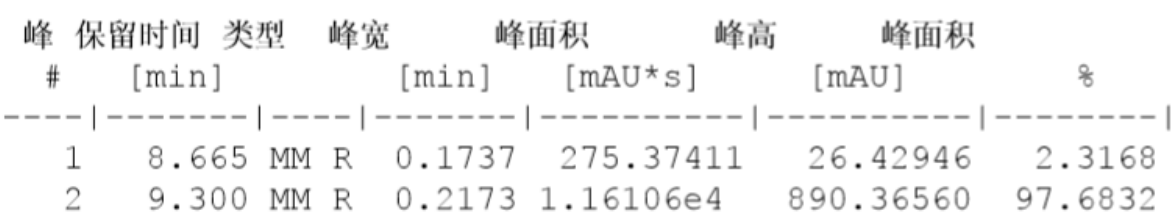




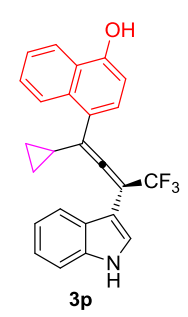

\section{HPLC spectrum of the racemate}

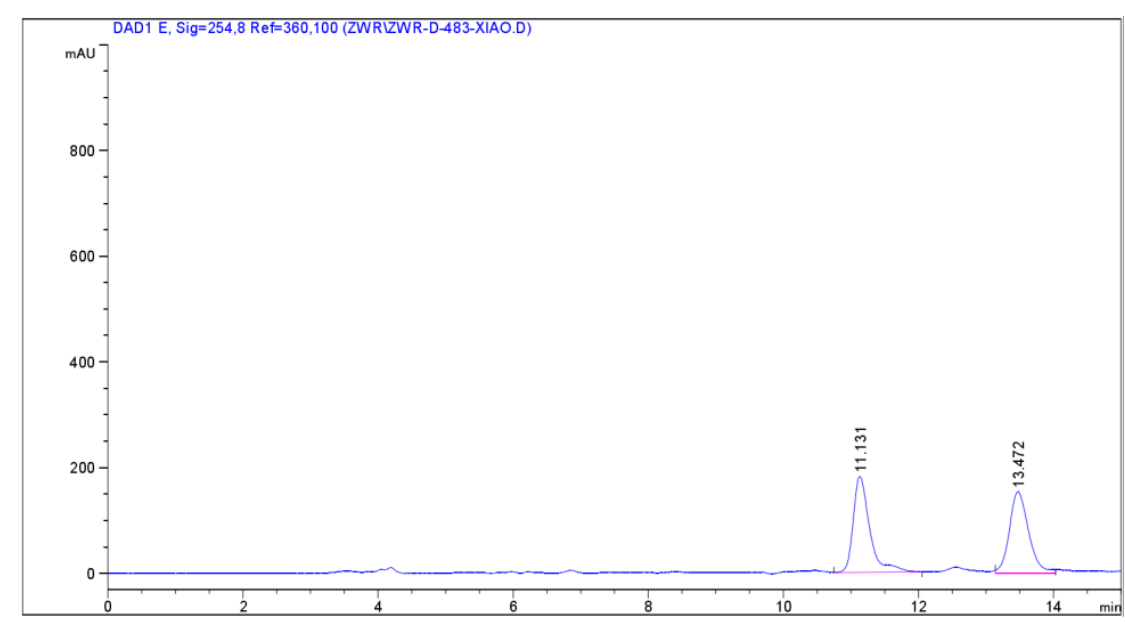

信号 1: DAD1 E， Sig=254,8 Ref=360,100

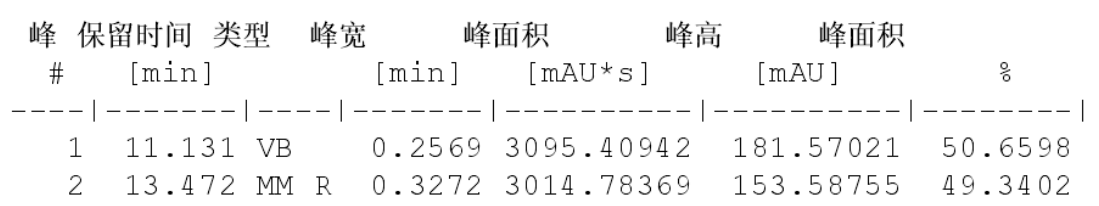

\section{HPLC spectrum of the chiral compound}

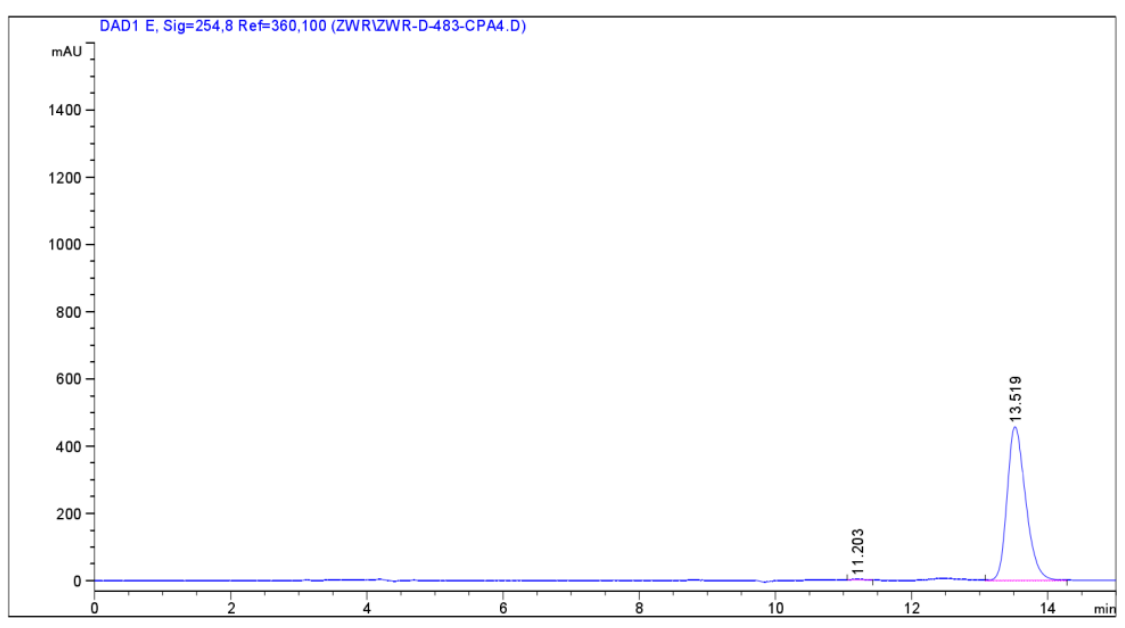

信号 1: DAD1 E， Sig=254,8 Ref=360,100

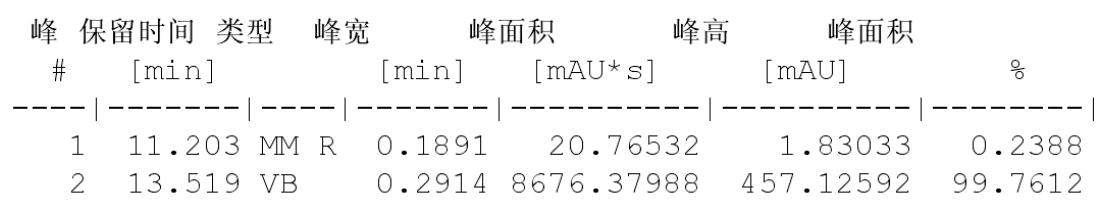




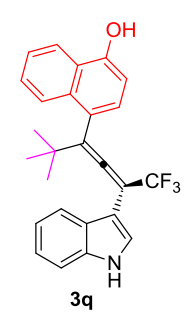

\section{HPLC spectrum of the racemate}

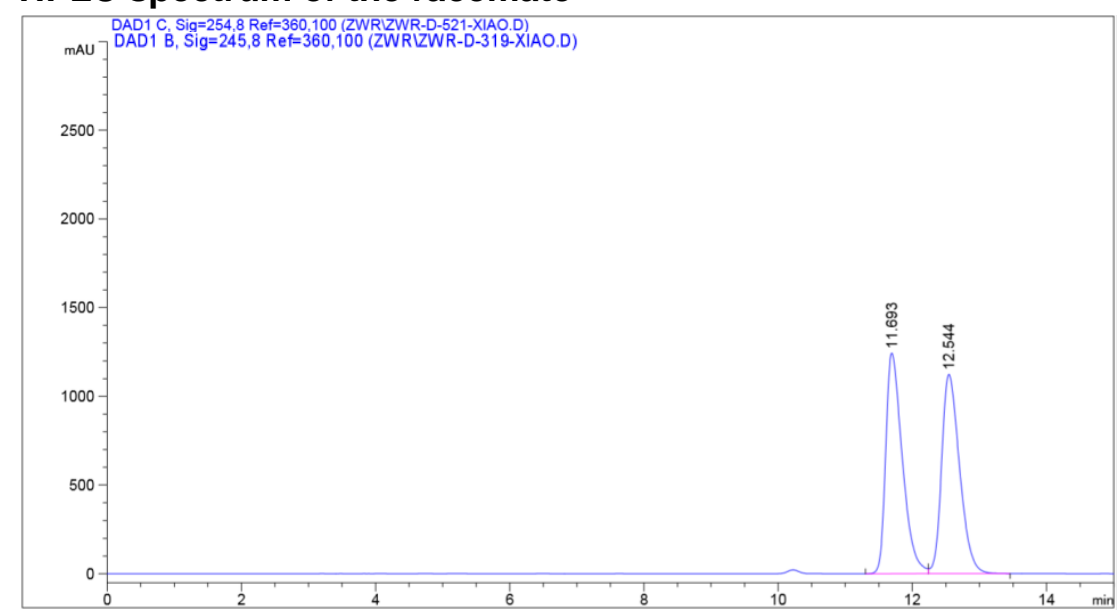

信号 1: DAD1 C, Sig=254,8 Ref=360,100

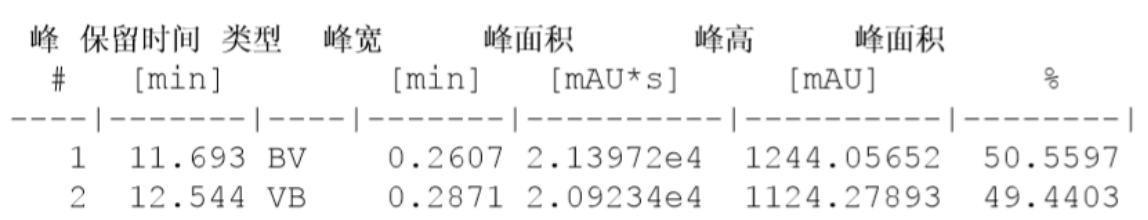

\section{HPLC spectrum of the chiral compound}

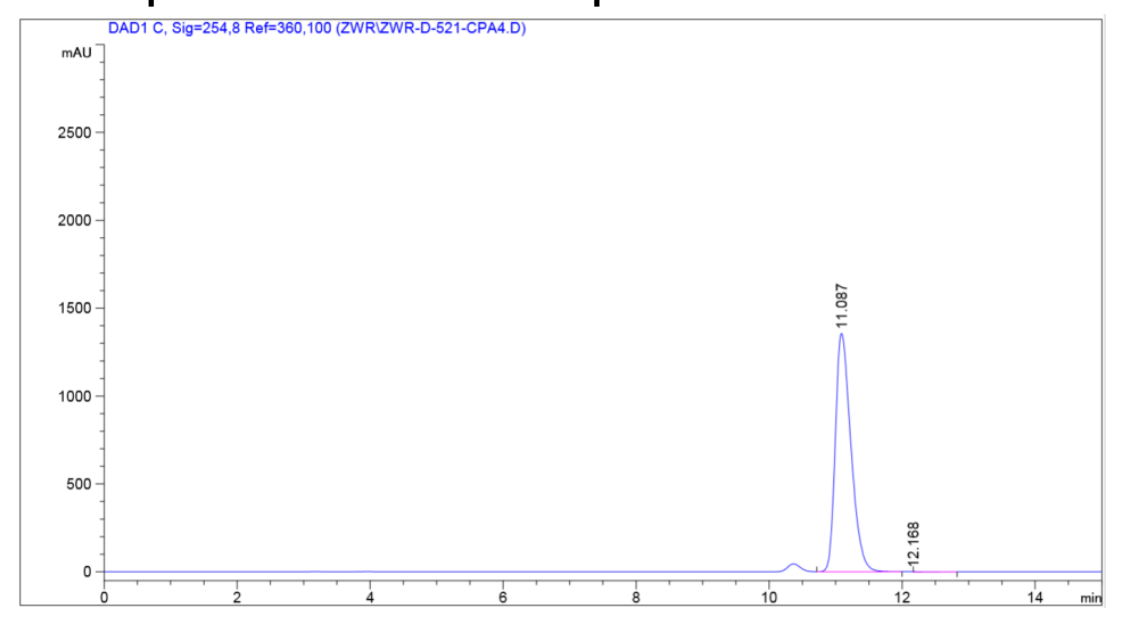

信号 1: DAD1 C, Sig=254,8 Ref=360,100

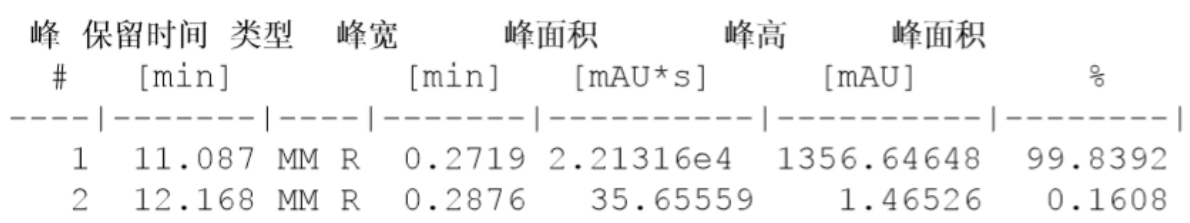




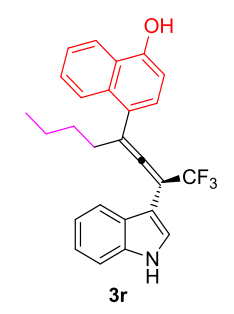

\section{HPLC spectrum of the racemate}

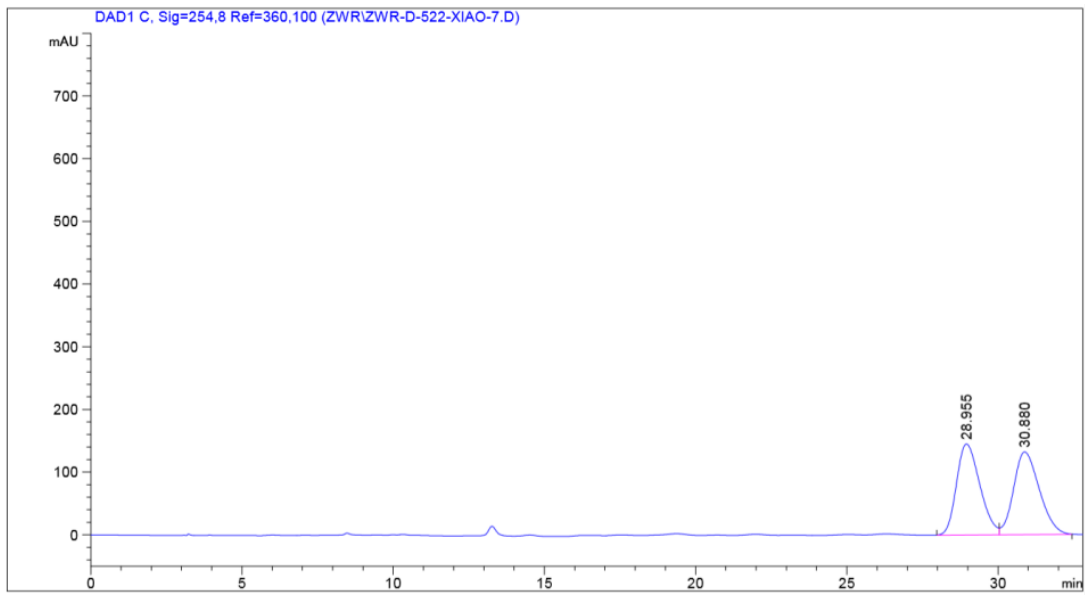

信号 1: DAD1 C, Sig=254, 8 Ref=360,100

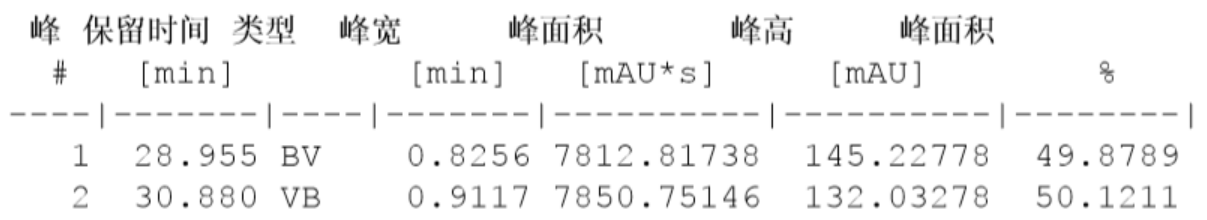

\section{HPLC spectrum of the chiral compound}

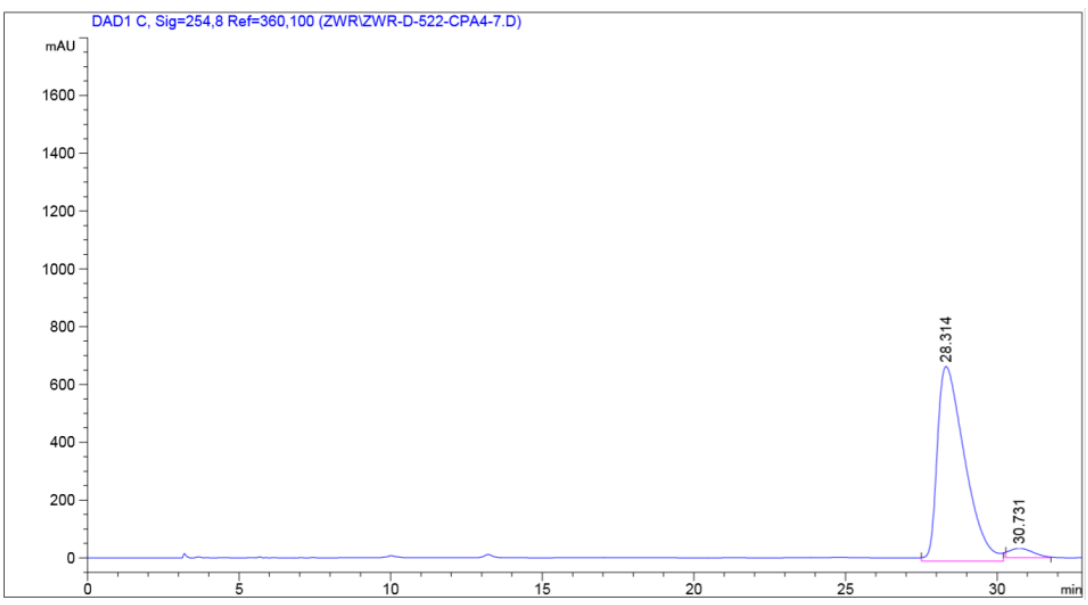

信号 1: DAD1 C, Sig=254, 8 Ref $=360,100$

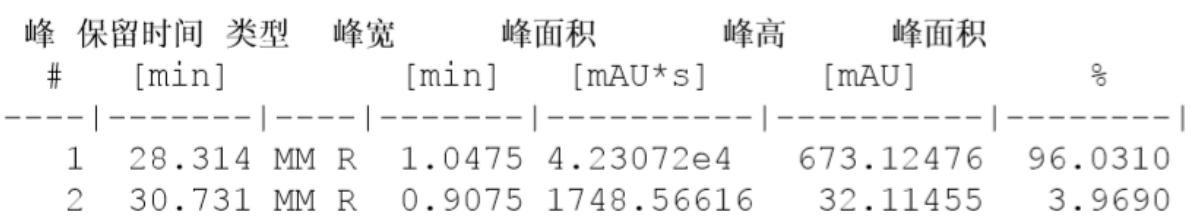




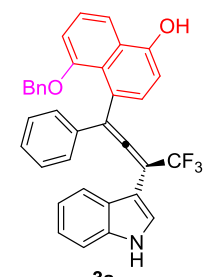

\section{HPLC spectrum of the racemate}

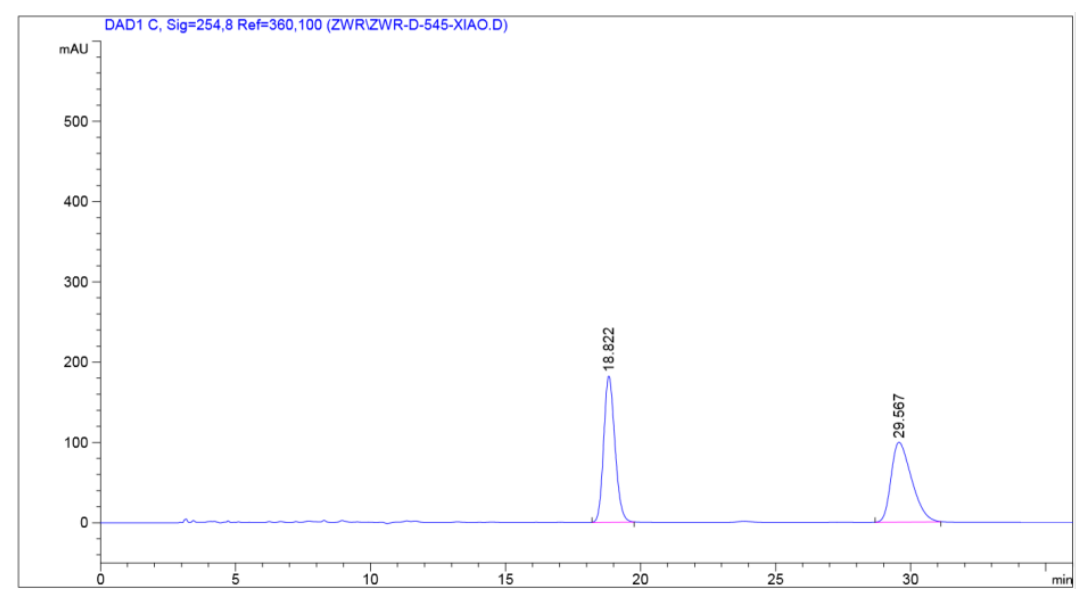

信号 1: DAD1 C, Sig=254,8 Ref=360,100

\begin{tabular}{|c|c|c|c|c|c|c|}
\hline 峰 & 保留时间 类型 & 峰宽 & 峰面积 & 峰高 & \multicolumn{2}{|l|}{ 峰面积 } \\
\hline \# & {$[\mathrm{min}]$} & {$[\mathrm{min}]$} & {$\left[\mathrm{mAU}{ }^{*} \mathrm{~s}\right]$} & & [mAU ] & $\%$ \\
\hline & $18.822 \mathrm{BE}$ & 0.448 & 45289.311 & & 182.32849 & 50.0607 \\
\hline & $29.567 \mathrm{BE}$ & 0.801 & 65276.479 & & 99.67080 & 49.9393 \\
\hline
\end{tabular}

\section{HPLC spectrum of the chiral compound}

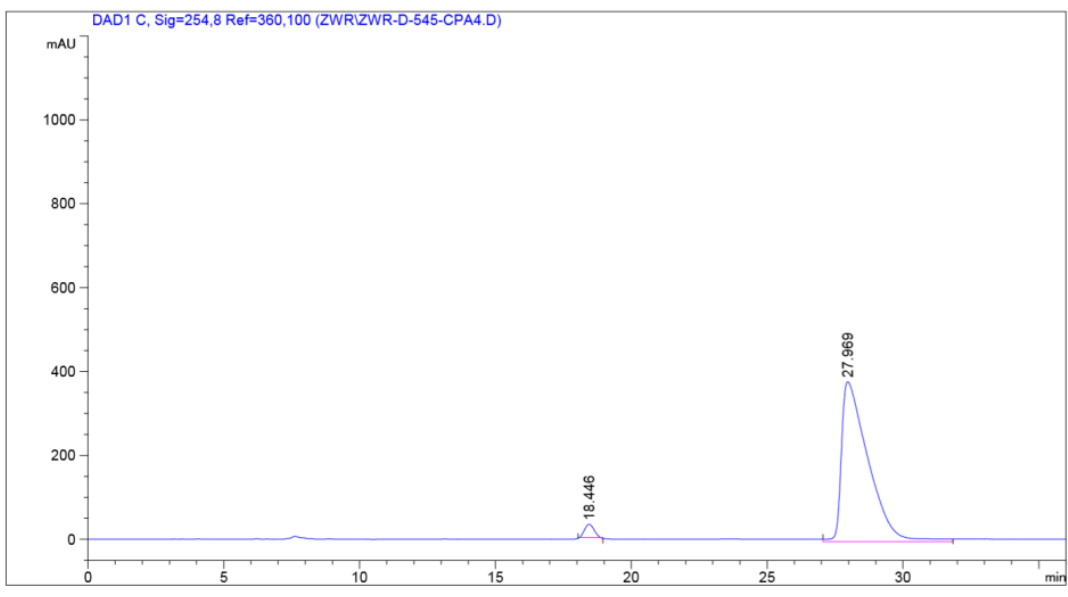

信号 1: DAD1 C, Sig=254, 8 Ref=360, 100

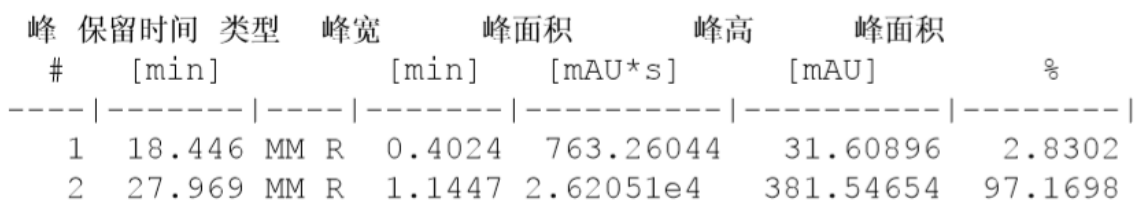




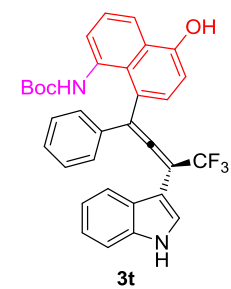

\section{HPLC spectrum of the racemate}

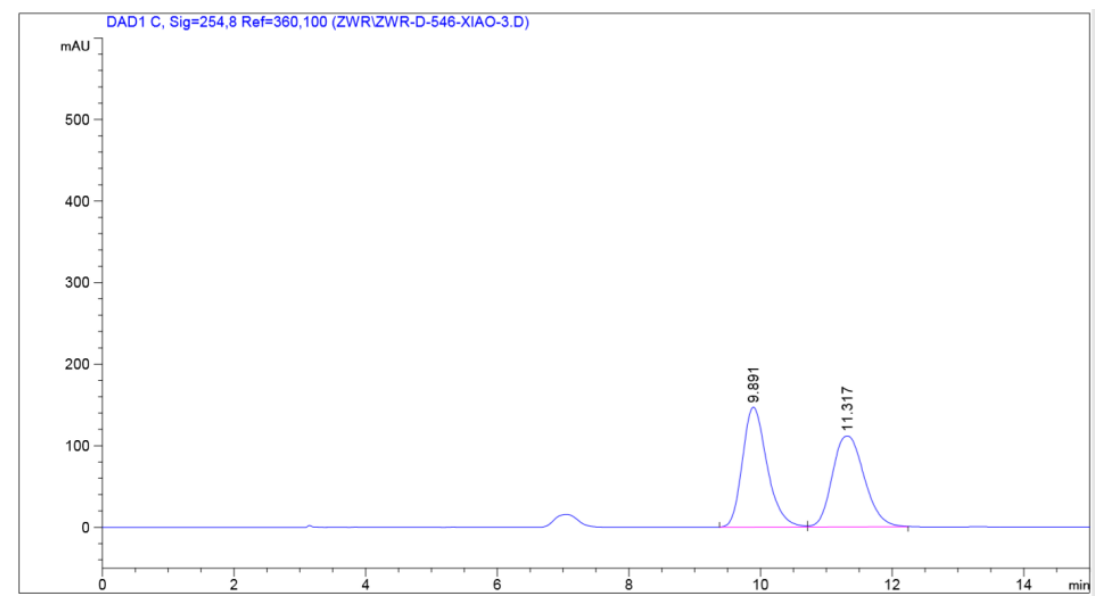

信号 1: DAD1 C, Sig=254,8 Ref=360,100

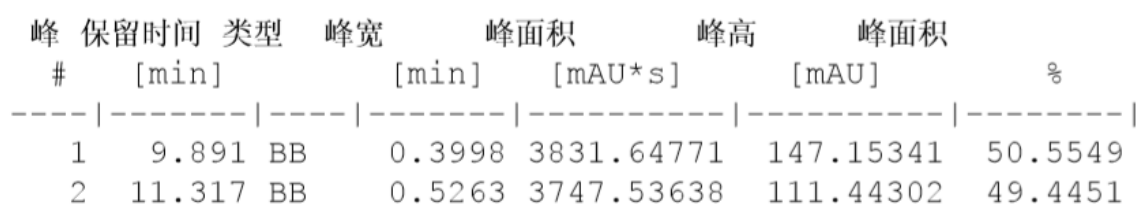

\section{HPLC spectrum of the chiral compound}

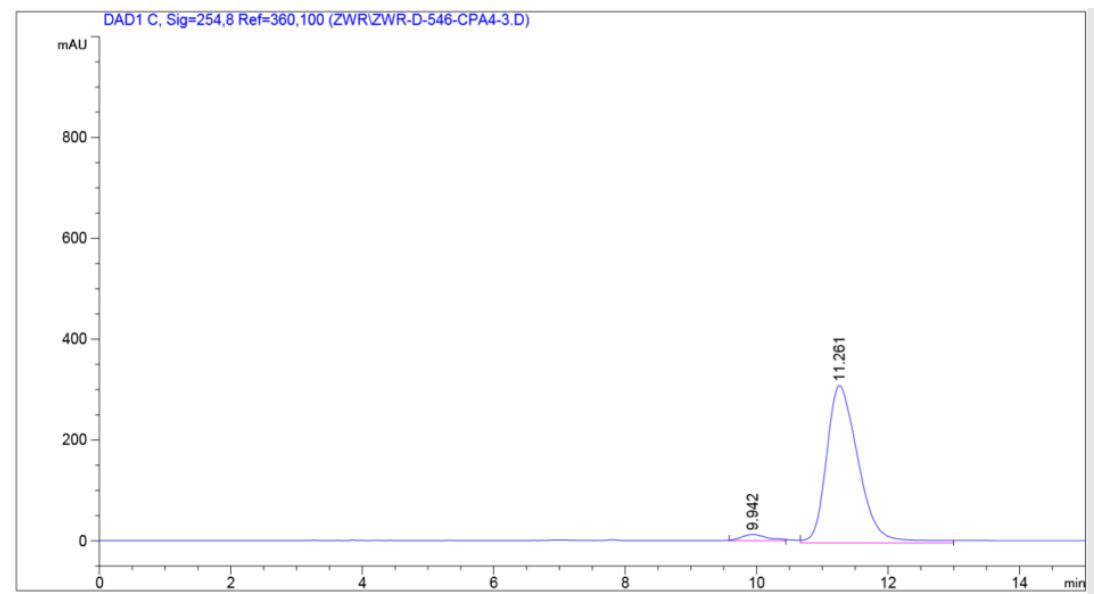

信号 1: DAD1 C, Sig=254,8 Ref=360,100

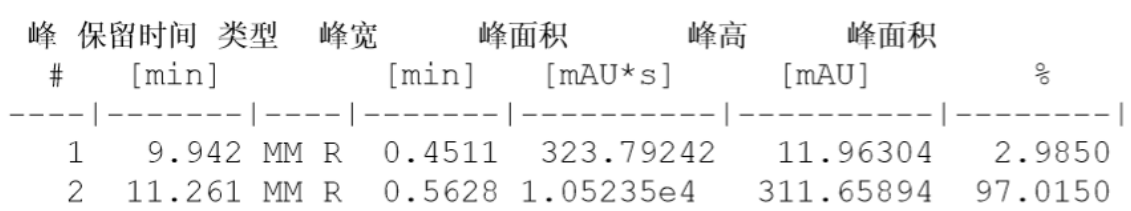




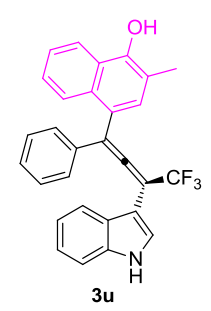

\section{HPLC spectrum of the racemate}

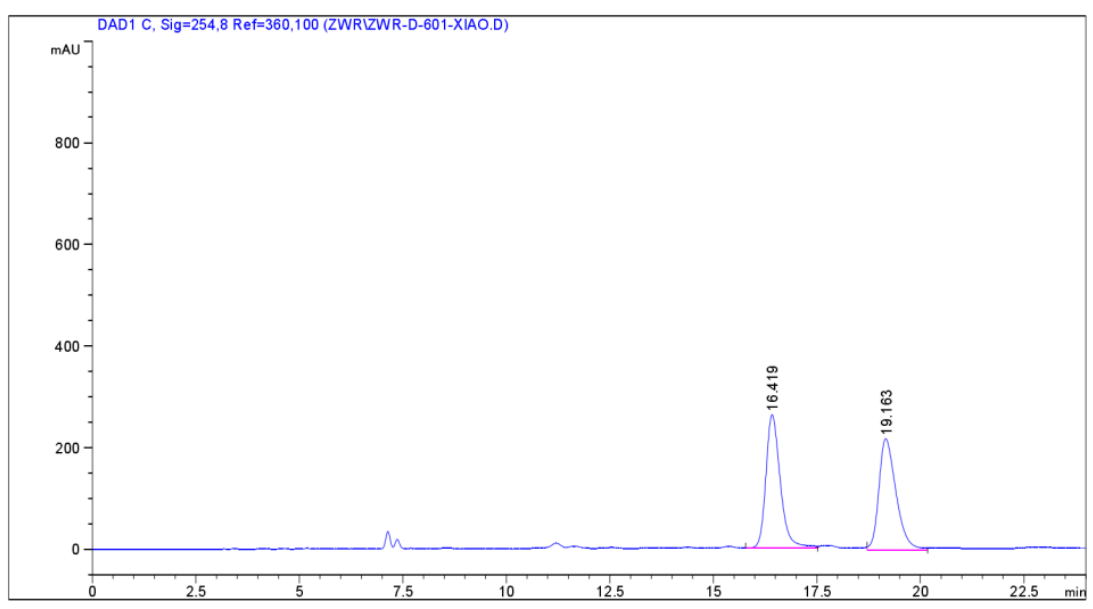

信号 1: DAD1 C, Sig=254,8 $\operatorname{Ref}=360,100$

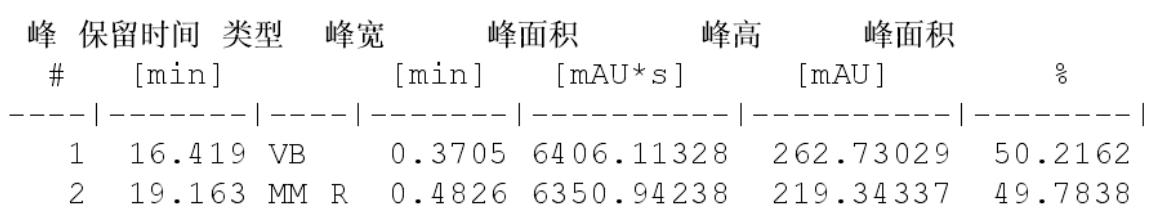

\section{HPLC spectrum of the chiral compound}

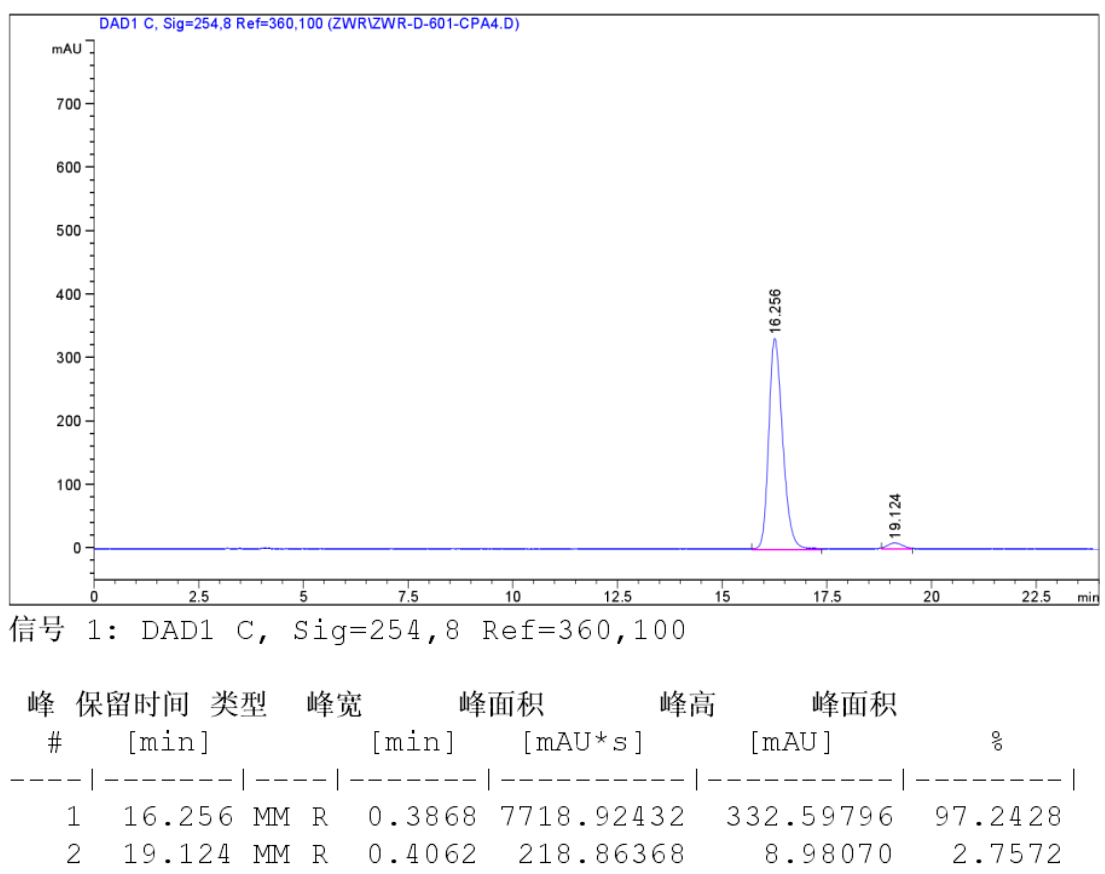




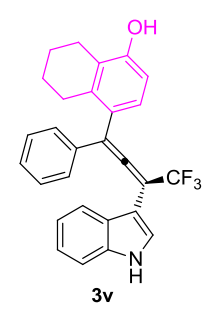

\section{HPLC spectrum of the racemate}

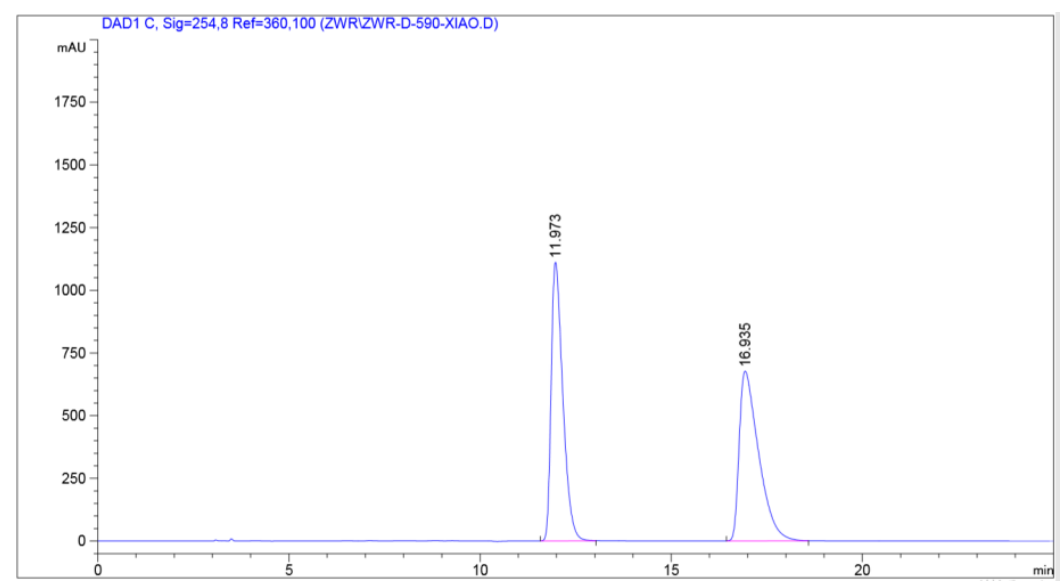

信号 1：DAD1 C, Sig=254, 8 Ref=360,100

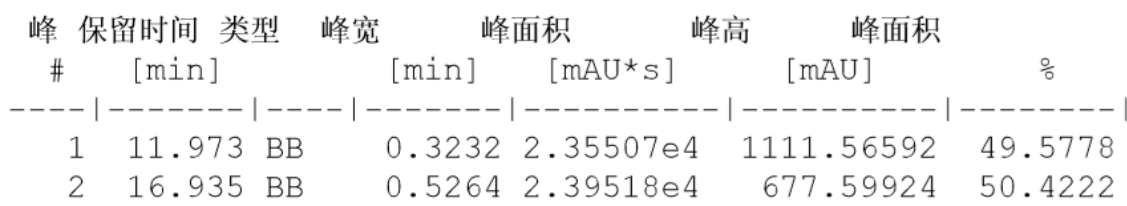

\section{HPLC spectrum of the chiral compound}

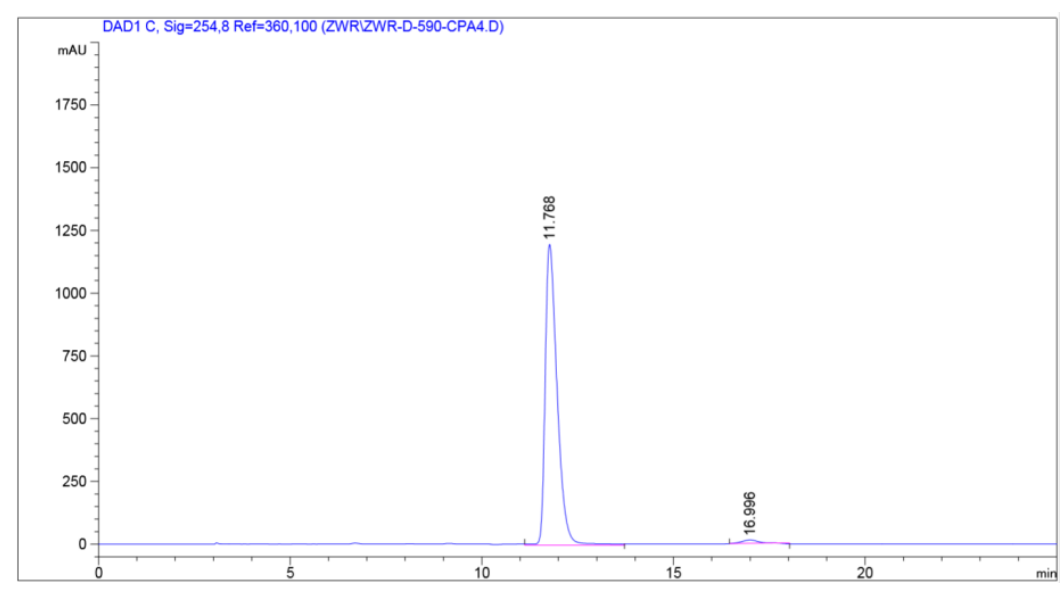

信号 1: DAD1 C, Sig=254, 8 Ref $=360,100$

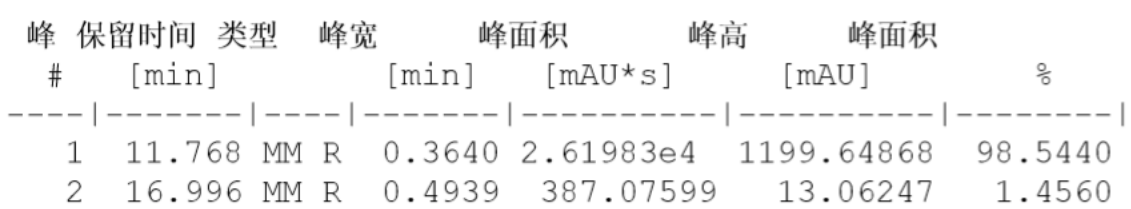




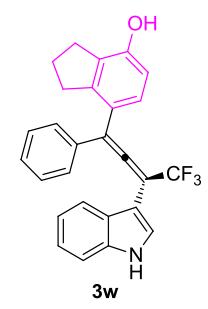

\section{HPLC spectrum of the racemate}

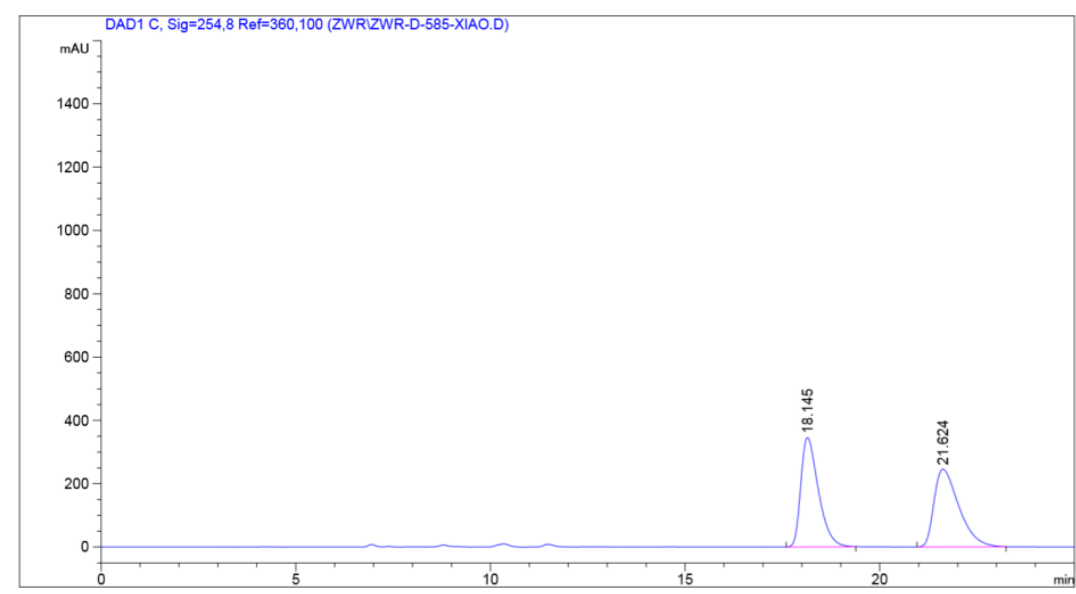

信号 1: DAD1 C, Sig=254,8 Ref=360,100

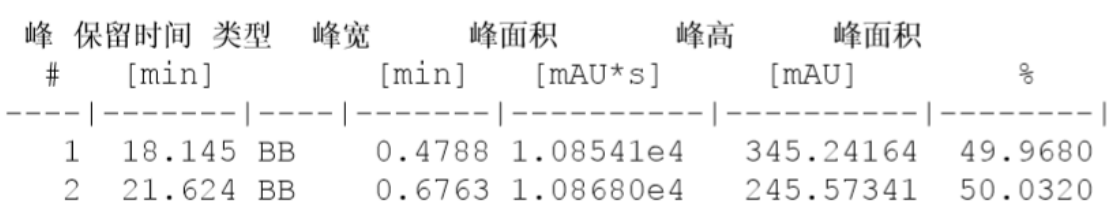

\section{HPLC spectrum of the chiral compound}

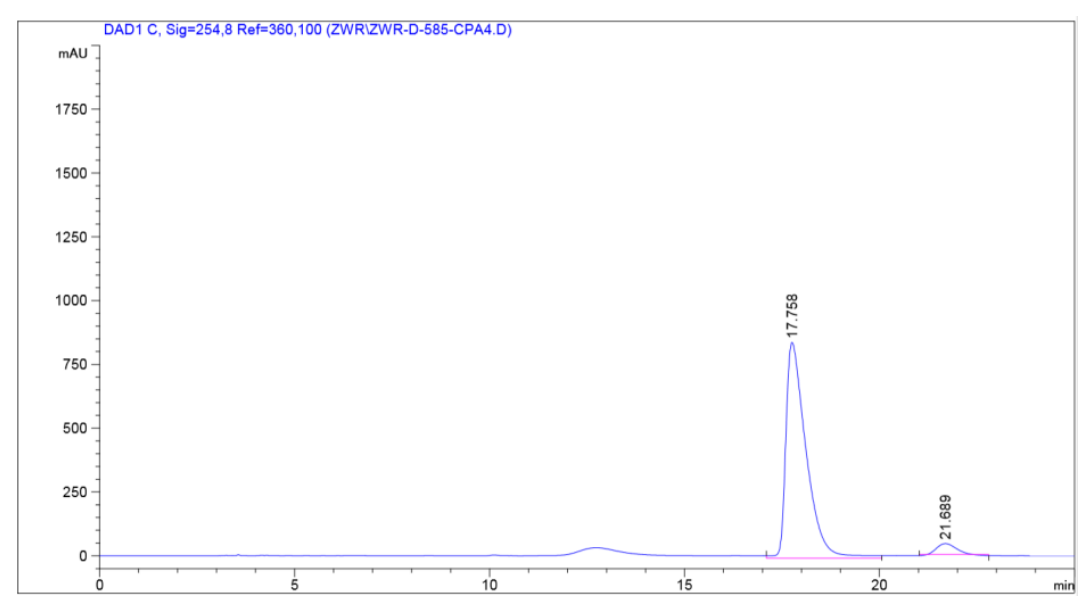

信号 1: DAD1 C, Sig=254,8 Ref=360, 100

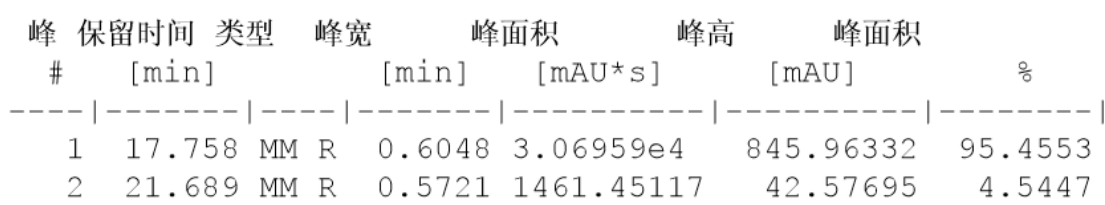




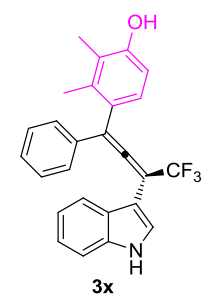

\section{HPLC spectrum of the racemate}

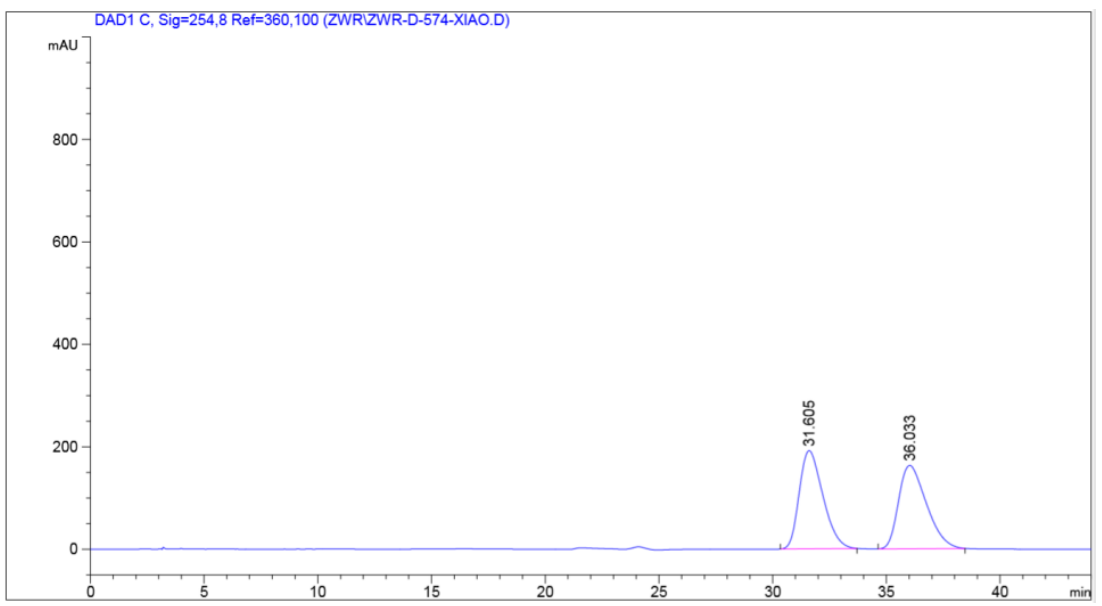

信号 1: DAD1 C, Sig=254, 8 Ref=360, 100

\begin{tabular}{|c|c|c|c|c|c|}
\hline 峰 & 保留时间 类型 & 峰宽 & 面积 & \multicolumn{2}{|l|}{ 峰面积 } \\
\hline \# & [min] & [min] & {$\left[\mathrm{mAU}{ }^{*} \mathrm{~s}\right]$} & [mAU] & g \\
\hline- & & -- & 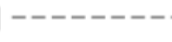 & $1--$ & \\
\hline 1 & $605 \mathrm{BE}$ & 1.1115 & $1.39421 \mathrm{e}$ & 191.92787 & 50.0824 \\
\hline 2 & $36.033 \mathrm{BE}$ & 1.2902 & $1.38962 \mathrm{e}$ & 163.06488 & 49.9176 \\
\hline
\end{tabular}

HPLC spectrum of the chiral compound

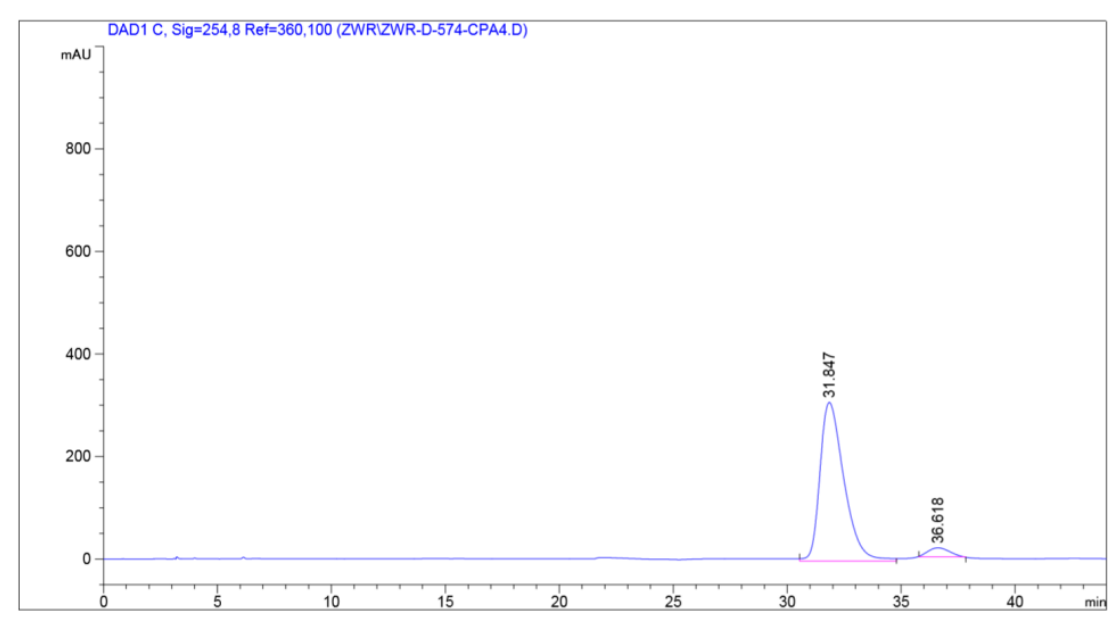

信号 1: DAD1 C, Sig=254,8 Ref=360,100

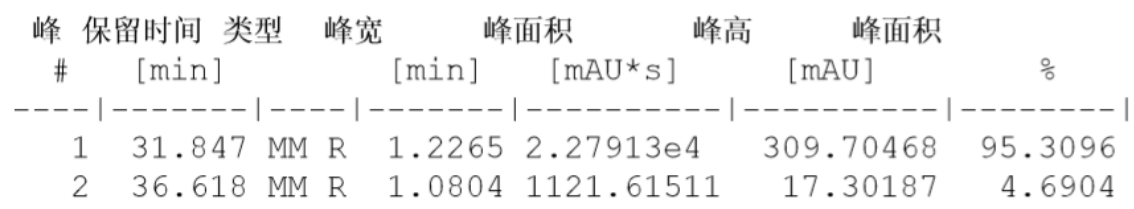




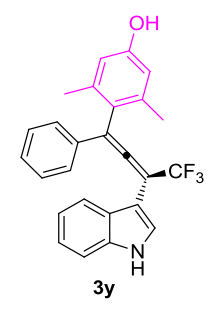

\section{HPLC spectrum of the racemate}

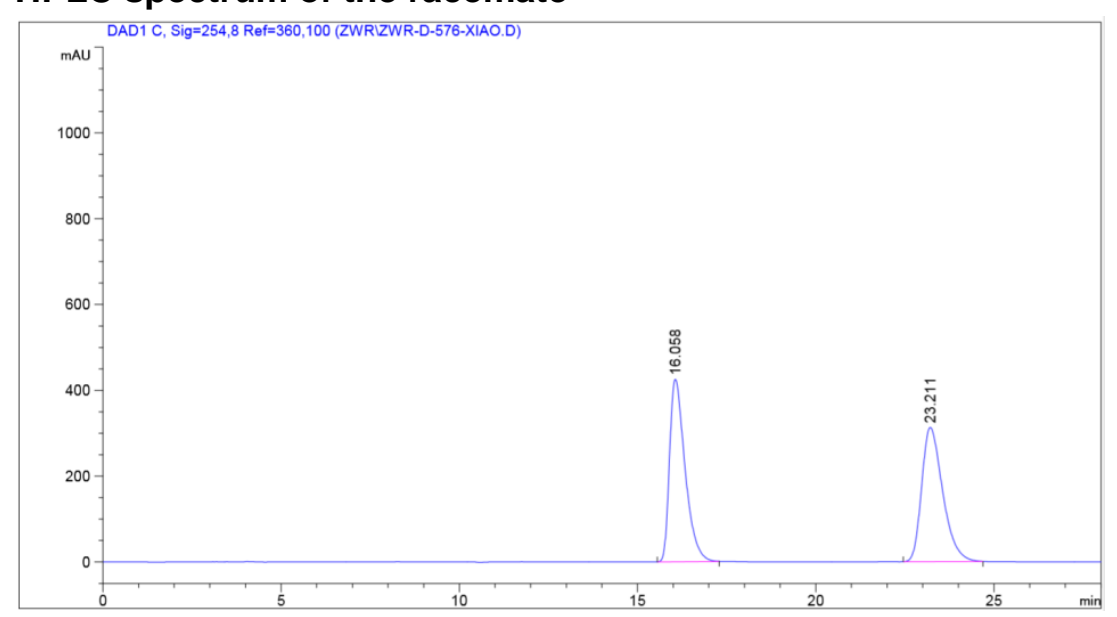

信号 1: DAD1 C, Sig=254, 8 Ref=360, 100

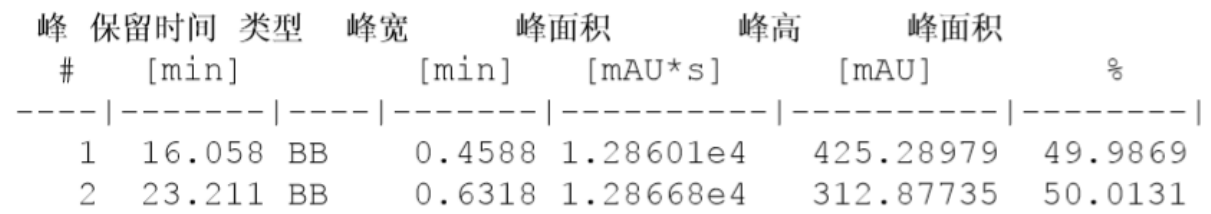

\section{HPLC spectrum of the chiral compound}

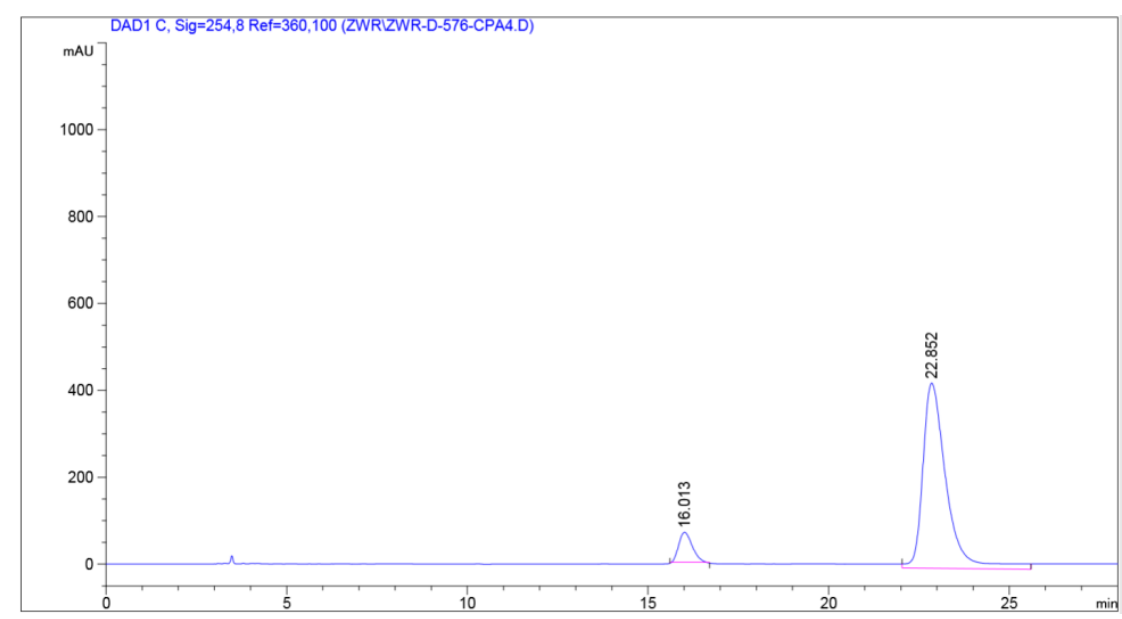

信号 1: DAD1 C, Sig=254, 8 Ref=360, 100

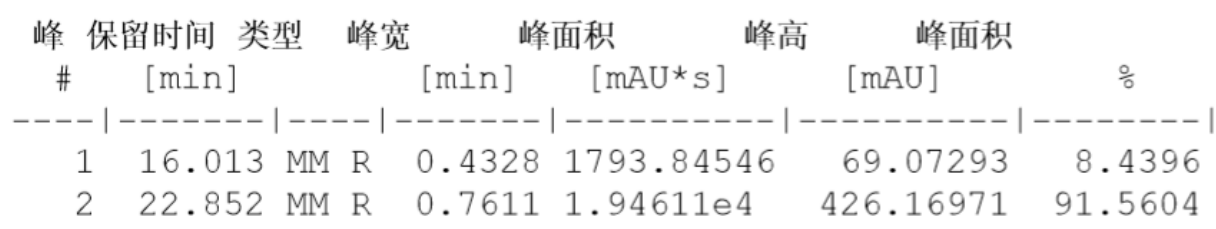




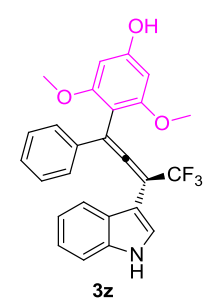

HPLC spectrum of the racemate

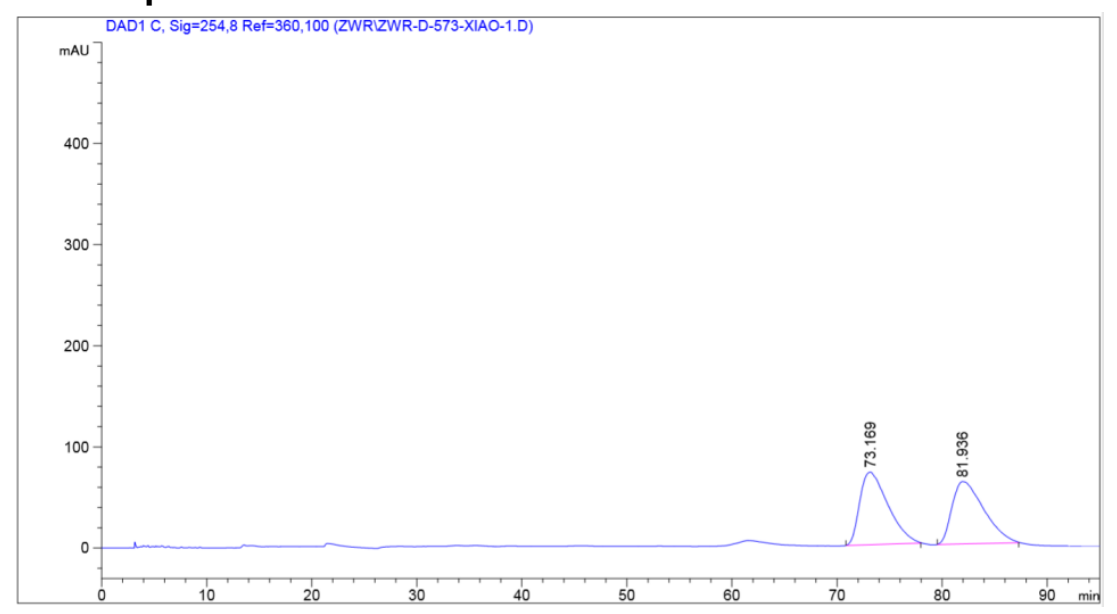

信号 1: DAD1 C, Sig=254,8 Ref=360,100

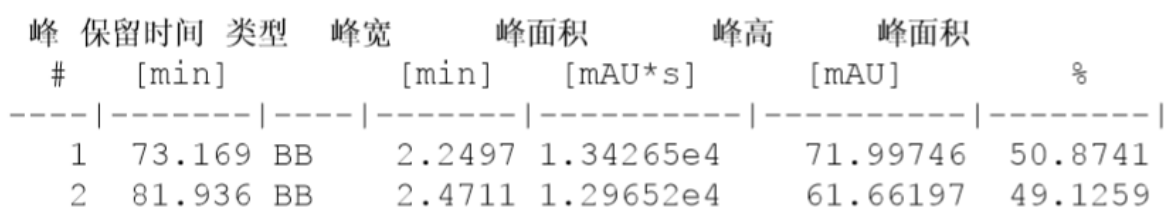

\section{HPLC spectrum of the chiral compound}

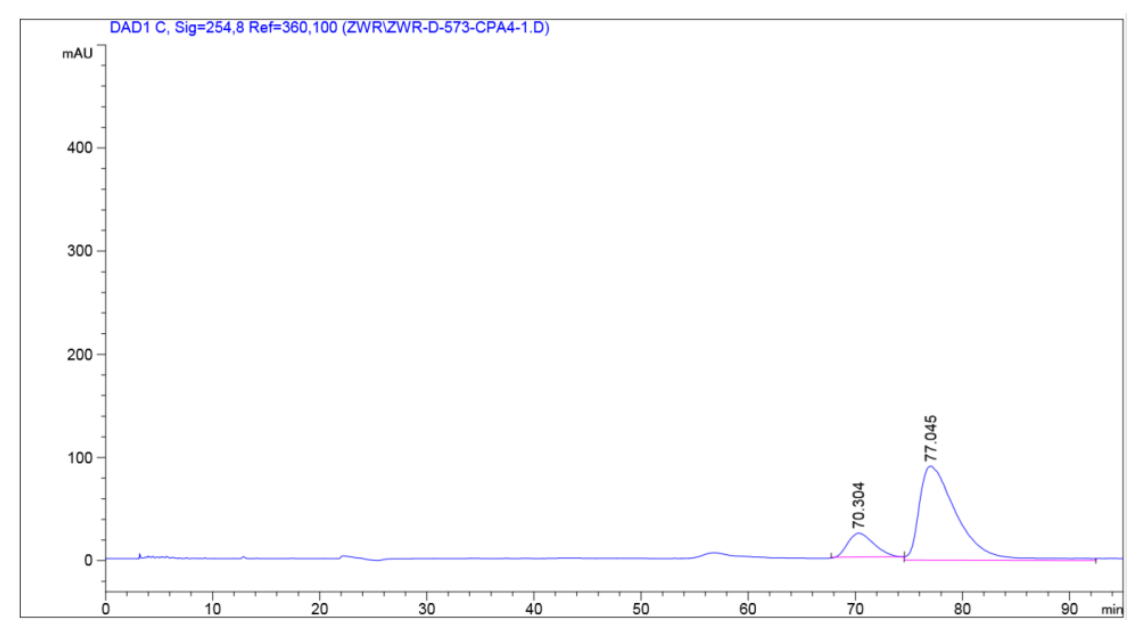

信号 1: DAD1 C, Sig=254, 8 Ref=360, 100

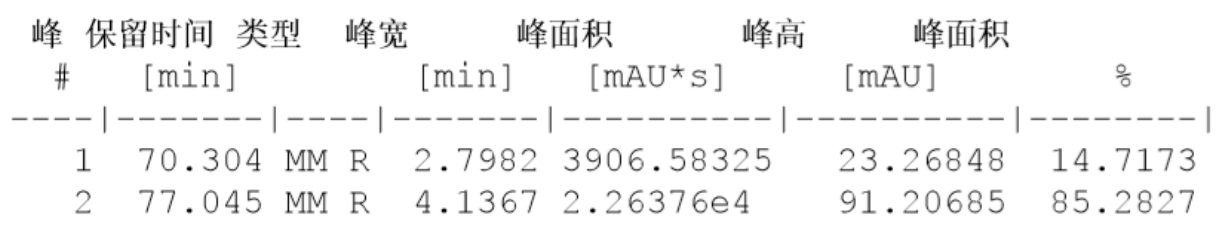




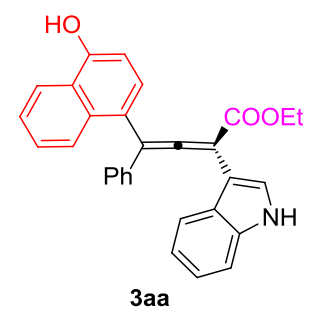

\section{HPLC spectrum of the racemate}

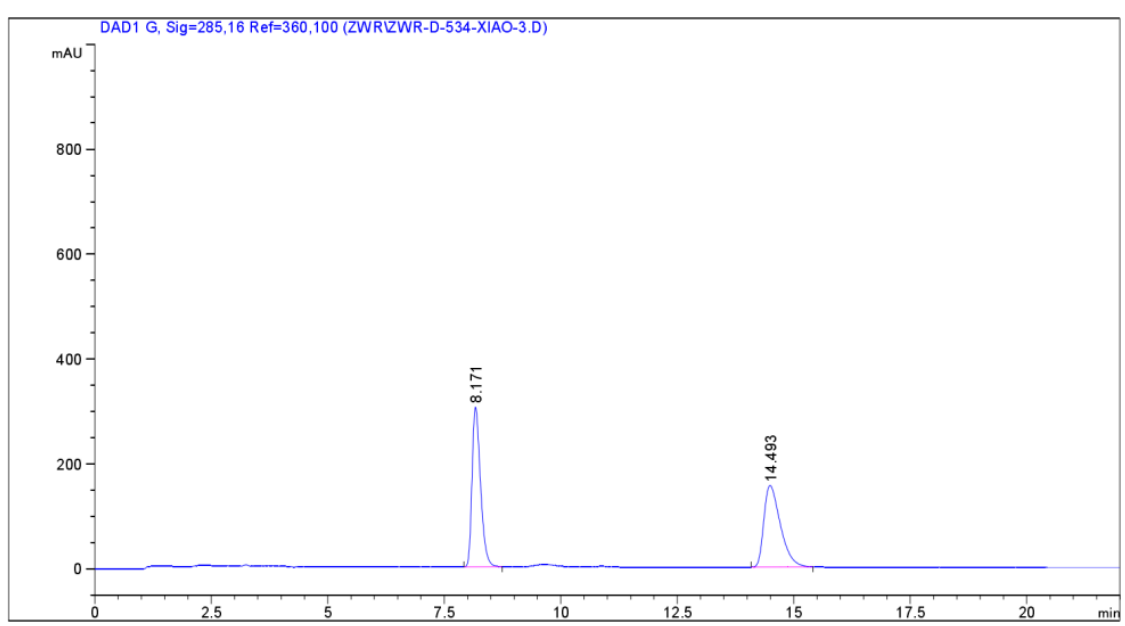

信号 1: DAD1 G, Sig=285,16 Ref=360,100

\begin{tabular}{|c|c|c|c|c|c|c|}
\hline 峰 & 保留时间 类型 & 峰宽 & 面积 & 峰高 & \multicolumn{2}{|l|}{ 峰面积 } \\
\hline \# & [min $]$ & [min $]$ & {$\left[\mathrm{mAU}{ }^{*} \mathrm{~s}\right]$} & & [mAU ] & $\%$ \\
\hline & & & & & & - \\
\hline 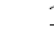 & $171 \mathrm{BH}$ & 0.1932 & 3845.168 & & 304.67520 & 50.0725 \\
\hline 2 & $14.493 \mathrm{BH}$ & 0.3775 & 3834.031 & & 155.57089 & 49.9275 \\
\hline
\end{tabular}

\section{HPLC spectrum of the chiral compound}

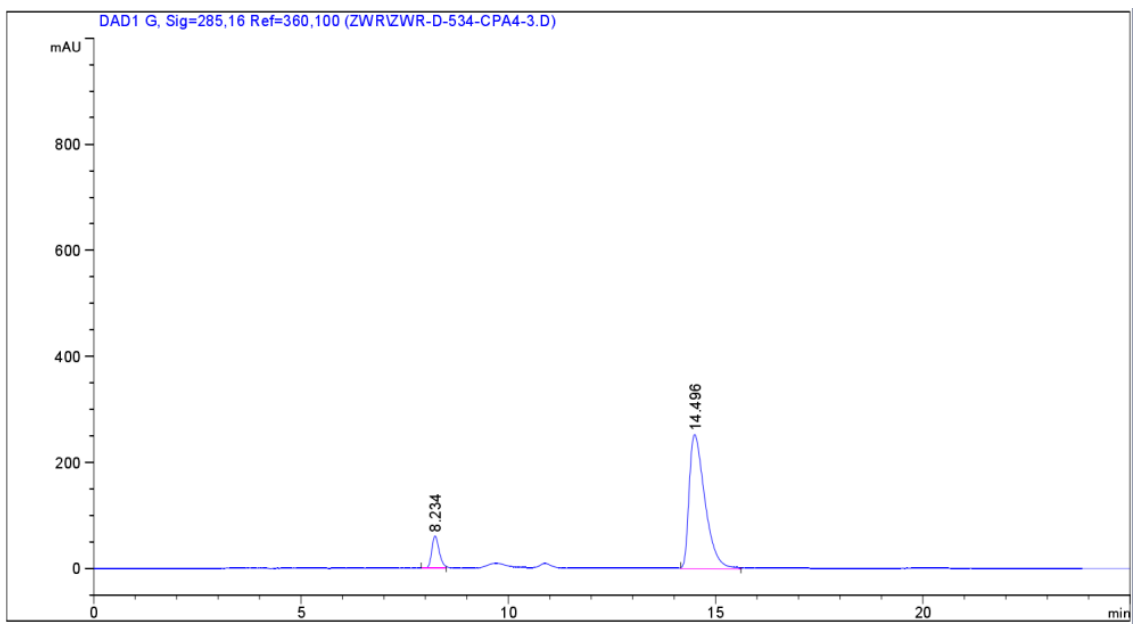

信号 1: DAD1 G， Sig=285,16 Ref=360,100

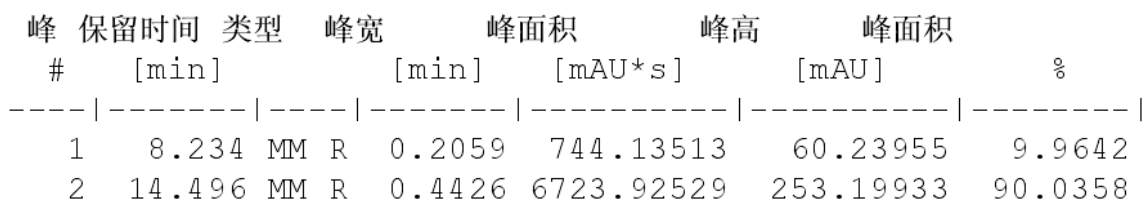




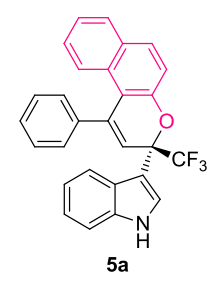

HPLC spectrum of the racemate

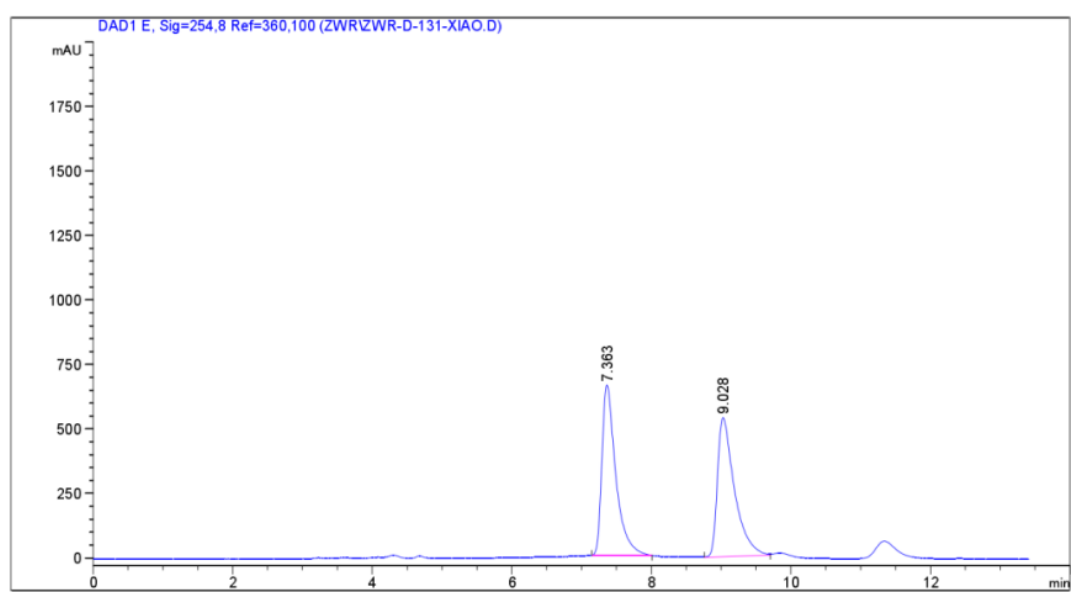

信号 1: DAD1 E， Sig=254,8 Ref=360,100

\begin{tabular}{|c|c|c|c|c|c|c|c|}
\hline 峰 & 保留时间 类型 & 峰宽 & & 备面积 & 峰高 & 峰面积 & \\
\hline \# & {$[\mathrm{min}]$} & & {$[\mathrm{min}]$} & {$[\mathrm{mAU} * \mathrm{~s}]$} & & {$[\mathrm{mAU}]$} & $\%$ \\
\hline & & & & & & & \\
\hline & & $\mathrm{R}$ & 0.2829 & 9153.225 & & 539.31946 & 50.080 \\
\hline
\end{tabular}

\section{HPLC spectrum of the chiral compound}

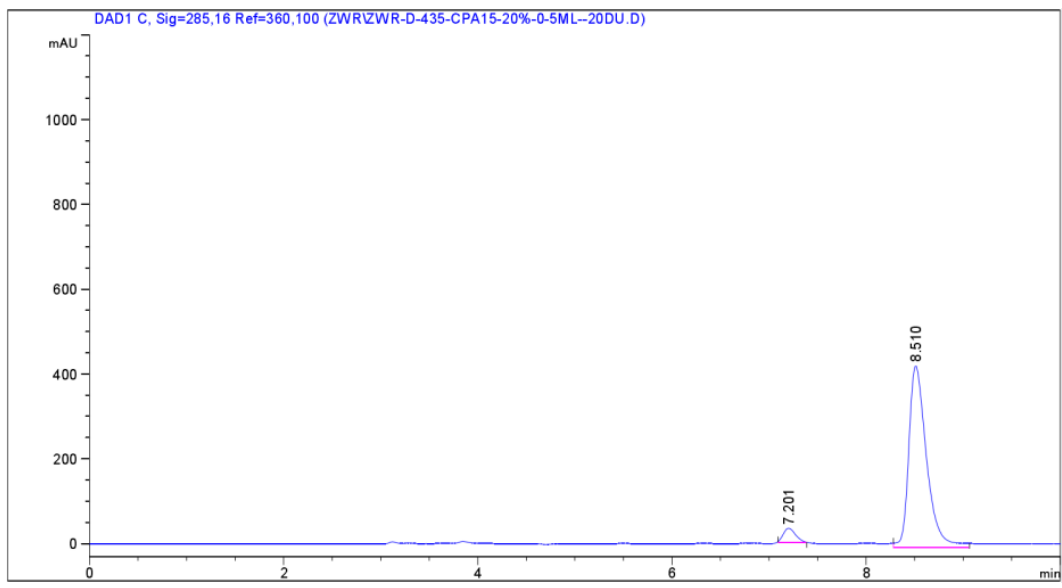

信号 1: DAD1 C, Sig=285,16 Ref=360,100

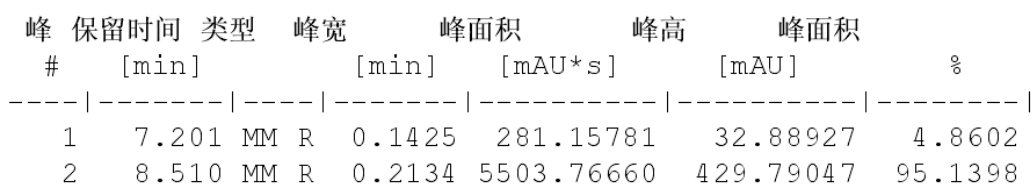




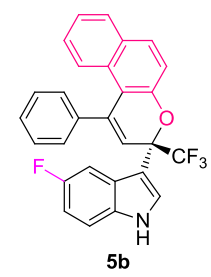

HPLC spectrum of the racemate

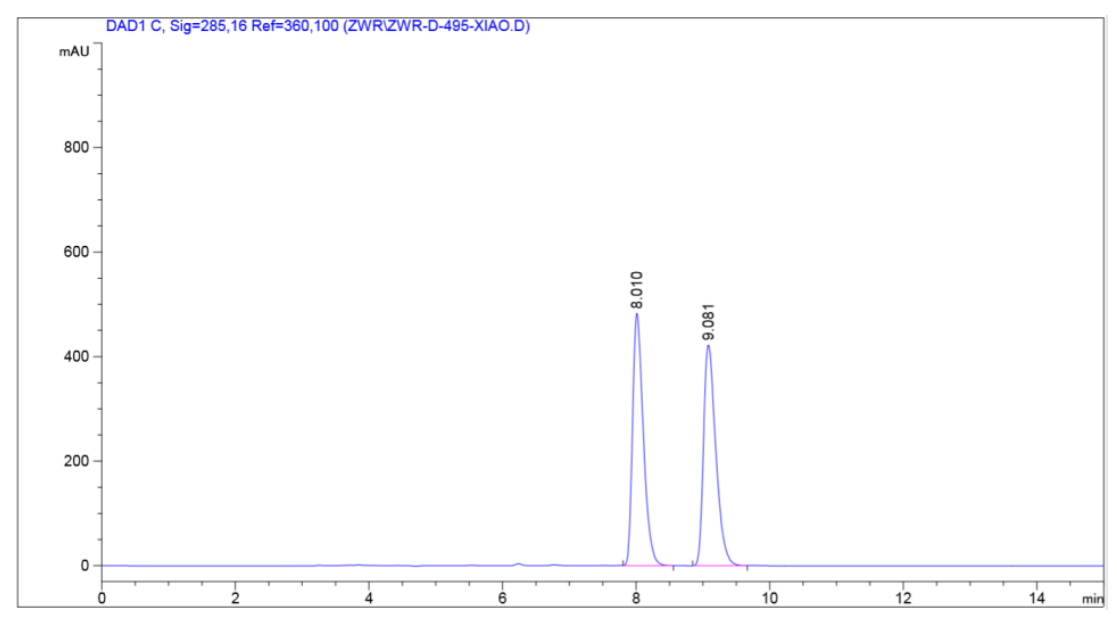

信号 1: DAD1 C, Sig=285,16 Ref=360,100

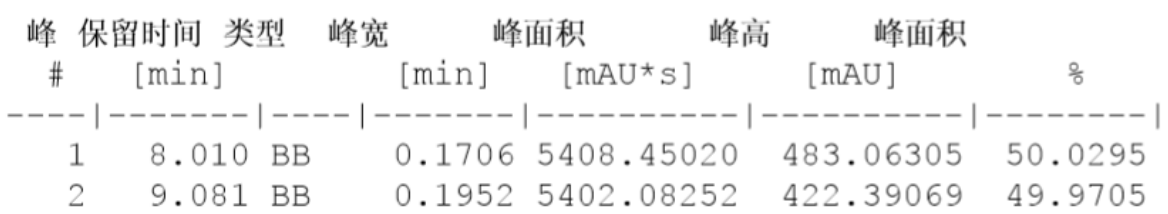

\section{HPLC spectrum of the chiral compound}

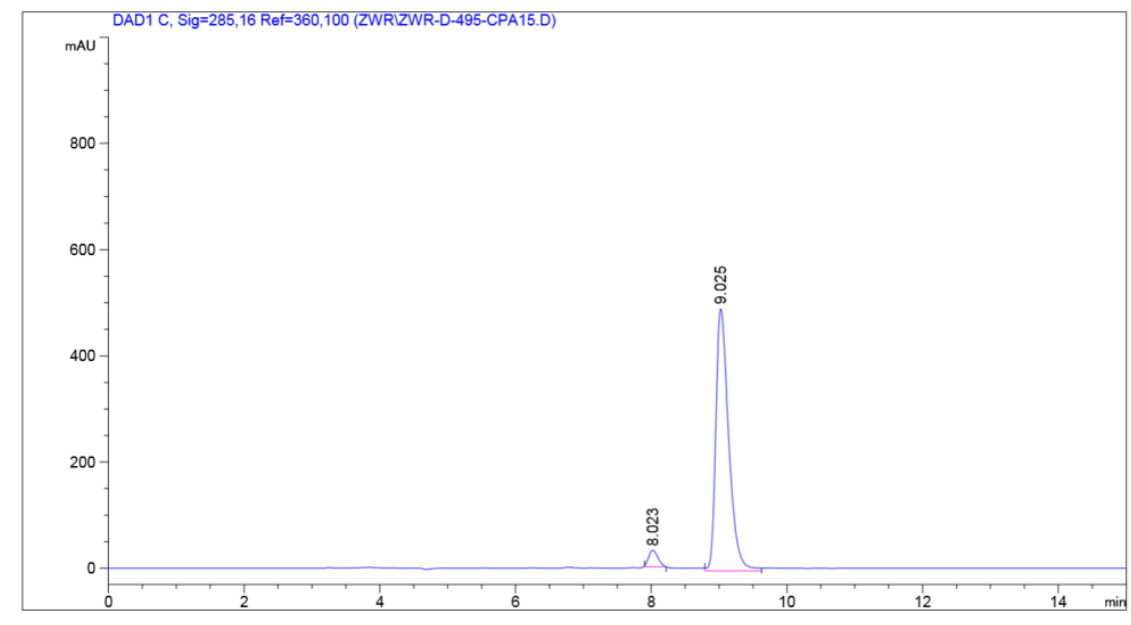

信号 1: DAD1 C, Sig=285,16 Ref=360,100

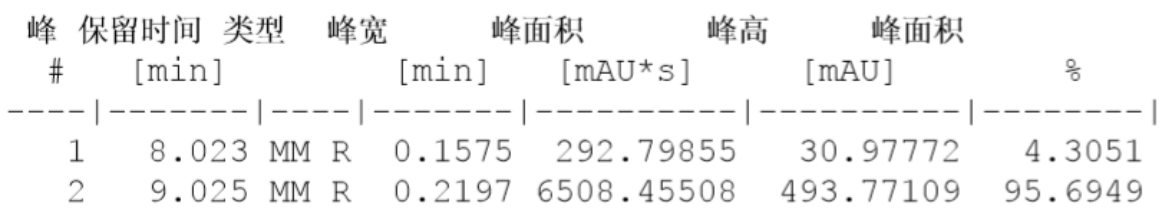




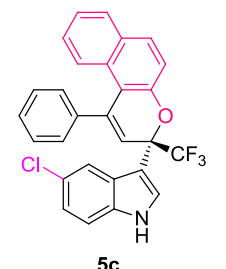

\section{HPLC spectrum of the racemate}

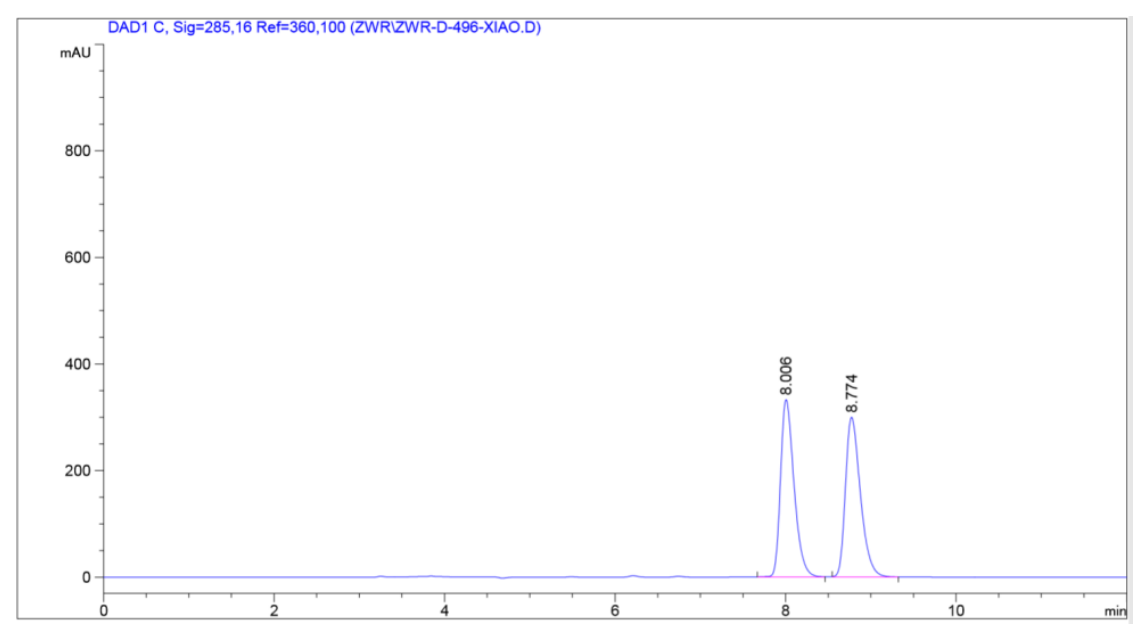

信号 1: DAD1 C, Sig=285, 16 Ref=360, 100

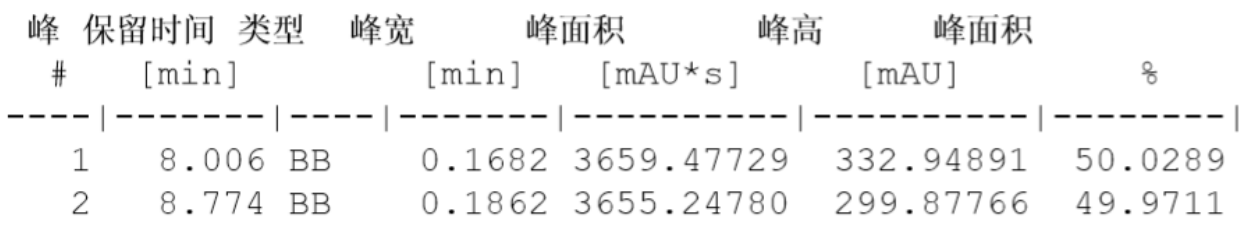

\section{HPLC spectrum of the chiral compound}

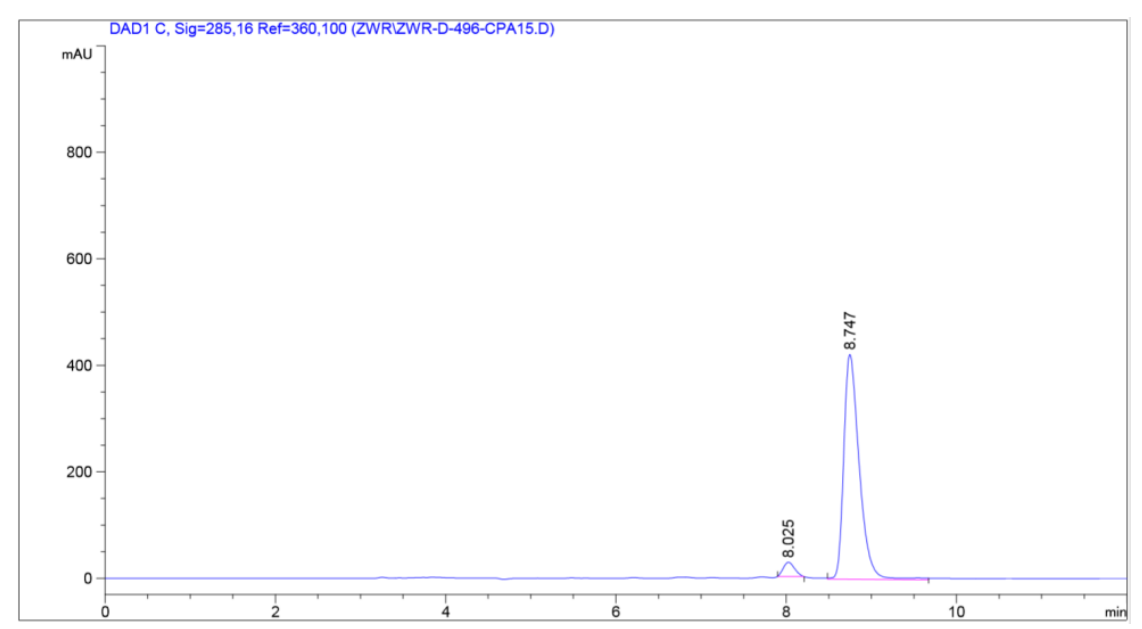

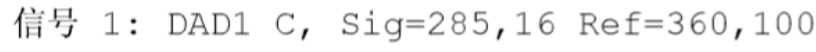

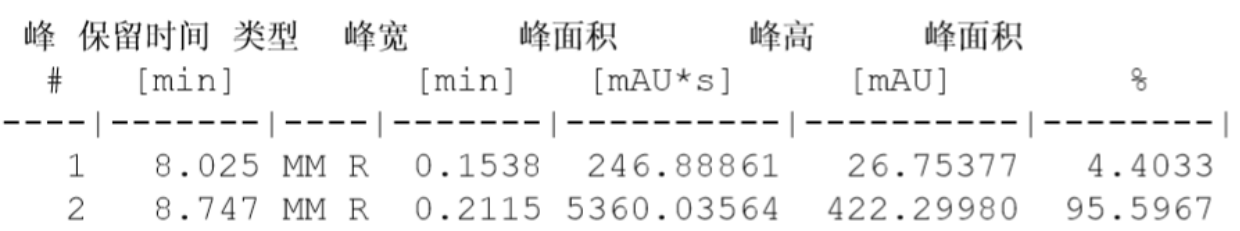




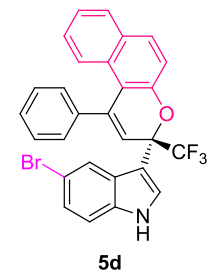

\section{HPLC spectrum of the racemate}

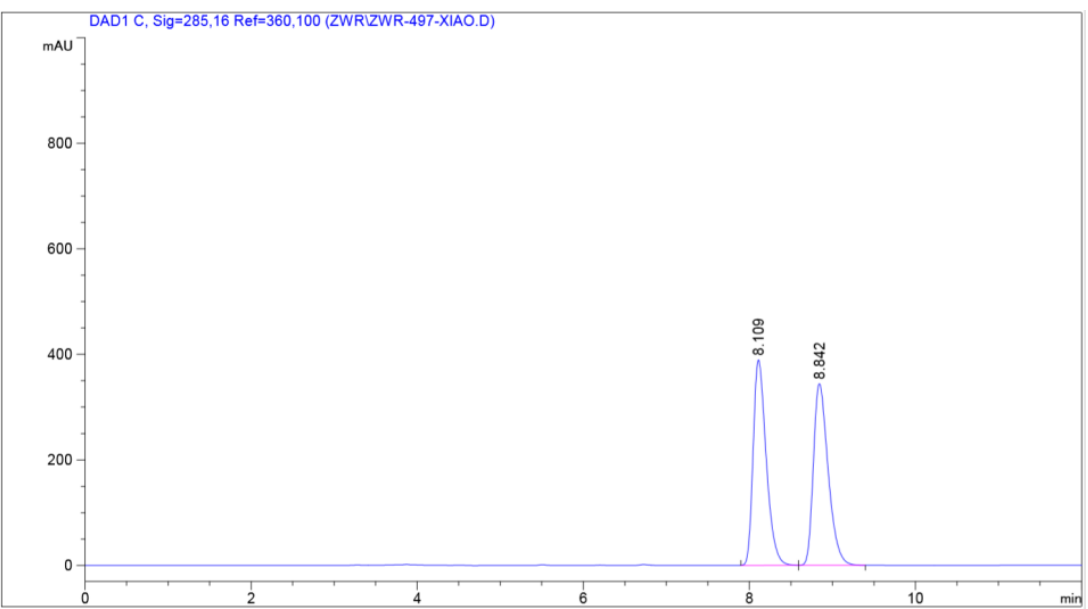

信号 1: DAD1 C, Sig=285,16 Ref=360,100

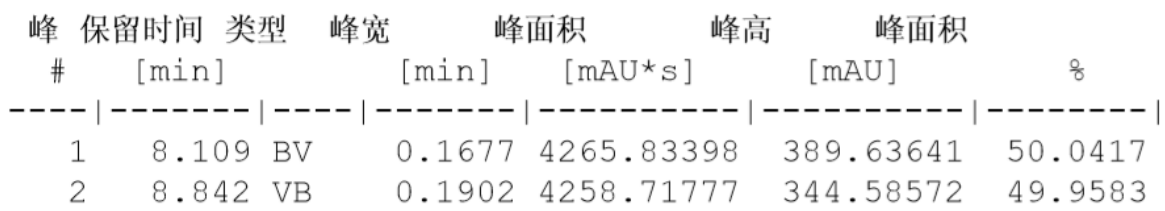

\section{HPLC spectrum of the chiral compound}

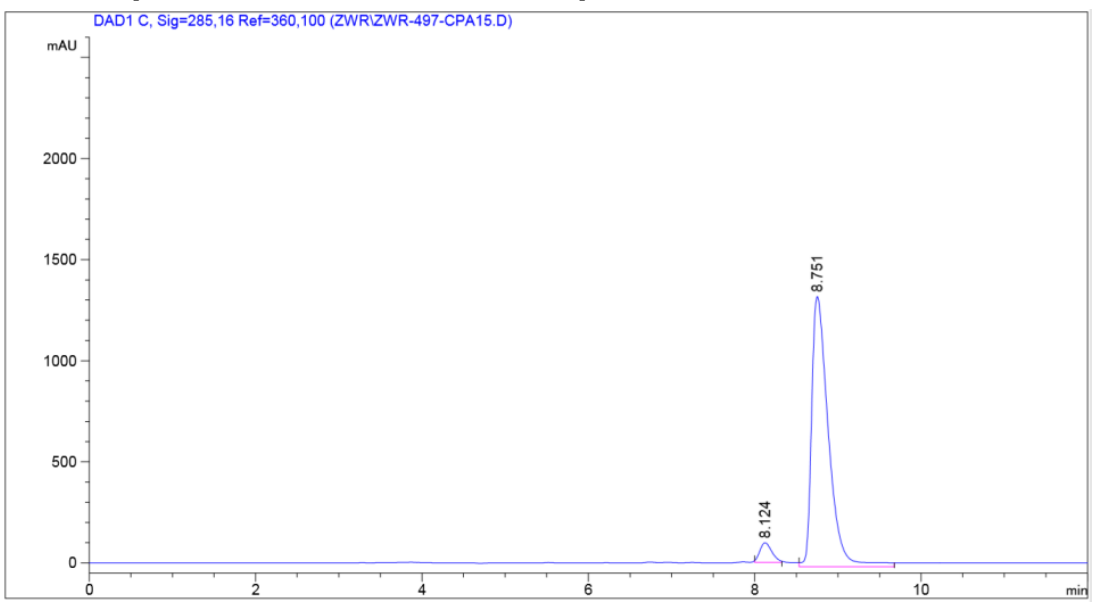

信号 1: DAD1 C, Sig=285,16 Ref=360,100

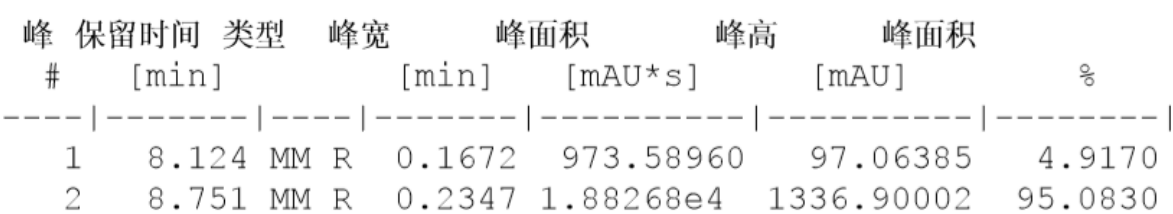




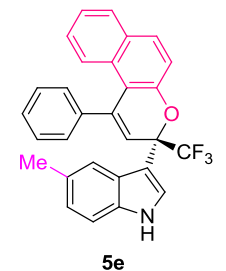

\section{HPLC spectrum of the racemate}

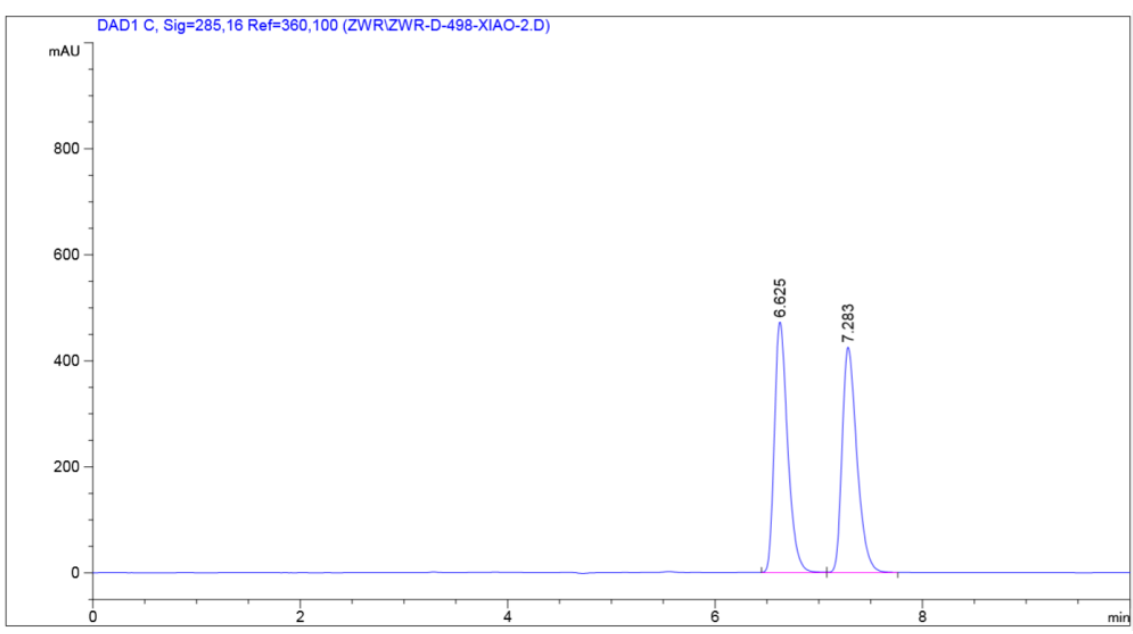

信号 1: DAD1 C, Sig=285,16 Ref=360,100

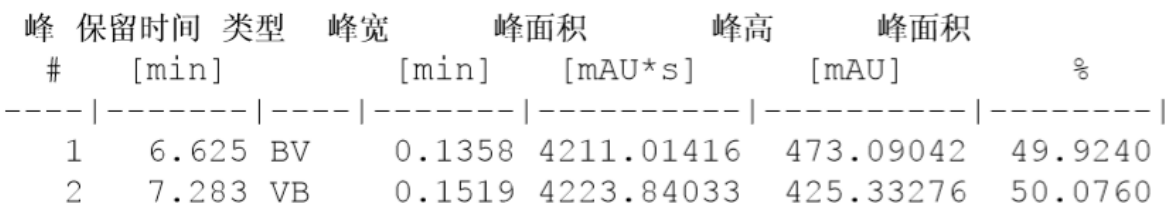

\section{HPLC spectrum of the chiral compound}

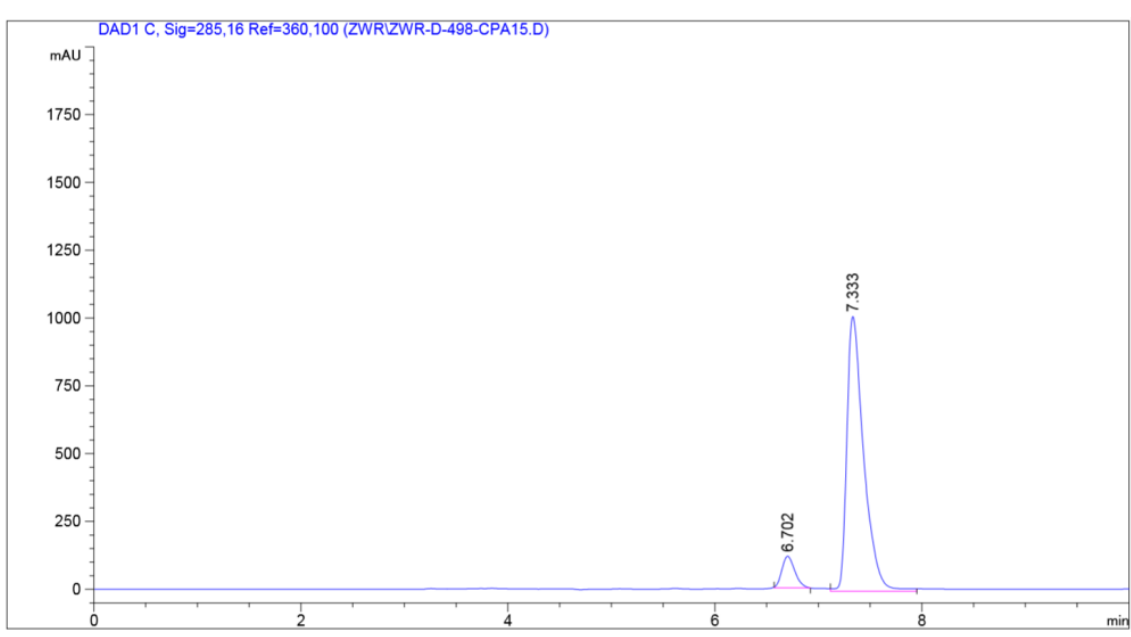

信号 1: DAD1 C, Sig=285,16 Ref=360,100

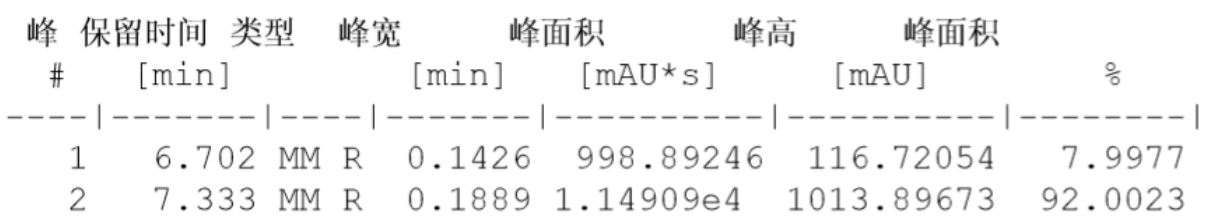




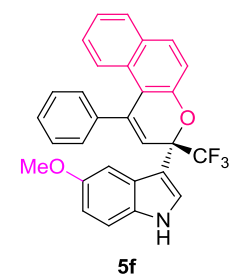

\section{HPLC spectrum of the racemate}

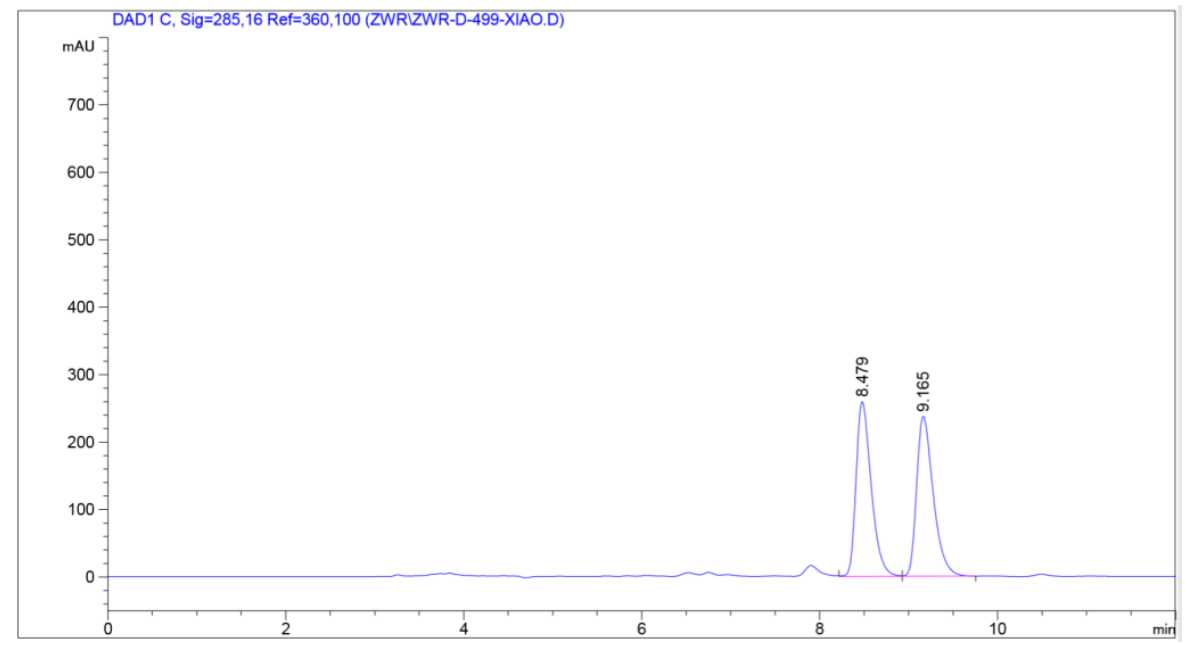

信号 1: DAD1 C, Sig=285,16 Ref=360, 100

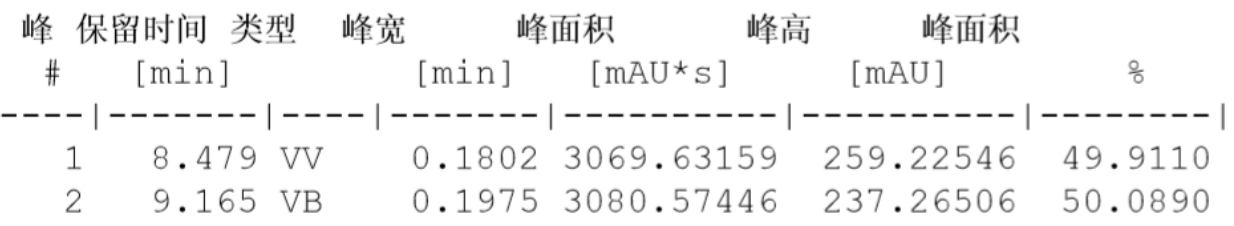

\section{HPLC spectrum of the chiral compound}

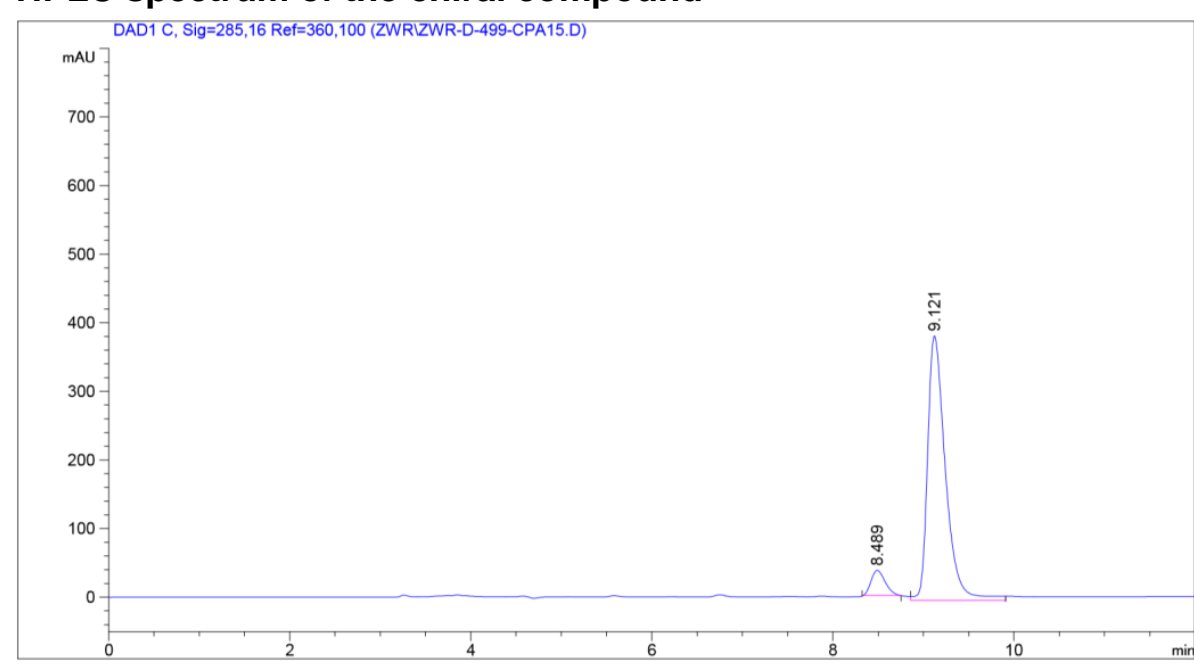

信号 1: DAD1 C, Sig=285, 16 Ref=360,100

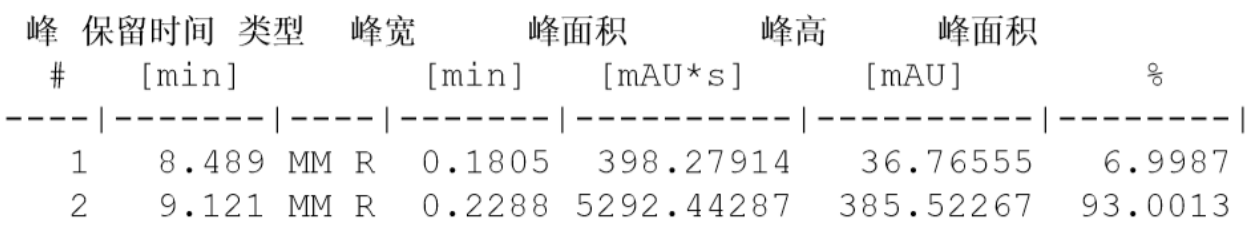




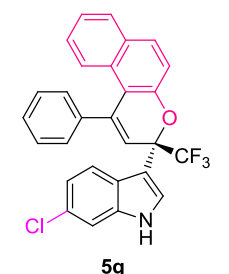

HPLC spectrum of the racemate

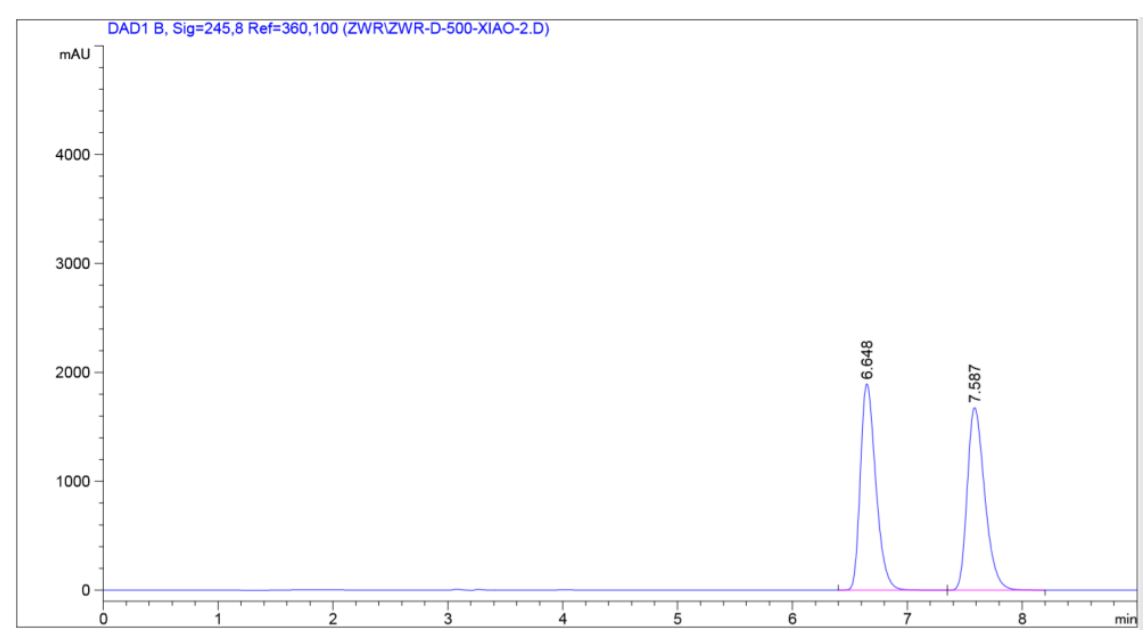

信号 1: DAD1 B, Sig=245, 8 Ref $=360,100$

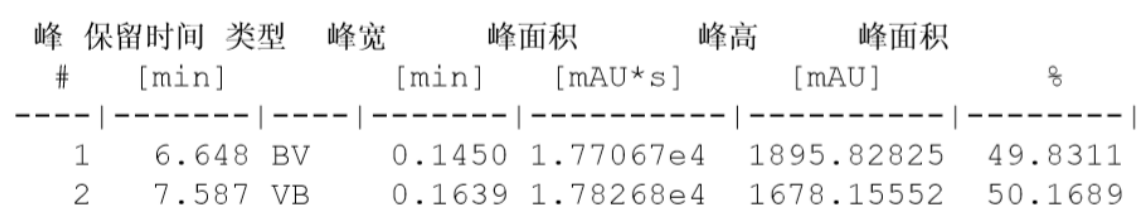

\section{HPLC spectrum of the chiral compound}

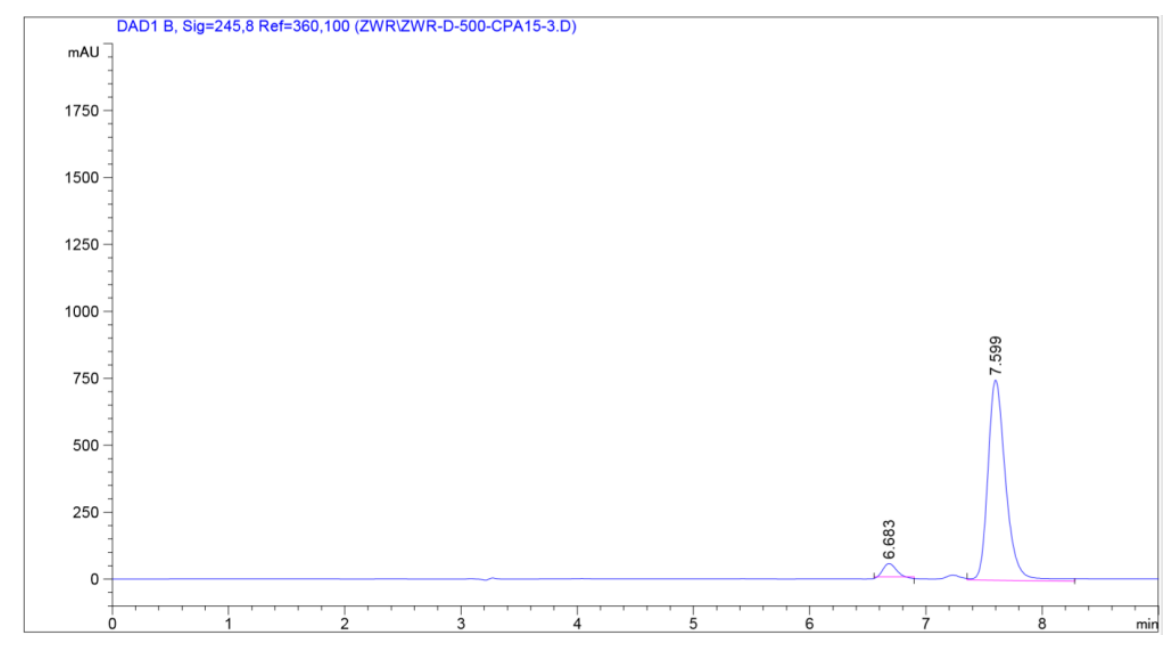

信号 1: DAD1 B, Sig=245, 8 Ref $=360,100$

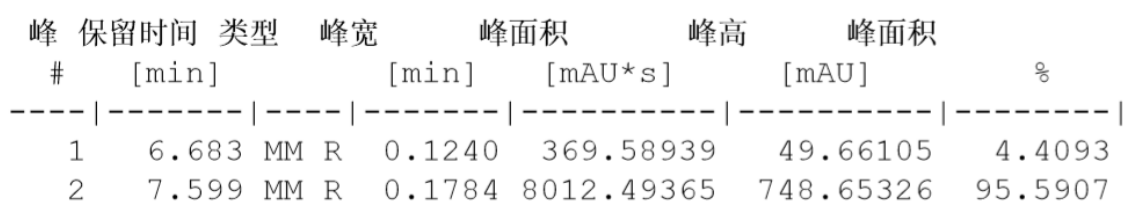




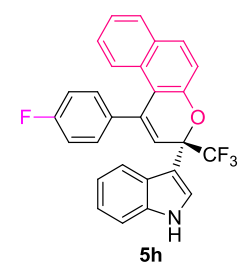

HPLC spectrum of the racemate

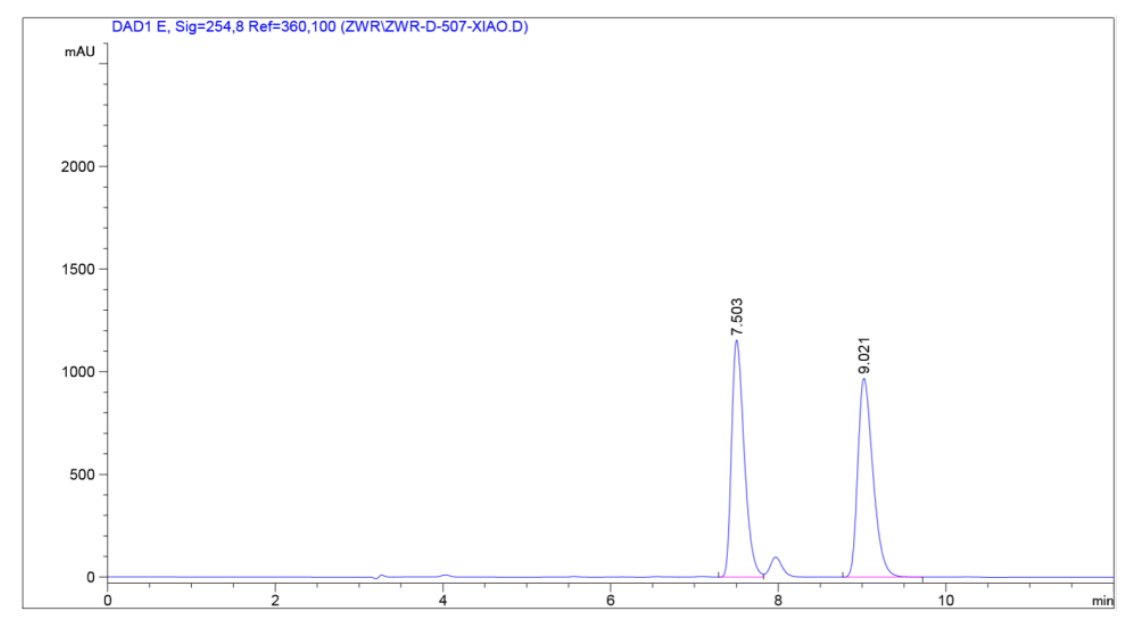

信号 1: DAD1 E， Sig=254,8 Ref=360,100

$\begin{array}{cccccc}\text { 峰 保留时间 类型 } & \text { 峰宽 } \\ \# & {[\mathrm{~min}]}\end{array}$

\section{HPLC spectrum of the chiral compound}

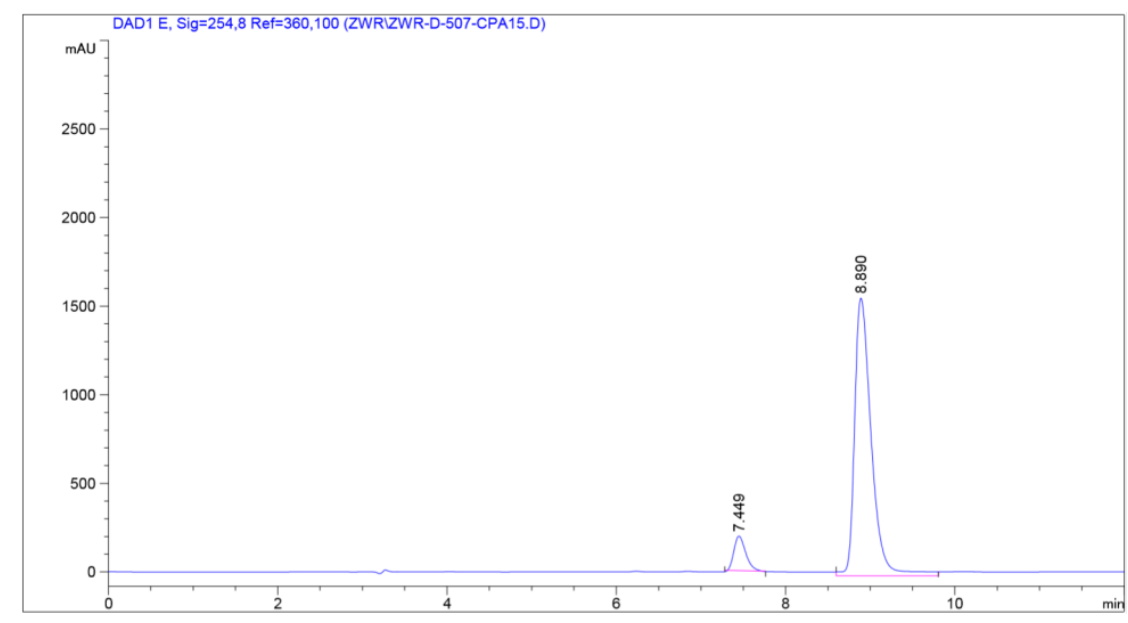

信号 1: DAD1 E, Sig=254,8 $\operatorname{Ref}=360,100$

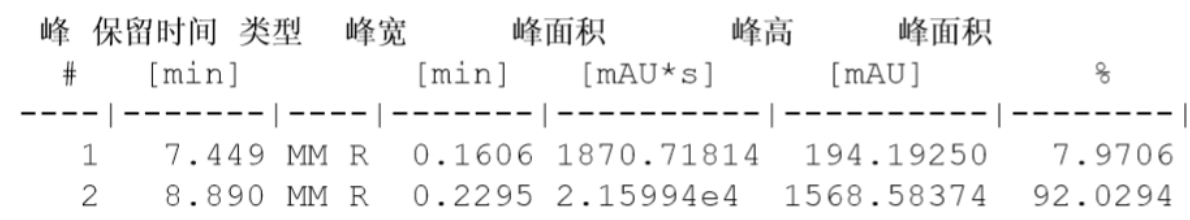




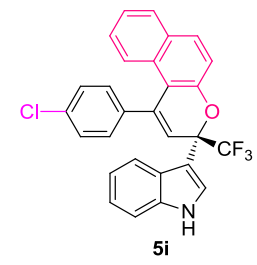

HPLC spectrum of the racemate

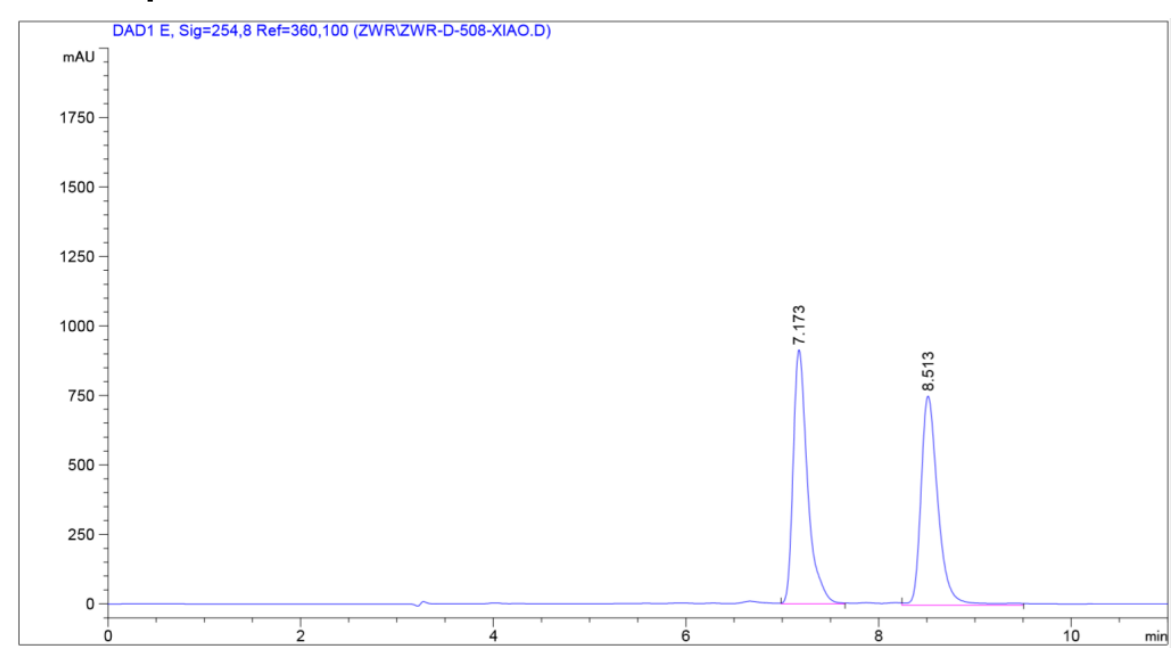

信号 1: DAD1 E， Sig=254,8 Ref=360,100

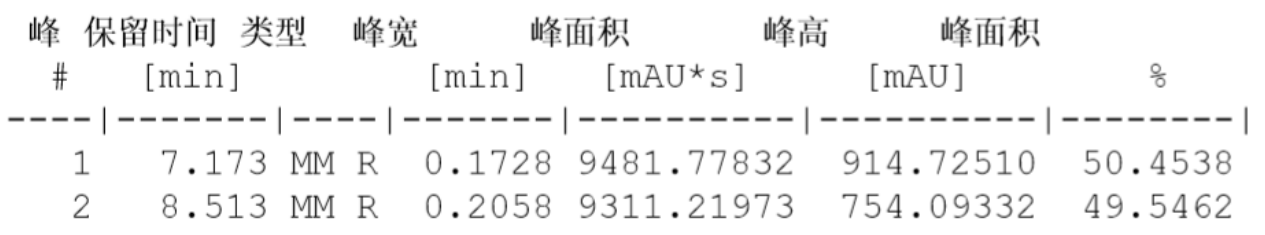

\section{HPLC spectrum of the chiral compound}

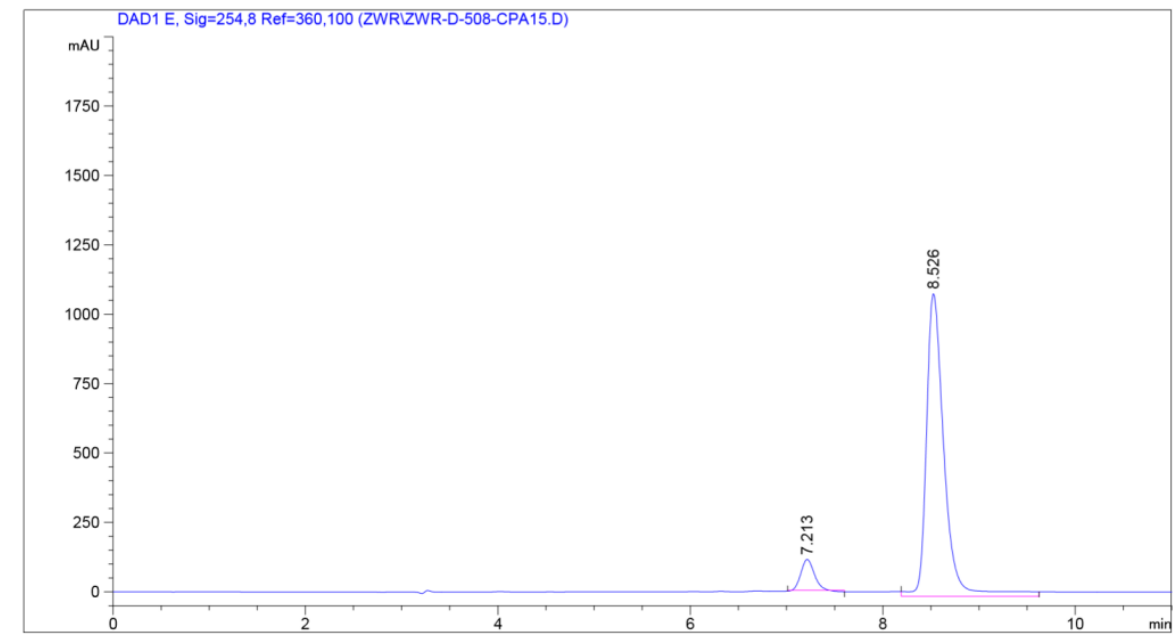

信号 1: DAD1 E， Sig=254,8 Ref=360,100

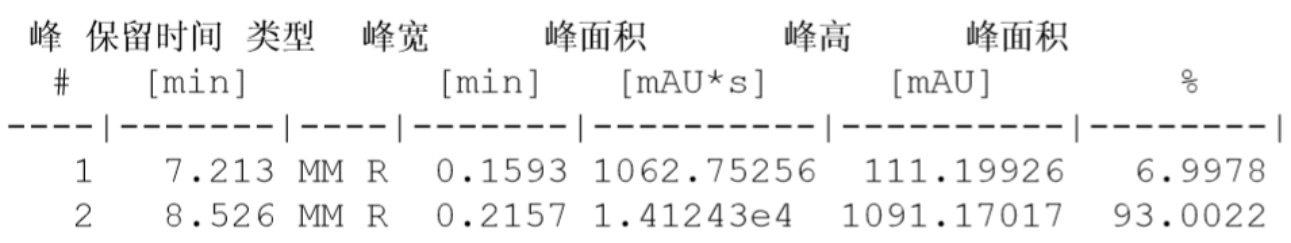




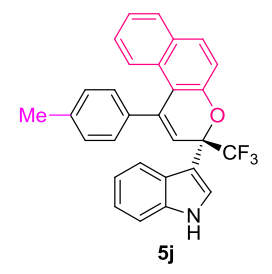

HPLC spectrum of the racemate

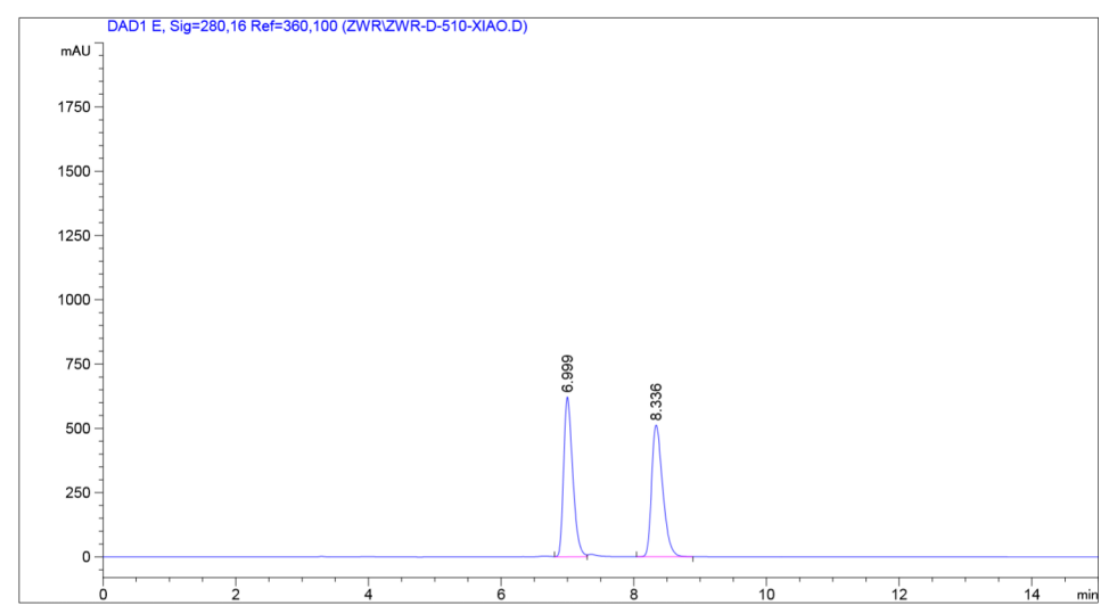

信号 1: DAD1 E， Sig=280,16 $\operatorname{Ref}=360,100$

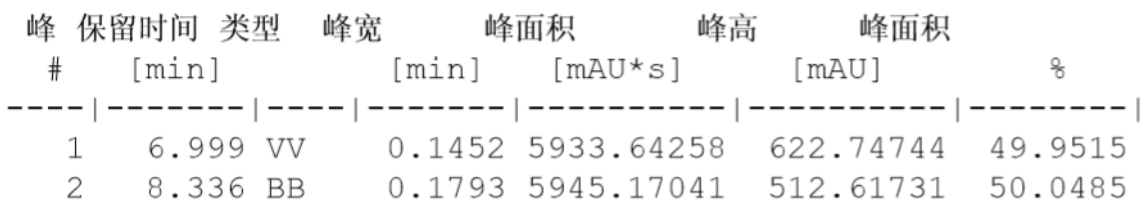

\section{HPLC spectrum of the chiral compound}

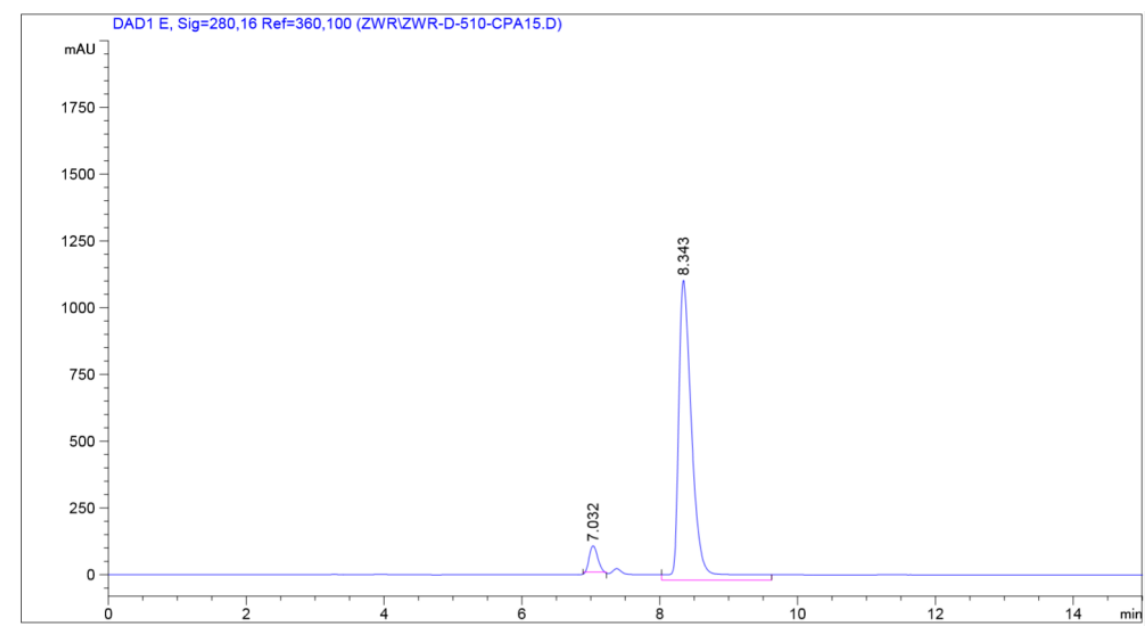

信号 1: DAD1 E, Sig=280,16 Ref=360,100

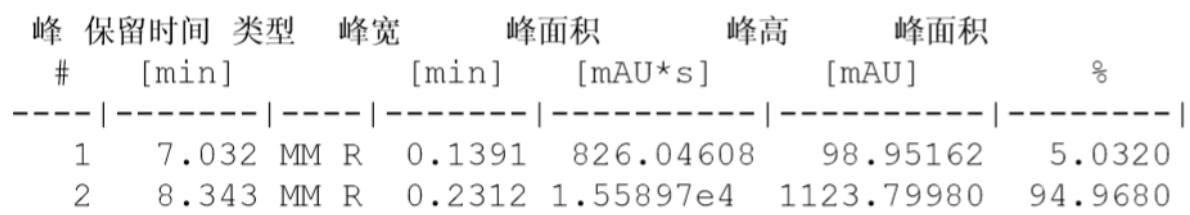




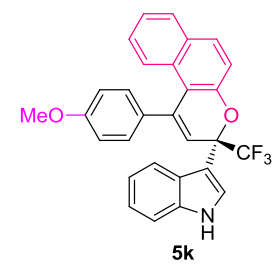

HPLC spectrum of the racemate

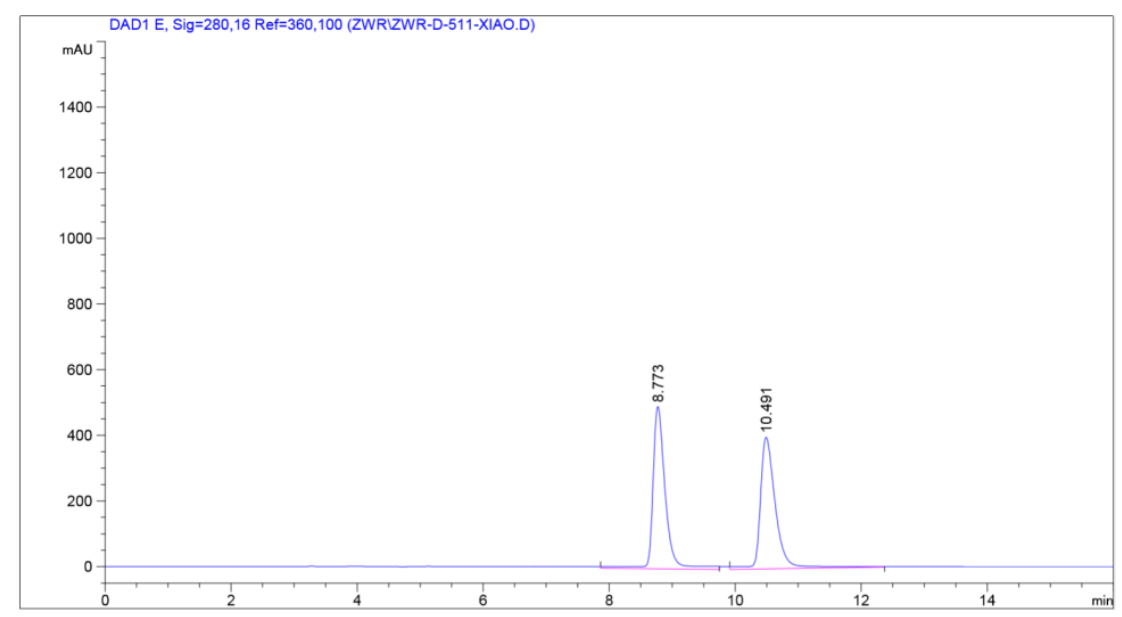

信号 1: DAD1 E, Sig=280,16 Ref=360,100

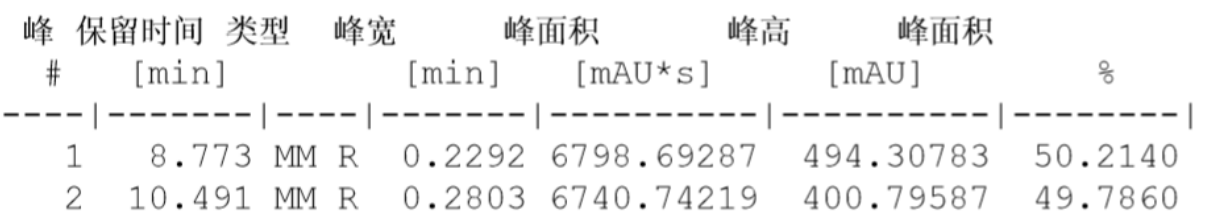

\section{HPLC spectrum of the chiral compound}

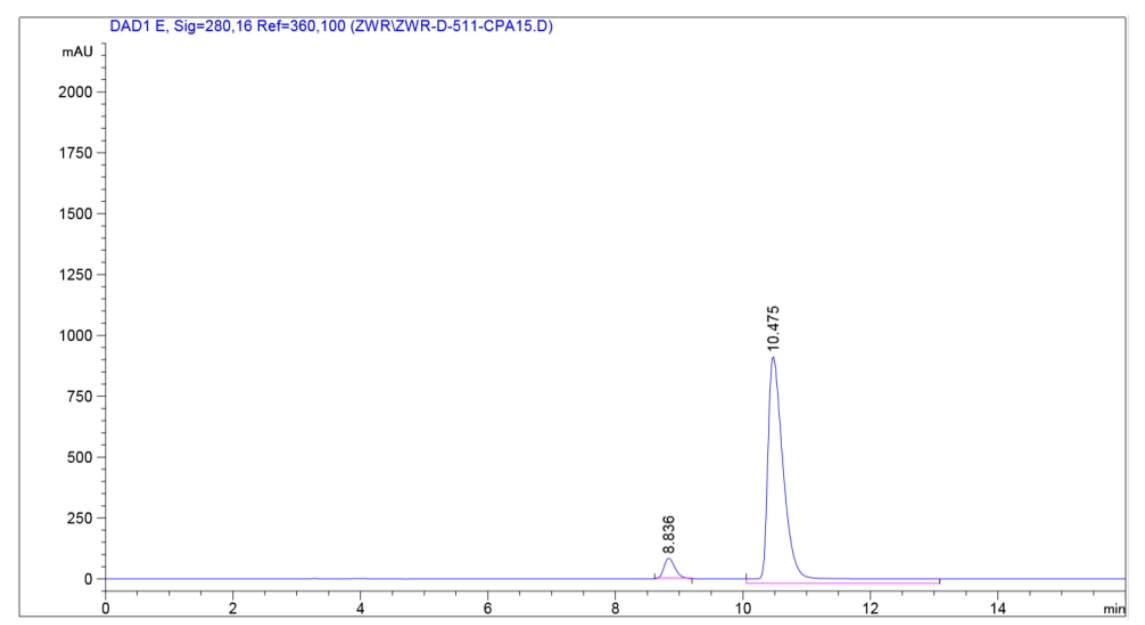

信号 1: DAD1 E, $\operatorname{Sig}=280,16$ Ref=360,100

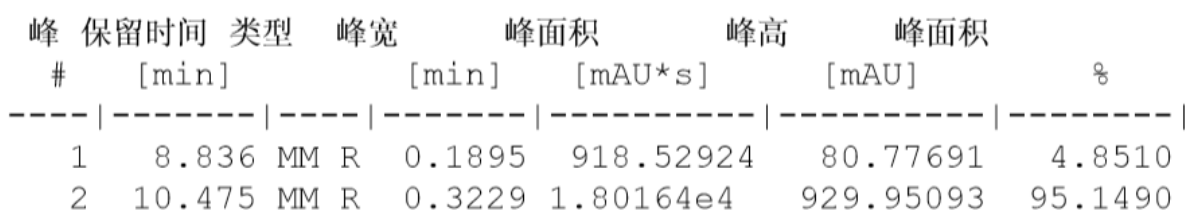




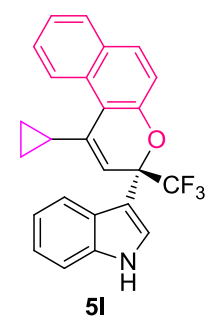

HPLC spectrum of the racemate

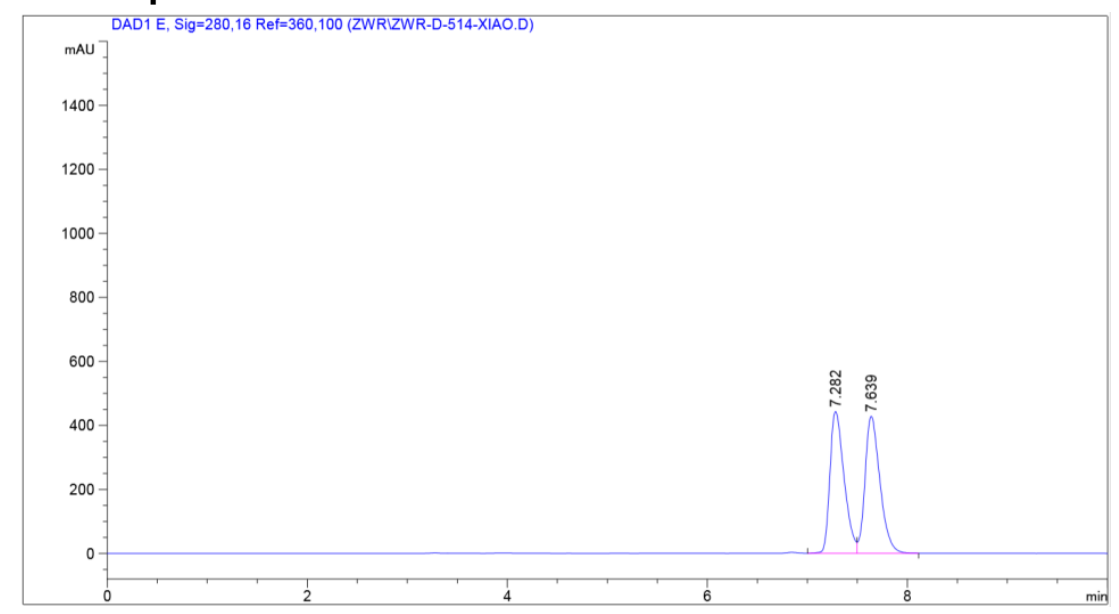

信号 1: DAD1 E， Sig=280,16 Ref=360,100

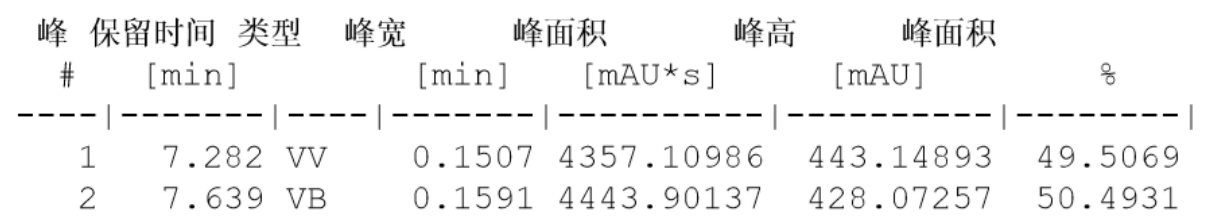

\section{HPLC spectrum of the chiral compound}

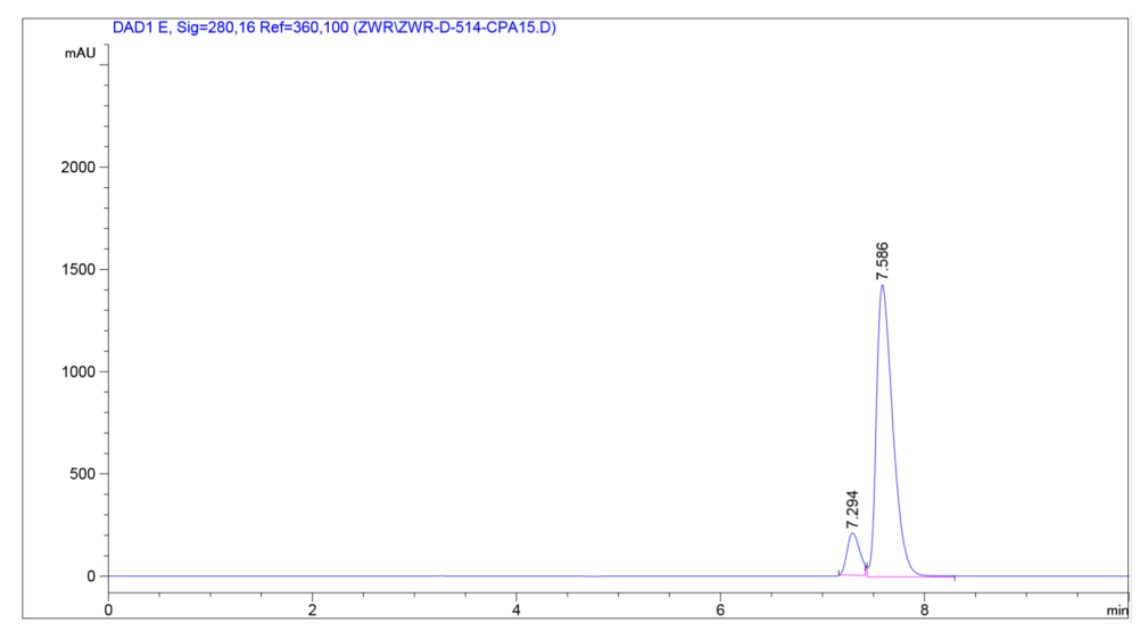

信号 1: DAD1 E, Sig=280,16 Ref=360,100

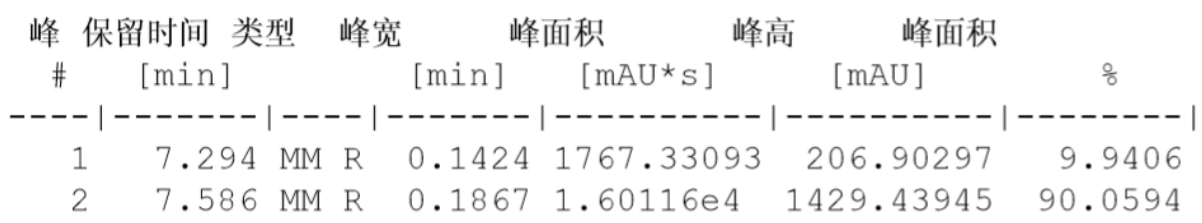




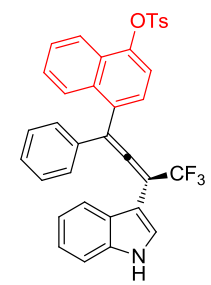

13

\section{HPLC spectrum of the racemate}

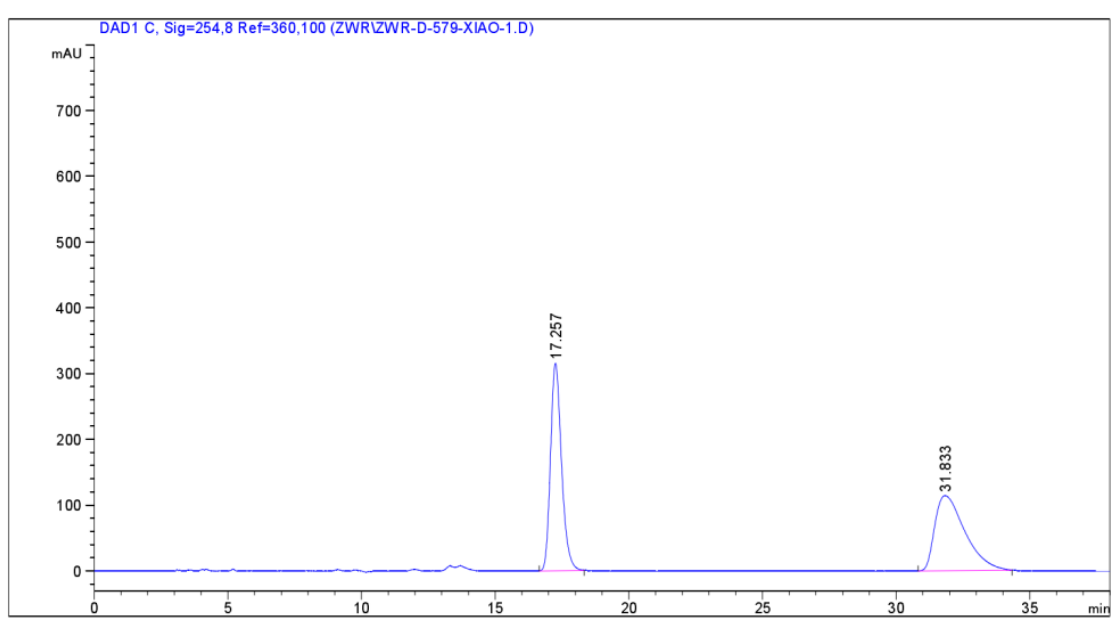

信号 1: DAD1 C, Sig=254,8 Ref $=360,100$

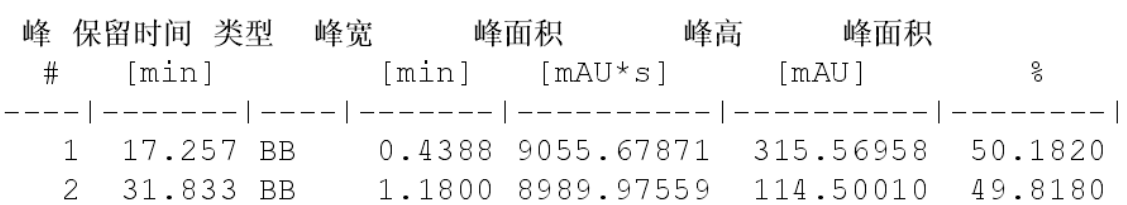

\section{HPLC spectrum of the chiral compound}

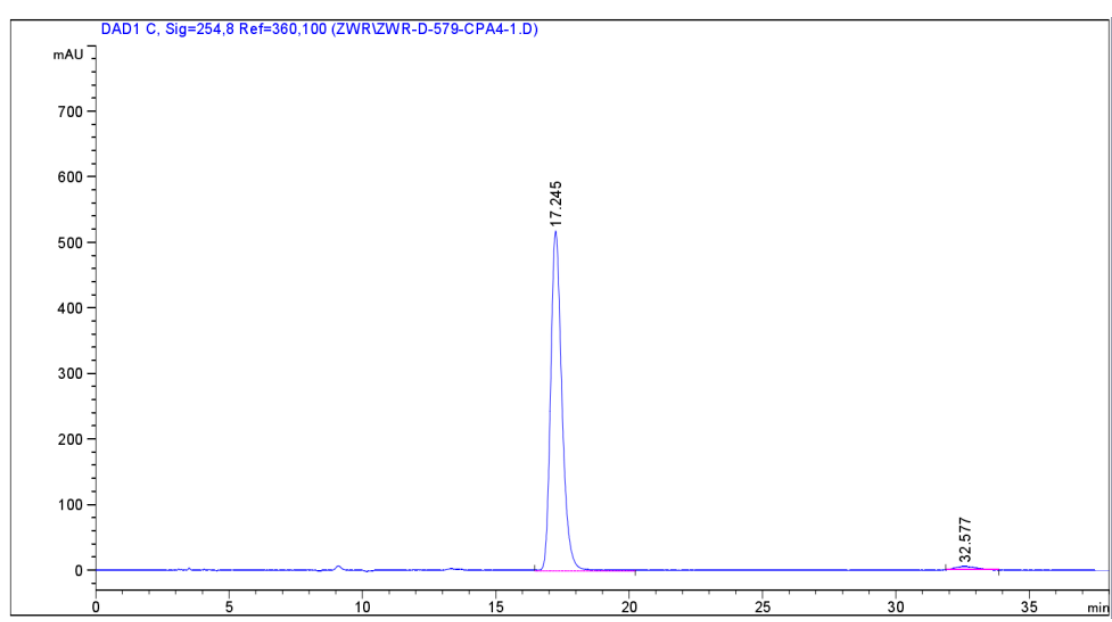

信号 1: DAD1 C, Sig=254,8 Ref=360,100

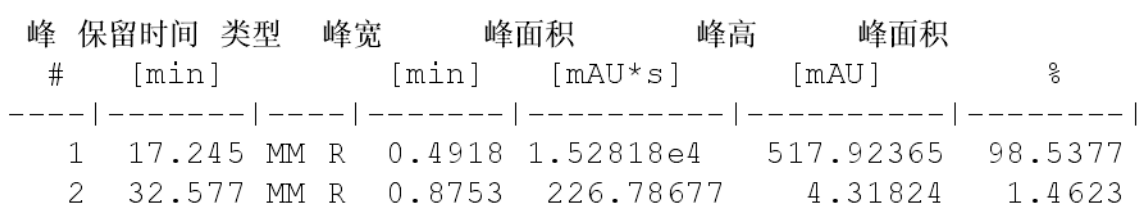




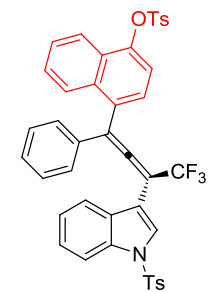

14

\section{HPLC spectrum of the racemate}

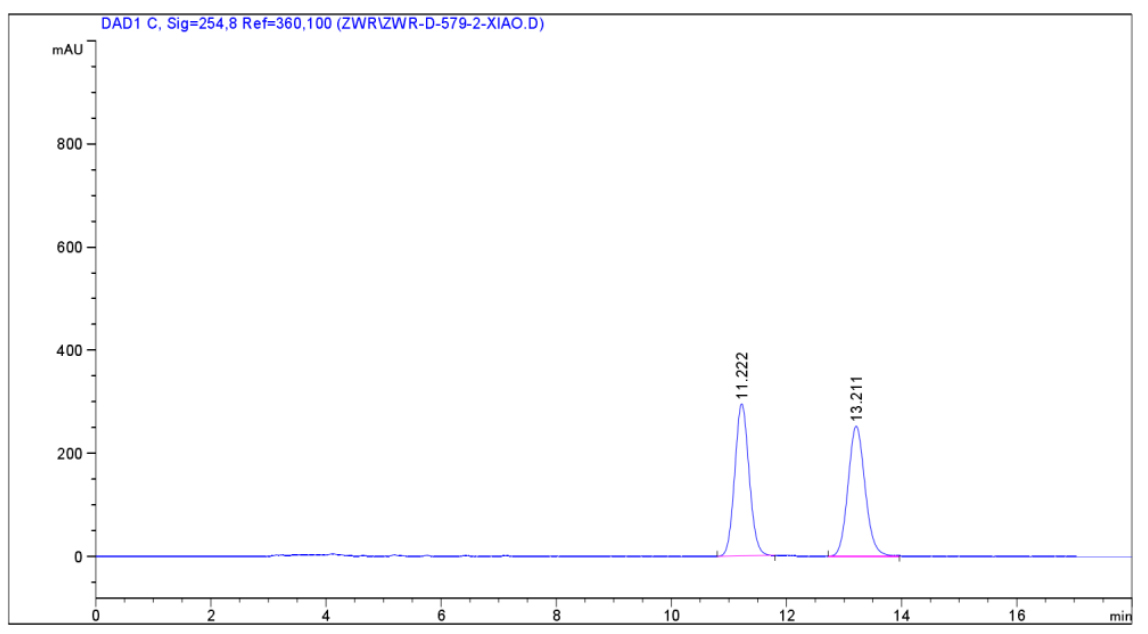

信号 1: DAD1 C, Sig=254,8 Ref=360,100

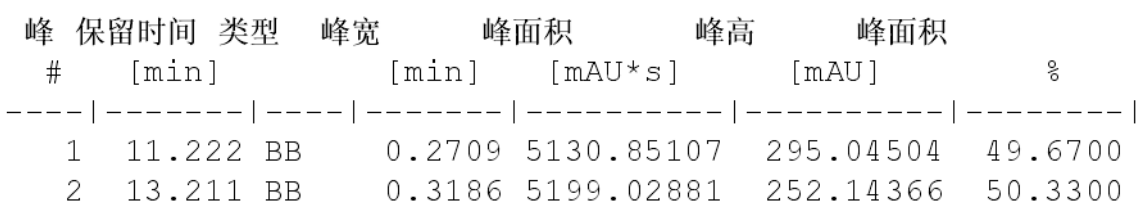

\section{HPLC spectrum of the chiral compound}

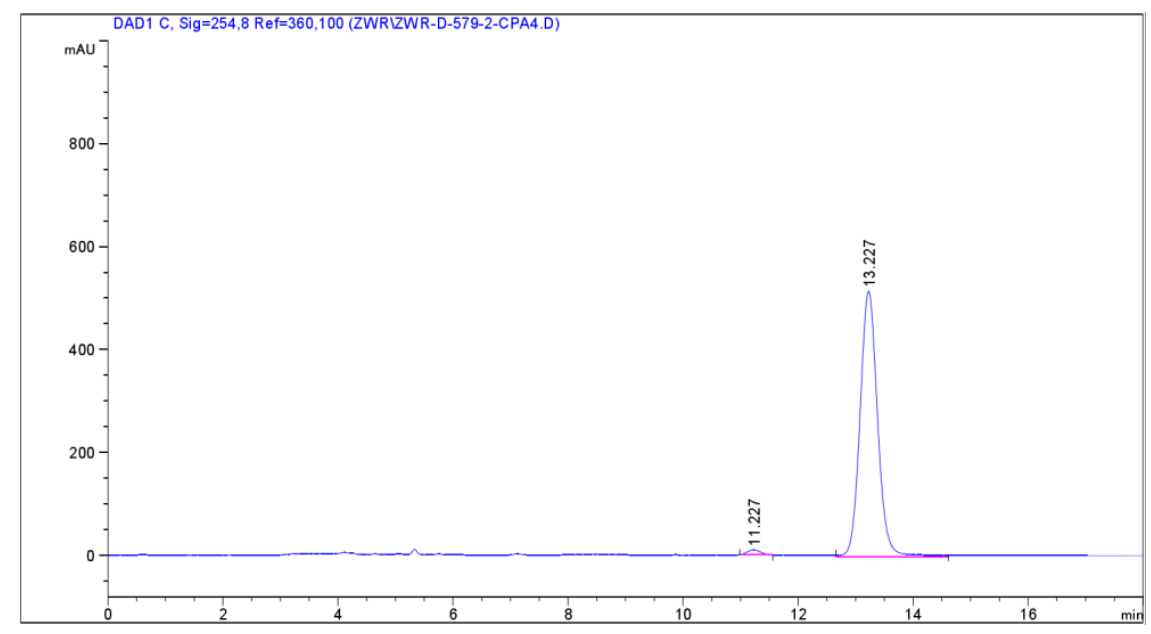

信号 1: DAD1 C, Sig=254,8 Ref=360,100

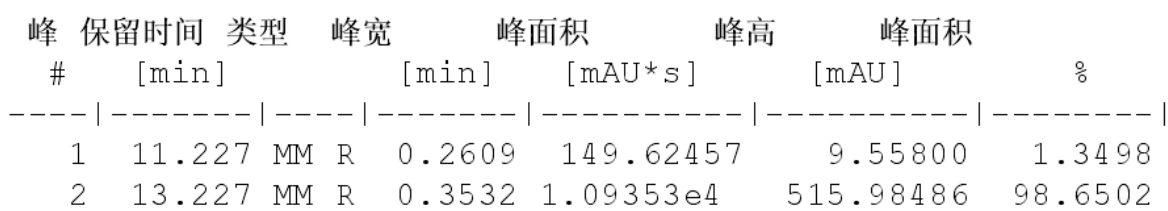




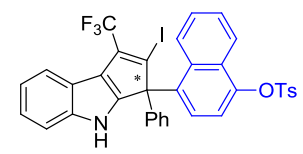

15

HPLC spectrum of the racemate

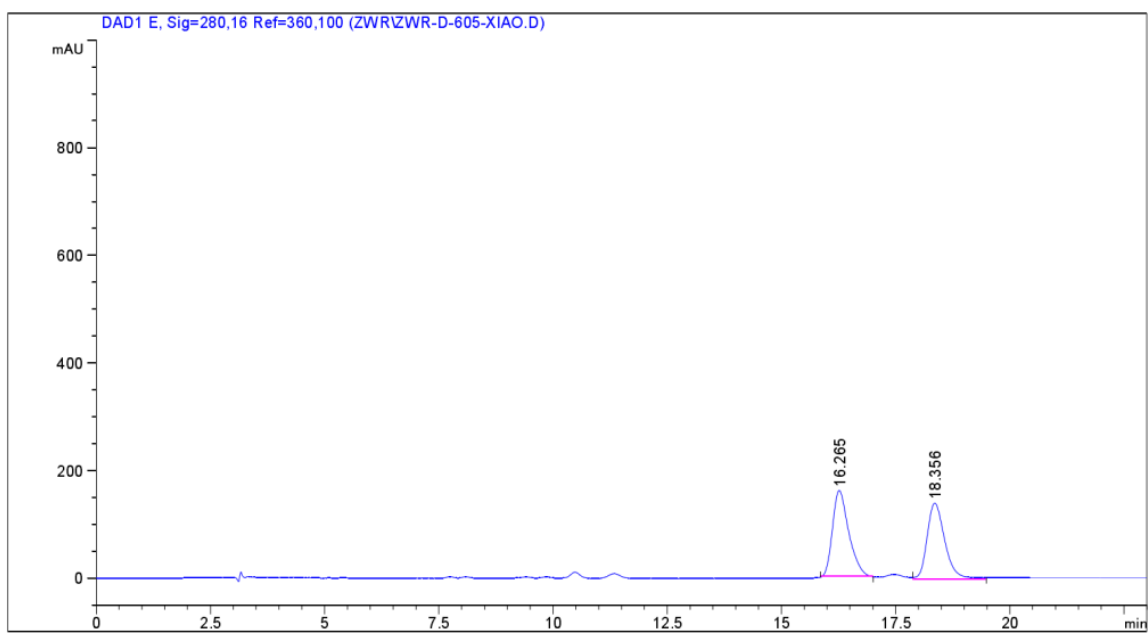

信号 1: DAD1 E, Sig=280,16 Ref=360,100

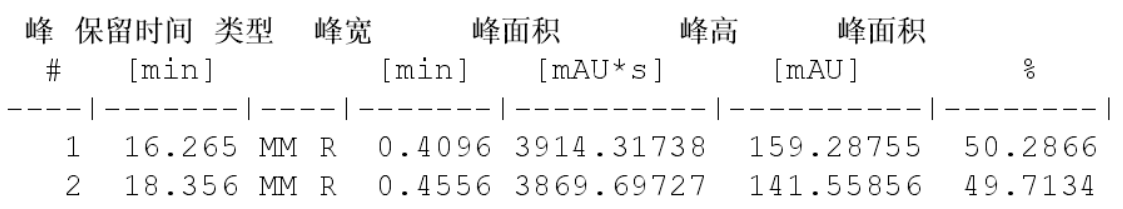

\section{HPLC spectrum of the chiral compound}

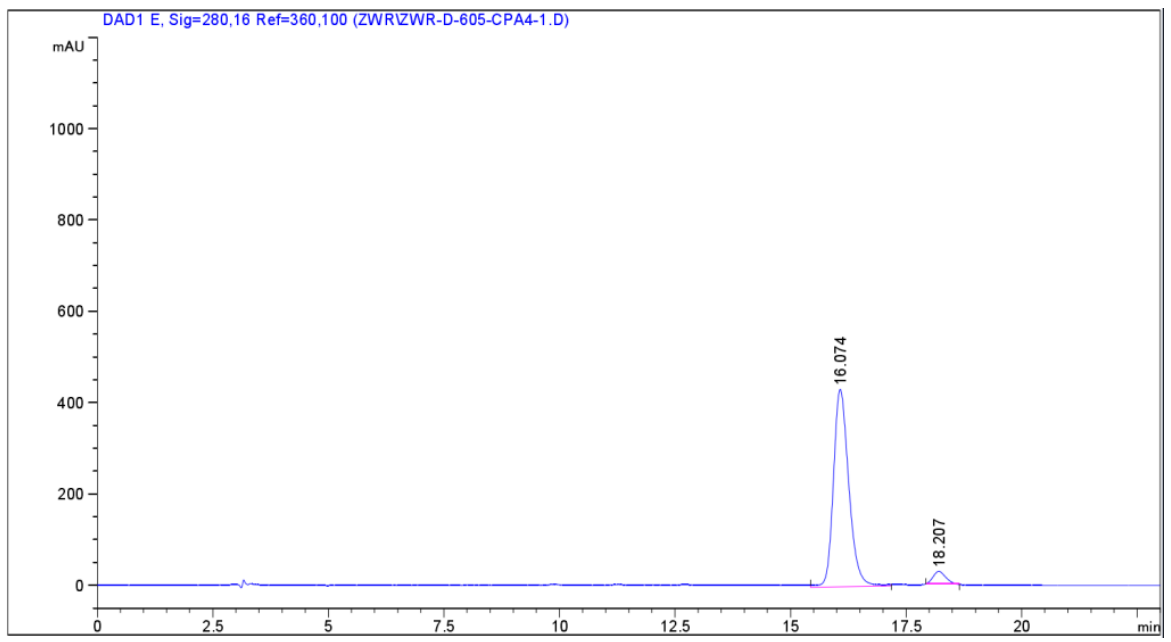

信号 1: DAD1 E, Sig=280,16 Ref=360,100

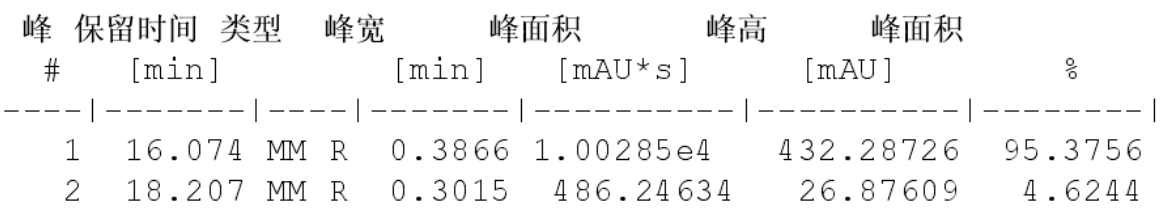




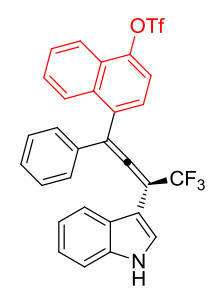

16

\section{HPLC spectrum of the racemate}

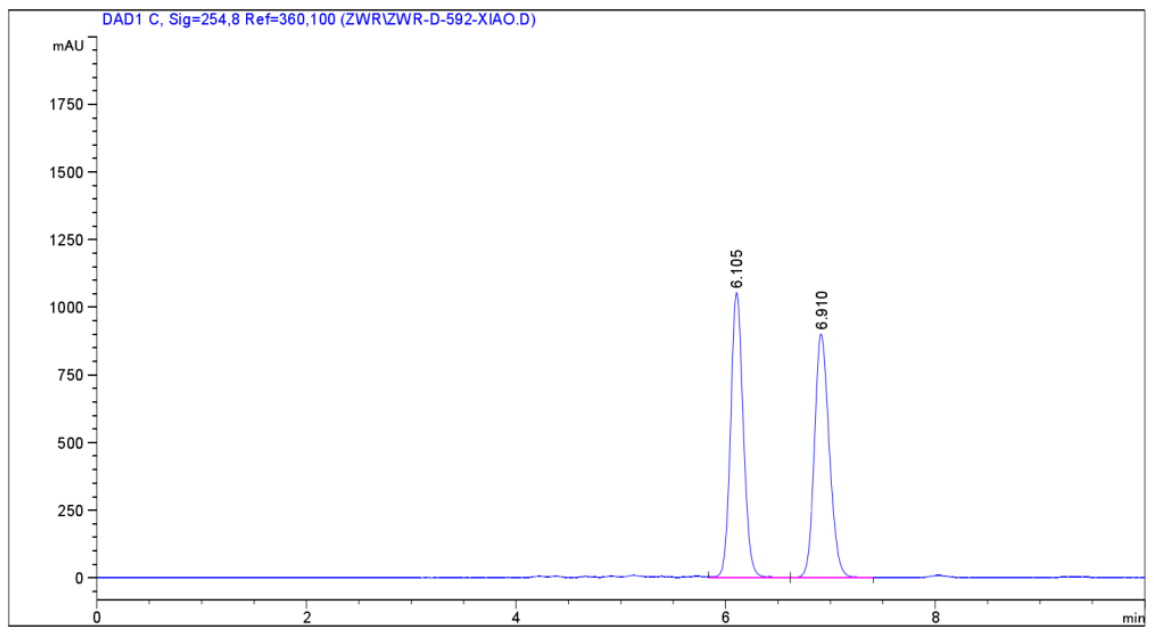

信号 1: DAD1 C, Sig=254,8 Ref=360,100

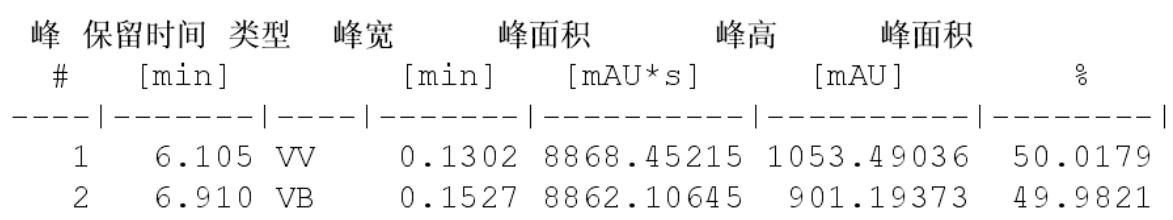

\section{HPLC spectrum of the chiral compound}

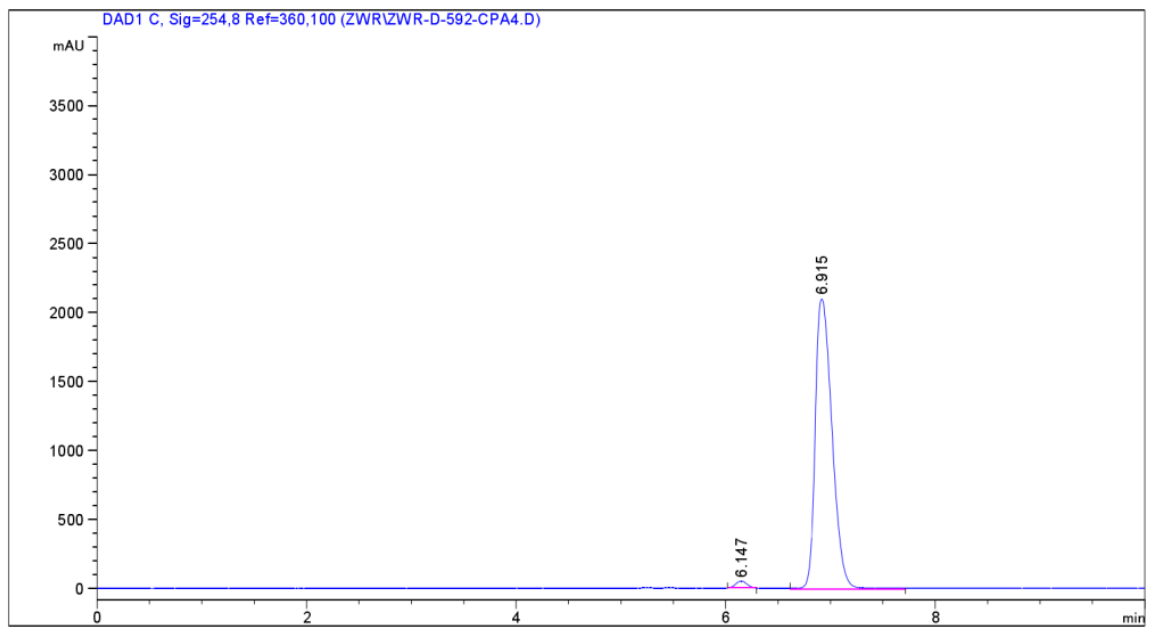

信号 1：DAD1 C， Sig=254,8 Ref=360,100

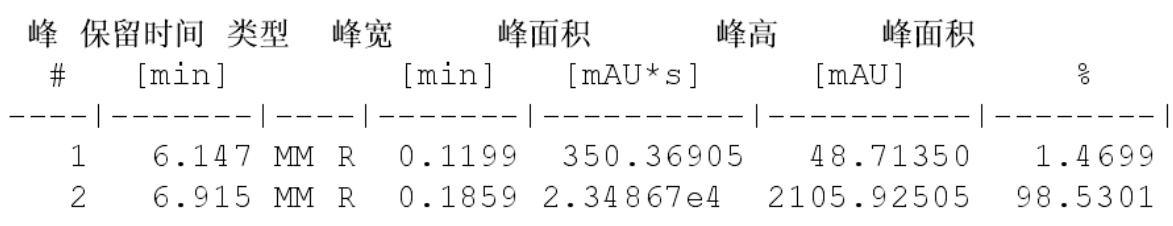




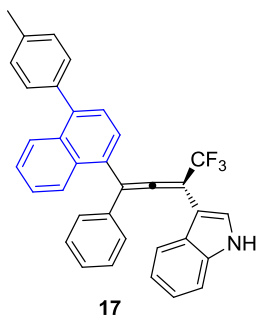

\section{HPLC spectrum of the racemate}

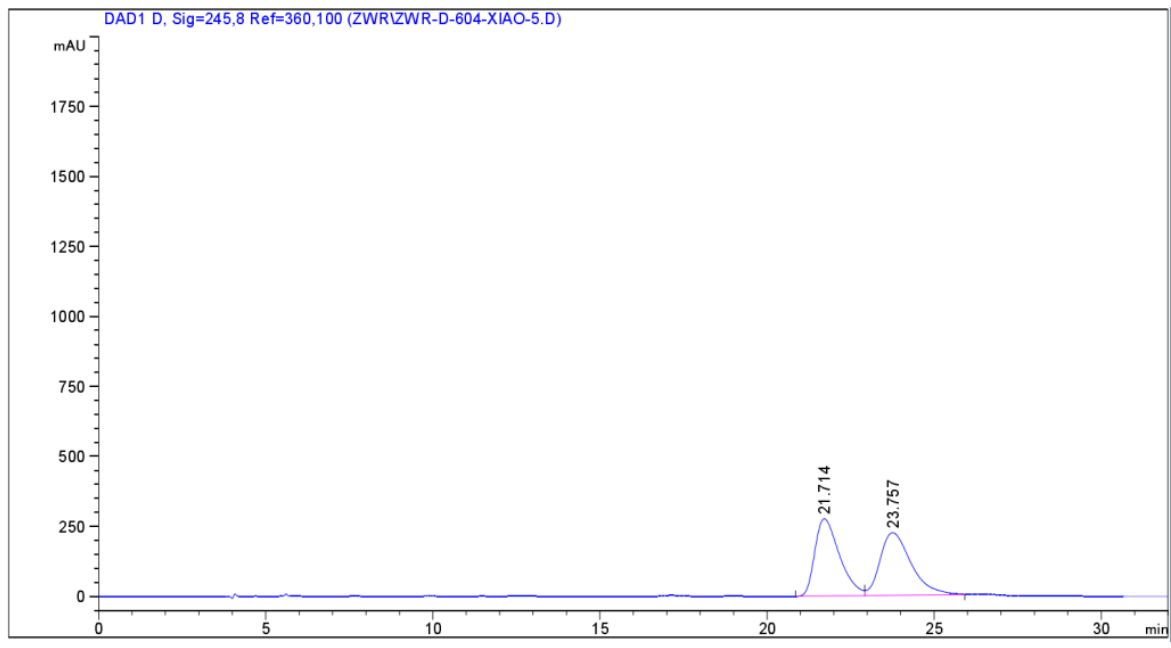

信号 1: DAD1 D, Sig=245,8 Ref=360,100

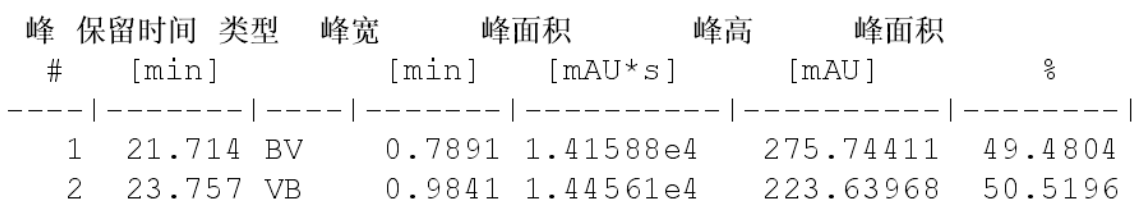

\section{HPLC spectrum of the chiral compound}

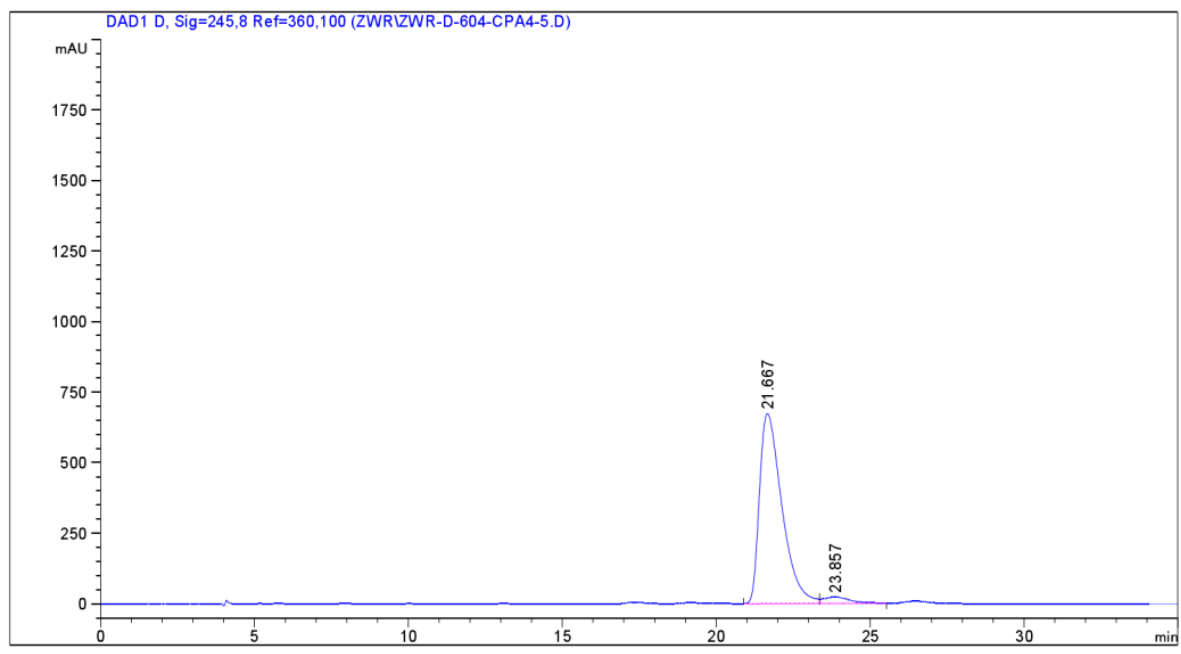

信号 1: DAD1 D， Sig=245,8 Ref $=360,100$

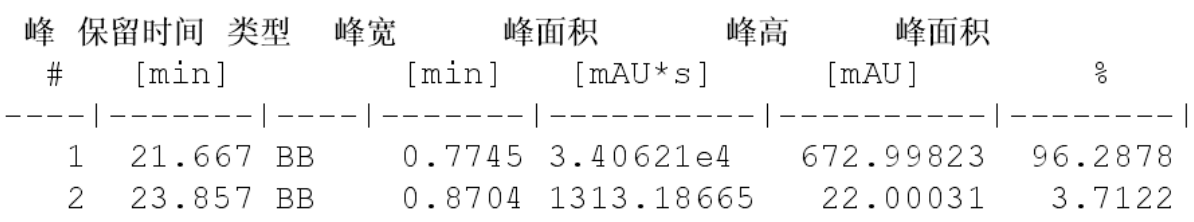


